University of Tennessee Health Science Center

UTHSC Digital Commons

\title{
Mesiodistal Crown Size in Relation to the Risk and Severity of Malocclusion
}

Jon Robert Zang-Bodis

University of Tennessee Health Science Center

Follow this and additional works at: https://dc.uthsc.edu/dissertations

Part of the Orthodontics and Orthodontology Commons

\section{Recommended Citation}

Zang-Bodis, Jon Robert, "Mesiodistal Crown Size in Relation to the Risk and Severity of Malocclusion" (2009). Theses and Dissertations (ETD). Paper 316. http://dx.doi.org/10.21007/etd.cghs.2009.0369.

This Thesis is brought to you for free and open access by the College of Graduate Health Sciences at UTHSC Digital Commons. It has been accepted for inclusion in Theses and Dissertations (ETD) by an authorized administrator of UTHSC Digital Commons. For more information, please contact jwelch30@uthsc.edu. 


\title{
Mesiodistal Crown Size in Relation to the Risk and Severity of Malocclusion
}

\begin{abstract}
From among numerous potential causes of dental malocclusion, tooth size has been implicated as one factor. Prior studies show that mesiodistally larger teeth increase the risk of malocclusion. The present report extends our understanding by testing for graded responses between crown size and the extent of dental malocclusion. Maximum mesiodistal crown dimensions of all 14 permanent tooth types (excluding third molars) were measured in 207 American white adolescents (routine orthodontic patients), and 10 measures of malocclusion (e.g., rotations, displacements, spacing) were recorded. Analysis of covariance (controlling for sexual dimorphism in tooth size) disclosed (1) significant positive associations between crown size and measures of crowding and (2) significant negative associations between crown size and measures of spacing. Of note, significant associations are widespread, involving all tooth types, both those emerging early and late. This systemic effect seems due to the intercorrelations among tooth dimensions and to the cumulative effects of crown sizes summed across multiple teeth-and this is borne out by multivariate models. Overall, tooth size probably is not commonly the paramount cause of malocclusion, but it is a readily documented influence, and its importance probably is increasing due to secular increases of crown sizes in response to diminished morbidity and improved nutrition.
\end{abstract}

\section{Document Type}

Thesis

Degree Name

Master of Dental Science (MDS)

Program

Orthodontics

Research Advisor

Edward F. Harris, Ph.D.

Keywords

Malocclusion, Tooth Size, Crowding, Mesiodistal, Crown Dimensions, Dental Cast

Subject Categories

Dentistry | Medicine and Health Sciences | Orthodontics and Orthodontology 


\author{
A Thesis \\ Presented for \\ The Graduate Studies Council \\ The University of Tennessee \\ Health Science Center
}

\begin{abstract}
In Partial Fulfillment
Of the Requirements for the Degree

Master of Dental Science

From The University of Tennessee
\end{abstract}

By

John Robert Zang-Bodis, D.D.S.

May 2009 
Copyright (c) 2009 by John Robert Zang-Bodis All rights reserved 


\section{ACKNOWLEGEMENTS}

Thanks should be given and gratitude expressed to the following:

Dr. Edward F. Harris, Research Advisor

Dr. Quinton C. Robinson, Committee Member

Dr. Richard A. Williams, Committee Member 


\begin{abstract}
From among numerous potential causes of dental malocclusion, tooth size has been implicated as one factor. Prior studies show that mesiodistally larger teeth increase the risk of malocclusion. The present report extends our understanding by testing for graded responses between crown size and the extent of dental malocclusion. Maximum mesiodistal crown dimensions of all 14 permanent tooth types (excluding third molars) were measured in 207 American white adolescents (routine orthodontic patients), and 10 measures of malocclusion (e.g., rotations, displacements, spacing) were recorded. Analysis of covariance (controlling for sexual dimorphism in tooth size) disclosed (1) significant positive associations between crown size and measures of crowding and (2) significant negative associations between crown size and measures of spacing. Of note, significant associations are widespread, involving all tooth types, both those emerging early and late. This systemic effect seems due to the intercorrelations among tooth dimensions and to the cumulative effects of crown sizes summed across multiple teeth - and this is borne out by multivariate models. Overall, tooth size probably is not commonly the paramount cause of malocclusion, but it is a readily documented influence, and its importance probably is increasing due to secular increases of crown sizes in response to diminished morbidity and improved nutrition.
\end{abstract}




\section{TABLE OF CONTENTS}

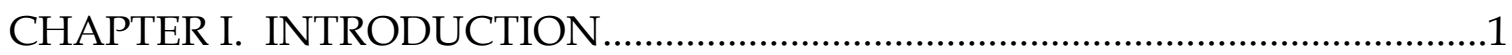

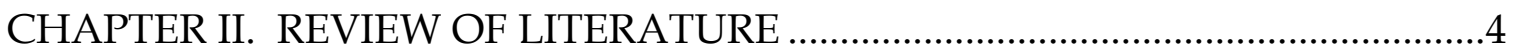

Buccal Segment Relationship ……………………......................................22

Bolton Tooth Size Discrepancies.........................................................................2

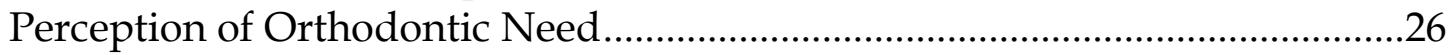

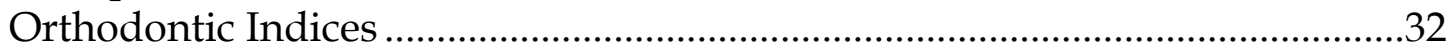

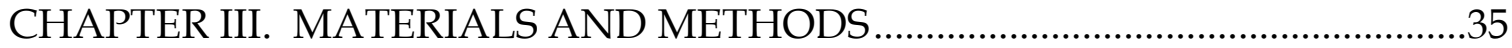

Nomenclature and Odontometric Method …………….........................................35

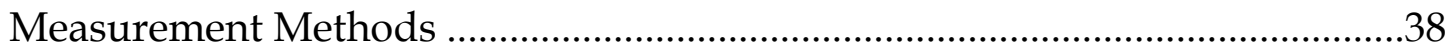

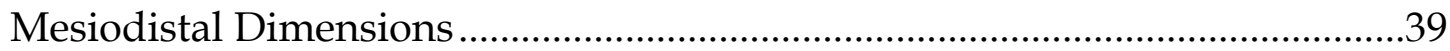

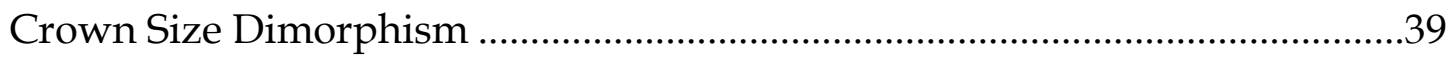

Quantifying Crowding ...................................................................................4

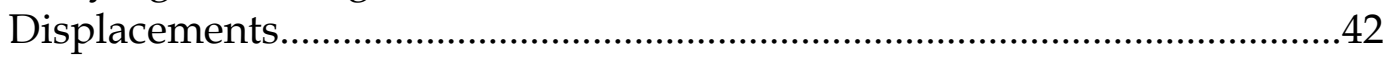

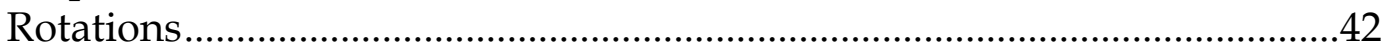

Mediolateral Overlap ..............................................................................42

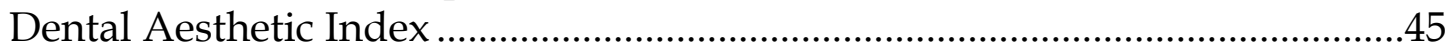

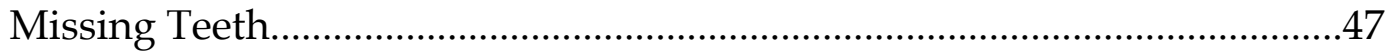

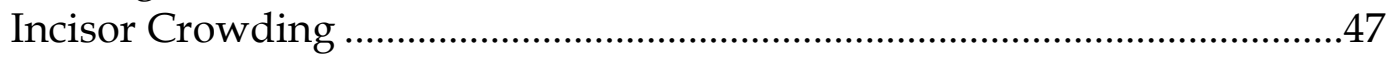

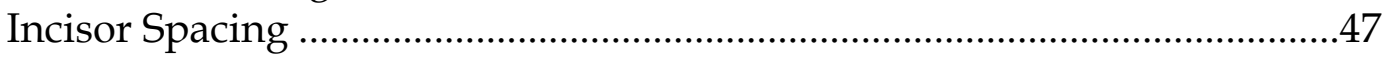

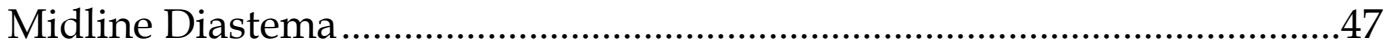

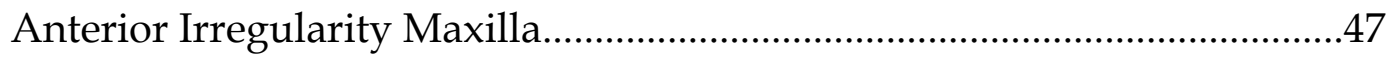

Anterior Irregularity Mandible .......................................................................51

Maxillary Overjet .......................................................................................51

Mandibular Overjet .......................................................................................

Anterior Openbite .........................................................................................51

Buccal Molar Relationship ...............................................................................51

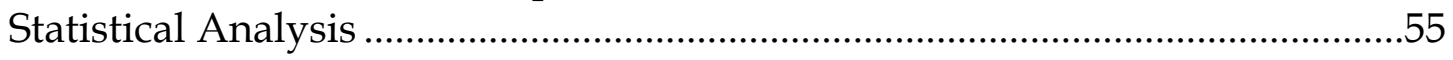

Statistical Models …………………………………......................................57

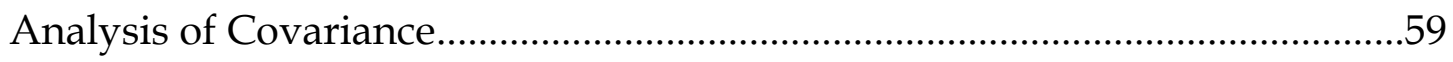

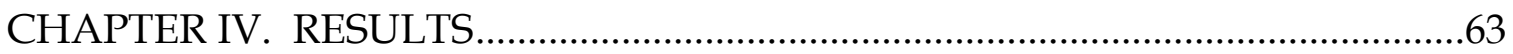

Maxillary Tooth Types ...................................................................................65

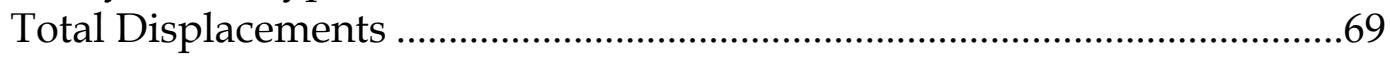

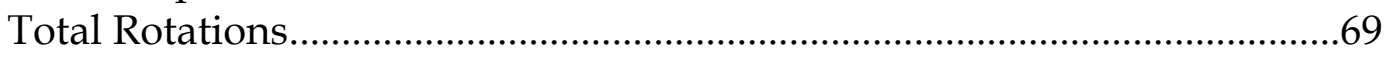

Total Overlapping ........................................................................................69

Total Displacements, Rotations, and Overlapping ........................................75

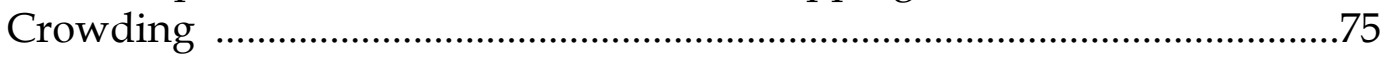

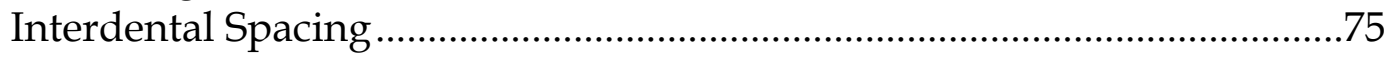




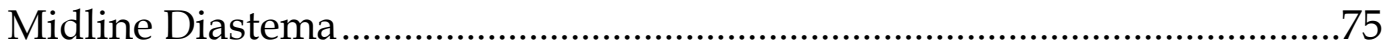

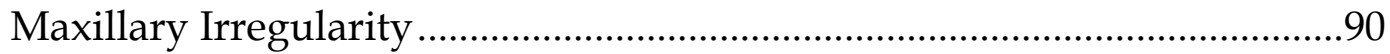

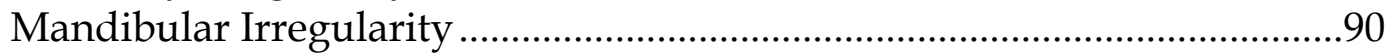

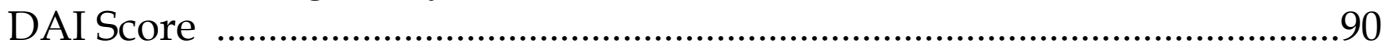

Mandibular Tooth Types ..................................................................................96

Total Displacements ..................................................................................96

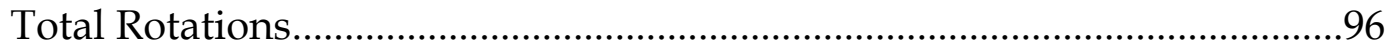

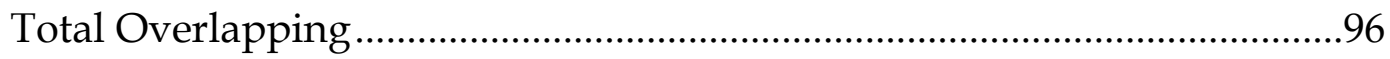

Total Displacements, Rotations, and Overlapping …....................................96

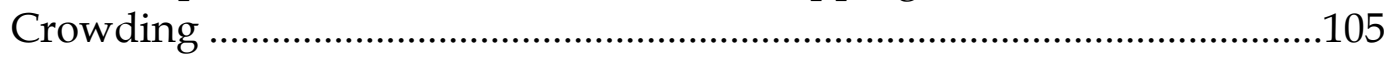

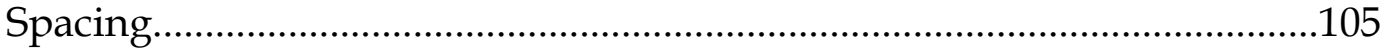

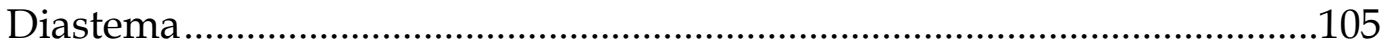

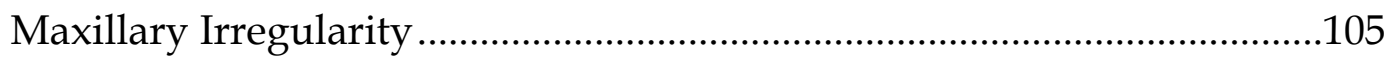

Mandibular Irregularity ….......................................................................116

Maxillary Overjet ...............................................................................116

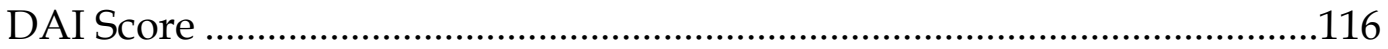

Predicting Crown Size from Malocclusion ..................................................116

Maxillary Crown Dimensions .......................................................................126

Mandibular Crown Dimensions .......................................................................133

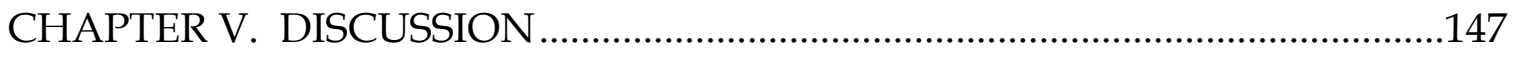

CHAPTER VI. SUMMARY AND CONCLUSIONS.............................................150

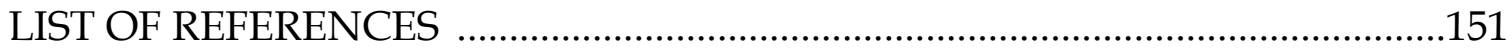

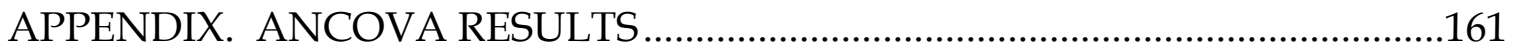

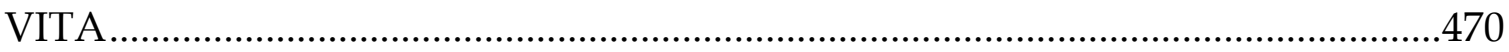




\section{LIST OF TABLES}

Table

1 Descriptive statistics of mesiodistal crown dimensions, by sex, and tests for sexual dimorphism.

2 Listing of the variables scored in the Dental Aesthetic Index......................56

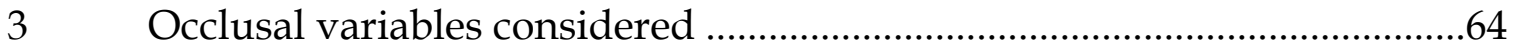

$4 \quad$ P-Values associated with test (F-Ratios) of the regression of crowding on tooth size in the maxilla

$5 \quad$ P-Values associated with test (F-Ratios) of the regression of crowding on tooth size in the mandible

6 Matrix of Pearson correlation coefficients for all 14 mesiodistal crown dimensions taken pairwise

$7 \quad$ Results of stepwise multiple regression analysis predicting summed mesiodistal widths of all 14 tooth types from measures of malocclusion

$8 \quad$ Results of stepwise multiple regression analysis predicting mesiodistal width of the maxillary central incisor from measures of malocclusion

$9 \quad$ Results of stepwise multiple regression analysis predicting mesiodistal width of the maxillary lateral incisor from measures of malocclusion

10 Results of stepwise multiple regression analysis predicting mesiodistal width of the maxillary canine from measures of malocclusion

11 Results of stepwise multiple regression analysis predicting mesiodistal width of the maxillary first premolar from measures of malocclusion 
12 Results of stepwise multiple regression analysis predicting mesiodistal width of the maxillary second premolar from measures of malocclusion

13 Results of stepwise multiple regression analysis predicting mesiodistal width of the maxillary first molar from measures of malocclusion.....

14 Results of stepwise multiple regression analysis predicting mesiodistal width of the maxillary second molar from measures of malocclusion

15 Results of stepwise multiple regression analysis predicting mesiodistal width of the mandibular central incisor from measures of malocclusion

16 Results of stepwise multiple regression analysis predicting mesiodistal width of the mandibular lateral incisor from measures of malocclusion

17 Results of stepwise multiple regression analysis predicting mesiodistal width of the mandibular canine from measures of malocclusion

18 Results of stepwise multiple regression analysis predicting mesiodistal width of the mandibular first premolar from measures of malocclusion

19 Results of stepwise multiple regression analysis predicting mesiodistal width of the mandibular second premolar from measures of malocclusion

20 Results of stepwise multiple regression analysis predicting mesiodistal width of the mandibular first molar from measures of malocclusion

21 Results of stepwise multiple regression analysis predicting mesiodistal width of the mandibular second molar from measures of malocclusion

22 Analysis of variance testing for a difference in summed tooth size among grades of spacing while controlling for sexual dimorphism 
A-1 ANCOVA results testing for associations between mesiodistal width of the maxillary central incisor and $\mathrm{U} 3$ displacements

A-2 ANCOVA results testing for associations between mesiodistal width of the maxillary central incisor and U2 displacements

A-3 ANCOVA results testing for associations between mesiodistal width of the maxillary central incisor and $\mathrm{U} 1$ displacements

A-4 ANCOVA results testing for associations between mesiodistal width of the maxillary central incisor and L3 displacements.....

A-5 ANCOVA results testing for associations between mesiodistal width of the maxillary central incisor and L2 displacements

A-6 ANCOVA results testing for associations between mesiodistal width of the maxillary central incisor and L1 displacements

A-7 ANCOVA results testing for associations between mesiodistal width of the maxillary central incisor and $\mathrm{U} 3$ rotations

A-8 ANCOVA results testing for associations between mesiodistal width of the maxillary central incisor and $\mathrm{U} 2$ rotations

A-9 ANCOVA results testing for associations between mesiodistal width of the maxillary central incisor and $\mathrm{U} 1$ rotations

A-10 ANCOVA results testing for associations between mesiodistal width of the maxillary central incisor and L3 rotations.

A-11 ANCOVA results testing for associations between mesiodistal width of the maxillary central incisor and L2 rotations

A-12 ANCOVA results testing for associations between mesiodistal width of the maxillary central incisor and L1 rotations

A-13 ANCOVA results testing for associations between mesiodistal width of the maxillary central incisor and U2-U3 overlap.

A-14 ANCOVA results testing for associations between mesiodistal width of the maxillary central incisor and U1-U2 overlap. 
A-15 ANCOVA results testing for associations between mesiodistal width of the maxillary central incisor and L2-L3 overlap.

A-16 ANCOVA results testing for associations between mesiodistal width of the maxillary central incisor and L1-L2 overlap

A-17 ANCOVA results testing for associations between mesiodistal width of the maxillary central incisor and total displacements

A-18 ANCOVA results testing for associations between mesiodistal width of the maxillary central incisor and total rotations

A-19 ANCOVA results testing for associations between mesiodistal width of the maxillary central incisor and maxillary R1-L1 overlap

A-20 ANCOVA results testing for associations between mesiodistal width of the maxillary central incisor and mandibular R1-L1 overlap

A-21 ANCOVA results testing for associations between mesiodistal width of the maxillary central incisor and total overlapping.

A-22 ANCOVA results testing for associations between mesiodistal width of the maxillary central incisor and total displacements, rotations, and overlapping

A-23 ANCOVA results testing for associations between mesiodistal width of the maxillary central incisor and crowding.

A-24 ANCOVA results testing for associations between mesiodistal width of the maxillary central incisor and spacing

A-25 ANCOVA results testing for associations between mesiodistal width of the maxillary central incisor and diastema.

A-26 ANCOVA results testing for associations between mesiodistal width of the maxillary central incisor and maxillary incisor irregularity

A-27 ANCOVA results testing for associations between mesiodistal width of the maxillary central incisor and mandibular incisor irregularity .181 
A-28 ANCOVA results testing for associations between mesiodistal width of the maxillary central incisor and maxillary overjet.

A-29 ANCOVA results testing for associations between mesiodistal width of the maxillary central incisor and mandibular overjet......

A-30 ANCOVA results testing for associations between mesiodistal width of the maxillary central incisor and openbite

A-31 ANCOVA results testing for associations between mesiodistal width of the maxillary central incisor and anteroposterior relationship

A-32 ANCOVA results testing for associations between mesiodistal width of the maxillary central incisor and DAI score

A-33 ANCOVA results testing for associations between mesiodistal width of the maxillary lateral incisor and U3 displacements

A-34 ANCOVA results testing for associations between mesiodistal width of the maxillary lateral incisor and U2 displacements

A-35 ANCOVA results testing for associations between mesiodistal width of the maxillary lateral incisor and U1 displacements

A-36 ANCOVA results testing for associations between mesiodistal width of the maxillary lateral incisor and L3 displacements

A-37 ANCOVA results testing for associations between mesiodistal width of the maxillary lateral incisor and L2 displacements.

A-38 ANCOVA results testing for associations between mesiodistal width of the maxillary lateral incisor and L1 displacements.

A-39 ANCOVA results testing for associations between mesiodistal width of the maxillary lateral incisor and $\mathrm{U} 3$ rotations.

A-40 ANCOVA results testing for associations between mesiodistal width of the maxillary lateral incisor and $\mathrm{U} 2$ rotations

A-41 ANCOVA results testing for associations between mesiodistal width of the maxillary lateral incisor and $\mathrm{U} 1$ rotations 
A-42 ANCOVA results testing for associations between mesiodistal width of the maxillary lateral incisor and L3 rotations.

A-43 ANCOVA results testing for associations between mesiodistal width of the maxillary lateral incisor and L2 rotations

A-44 ANCOVA results testing for associations between mesiodistal width of the maxillary lateral incisor and L1 rotations

A-45 ANCOVA results testing for associations between mesiodistal width of the maxillary lateral incisor and U2-U3 overlap.

A-46 ANCOVA results testing for associations between mesiodistal width of the maxillary lateral incisor and U1-U2 overlap.

A-47 ANCOVA results testing for associations between mesiodistal width of the maxillary lateral incisor and L2-L3 overlap

A-48 ANCOVA results testing for associations between mesiodistal width of the maxillary lateral incisor and L1-L2 overlap

A-49 ANCOVA results testing for associations between mesiodistal width of the maxillary lateral incisor and total displacements

A-50 ANCOVA results testing for associations between mesiodistal width of the maxillary lateral incisor and total rotations

A-51 ANCOVA results testing for associations between mesiodistal width of the maxillary lateral incisor and maxillary R1-L1 overlap. 200

A-52 ANCOVA results testing for associations between mesiodistal width of the maxillary lateral incisor and mandibular R1-L1 overlap

A-53 ANCOVA results testing for associations between mesiodistal width of the maxillary lateral incisor and total overlapping

A-54 ANCOVA results testing for associations between mesiodistal width of the maxillary lateral incisor and total displacements, rotations, and overlapping. 
A-55 ANCOVA results testing for associations between mesiodistal width of the maxillary lateral incisor and crowding.

A-56 ANCOVA results testing for associations between mesiodistal width of the maxillary lateral incisor and spacing

A-57 ANCOVA results testing for associations between mesiodistal width of the maxillary lateral incisor and diastema.

A-58 ANCOVA results testing for associations between mesiodistal width of the maxillary lateral incisor and maxillary incisor irregularity

A-59 ANCOVA results testing for associations between mesiodistal width of the maxillary lateral incisor and mandibular incisor irregularity.

A-60 ANCOVA results testing for associations between mesiodistal width of the maxillary lateral incisor and maxillary overjet.

A-61 ANCOVA results testing for associations between mesiodistal width of the maxillary lateral incisor and mandibular overjet. .204

A-62 ANCOVA results testing for associations between mesiodistal width of the maxillary lateral incisor and openbite .204

A-63 ANCOVA results testing for associations between mesiodistal width of the maxillary lateral incisor and anteroposterior relationship

A-64 ANCOVA results testing for associations between mesiodistal width of the maxillary lateral incisor and DAI score .205

A-65 ANCOVA results testing for associations between mesiodistal width of the maxillary canine and U3 displacements .206

A-66 ANCOVA results testing for associations between mesiodistal width of the maxillary canine and $\mathrm{U} 2$ displacements 207

A-67 ANCOVA results testing for associations between mesiodistal width of the maxillary canine and U1 displacements 
A-68 ANCOVA results testing for associations between mesiodistal width of the maxillary canine and L3 displacements

A-69 ANCOVA results testing for associations between mesiodistal width of the maxillary canine and L2 displacements

A-70 ANCOVA results testing for associations between mesiodistal width of the maxillary canine and L1 displacements

A-71 ANCOVA results testing for associations between mesiodistal width of the maxillary canine and $\mathrm{U} 3$ rotations

A-72 ANCOVA results testing for associations between mesiodistal width of the maxillary canine and $\mathrm{U} 2$ rotations

A-73 ANCOVA results testing for associations between mesiodistal width of the maxillary canine and $\mathrm{U} 1$ rotations

A-74 ANCOVA results testing for associations between mesiodistal width of the maxillary canine and L3 rotations

A-75 ANCOVA results testing for associations between mesiodistal width of the maxillary canine and L2 rotations

A-76 ANCOVA results testing for associations between mesiodistal width of the maxillary canine and L1 rotations

A-77 ANCOVA results testing for associations between mesiodistal width of the maxillary canine and U2-U3 overlap

A-78 ANCOVA results testing for associations between mesiodistal width of the maxillary canine and U1-U2 overlap

A-79 ANCOVA results testing for associations between mesiodistal width of the maxillary canine and L2-L3 overlap .220

A-80 ANCOVA results testing for associations between mesiodistal width of the maxillary canine and L1-L2 overlap

A-81 ANCOVA results testing for associations between mesiodistal width of the maxillary canine and total displacements 
A-82 ANCOVA results testing for associations between mesiodistal width of the maxillary canine and total rotations.

A-83 ANCOVA results testing for associations between mesiodistal width of the maxillary canine and maxillary R1-L1 overlap

A-84 ANCOVA results testing for associations between mesiodistal width of the maxillary canine and mandibular R1-L1 overlap

A-85 ANCOVA results testing for associations between mesiodistal width of the maxillary canine and total overlapping.

A-86 ANCOVA results testing for associations between mesiodistal width of the maxillary canine and total displacements, rotations and overlapping. .223

A-87 ANCOVA results testing for associations between mesiodistal width of the maxillary canine and crowding.....

A-88 ANCOVA results testing for associations between mesiodistal width of the maxillary canine and spacing.

A-89 ANCOVA results testing for associations between mesiodistal width of the maxillary canine and diastema

A-90 ANCOVA results testing for associations between mesiodistal width of the maxillary canine and maxillary incisor irregularity .225

A-91 ANCOVA results testing for associations between mesiodistal width of the maxillary canine and mandibular incisor irregularity ........225

A-92 ANCOVA results testing for associations between mesiodistal width of the maxillary canine and maxillary overjet 225

A-93 ANCOVA results testing for associations between mesiodistal width of the maxillary canine and mandibular overjet

A-94 ANCOVA results testing for associations between mesiodistal width of the maxillary canine and openbite.

A-95 ANCOVA results testing for associations between mesiodistal width of the maxillary canine and anteroposterior relationship .226 
A-96 ANCOVA results testing for associations between mesiodistal width of the maxillary canine and DAI score.

A-97 ANCOVA results testing for associations between mesiodistal width of the maxillary first premolar and U3 displacements.

A-98 ANCOVA results testing for associations between mesiodistal width of the maxillary first premolar and $\mathrm{U} 2$ displacements.

A-99 ANCOVA results testing for associations between mesiodistal width of the maxillary first premolar and U1 displacements.

A-100 ANCOVA results testing for associations between mesiodistal width of the maxillary first premolar and L3 displacements

A-101 ANCOVA results testing for associations between mesiodistal width of the maxillary first premolar and L2 displacements

A-102 ANCOVA results testing for associations between mesiodistal width of the maxillary first premolar and L1 displacements. .233

A-103 ANCOVA results testing for associations between mesiodistal width of the maxillary first premolar and $\mathrm{U} 3$ rotations

A-104 ANCOVA results testing for associations between mesiodistal width of the maxillary first premolar and $\mathrm{U} 2$ rotations

A-105 ANCOVA results testing for associations between mesiodistal width of the maxillary first premolar and $\mathrm{U} 1$ rotations .236

A-106 ANCOVA results testing for associations between mesiodistal width of the maxillary first premolar and L3 rotations

A-107 ANCOVA results testing for associations between mesiodistal width of the maxillary first premolar and L2 rotations .238

A-108 ANCOVA results testing for associations between mesiodistal width of the maxillary first premolar and L1 rotations

A-109 ANCOVA results testing for associations between mesiodistal width of the maxillary first premolar and U2-U3 overlap 
A-110 ANCOVA results testing for associations between mesiodistal width of the maxillary first premolar and U1-U2 overlap

A-111 ANCOVA results testing for associations between mesiodistal width of the maxillary first premolar and L2-L3 overlap

A-112 ANCOVA results testing for associations between mesiodistal width of the maxillary first premolar and L1-L2 overlap

A-113 ANCOVA results testing for associations between mesiodistal width of the maxillary first premolar and total displacements

A-114 ANCOVA results testing for associations between mesiodistal width of the maxillary first premolar and total rotations

A-115 ANCOVA results testing for associations between mesiodistal width of the maxillary first premolar and maxillary R1-L1 overlap .......244

A-116 ANCOVA results testing for associations between mesiodistal width of the maxillary first premolar and mandibular R1-L1 overlap.

A-117 ANCOVA results testing for associations between mesiodistal width of the maxillary first premolar and total overlapping

A-118 ANCOVA results testing for associations between mesiodistal width of the maxillary first premolar and total displacements, rotations, and overlapping.

A-119 ANCOVA results testing for associations between mesiodistal width of the maxillary first premolar and crowding

A-120 ANCOVA results testing for associations between mesiodistal width of the maxillary first premolar and spacing

A-121 ANCOVA results testing for associations between mesiodistal width of the maxillary first premolar and diastema

A-122 ANCOVA results testing for associations between mesiodistal width of the maxillary first premolar and maxillary incisor irregularity. 
A-123 ANCOVA results testing for associations between mesiodistal width of the maxillary first premolar and mandibular incisor irregularity.....

A-124 ANCOVA results testing for associations between mesiodistal width of the maxillary first premolar and maxillary overjet

A-125 ANCOVA results testing for associations between mesiodistal width of the maxillary first premolar and mandibular overjet .248

A-126 ANCOVA results testing for associations between mesiodistal width of the maxillary first premolar and openbite

A-127 ANCOVA results testing for associations between mesiodistal width of the maxillary first premolar and anteroposterior relationship

A-128 ANCOVA results testing for associations between mesiodistal width of the maxillary first premolar and DAI score.

A-129 ANCOVA results testing for associations between mesiodistal width of the maxillary second premolar and U3 displacements .250

A-130 ANCOVA results testing for associations between mesiodistal width of the maxillary second premolar and $\mathrm{U} 2$ displacements .251

A-131 ANCOVA results testing for associations between mesiodistal width of the maxillary second premolar and U1 displacements

A-132 ANCOVA results testing for associations between mesiodistal width of the maxillary second premolar and L3 displacements

A-133 ANCOVA results testing for associations between mesiodistal width of the maxillary second premolar and L2 displacements

A-134 ANCOVA results testing for associations between mesiodistal width of the maxillary second premolar and L1 displacements .255

A-135 ANCOVA results testing for associations between mesiodistal width of the maxillary second premolar and $\mathrm{U} 3$ rotations .256

A-136 ANCOVA results testing for associations between mesiodistal width of the maxillary second premolar and $\mathrm{U} 2$ rotations .257 
A-137 ANCOVA results testing for associations between mesiodistal width of the maxillary second premolar and $\mathrm{U} 1$ rotations

A-138 ANCOVA results testing for associations between mesiodistal width of the maxillary second premolar and L3 rotations

A-139 ANCOVA results testing for associations between mesiodistal width of the maxillary second premolar and L2 rotations

A-140 ANCOVA results testing for associations between mesiodistal width of the maxillary second premolar and L1 rotations

A-141 ANCOVA results testing for associations between mesiodistal width of the maxillary second premolar and U2-U3 overlap .262

A-142 ANCOVA results testing for associations between mesiodistal width of the maxillary second premolar and U1-U2 overlap .263

A-143 ANCOVA results testing for associations between mesiodistal width of the maxillary second premolar and L2-L3 overlap

A-144 ANCOVA results testing for associations between mesiodistal width of the maxillary second premolar and L1-L2 overlap

A-145 ANCOVA results testing for associations between mesiodistal width of the maxillary second premolar and total displacements.

A-146 ANCOVA results testing for associations between mesiodistal width of the maxillary second premolar and total rotations .266

A-147 ANCOVA results testing for associations between mesiodistal width of the maxillary second premolar and maxillary R1-L1 overlap 266

A-148 ANCOVA results testing for associations between mesiodistal width of the maxillary second premolar and mandibular R1-L1 overlap

A-149 ANCOVA results testing for associations between mesiodistal width of the maxillary second premolar and total overlapping .267 
A-150 ANCOVA results testing for associations between mesiodistal width of the maxillary second premolar and total displacements, rotations, and overlapping......

A-151 ANCOVA results testing for associations between mesiodistal width of the maxillary second premolar and crowding

A-152 ANCOVA results testing for associations between mesiodistal width of the maxillary second premolar and spacing .268

A-153 ANCOVA results testing for associations between mesiodistal width of the maxillary second premolar and diastema. .268

A-154 ANCOVA results testing for associations between mesiodistal width of the maxillary second premolar and maxillary incisor irregularity.

A-155 ANCOVA results testing for associations between mesiodistal width of the maxillary second premolar and mandibular incisor irregularity

A-156 ANCOVA results testing for associations between mesiodistal width of the maxillary second premolar and maxillary overjet

A-157 ANCOVA results testing for associations between mesiodistal width of the maxillary second premolar and mandibular overjet

A-158 ANCOVA results testing for associations between mesiodistal width of the maxillary second premolar and openbite.

A-159 ANCOVA results testing for associations between mesiodistal width of the maxillary second premolar and anteroposterior relationship 270

A-160 ANCOVA results testing for associations between mesiodistal width of the maxillary second premolar and DAI score

A-161 ANCOVA results testing for associations between mesiodistal width of the maxillary first molar and U3 displacements.

A-162 ANCOVA results testing for associations between mesiodistal width of the maxillary first molar and U2 displacements 
A-163 ANCOVA results testing for associations between mesiodistal width of the maxillary first molar and U1 displacements

A-164 ANCOVA results testing for associations between mesiodistal width of the maxillary first molar and L3 displacements

A-165 ANCOVA results testing for associations between mesiodistal width of the maxillary first molar and L2 displacements

A-166 ANCOVA results testing for associations between mesiodistal width of the maxillary first molar and L1 displacements

A-167 ANCOVA results testing for associations between mesiodistal width of the maxillary first molar and U3 rotations .278

A168 ANCOVA results testing for associations between mesiodistal width of the maxillary first molar and $\mathrm{U} 2$ rotations

A-169 ANCOVA results testing for associations between mesiodistal width of the maxillary first molar and U1 rotations 280

A-170 ANCOVA results testing for associations between mesiodistal width of the maxillary first molar and L3 rotations

A-171 ANCOVA results testing for associations between mesiodistal width of the maxillary first molar and L2 rotations

A-172 ANCOVA results testing for associations between mesiodistal width of the maxillary first molar and L1 rotations

A-173 ANCOVA results testing for associations between mesiodistal width of the maxillary first molar and U2-U3 overlap .284

A-174 ANCOVA results testing for associations between mesiodistal width of the maxillary first molar and U1-U2 overlap .285

A-175 ANCOVA results testing for associations between mesiodistal width of the maxillary first molar and L2-L3 overlap .286

A-176 ANCOVA results testing for associations between mesiodistal width of the maxillary first molar and L1-L2 overlap .287 
A-177 ANCOVA results testing for associations between mesiodistal width of the maxillary first molar and total displacements. .288

A-178 ANCOVA results testing for associations between mesiodistal width of the maxillary first molar and total rotations

A-179 ANCOVA results testing for associations between mesiodistal width of the maxillary first molar and maxillary R1-L1 overlap

A-180 ANCOVA results testing for associations between mesiodistal width of the maxillary first molar and mandibular R1-L1 overlap

A-181 ANCOVA results testing for associations between mesiodistal width of the maxillary first molar and total overlapping.

A-182 ANCOVA results testing for associations between mesiodistal width of the maxillary first molar and total displacements, rotations, and overlapping.

A-183 ANCOVA results testing for associations between mesiodistal width of the maxillary first molar and crowding.

A-184 ANCOVA results testing for associations between mesiodistal width of the maxillary first molar and spacing.

A-185 ANCOVA results testing for associations between mesiodistal width of the maxillary first molar and diastema

A-186 ANCOVA results testing for associations between mesiodistal width of the maxillary first molar and maxillary incisor irregularity .....291

A-187 ANCOVA results testing for associations between mesiodistal width of the maxillary first molar and mandibular incisor irregularity

A-188 ANCOVA results testing for associations between mesiodistal width of the maxillary first molar and maxillary overjet

A-189 ANCOVA results testing for associations between mesiodistal width of the maxillary first molar and mandibular overjet

A-190 ANCOVA results testing for associations between mesiodistal width of the maxillary first molar and openbite .292 
A-191 ANCOVA results testing for associations between mesiodistal width of the maxillary first molar and anteroposterior relationship.......292

A-192 ANCOVA results testing for associations between mesiodistal width of the maxillary first molar and DAI score

A-193 ANCOVA results testing for associations between mesiodistal width of the maxillary second molar and U3 displacements

A-194 ANCOVA results testing for associations between mesiodistal width of the maxillary second molar and $\mathrm{U} 2$ displacements

A-195 ANCOVA results testing for associations between mesiodistal width of the maxillary second molar and U1 displacements .296

A-196 ANCOVA results testing for associations between mesiodistal width of the maxillary second molar and L3 displacements

A-197 ANCOVA results testing for associations between mesiodistal width of the maxillary second molar and L2 displacements. 298

A-198 ANCOVA results testing for associations between mesiodistal width of the maxillary second molar and L1 displacements.

A-199 ANCOVA results testing for associations between mesiodistal width of the maxillary second molar and U3 rotations.

A-200 ANCOVA results testing for associations between mesiodistal width of the maxillary second molar and $\mathrm{U} 2$ rotations

A-201 ANCOVA results testing for associations between mesiodistal width of the maxillary second molar and $\mathrm{U} 1$ rotations

A-202 ANCOVA results testing for associations between mesiodistal width of the maxillary second molar and L3 rotations 303

A-203 ANCOVA results testing for associations between mesiodistal width of the maxillary second molar and $\mathrm{L} 2$ rotations

A-204 ANCOVA results testing for associations between mesiodistal width of the maxillary second molar and L1 rotations. 
A-205 ANCOVA results testing for associations between mesiodistal width of the maxillary second molar and U2-U3 overlap. 306

A-206 ANCOVA results testing for associations between mesiodistal width of the maxillary second molar and U1-U2 overlap

A-207 ANCOVA results testing for associations between mesiodistal width of the maxillary second molar and L2-L3 overlap

A-208 ANCOVA results testing for associations between mesiodistal width of the maxillary second molar and L1-L2 overlap

A-209 ANCOVA results testing for associations between mesiodistal width of the maxillary second molar and total displacements.

A-210 ANCOVA results testing for associations between mesiodistal width of the maxillary second molar and total rotations

A-211 ANCOVA results testing for associations between mesiodistal width of the maxillary second molar and maxillary R1-L1 overlap

A-212 ANCOVA results testing for associations between mesiodistal width of the maxillary second molar and mandibular R1-L1 overlap

A-213 ANCOVA results testing for associations between mesiodistal width of the maxillary second molar and total overlapping

A-214 ANCOVA results testing for associations between mesiodistal width of the maxillary second molar and total displacements, rotations, and overlapping

A-215 ANCOVA results testing for associations between mesiodistal width of the maxillary second molar and crowding.

A-216 ANCOVA results testing for associations between mesiodistal width of the maxillary second molar and spacing

A-217 ANCOVA results testing for associations between mesiodistal width of the maxillary second molar and diastema 
A-218 ANCOVA results testing for associations between mesiodistal width of the maxillary second molar and maxillary incisor irregularity.....

A-219 ANCOVA results testing for associations between mesiodistal width of the maxillary second molar and mandibular incisor irregularity

A-220 ANCOVA results testing for associations between mesiodistal width of the maxillary second molar and maxillary overjet.

A-221 ANCOVA results testing for associations between mesiodistal width of the maxillary second molar and mandibular overjet.

A-222 ANCOVA results testing for associations between mesiodistal width of the maxillary second molar and openbite

A-223 ANCOVA results testing for associations between mesiodistal width of the maxillary second molar and anteroposterior relationship 314

A-224 ANCOVA results testing for associations between mesiodistal width of the maxillary second molar and DAI score

A-225 ANCOVA results testing for associations between mesiodistal width of the mandibular central incisor and U3 displacements

A-226 ANCOVA results testing for associations between mesiodistal width of the mandibular central incisor and U2 displacements

A-227 ANCOVA results testing for associations between mesiodistal width of the mandibular central incisor and U1 displacements

A-228 ANCOVA results testing for associations between mesiodistal width of the mandibular central incisor and L3 displacements

A-229 ANCOVA results testing for associations between mesiodistal width of the mandibular central incisor and L2 displacements

A-230 ANCOVA results testing for associations between mesiodistal width of the mandibular central incisor and L1 displacements 
A-231 ANCOVA results testing for associations between mesiodistal width of the mandibular central incisor and U3 rotations

A-232 ANCOVA results testing for associations between mesiodistal width of the mandibular central incisor and $\mathrm{U} 2$ rotations

A-233 ANCOVA results testing for associations between mesiodistal width of the mandibular central incisor and U1 rotations

A-234 ANCOVA results testing for associations between mesiodistal width of the mandibular central incisor and L3 rotations.

A-235 ANCOVA results testing for associations between mesiodistal width of the mandibular central incisor and L2 rotations .326

A-236 ANCOVA results testing for associations between mesiodistal width of the mandibular central incisor and L1 rotations.

A-237 ANCOVA results testing for associations between mesiodistal width of the mandibular central incisor and U2-U3 overlap. 328

A-238 ANCOVA results testing for associations between mesiodistal width the mandibular central incisor and U1-U2 overlap

A-239 ANCOVA results testing for associations between mesiodistal width of the mandibular central incisor and L2-L3 overlap.

A-240 ANCOVA results testing for associations between mesiodistal width of the mandibular central incisor and L1-L2 overlap.

A-241 ANCOVA results testing for associations between mesiodistal width of the mandibular central incisor and total displacements

A-242 ANCOVA results testing for associations between mesiodistal width of the mandibular central incisor and total rotations .332

A-243 ANCOVA results testing for associations between mesiodistal width of the mandibular central incisor and maxillary R1-L1 overlap.

A-244 ANCOVA results testing for associations between mesiodistal width of the mandibular central incisor and mandibular R1-L1 overlap. .333 
A-245 ANCOVA results testing for associations between mesiodistal width of the mandibular central incisor and total overlapping. .333

A-246 ANCOVA results testing for associations between mesiodistal width of the mandibular central incisor and total displacements, rotations, and overlapping

A-247 ANCOVA results testing for associations between mesiodistal width of the mandibular central incisor and crowding. .334

A-248 ANCOVA results testing for associations between mesiodistal width of the mandibular central incisor and spacing .334

A-249 ANCOVA results testing for associations between mesiodistal width of the mandibular central incisor and diastema

A-250 ANCOVA results testing for associations between mesiodistal width of the mandibular central incisor and maxillary incisor irregularity

A-251 ANCOVA results testing for associations between mesiodistal width of the mandibular central incisor and mandibular incisor irregularity.

A-252 ANCOVA results testing for associations between mesiodistal width of the mandibular central incisor and maxillary overjet

A-253 ANCOVA results testing for associations between mesiodistal width of the mandibular central incisor and mandibular overjet.

A-254 ANCOVA results testing for associations between mesiodistal width of the mandibular central incisor and openbite .336

A-255 ANCOVA results testing for associations between mesiodistal width of the mandibular central incisor and anteroposterior relationship.

A-256 ANCOVA results testing for associations between mesiodistal width of the mandibular central incisor and DAI score

A-257 ANCOVA results testing for associations between mesiodistal width of the mandibular lateral incisor and U3 displacements 
A-258 ANCOVA results testing for associations between mesiodistal width of the mandibular lateral incisor and U2 displacements

A-259 ANCOVA results testing for associations between mesiodistal width of the mandibular lateral incisor and U1 displacements

A-260 ANCOVA results testing for associations between mesiodistal width of the mandibular lateral incisor and L3 displacements.

A-261 ANCOVA results testing for associations between mesiodistal width of the mandibular lateral incisor and L2 displacements.

A-262 ANCOVA results testing for associations between mesiodistal width of the mandibular lateral incisor and L1 displacements.

A-263 ANCOVA results testing for associations between mesiodistal width of the mandibular lateral incisor and $\mathrm{U} 3$ rotations.

A-264 ANCOVA results testing for associations between mesiodistal width of the mandibular lateral incisor and $\mathrm{U} 2$ rotations.

A-265 ANCOVA results testing for associations between mesiodistal width of the mandibular lateral incisor and $\mathrm{U} 1$ rotations

A-266 ANCOVA results testing for associations between mesiodistal width of the mandibular lateral incisor and L3 rotations

A-267 ANCOVA results testing for associations between mesiodistal width of the mandibular lateral incisor and L2 rotations

A-268 ANCOVA results testing for associations between mesiodistal width of the mandibular lateral incisor and L1 rotations

A-269 ANCOVA results testing for associations between mesiodistal width of the mandibular lateral incisor and U2-U3 overlap. .350

A-270 ANCOVA results testing for associations between mesiodistal width of the mandibular lateral incisor and U1-U2 overlap.

A-271 ANCOVA results testing for associations between mesiodistal width of the mandibular lateral incisor and L2-L3 overlap. 
A-272 ANCOVA results testing for associations between mesiodistal width of the mandibular lateral incisor and L1-L2 overlap. .353

A-273 ANCOVA results testing for associations between mesiodistal width of the mandibular lateral incisor and total displacements

A-274 ANCOVA results testing for associations between mesiodistal width of the mandibular lateral incisor and total rotations.

A-275 ANCOVA results testing for associations between mesiodistal width of the mandibular lateral incisor and maxillary R1-L1 overlap. .354

A-276 ANCOVA results testing for associations between mesiodistal width of the mandibular lateral incisor and mandibular R1-L1 overlap

A-277 ANCOVA results testing for associations between mesiodistal width of the mandibular lateral incisor and total overlapping .355

A-278 ANCOVA results testing for associations between mesiodistal width of the mandibular lateral incisor and total displacements, rotations, and overlapping.

A-279 ANCOVA results testing for associations between mesiodistal width of the mandibular lateral incisor and crowding...

A-280 ANCOVA results testing for associations between mesiodistal width of the mandibular lateral incisor and spacing

A-281 ANCOVA results testing for associations between mesiodistal width of the mandibular lateral incisor and diastema .356

A-282 ANCOVA results testing for associations between mesiodistal width of the mandibular lateral incisor and maxillary incisor irregularity

A-283 ANCOVA results testing for associations between mesiodistal width of the mandibular lateral incisor and mandibular incisor irregularity .357

A-284 ANCOVA results testing for associations between mesiodistal width of the mandibular lateral incisor and maxillary overjet. .357 
A-285 ANCOVA results testing for associations between mesiodistal width of the mandibular lateral incisor and mandibular overjet

A-286 ANCOVA results testing for associations between mesiodistal width of the mandibular lateral incisor and openbite

A-287 ANCOVA results testing for associations between mesiodistal width of the mandibular lateral incisor and anteroposterior relationship .358

A-288 ANCOVA results testing for associations between mesiodistal width of the mandibular lateral incisor and DAI score.

A-289 ANCOVA results testing for associations between mesiodistal width of the mandibular canine and U3 displacements 360

A-290 ANCOVA results testing for associations between mesiodistal width of the mandibular canine and $\mathrm{U} 2$ displacements

A-291 ANCOVA results testing for associations between mesiodistal width of the mandibular canine and U1 displacements

A-292 ANCOVA results testing for associations between mesiodistal width of the mandibular canine and L3 displacements

A-293 ANCOVA results testing for associations between mesiodistal width of the mandibular canine and L2 displacements .364

A-294 ANCOVA results testing for associations between mesiodistal width of the mandibular canine and L1 displacements 365

A-295 ANCOVA results testing for associations between mesiodistal width of the mandibular canine and $\mathrm{U} 3$ rotations

A-296 ANCOVA results testing for associations between mesiodistal width of the mandibular canine and $\mathrm{U} 2$ rotations

A-297 ANCOVA results testing for associations between mesiodistal width of the mandibular canine and $\mathrm{U} 1$ rotations

A-298 ANCOVA results testing for associations between mesiodistal width of the mandibular canine and L3 rotations 
A-299 ANCOVA results testing for associations between mesiodistal width of the mandibular canine and $\mathrm{L} 2$ rotations

A-300 ANCOVA results testing for associations between mesiodistal width of the mandibular canine and L1 rotations

A-301 ANCOVA results testing for associations between mesiodistal width of the mandibular canine and U2-U3 overlap

A-302 ANCOVA results testing for associations between mesiodistal width of the mandibular canine and U1-U2 overlap

A-303 ANCOVA results testing for associations between mesiodistal width of the mandibular canine and L2-L3 overlap

A-304 ANCOVA results testing for associations between mesiodistal width of the mandibular canine and L1-L2 overlap. .375

A-305 ANCOVA results testing for associations between mesiodistal width of the mandibular canine and total displacements.

A-306 ANCOVA results testing for associations between mesiodistal width of the mandibular canine and total rotations

A-307 ANCOVA results testing for associations between mesiodistal width of the mandibular canine and maxillary R1-L1 overlap

A-308 ANCOVA results testing for associations between mesiodistal width of the mandibular canine and mandibular R1-L1 overlap

A-309 ANCOVA results testing for associations between mesiodistal width of the mandibular canine and total overlapping.

A-310 ANCOVA results testing for associations between mesiodistal width of the mandibular canine and total displacements, rotations and overlapping.

A-311 ANCOVA results testing for associations between mesiodistal width of the mandibular canine and crowding

A-312 ANCOVA results testing for associations between mesiodistal width of the mandibular canine and spacing. .378 
A-313 ANCOVA results testing for associations between mesiodistal width of the mandibular canine and diastema

A-314 ANCOVA results testing for associations between mesiodistal width of the mandibular canine and maxillary incisor irregularity

A-315 ANCOVA results testing for associations between mesiodistal width of the mandibular canine and mandibular incisor irregularity

A-316 ANCOVA results testing for associations between mesiodistal width of the mandibular canine and maxillary overjet

A-317 ANCOVA results testing for associations between mesiodistal width of the mandibular canine and mandibular overjet

A-318 ANCOVA results testing for associations between mesiodistal width of the mandibular canine and openbite.

A-319 ANCOVA results testing for associations between mesiodistal width of the mandibular canine and anteroposterior relationship.

A-320 ANCOVA results testing for associations between mesiodistal width of the mandibular canine and DAI score.

A-321 ANCOVA results testing for associations between mesiodistal width of the mandibular first premolar and U3 displacements

A-322 ANCOVA results testing for associations between mesiodistal width of the mandibular first premolar and U2 displacements

A-323 ANCOVA results testing for associations between mesiodistal width of the mandibular first premolar and U1 displacements

A-324 ANCOVA results testing for associations between mesiodistal width of the mandibular first premolar and L3 displacements.

A-325 ANCOVA results testing for associations between mesiodistal width of the mandibular first premolar and L2 displacements.

A-326 ANCOVA results testing for associations between mesiodistal width of the mandibular first premolar and L1 displacements. 
A-327 ANCOVA results testing for associations between mesiodistal width of the mandibular first premolar and $\mathrm{U} 3$ rotations 388

A-328 ANCOVA results testing for associations between mesiodistal width of the mandibular first premolar and U2 rotations

A-329 ANCOVA results testing for associations between mesiodistal width of the mandibular first premolar and U1 rotations

A-330 ANCOVA results testing for associations between mesiodistal width of the mandibular first premolar and L3 rotations

A-331 ANCOVA results testing for associations between mesiodistal width of the mandibular first premolar and L2 rotations

A-332 ANCOVA results testing for associations between mesiodistal width of the mandibular first premolar and L1 rotations

A-333 ANCOVA results testing for associations between mesiodistal width of the mandibular first premolar and U2-U3 overlap

A-334 ANCOVA results testing for associations between mesiodistal width of the mandibular first premolar and U1-U2 overlap

A-335 ANCOVA results testing for associations between mesiodistal width of the mandibular first premolar and L2-L3 overlap.

A-336 ANCOVA results testing for associations between mesiodistal width of the mandibular first premolar and L1-L2 overlap.

A-337 ANCOVA results testing for associations between mesiodistal width of the mandibular first premolar and total displacements .398

A-338 ANCOVA results testing for associations between mesiodistal width of the mandibular first premolar and total rotations. .398

A-339 ANCOVA results testing for associations between mesiodistal width of the mandibular first premolar and maxillary R1-L1 overlap.

A-340 ANCOVA results testing for associations between mesiodistal width of the mandibular first premolar and mandibular R1-L1 overlap. 
A-341 ANCOVA results testing for associations between mesiodistal width of the mandibular first premolar and total overlapping.

A-342 ANCOVA results testing for associations between mesiodistal width of the mandibular first premolar and total displacements, rotations, and overlapping

A-343 ANCOVA results testing for associations between mesiodistal width of the mandibular first premolar and crowding

A-344 ANCOVA results testing for associations between mesiodistal width of the mandibular first premolar and spacing.....

A-345 ANCOVA results testing for associations between mesiodistal width of the mandibular first premolar and diastema

A-346 ANCOVA results testing for associations between mesiodistal width of the mandibular first premolar and maxillary incisor irregularity

A-347 ANCOVA results testing for associations between mesiodistal width of the mandibular first premolar and mandibular incisor irregularity.

A-348 ANCOVA results testing for associations between mesiodistal width of the mandibular first premolar and maxillary overjet

A-349 ANCOVA results testing for associations between mesiodistal width of the mandibular first premolar and mandibular overjet

A-350 ANCOVA results testing for associations between mesiodistal width of the mandibular first premolar and openbite

A-351 ANCOVA results testing for associations between mesiodistal width of the mandibular first premolar and anteroposterior relationship

A-352 ANCOVA results testing for associations between mesiodistal width of the mandibular first premolar and DAI score.

A-353 ANCOVA results testing for associations between mesiodistal width of the mandibular second premolar and U3 displacements. 
A-354 ANCOVA results testing for associations between mesiodistal width of the mandibular second premolar and $\mathrm{U} 2$ displacements. .405

A-355 ANCOVA results testing for associations between mesiodistal width of the mandibular second premolar and U1 displacements..........406

A-356 ANCOVA results testing for associations between mesiodistal width of the mandibular second premolar and L3 displacements

A-357 ANCOVA results testing for associations between mesiodistal width of the mandibular second premolar and L2 displacements

A-358 ANCOVA results testing for associations between mesiodistal width of the mandibular second premolar and L1 displacements

A-359 ANCOVA results testing for associations between mesiodistal width of the mandibular second premolar and U3 rotations.

A-360 ANCOVA results testing for associations between mesiodistal width of the mandibular second premolar and $\mathrm{U} 2$ rotations.

A-361 ANCOVA results testing for associations between mesiodistal width of the mandibular second premolar and U1 rotations

A-362 ANCOVA results testing for associations between mesiodistal width of the mandibular second premolar and L3 rotations.

A-363 ANCOVA results testing for associations between mesiodistal width of the mandibular second premolar and L2 rotations.

A-364 ANCOVA results testing for associations between mesiodistal width of the mandibular second premolar and L1 rotations

A-365 ANCOVA results testing for associations between mesiodistal width of the mandibular second premolar and U2-U3 overlap

A-366 ANCOVA results testing for associations between mesiodistal width of the mandibular second premolar and U1-U2 overlap

A-367 ANCOVA results testing for associations between mesiodistal width of the mandibular second premolar and L2-L3 overlap 
A-368 ANCOVA results testing for associations between mesiodistal width of the mandibular second premolar and L1-L2 overlap

A-369 ANCOVA results testing for associations between mesiodistal width of the mandibular second premolar and total displacements.......420

A-370 ANCOVA results testing for associations between mesiodistal width of the mandibular second premolar and total rotations

A-371 ANCOVA results testing for associations between mesiodistal width of the mandibular second premolar and maxillary R1-L1 overlap.

A-372 ANCOVA results testing for associations between mesiodistal width of the mandibular second premolar and mandibular R1-L1 overlap

A-373 ANCOVA results testing for associations between mesiodistal width of the mandibular second premolar and total overlapping

A-374 ANCOVA results testing for associations between mesiodistal width of the mandibular second premolar and total displacements, rotations, and overlapping

A-375 ANCOVA results testing for associations between mesiodistal width of the mandibular second premolar and crowding

A-376 ANCOVA results testing for associations between mesiodistal width of the mandibular second premolar and spacing

A-377 ANCOVA results testing for associations between mesiodistal width of the mandibular second premolar and diastema

A-378 ANCOVA results testing for associations between mesiodistal width of the mandibular second premolar and maxillary incisor irregularity

A-379 ANCOVA results testing for associations between mesiodistal width of the mandibular second premolar and mandibular incisor irregularity 
A-380 ANCOVA results testing for associations between mesiodistal width of the mandibular second premolar and maxillary overjet

A-381 ANCOVA results testing for associations between mesiodistal width of the mandibular second premolar and mandibular overjet .......424

A-382 ANCOVA results testing for associations between mesiodistal width of the mandibular second premolar and openbite.

A-383 ANCOVA results testing for associations between mesiodistal width of the mandibular second premolar and anteroposterior relationship

A-384 ANCOVA results testing for associations between mesiodistal width of the mandibular second premolar and DAI score

A-385 ANCOVA results testing for associations between mesiodistal width of the mandibular first molar and U3 displacements.

A-386 ANCOVA results testing for associations between mesiodistal width of the mandibular first molar and $\mathrm{U} 2$ displacements.

A-387 ANCOVA results testing for associations between mesiodistal width of the mandibular first molar and $\mathrm{U} 1$ displacements.

A-388 ANCOVA results testing for associations between mesiodistal width of the mandibular first molar and L3 displacements

A-389 ANCOVA results testing for associations between mesiodistal width of the mandibular first molar and L2 displacements

A-390 ANCOVA results testing for associations between mesiodistal width of the mandibular first molar and L1 displacements

A-391 ANCOVA results testing for associations between mesiodistal width of the mandibular first molar and U3 rotations

A-392 ANCOVA results testing for associations between mesiodistal width of the mandibular first molar and $\mathrm{U} 2$ rotations

A-393 ANCOVA results testing for associations between mesiodistal width of the mandibular first molar and U1 rotations 
A-394 ANCOVA results testing for associations between mesiodistal width of the mandibular first molar and L3 rotations

A-395 ANCOVA results testing for associations between mesiodistal width of the mandibular first molar and L2 rotations

A-396 ANCOVA results testing for associations between mesiodistal width of the mandibular first molar and L1 rotations

A-397 ANCOVA results testing for associations between mesiodistal width of the mandibular first molar and U2-U3 overlap

A-398 ANCOVA results testing for associations between mesiodistal width of the mandibular first molar and U1-U2 overlap

A-399 ANCOVA results testing for associations between mesiodistal width of the mandibular first molar and L2-L3 overlap

A-400 ANCOVA results testing for associations between mesiodistal width of the mandibular first molar and L1-L2 overlap

A-401 ANCOVA results testing for associations between mesiodistal width of the mandibular first molar and total displacements.

A-402 ANCOVA results testing for associations between mesiodistal width of the mandibular first molar and total rotations

A-403 ANCOVA results testing for associations between mesiodistal width of the mandibular first molar and maxillary R1-L1 overlap

A-404 ANCOVA results testing for associations between mesiodistal width of the mandibular first molar and mandibular R1-L1 overlap

A-405 ANCOVA results testing for associations between mesiodistal width of the mandibular first molar and total overlapping.

A-406 ANCOVA results testing for associations between mesiodistal width of the mandibular first molar and total displacements, rotations, and overlapping

A-407 ANCOVA results testing for associations between mesiodistal width of the mandibular first molar and crowding.... 
A-408 ANCOVA results testing for associations between mesiodistal width of the mandibular first molar and spacing.

A-409 ANCOVA results testing for associations between mesiodistal width of the mandibular first molar and diastema

A-410 ANCOVA results testing for associations between mesiodistal width of the mandibular first molar and maxillary incisor irregularity

A-411 ANCOVA results testing for associations between mesiodistal width of the mandibular first molar and mandibular incisor irregularity......

A-412 ANCOVA results testing for associations between mesiodistal width of the mandibular first molar and maxillary overjet

A-413 ANCOVA results testing for associations between mesiodistal width of the mandibular first molar and mandibular overjet

A-414 ANCOVA results testing for associations between mesiodistal width of the mandibular first molar and openbite

A-415 ANCOVA results testing for associations between mesiodistal width of the mandibular first molar and anteroposterior relationship

A-416 ANCOVA results testing for associations between mesiodistal width of the mandibular first molar and DAI score.

A-417 ANCOVA results testing for associations between mesiodistal width of the mandibular second molar and $\mathrm{U} 3$ displacements.

A-418 ANCOVA results testing for associations between mesiodistal width of the mandibular second molar and U2 displacements

A-419 ANCOVA results testing for associations between mesiodistal width of the mandibular second molar and U1 displacements

A-420 ANCOVA results testing for associations between mesiodistal width of the mandibular second molar and L3 displacements 
A-421 ANCOVA results testing for associations between mesiodistal width of the mandibular second molar and L2 displacements

A-422 ANCOVA results testing for associations between mesiodistal width of the mandibular second molar and L1 displacements

A-423 ANCOVA results testing for associations between mesiodistal width of the mandibular second molar and $\mathrm{U} 3$ rotations

A-424 ANCOVA results testing for associations between mesiodistal width of the mandibular second molar and $\mathrm{U} 2$ rotations

A-425 ANCOVA results testing for associations between mesiodistal width of the mandibular second molar and $\mathrm{U} 1$ rotations

A-426 ANCOVA results testing for associations between mesiodistal width of the mandibular second molar and L3 rotations

A-427 ANCOVA results testing for associations between mesiodistal width of the mandibular second molar and L2 rotations.

A-428 ANCOVA results testing for associations between mesiodistal width of the mandibular second molar and L1 rotations

A-429 ANCOVA results testing for associations between mesiodistal width of the mandibular second molar and U2-U3 overlap.

A-430 ANCOVA results testing for associations between mesiodistal width of the mandibular second molar and U1-U2 overlap.

A-431 ANCOVA results testing for associations between mesiodistal width of the mandibular second molar and L2-L3 overlap

A-432 ANCOVA results testing for associations between mesiodistal width of the mandibular second molar and L1-L2 overlap 463

A-433 ANCOVA results testing for associations between mesiodistal width of the mandibular second molar and total displacements .464

A-434 ANCOVA results testing for associations between mesiodistal width of the mandibular second molar and total rotations 
A-435 ANCOVA results testing for associations between mesiodistal width of the mandibular second molar and maxillary R1-L1 overlap.

A-436 ANCOVA results testing for associations between mesiodistal width of the mandibular second molar and mandibular R1-L1 overlap. .465

A-437 ANCOVA results testing for associations between mesiodistal width of the mandibular second molar and total overlapping

A-438 ANCOVA results testing for associations between mesiodistal width of the mandibular second molar and total displacements, rotations, and overlapping .465

A-439 ANCOVA results testing for associations between mesiodistal width of the mandibular second molar and crowding .466

A-440 ANCOVA results testing for associations between mesiodistal width of the mandibular second molar and spacing 466

A-441 ANCOVA results testing for associations between mesiodistal width of the mandibular second molar and diastema

A-442 ANCOVA results testing for associations between mesiodistal width of the mandibular second molar and maxillary incisor irregularity

A-443 ANCOVA results testing for associations between mesiodistal width of the mandibular second molar and mandibular incisor irregularity......

A-444 ANCOVA results testing for associations between mesiodistal width of the mandibular second molar and maxillary overjet.

A-445 ANCOVA results testing for associations between mesiodistal width of the mandibular second molar and mandibular overjet .468

A-446 ANCOVA results testing for associations between mesiodistal width of the mandibular second molar and openbite .468 
A-447 ANCOVA results testing for associations between mesiodistal width of the mandibular second molar and anteroposterior relationship

A-448 ANCOVA results testing for associations between mesiodistal width of the mandibular second molar and DAI score. 


\section{LIST OF FIGURES}

Figure

Page

1 Plot of mean mesiodistal tooth crown diameters by sex, showing the 3 to $6 \%$ sexual dimorphism in tooth size of contemporary American whites

2 Mesiodistal tooth crown dimensions where their sample of adolescents with little crowding had smaller mesiodistal crown dimensions than their other group with at least 4 millimeters of crowding

3 Results showing the mean crown diameters of the six primary teeth were significantly larger in the crowded group than those in the noncrowded group

4 Plot of mean mesiodistal tooth width in the different malocclusion

groups

5 Plot of maxillary mean individual tooth sizes for the malocclusion and bimaxillary groups

6 Plot of mandible mean individual tooth sizes for the malocclusion and bimaxillary groups

$7 \quad$ Summation of the 12 mesiodistal crown diameters in the maxilla (M1 through M1) in males depending on whether the person exhibited excellent occlusion or crowding....

8 Summation of the 12 mesiodistal crown diameters in the maxilla (M1 through M1) in females depending on whether the person exhibited excellent occlusion or crowding...

9 There was no statistically significant difference in tooth size between the crowded and noncrowded samples for boys

10 There was no statistically significant difference in tooth size between the crowded and noncrowded samples for girls

11 Plot of mean mandibular incisor crown dimensions of females. 
12 Results showing (A) that mesiodistal incisor diameters were significantly smaller in the perfect-alignment group, (B) buccolingual widths were significantly larger

13 Plot of mean buccolingual crown width of right side primary teeth as shown by those crowded and those with spaced arch

14 Plot of mean mesiodistal primary crown width of right side teeth as shown by those crowded and those with spaced $\operatorname{arch}$

15 Plot of mean overall and anterior ratios for males and females in Class I, Class II, and Class III .25

16 Examples of the four permanent human tooth types showing the axis along which maximum mesiodistal crown diameters were measured using electronic-readout sliding calipers

17 Schematic depictions of the occlusal view of the maxillary right quadrant

18 Schematic examples of how tooth rotations were scored

19 Labial views of the six mandibular anterior teeth. "Overlap" was scored on a three-grade scheme at each of the five contacts shown in this schematic.

20 Occlusal views of the dental arches of an orthodontic patient at pretreatment who exhibits severe incisor crowding....

21 Schematic depiction of a diastema between the maxillary central incisors

22 Schematic view of a maxillary dental arch................................................50

23 Depictions of horizontal incisor relationships between the arches .............52

24 Depiction of the lateral view of the lower face showing an anterior openbite, where the incisal edges of the central incisors do not overlap vertically

25 Buccal segment relationship refers to the parasagittal relationship of the permanent first molars 
26 Hypothetical plot of a positive linear association between tooth size and irregularity.

$27 \quad$ Hypothetical plot showing a steeper slope for one sex than the other

28 Plot between mesiodistal tooth size of U1 and total displacement scores.

29 Plot between mesiodistal tooth size of U2 and total displacement scores.

30 Plot between mesiodistal tooth size of U3 and total displacement scores.

31 Plot between mesiodistal tooth size of $\mathrm{U} 1$ and total rotations ......................72

32 Plot between mesiodistal tooth size of U1 and total overlap .......................73

33 Plot between mesiodistal tooth size of U2 and total overlap .......................74

34 Plot between mesiodistal tooth size of U1 and total displacements plus rotations plus overlap ..............................................................................

35 Plot between mesiodistal tooth size of $\mathrm{U} 2$ and total $\mathrm{D}+\mathrm{R}+\mathrm{O}$ scores.

36 Plot between mesiodistal tooth size of $\mathrm{U} 1$ and total $\mathrm{D}+\mathrm{R}+\mathrm{O}$ scores .78

37 Plot between mesiodistal tooth size of U1 and crowding score. .79

38 Plot between mesiodistal tooth size of U2 and crowding score.

39 Plot between mesiodistal tooth size of U3 and crowding score.

40 Plot between mesiodistal tooth size of U4 and crowding score. .82

41 Plot between mesiodistal tooth size of U1 and spacing score

42 Plot between mesiodistal tooth size of U2 and spacing score

43 Plot between mesiodistal tooth size of U3 and spacing score .85 
44 Plot between mesiodistal tooth size of U4 and spacing score .......................86

45 Plot between mesiodistal tooth size of U1 and diastema..............................87

46 Plot between mesiodistal tooth size of U3 and diastema..............................88

$47 \quad$ Plot between mesiodistal tooth size of U5 and diastema................................89

48 Plot between mesiodistal tooth size of U1 and maxillary

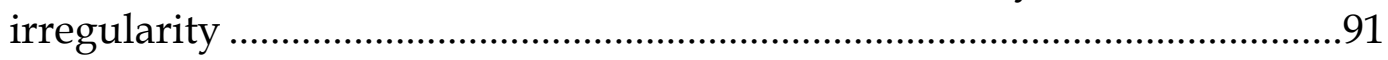

49 Plot between mesiodistal tooth size of U2 and maxillary irregularity

50 Plot between mesiodistal tooth size of U3 and maxillary irregularity

51 Plot between mesiodistal tooth size of U1 and mandibular irregularity

52 Plot between mesiodistal tooth size of U1 and DAI score .95

53 Plot between mesiodistal tooth size of L1 and total displacement score

54 Plot between mesiodistal tooth size of L2 and total displacement score .98

55 Plot between mesiodistal tooth size of L1 and total rotations. .99

56 Plot between mesiodistal tooth size of L2 and total rotations 100

57 Plot between mesiodistal tooth size of L1 and total overlap .101

58 Plot between mesiodistal tooth size of L2 and total overlap .102

59 Plot between mesiodistal tooth size of L1 and total displacements plus rotations plus overlapping. .103

60 Plot between mesiodistal tooth size of L2 and total displacements plus rotations plus overlapping. .104

61 Plot between mesiodistal tooth size of L1 and crowding score .106 
62 Plot between mesiodistal tooth size of L2 and crowding score .................107

63 Plot between mesiodistal tooth size of L3 and crowding score .................108

64 Plot between mesiodistal tooth size of L4 and crowding score .................109

65 Plot between mesiodistal tooth size of L1 and spacing score.....................110

66 Plot between mesiodistal tooth size of L2 and spacing score.....................111

67 Plot between mesiodistal tooth size of L1 and diastema ...........................112

68 Plot between mesiodistal tooth size of L2 and diastema ...........................113

69 Plot between mesiodistal tooth size of L1 and maxillary irregularity

70 Plot between mesiodistal tooth size of L2 and maxillary irregularity

71 Plot between mesiodistal tooth size of L1 and mandibular irregularity

72 Plot between mesiodistal tooth size of L2 and mandibular irregularity

73 Plot between mesiodistal tooth size of L1 and maxillary overjet

74 Plot between mesiodistal tooth size of L2 and maxillary overjet

75 Plot between mesiodistal tooth size of L1 and DAI score.

76 Plot between mesiodistal tooth size of L2 and DAI score.

77 Plot showing the relationship between the three grades of "spacing" against the summed mesiodistal sizes of all 14 tooth types 


\section{CHAPTER I. INTRODUCTION}

Dental malocclusions are commonly the result of a space discrepancy; that is, space available in the dental arch is less than the space required to accommodate the teeth (Nance 1947). This tooth-size arch-size discrepancy (TSASD) may result in malocclusion and represents one factor to be considered when a patient seeks orthodontic treatment. On reflection, it is apparent that TSASD can develop due to: (A) an excess of tooth dimension (notably mesiodistal crown dimensions), (B) a deficit of arch perimeter, or (C) some combination of the two. Most studies have been on arch size and arch development, but some have tested whether crown dimensions are in themselves a risk factor for TSASD. Some of these latter investigations concluded that those with a crowded dentition (i.e., TSASD) have larger teeth (e.g., Fastlicht 1970; Norderval 1975; Keene 1979; Doris et al. 1981; Gilmore and Little 1984; Melo 2001), but other studies did not find a significant tooth size differences between those with crowding and those without (e.g., Mills 1964; Howe et al. 1983; Radnzic 1988; Laino 2003).

Several studies that have tested for a relationship between dental crowding and tooth size did not consider the effects of sexual dimorphism in tooth crown dimensions when reporting their results (Neff 1949; Horowitz 1958; Sperry 1977). It is well documented that males have larger tooth crown dimensions than females (e.g., Moorrees 1957; Garn et al. 1967) as statistical averages. In contemporary America, white male averages exceed those for females by about 3 to $6 \%$ depending on the tooth type (with the canines exhibiting the greatest sex difference). An example of this sexual dimorphism is shown in Figure 1, where means of a sample of American whites are plotted. For each of the 14 tooth types the male mean exceeds that for females. Consequently, when searching for an association between tooth size and TSASD, it is critical that the researcher's study design account for sex differences. Otherwise, by manipulating (or not controlling for) the subject's sex, the effect of tooth size in crowding can artificially be accentuated or eliminated simply by altering the male-female ratios in the samples. This failure to control for sexual dimorphism has led to several studies where the conclusions are confounded to unknown extents. That is, the risk of TSASD is confounded with sexual dimorphism, so no definitive interpretation of the hypothesis is possible.

The purpose of the present study is to determine whether mesiodistal crown size differs as a function of the risk and severity of malocclusion. The intent is to test for sex-specific graded dose-responses between crown size and the severity of the dental malocclusion. Prior studies (e.g., Agenter 2008) have 


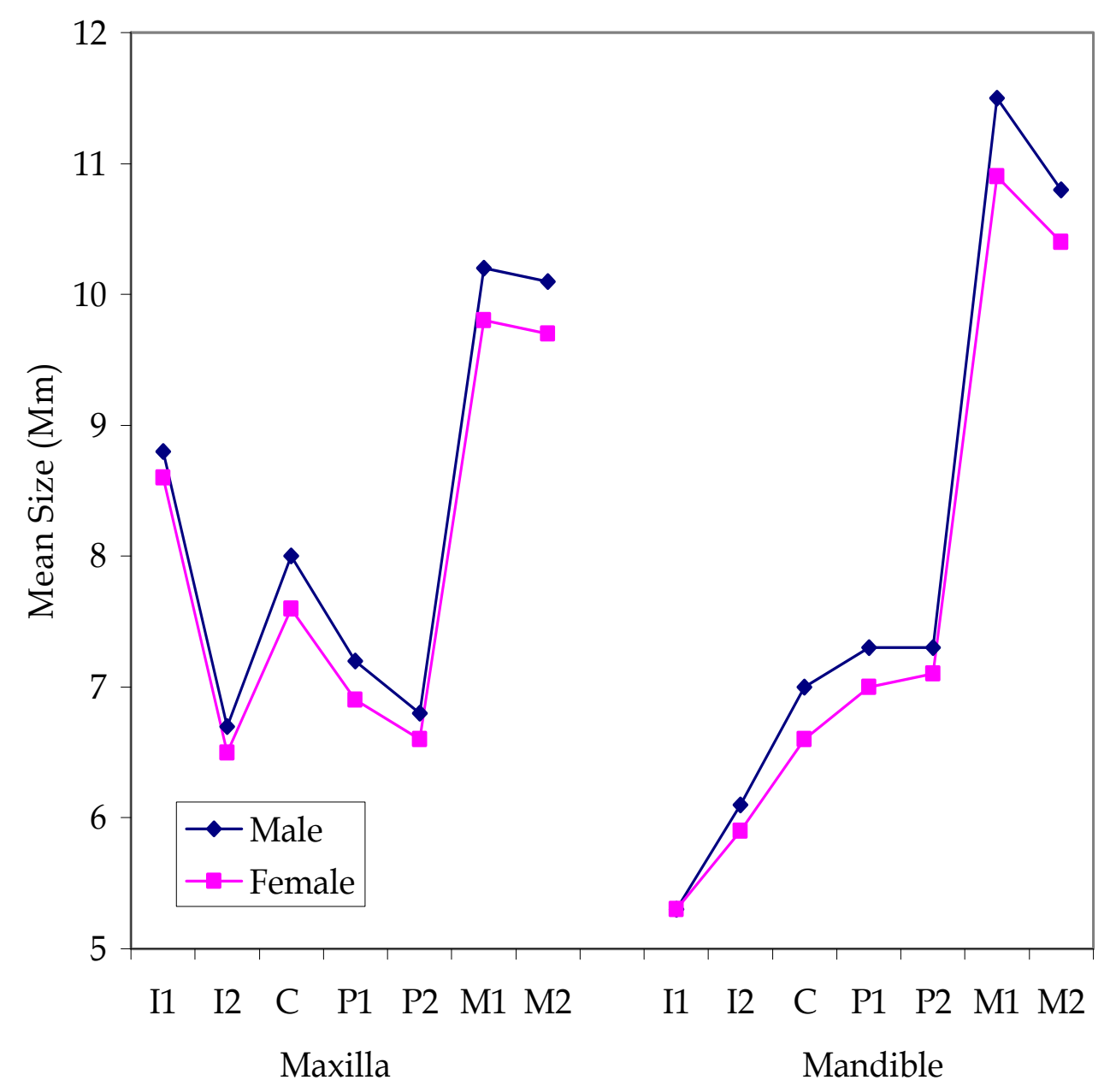

Figure 1. Plot of mean mesiodistal tooth crown diameters by sex, showing the 3 to $6 \%$ sexual dimorphism in tooth size of contemporary American whites.

Data from Garn SM, Lewis AB, Swindler DR. Genetic control of sexual dimorphism in tooth size. J Dent Res 1967;46:963-72. 
documented smaller tooth sizes in those with naturally occurring good occlusions (compared to a sample with malocclusion that received treatment). It probably is artificial to suppose that the relationship actually is dichotomousthat one either has smaller teeth and no occlusal deviations or larger teeth and occlusal deviations - but what is the nature of the dose-response (where "dose" is tooth size and "response" is severity of the malocclusion). It is necessary to evaluate boys and girls separately, not just because of the confounding issue of sexual dimorphism mentioned above, but also because girls are much more likely to seek orthodontic treatment because of their (and their parents) heightened esthetic concern. 


\section{CHAPTER II. REVIEW OF LITERATURE}

The causes of malocclusion are multiple and varied. Heredity, ethnicity, bone size and growth, dietary consistency, function, and numerous other factors may contribute to a person's malocclusion. Various researchers have reviewed the common causes of malocclusion (e.g., Proffit 1986; Harris and Johnson 1991; Mossey 1999), though it usually is difficult to identify a single source for any given individual.

Some authors have suggested that the presence of malocclusion in a patient may be related to tooth size. For example, the orthodontic records of 80 patients were examined in the Doris et al. 1981 study. There were equal numbers of males and females and all were North American Caucasians between 11 to 18 years of age. This sample was divided into two groups of 40, each based on the amount of dental crowding in the upper and lower arches. Group I was composed of those with $4 \mathrm{~mm}$ or less of crowding; those with more than $4 \mathrm{~mm}$ of crowding were assigned to Group II. Measurements of the central and lateral incisors, canines, and first and second premolars on each side and in both arches were recorded. The total mean mesiodistal tooth size was larger in the group with crowded arches. These results are graphed in Figure 2. Inspection of this graph suggests that, in the maxilla, the tooth type contributing most to the difference was the lateral incisor. In the mandible, in contrast, the two groupings are most disparate for the canine and the two premolars. These authors concluded that the size of teeth is one factor affecting whether or not a dental arch will be crowded. In their analysis, Doris and coworkers combined the sexes, making it impossible to determine whether the results were due of the effect of sexual dimorphism. It needs to be appreciated here that "balancing" the malefemale ratio of subjects does not guarantee that the effect of sexual dimorphism is eliminated.

Melo et al. (2001) studied the dental casts of 23 subjects from 3 to 15 years of age at the primary dentition stage and at mixed dentition stage from a sample of 130 pairs of Japanese twins. These longitudinal records were used to compare primary tooth crown size differences in the same subjects in whom, at an older age, they could assess the degree of incisor irregularity in the permanent dentition. Just one set of records was selected from each twin pair for analysis. Those with $4 \mathrm{~mm}$ or more of crowding formed one group, and those with less than $4 \mathrm{~mm}$ of crowding formed another group. Males and females were combined in the analysis, so sexual dimorphism was not controlled in the study design. The results are graphed in Figure 3. Melo and coworkers concluded that larger primary tooth size is one discernible factor in the development of dental crowding. 


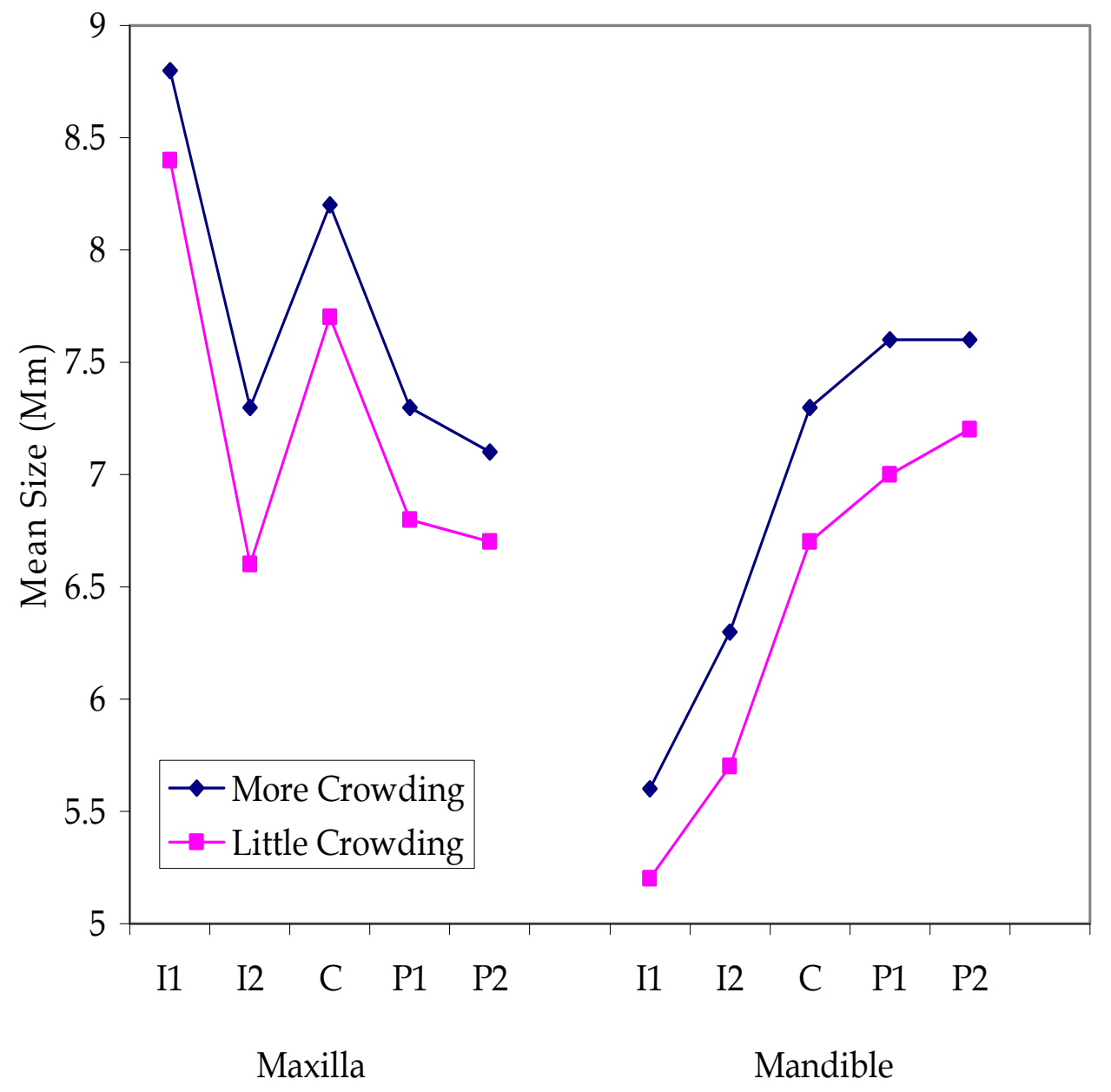

Figure 2. Mesiodistal tooth crown dimensions where their sample of adolescents with little crowding had smaller mesiodistal crown dimensions than their other group with at least 4 millimeters of crowding.

Data from Doris JM, Dernard BW, Kuftinec MM. A biometric study of tooth size and dental crowding. Am J Orthod 1981;79:326-36. 


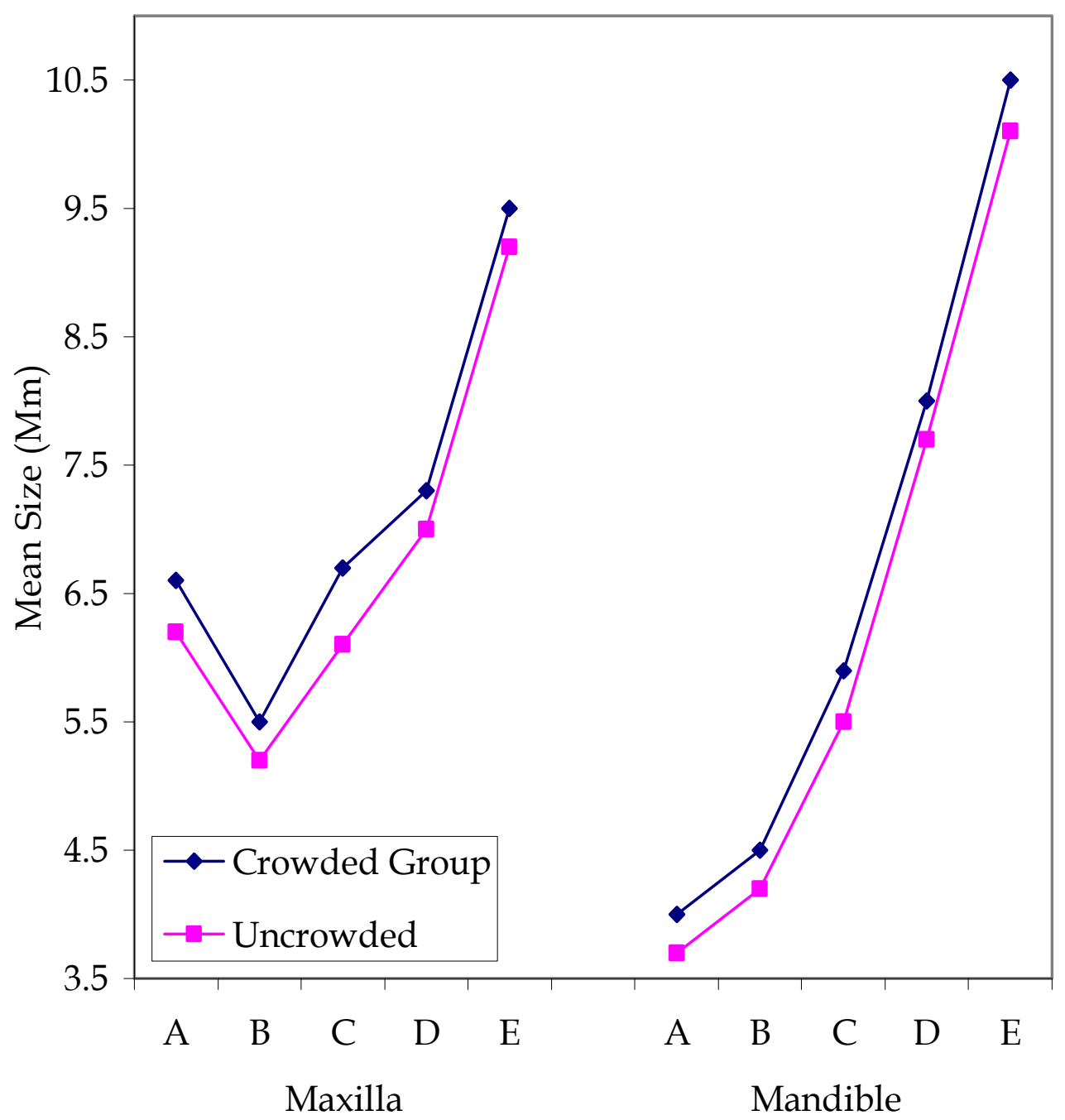

Figure 3. Results showing the mean crown diameters of the six primary teeth were significantly larger in the crowded group than those in the noncrowded group.

Data from Melo L, Ono Y, Takagi Y. Indicators of mandibular dental crowding in mixed dentition. Pediatr Dent 2001;23:118-22. 
Al-Khateeb (2006) studied mesiodistal crown dimensions among different malocclusion groups in a Jordanian population. Dental impressions of 140 adolescents, 13 to 15 years of age with a Class I, Class II or Class III malocclusion. There were 34 males and females with Class I malocclusions, 70 males and females with Class II malocclusions, and 36 males and females with Class III malocclusions. They found that those with Class III malocclusions had larger mesiodistal crown dimensions than the other occlusal categories and that the mandibular arch width and arch length for the Class III malocclusion were greater than those of the Class II malocclusions. These tooth size results are graphed in Figure 4. Of note, their analysis pooled boys and girls, so their results, purportedly due to differences among types of malocclusions-were confounded by sexual dimorphism.

Comparing tooth size discrepancies in different malocclusion groups, $\mathrm{Nie}$ and Lin (1999) studied the dental casts of 360 patients from the clinic in the Department of Orthodontics, Beijing Medical University, China. The subjects were born and living in China, and were between 13 and 23 years of age. The subjects were grouped by Angle's classification: Class I (30 males and 30 females), Class II (60 males and 60 females), Class III (60 males and 60 females), normal occlusion (30 males and 30 females). The maximum mesiodistal diameters of all the teeth on each cast (except for third molars) were measured using the three dimension measuring machine. The statistical tests disclosed no significant sexual dimorphism of the ratios for any of the six groups. These results suggest that the tooth size discrepancy between maxillary and mandibular teeth may be one of the important factors in the cause of malocclusion.

In another study, Bernabé and Flores-Mir (2006) compared the mesiodistal and buccolingual tooth crown sizes as well as the respective crown proportions in three groups that they labeled: mild (between -0.1 and $-5 \mathrm{~mm}$ of crowding), moderate (-5.1 $\mathrm{mm}$ or more of crowding), and no crowding (zero or no crowding). This study analyzed 200 Peruvian students 12 to 16 years of age. Crowding was defined as the difference in millimeters between the arch perimeter and the MD tooth size sum. The mesiodistal and buccolingual crown sizes were measured, and each dental arch was classified as no crowding (zero or a positive discrepancy), mild crowding $(<5 \mathrm{~mm})$, or moderate crowding ( $>5$ $\mathrm{mm}$ ) based on Little's Irregularity Index (Little 1975). When all upper mesiodistal tooth sizes were grouped together and analyzed, a statistically significant average difference was found among moderate, mild, and noncrowded dental arches. When all lower mesiodistal tooth dimensions were summed, a significant average difference was found among moderate, mild, and noncrowded dental arches. Their results showed the mesiodistal crown sizes in the moderate crowded arch were always larger than those in the mildly crowded 


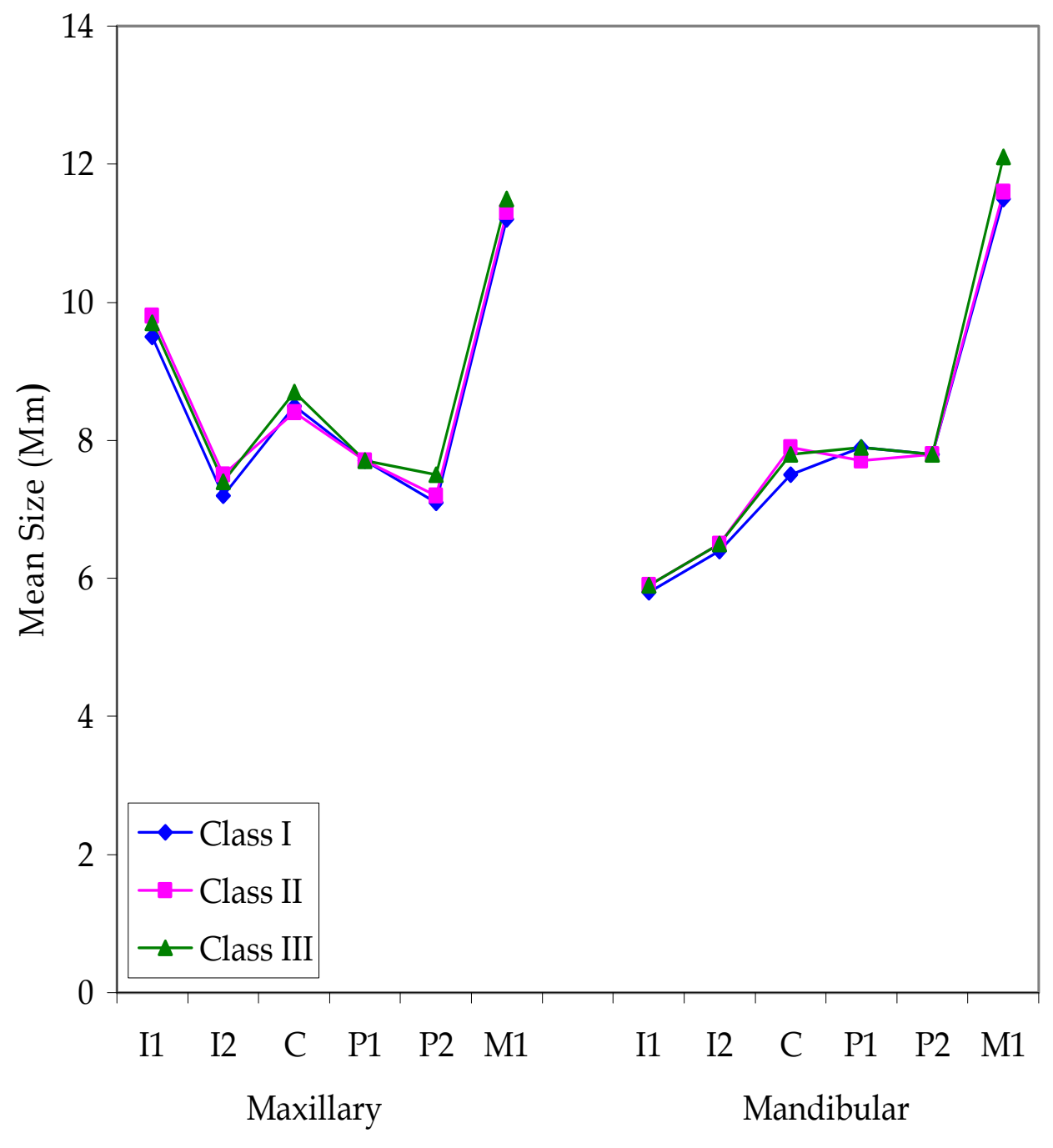

Figure 4. Plot of mean mesiodistal tooth width in the different malocclusion groups.

Data from Al-Khateeb S, Abu Alhaija E. Tooth size discrepancies and arch parameters among different malocclusions in a Jordanian sample. Angle Orthod 2006;76: $459-65$. 
arch, and these were larger than in the noncrowded arches. The most statistically significant differences were between those with no crowding and those with moderate crowding. The comparison of the combined BL tooth sizes within both dental arches did not indicate significant statistical differences for the upper arch or the lower arch. When considering the mesiodistal tooth sizes, significant differences among crowding groups existed in all upper teeth and in the lower central incisor, second, and first premolar. The evaluation of crown proportions between groups indicated combined differences for both dental arches, though not as significant as that for mesiodistal tooth sizes. In reporting their findings, the authors combined the sexes, which confounds interpretations.

A study that investigated bimaxillary dental protrusion as it relates to tooth size was done by McCann and Burden (1996). They studied the dental casts of 30 white orthodontic patients (14 males and 16 females) between the ages of 9 and 21 years of age, all with an Angle Class I bimaxillary protrusion; and a group of 30 white patients (14 males and 16 females) between 9 and 28 years of age exhibiting a variety of malocclusions without bimaxillary protrusion. Bimaxillary dental protrusion was considered present if the interincisal angle was less than 125 degrees, the maxillary incisors proclined beyond $115^{\circ}$ to the maxillary plane and the mandibular incisors proclined beyond 99 degrees to the mandibular plane. Their results (graphed in Figures 5 and 6) showed that, for both males and females, the sum of mesiodistal crown sizes were significantly greater for the bimaxillary group than for the malocclusion group. On average tooth size for the overall maxillary and mandibular dentition was $6 \%$ larger in the bimaxillary sample than in the malocclusion group.

The correlation of tooth size and dental crowding as well as face size and shape was considered in a study by Adams (1982). Adams studied the dental casts of 138 boys and girls between 15 and 16 years of age. These were divided into two groups, 47 with excellent occlusion ( 22 boys and 25 girls), and 91 with incisor crowding ( 46 boys and 45 girls). The selection of the subjects was visual and crowding was not quantified. Comparisons were made, (1) between boys and girls having excellent occlusion, (2) between boys and girls with crowded dentitions, (3) between boys with excellent occlusions and girls with crowded dentitions, and (4) between girls with excellent occlusions and girls with crowded dentitions. A comparison was also made for all these groups between the total tooth substance dimension in the upper arch and the total tooth substance in the lower arch. For all the comparisons, the mean total mesiodistal diameter of the teeth was larger in crowded dentitions than in excellent occlusions (Figures 7 and 8).

In trying to discern some of the causes of mandibular crowding, Fastlicht (1970) looked at numerous aspects including age, sex, and mesiodistal sizes of 


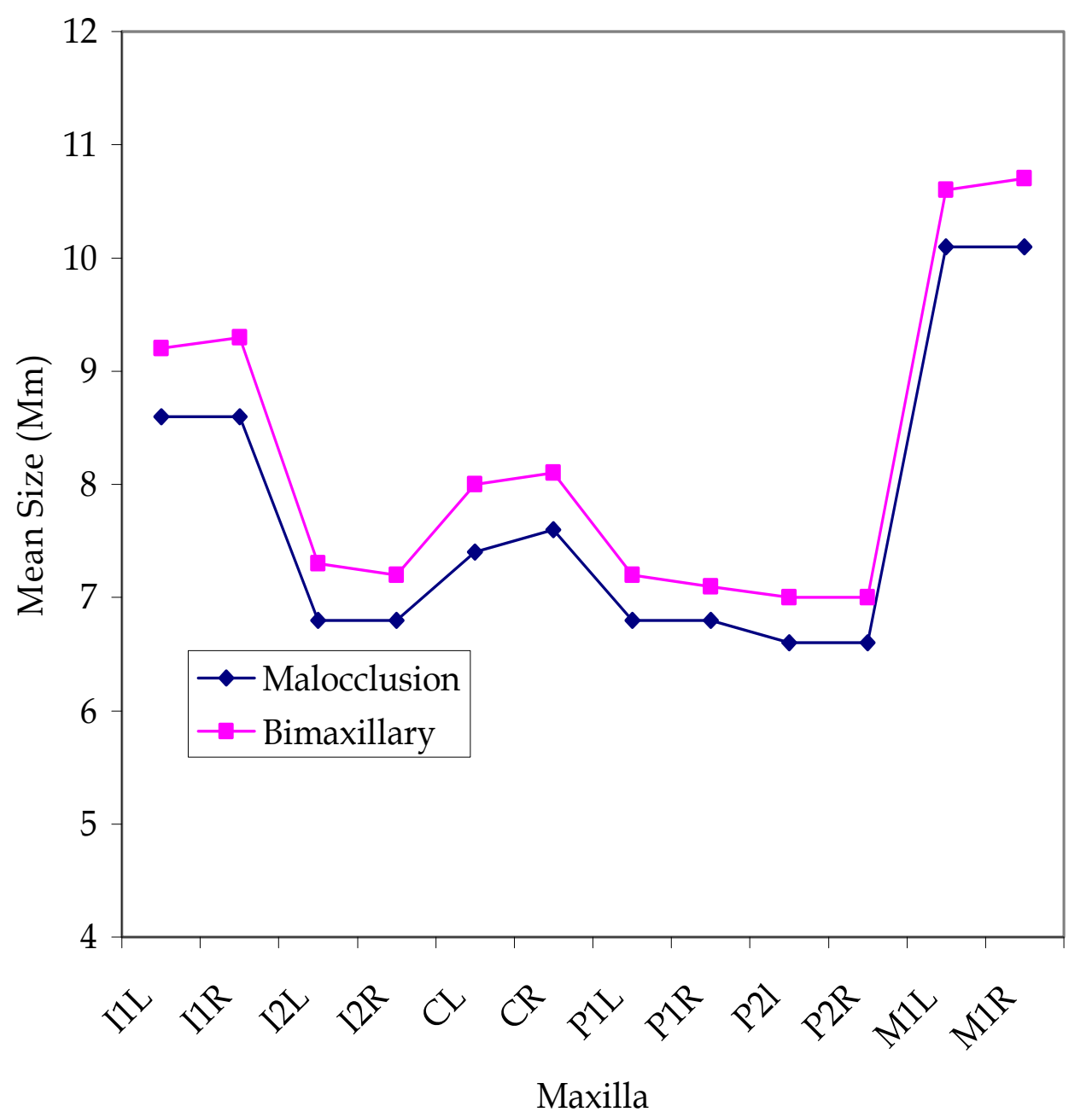

Figure 5. Plot of maxillary mean individual tooth sizes for the malocclusion and bimaxillary groups.

Data from McCann J, Burden DJ. An investigation of tooth size in Northern Irish people with bimaxillary dental protrusion. Eur J Orthod 1996;18:617-21. 


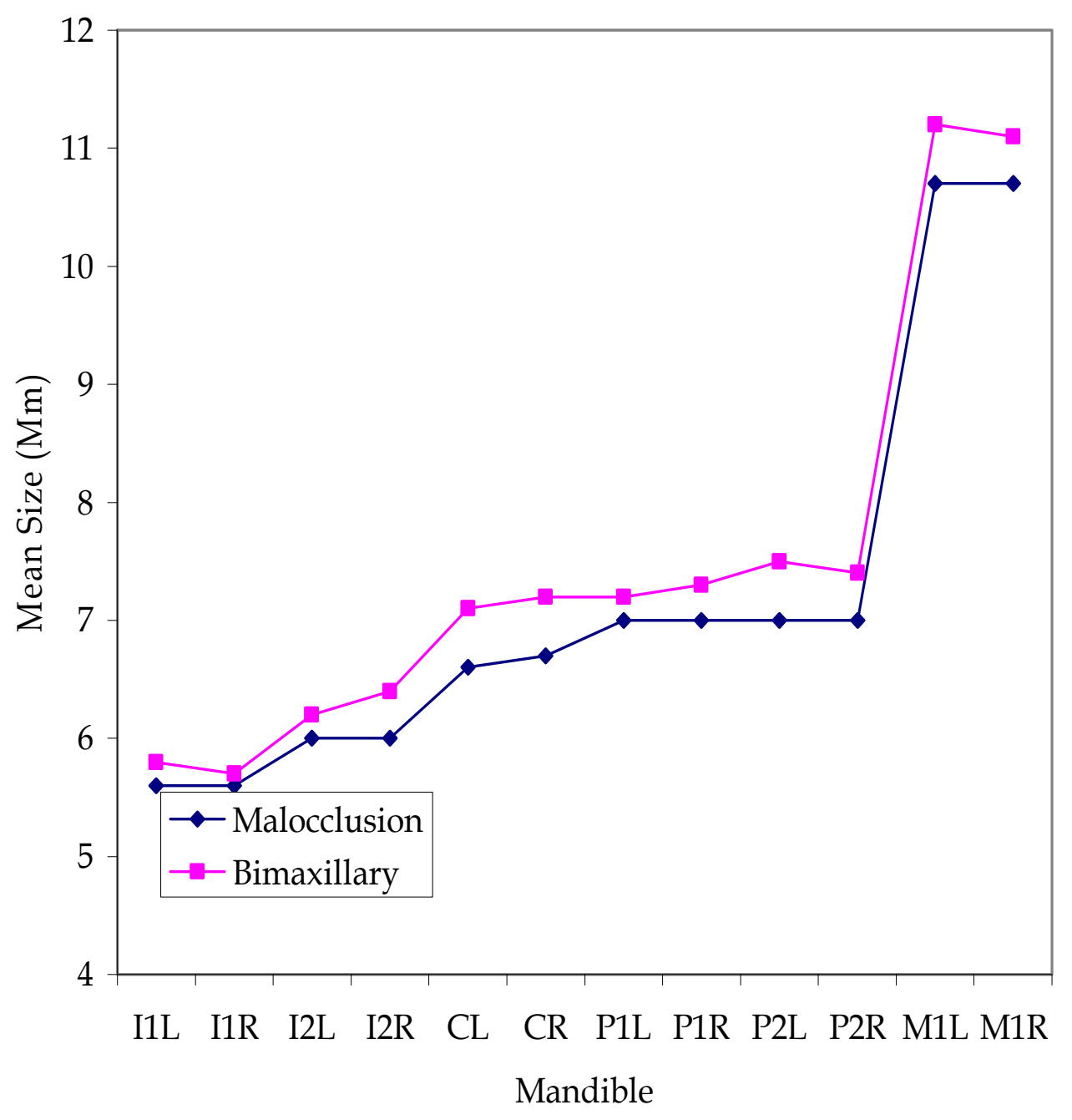

Figure 6. Plot of mandible mean individual tooth sizes for the malocclusion and bimaxillary groups.

Data from McCann J, Burden DJ. An investigation of tooth size in Northern Irish people with bimaxillary dental protrusion. Eur J Orthod 1996;18:617-21. 


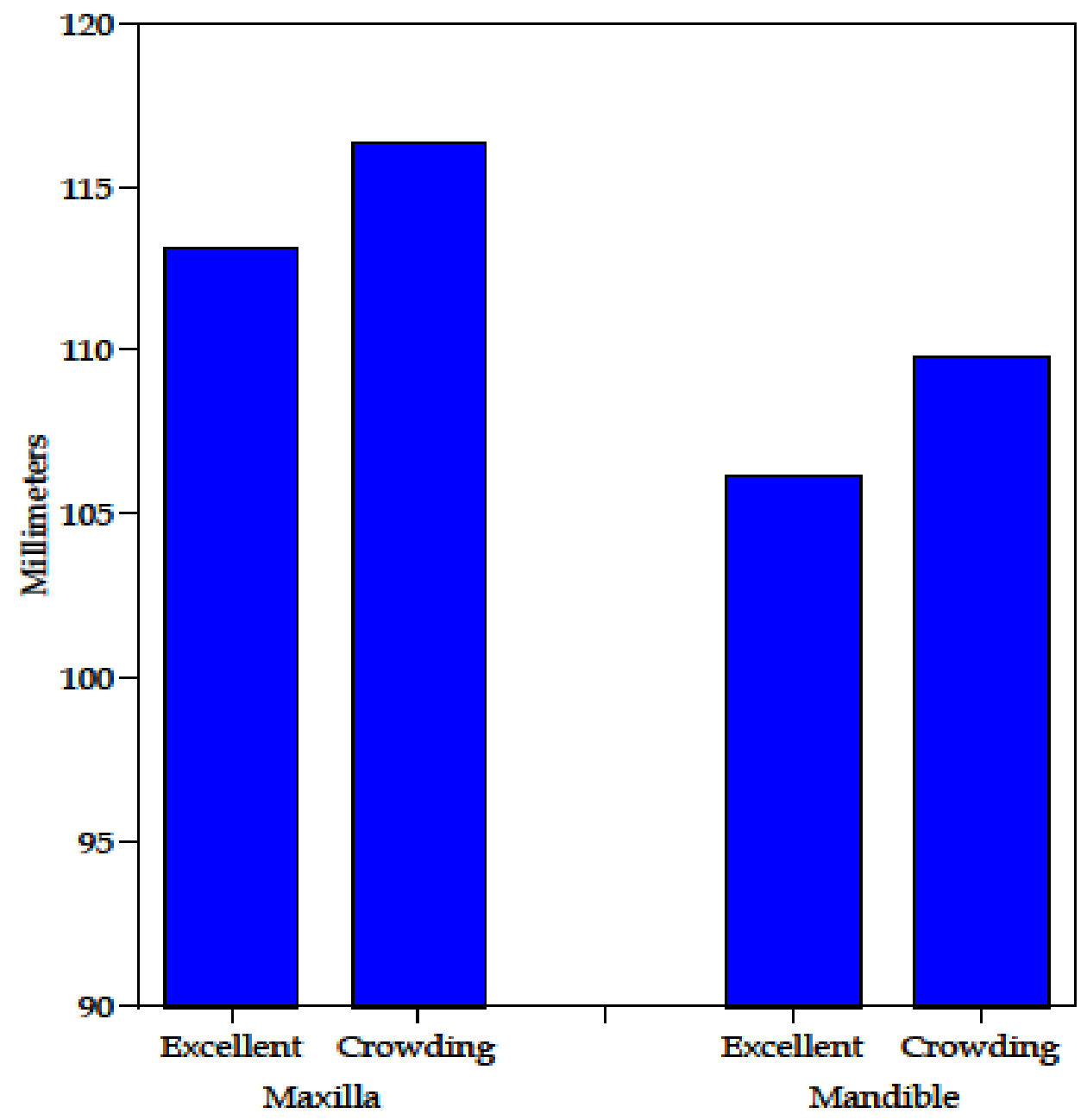

Figure 7. Summation of the 12 mesiodistal crown diameters in the maxilla (M1 through M1) in males depending on whether the person exhibited excellent occlusion or crowding. Differences between groups were significant statistically.

Data from Adams CP. A comparison of 15 year old children with excellent occlusion and with crowding of the teeth, Angle Class I malocclusion, in respect of face size and shape and tooth size. Swed Dent J Suppl 1982;15:11-26. 


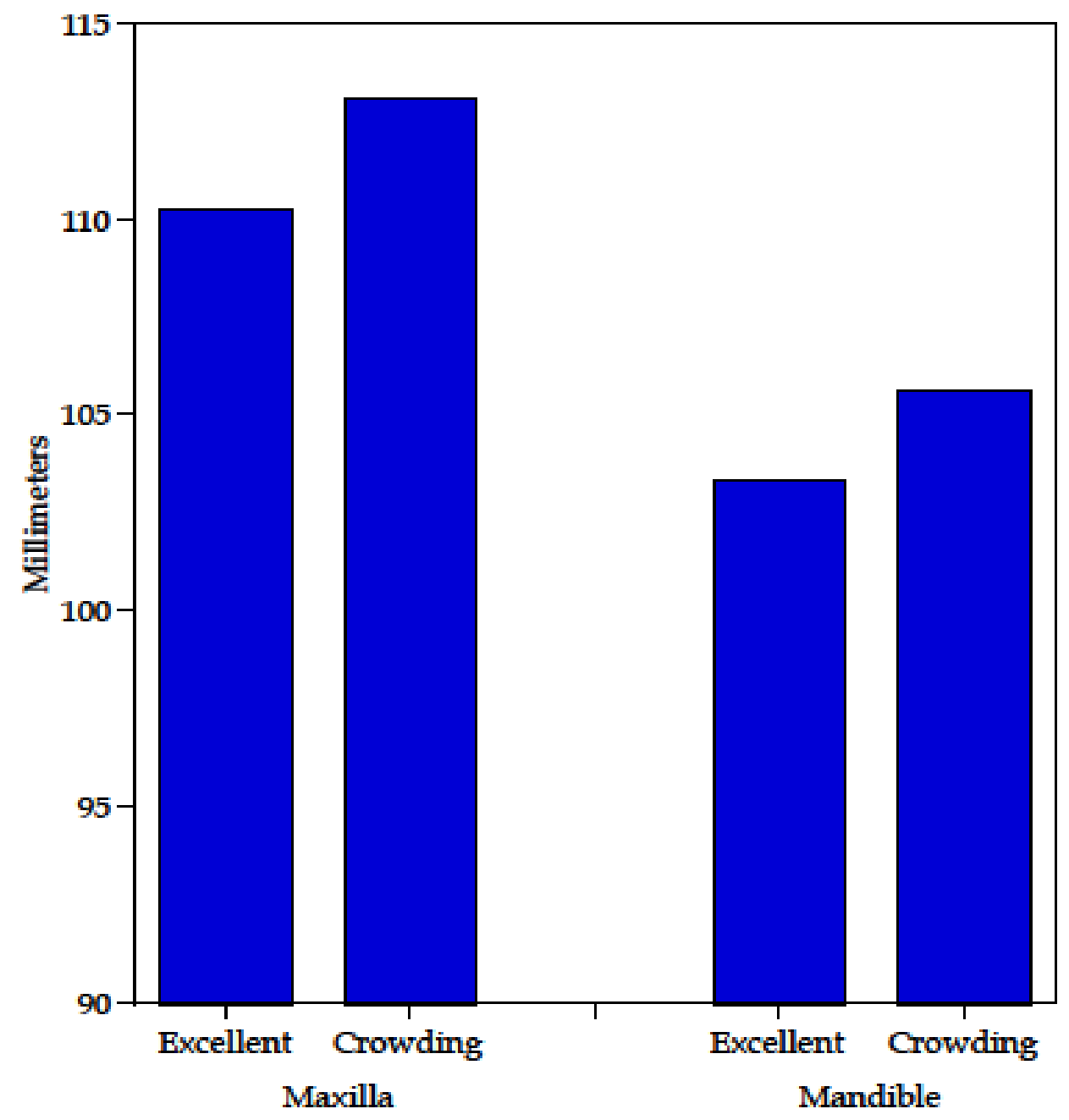

Figure 8. Summation of the 12 mesiodistal crown diameters in the maxilla (M1 through M1) in females depending on whether the person exhibited excellent occlusion or crowding. Differences between groups were significant statistically.

Data from Adams CP. A comparison of 15 year old children with excellent occlusion and with crowding of the teeth, Angle Class I malocclusion, in respect of face size and shape and tooth size. Swed Dent J Suppl 1982;15:11-26 
the tooth crowns. He compared two groups. One group of 28 cases (13 males and 15 females) between 14 and 24 years of age had received orthodontic treatment at the University of Michigan. They had been diagnosed as Class II malocclusions. The second group of 28 cases (13 males and 15 females) between 11 and 42 years of age had not received orthodontic treatment and were considered to have esthetically well aligned upper dental arches. Fastlicht found that both the maxillary and mandibular average for crowding was larger in the untreated group. This difference between the mesiodistal widths of the mandibular and the maxillary incisors with crowding was highly significant in both tests.

In order to gather evidence regarding crowding, Lundström (1951) used the dental casts of 139 Swedish males with a mean age of 13 years of age and varying amounts of dental crowding. The connection between the degree of crowding and the arch perimeter was investigated, as well as the sum of the widths of the teeth from M1 through M1 in the upper anterior teeth. He concluded that the larger the tooth-size, the greater risk of tooth crowding, that jaws with small teeth were less likely to be crowded than those with large teeth, and that as the size of the dental arch decreases crowding increases.

Other authors have investigated the relationship of malocclusion and tooth size and reached different conclusions. Howe et al. (1983) studied tooth size and jaw size as it contributes to dental crowding. The dental casts of 104 subjects from 9 to 44 years of age obtained from the University of Michigan Elementary and Secondary Growth Study and from private practices were assigned to one of two groups, namely those with normal occlusions and no dental crowding and those with significant dental crowding. Statistical analysis apparently was based on a series of t-tests. Howe et al. reported no statistically significant difference between the crowded and non-crowded groups, and this negative finding is in concert with the visual impression of "no difference" supplied by the sex-specific graphs in Figures 9 and 10. Of note, these authors did remove the effects of sexual dimorphism from their data (by performing sexspecific comparisons). We have no interpretation as to why their finding of "no difference" contrasts with the other reports reviewed here.

Tooth size and arch width and length relating to crowding were studied by Mills (1964). His study group was selected from midshipmen at the United States Naval Academy. This sample of males $(n=230)$, included members from all 50 states and various socioeconomic levels. They were between 17 and 21 years of age, and none had a history of orthodontic treatment. Mills found no significant difference in tooth size or arch length between crowded and uncrowded arches. 


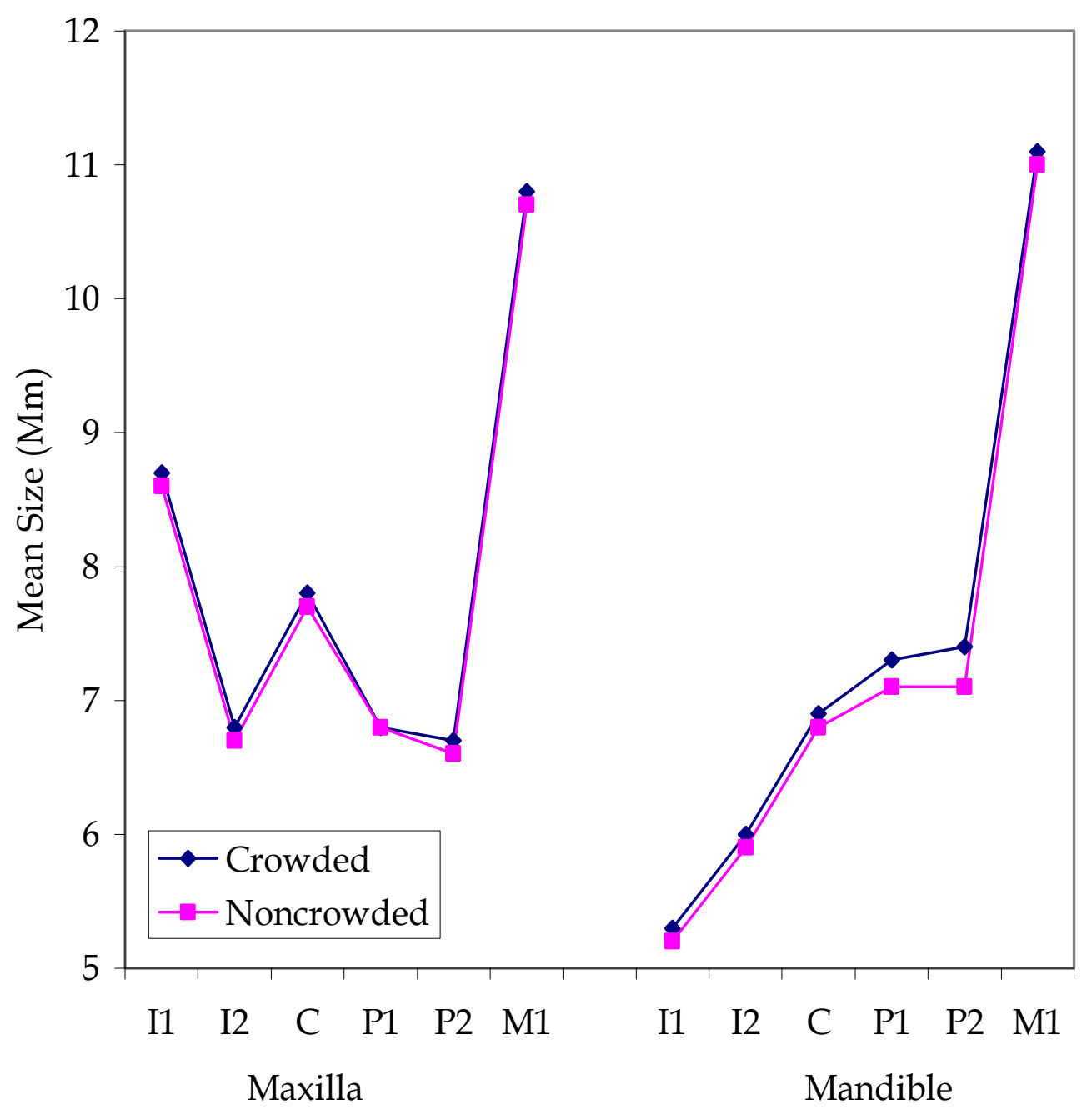

Figure 9. There was no statistically significant difference in tooth size between the crowded and noncrowded samples for boys.

Data from Howe RP, McNamara JA Jr, O'Connor KA. An examination of dental crowding and its relationship to tooth size and arch dimensions. Am J Orthod 1983;83:363-73. 


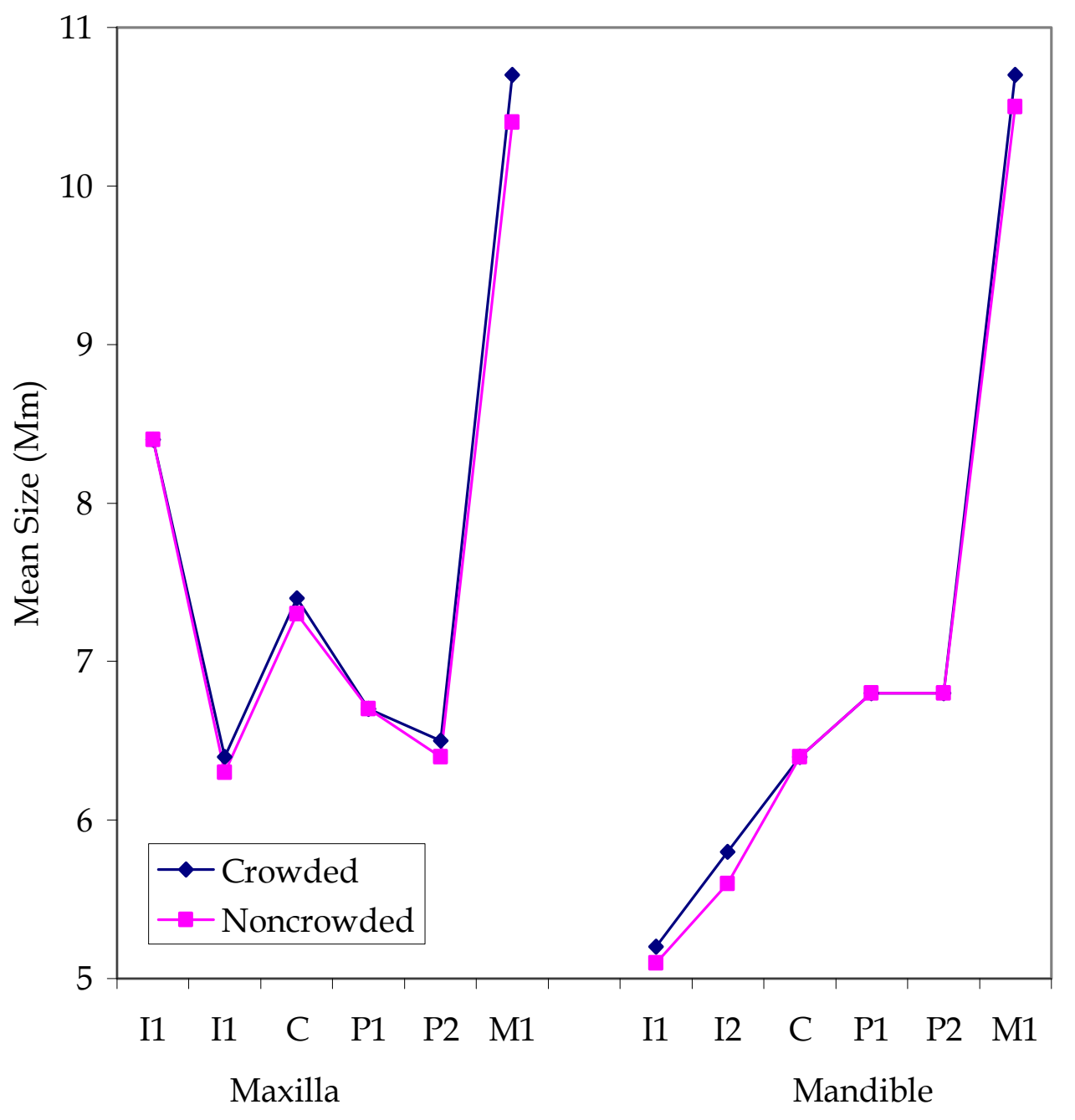

Figure 10. There was no statistically significant difference in tooth size between the crowded and noncrowded samples for girls.

Data from Howe RP, McNamara JA Jr, O'Connor KA. An examination of dental crowding and its relationship to tooth size and arch dimensions. Am J Orthod 1983;83:363-73. 
An influential study as regards tooth size and the risk of malocclusion was by Peck and Peck (1972). They contrasted tooth crown diameters of two groups of Caucasian women, (1) a sample $(n=45)$ with no mandibular incisor irregularity and (2) a sample $(\mathrm{n}=70)$ simply described as "unselected" for incisor irregularity. The key result (Figure 11) was that mean mesiodistal incisor widths were significantly narrower in the good-occlusion sample.

Peck and Peck also measured the mesiodistal and buccolingual incisor diameters of the mandibular incisors and, of interest, these averages were larger in the perfect-alignment sample (Figure 12). This led the authors to infer that the risk of malocclusion is heightened by a difference in incisor crown shape; that the $\mathrm{BL} / \mathrm{MD}$ index is larger in people with mandibular irregularity.

Though there are numerous studies investigating the causes of crowding in permanent dentition, but the causes of crowding in primary dentition have not been explored in much detail. An investigation into dental crowding in the primary dentition and its relationship to arch and crown dimensions was done by Tsai (2003). His sample consisted of 61 Taiwanese children between 4 and 5 years of age. These were put into two groups, those with anterior crowding in both dental arches $(\mathrm{n}=27)$, and those with anterior spacing in both dental arches $(\mathrm{n}=34)$. Each child had all primary teeth present in each arch, and measurements were made on the dental casts. Tsai reported that the mesiodistal crown widths of all tooth types were larger in the crowded arches, but there was no significant difference in the mean mesiodistal crown width or crown shape of the primary teeth between the two groups. These results are graphed in Figures 13 and 14. Also, there was no significant difference between the groups in terms of arch length, crown width, or crown shape, but arch width of both arches in the crowded group were generally smaller that those in the uncrowded group. This indicated to Tsai that dental crowding of the primary dentition is associated with small dental arch widths rather than large teeth.

Corruccini (1990) reevaluated P. R. Begg's (1954) study that asserted that the disappearance of interproximal attrition and modern man's processed diet have resulted in teeth remaining too large for the arches. Corruccini used the dental casts of modern Australian aborigines, the first generation lacking notable interproximal attrition due to a more refined diet, to analyze primary and permanent tooth size and arch size in the absence of attritional tooth reduction. The casts in this sample were from Aborigines who grew up at a government settlement that provided them food that consisted mostly of flour, sugar and hot stew. The casts were obtained between 1951 and 1971 of 50 subjects ( 25 boys and 25 girls) between 13 and 15 years of age. From this group, 15 were considered to have malocclusion (7 Class I and 8 Class II or Class III). Analyzing numerous 


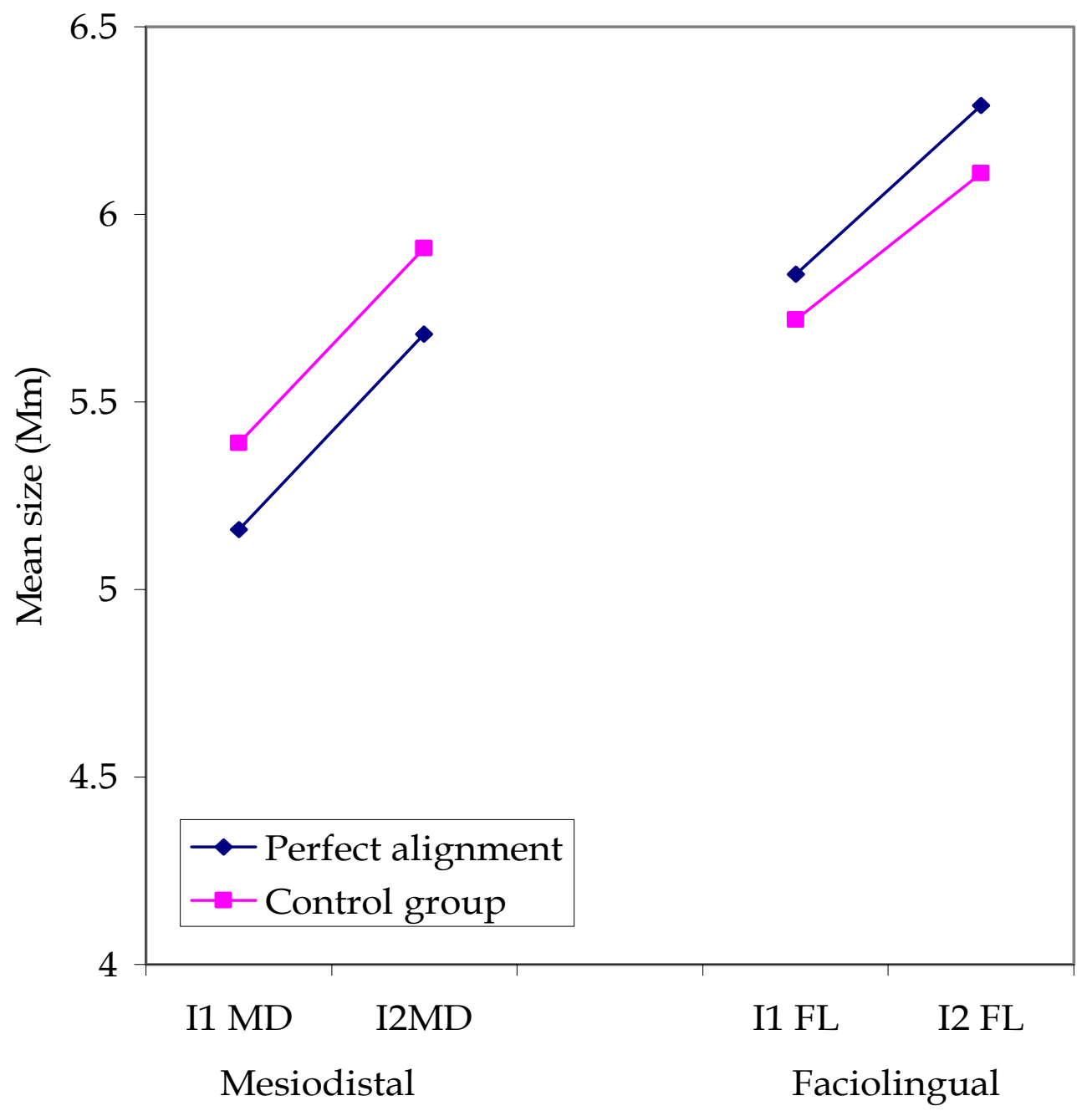

Figure 11. Plot of mean mandibular incisor crown dimensions of females.

Data from Peck S, Peck H. Crown dimensions and mandibular incisor alignment. Angle Orthod 1972;42:148-53. 


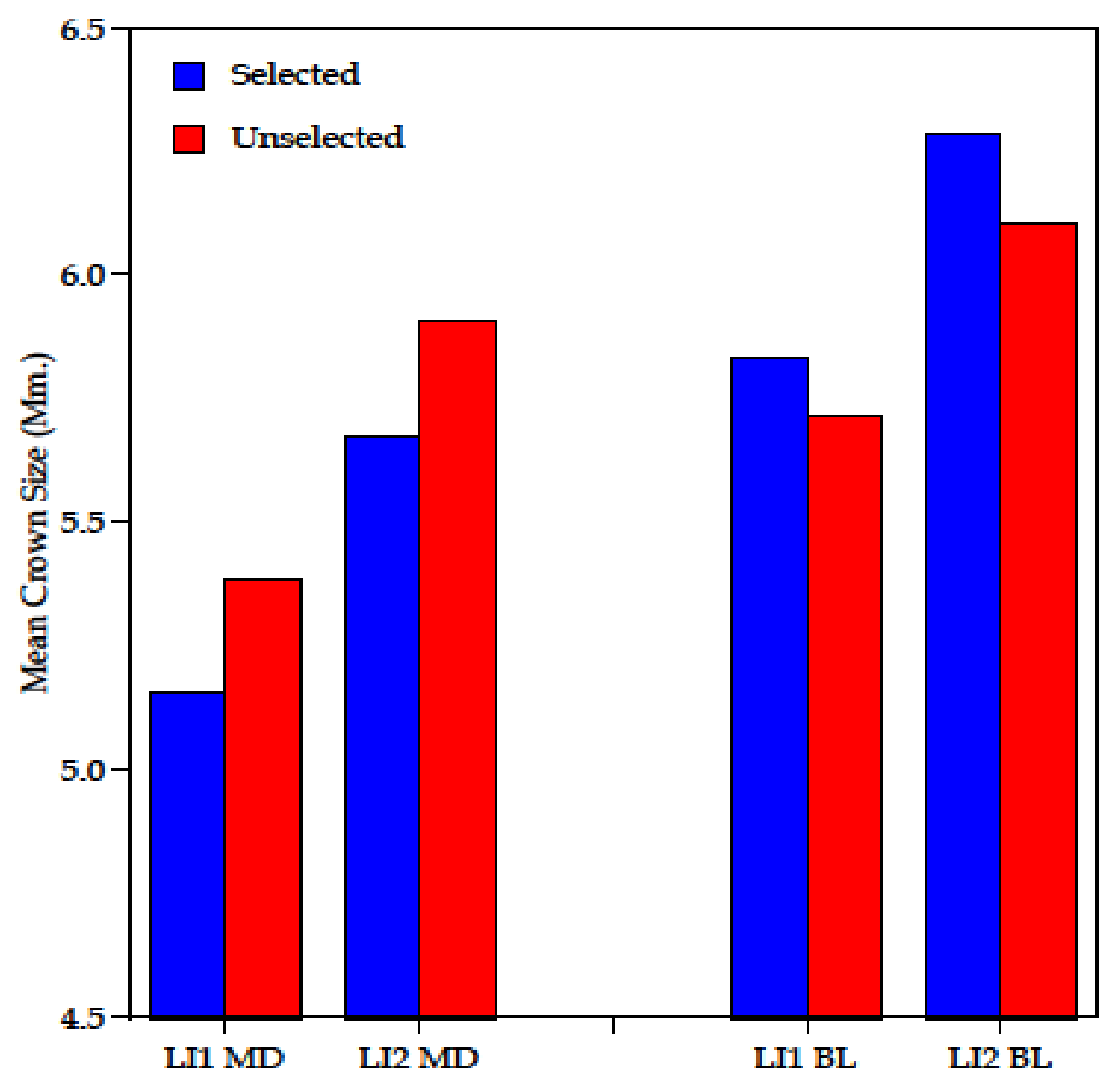

Figure 12. Results showing (A) that mesiodistal incisor diameters were significantly smaller in the perfect-alignment group, (B) buccolingual widths were significantly larger.

Data from Peck S, Peck H. Crown dimensions and mandibular incisor alignment. Angle Orthod 1972;42:148-53. 


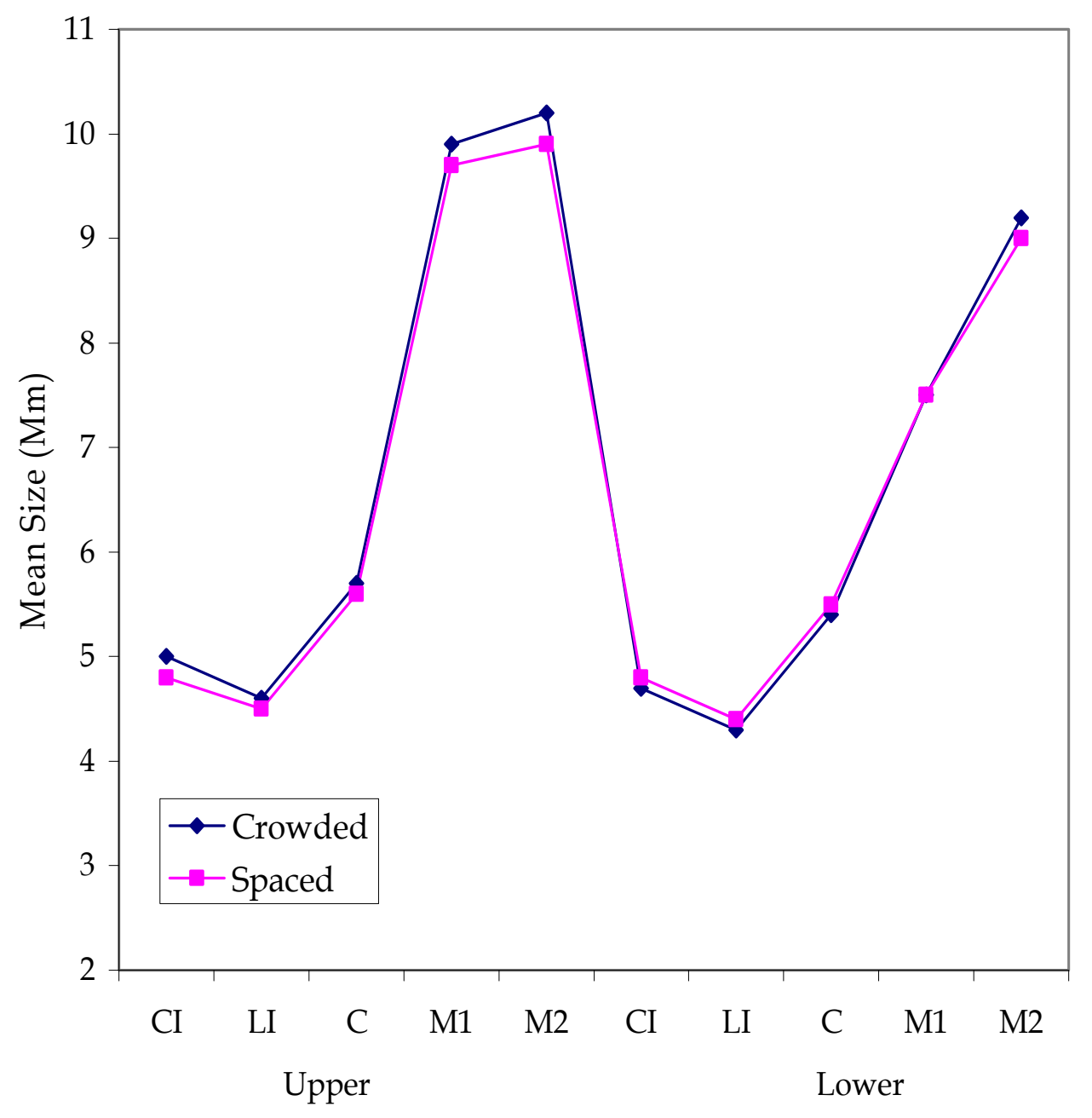

Figure 13. Plot of mean buccolingual crown width of right side primary teeth as shown by those crowded and those with spaced arch.

Data from Tsai HH. Dental crowding in primary dentition and its relationship to arch and crown dimensions. J Dent Child 2003;70:164-9. 


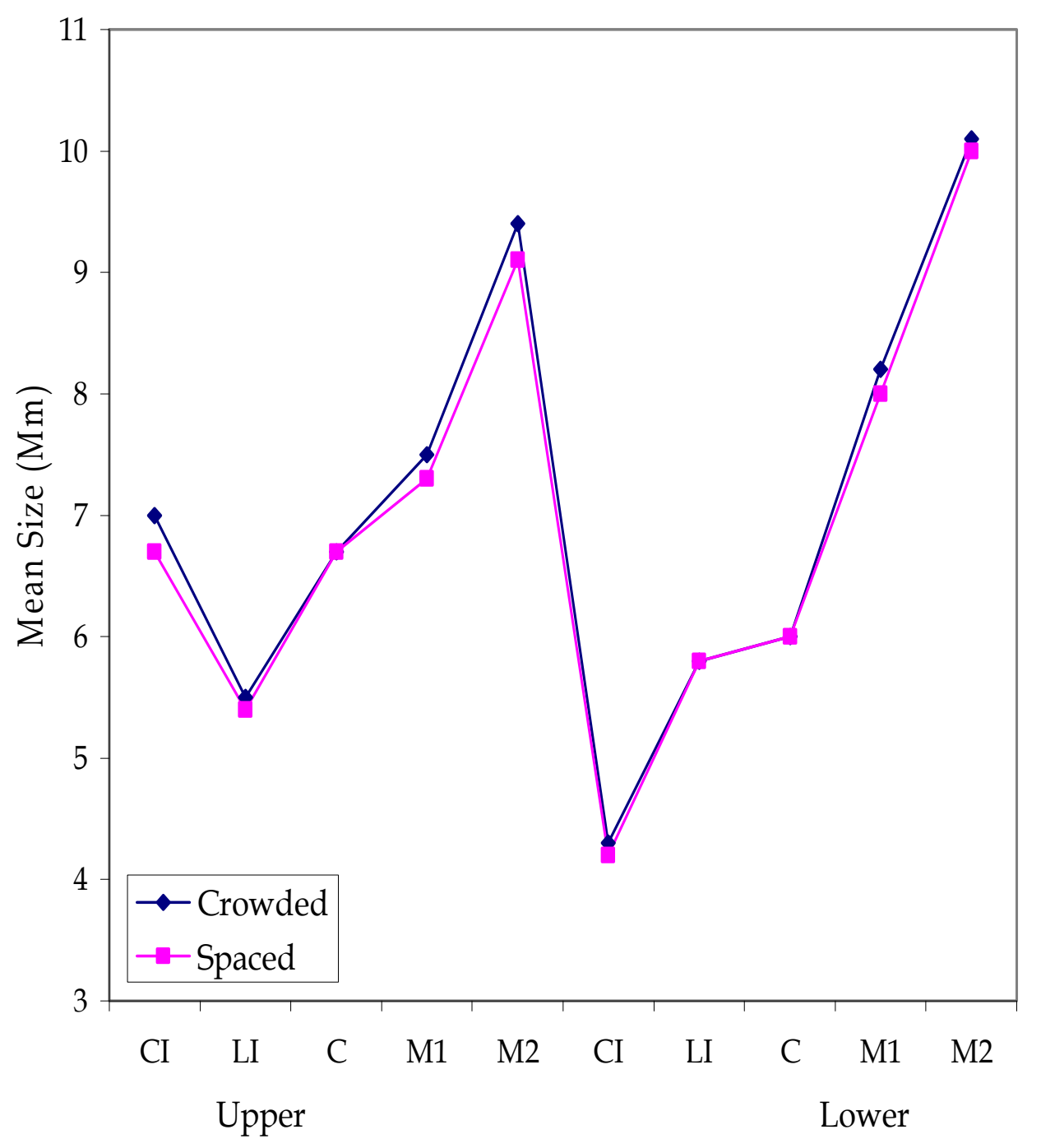

Figure 14. Plot of mean mesiodistal primary crown width of right side teeth as shown by those crowded and those with spaced arch.

Data from Tsai HH. Dental crowding in primary dentition and its relationship to arch and crown dimensions. J Dent Child 2003;70:164-9. 
variables, Corruccini reported that mesiodistally longer teeth did not relate to dental crowding and that lack of space did not significantly relate to crowding or other malocclusions. He did report that narrowness of the maxilla related significantly to malocclusion and that it played a major role in tooth size arch size discrepancy.

Though numerous studies have examined contributing causes for dental crowding, no single factor has emerged. Rather, it seems that the causes are multifactoral. Shah et al. (2003) investigated the correlation between the shape of mandibular incisor crown and crowding. They studied the dental casts of 50 white adults ( 25 males and 25 females) between 18 and 29 years of age, who were dental undergraduates and staff members of the Charles Clifford Dental Hospital in Sheffield, United Kingdom. None of the subjects had had orthodontic treatment, and 39 were considered to be Class I, 8 Class II, and 3 Class III. Lower incisor crowding was quantified with Little's irregularity index and anterior tooth size arch length discrepancy (TSASD). No predictor of lower incisor crowding could be established from mandibular incisor crown shape in this study.

\section{Buccal Segment Relationship}

Tooth size has been studied as it relates to differences between Angle's various malocclusion classes and as it relates to differences between the sexes. In view of the fact that tooth size in a malocclusion may be influenced by sex, and larger or smaller teeth may influence malocclusion, the interaction of both these areas is an area to consider.

Ayra et al. (1974) tested for differences in tooth crown size between occlusion categories. The study used dental records from the University of Oregon Dental School, consisting of 95 boys and girls of Northwest European ancestry between the ages of 4.5 and 14 years. The group of 45 boys and girls had normal occlusion, and the remainder had Angle Class II malocclusions. Mesiodistal crown diameters were measured for all teeth anterior to and including the first permanent molars, and tooth size was considered to be the mean of the tooth sizes from the left and right sides of the arch. The authors found that all tooth types except the mandibular central incisor were significantly different between boys and girls. When the mean tooth sizes were compared between the Angle Class I and Angle Class II groups, no significant difference was observed, regardless of sex. 


\section{Bolton Tooth Size Discrepancies}

Analysis of the proportionality of the maxillary and mandibular teeth is a useful diagnostic tool for use in treatment planning. Bolton (1958) introduced two indexes. The total Bolton index is obtained by dividing the mesiodistal size of the 12 mandibular teeth (first molar to first molar) by the mesiodistal size of the 12 maxillary teeth. The ratio between the two sums is the percentage relationship of mandibular arch length to maxillary arch length. Bolton also devised an anterior index, obtained by dividing the mesiodistal size of the 6 mandibular anterior teeth (canine to canine) by the mesiodistal size of the 6 maxillary anterior teeth. The ratio between these two sums is the percentage relationship of mandibular anterior width to maxillary anterior width. If the ratio was greater than one standard deviation from his reported mean values, Bolton suggested the possible need for treatment.

Crosby and Alexander (1989) studied the frequency of mesiodistal crown size discrepancies in the malocclusions groups and compared them with Bolton's means and standard deviations. The dental casts of 109 patients with varying malocclusions (Angle Class I, Angle Class II) were obtained from a private orthodontic practice, and Bolton's analysis was performed on each set of casts. The frequency of mesiodistal tooth size discrepancies in the malocclusion groups was compared with Bolton's means and standard deviations. The results, which did not differ between sexes, showed that no specific malocclusion group contained a larger percentage of tooth size discrepancies.

When considering tooth sizes among different malocclusion groups, Basaran et al. (2006) found no statistically significant difference in Bolton's tooth size ratios between classes. Another study looked at intermaxillary tooth size discrepancies among different malocclusion groups (Alkofide and Hashim 2002). They analyzed the pretreatment casts of 240 male and female Saudi orthodontic patients 13 to 20 years of age. There was a mixture of Class I, Class II and Class III malocclusions. When tooth size ratios were compared, there was a significant difference between normal occlusion and Class III malocclusion for the anterior ratio, and no significant difference among the other malocclusion groups for the overall ratio and anterior ratio.

Freeman et al. (1996) evaluated the percentage of orthodontic patients with an interarch tooth size discrepancy in a study of 157 patients from the U.S. Army Orthodontic Residency program at Fort Meade, Maryland. The sample included 89 females and 68 males (115 white, 27 black, and 15 of other ethnic origins). They evaluated the percentage of patients with a significant tooth size discrepancy, defined as a value outside of 2 standard deviations from Bolton's mean. They defined a "significant" discrepancy as those below 87.5 or above 
95.1 for the total ratio, and those below 73.9 or above 80.5 for the anterior ratio. The results for the total ratio showed $21(13.4 \%)$ of the 157 patients with ratios outside the 2 standard deviation from Bolton's mean. Of the anterior ratio 48 (30.6\%) of the 157 patients fell outside the 2 standard deviation from Bolton's mean. These results are similar to those found by Crosby and Alexander (1989) who found that $23 \%$ of their sample exceeded the total ratio by 2 standard deviations. This suggests that tooth size analysis in orthodontic treatment would be of benefit in outlining treatment goals.

The study by Laino et al. (2003) explored the prevalence of tooth size discrepancies as related to skeletal malocclusion in a sample from the Campanian region of Italy. They examined the dental casts of 94 patients who were in treatment in the orthodontic department at the University of Naples. The 38 males and 56 females were assigned to one of three groups based on the values of Steiner cephalometric analysis: Class I (26 males and 31 females), Class II (6 males and 18 females), or Class III ( 7 males and 6 females). The mesiodistal diameters of teeth were measured with digital electronic calipers. Based on multiple linear regression analysis, they concluded that there is no evidence of a difference in tooth-size discrepancies among the malocclusion groups.

Determining whether tooth size arch size discrepancy and interarch tooth size relationships differ among ethnic populations was the focus of a study by Paredes et al. (2006). They looked at whether Bolton's ratios applied to a Spanish population. They used the dental casts of 100 cases (30 females and 70 males) between 11 and 20 years of age, from the Orthodontic Department of the University of Valencia, Spain. Each had optimal occlusion (Class I with no arch discrepancy). The mesiodistal sizes of all of the tooth crowns, excluding the second and third molars, were measured from casts. They found that the anterior ratio values were larger than the Bolton standards, indicting that the relationship between the sizes of the mandibular and maxillary teeth depends of the population. They also found no statistically significant difference between the mean anterior and total tooth width ratios for males and females.

Determining whether there is a discernible difference in intermaxillary tooth crown dimensions among different malocclusion groups in Saudi patients was the focus of a study by Alkofide and Hashim (2002). They examined the pretreatment dental casts of 240 patients from the orthodontic clinic at the dental college of King Saud University. The cases were between 13 and 20 years of age and grouped by Angle's classification; 60 with normal occlusion, 60 Class I, 60 Class II and 60 Class III malocclusion with both sexes evenly distributed in each class. They found (graphed on Figure 15) when mean tooth ratios were compared, there was a significant statistical difference between normal occlusion and Class III malocclusion for the anterior ratio but no significant difference 


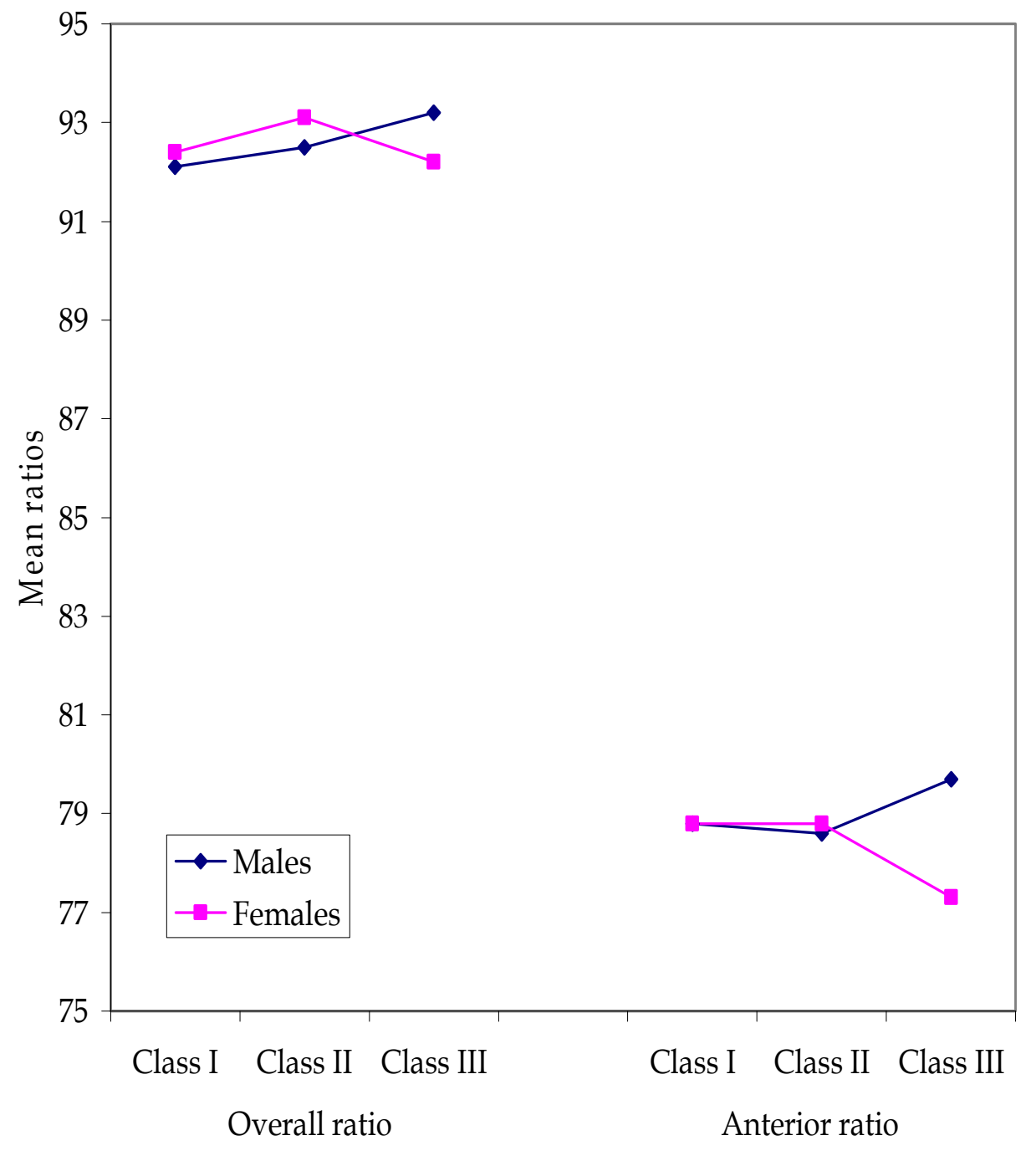

Figure 15. Plot of mean overall and anterior ratios for males and females in Class I, Class II, and Class III.

Data from Alkofide E, Hashim H. Intermaxillary tooth size discrepancies among different malocclusion classes: a comparative study. J Clin Ped Dent 2002;26:383-7. 
between the other malocclusion classes for the overall ratio and the anterior ratio. In addition, no statistical significant difference was observed between males and females. When the mean values for the overall and anterior ratios of this study were compared to Bolton's, a significant difference was found in all the malocclusion classes.

An investigation of Bolton's analysis as it applies to tooth size and different ethnic populations (black, Hispanic, and white), was studied by Smith et al. (2000). The pre-orthodontic records of 180 cases (30 males and 30 females) from each of 3 populations (black, Hispanic and white) were analyzed. The subjects were between 12 and 38 years of age. The results indicated significant differences in the overall, anterior, and posterior interarch ratios between the black, Hispanic, and white populations and the relationship between the sizes of the mandibular and maxillary teeth are dependent on population, gender, and arch segment length.

\section{Perception of Orthodontic Need}

Studies show that girls and their parents have a lower threshold for seeking orthodontic treatment than boys and their parents ( $\mathrm{O}^{\prime}$ Brien et al. 1996; Sheats et al. 1998; Mendall et al. 1999; Birkeland et al. 2000; Esa et al. 2001). This psychosocial sex difference is what explains (1) the 2-to-1 sex difference in orthodontic practices versus (2) the general lack of sex differences in national objective surveys of orthodontic need. Yet the evidence of influence of sex on both normative and perceived need for orthodontic treatment in studies is inconsistent.

For most prospective orthodontic patients, the decision to seek orthodontic treatment reflects a combination of their expectations and those of their dentist or orthodontist. O'Brien et al. (1996) investigated factors that influenced the uptake of orthodontic treatment for children referred by their general dentist. Data was collected on 162 referred patients (54\% female, $46 \%$ male) whose mean age was 12.7 years. Of the children referred, 87 were accepted for treatment (54 girls and 33 boys). Analysis indicated that the sex of the patient and the need for treatment (measured by the IOTN) were the most significant predictors.

Mandall et al. (1999) investigated orthodontic esthetic self-perception and the perceived esthetic impact of malocclusion. They matched 434 boys and girls (14 to 15 years of age from Manchester England) for age, sex, social class and ethnicity. Five questions were asked to assess the degree of concern the child perceived because of the arrangement of his or her occlusion. In addition, the 
children were asked to identify which photograph of the IOTN AC most clearly matched their orthodontic esthetic self-perception of the appearance of their anterior teeth. The scores for all questions and the child's perceived AC score were totaled to give an overall perceived oral aesthetic impact score for each child. They referred to this as the OASIS score (Orthodontic Aesthetic Subjective Impact Score). Most children (94\% of 334) perceived their anterior tooth arrangement to be acceptable (IOTN AC scored between 1 and 4), while only $54 \%$ of the IOTN AC scores from the children agreed with the examiner's score of the need for treatment. Mandall's data showed that ethnicity and social deprivation did not influence a child's orthodontic esthetic self-perceived AC score or their self-perceived need for orthodontic treatment. The authors reported that Asians and females had a higher orthodontic treatment need based on dental health grounds than Caucasians and males, despite having a lower esthetic need for treatment.

Birkeland et al. (2000) studied the relationship in children between occlusion, satisfaction with dental appearance, self-esteem, and their perceived need for treatment at ages 11 and 15 years of age, in a treated and untreated group. They also evaluated the degree of satisfaction of the children and their parents. The study consisted of 359 children (age 11 in 1993) from Bergen, Norway. Then in 1996 the same group of children (now 15 years of age) and their parents were invited for a follow-up study (224 participated). The dental casts were assessed using the Dental Health Component (DHC) and Aesthetic Component (AC) of the IOTN. In addition, questionnaires (regarding satisfaction with dental appearance and desire for orthodontic treatment) were completed separately by the parents and the children. The Global Negative SelfEvaluation Scale (GSE) was used to measure the child's self-esteem. Significantly more girls than boys had been treated with fixed appliances between the two examinations. However, when combining treatment with fixed and removable appliances there was no sex difference (evidently because more boys had been treated with removable devices). The fixed appliance group showed the greatest improvements both for AC and DHC grades. For the untreated group, significant increases in mean AC grades, DHC grades, and PAR scores were found from T1 to T2. Treatment with fixed appliances (as opposed to no treatment or treatment with removable appliances) had the greatest influence on the satisfaction level. At T1, no sex difference was discovered, while at T2, boys showed greater satisfaction with their own dental appearance than girls in the untreated and the removable appliance groups. The sex difference was more evident at 15 than at 11 years of age, as more girls than boys had developed negative self-evaluation. In general, there was a high degree of satisfaction with orthodontic treatment results by both children $(95.4 \%)$ and by parents $(95.6 \%)$. About $45 \%$ of the children were of the opinion that orthodontic treatment would 
have a positive influence on their future possibilities in life and that treatment was important for their self-confidence.

Determining the prevalence of malocclusion and orthodontic treatment need in a sample of New Zealand children was the focus of a study by Johnson and Harkness (2000). They examined 294 Caucasian children (153 boys; 141 girls) between 9 and 11 years of age in the mixed dentition. A DAI score was calculated for each child and on the basis of that score they were divided into four orthodontic treatment-need categories. About $20 \%$ of the children fell into the little or no treatment need category, $25 \%$ in the treatment elective category, $22 \%$ in the treatment highly desirable category, and $33 \%$ in the treatment mandatory category. A criticism of the DAI has been its inability to assess malocclusion in the mixed dentition, as it was developed for use on subjects in the permanent dentition. Considering this, the high proportion of subjects with handicapping malocclusions in this study may be due to the large number of subjects in the mixed dentition who have temporary occlusal conditions. In this study, the mean DAI scores for the boys and girls differed significantly until the missing teeth component was omitted, then the significant sex difference was lost.

The perception of orthodontic need and if it changes over time was the focus of a study by Stenvik et al. (1999). They compared the attitudes to malocclusion and orthodontic treatment in young and middle-aged adults. The sample included a total of 123 individuals, 50 orthodontically untreated young adults ( 25 males; 25 females) between 17 and 18 years of age, and 73 untreated middle-aged adults (38 females; 35 males) 38 years of age living in Oslo, Norway. Dental casts were used to group subjects according to the IOTN grades. No significant difference between sexes appeared for any of the variables (except for the question about importance of teeth for facial appearance). A high percentage ( $90 \%$ of 18 year olds, and $86 \%$ of 35 year olds) felt that well aligned teeth were important for overall facial appearance. Dissatisfaction with their dental arrangement was reported by $20 \%$ of the 18 year olds and $15 \%$ of the 35 year olds. Desire for treatment was about the same for 18 and 35 year olds. When comparing patients with similarly scored malocclusions the results in the obvious-need category show that $54 \%$ of 18 year olds and $21 \%$ of 35 year olds were dissatisfied. Results show a decline in concern for borderline malocclusion at midlife compared to the concern in early adulthood.

Evaluating malocclusion and orthodontic treatment need was the focus of a study by Esa et al. (2001). They also assessed the relationship between malocclusion and socio-demographic variables, the perceptions of need for orthodontic treatment, esthetic perception and social functioning. The sample consisted of 1,519 Malaysian children (770 males; 742 females) between 12 and 13 
years of age. In addition, a self-administered questionnaire covering sociodemographic information, perceived need of treatment and satisfaction with dental appearance was given to each child. The child's sex and regional differences were found to be significantly related to DAI. Girls tended to have lower DAI scores than boys. This study showed a consistent relationship between the DAI score and the subjects' desire for orthodontic treatment, as well as satisfaction with dental appearance and social functioning.

Another study investigated the correlations between the prevalence of malocclusion and the need for orthodontic treatment (Willens et al. 2001). In this study the sample included the records from 1,477 (641 males; 836 females) patients from a university orthodontic department in Belgium. From these patients' records, three groups were formed; Group 1 (220 patients) received no treatment, Group 2 (124 patients) stopped their treatment prematurely, and Group 3 (1,133 patients) which completed their orthodontic treatment. In this study, a clear need for orthodontic treatment was found in $63 \%$ of the cases according to the DHC component of the IOTN. The mean DHC grade of 3.7 was comparable to other studies of university orthodontic populations, and for nonuniversity orthodontic populations of the same geographical region. In addition, spacing and tooth-size discrepancy (TSD) was investigated. The results reported showed $34.1 \%$ of the orthodontic patients, in this study, had a tooth size discrepancy of $1 \mathrm{~mm}$ or more indicating that considering tooth size analysis in the treatment plan would be prudent.

The age range of the sample can affect the outcome of the results. In a study by Chi et al. (2000) the DAI scores of 150 New Zealand children 13 year old were used to assess the prevalence of orthodontic treatment need. Those results were then compared to the results of a study of those same children at 10 years of age. In the original group, surveyed in 1995, 294 children, 10 years of age were examined, of those, 183 (62.2\%) agreed to be re-examined as 13 year olds, in 1998. Methods for patient examination were identical in the two studies. The DAI was administered again in 1998. There was no sex significant difference between the DAI scores for the 13 year old boys and girls. The mean change in the DAI scores for the children examined at both 10 and 13 years of age was $-0.7(\mathrm{sd}=7.1)$. About half of the 150 children examined at 10 years of age were in the same treatment-need category at 13 years of age; 30 moved into a higher treatmentneed category, and the other 47 moved into a lesser treatment-need category. When considering the results by treatment need category the authors reported that fewer 13 year olds $(27 \%)$ had a mandatory treatment need than when they were 10 years old $(33 \%)$. This was also true in the desirable treatment category, $20 \%$ for 13 year olds compared to $23 \%$ for 10 year olds. When the DAI scores, as opposed to the treatment need categories were examined, only $7 \%$ of the children were assigned the same score at both 10 and 13 years of age (52\% received a 
higher score; $41 \%$ received a lower score). When the four treatment need categories were analyzed, agreement occurred in $49 \%$ of the children assessed at both ages, compared with the same children when the DHC component of the IOTN was used. When the individual children's scores were compared, agreement occurred $7 \%$ of the time. The authors found the results of malocclusion indices (DAI and IOTN) are affected by the developmental changes with growth.

The idea that some groups are over-represented among patients who receive orthodontic treatment was the focus of a study by Kisely et al. (1997). The use of a reliable and valid assessment scale such as the Index of Orthodontic Treatment Need (IOTN) to identify those patients most likely to benefit from treatment was thought to enhance consistency and provide an objective measure. Though the IOTN ranks malocclusions in terms of dental health and esthetic impairment, Kisely et al. thought it needed to be supplemented by assessments of the timing and complexity of treatment required because these are areas that the IOTN does not cover. The sample consisted of 400 patients (62\% female; $38 \%$ male) with a mean age of 12.4 years. All patients were interviewed using the "Pathways Encounter Form" and had a dental assessment that included the IOTN, an assessment of complexity of, treatment and timing of the referral. The authors found no particular socio-demographic or geographic variable associated with a fast or slow pathway, and that severity (measured by an IOTN score greater than 3) was not significantly associated with length of time for obtaining orthodontic treatment. When considering the results, the gender and socio-demographic balance of the sample needs to be noted. Though there is a uniform prevalence of orthodontic anomalies in the population, $62 \%$ of the subjects were female and $25 \%$ were from social classes I and V (the latter is double the figure expected if the sample reflected the socio-demographic profile of the area). These results in this study showed that orthodontic treatment was not equitably provided and was not based on objective need.

Investigating self-awareness of malocclusion and actual clinical status of orthodontic need was the focus of a study by Sheats et al. (1998). The authors conducted orthodontic screening examinations of 861 eighth-grade children (54\% female; $46 \%$ male) in Florida. Visual examinations were done and wax bite impressions were taken for calculation of the Peer Assessment Rating (PAR) index. In addition, each child was asked four questions (and two follow-up questions when appropriate) regarding self-perception of this occlusion and the need for orthodontic treatment. Those with braces or a history of orthodontic treatment were excluded. The results showed that $64 \%$ of the group expressed no self-perceived need for braces while $25 \%$ were not satisfied with the way their teeth looked. Results showed that females were significantly more likely than males to perceive a need for non-removable orthodontic devices. In addition, 
girls were less likely to be satisfied with their dental appearance and were more likely to have a perception of overjet. Girls also expressed more concerns than boys about anterior crowding, but not for increased overjet in those cases where there was a perception of "crooked teeth" or teeth that "stuck out." Race differences were not demonstrated. It was also found that while $57 \%$ of the subjects were classified as having no treatment need, $64 \%$ of the subjects judged themselves as having no need for braces. With other variables held constant, those who were satisfied with the way their teeth looked, were one-sixth less likely to perceive a need for braces than those who were not happy with they way their teeth looked. The results show a discrepancy between actual clinical findings and a child's demand for orthodontic treatment.

A longitudinal study to examine factors influencing the decision for orthodontic treatment was done by Birkeland at al. (1999). They evaluated the attitudes of 11 and 15 year old and their parents which influenced their decisions about orthodontic treatment. The sample consisted of 359 children who were 11 years old in 1993 (T1). Then in 1997 (T2), 293 of those same children (now 15 years old) agreed to participate in a follow-up study. Initially (T1), prevalence of malocclusion was recorded based on assessment of casts using the Dental Health Component (DHC) and Aesthetic Component (AC) grades of the Index of Orthodontic Treatment Need (IOTN). Of that number, 74 (20\%) were under orthodontic treatment at that time. Additionally, parents and children separately, completed a questionnaire regarding their orthodontic concerns. The Global Negative Self-Evaluations scale (GSF) was given to each child to measure self-esteem. Then again in 1997 (T2) separate child and parent questionnaires were given, and the children were clinically examined and had dental impressions taken. The results showed that between $\mathrm{T} 1$ and $\mathrm{T} 2$ the majority $(78.9 \%)$ changed only one DHC grade. The parents' desire for treatment of their child decreased from $53 \%$ at $\mathrm{T} 1$ to $24 \%$ at $\mathrm{T} 2$, with the children's answers showing the same tendency, even though most children (83\%) and parents $(87 \%)$ felt that well-aligned teeth were important for overall facial appearance. Esthetics was the most frequent reason given for treatment by both children $(55 \%)$ and their parents $(37.5 \%)$. Of the group 128 children had completed or were undergoing orthodontic treatment. The results for treatment satisfaction were high, with $95 \%$ of treated children and $93 \%$ of their parent satisfied with their orthodontic results. Neither gender, self-esteem, a negative attitude to public funding or previous orthodontic treatment experience of parent had any influence on treatment uptake in this study. This study did not show an expected increase in the desire for treatment with the general increase in concern about appearance in teens.

Measuring the distribution, prevalence and severity of malocclusion and treatment need in a sample of Nigerian children was the focus of a study by 
Otuyemi et al. (1999). They used the Dental Aesthetic Index (DAI) to assess whether malocclusion was affected by age, gender, and socio-economic background. In this study sample, there were 703 children (328 males and 375 females) from Nigeria between 12 and 18 years of age. None of the children had a history of orthodontic treatment. Malocclusions were assessed using the DAI and all ten components were measured. The authors found no statistically significant differences in DAI scores between age groups, gender and socioeconomic background. Most of the children $(77 \%)$ had dental appearances indicated as "slight" or "not indicated" orthodontic treatment needed. About $13 \%$ were in the "elective" category, $6 \%$ were in the "highly desirable category and $4 \%$ fell in the mandatory category. When comparing the cumulative percentage frequency of DAI scores of various populations, Nigerian adolescents were found to have better dental aesthetics and less need for orthodontic treatment than American, Japanese, and Australian adolescents.

Kerosuo et al. (2004) studied the association between normative and selfperceived orthodontic treatment need in Arab high school students. The authors also evaluated the influence of sex, socioeconomic status, area of living and satisfaction with dental appearance on treatment need. The sample consisted of 139 students (70 girls and 69 boys) between 14 and 18 years of age for urban and rural areas of Kuwait. Data was collected from a questionnaire and clinical examination with treatment need assessed according to the IOTN. The IOTN grade combined the DHC and AC components and was determined for each subject according to the component that had the higher grade in the clinical inspection. The results reported definite need for orthodontic treatment (IOTN $4-5$ ) in $28 \%$ of the subjects. Self-reported treatment need among the same subjects was $34 \%$. There was agreement between normative and self-perceived treatment need in $77 \%$ of the subjects, showing that IOTN and its components DHC and AC correlated positively for this. In this study normative treatment need (IOTN) did not significantly differ between males and females. Girls tend to pursue treatment more than boys, sex differences regarding normative treatment need might result from and uneven distribution of orthodontic treatment between the sexes.

\section{Orthodontic Indices}

Orthodontic indices are used when considering the need for orthodontic treatment. Numerous authors (e.g., Helm 1977; Stricker et al. 1979; Brook and Shaw 1989) have studied factors involved in defining the need for orthodontic treatment. All emphasize a combination of psychological and dental esthetic factors, when considering the need for orthodontic treatment. 
Considering this, there arose a need for an orthodontic index that not only included the biological aspects of malocclusion but the psychological aspects also. The Dental Aesthetic Index (DAI) was developed by Cons et al. (1986) to be responsive to not only malocclusion conditions but the psychological harm that may occur if untreated. By linking the physical measurements of the traits associated with malocclusion with the public's perception of dental esthetics using a regression equation, a score is obtained that when placed on a scale gives an indication of the variance from the norm of acceptable dental appearance. The farther the score is from the norm, the more severe the dental appearance and the greater the likelihood that a physical or psychological handicap may occur if not treated.

Adding to this, Jenny et al. (1993) established a cut-off score that defined a handicapping malocclusion. In a study involving 1306 study casts of subjects between 15 and 18 years of age, orthodontists were asked to separate those with non-handicapping from those with handicapping occlusal conditions. The orthodontists' decisions were correlated with DAI scores resulting in a score of 36 as the point of the scale separating those handicapping malocclusion from non-handicapping (the greater the score the greater the severity).

Another study (Jenny and Cons, 1996) took the DAI information one step farther by establishing other points on the scale for decision-making purposes. By looking at the frequency distribution of the DAI scores and those from the Jenny et al. (1993) study along with data from the National Center for Health Statistics, points of severity along the scale were established along with percent of the NCHS sample encompassed by each interval of the DAI scale. A score of 25 or lower indicates normal or minor malocclusion with no treatment needed. A score of 26 to 30 on the DAI indicates a definite malocclusion with treatment elective. A score of 31 to 36 indicates a severe malocclusion with treatment highly desirable, while a score of 36 or higher indicates a very severe malocclusion with treatment mandatory.

Brook and Shaw (1989) also developed a system for scoring malocclusions, a system that they labeled the Index of Treatment Need (IOTN). The index consists of five grades, (1) no need for treatment, (2) mild or little need for treatment, (3) moderate or borderline need for treatment, (4) severe, meaning a need for treatment and (5) extreme, meaning definite need for treatment. The index includes a dental occlusion and alignment component as well as an esthetic component that evaluates dental appearance against an ordinal scale of ten standard photographs. A consensus of a panel of orthodontists was used to determine the degree of severity of the various malocclusions. 
There are various ways to measure malocclusion to classify its severity. The one outlined in this investigation along will the DAI and the IOTN were used to show a continuum of malocclusion severity as it relates to tooth size. The hypothesis is that there will be a discernible statistical correlation between tooth size and severity of malocclusion. The anticipated relationship is that, as tooth size increases so does the severity of the malocclusion within each sex. 


\section{CHAPTER III. MATERIALS AND METHODS}

The sample for this study consisted of North American white adolescent boys and girls, between 11 and 25 years of age. There were 107 boys and 100 girls in the sample. Mean age was 16.2 years ( $\mathrm{sd}=4.6$ years). Most subjects were selected from orthodontic cases treated in the dental school, but the adults were dental students who had not been treated orthodontically. All cases with a condition known to affect tooth size were excluded. The dental records were selected based on the following criteria: all 24 teeth fully erupted and intact (ignoring second and third molars), and all had good quality pretreatment dental casts.

With the intent of increasing the range (and within-sample variability) of the data, we included 38 casts of males with naturally-occurring good occlusions. These were collected from dental students and have been reported by Agenter (2008). Only two cases of women with naturally-occurring good occlusions were included in the present sample. Consequently, there is an inherent bias in any biological interpretation of sexual dimorphism with regard to the incidence of severity of malocclusion because we have intentionally and knowingly "loaded" the sample with men who have effectively no dentoalveolar malocclusion. The effect of "sex" in the ANCOVA and other models analyzed here should only be viewed as accounting for a "nuisance variable," not as any reliable measure of whether the two sexes differ in the severity of malocclusion.

Teeth on one side of the midline of the upper and lower arches were measured (7 teeth in each arch, the central incisor through the first molar), for a total of 14 measurements per subject. Maximum mesio-distal crown measurements (Seipel 1946) were taken using digital-readout, sliding calipers using the methods described by Moorrees (1959). The beaks of the calipers had been machined to fit well into the embrasures.

\section{Nomenclature and Odontometric Method}

Various naming, lettering, and coding systems have been used to refer to specific tooth types (reviewed by Peck and Peck 1993). A combination of letters

and numbers was used in the present study corresponding to conventions in the anthropological and genetic literature. The system in the present study was to refer to the four tooth types by their initials, namely incisor (I), canine (C), premolar $(\mathrm{P})$, and molar $(\mathrm{M})$, and to code a tooth's location within each morphogenetic complex by its position, mesial to distal (e.g., Dahlberg 1945, 1951). Arcade and side of the body, where applicable, are written-out for clarity. 
It is tedious and space-consuming to constantly write-out the full names of each of the 14 tooth types evaluated here, and it is expedient to use a coding system (Peck and Peck 1993; Harris 2005). Codes used here are (1) to denote the dental arch as maxillary ( $\mathrm{U}$ for upper) and mandibular (L for lower), (2) to distinguish left $(\mathrm{L})$ and right $(\mathrm{R})$ sides of the arch when necessary, and (3) to use Palmer notation to code the teeth based on their positions and sequence from front to back namely

1. central incisor

2. lateral incisor

3. canine

4. first premolar

5. second premolar

6. first molar

7. second molar

Aspects of the tooth's crown are referred to by using conventional anatomical descriptions, namely mesial, distal, buccal, and lingual (e.g., Zeisz and Nuckolls 1949; Kraus et al. 1969; Ash 1993). A tooth's two major occlusal axes are mesiodistal (MD) and buccolingual (BL). These directions are, more specifically, mediolateral and faciolingual for the incisors, but, by convention, the terms MD and BL are used throughout this work. Following conventions (e.g., Moorrees 1957, 1959), mesiodistal crown diameters are termed lengths while buccolingual crown diameters are termed widths.

Since each tooth crown has a complex three-dimensional morphology, obtaining "maximum" MD and BL dimensions requires experience and systemization (Seipel 1946; Kieser et al. 1990). There is some art as well as science in obtaining homologous tooth dimensions within a tooth type among individuals. General statements can be made regarding methodology, but one needs to be cautious when comparing among investigators. The three mostfrequently cited descriptions of how to measure maximum MD and BL crown dimensions are those of Lundström (1948), Selmer-Olsen (1949), and Moorrees (1957). Mesiodistal crown length is defined as the maximum distance between a crown's mesial and distal anatomic contact points when a tooth is in its normal position (not rotated or otherwise deviated from its ideal position in the dental arch). The measurement was made with sliding calipers held perpendicular to the tooth's long axis, which should be approximately parallel with the occlusal plane. Adjustments were made to account for tooth rotations and shifts in axial inclinations (Seipel 1946). The measurement should not be influenced by positions of adjacent teeth. Figure 16 illustrates the orientation of maximum MD dimensions on the four maxillary tooth types. Maximum width normally is at the occlusal edge of incisors, but lower, at the height of contour in premolars, 

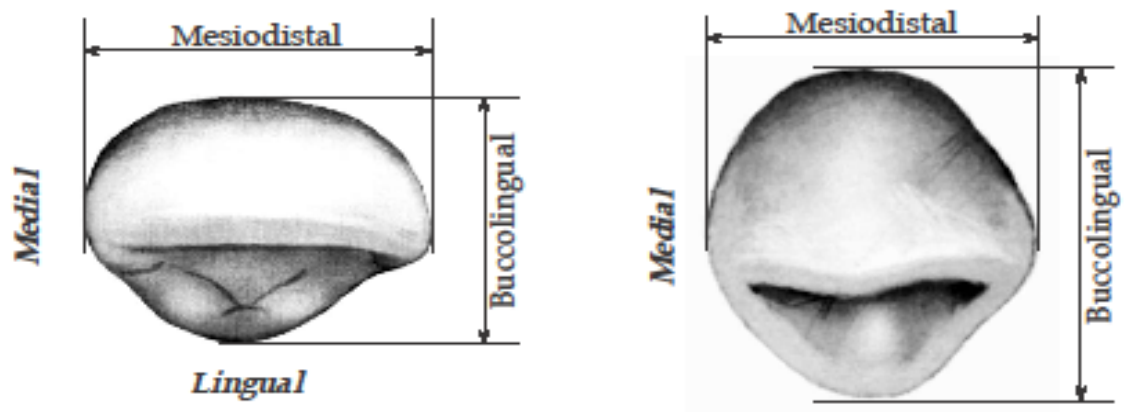

\section{Lingual}
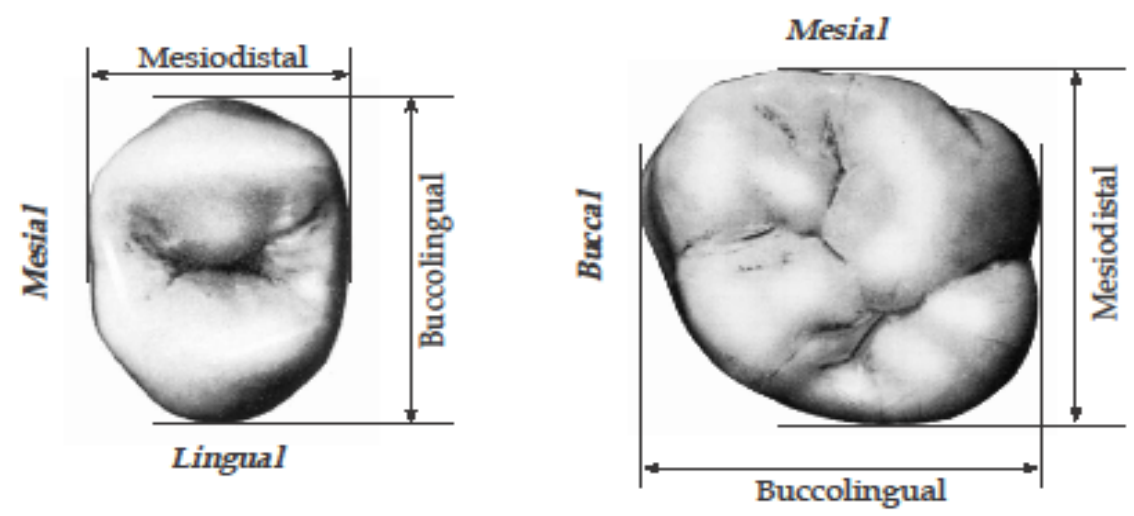

Figure 16. Examples of the four permanent human tooth types showing the axis along which maximum mesiodistal crown diameters were measured using electronic-readout sliding calipers.

Maxillary teeth are illustrated here, incisor (top left), canine (top right), premolar (bottom left), and molar (bottom right). 
and lower yet, at or near the gingival border (and dentinoenamel junction) on canines and molars.

\section{Measurement Methods}

Obtaining dental measurements involves several judgments about landmark locations, and these judgments can affect the acquired dimensions of a sample, both systematically (due to one's measurement style and one's interpretations of dimensions' definitions) and in terms of sample variability (due to interobserver measurement inconsistencies). Kieser and coworkers (1990, 1991) have explored within- and among-observer variations in tooth size determinations, and several other investigators have examined the problem in other areas of human biology (e.g., Houston 1983). Useful articles dealing with non-dental topics are by Gavan (1950), van der Linden et al. (1970), Utermohle et al. (1983), and Knapp (1992).

Additional concerns occur when one is measuring dental casts rather than real teeth because casting errors in the monochromatic dental material need to be distinguished from actual tooth anatomy. Notably, casting defects that result in blebs of dental stone on a tooth can artificially inflate crown dimensions. Another common problem involves gingival distortions that are incorporated into the dental casts. These are of two sorts, (1) stripping (gingival recession, particularly adjacent to a tooth root's labial and lingual margins) and (2) gingival hyperplasia, where the gingival is inflamed or otherwise hyperplastic, so it covers essential tooth crown landmarks near the cementoenamel junction. Obviously, it is important not to confound measurements of crown size by including artifacts of the gingival morphology that can, variably, increase or decrease the true crown dimensions.

The comments in this section are provided to give the reader a better sense of how the size determinations were obtained in the present study. That is, it is commonplace for researchers in this field to cite published sources, such as Seipel (1946) or Moorrees (1957), as containing definitions of the measurements employed. These definitions are, however, very general. As an example, Moorrees (1957:78) states that a tooth's mesiodistal diameter is "the greatest mesiodistal dimension of the tooth crown, measured parallel to the occlusal and labial surfaces." His operational definition of the BL diameter is equally generic, "the greatest distance between the labial and lingual surfaces of the tooth crown in a plane perpendicular to that in which the mesiodistal diameter was measured" (1957:80). 


\section{Mesiodistal Dimensions}

Maximum MD diameter of the incisor typically occurs at its occlusal edges so the caliper beaks can be positioned just below the line angles of the tooth, perpendicular to the crown's occlusogingival long axis (which typically is normal to the occlusal plane). On dental casts, this often requires that the sharpened beaks of the calipers be pressed into the embrasures, so the measurements are somewhat less than would be obtained from actual teeth, especially isolated teeth where the beaks can truly span the mesial and distal margin of the tooth's crown.

Maximum MD distances of the canines, premolars, and molars occur at the heights of contour. These heights generally are midway up the crown's facial outline, but may be more gingival in some tooth forms. It is important to hold the calipers parallel with the occlusal plane (specifically, perpendicular to each tooth crown's occlusogingival long axis) and to accommodate to a rotated tooth's mesiodistal occlusal axis. When adjacent teeth have a tight contact, the points of the caliper beaks need to be pressed into the embrasures. One should recognize, though, that such dimensions will be smaller than for isolated teeth (and those cases with interdental spacing). Teeth with mesial and/or distal wear facets due to abrasion against adjacent teeth will yield smaller dimensions. There also is the issue that the measurer should not position the calipers to include the actual heel of the terminal molar. This would angulate the calipers relative to the tooth's mesiodistal axis. Instead, beaks need to be positioned so the measurement if made parallel with the tooth row (unless, of course, the molar itself is rotated). Normally, the MD dimension of the terminal molar will be from the buccal side of the embrasure distal to a point on the metacone (distobuccal cusp) such that the calipers' beaks are at right angles to the tooth row (and with the calipers at the height of contour for that tooth and perpendicular to the crown's occlusogingival axis).

\section{Crown Size Dimorphism}

Sexual dimorphism in tooth crown dimensions needs to be accounted for in the statistical analysis, but it is not central to this research project. Consequently, it is described here (rather than in Results), largely to confirm that it is a pertinent source of variation. Descriptive statistics are listed in Table 1, along with the results of one-way ANOVA tests.

Most of these mesiodistal dimensions are significantly different between the sexes. Exceptions are (1) the maxillary lateral incisor, (2) the maxillary first 
Table 1. Descriptive statistics of mesiodistal crown dimensions, by sex, and tests for sexual dimorphism.

\begin{tabular}{|c|c|c|c|c|c|c|c|c|c|c|}
\hline \multirow[b]{2}{*}{ Tooth Type } & \multicolumn{3}{|c|}{ Males } & \multicolumn{3}{|c|}{ Females } & \multirow{2}{*}{$\begin{array}{c}\mathrm{r}^{2} \\
\text { (Percent) }\end{array}$} & \multirow{2}{*}{$\begin{array}{c}\text { Percent } \\
\text { Dimorph. }\end{array}$} & \multirow[b]{2}{*}{ F-Ratio } & \multirow[b]{2}{*}{ P-Value } \\
\hline & $\mathrm{n}$ & $\bar{x}$ & se & $\mathrm{n}$ & $\bar{x}$ & se & & & & \\
\hline \multicolumn{11}{|c|}{ Maxilla } \\
\hline Central Incisor & 107 & 8.82 & 0.05 & 100 & 8.53 & 0.05 & 6.59 & 3.35 & 14.47 & 0.0002 \\
\hline Lateral Incisor & 107 & 6.69 & 0.06 & 100 & 6.57 & 0.06 & 1.03 & 1.84 & 2.13 & 0.1462 \\
\hline Canine & 107 & 7.98 & 0.04 & 100 & 7.65 & 0.04 & 12.81 & 4.34 & 30.13 & $<0.0001$ \\
\hline First Premolar & 107 & 6.96 & 0.04 & 100 & 6.88 & 0.04 & 0.89 & 1.15 & 1.84 & 0.1765 \\
\hline Second Premolar & 107 & 6.75 & 0.04 & 100 & 6.61 & 0.04 & 2.93 & 2.26 & 6.19 & 0.0137 \\
\hline First Molar & 107 & 10.48 & 0.05 & 100 & 10.20 & 0.05 & 6.60 & 2.80 & 14.47 & 0.0002 \\
\hline Second Molar & 107 & 9.87 & 0.06 & 100 & 9.62 & 0.06 & 4.65 & 2.60 & 9.99 & 0.0018 \\
\hline \multicolumn{11}{|c|}{ Mandible } \\
\hline Central Incisor & 107 & 5.47 & 0.03 & 100 & 5.33 & 0.04 & 4.05 & 2.73 & 8.65 & 0.0036 \\
\hline Lateral Incisor & 107 & 5.97 & 0.04 & 100 & 5.84 & 0.04 & 2.38 & 2.16 & 4.99 & 0.0265 \\
\hline Canine & 107 & 6.92 & 0.04 & 100 & 6.55 & 0.04 & 15.88 & 5.58 & 38.70 & $<0.0001$ \\
\hline First Premolars & 107 & 7.14 & 0.04 & 100 & 7.02 & 0.04 & 2.08 & 1.69 & 4.36 & 0.0380 \\
\hline Second Premolar & 107 & 7.24 & 0.04 & 100 & 7.13 & 0.04 & 1.52 & 1.54 & 3.16 & 0.0768 \\
\hline First Molar & 107 & 11.23 & 0.05 & 100 & 10.89 & 0.06 & 8.35 & 3.08 & 18.67 & $<0.0001$ \\
\hline Second Molar & 107 & 10.35 & 0.06 & 100 & 10.13 & 0.06 & 3.19 & 2.17 & 6.76 & 0.0100 \\
\hline \multicolumn{11}{|c|}{ Combined Dimensions } \\
\hline Maxillary Sum & 107 & 57.55 & 0.25 & 100 & 56.05 & 0.26 & 7.83 & 2.68 & 17.41 & $<0.0001$ \\
\hline Mandibular Sum & 107 & 54.31 & 0.23 & 100 & 52.89 & 0.24 & 7.97 & 2.69 & 17.75 & $<0.0001$ \\
\hline Grand Sum & 107 & 111.86 & 0.47 & 100 & 108.94 & 0.48 & 8.40 & 2.68 & 18.79 & $<0.0001$ \\
\hline
\end{tabular}


premolar, and (3) the mandibular second premolar. Percent of sexual dimorphism ranges from a low of $1 \%$ (UI2) up to about $6 \%$.

Overall, sex differences in these American whites average about $3 \mathrm{~mm}$ (see "grand sum") for the 14 tooth types, which translates to about $6 \mathrm{~mm}$ for the full arch perimeters. The statistical impact of accounting for "sex" in the analytical models $\mathrm{r}^{2}$ (i.e., how much of the variation in tooth size is accounting for by the subject's sex) can be an important percentage of the totals. Overall, $\mathrm{r}^{2}$ is over $8 \%$ for the sum of all tooth types; individually, it ranges up to $16 \%$ for the mandibular canine. In other words, not accounting for subject's sex could emphatically confound interpretations of the statistical results.

\section{Quantifying Crowding}

A key issue in this study was how to quantify the severity of malocclusion specifically as regards tooth-size arch-size discrepancies (TSASD). What was desired here was an expedient means of arranging cases, from those with no TSASD along a continuum up to those with severe TSASD. The scoring had to take into account the potential variations in tooth position in all three planes of space (plus axial rotations), but had to be simple enough that a lot of cases could be assessed. On the other hand, skeletal malrelationships should not enter the equation since they have separate etiologies (Harris and Smith 1980; Harris and Johnson 1991) and, while important, skeletal relationships do not pertain to how the teeth per se are accommodated into the existing arch space. There are, of course, conventional methods of quantifying occlusal variations (e.g., Little 1976; Harris et al. 1987), but they can be insensitive to capturing the degree of dental crowding and, moreover, can be time-consuming, which detracts from elaborating the sample size of a study.

We developed a semi-continuous scale of malocclusion that (1) focuses on the anterior dental segment, where most rotations and displacements occur (Grainger 1967; Kelly and Harvey 1977) and (2) is based on expedient, visual assessments (ordinal grades), while preserving a broad range of inter-individual variation. The methods borrows heavily on the TPI (treatment priority index) developed by the National Center for Health Statistics (Kelly and Harvey 1977). There are three aspects of the index that we collectively label as "crowding," namely (A) tooth displacements, (B) axial tooth rotations, and (C) mesiodistal overlapping of teeth. Just the maxillary six teeth (2 canines and 4 incisors) are scored for these three variables. 


\section{Displacements}

Each of the six maxillary anterior teeth (I1, I2, C in both quadrants) was scored for its degree of displacement buccolingually out of the idealized arch form. Each displacement was given an ordinal score of 0, 1, or 2, and it was not important whether the displacement involves labioversion or linguoversion (Figure 17):

0 . The tooth's buccolingual position is in the idealized arch form.

1. The tooth is displaced (buccally or lingually), but less than $2 \mathrm{~mm}$.

2. The tooth is displaced $2 \mathrm{~mm}$ or more, either buccally or lingually.

Scores of the six displacements are summed, with a potential score between 0 and 12.

\section{Rotations}

Teeth can be in the desired position labiolingually and mesiodistally, but rotated on their long axis. Typically, additional arch space is required to derotate a tooth into its idealized position. The axial rotation of each of the six maxillary anterior teeth was scored (Figure 18):

0. Rotation is normal relative to the idealized arch from, without torsiversion.

1. The tooth is rotated on its long axis, but less than 30 degrees.

2. The tooth is rotated on its long axis more than 30 degrees.

Scores of the six rotations are summed, with a potential range from 0 to 12 .

\section{Mediolateral Overlap}

The intent here was to quantify the amount of mesiodistal tooth crown size that cannot be accommodated into the arch form without treatment. The mediolateral overlap of a tooth is the extent to which tooth crowns overlap one another in the arch by virtue of their buccolingual displacements. For example, the maxillary lateral incisors often erupt lingual to the central incisors, so the mesial aspect of the lateral tooth overlaps the lateral aspect of the central. The greater the overlap, the more arch space that has to be obtained during treatment 


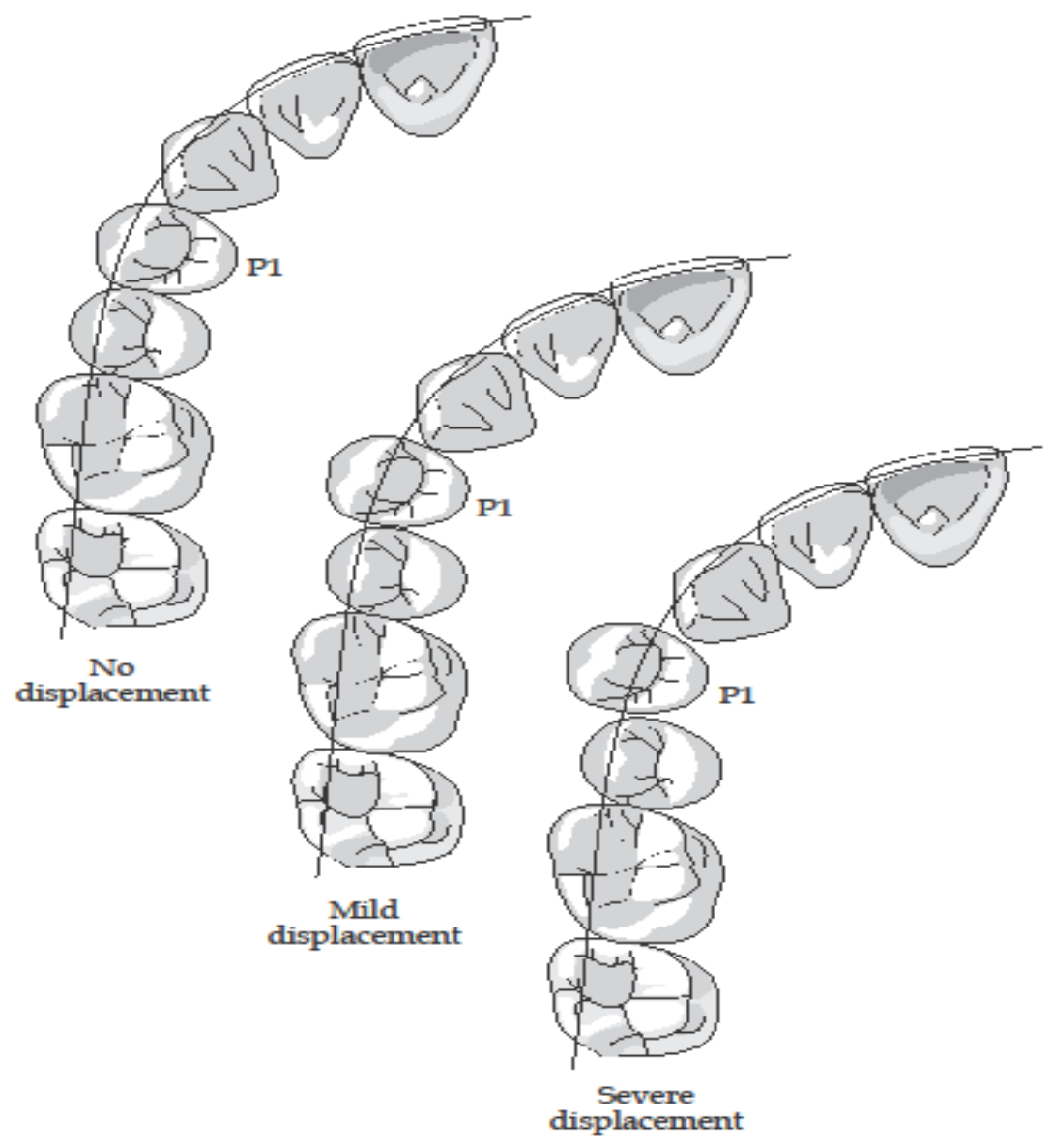

Figure 17. Schematic depictions of the occlusal view of the maxillary right quadrant.

Left: An arch form (heavy line) is shown that approximates the best fit to the buccal cusps of the teeth. None of the teeth is displaced. Middle: The first premolar is shown to be slightly $(<2 \mathrm{~mm})$ displaced from the overall arch form. Right: Severely displaced second premolar $(>2 \mathrm{~m})$. 


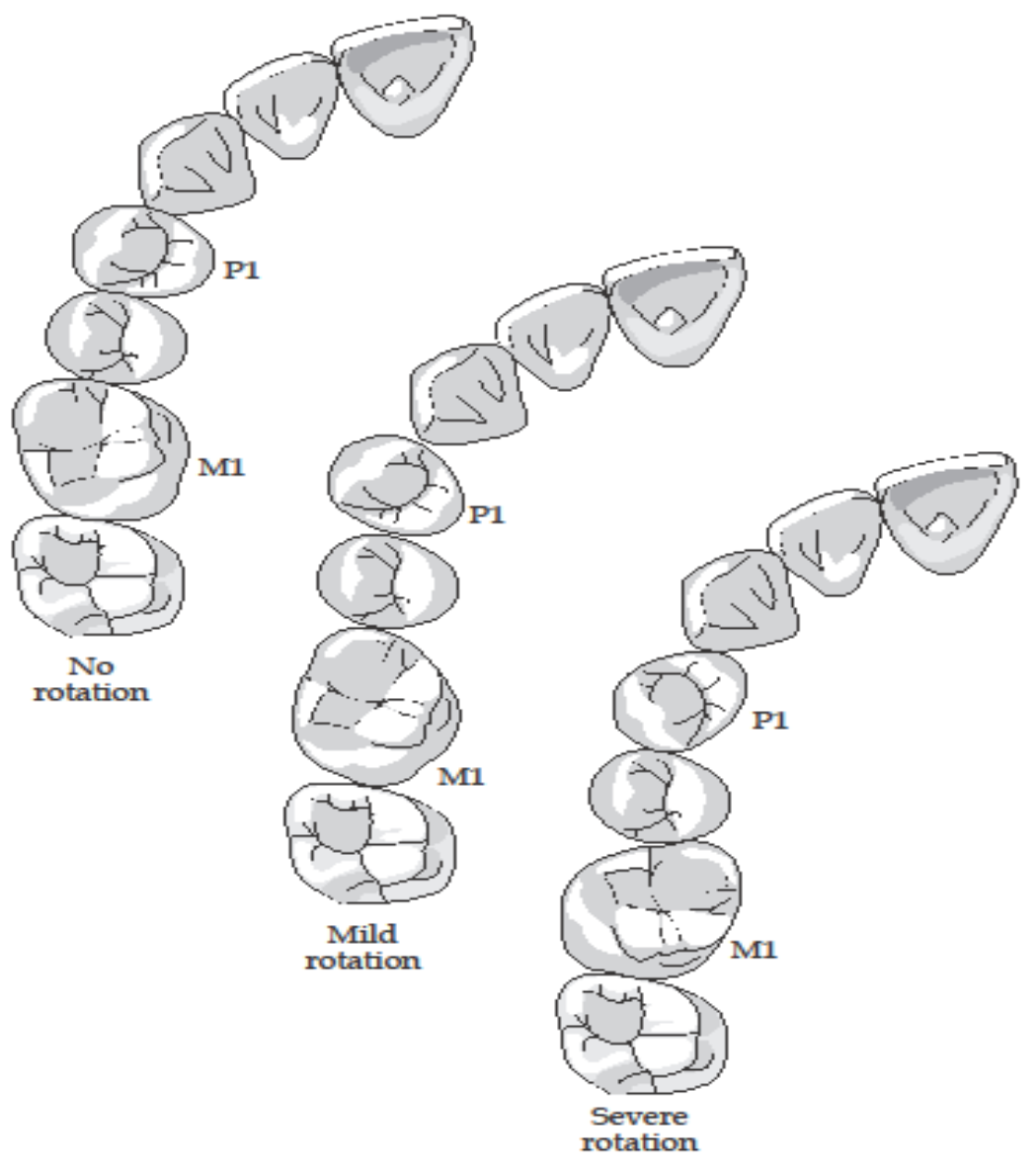

Figure 18. Schematic examples of how tooth rotations were scored.

Left: All teeth in the quadrant are in their idealized positions. Middle: First premolar is mildly rotated (clockwise rotation in this figure) and the first molar is mildly rotated (also clockwise). Right: First premolar is severely rotated (counterclockwise) as is the first molar, with about 90 degrees of clockwise rotation. 
to resolve the TSASD. The mediolateral overlap of each of the six maxillary anterior teeth was scored as:

0 . No overlap (though there may be displacement and/or rotation).

1. Up to $2 \mathrm{~mm}$ of overlap, from visual assessment of the mediol and lateral aspects of each tooth.

2. Over $2 \mathrm{~mm}$ of overlap, assessing both the mediol and lateral aspects.

Summation of displacements, rotations, and dental overlap provides a score from 0 (perfect arch form) to a potential upper limit of 36. This "crowding" provides a semi-quantitative scale of the severity of TSASD that is quick and easy to score (Figure 19). Refinements, not considered here, would be to devise a weighting scheme since there probably is considerable left-right symmetry to the malocclusion that, statistically, involves redundancy of information, as well as redundancy among the three components to the score (displacements, rotations, overlapping). So, too, this score only applies to the maxillary arch.

\section{Dental Aesthetic Index}

The Dental Aesthetic Index (DAI) developed by Cons et. al. (1996) is aimed at integrating the psychological and physical elements of malocclusion, linking the physical measurements of malocclusion with the public perception of dental esthetics arithmetically. The DAI is formulated as a regression equation with 10 predictive variables (Jenny and Cons 1996). A subject (or his dental casts) is scored for each of 10 variables. These scores are multiplied by their regression coefficients (weights), then added together along with a constant (the intercept of the equation). The sum is the DAI score. After a score had been obtained, it can be placed on a scale that spans a range from most to least esthetic dental appearance. The farther a DAI score is from the norm of acceptable dental appearance the greater the chances that the malocclusion could be socially or physically handicapping if left untreated.

The DAI (dental aesthetic index) was developed by Jenny and Cons (1996) as a means of quantifying the impact of the dentition on facial esthetics. A total of 10 variables are scored, and these can be done with equal precision on subjects themselves, or on their dental casts. A multiple regression coefficient is provided by these authors that yields a value; the lower the value, the more esthetic the dentition is gauged to be. Conversely, the higher the number, the more the dentition is felt to harm a person's facial esthetics. The ten components of the DAI are scored as follows: 


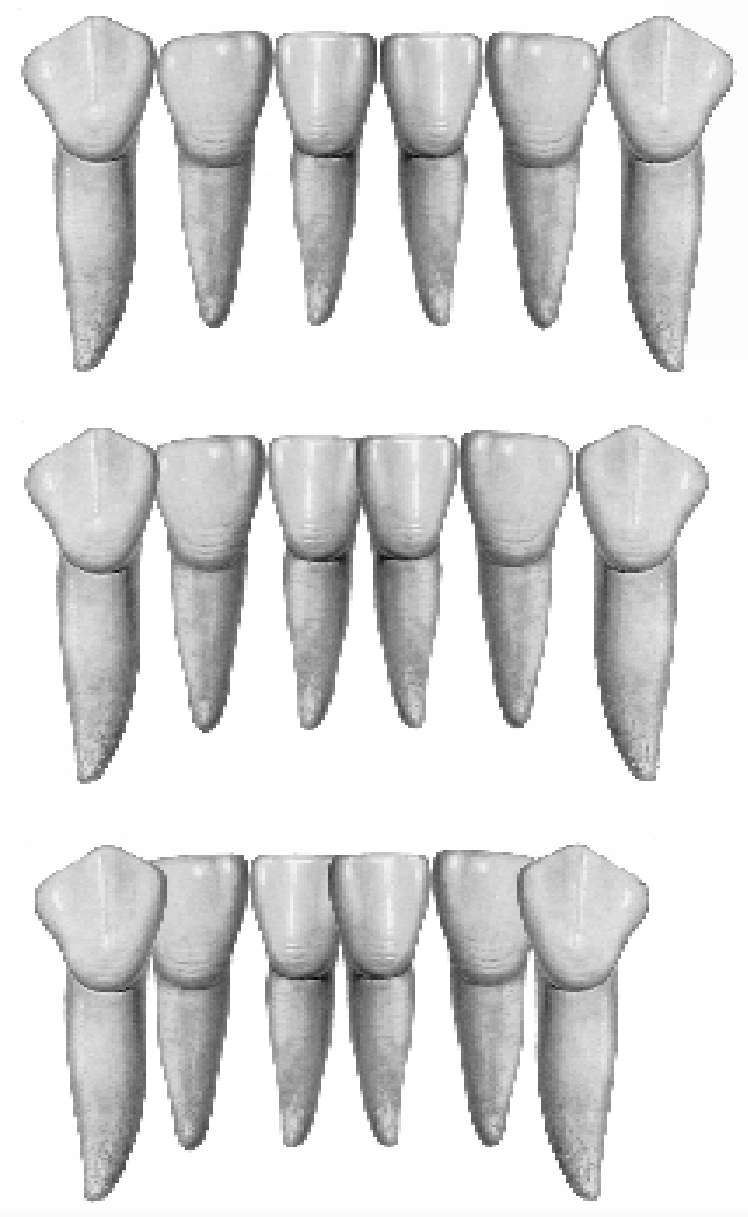

Figure 19. Labial views of the six mandibular anterior teeth. "Overlap" was scored on a three-grade scheme at each of the five contacts shown in this schematic.

Top: All six teeth are correctly approximated (overlap scores of 0$)$. Middle: The overlap scores are zero, except for the central incisors with minor overlap (score+1). Bottom: There is moderate overlap of at least $2 \mathrm{~mm}$ between the canine and lateral incisor in each quadrant, and minor overlap (1 to 2 $\mathrm{mm}$ ) between the central incisors. 


\section{Missing Teeth}

Missing teeth can arise from a variety of causes, such as congenital absence, trauma, caries, or periodontal involvement. In the adolescent age group, cases would most commonly result from congenital absence. The JennyCons system counts the number of missing incisors, canines, and premolars in the two arches. In fact, no instance of a missing tooth was encountered in the sample studied in the present investigation.

\section{Incisor Crowding}

Crowding is scored using a simple three-grade ordinal scale. Either there was no crowding (score 0), which, predictably, was uncommon in the present study; there was crowding in one jaw (score 1); or there was crowding in both jaws (score 2). An example of severe incisor crowding is illustrated in Figure 20.

\section{Incisor Spacing}

Analogous to crowding, interdental spacing among the incisors was scored using a three-grade ordinal scale. Either there was no spacing in either arch (score 0); there was spacing in one arch (score 1); or there was spacing in both arches (score 2).

\section{Midline Diastema}

This is the mediolateral width of the diastema that may occur between the maxillary central incisors. Width of the diastema is measured millimetrically with sliding calipers; when a diastema is absent, the score is zero. Figure 21 is a schematic depiction of a diastema between the maxillary central incisors.

\section{Anterior Irregularity Maxilla}

The anatomic contacts of the incisors and canines should be closely approximated in an ideal arch. In the Jenny-Cons system, the single largest maxillary discrepancy between the contacts of the adjacent teeth is scored using sliding calipers (Figure 22). This differs from the familiar orthodontic measure of incisor irregularity (Little 1976), where all of the open contacts are considered. 


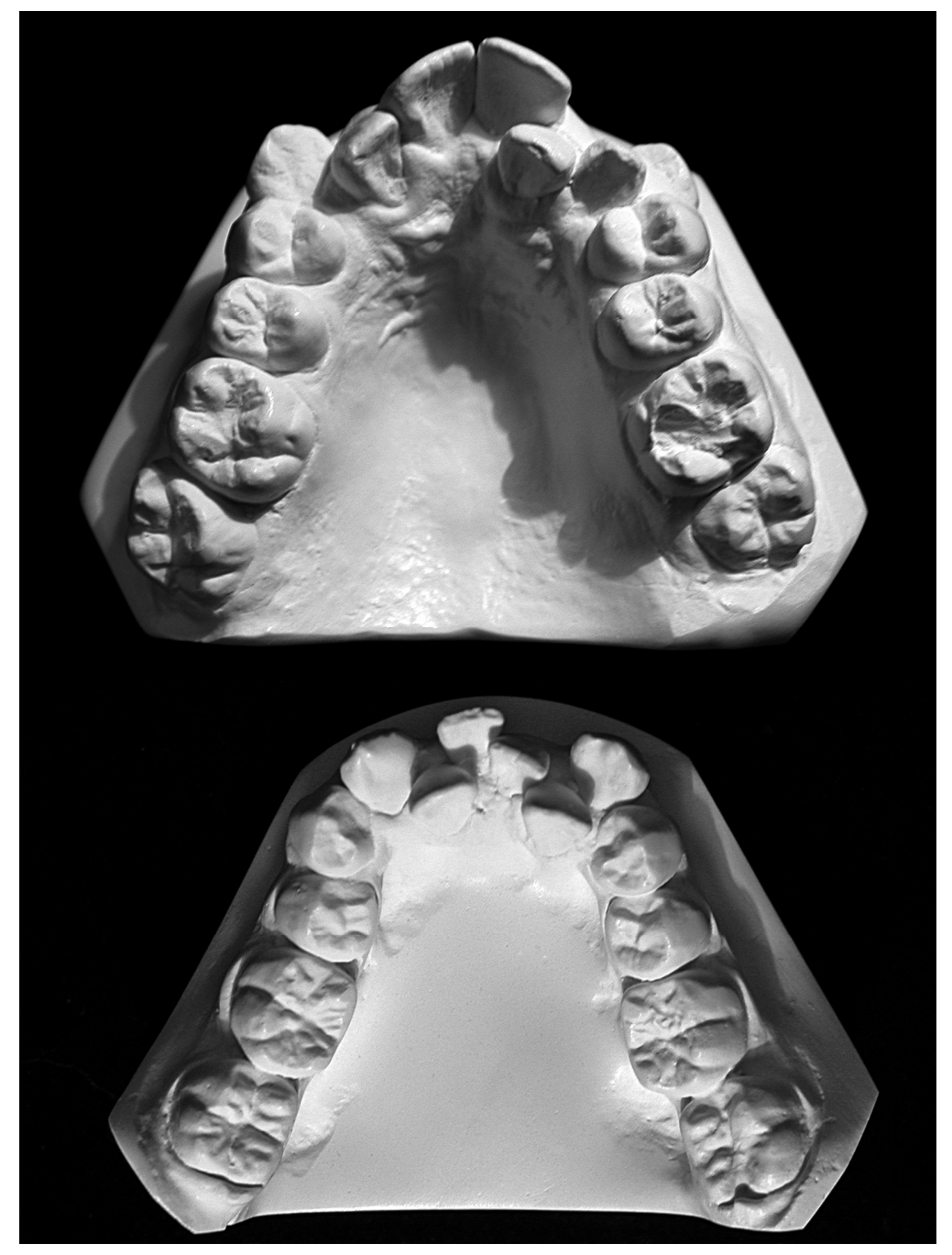

Figure 20. Occlusal views of the dental arches of an orthodontic patient at pretreatment who exhibits severe incisor crowding. 

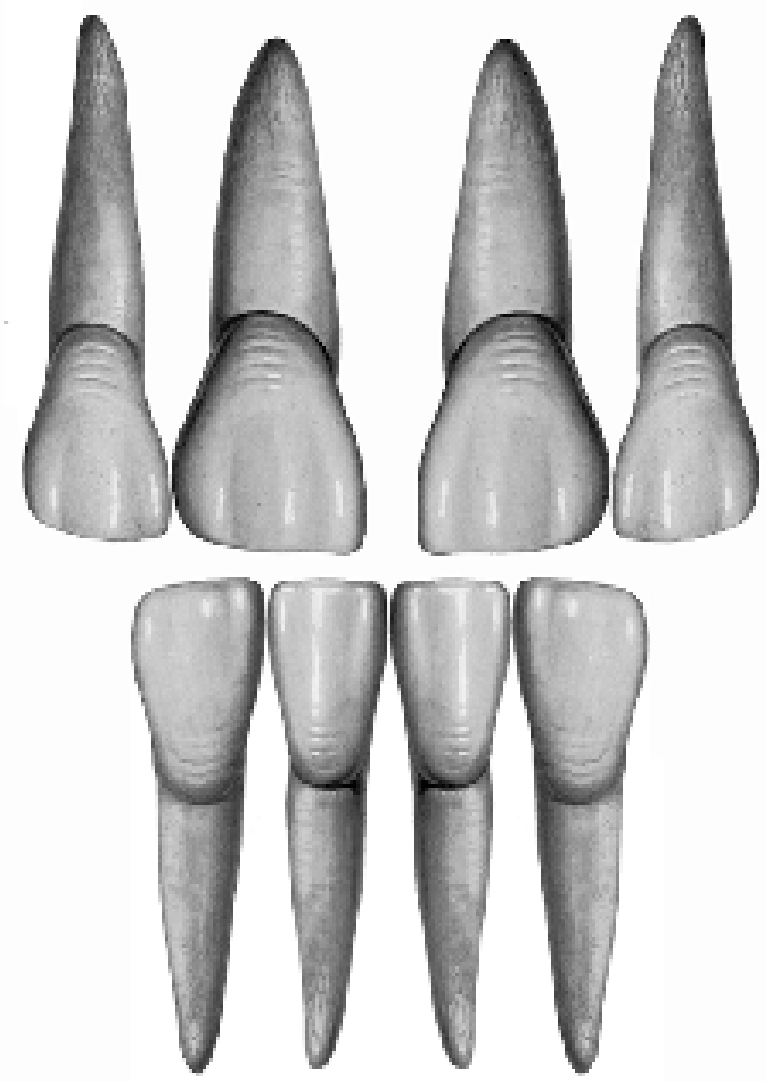

Figure 21. Schematic depiction of a diastema between the maxillary central incisors. 


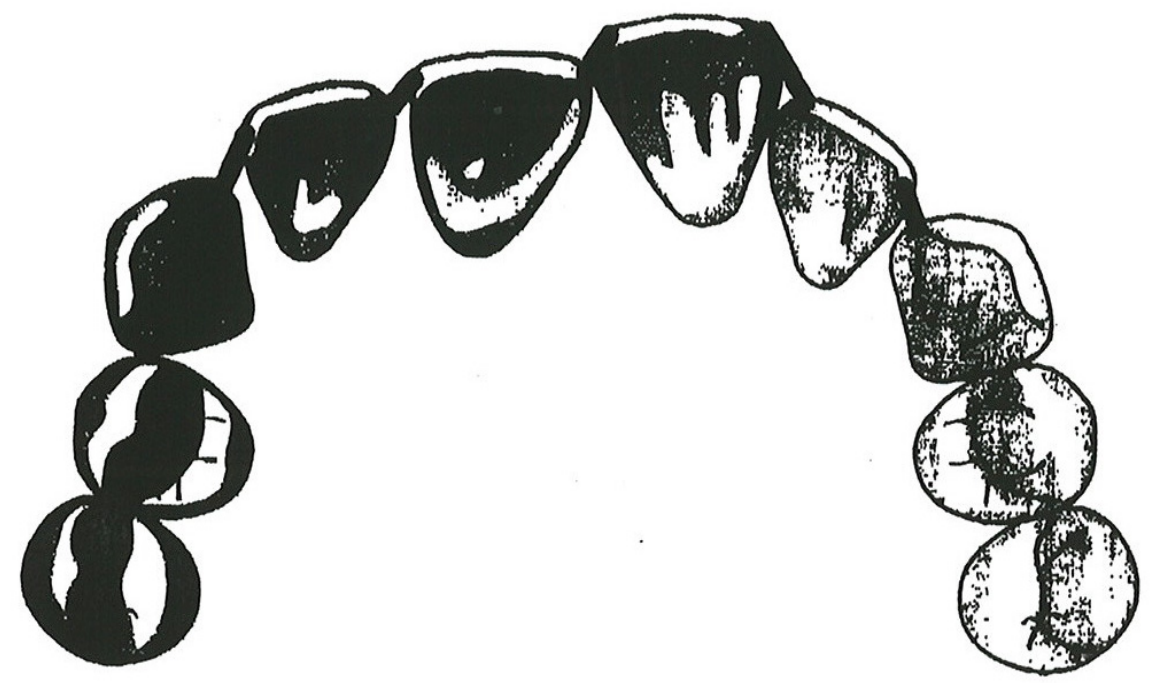

Figure 22. Schematic view of a maxillary dental arch. Heavy lines depict the irregularity between anterior teeth, where the anatomic contacts are not approximated.

In the Jenny-Cons scoring system, only the largest discrepancy in an arch is recorded, in this case the distance between the contacts of the left central and lateral incisors. 


\section{Anterior Irregularity Mandible}

The single largest mandibular discrepancy among the incisor and canine tooth types is scored. This millimetric value is entered into the DAI equation.

\section{Maxillary Overjet}

This is a millimetric value, where the maxillary and mandibular casts are placed in maximum interdigitation, and overjet from the labial of the most prominent maxillary central incisor to the homologous mandibular incisor is measured along the occlusal plane. This is illustrated in Figure 23.

\section{Mandibular Overjet}

When there is mandibular prognathism such that the anterior teeth are in crossbite, then this value is measured (and "maxillary overjet" is ignored). Mandibular overjet ("underjet") is measured millimetrically with sliding calipers from the labial surface of the most prominent mandibular incisor to the labial surface of the homologous maxillary incisor (Figure 23).

\section{Anterior Openbite}

This vertical measurement is included when the incisors do not overlap vertically (Figure 24). Openbite is the maximum millimetric distance between the opposing incisal edges measured perpendicular to the occlusal plane.

\section{Buccal Molar Relationship}

This is scored along a three-grade ordinal scale. The more-deviant side of the arch is recorded, and if there is a Class I molar relationship, the score is 0. This occurs when the mesiobuccal cusp of the maxillary first molar is located against the buccal groove of the mandibular first molar when viewed in norma lateralis. When, instead, the cusp-to-groove relationship is off by up to $1 / 2$-cusp either mesially or distally, the score is 1 . As the third option, if the deviation exceeds one-half cusp, the score is 2 . Buccal molar relationships of the permanent first molars are illustrated in Figure 25.

In the present study, we used each of these ten constituent variables individually as measures of dental malocclusions (excepting "missing teeth" that 

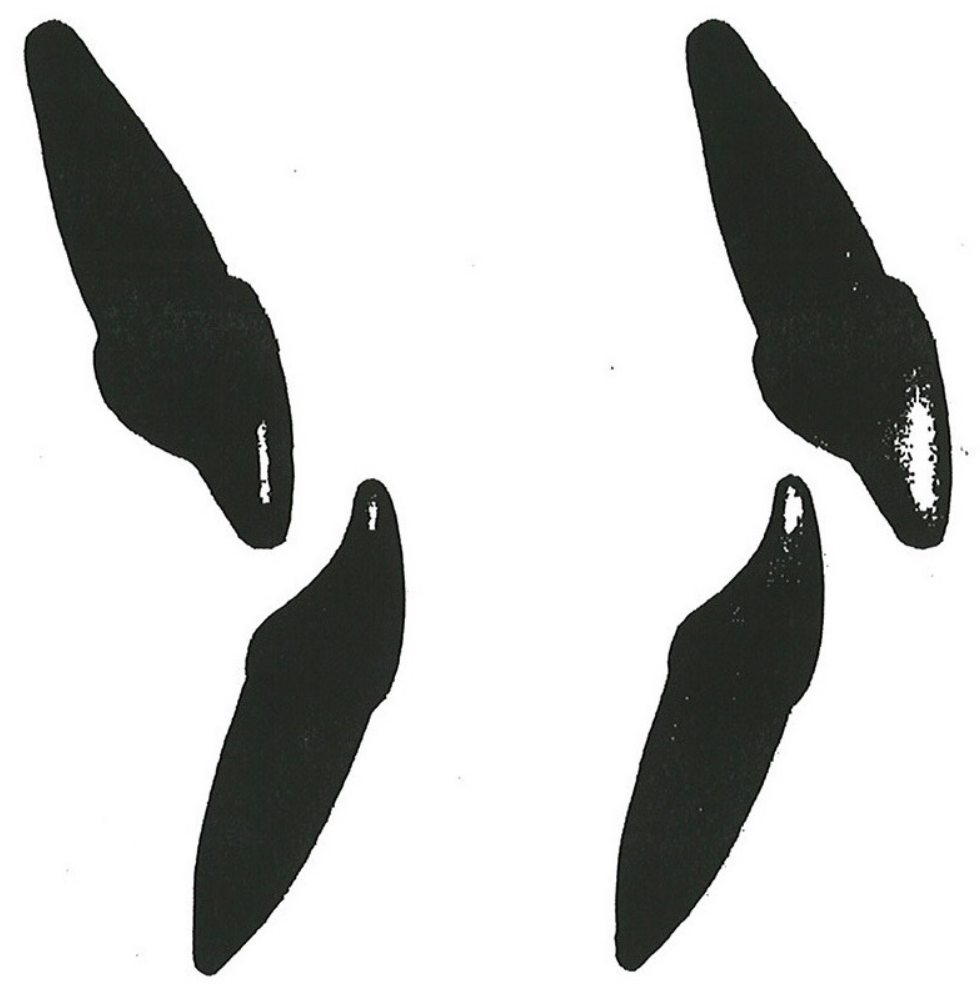

Figure 23. Depictions of horizontal incisor relationships between the arches.

Left: An anterior crossbite termed here a negative overjet. Right: The incisor overjet is the horizontal distance between the labial incisal surfaces of the upper and lower incisors parallel with the occlusal plane. 

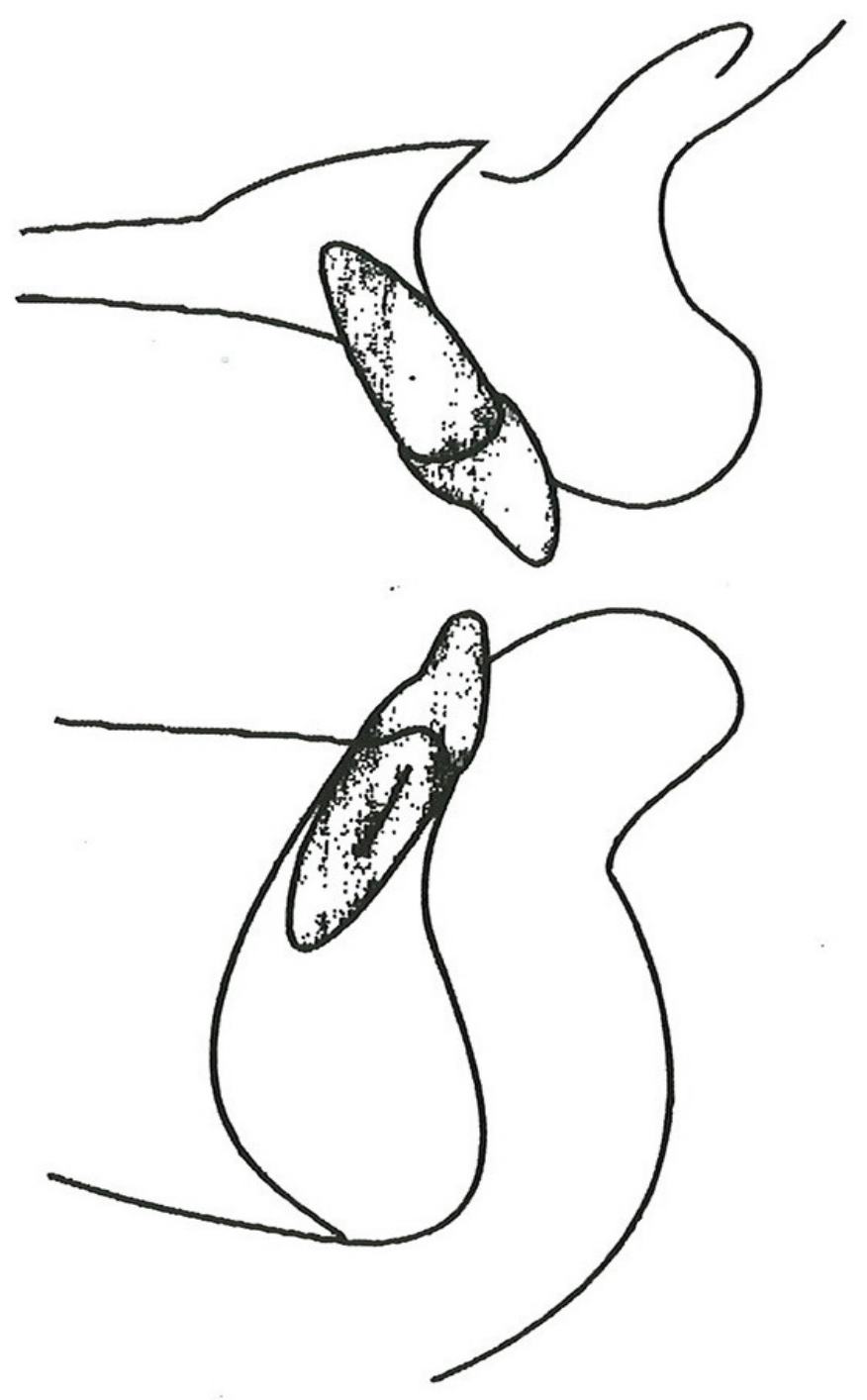

Figure 24. Depiction of the lateral view of the lower face showing an anterior openbite, where the incisal edges of the central incisors do not overlap vertically. 

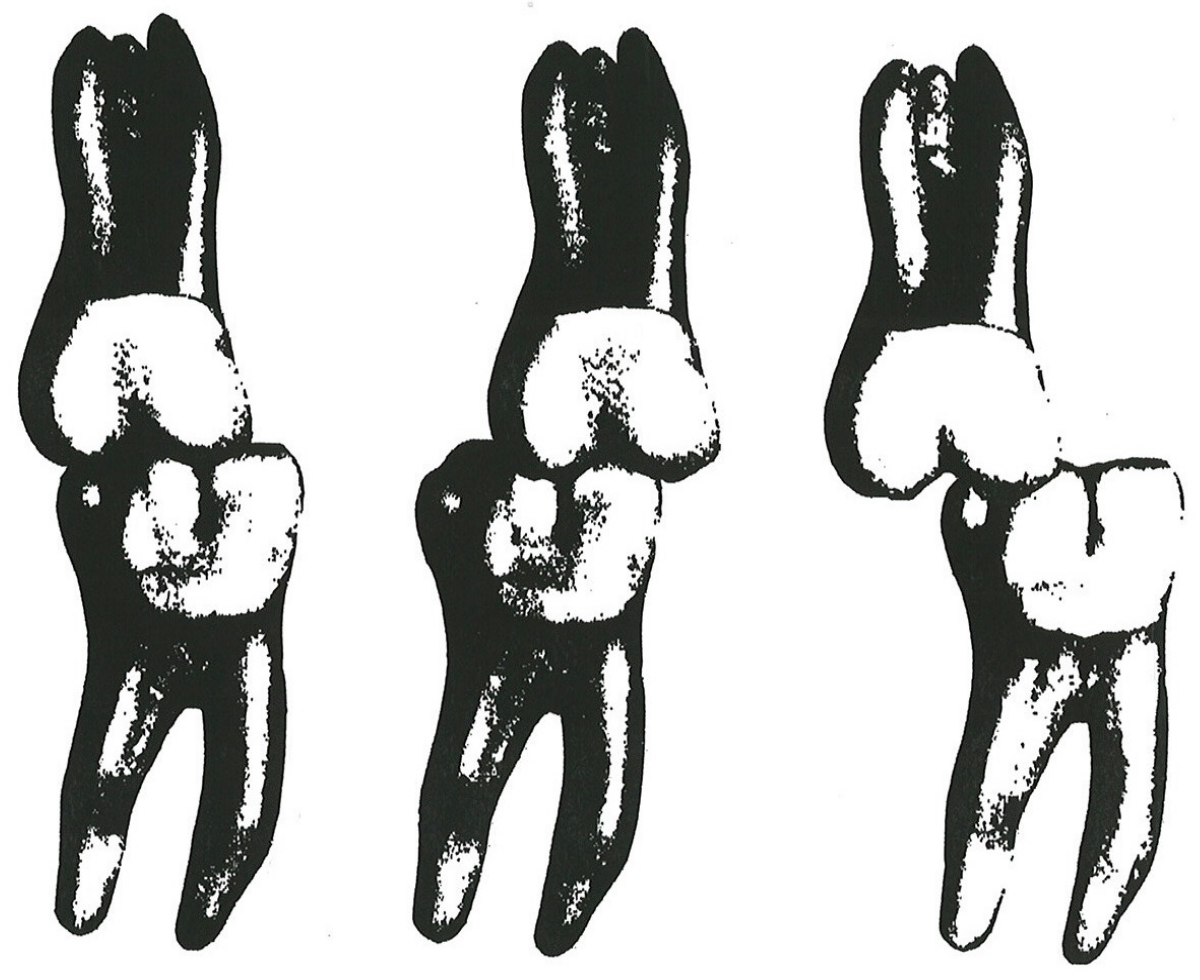

Figure 25. Buccal segment relationship refers to the parasagittal relationship of the permanent first molars.

Left: normal (Class 1) molar relationship has the mesiobuccal cusp of the maxillary molar aligned with the buccal groove of the mandibular molar. Middle: Full-step distoclusion (Class II) has the mesiobuccal cusp of the maxillary molar a full cusp mesial of the buccal groove of the lower molar. Right: Illustration of full-step mesioclusion (Class III). Most deviations from a Class I relationship involve less than the full-cusp divergences shown here. 
did not occur). The DAI score, weighted by the coefficients listed in Table 2 also was tested as a predictor of tooth size.

\section{Statistical Analysis}

The questions asked of these data are fairly straightforward; one batch of issues is purely descriptive, namely, by Angle's classification and/or sex, what are the normative dimensions of the teeth? What are the size differences between the incisor tooth types? How much left-right asymmetry is there? A second battery of questions involves understanding and partitioning the variation in the sample. As examples: How much sexual dimorphism is there, and does this differ between crown and root dimensions?

Once the tooth sizes were obtained, the measurements were collated in Excel and statistical analysis was performed using the JMP statistical package. Distances calculated in Photoshop ${ }^{\circledR} 6.0$ were transcribed onto data forms and then entered into a Microsoft ${ }^{\circledR}$ Excel ${ }^{\circledR}$ spreadsheet (Microsoft, Seattle, WA), where the ratios were calculated. The Excel@ document was then loaded into JMP (SAS Corporation, Cary, NC) where statistical analysis was performed.

Descriptive statistics were calculated as defined by Sokal and Rohlf (1995), namely sample size $(\mathrm{n})$, arithmetic mean $(\overline{\mathrm{x}})$, standard deviation $(\mathrm{sd})$, sample variance $\left(\mathrm{s}^{2}\right)$, standard error of the mean (se), skewness $\left(\mathrm{g}_{1}\right)$, and kurtosis $\left(\mathrm{g}_{2}\right)$. Regarding skewness and kurtosis, statistical packages commonly fail to provide inferential tests of whether $\mathrm{g}_{1}$ or $\mathrm{g}_{2}$ differ significantly from normality. Inspection of these raw statistics themselves is not particularly informative. Following Sokal and Rohlf (1995, p 138), the standard error for skewness is

$$
\mathrm{se}_{\mathrm{g}_{1}}=\sqrt{\frac{6 n(\mathrm{n}-1)}{(\mathrm{n}-2)(\mathrm{n}+1)(\mathrm{n}+3)}}
$$

where $\mathrm{n}$ is the sample sizes, and the standard error for kurtosis is

$$
\mathrm{se}_{\mathrm{g}_{2}}=\sqrt{\frac{24 \mathrm{n}(\mathrm{n}-1)^{2}}{(\mathrm{n}-3)(\mathrm{n}-2)(\mathrm{n}+3)(\mathrm{n}+5)}}
$$

an interesting feature of the tests of whether skewness or kurtosis departs from normality is that they are each evaluated at infinite degrees of freedom regardless of the actual size of the samples. 
Table 2. Listing of the variables scored in the Dental Aesthetic Index. ${ }^{1}$

\begin{tabular}{lc}
\hline Variable & Weight \\
\hline 1. Number of missing teeth & 5.76 \\
2. Incisor crowding & 1.15 \\
3. Incisor spacing & 1.31 \\
4. Midline diastema & 3.13 \\
5. Largest anterior irregularity, maxilla & 1.34 \\
6. Largest anterior irregularity, mandible & 0.75 \\
7. Anterior maxillary overjet $(\mathrm{mm})$ & 1.62 \\
8. Anterior mandibular overject $(\mathrm{mm})$ & 3.68 \\
9. Anterior openbite (mm) & 3.69 \\
10. Buccal molar relationship & 2.69 \\
\hline
\end{tabular}

${ }^{1}$ Numbers in right column are the regression weighting coefficients. Along with the Y-intercept of 13.36, these weights yield the DAI. 
Exploratory data methods (Tukey 1977) were used to identify statistical outliers. Percentage sexual dimorphism was calculated from this formula:

$$
\left(\frac{\left(\bar{x}_{M}-\bar{x}_{F}\right)}{\bar{x}_{F}}\right) 100
$$

so the percentage is read as the degree to which the male average exceeds the mean size of females.

Statistics were generated using JMP version 7.0.02 (SAS Institute Inc., Cary, NC). Tests were two-tail, and the conventional level of statistical significance (alpha $=0.05$ ) was used throughout.

\title{
Statistical Models
}

ANCOVA models are useful in the present study because they can be used to test for an association between two variables, while controlling for extraneous variables (e.g., patient's sex, measurements of homologous left-right traits), thus (1) greatly reducing the number of tests that have to be performed (and interpreted), (2) preserving degrees of freedom, and (3) testing for statistical interactions among the variables evaluated in combination. Essentially two ANCOVA models are used, both search for an association between mesiodistal crown size and some measure of dentoalveolar malocclusion. Tests were run using the JMP statistical package (SAS Institute Inc., Cary, NC), which used a generalized linear model approach for calculation. One, simple model uses patient's sex as a covariate, so (1) males and females can be combined in the same test while (2) testing for heterogeneity of slopes - whether the association is significantly different in the two sexes. The form of the table is this (Winer et al. 1991):

\author{
Intercept \\ Tooth size \\ Sex \\ Tooth size-by-Sex Interaction
}

where Intercept is the Y-intercept, Tooth size is mesiodistal crown size of one of the 14 tooth types, Sex is whether the patient is male or female, and the Interaction term tests whether the association (slope of the regression line) is statistically different between the two sexes. If the interaction term is significant, then the main effects of the model are biased, and the analysis should be run on a sex-specific basis. 
The other common model used here is a mixed-effect design where some measure of malocclusion is measured on both the right and left quadrants, so they need to be viewed as repeated measures:

\author{
Among Subjects \\ Intercept \\ Tooth Size \\ Sex \\ Tooth size-by-Sex Interaction \\ Within Subjects \\ Side \\ Side-x-Tooth size Interaction \\ Side- $x-$ Sex \\ Side- $x$-Tooth size- $x$-Sex
}

Here - as with the other mixed models (e.g., Winer et al. 1991) - there are two separate error terms (one among subjects and one within subjects). The Intercept is the Y-intercept of the regression line. Tooth size is the mesiodistal crown size of one of the 14 tooth types. "Sex" is a test of significant sexual dimorphism in average tooth size between males and females. The tooth size-bysex interaction term assesses whether the slopes differ significantly by sex.

Within subjects, Side is used when a trait (e.g., BSR, rotations, displacements) measured in each quadrant is systematically different on one side (i.e., directional asymmetry). Importantly, Side also extracts the variance due to systematic leftright differences from the error term, which enhances the chance of finding a difference in the other effects if they occur. The two first-order interaction terms and the one second-order term provide tests of the additivity of the model. Additivity is an assumption of the covariance model; when it is violated, individual tests should be run on each category of that covariate.

At their simplest, analysis of covariance (ANCOVA) models test for a linear relationship between the predictor and the outcome variables. Unless noted, a more complex (curvilinear) model did not provide a significantly better fit than a straight line. When indicated, a curvilinear model of the form $\left(X+X^{2}\right)$ was tested to see whether extreme values of tooth size were associated with exaggerated (nonlinear) levels of malocclusion. The following section builds on the comments just provided. 


\section{Analysis of Covariance}

A central question in the present study is whether there is an association between tooth crown size and the severity of the malocclusion (where "malocclusion" is some measure of crowding, displacement, overlap, and/or irregularity). A simple example is shown in Figure 26, where (hypothetherally) there is a positive statistical association between mesiodistal width of the maxillary central incisor and incisor irregularity. Such a statistical association can be a test for using linear regression (e.g., Freund and Littell 1991).

Since, however, tooth size is sexually dimorphic, it is necessary to account for "sex" of the subject, and this can be done by analyzing males and females separately (which is inefficient), or, preferably, including "sex" in the regression model using analysis of covariance (ANCOVA). "Sex" in the analysis is used here as "indicator variable" (Freund and Littell 1991); it is a dichotomous qualitative variable that accounts in these tests for the offset in tooth size (males $>$ female) between the two sexes. An indicator variable is identical to what previously was termed a dummy variable. There are a couple of concerns here, the main one being whether the relationships of incisor irregularity on tooth size are the same in both sexes. Statistically, the concern here is whether the slopes of the regression coefficients are the same in the two sexes. For example, one possibility is that the slope is significantly steeper in one sex than the other, as illustrated in Figure 27. It is possible (A) that the two slopes differ significantly in their steepness, (B) that they are of opposite signs, or (C) that the slope differs significantly from zero (either positively or negatively) for one sex, but is flat ( $\mathrm{r}=$ 0 ) for the other sex. These three possibilities - that is whether there is significant heterogeneity of slopes - can be assessed from inspection of the size-bymalocclusion interaction term in the ANCOVA model. When the interaction term is significant statistically, then the main effects are biased and other regression models need to be applied instead.

In the ANCOVA design used here, there are three inferential tests, namely (1) whether the interaction term is significant, (2) whether "sex" significantly affects the best-fit regression lines Y-intercept, and (3) whether the association between the predictor variable (tooth crown dimension) and the outcome variable (a measure of malocclusion) is significant statistically. The second of these tests ( $a$ "sex" effect) assesses whether there is a statistically significant vertical offset (i.e., the Y-intercept of the regression line) between the two sexes.

The ANCOVA model used here can, usefully, be made a bit more complex since homologous measures of malocclusion of the right and left teeth were measured in this study, but there is no systematic side difference in the extent of the malocclusions. Instead, malocclusions in the left and right hemispheres can 


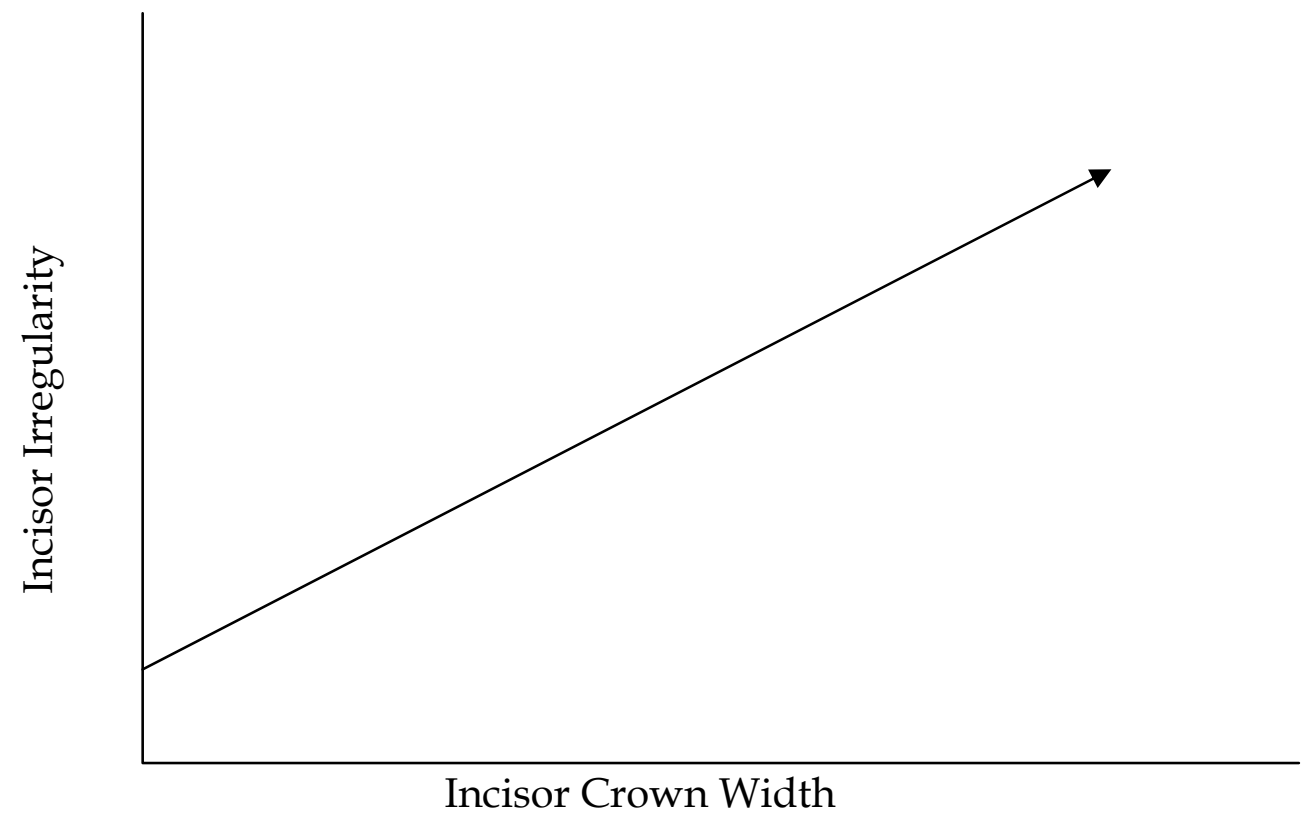

Figure 26. Hypothetical plot of a positive linear association between tooth size and irregularity. 


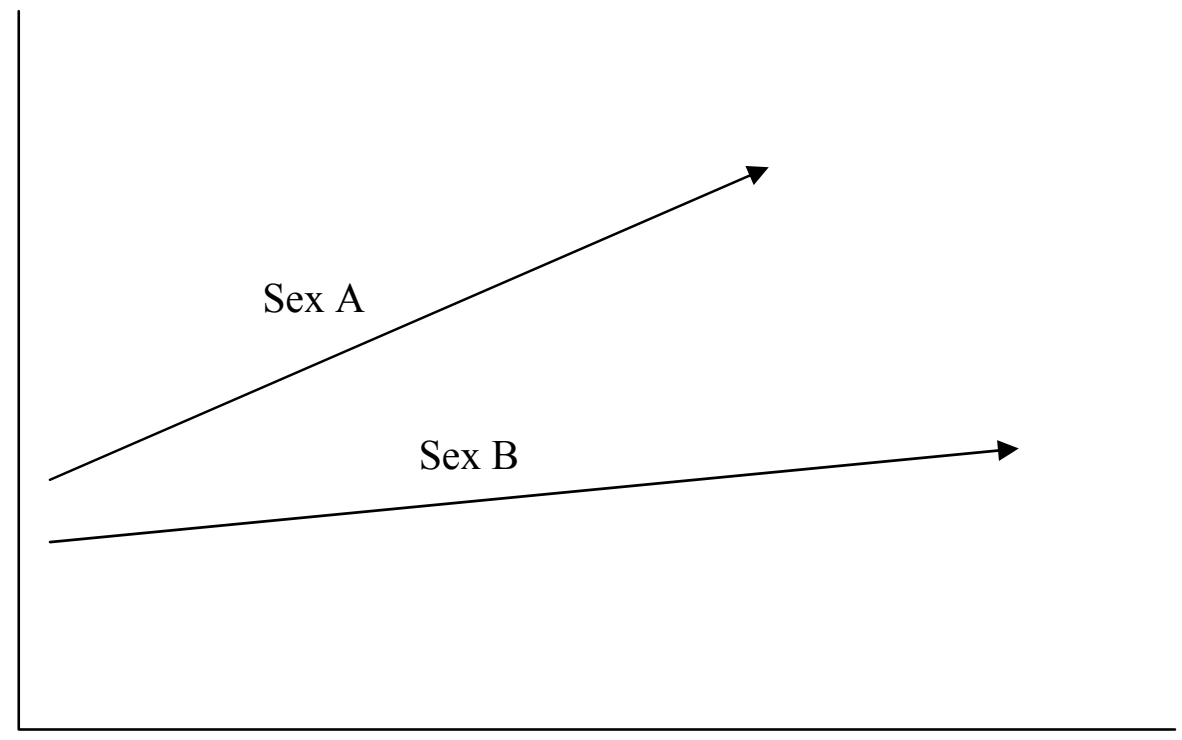

Figure 27. Hypothetical plot showing a steeper slope for one sex than the other. 
best be viewed as duplicates. The number of tests is cut in half when both sides of the dentition are used in an ANCOVA model, with measures on the left and sides viewed as repeated measures. Not only does this reduce the number of tests by half (thus reducing the risk of type II statistical errors), it also provides internal tests for directional asymmetry in severity of dentoavleolar malocclusions (e.g., Harris and Bodford 2007). This model can be handled in the JMP statistical package, though, of course, there is a different error mean squares for testing the repeated measure term.

As with ANCOVA designs in general, there are various assumptions of the model (Sokal and Rohlf 1995). Perhaps foremost is the assumption of homogeneity of the slopes of the regression lines (i.e., that they are parallel), but, as noted, this can be tested explicitly by the interaction term. Second is linearity of the response. We did not overtly test for the fit of other, nonlinear models, but the computer-generated plots were perused, looking for systematic regions of poor fit, where a curvilinear model might provide a better fit.

Another assumption of the analysis of covariance is that the covariate is measured without error and is under the researcher's control. The covariate in these tests is tooth size, so this assumption (as is common) is not strictly met. There doubtlessly, is some error in the tooth size measurements (e.g., Kieser and Groeneveld 1990, 1991), and they are not under the control of the investigator. 


\section{CHAPTER IV. RESULTS}

A total of 33 measures of malocclusion are described in the Methods section. Several of these are of little interest in themselves but were measured with the intent of combining them into more comprehensive assessments. For example, tooth displacements out of the idealized dental arch (i.e., ectopia) was quantified for 12 teeth (the anterior six teeth in each arch). Each of these was measured on an ordinal scale $(0,1$, or 2$)$. However, the sum of these scores provides a quasi-continuous variable termed "total displacements." Similarly, total rotations and total overlapping also were derived. Eleven other variables suggested by Jenny and Cons (1996) also provide generalized measures of malocclusion. Altogether, there are 14 measures that collectively quantify several aspects of malocclusion in the sense of greater-or-lesser failures of teeth to erupt into occlusion as gauged within and between the arches. These 14 are listed in Table 3.

The driving question in this thesis was whether there is a graded response between mesiodistal size of a tooth and the extent of the malocclusion. As described previously, maximum mesiodistal crown dimensions were obtained for each of the 14 tooth types in the two arches, central incisor through second molar. Is there a statistical association between tooth size and the extent of a subject's malocclusion? This was tested in a series of ANCOVA tests, where prime interest was on whether the regression of a measure of malocclusion on tooth size was statistically significant while controlling for ancillary variables such as sex and, for bilaterally symmetric variables, side of the dental arch. Testing for graded "dose-responses" between MD crown size and the extent (severity) of the malocclusion extends prior research (e.g., Peck and Peck 1972; Agenter 2008) that tooth size is larger in samples of people with malocclusions. In other words, the goal here was to move beyond artificially dichotomous comparisons between "no malocclusion" versus "malocclusion" to look, more realistically, at severity of malocclusion as a continuum that ranges from mild to handicapping.

For completeness, the ANCOVA tests (14 tooth dimensions and 32 measures of malocclusion) all are listed in the Appendix. Here we discuss the key results of associations between tooth sizes and what we deem are the 14 pertinent measures of malocclusion.

The ANCOVA statistical models (Appendix) are more or less complex, each with multiple F-ratios, but the purpose of these elaborations was to remove potential sources of extraneous variation from the residual mean squares in order to improve chances of finding significant tooth size malocclusion 
Table 3. Occlusal variables considered.

\begin{tabular}{lll}
\hline Feature & Scoring Method \\
\hline 1. & Total displacements & Sum of Ordinal Scores (3grades) \\
2. & Total rotations & Sum of Ordinal Scores (3grades) \\
3. & Total overlapping & Sum of Ordinal Scores (3grades) \\
4. $\quad$ Total displacements, rotations, and & Sum of Ordinal Scores (3grades) \\
overlapping & \\
5. Crowding & Ordinal Scores (3grades) \\
6. Spacing & Ordinal Scores (3grades) \\
7. Diastema & Ratio (millimeter) \\
8. Maxillary irregularity & Ratio (millimeter) \\
9. Mandibular irregularity & Ratio (millimeter) \\
10. Maxillary overjet & Ratio (millimeter) \\
11. Mandibular overjet & Ratio (millimeter) \\
12. Openbite & Ratio (millimeter) \\
13. AP relationship & Ordinal Scores (3grades) \\
14. DAI score & Weighted Sum \\
\hline
\end{tabular}


relationships if they exist. Consequently-and with some degree of simplification - we only focus here on the F-ratios for the regression aspect of the ANCOVA tests, and these are summarized for the maxillary tooth types (Table 4) and the mandibular tooth types (Table 5). Again, the ANCOVA models are described in the Methods chapter, and full details of all tests are provided in the Appendix.

\section{Maxillary Tooth Types}

The P-values and the F-tests between each of the seven maxillary tooth types are collected in Table 4 . Full results of the ANOVA results are provided in the Appendix. This one page table involves much less effect than searching through the full results. What is shown here is the P-value of the F-ratio testing whether the association (linear regression) between tooth size and a measure of malocclusion.

This situation is readily illustrated using the first test in Table 4, between the maximum mesiodistal size of the maxillary central incisor (U1) and severity of the total displacement score. Regression of total displacements on U1 size is highly significant, which is the P-value listed in the table. Significance does not disclose the nature of the association, but Figure 28 makes it evident that broader $\mathrm{U} 1$ teeth are associated with greater displacements. The full ANOVA table in the Appendix shows that, additionally, there is a significant sex difference (i.e., larger dimensions in males than females), but there is no tooth-size by displacement interaction.

A few generalities can be drawn from the results in Table 4: One, many of the statistical associations are significant $($ alpha $=0.05)$, and many are highly significant $(\mathrm{P}<0.01)$, indicating that tooth sizes commonly are reflected in the severity of tooth-based malocclusions. Two, the significant associations are more common in the anterior segment of the arch, but some variables (e.g., overlapping and the combination of displacements, rotations, and overlapping) have significant associations with most maxillary tooth types. One interpretation here is simply that, indeed, crown dimensions throughout the arch affect severity of the malocclusion.

A second, seemingly more parsimonious interpretation is that crown dimensions among all tooth types are positively intercorrelated (e.g., Harris and Bailit 1988) and that some of the associations in Table 4 developed indirectly from these intertooth correlations. It depends on multivariate approaches (later in this chapter) to distinguish between these two scenarios. Three, some of 
Table 4. P-Values associated with test (F-Ratios) of the regression of crowding on tooth size in the maxilla.

\begin{tabular}{|c|c|c|c|c|c|c|c|}
\hline Variable & U1 & $\mathrm{U} 2$ & U3 & $\mathrm{U} 4$ & U5 & U6 & $\mathrm{U} 7$ \\
\hline $\begin{array}{l}\text { Total } \\
\text { displacements }\end{array}$ & $<0.0001$ & 0.0030 & 0.0006 & 0.1517 & 0.7024 & 0.0390 & 0.0702 \\
\hline $\begin{array}{l}\text { Total } \\
\text { rotations }\end{array}$ & 0.0016 & 0.0464 & 0.0132 & 0.0459 & 0.0254 & 0.3356 & 0.5983 \\
\hline $\begin{array}{l}\text { Total } \\
\text { overlapping }\end{array}$ & 0.0001 & $<0.0001$ & $<0.0001$ & 0.0054 & 0.0186 & 0.0139 & 0.0518 \\
\hline $\begin{array}{l}\text { Total } \\
\text { displacements, } \\
\text { rotations, and } \\
\text { overlapping }\end{array}$ & $<0.0001$ & 0.0002 & $<0.0001$ & 0.0167 & 0.0666 & 0.0272 & 0.0807 \\
\hline Crowding & $<0.0001$ & $<0.0001$ & $<0.0001$ & $<0.0001$ & 0.0009 & 0.0087 & 0.0353 \\
\hline Spacing & 0.0002 & $<0.0001$ & 0.0001 & $<0.0001$ & 0.0013 & 0.0169 & 0.0060 \\
\hline Diastema & 0.0037 & 0.0080 & 0.0065 & 0.0235 & 0.0174 & 0.3552 & 0.0511 \\
\hline $\begin{array}{l}\text { Maxillary } \\
\text { irregularity }\end{array}$ & 0.0033 & 0.0006 & 0.0023 & 0.1863 & 0.3834 & 0.0294 & 0.2129 \\
\hline $\begin{array}{l}\text { Mandibular } \\
\text { irregularity }\end{array}$ & $<0.0001$ & 0.0043 & 0.0072 & 0.0333 & 0.5911 & 0.0221 & 0.0854 \\
\hline $\begin{array}{l}\text { Maxillary } \\
\text { overjet }\end{array}$ & 0.0005 & 0.3360 & 0.0207 & 0.0544 & 0.0441 & 0.5124 & 0.7633 \\
\hline $\begin{array}{l}\text { Mandibular } \\
\text { overjet }\end{array}$ & 0.1151 & 0.4743 & 0.1280 & 0.3615 & 0.5897 & 0.8752 & 0.2853 \\
\hline Openbite & 0.5755 & 0.2432 & 0.2555 & 0.5366 & 0.4407 & 0.4833 & 0.3663 \\
\hline AP relationship & 0.7609 & 0.0695 & 0.2957 & 0.1799 & 0.6431 & 0.7499 & 0.8244 \\
\hline DAI score & 0.0100 & 0.2194 & 0.0247 & 0.4172 & 0.5363 & 0.4091 & 0.9515 \\
\hline
\end{tabular}

Full results of the ANCOVA models are provided in the Appendix. 
Table 5. P-Values associated with test (F-Ratios) of the regression of crowding on tooth size in the mandible.

\begin{tabular}{|c|c|c|c|c|c|c|c|}
\hline Variable & L1 & L2 & L3 & $\mathrm{L} 4$ & L5 & L6 & L7 \\
\hline $\begin{array}{l}\text { Total } \\
\text { displacements }\end{array}$ & 0.0004 & $<0.0001$ & 0.0273 & 0.1177 & 0.2808 & 0.1386 & 0.4212 \\
\hline $\begin{array}{l}\text { Total } \\
\text { rotations }\end{array}$ & $<0.0001$ & $<0.0001$ & 0.0189 & 0.0143 & 0.0715 & 0.2488 & 0.5299 \\
\hline $\begin{array}{l}\text { Total } \\
\text { overlapping }\end{array}$ & $<0.0001$ & $<0.0001$ & 0.0048 & 0.0037 & 0.3720 & 0.0799 & 0.2378 \\
\hline $\begin{array}{l}\text { Total } \\
\text { displacements, } \\
\text { rotations, and } \\
\text { overlapping }\end{array}$ & $<0.0001$ & $<0.0001$ & 0.0042 & 0.0081 & 0.0581 & 0.0840 & 0.5347 \\
\hline Crowding & $<0.0001$ & $<0.0001$ & $<0.0001$ & $<0.0001$ & 0.0158 & 0.0746 & 0.0582 \\
\hline Spacing & $<0.0001$ & $<0.0001$ & $<0.0001$ & $<0.0001$ & 0.0003 & 0.0024 & 0.0078 \\
\hline Diastema & 0.0056 & 0.0062 & 0.0017 & 0.0015 & 0.0585 & 0.0764 & 0.0214 \\
\hline $\begin{array}{l}\text { Maxillary } \\
\text { irregularity }\end{array}$ & 0.0122 & 0.0006 & 0.1688 & 0.2977 & 0.1490 & 0.1487 & 0.8936 \\
\hline $\begin{array}{l}\text { Mandibular } \\
\text { irregularity }\end{array}$ & $<0.0001$ & $<0.0001$ & 0.0413 & 0.0476 & 0.1670 & 0.0513 & 0.0261 \\
\hline $\begin{array}{l}\text { Maxillary } \\
\text { overjet }\end{array}$ & 0.0380 & 0.0274 & 0.0654 & 0.3181 & 0.1974 & 0.6994 & 0.3142 \\
\hline $\begin{array}{l}\text { Mandibular } \\
\text { overjet }\end{array}$ & 0.0766 & 0.0024 & 0.3148 & 0.8549 & 0.8705 & 0.2549 & 0.0432 \\
\hline Openbite & 0.7494 & 0.7100 & 0.6068 & 0.7931 & 0.6946 & 0.9967 & 0.1595 \\
\hline AP relationship & 0.2861 & 0.4115 & 0.6296 & 0.2836 & 0.4599 & 0.7056 & 0.0836 \\
\hline DAI score & 0.0409 & 0.0090 & 0.2906 & 0.9854 & 0.5230 & 0.7082 & 0.1219 \\
\hline
\end{tabular}

Full results of the ANCOVA models are provided in the Appendix. 


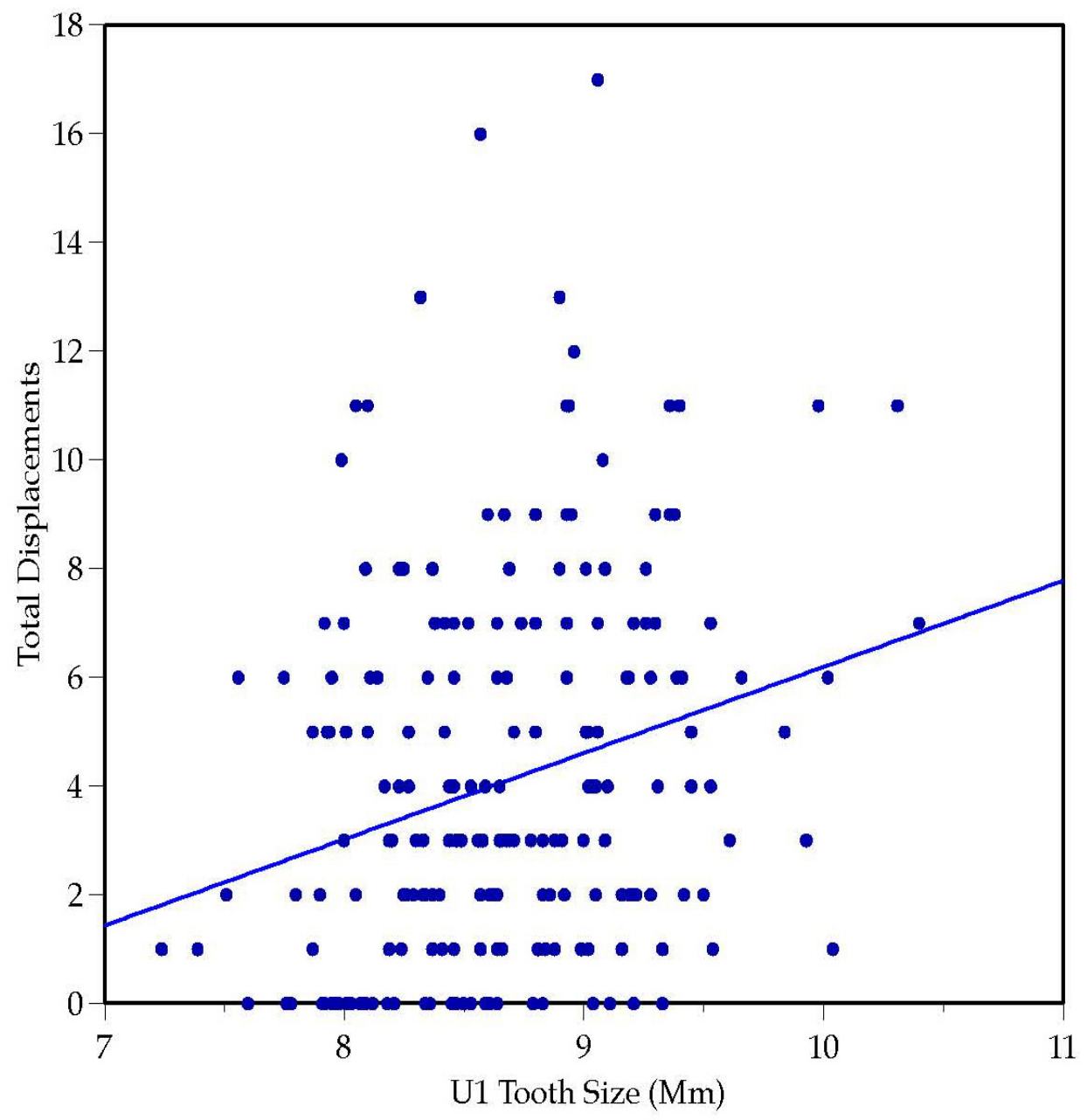

Figure 28. Plot between mesiodistal tooth size of U1 and total displacement scores. 
the 14 variables tested are consistently independent of tooth size, notably mandibular overjet, openbite, and AP relationships. Of the 14 variables tested, these three are distinguished in that they depend on skeletal relationships rather than tooth relationships per se (see Harris and Johnson 1991, and Harris 2008).

The following presentation of results is organized by occlusal trait.

\section{Total Displacements}

There are highly significant, positive associations between displacement scores and mesiodistal crown dimensions for U1 (Figure 28), U2 (Figure 29), and U3 (Figure 30). Distal to the canine, the associations are weak and not significant statistically.

\section{Total Rotations}

Statistically significant, positive associations occur throughout much of the arch. Somewhat erratically, the associations are statistically significant $(\mathrm{P}<$ 0.05 ) from a high at the central incisor (Figure 31) through the second premolar. In each case, the associations are positive, meaning that larger teeth size tend to occur with more rotations. In contrast, the molars have no relationship to rotation scores.

\section{Total Overlapping}

As with the rotation scores just examined, overlapping is significantly associated with MD crown lengths. Here, the positive associations achieve statistical significance throughout the whole arcade (though U7, the terminal tooth is only marginally significant, $\mathrm{P}=0.05$ ). Strengths of the associations are strongest for U1 (Figure 32) and U2 (Figure 33). The nature of the associations is that mesiodistally larger teeth are associated with greater scores for dental overlapping.

In all of the results presented in this chapter, tooth size is a fixed entity so the necessary scenario is that large tooth size is the etiological cause of the dental malocclusion, In other words, the malocclusion - variously expressed as ectopic, rotated, and overlapping teeth - is the consequence of mesiodistally large crown sizes. It also is noteworthy that all of the significant results described here show a graded response; it is not just that large teeth are a risk 


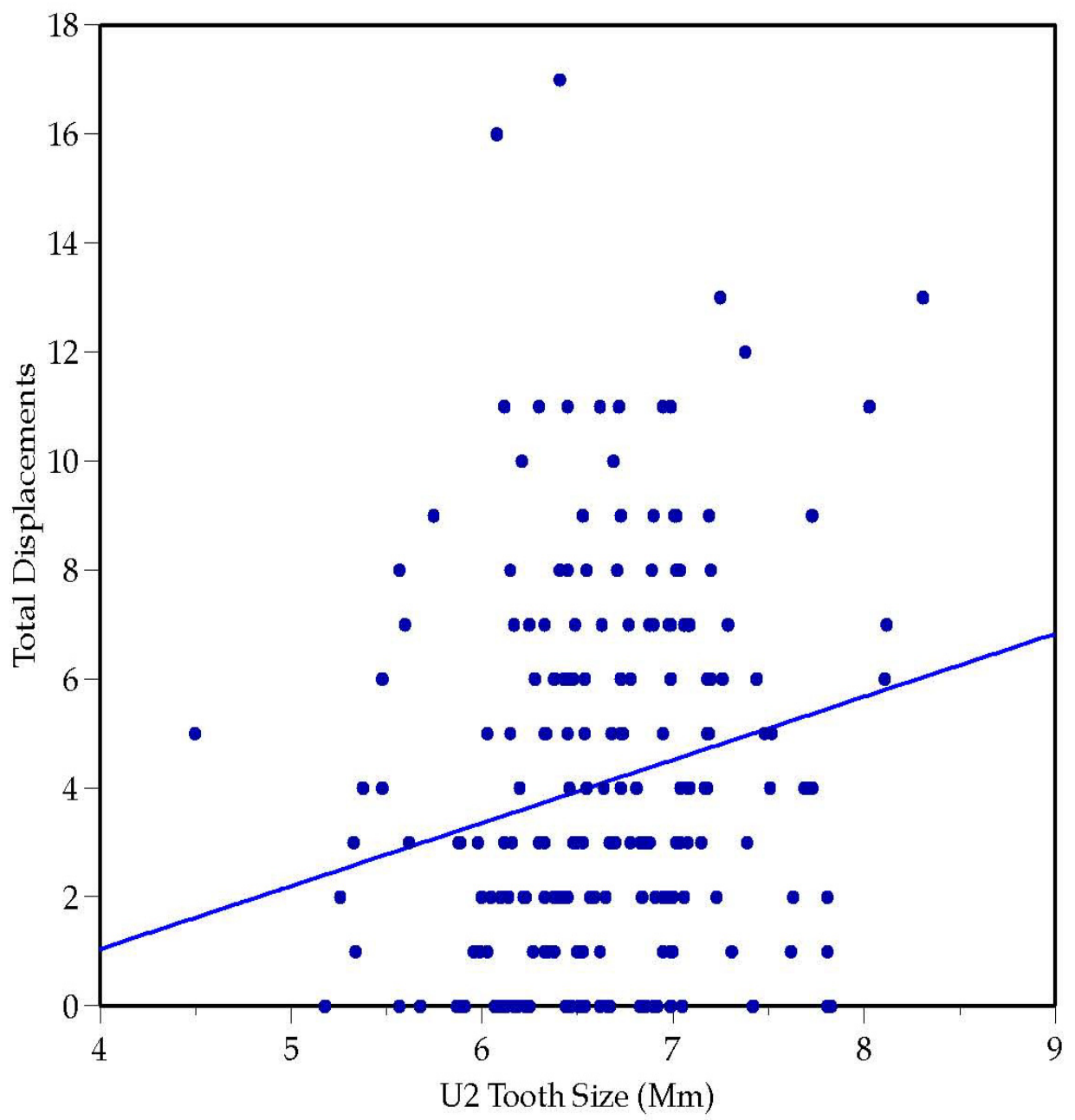

Figure 29. Plot between mesiodistal tooth size of U2 and total displacement scores.

As tooth size increases, so does the average total displacement score. 


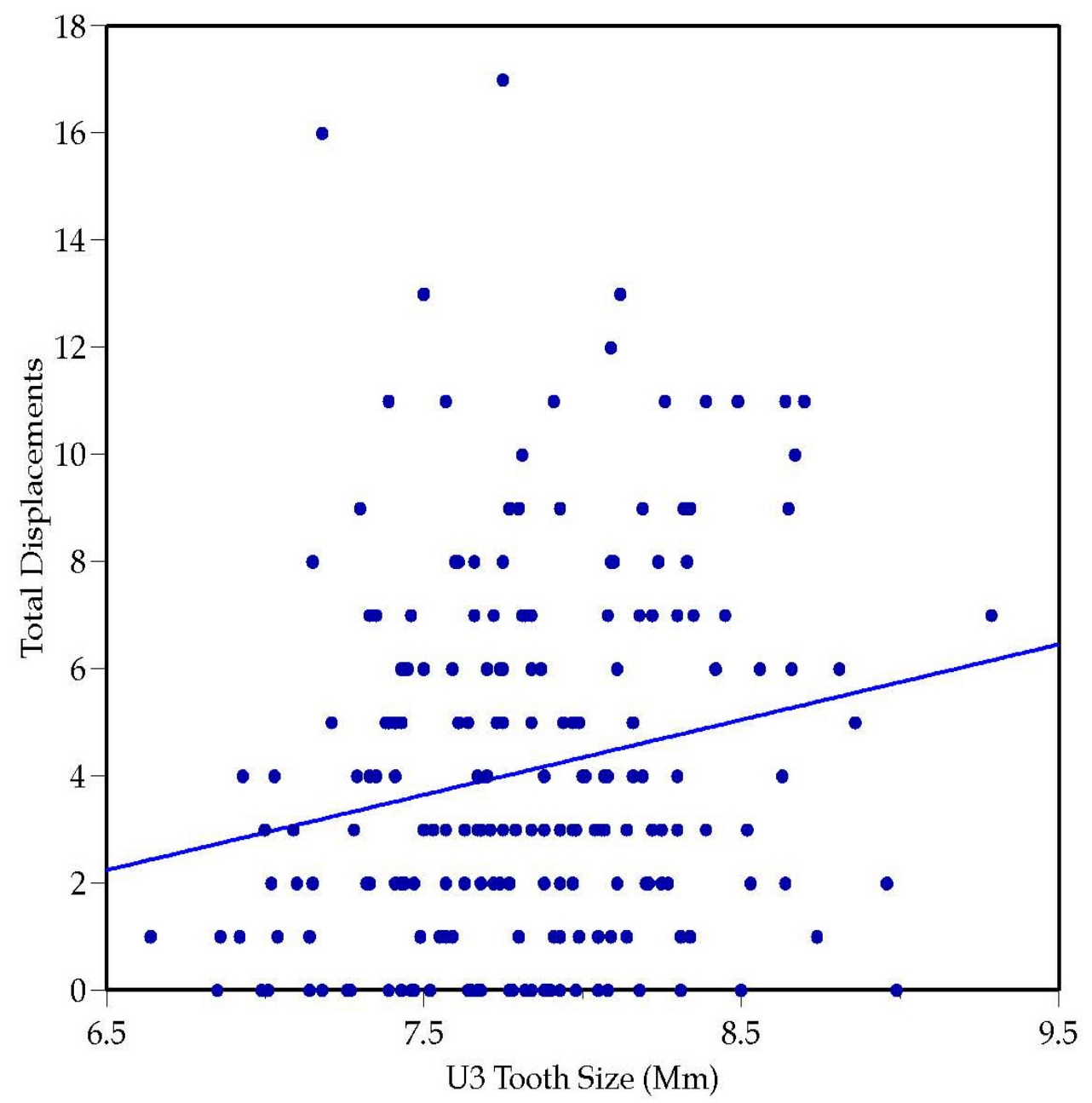

Figure 30. Plot between mesiodistal tooth size of U3 and total displacement scores.

As tooth size increases, so does the average total displacement score. 


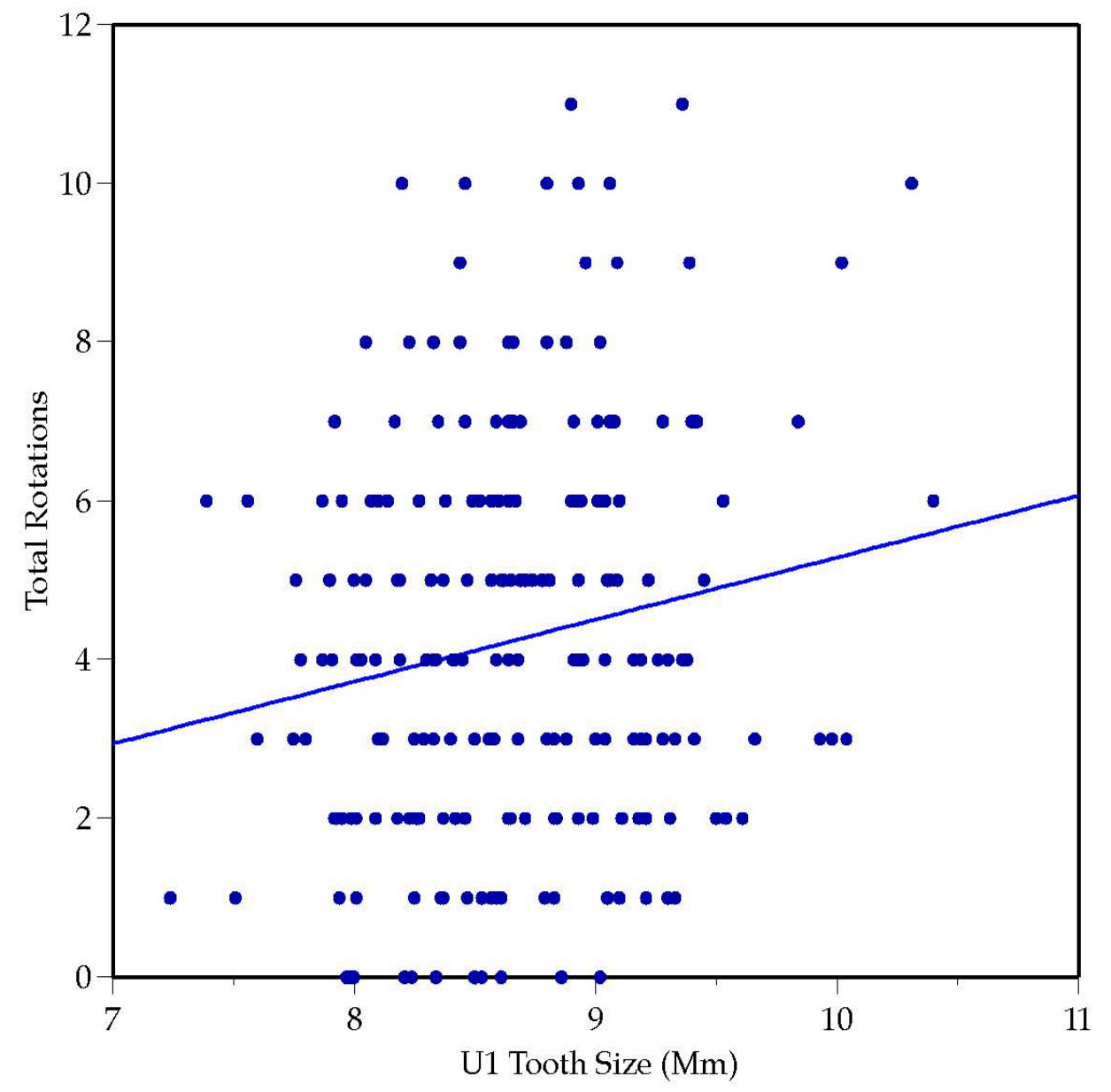

Figure 31. Plot between mesiodistal tooth size of U1 and total rotations.

As tooth size increases, so does the average total rotations. 


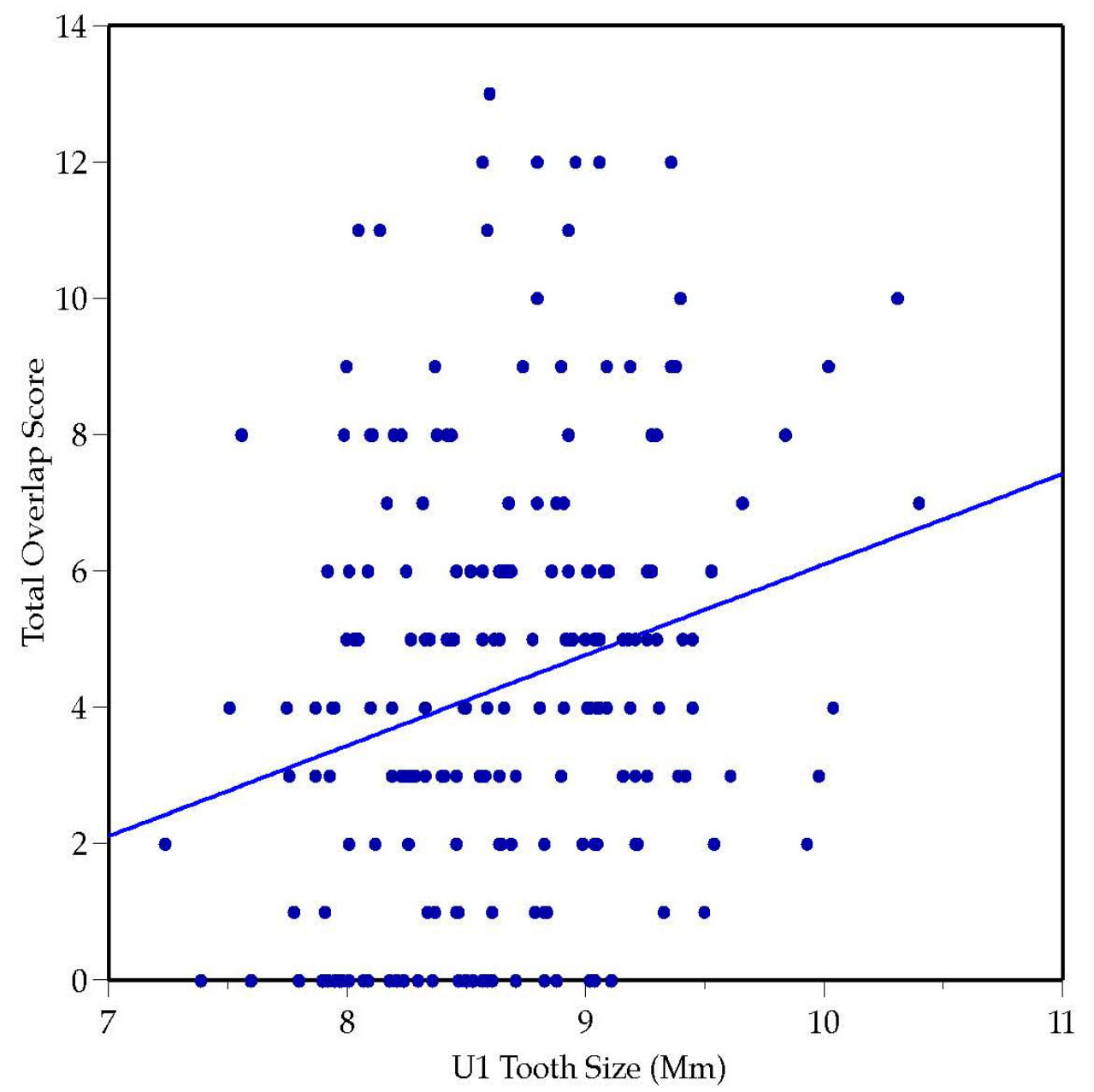

Figure 32. Plot between mesiodistal tooth size of U1 and total overlap.

As tooth size increases, so does the average total overlap score. 


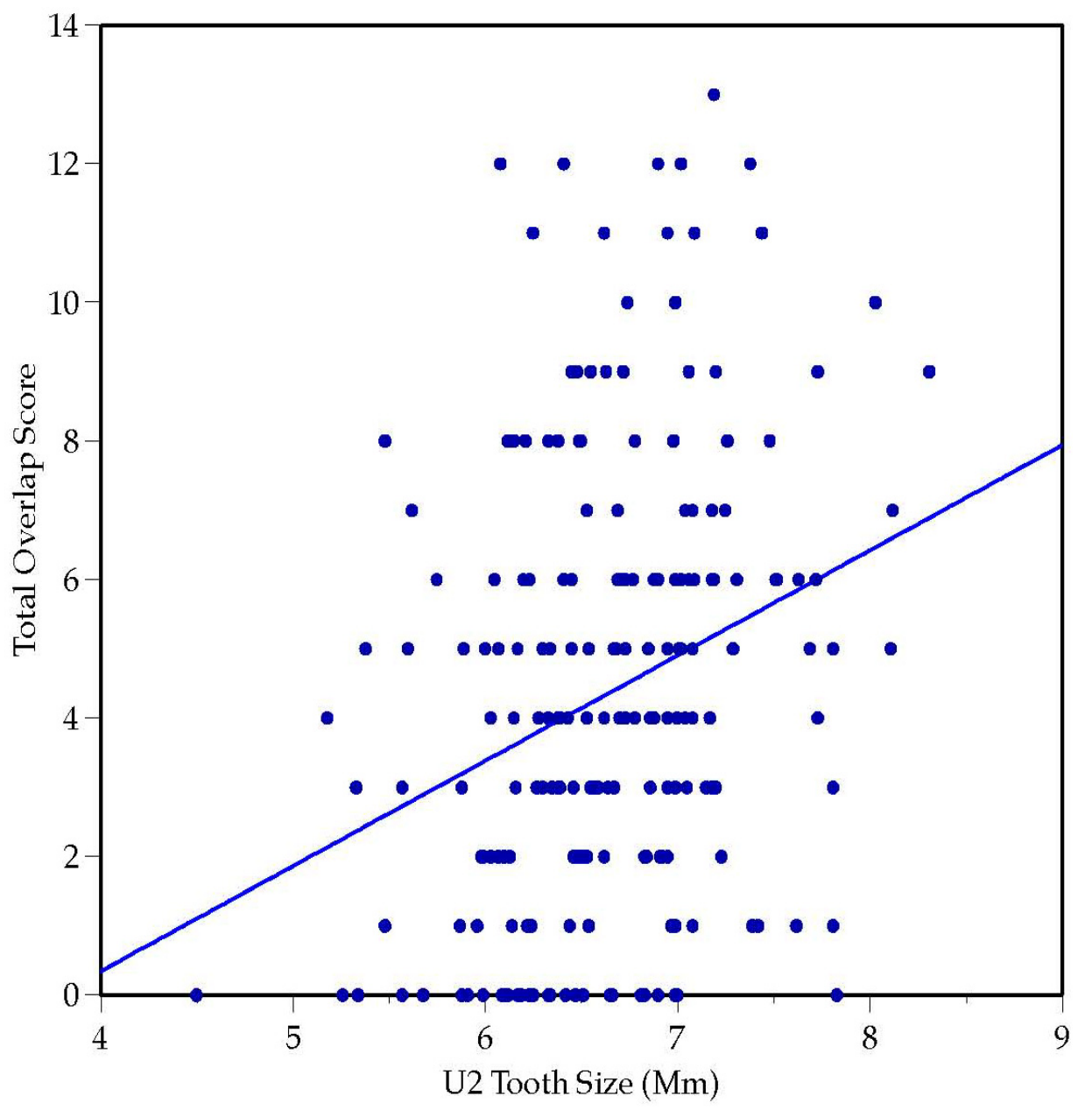

Figure 33. Plot between mediodistal tooth size of U2 and total overlap.

As tooth size increases, so does the average total overlap score. 
factor for malpositional teeth but that the greater the crown size the greater the extent of the malpositions.

\section{Total Displacements, Rotations, and Overlapping}

This composite measure of three sorts of dental malocclusions is significantly associated with MD crown dimensions throughout most of the tooth row; of the seven teeth tested, only U5 and U7 have non-significant Pvalues; and these are suggestive $(0.10>\mathrm{P}>0.05)$. The stronger associations occur among the anterior tooth types, and the data are plotted for U1 (Figure 34), U2 (Figure 35), and U3 (Figure 36).

\section{Crowding}

Mesiodistal crown sizes are highly significantly predictive of crowding; the P-values are highly significant through all tooth types (excepting U7, where P $=0.03$ ). The associations all are positive, meaning that larger crown sizes are associated with greater crowding. Examples are illustrated for U1 (Figure 37), U2 (Figure 38), U3 (Figure 39), and U4 (Figure 40).

\section{Interdental Spacing}

Interdental spacing is of particular interest here because it is the antithesis of the crowding just reviewed. With crowding, tooth sizes exceed the available arch size. With spacing, the opposite relationship occurs, where there is a deficiency of tooth size. Here, (1) the significant associations are widespread, involving all seven tooth types, and (2) the associations are negative. The negative associations are illustrated in Figure 41 (for U1), Figure 42 (for U2), Figure 43 (for U3), and Figure 44 (for U4). With smaller crown sizes there is greater spacing, so the associations are negative.

\section{Midline Diastema}

Both the typical width and the incidence of this feature diminish as mesiodistal crown width increases. Moreover, several tooth types (U1 through U5) are significantly predictive of diastema width. As examples, the relationship is evident for U1 (Figure 45), and for the canine (Figure 46), but also in the midarch such as the second premolar (Figure 47). The indication here is that small 


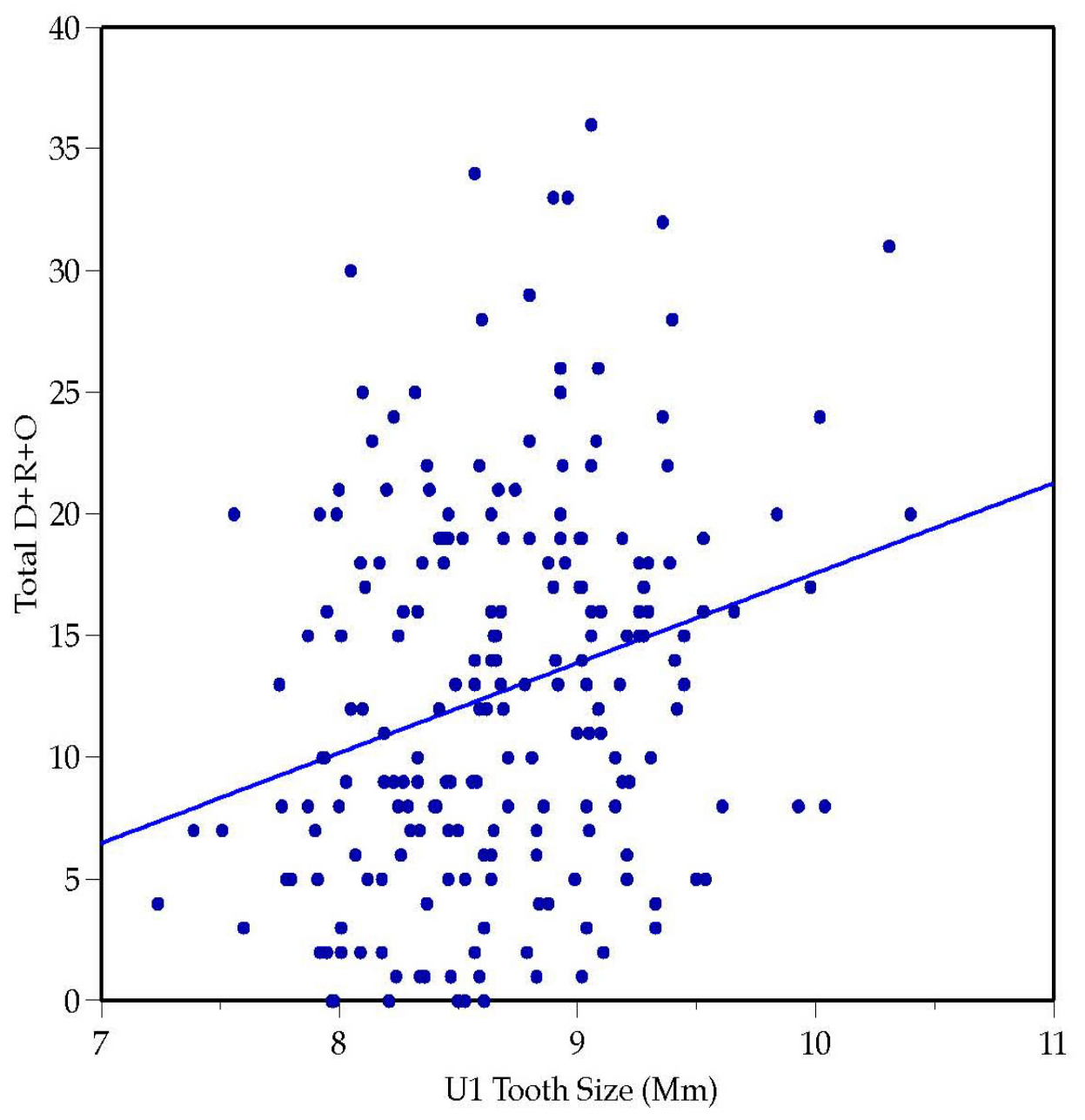

Figure 34. Plot between mesiodistal tooth size of U1 and total displacements plus rotations plus overlap.

As tooth size increases, so does the average total $\mathrm{D}+\mathrm{R}+\mathrm{O}$ score. 


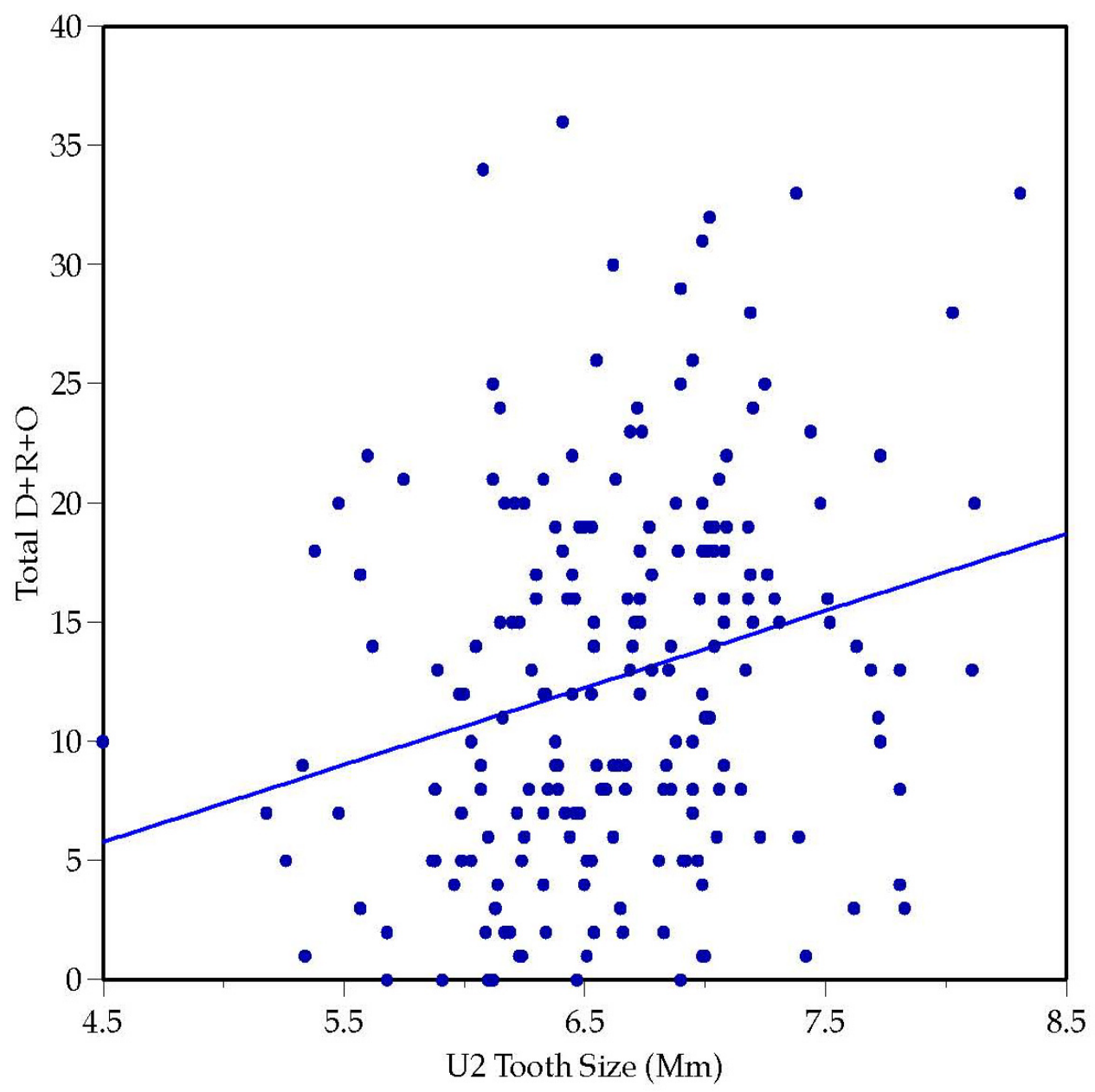

Figure 35. Plot between mesiodistal tooth size of U2 and total $\mathrm{D}+\mathrm{R}+\mathrm{O}$ scores.

As tooth size increases, so does the average total $\mathrm{D}+\mathrm{R}+\mathrm{O}$ score. 


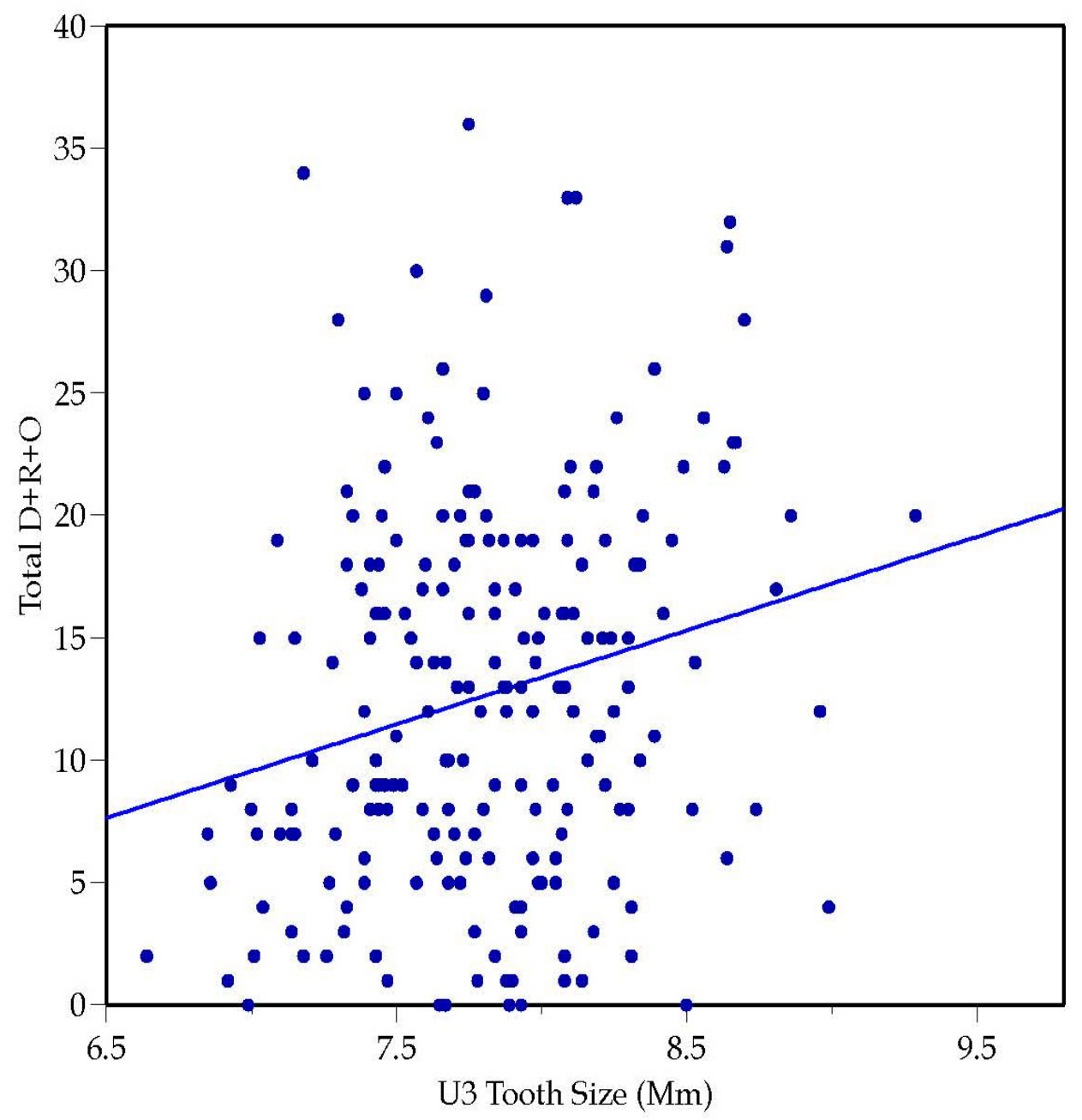

Figure 36. Plot between mesiodistal tooth size of U1 and total $\mathrm{D}+\mathrm{R}+\mathrm{O}$ scores.

As tooth size increases, so does the average total $D+R+O$ 


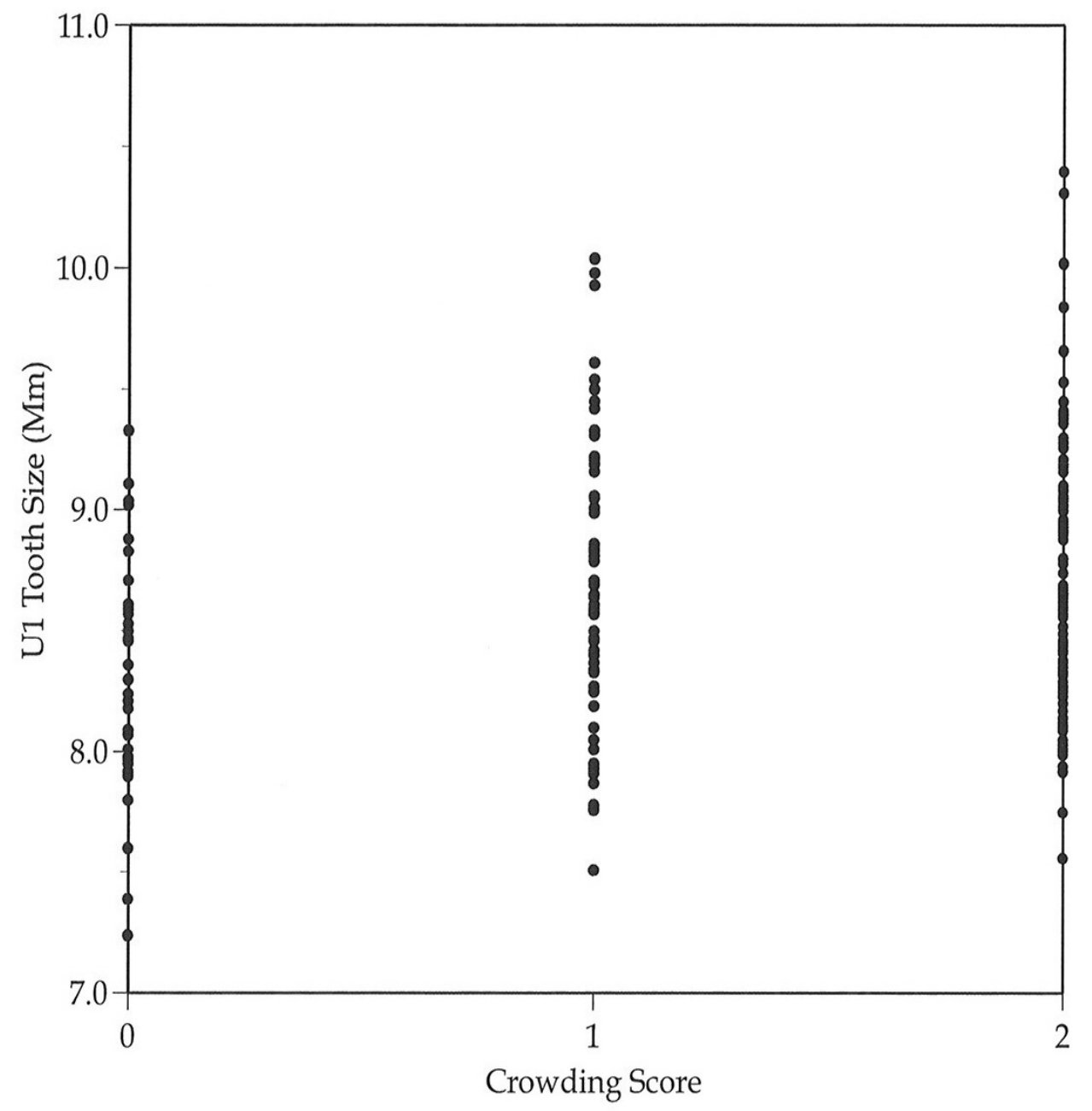

Figure 37. Plot between mesiodistal tooth size of U1 and crowding score.

As tooth size increases, average crowding also increases. 


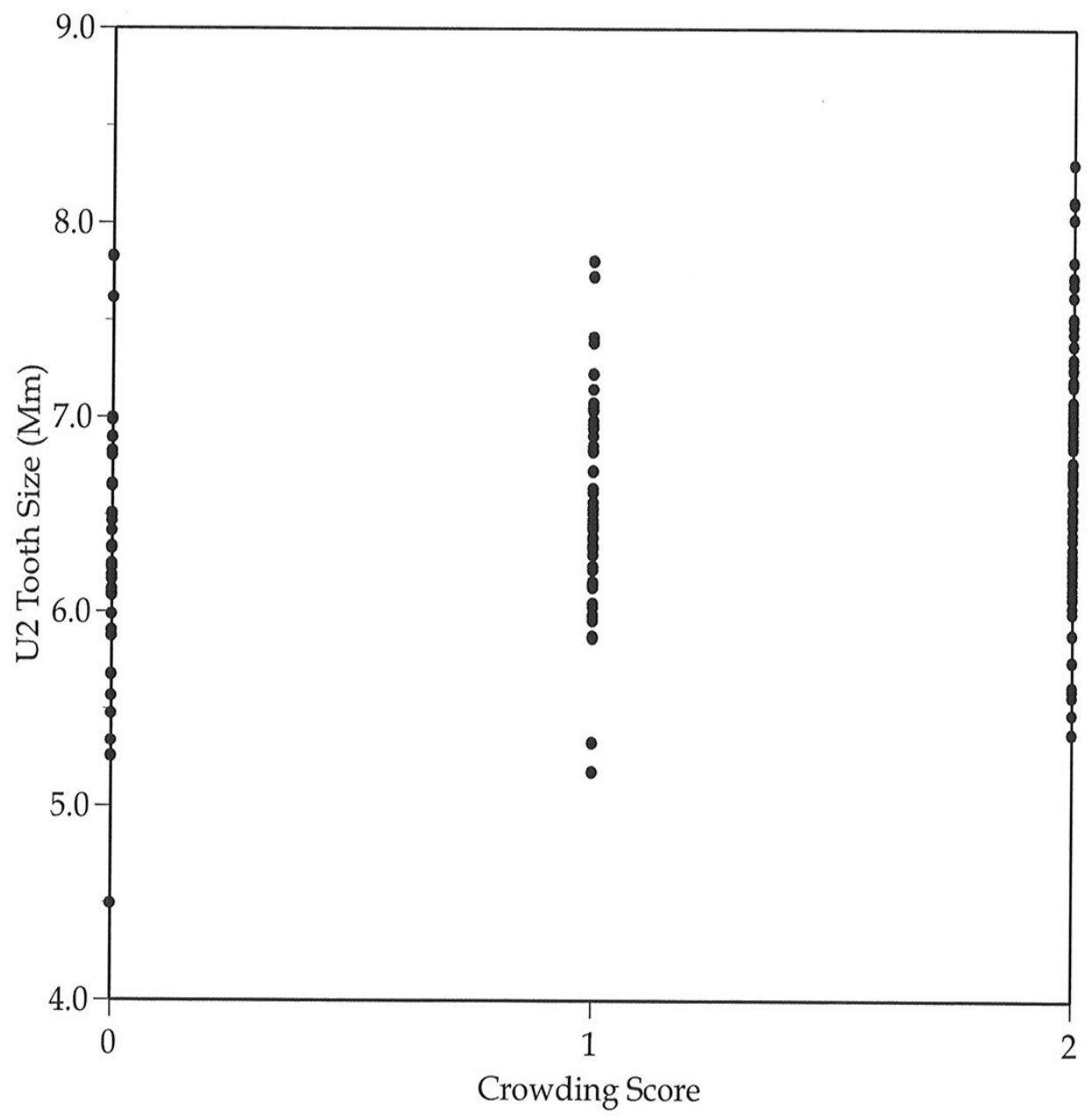

Figure 38. Plot between mesiodistal tooth size of U2 and crowding score.

As tooth size increases, average crowding also increases. 


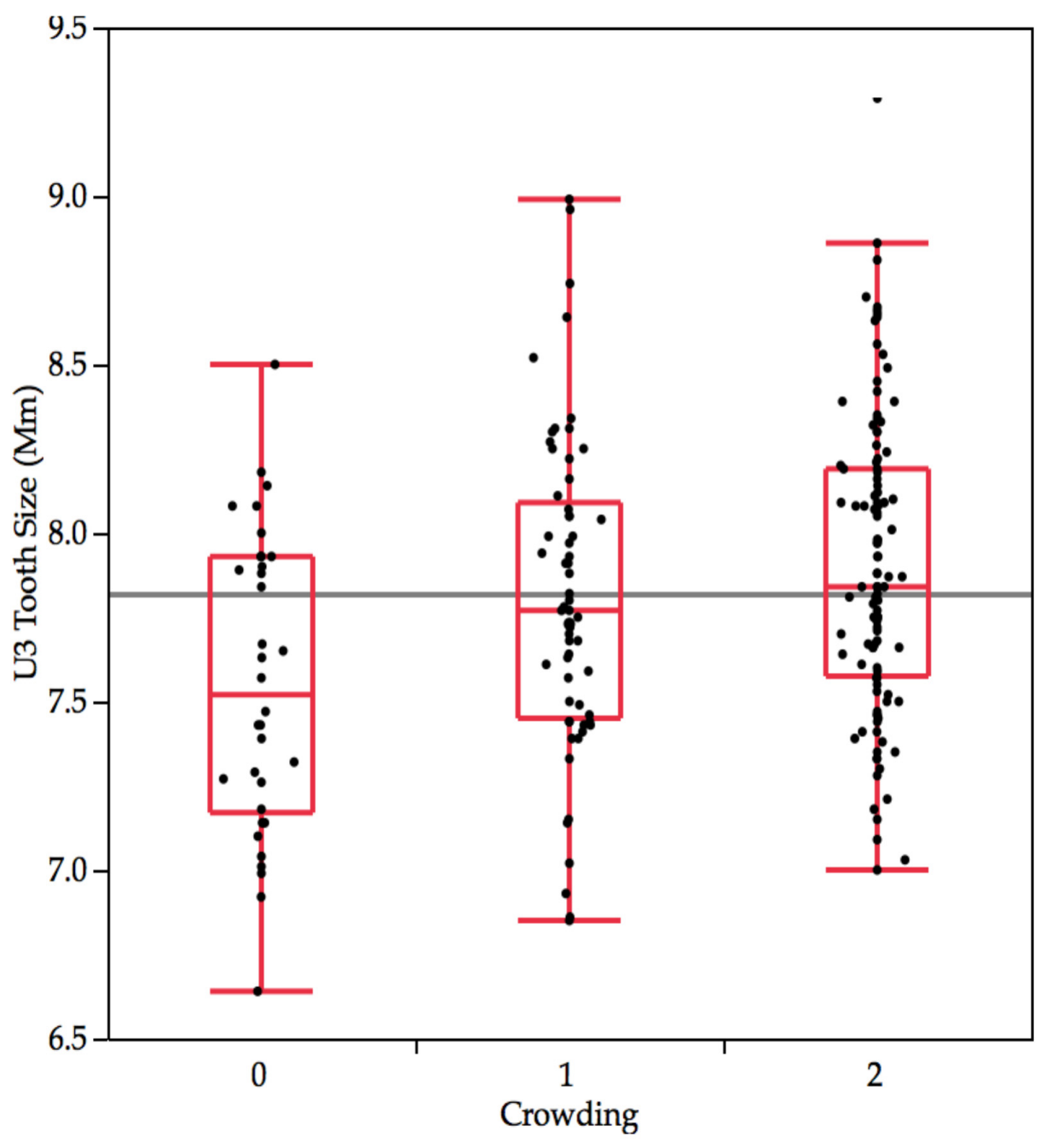

Figure 39. Plot between mesiodistal tooth size of U3 and crowding score.

As tooth size increases, average crowding also increases. 


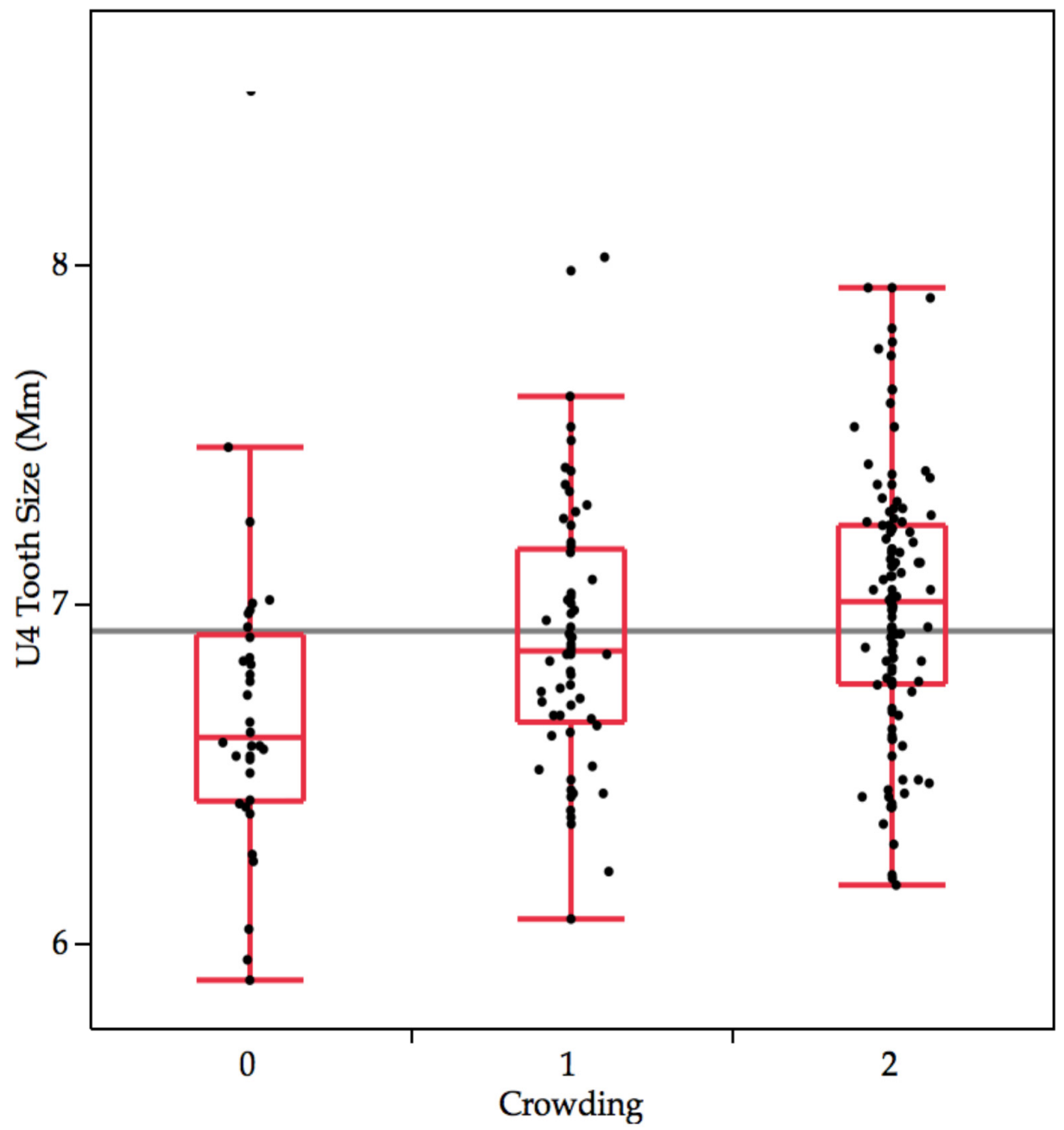

Figure 40. Plot between mesiodistal tooth size of U4 and crowding score.

As tooth size increases, average crowding also increases. 


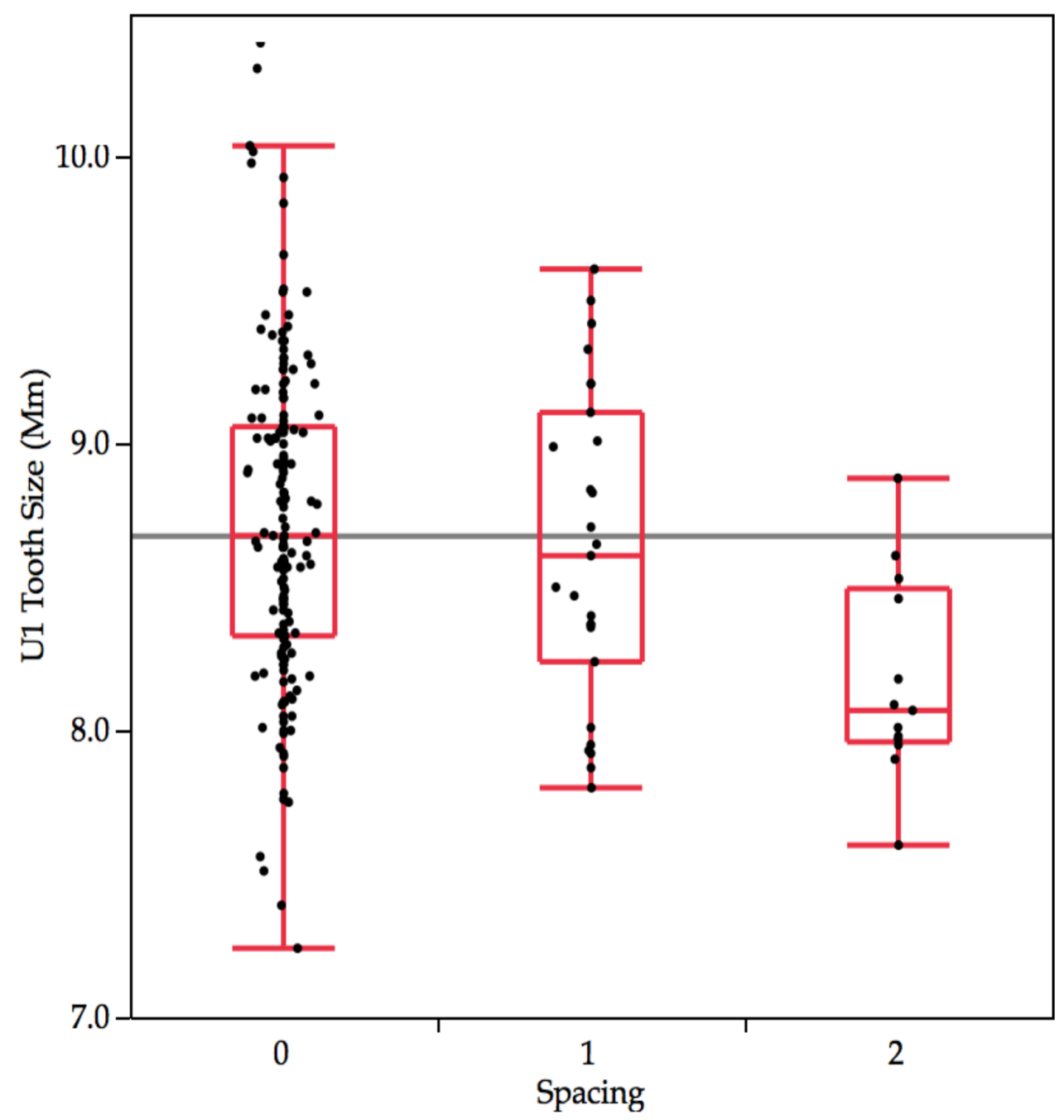

Figure 41. Plot between mesiodistal tooth size of U1 and spacing score.

As tooth size increases, average spacing decreases. 


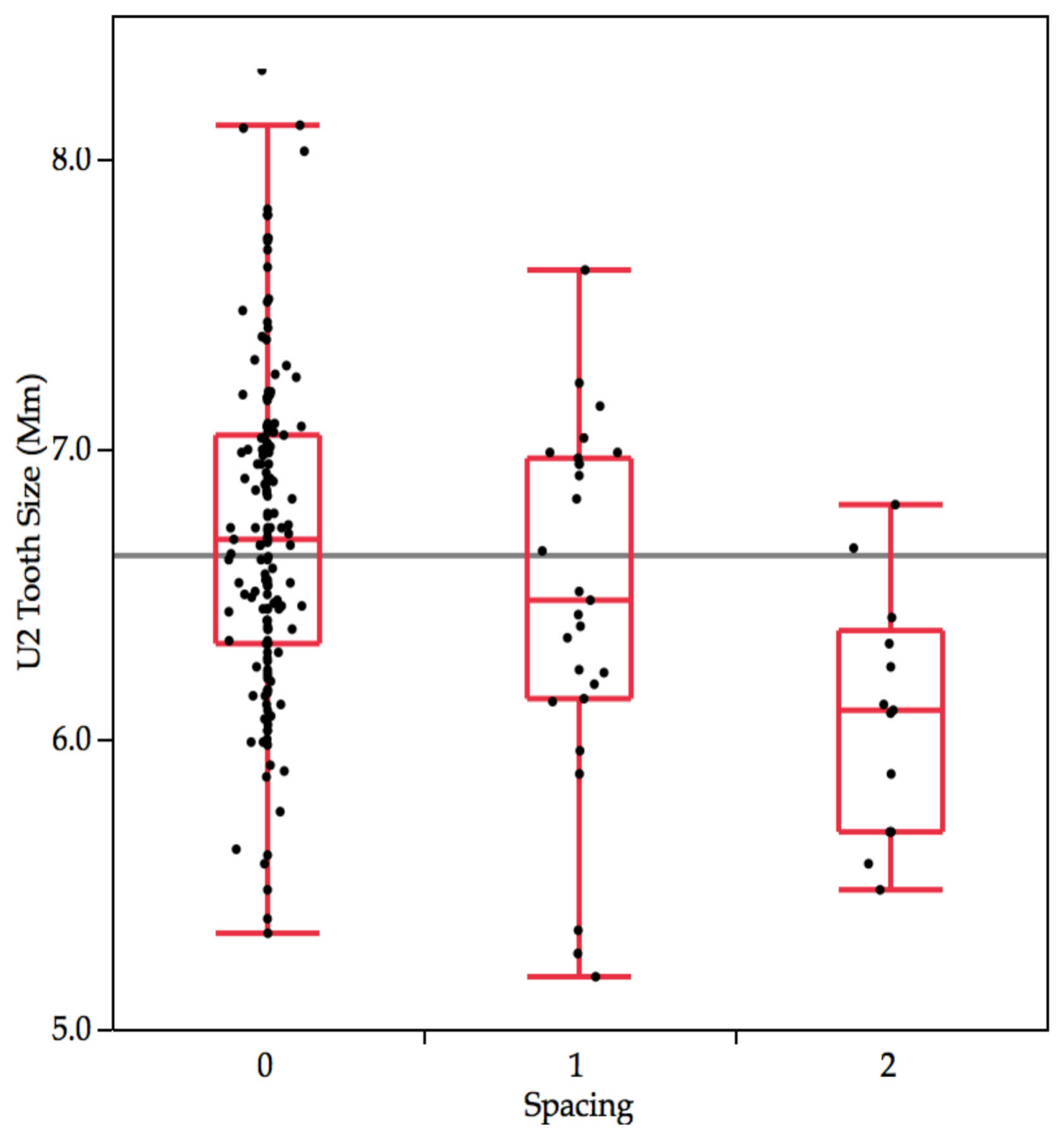

Figure 42. Plot between mesiodistal tooth size of U2 and spacing score.

As tooth size increases, average spacing decreases. 


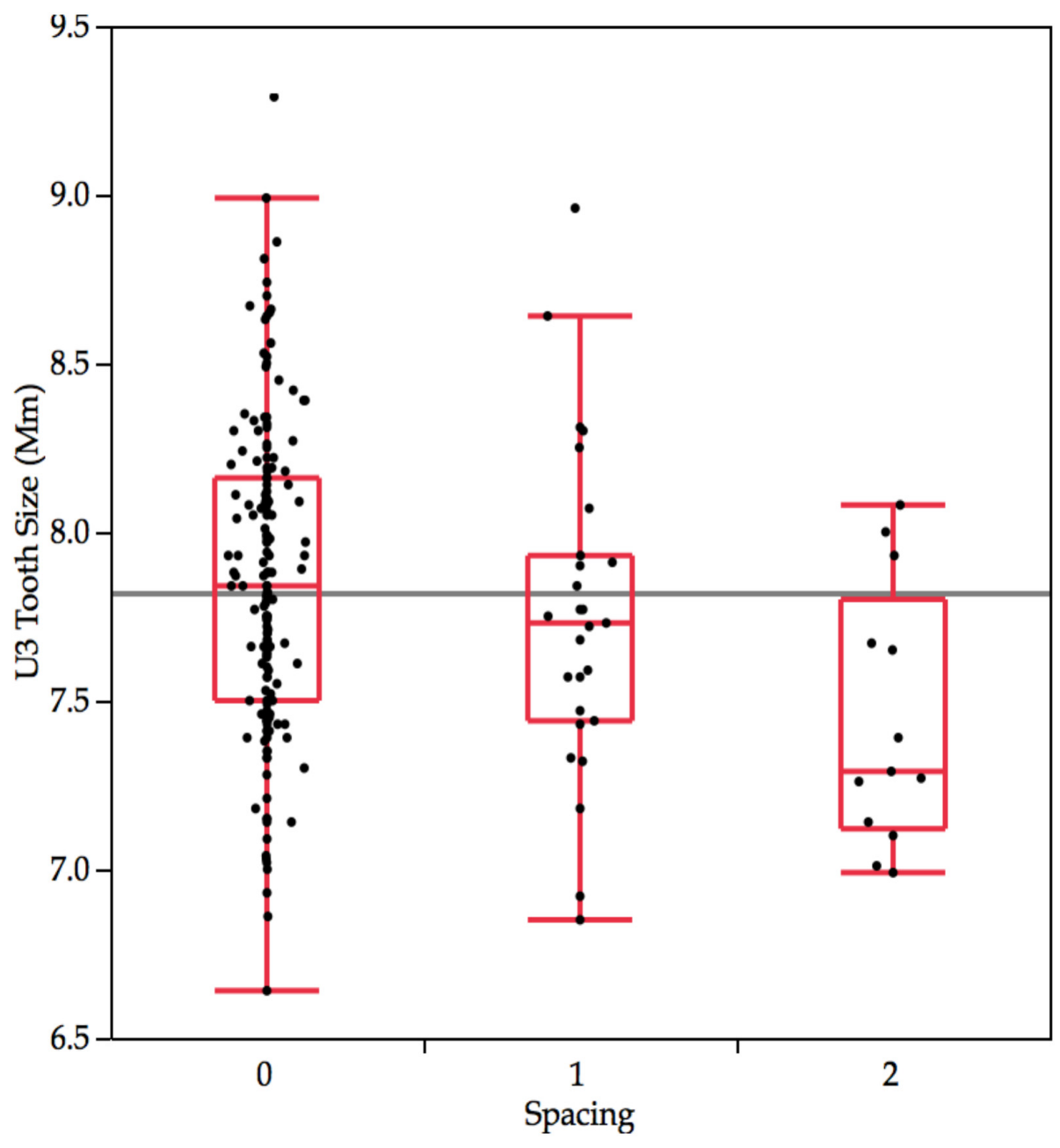

Figure 43. Plot between mesiodistal tooth size of U3 and spacing score.

As tooth size increases, average spacing decreases. 


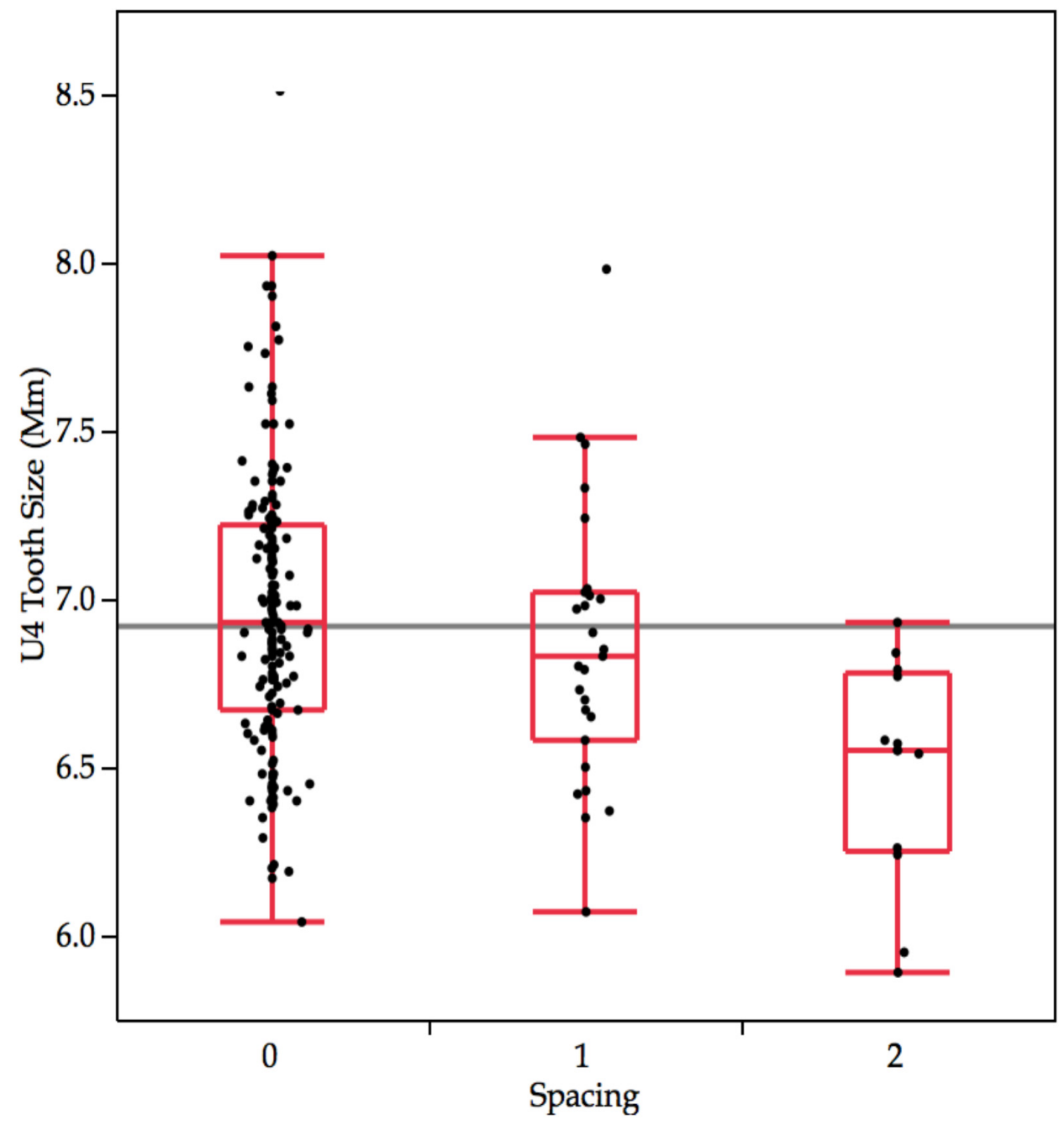

Figure 44. Plot between mesiodistal tooth size of U4 and spacing score.

As tooth size increases, average spacing decreases. 


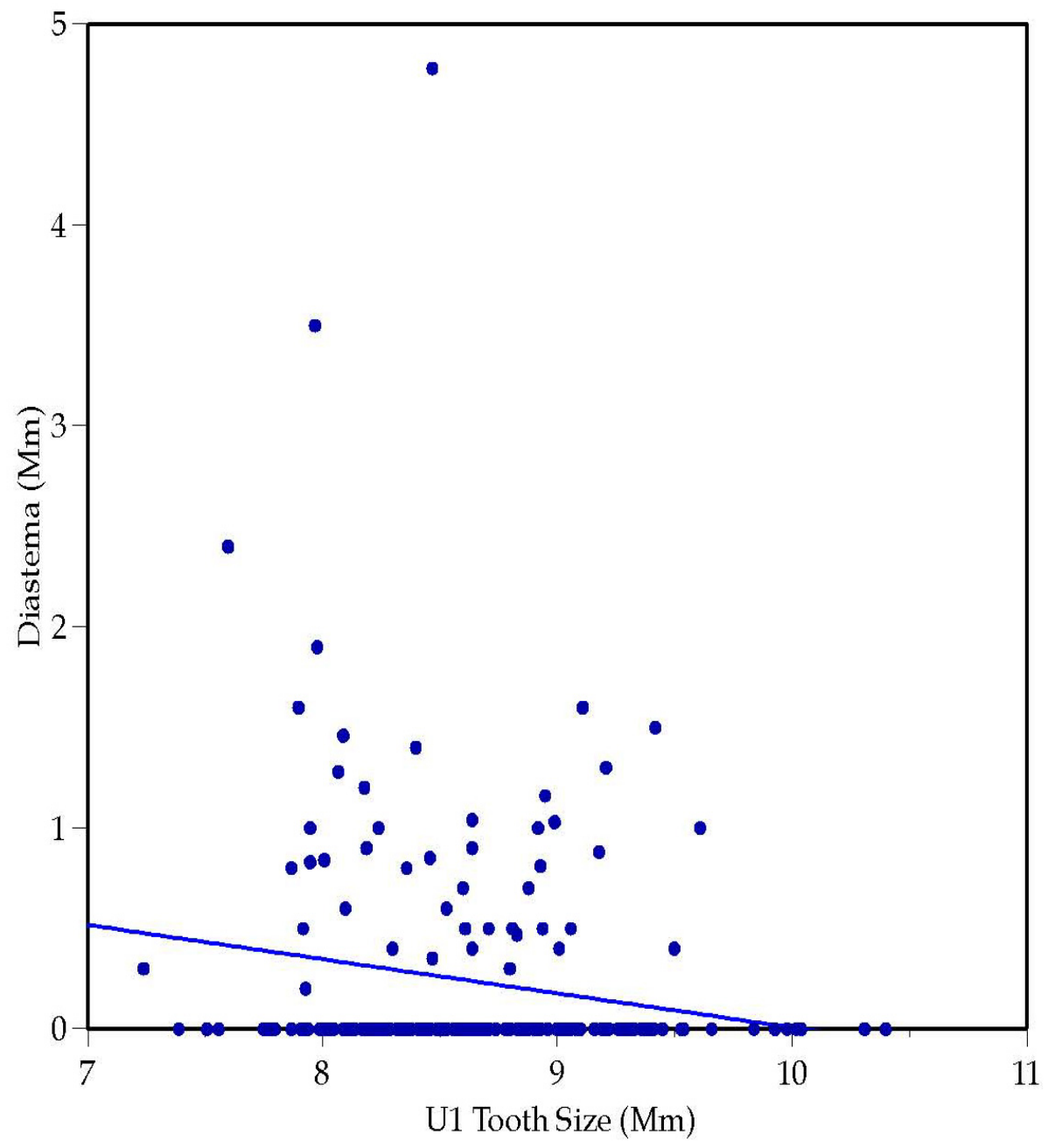

Figure 45. Plot between mesiodistal tooth size of U1 and diastema.

As tooth size increases, diastema spaces diminish. 


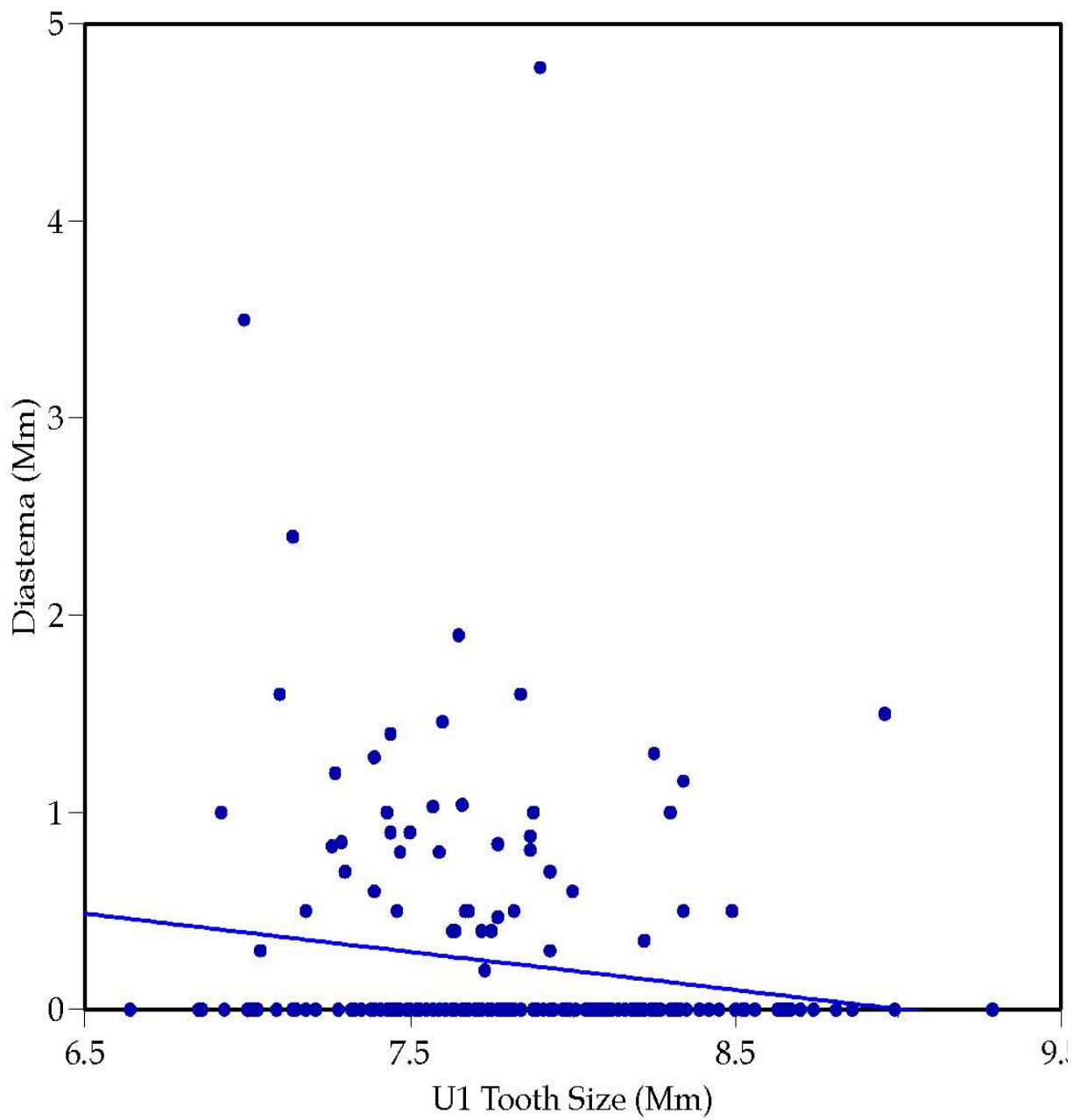

Figure 46. Plot between mesiodistal tooth size of U3 and diastema. As tooth size increases, diastema spaces diminish. 


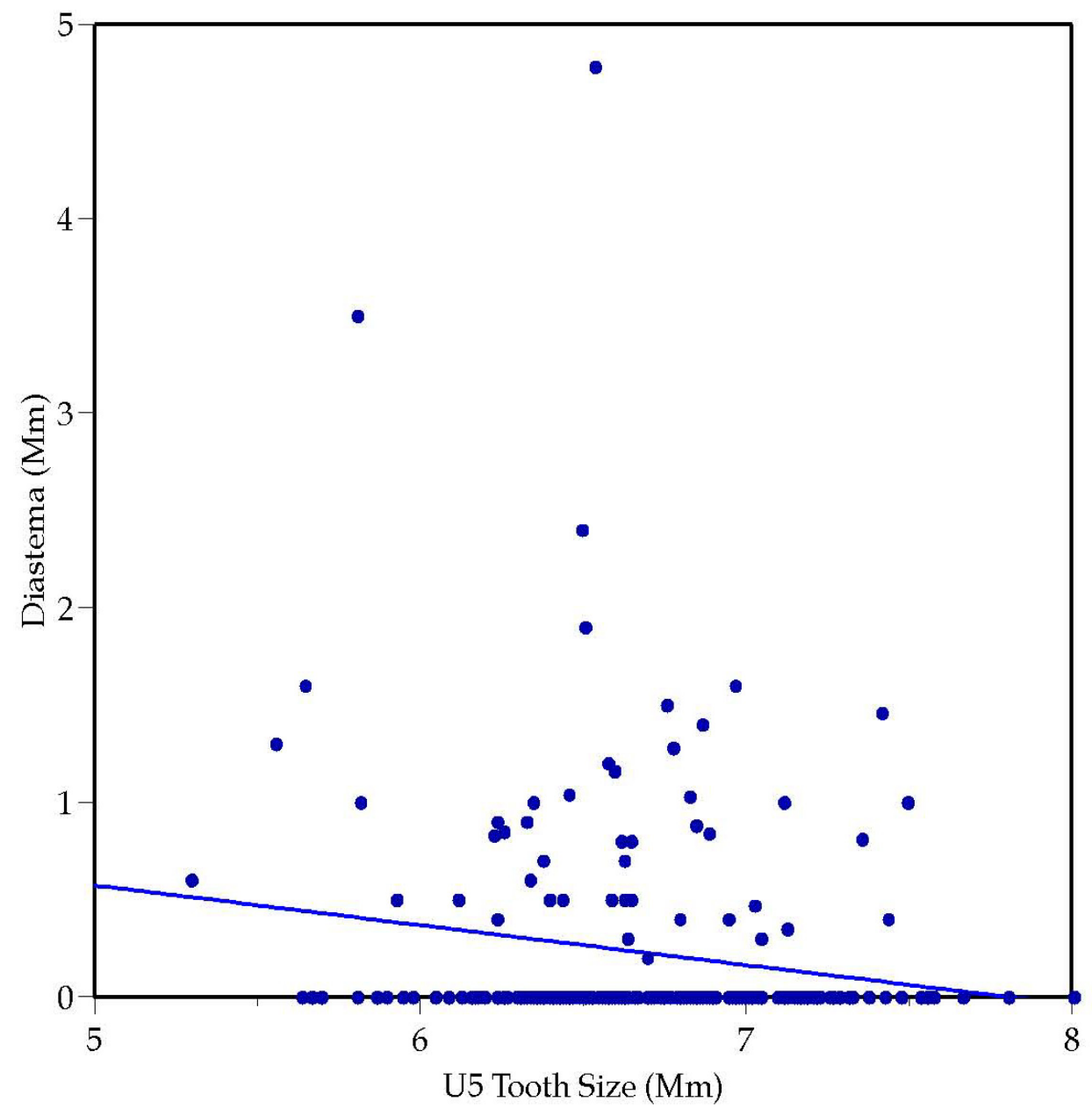

Figure 47. Plot between mesiodistal tooth size of U5 and diastema.

As tooth size increases, diastema spaces diminish. 
crowns "lend themselves" to arch length excesses, but as crowns get larger across the population distribution, arch length is at a premium and the size and incidence of diastemas decrease.

\section{Maxillary Irregularity}

There are several tooth types showing significant, positive associations between crown size and maxillary irregularity (Table 4), and the strongest relationships are for the anterior teeth. The relationship is graphed for U1 (Figure 48), U2 (Figure 49), U3 (Figure 50). Associations are weak or nonsignificant for the premolars and molars. Indeed, we suspect that the marginally significant situations (U6) may be indirect effects, where U6 size is positively correlated with U1 size, so both dimensions show a simple association with irregularity.

\section{Mandibular Irregularity}

Of the seven maxillary tooth types tested (Table 4), only U1 is strongly associated with incisor irregularity in the lower arch (Figure 51). The mode of operation between the arches may be that mesiodistally broad U1 also tend to be big faciolingually, which may tend to push the lower incisors out of alignment. Too, the scenario may be that large U1 tend to occur with large L1, which in turn increases the risk of lower incisor irregularity.

\section{DAI Score}

One might anticipate that this composite measure of esthetic dental problems (Jenny and Cons 1996) would be strongly and broadly intercorrelated with tooth crown dimensions, but it is not (Table 4). Indeed, only the central incisor (of the seven maxillary teeth) exhibits a significant association (Figure 52). The apparent reasons for the weak associations are twofold. One, the DAI is explicitly an esthetic index, so features are included that do not involve dental malocclusions. Mandibular overjet (i.e., mandibular prognathism), anterior overbite, and AP (anterior-posterior) relationship are obvious cases in point since they are shown to be uncorrelated with crown size. Two, the DAI is a weighted index, and the weighting coefficients are based on a person's esthetic conditions, not on dental malocclusions per se. 


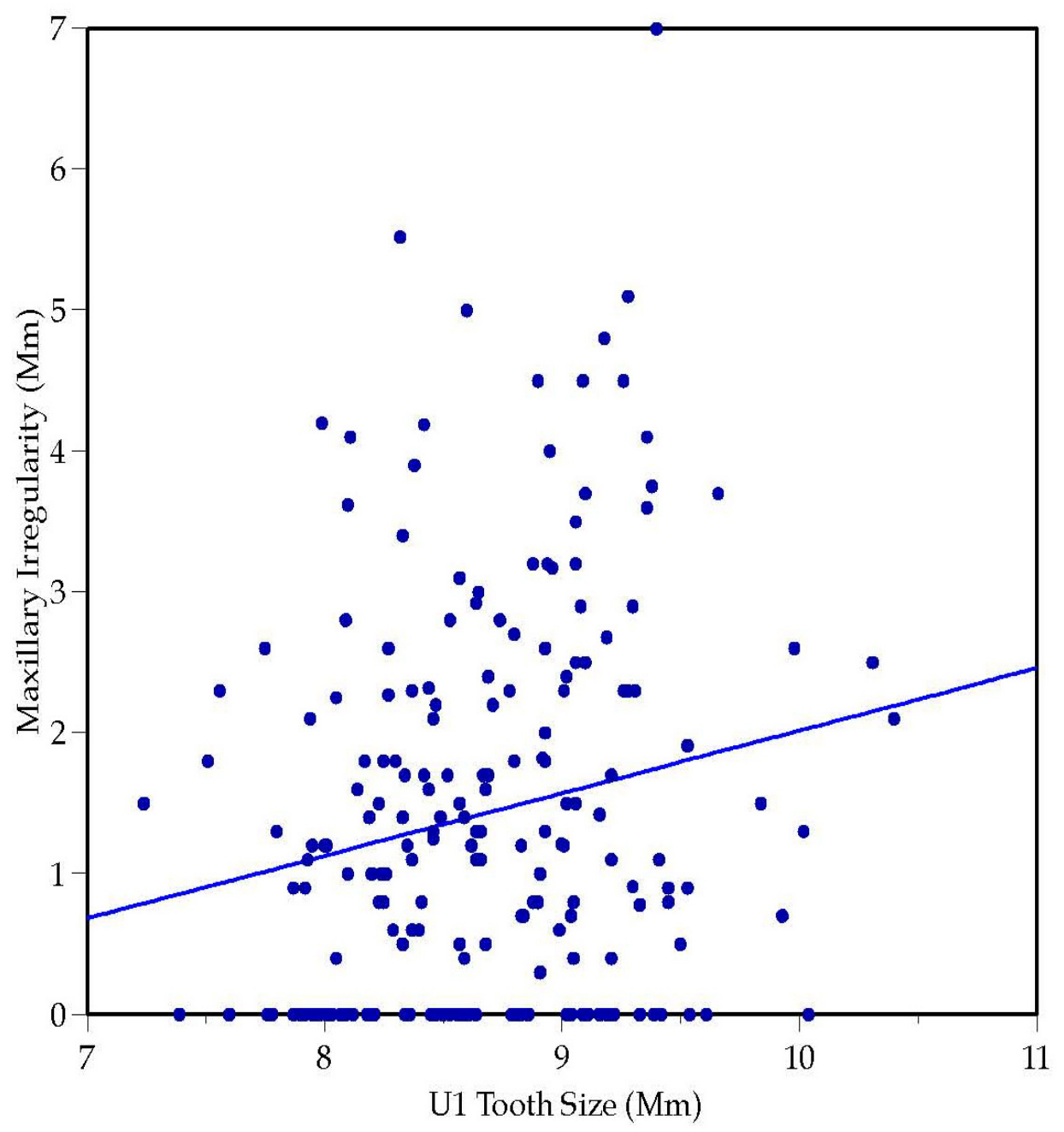

Figure 48. Plot between mesiodistal tooth size of U1 and maxillary irregularity.

As tooth size increases, so does maxillary irregularity. 


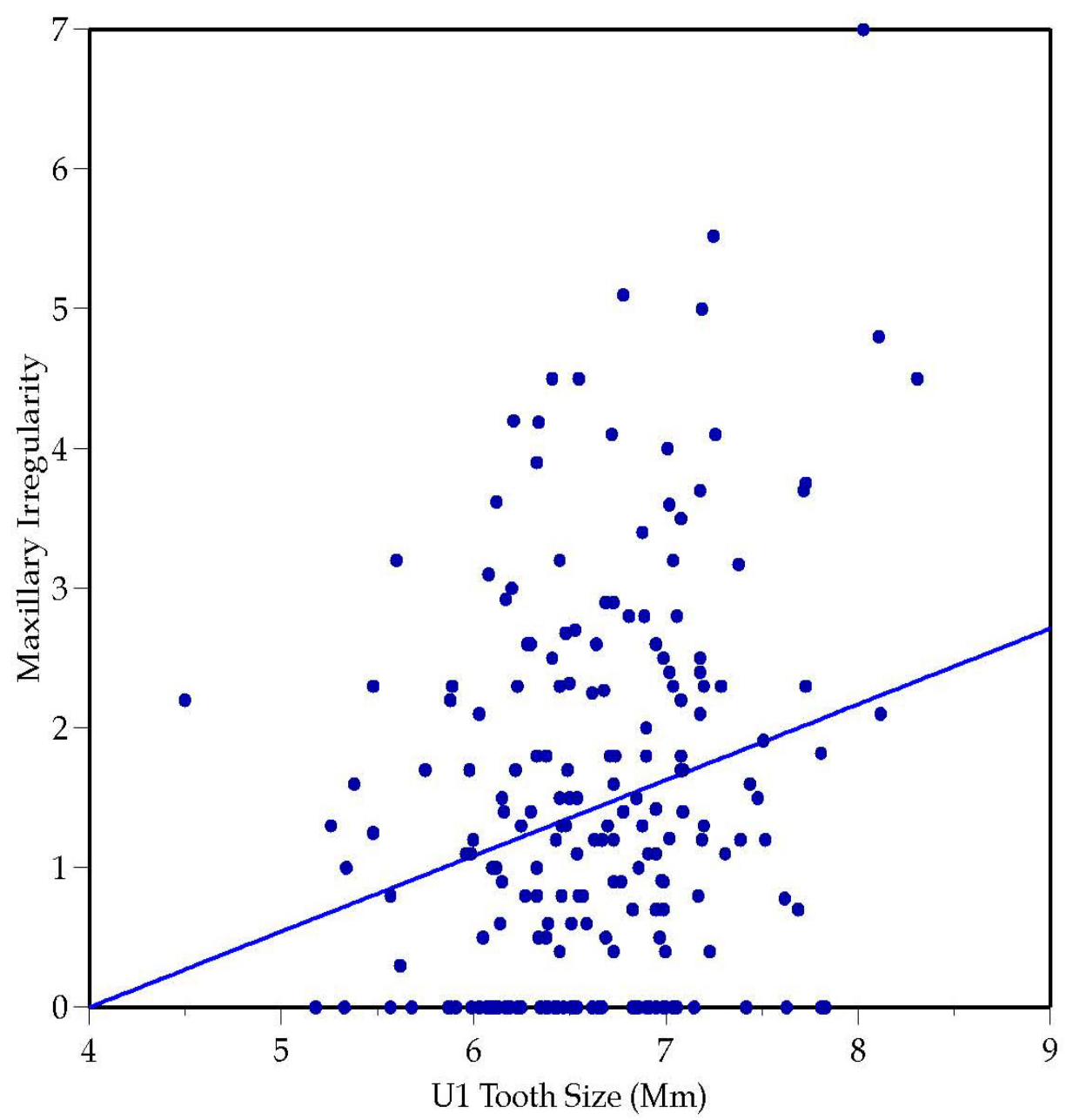

Figure 49. Plot between mesiodistal tooth size of U2 and maxillary irregularity.

As tooth size increases, so does maxillary irregularity. 


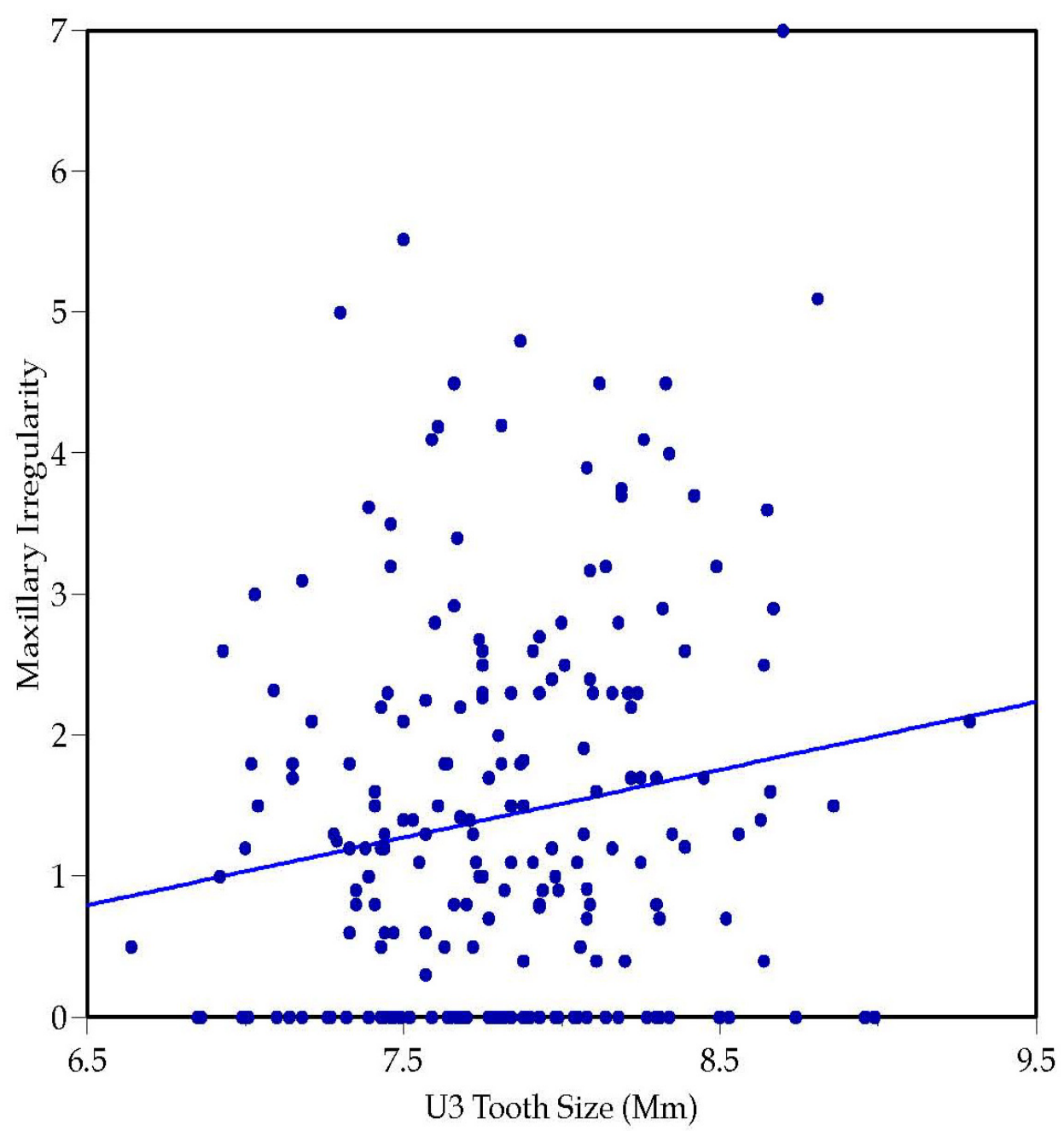

Figure 50. Plot between mesiodistal tooth size of U3 and maxillary irregularity.

As tooth size increases, so does maxillary irregulalrity. 


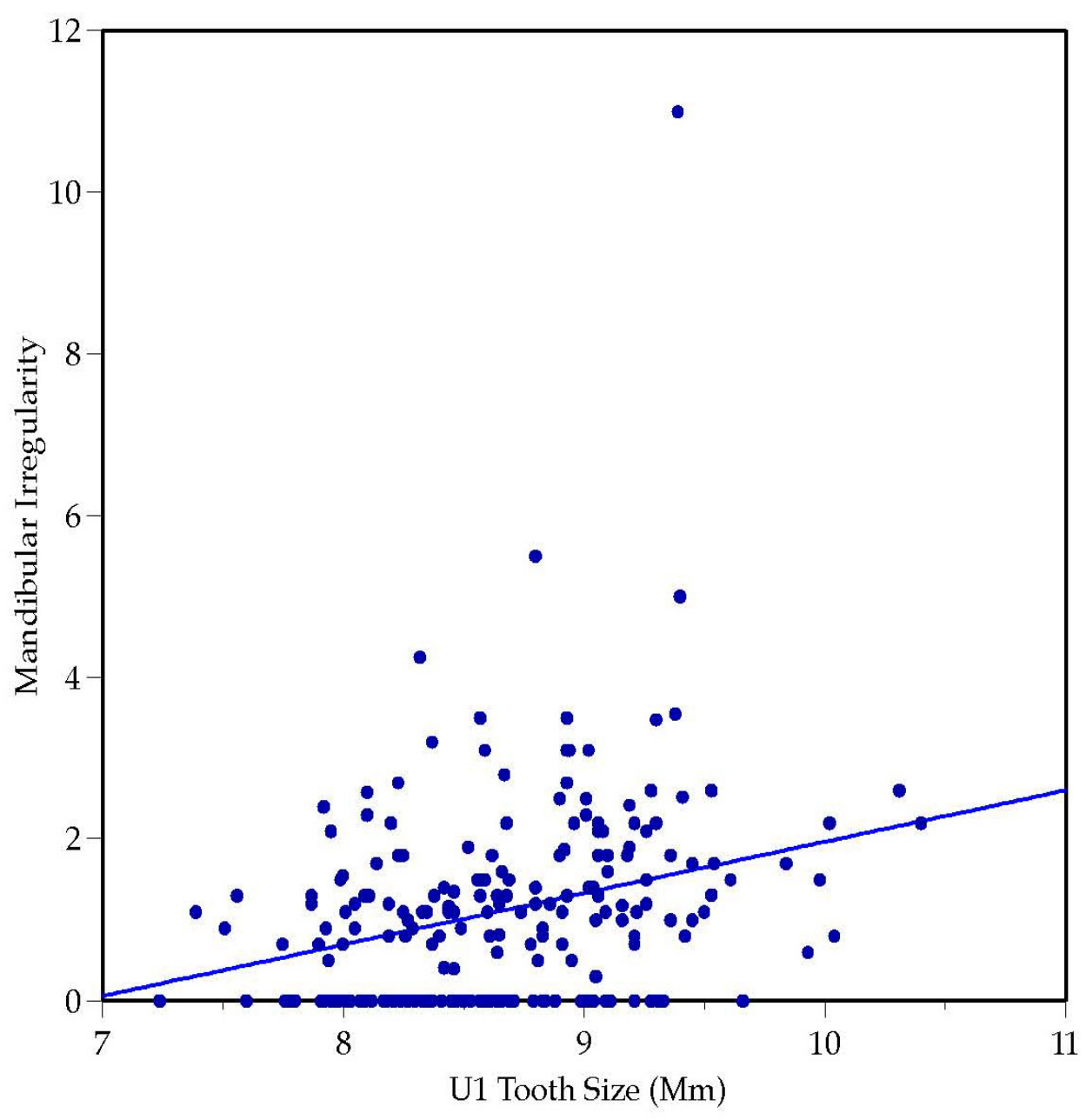

Figure 51. Plot between mesiodistal tooth size of U1 and mandibular irregularity.

As tooth size increases, so does irregularity of the lower arch. 


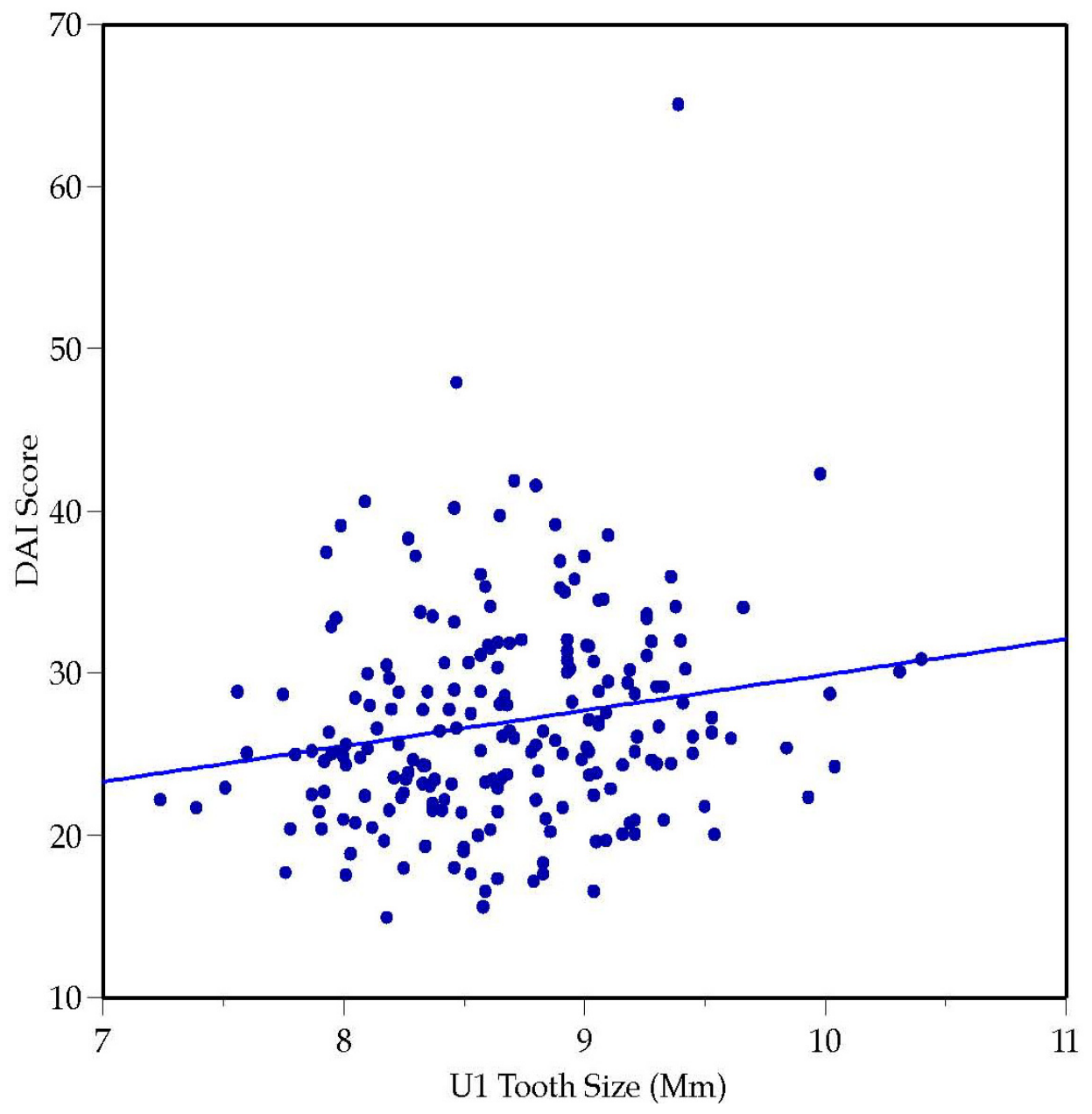

Figure 52. Plot between mesiodistal tooth size of U1 and DAI score.

As tooth size increases, so does the average DAI score. 


\section{Mandibular Tooth Types}

A table of P-Values associated with the F-Ratios of the regression of occlusal status on mandibular mesiodistal tooth size is in Table 5. This parallels the findings for the maxillary teeth and, again, some generalities can be drawn. One, there are a fewer statistically significant associations here than for the maxillary variables, and the difference is primarily due to the lack of associations with the posterior teeth. Unlike the table of maxillary associations, significant relationships between crown size and occlusal variations are clustered in the anterior region of the arch. Two, the sources of malocculsions that depend primarily on relationships of the supporting bony structures (mandibular overjet, openbite, AP relationship) are seldom significantly correlated with crown size.

\section{Total Displacements}

There are highly significant, positive associations between anterior crown sizes and total displacement. This is evident for L1 (Figure 53) and L2 (Figure 54). Mesiodistally broader teeth are associated with greater displacement scores.

\section{Total Rotations}

Four of the seven associations are significant statistically, and the stronger associations are with L1 (Figure 55) and L2 (Figure 56). Also of the associations are positive: Broader tooth crowns are associated with greater rotation scores.

\section{Total Overlapping}

Significant, positive associations occur between tooth size and the extent of overlapping, particularly in the teeth of the anterior and mid-arch segments. The relationships are illustrated here for L1 (Figure 57) and L2 (Figure 58) where the associations are strongest.

\section{Total Displacements, Rotations, and Overlapping}

This composite of different sorts of malocclusions is positively associated with mesiodistal crown sizes in the mandible, especially for the incisors and canine. The strongest associations are for L1 (Figure 59) and L2 (Figure 60). 


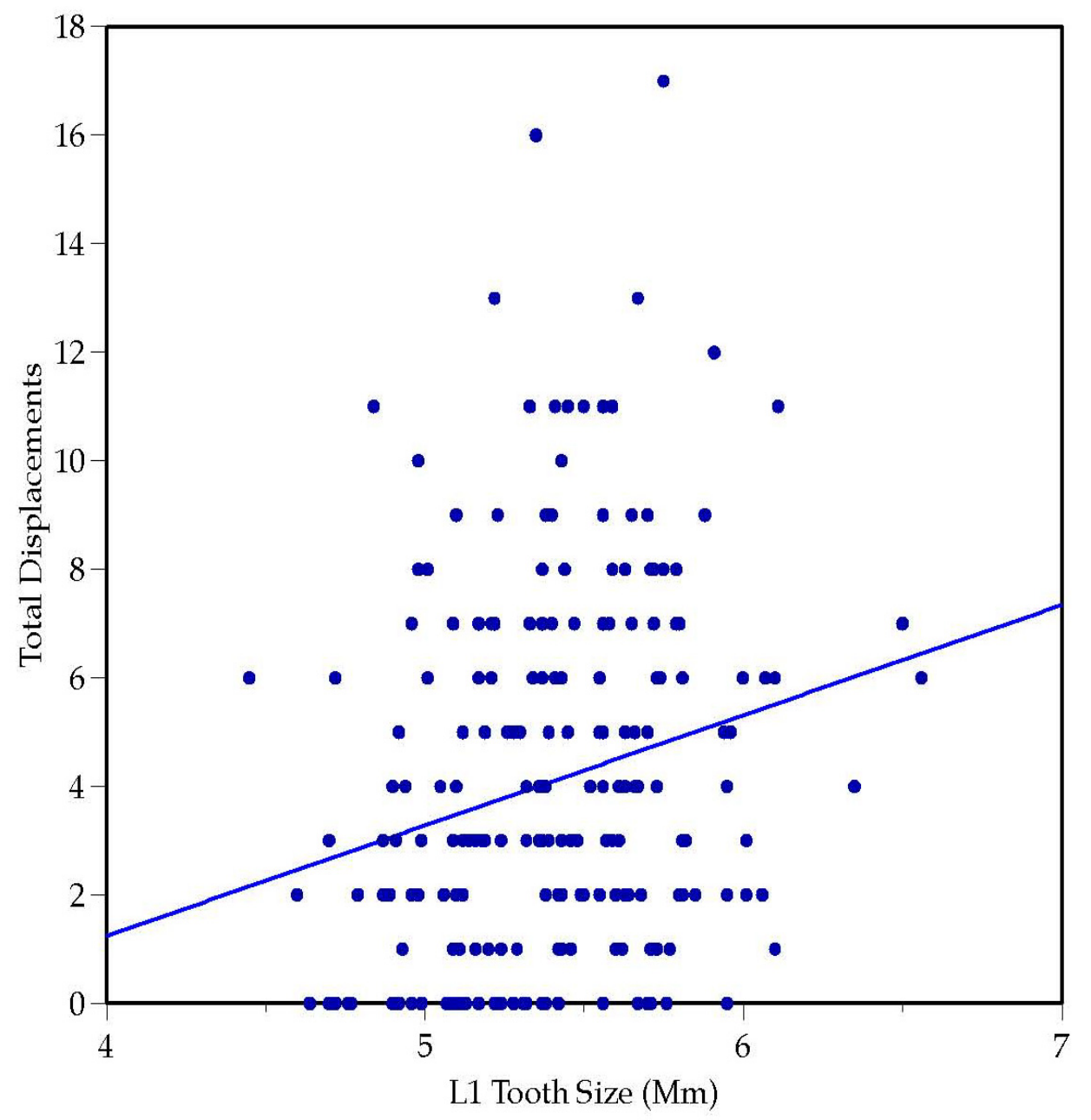

Figure 53. Plot between mesiodistal tooth size of L1 and total displacement score.

As tooth size increases, so does the average total displacement score. 


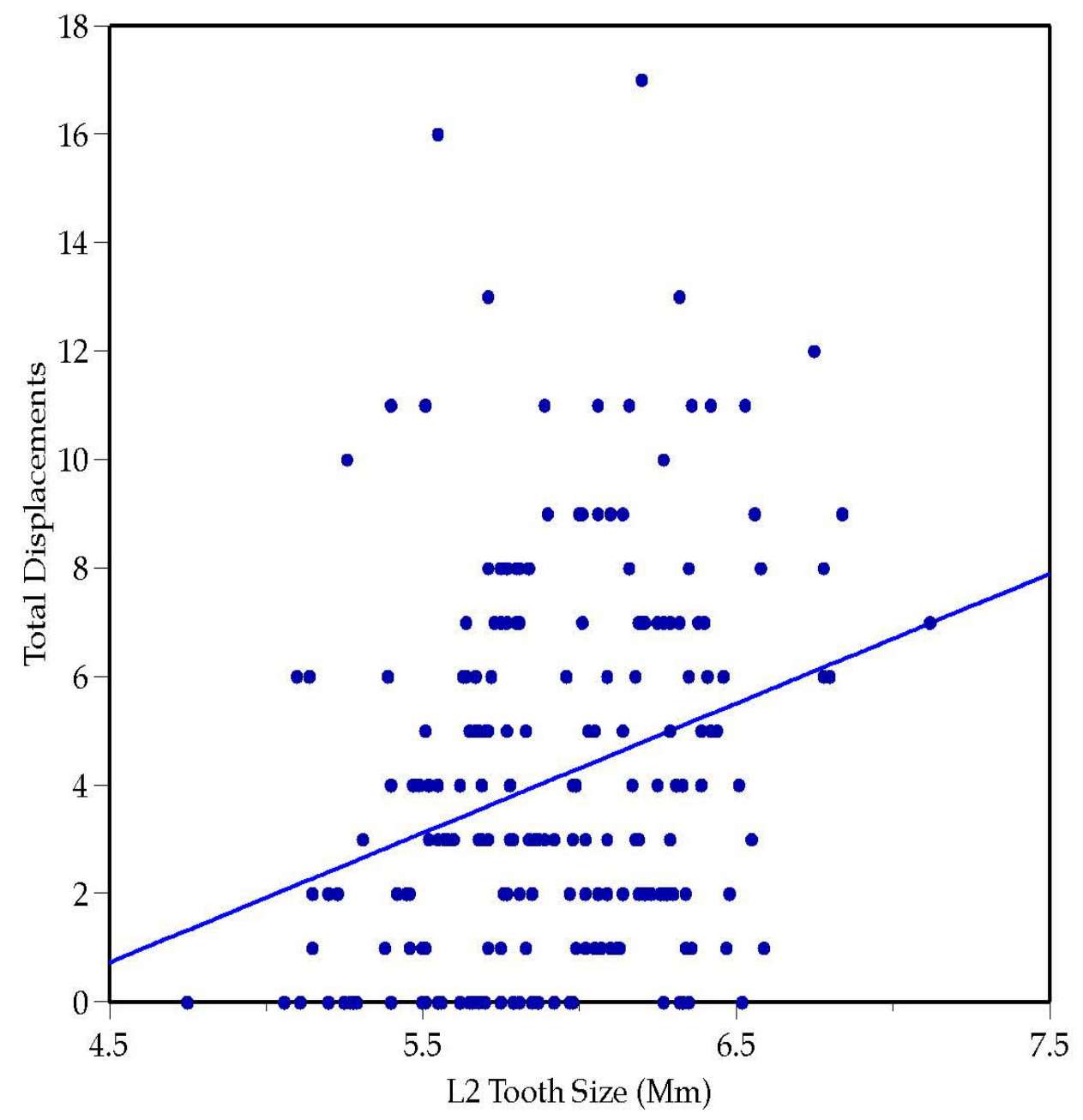

Figure 54. Plot between mesiodistal tooth size of L2 and total displacement score.

As tooth size increases, so does the average total displacement score. 


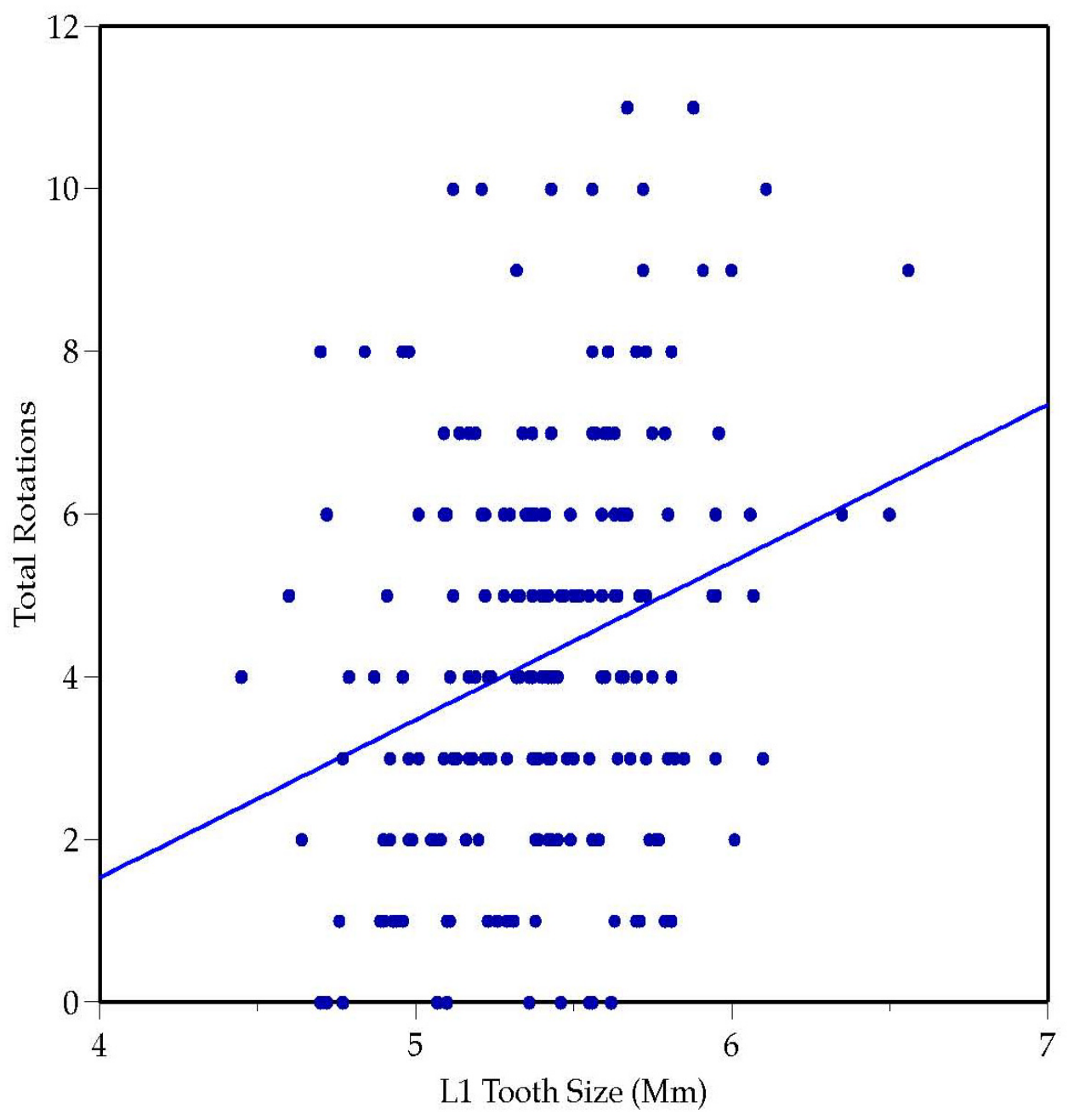

Figure 55. Plot between mesiodistal tooth size of L1 and total rotations.

As tooth size increases, so does the average total rotations. 


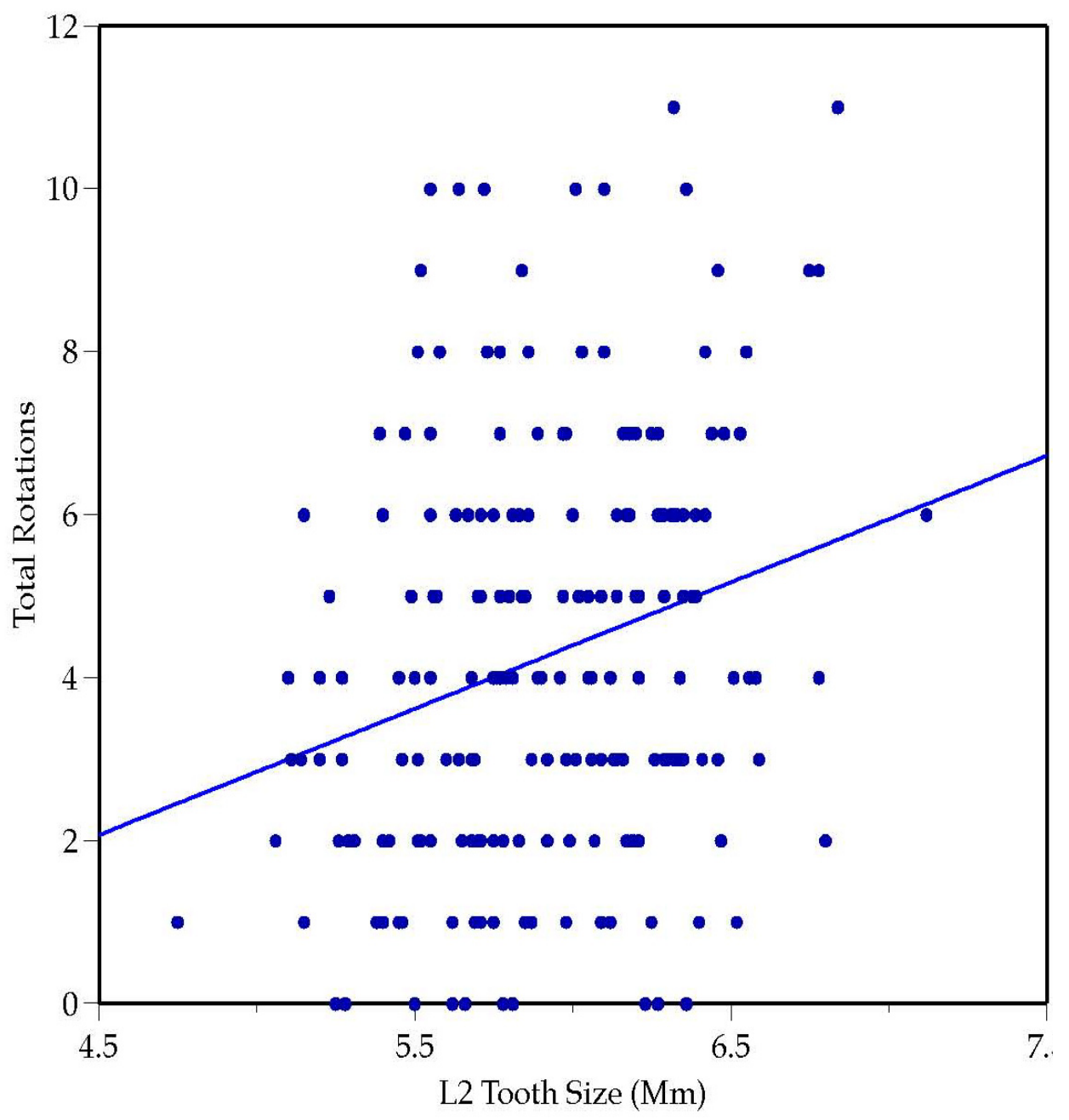

Figure 56. Plot between mesiodistal tooth size of L2 and total rotations.

As tooth size increases, so does the average total rotations. 


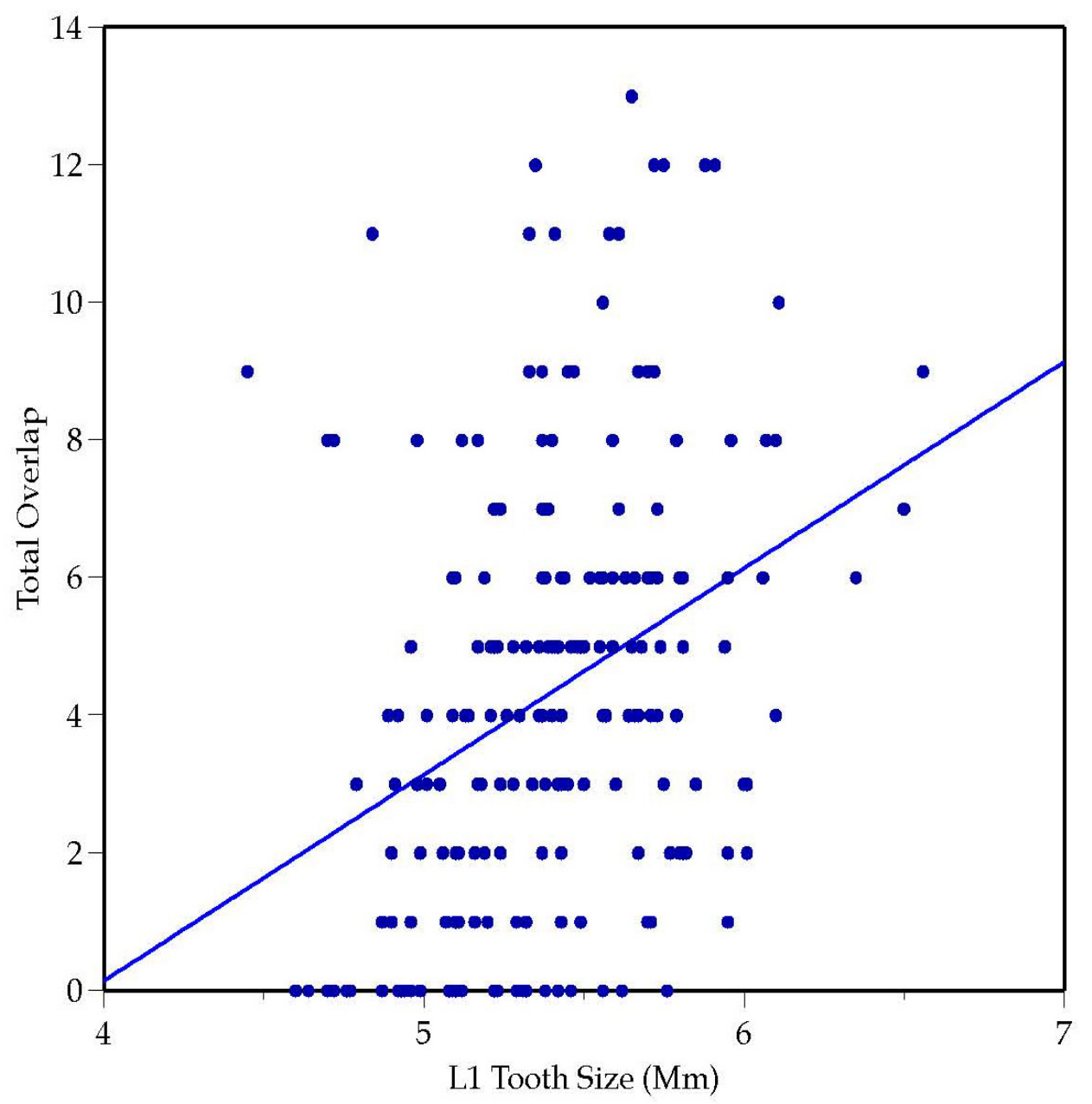

Figure 57. Plot between mesiodistal tooth size of L1 and total overlap.

As tooth size increases. so does the average total overlap score. 


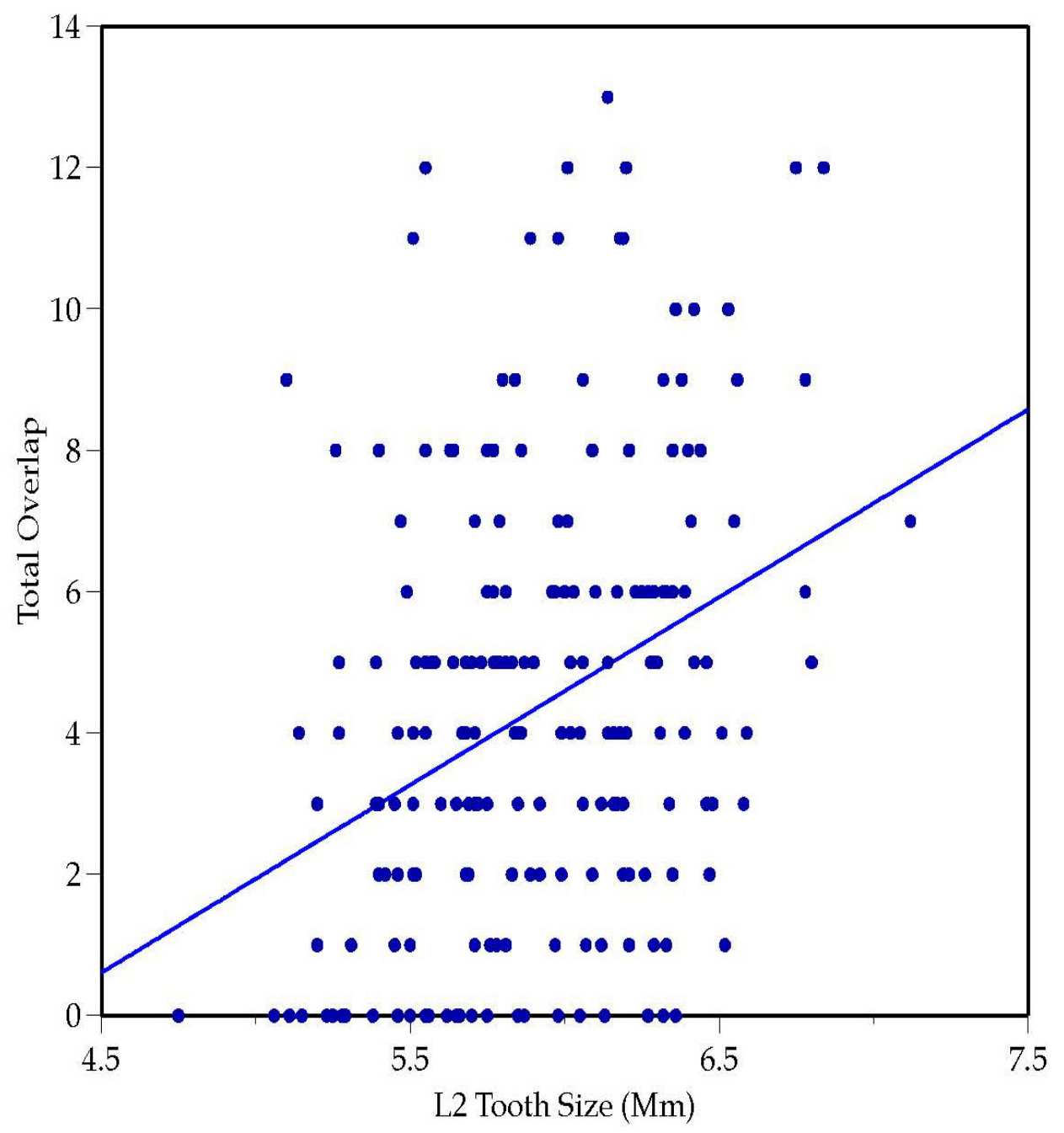

Figure 58. Plot between mesiodistal tooth size of L2 and total overlap.

As tooth size increases, so does the average total overlap score. 


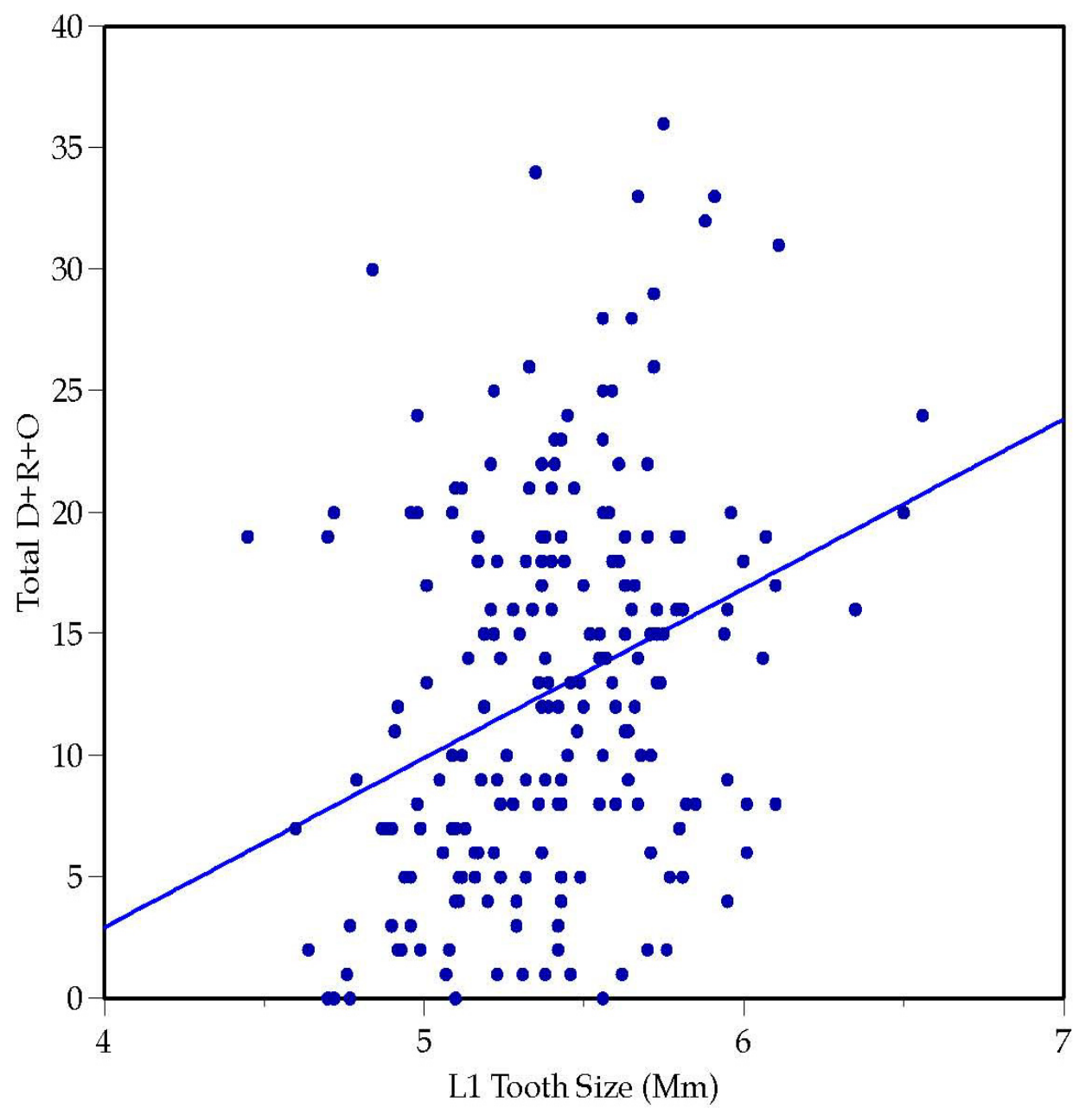

Figure 59. Plot between mesiodistal tooth size of L1 and total displacements plus rotations plus overlapping.

As tooth size increases so does the average total $\mathrm{D}+\mathrm{R}+\mathrm{O}$ score. 


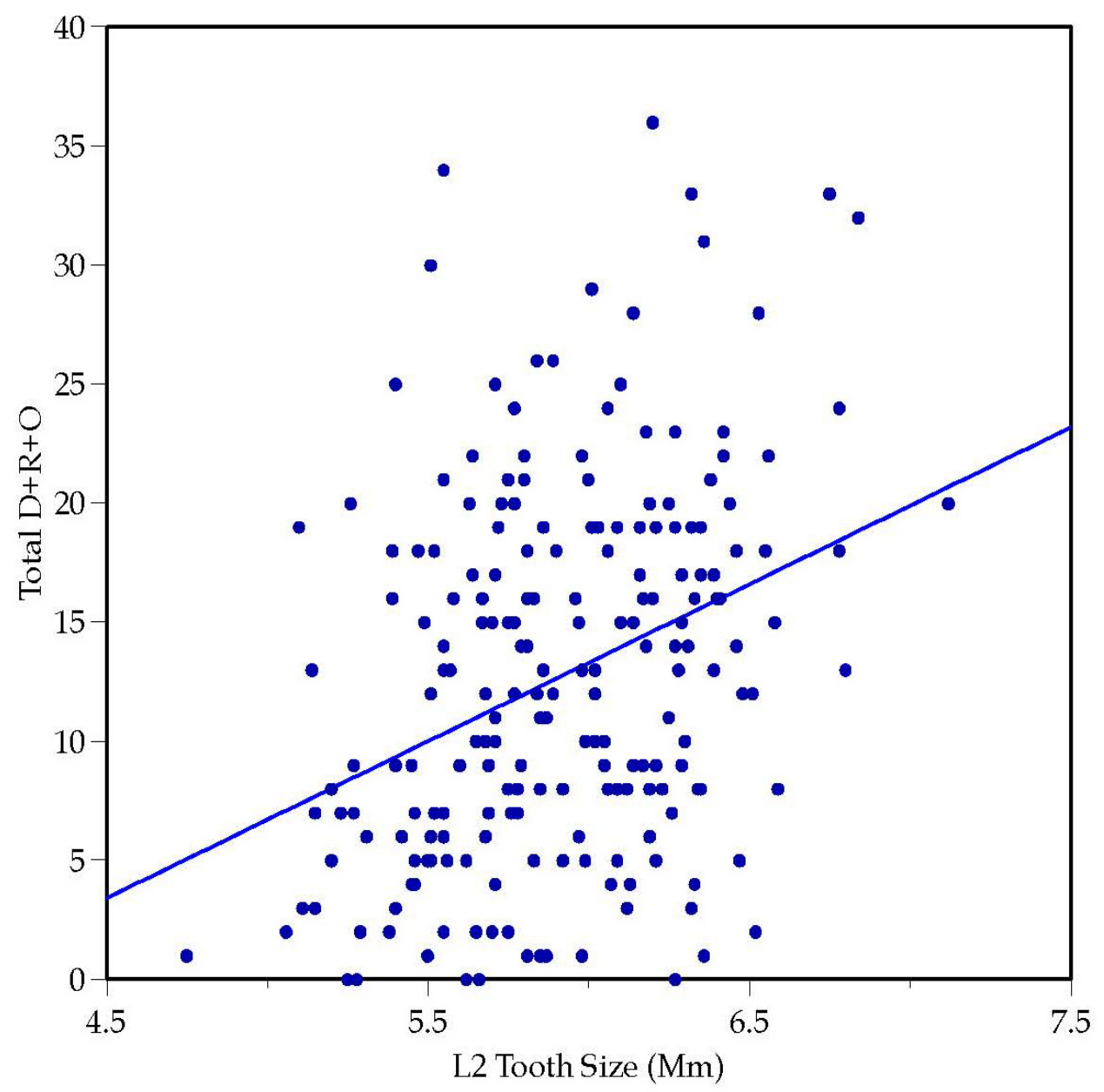

Figure 60. Plot between mesiodistal tooth size of L2 and total displacements plus rotations plus overlapping.

As tooth size increases, so does the average total $\mathrm{D}+\mathrm{R}+\mathrm{O}$ score. 


\section{Crowding}

Highly significant, positive associations $(\mathrm{P}<0.0001)$ occur between the extent of crowding and (1) L1 (Figure 61), (2) L2 (Figure 62), (3) L3 (Figure 63), and (4) L4 (Figure 64). Just as elsewhere, broader mesiodistal tooth crowns are associated with greater crowding. The three distal tooth types (L5, L6, L7) are comparatively weakly associated with this measure of crowding.

\section{Spacing}

Interdental spacing is unique among the mandibular variables tested in that (1) the associations with tooth size are negative and (2) all seven tooth types are highly significantly correlated with spacing. The relationships are negative (Figure 65 and 66) because the smaller the crown widths the greater the spacing tends to be.

It is fairly commonplace to find all seven tooth types significantly correlated with a measure of malocclusion in the maxillary (Table 4). The trend in the mandible (Table 5) is that the two molar tooth types are unrelated.

\section{Diastema}

The relationship for the diastema parallel that for interdental spacing: smaller crown sizes are associated with diastemas; larger teeth significantly deduce the likelihood of a diastema. In other words, the relationships are negative, and this is illustrated in Figure 67 (L1) and Figure 68 (L2).Comparable to the situation for spacing most of the seven tooth types exhibit a significant association with diastema. Perhaps this is due to the interrelationships across crown sizes (e.g., Moorrees and Reed 1964), so some of the statistical associations may be indirect.

\section{Maxillary Irregularity}

There are highly significant, positive associations between mandibular incisor width, but teeth distal of the incisors are independent of irregularity. The association for L1 and irregularity is shown in Figure 69; that for L2 is shown in Figure 70. 


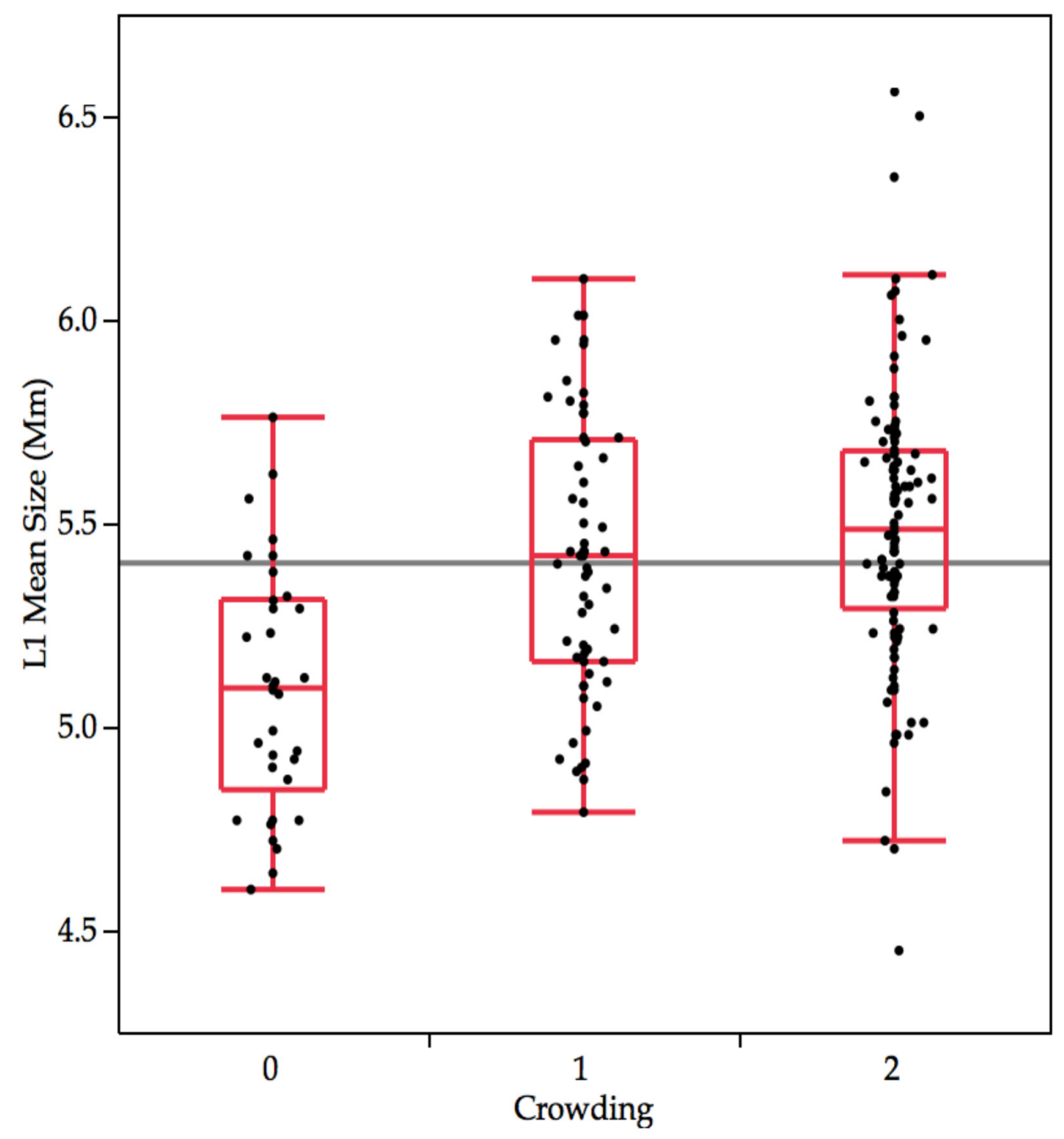

Figure 61. Plot between mesiodistal tooth size of L1 and crowding score.

As tooth size increases, average crowding also increases. 


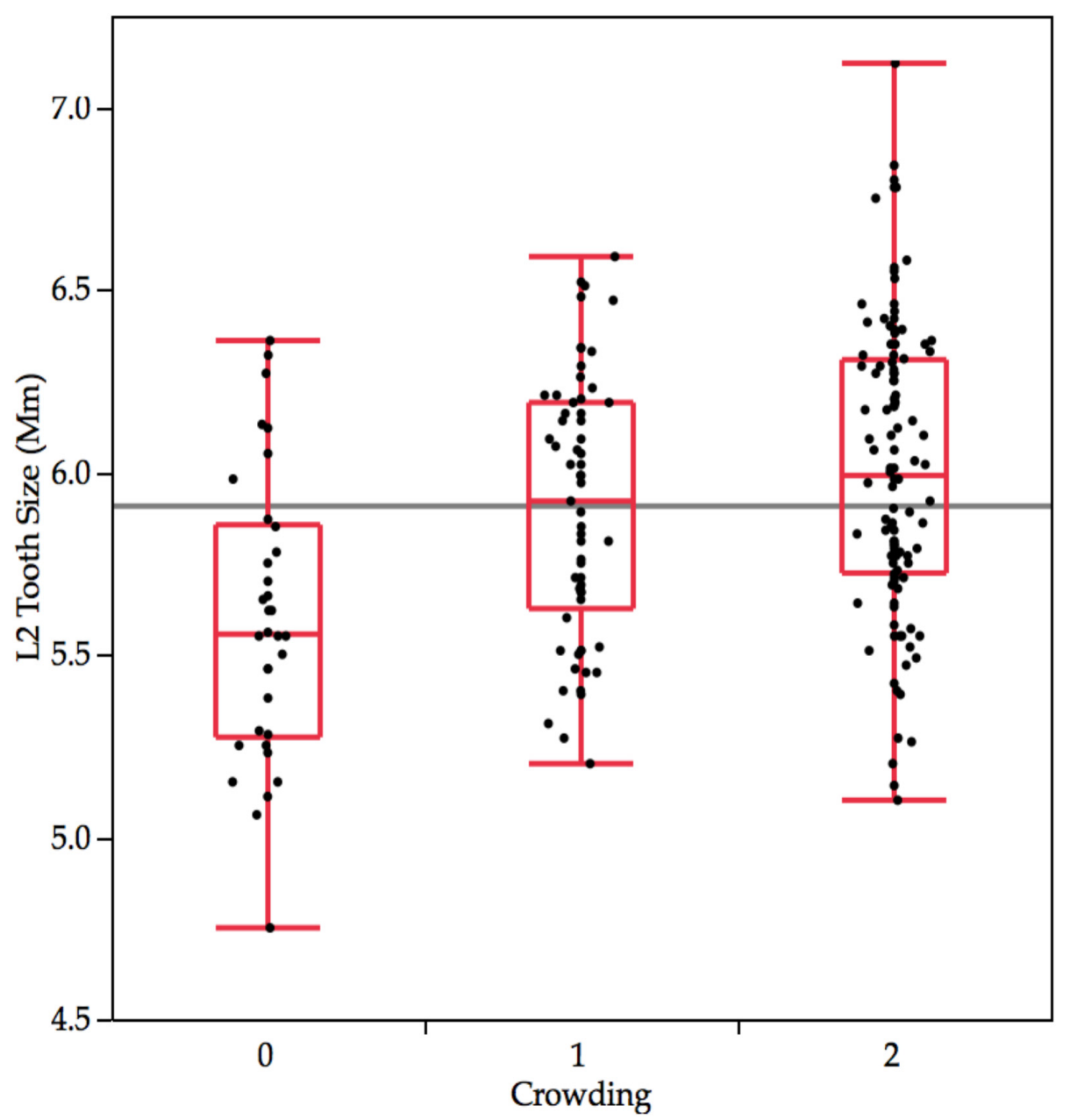

Figure 62. Plot between mesiodistal tooth size of L2 and crowding score.

As tooth size increases, average crowding also increases. 


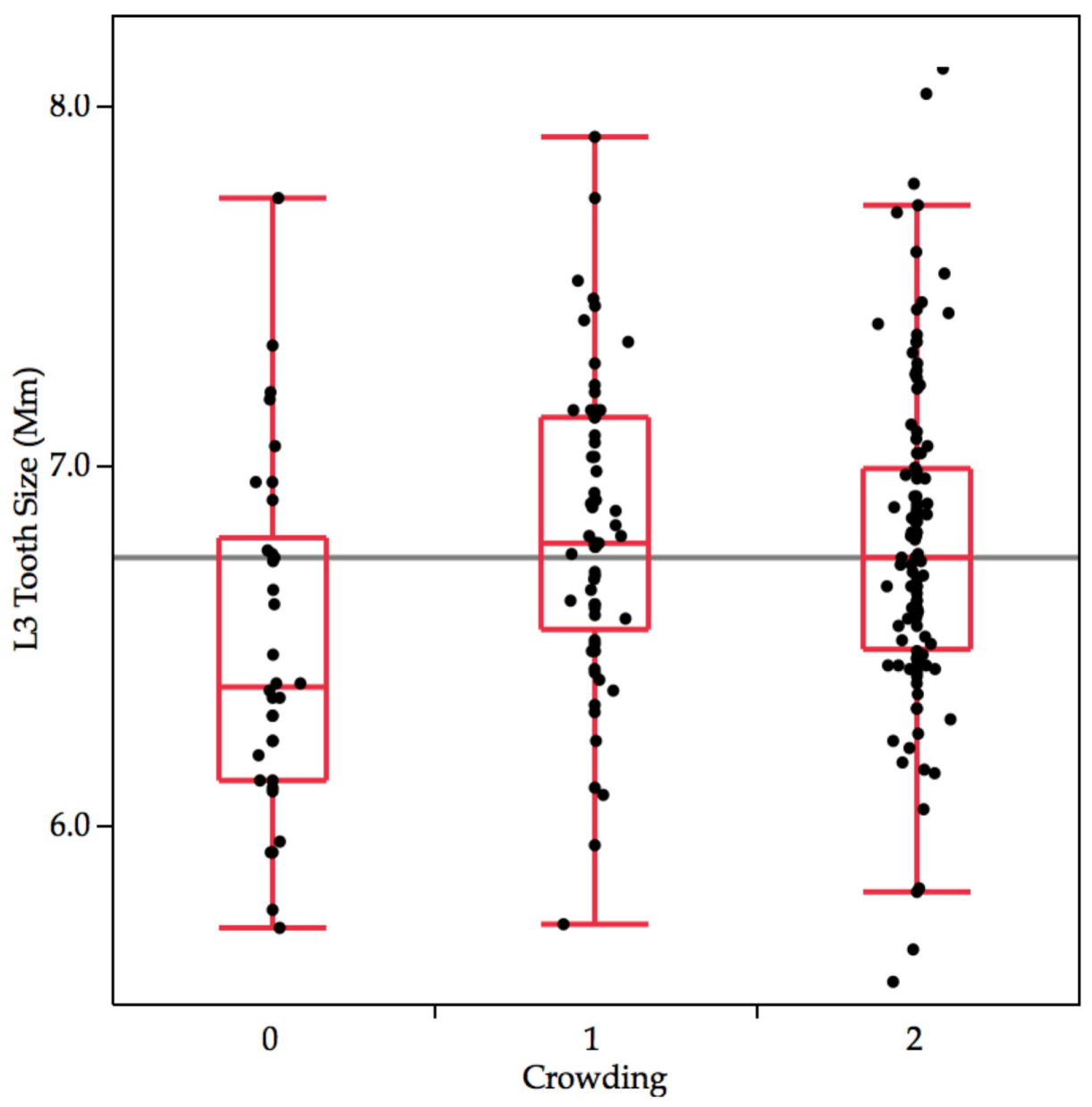

Figure 63. Plot between mesiodistal tooth size of L3 and crowding score.

As tooth size increases, average crowding also increases. 


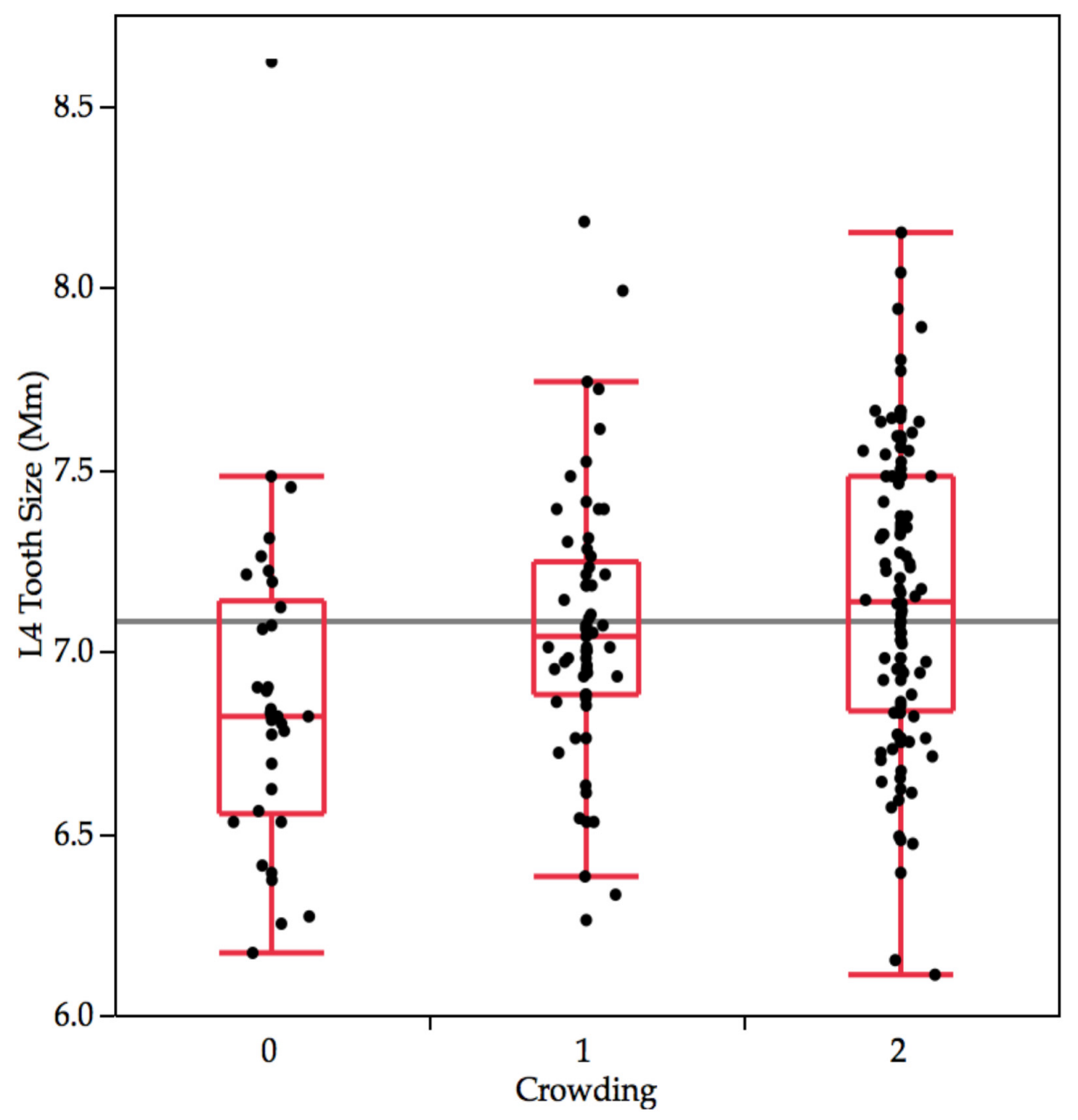

Figure 64. Plot between mesiodistal tooth size of L4 and crowding score.

As tooth size increases, average crowding also increases. 


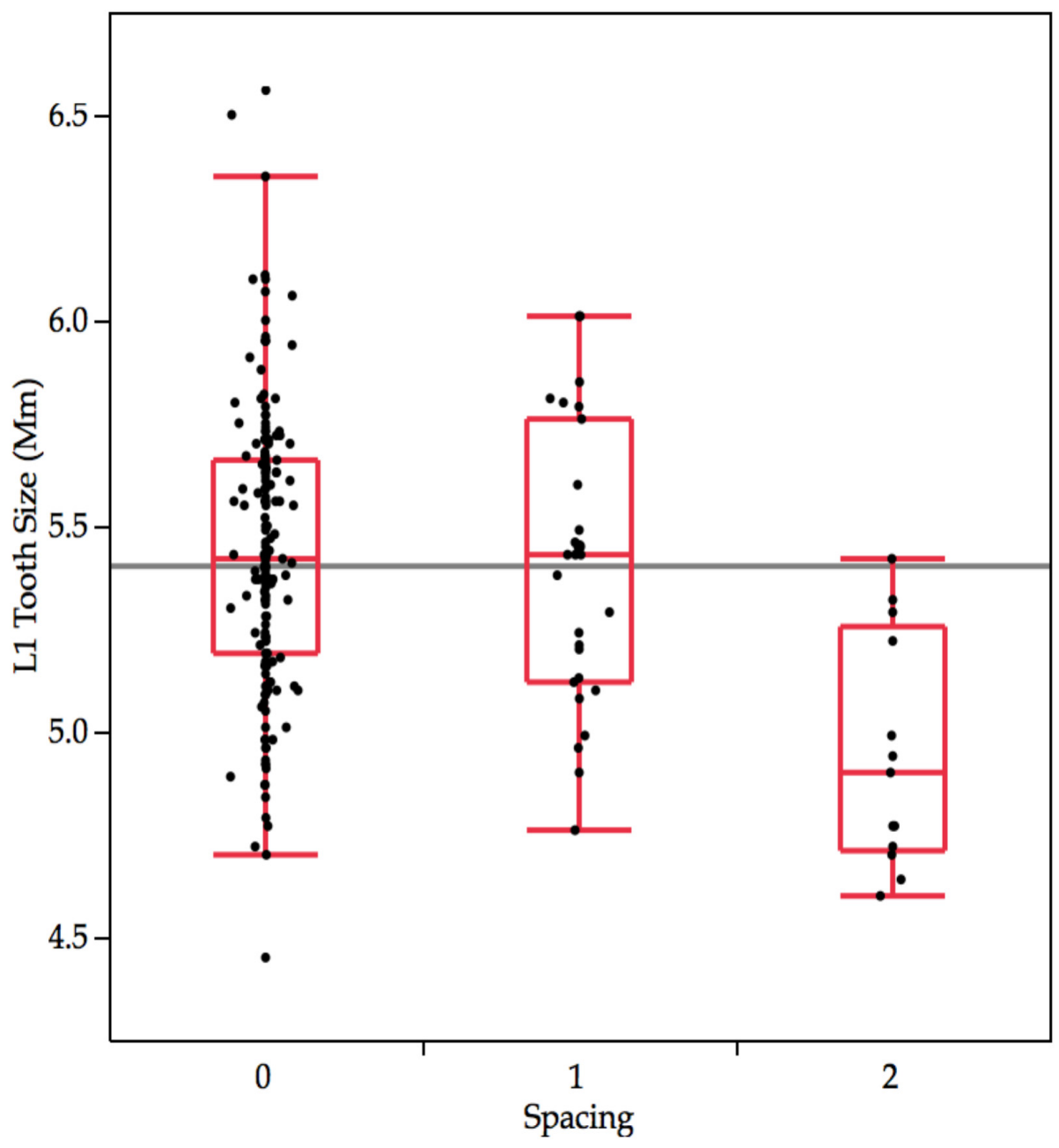

Figure 65. Plot between mesiodistal tooth size of L1 and spacing score.

As tooth size increases, average spacing decreases. 


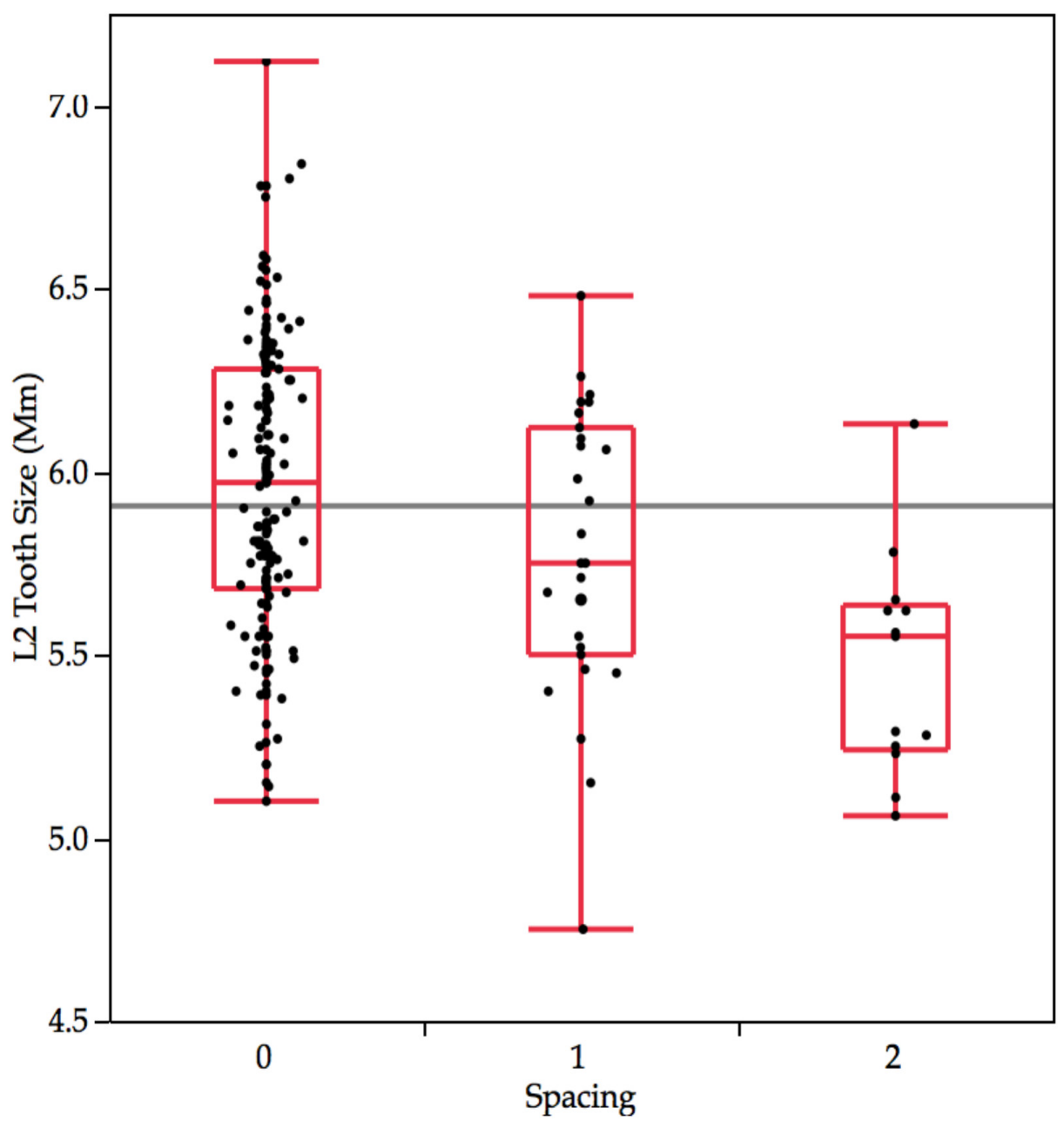

Figure 66. Plot between mesiodistal tooth size of L2 and spacing score.

As tooth size increases, average spacing decreases. 


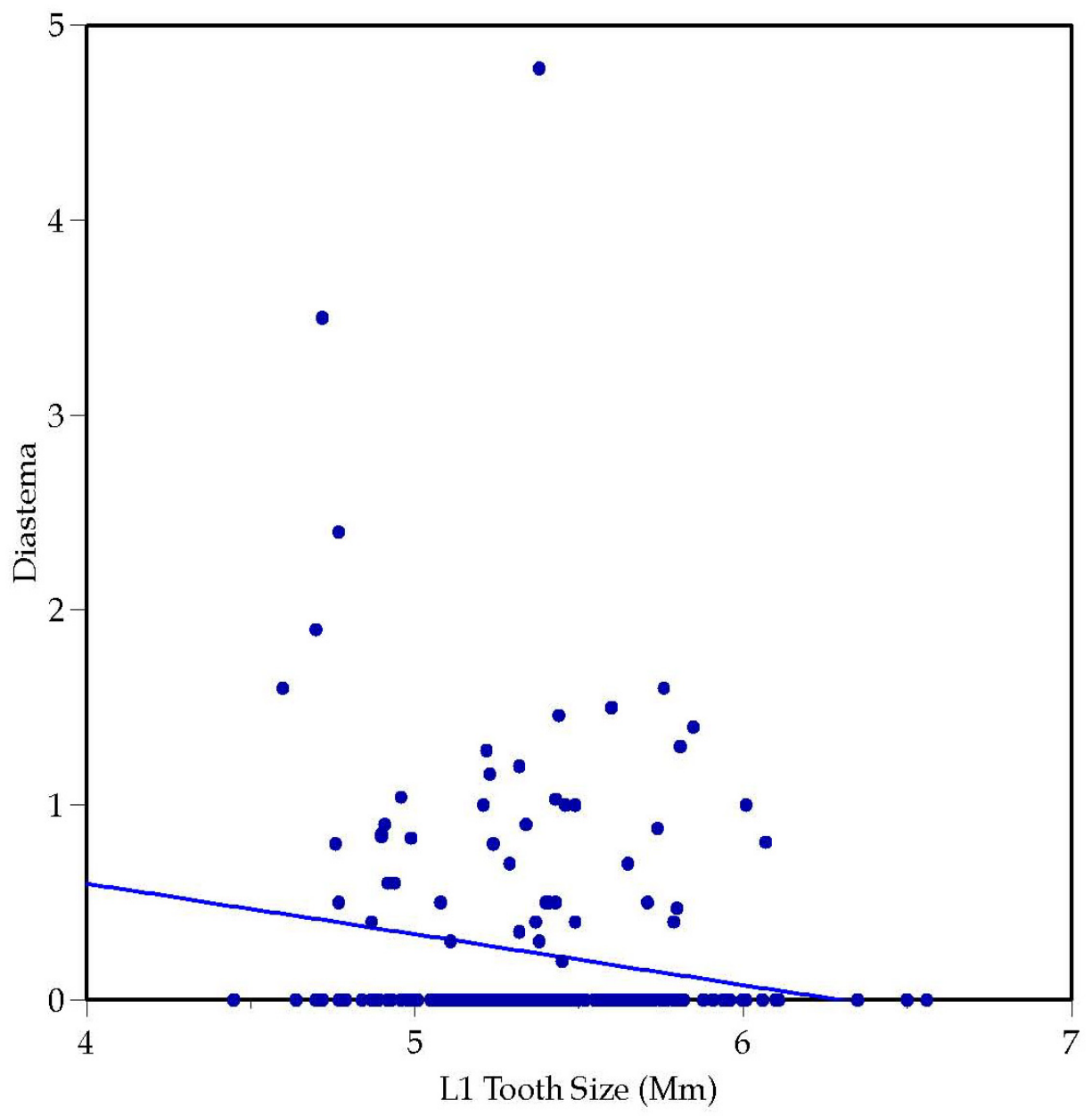

Figure 67. Plot between mesiodistal tooth size of L1 and diastema. As tooth size increases, average diastema diminishes. 


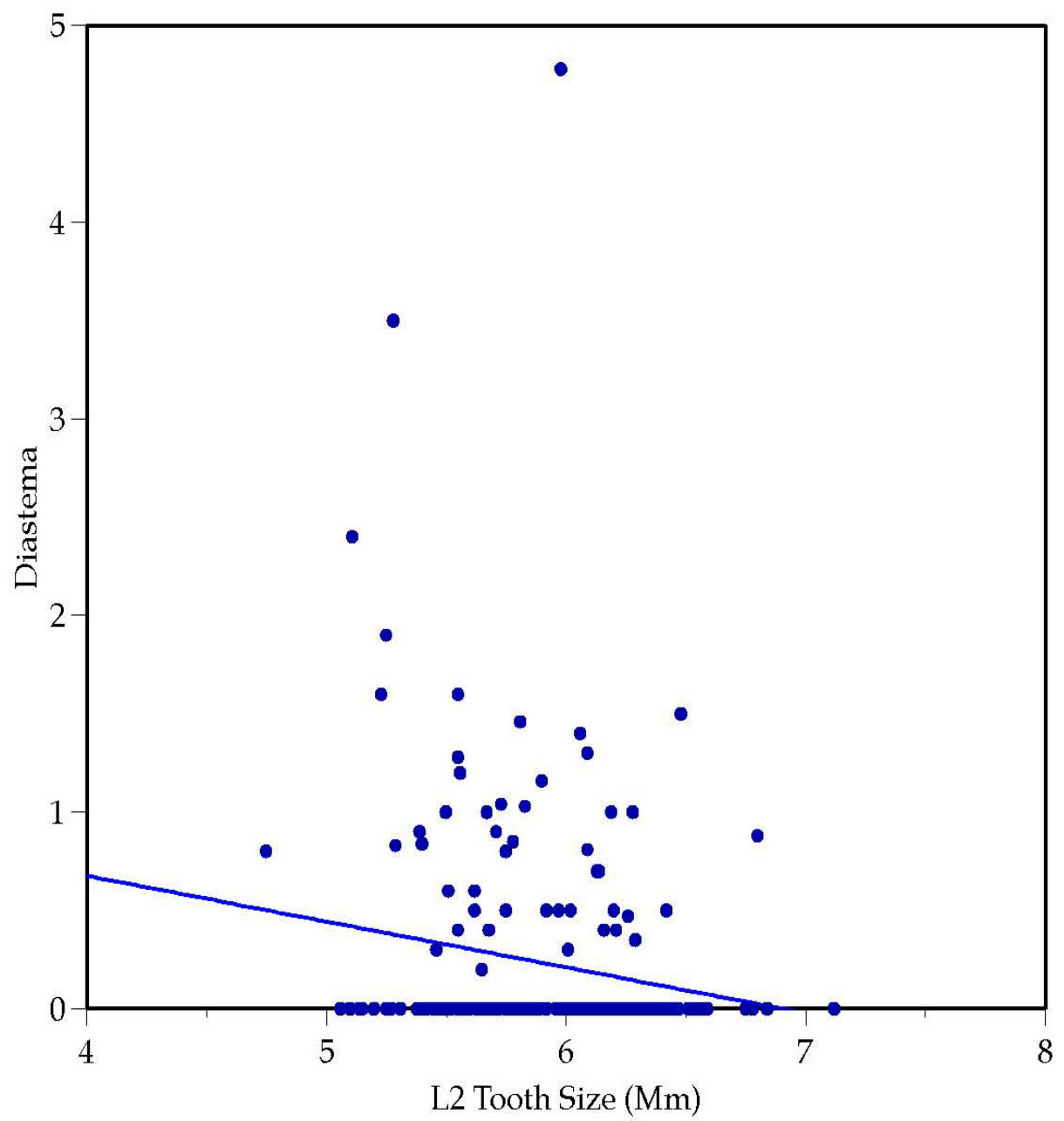

Figure 68. Plot between mesiodistal tooth size of L2 and diastema. As tooth size increases, average diastema diminishes. 


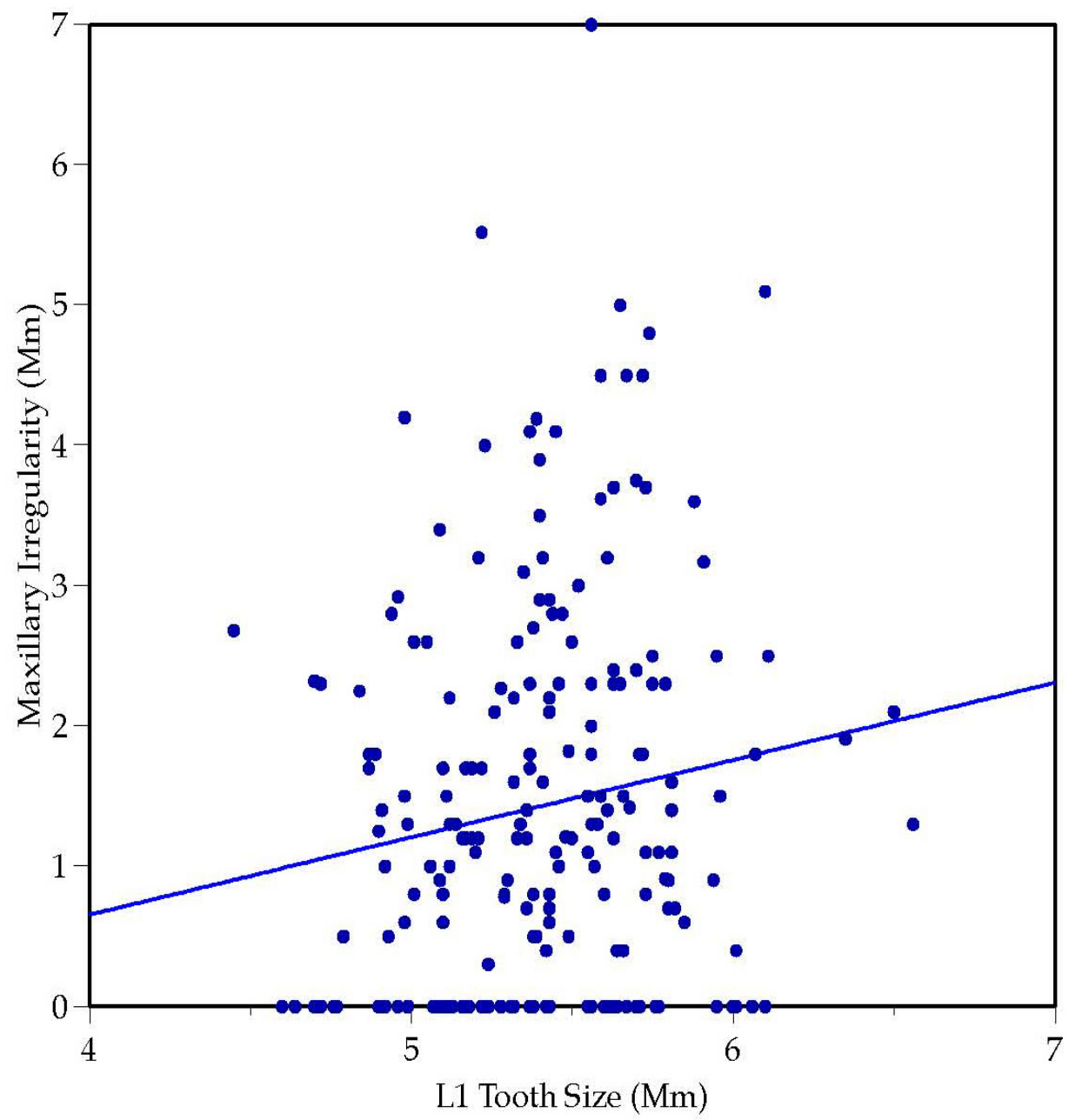

Figure 69. Plot between mesiodistal tooth size of L1 and maxillary irregularity.

As tooth size increases, so does the typical irregularity. 


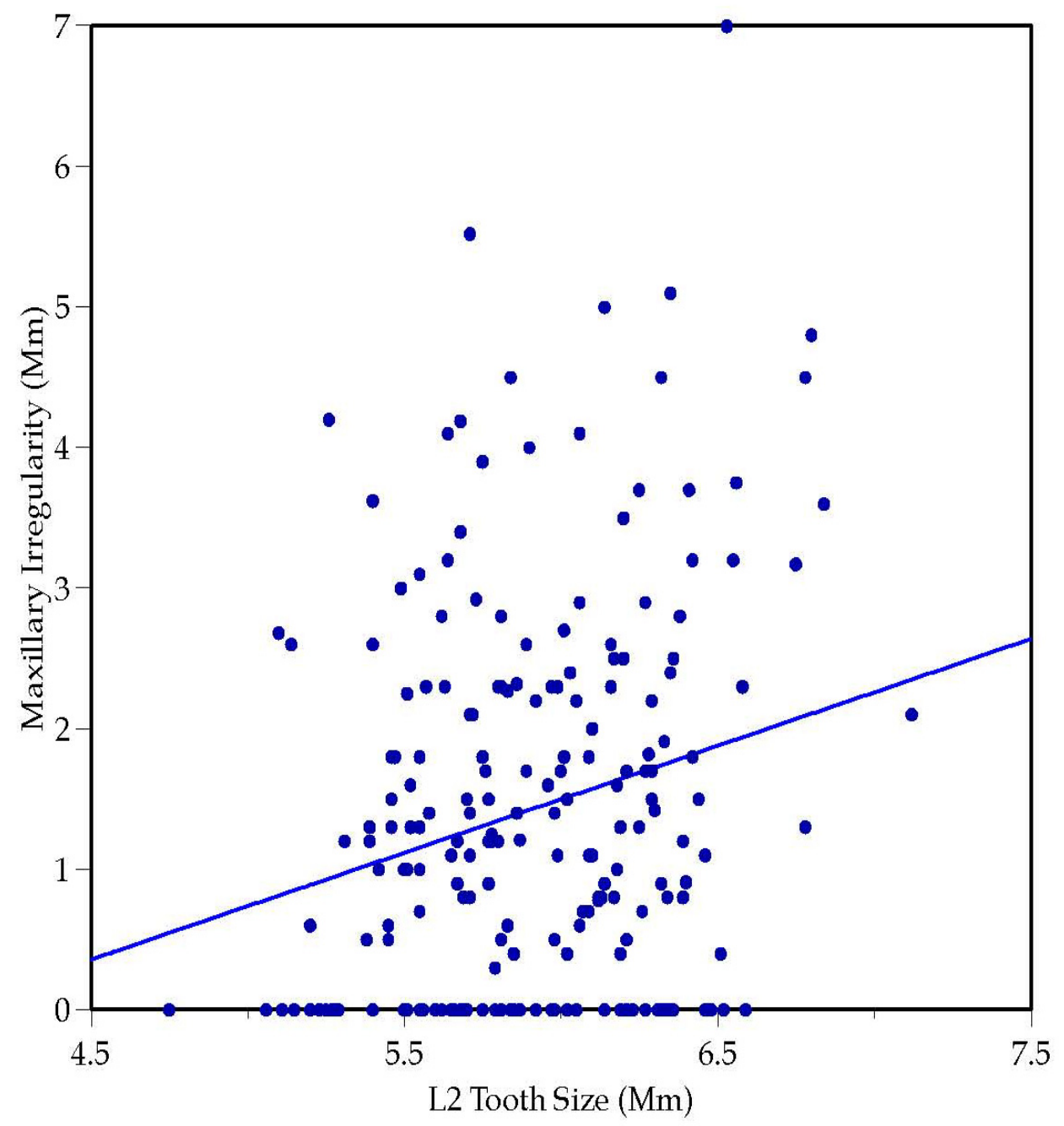

Figure 70. Plot between mesiodistal tooth size of L2 and maxillary irregularity.

As tooth size increases, so does the typical irregularity. 


\section{Mandibular Irregularity}

The pattern of associations for the lower arch parallel those in the maxillary: there are highly significant, positive associations between incisor width and mandibular irregularity. These are graphed for L1 (Figure 71) and L2 (Figure 72). Interestingly, the relationships are much weaker for the teeth distal to the incisors.

\section{Maxillary Overjet}

Extent of the overjet is mildly correlated with mandibular incisor widths $(0.05>\mathrm{P}>0.01)$, but not with any tooth types from the canine back. The associations are positive as shown for L1 (Figure 73) and L2 (Figure 74), but the causes of the association is unclear. Perhaps it is an indirect association brought about by the pervasive intertooth size correlations (e.g., Harris and Bailit 1988).

\section{DAI Score}

Associations between DAI and maxillary crown sizes are modest at best, and the same holds for these mandibular crown sizes (Table 5). Just the two incisor tooth types are significantly associated (Figure 75 and 76). As before, we attribute these modest associations to the fact that the DAI was not specifically intended as a measure of occlusal variations but, rather, as an instrument for quantifying facial esthetics.

\section{Predicting Crown Size from Malocclusion}

Prior sections in this chapter examined the bivariate associations among crown sizes and measures of malocclusions. While informative, there is considerable redundancy in the outcomes, primarily because (1) tooth crown dimensions are statistically intercorrelated (e.g., Moorrees and Reed 1964; Potter et al. 1968) and (2) the measures of malocclusion are themselves statistically interdependent. For example, Table 6 lists the correlation matrix for all 14 tooth types taken pair wise. With the sample size of 207 individuals (sexes pooled), the Pearson correlation coefficient only needs to be at least $r \geq 0.14$ to be significant at alpha of 0.05 , which means that all 91 correlations are significantly different from zero. This means, for example, that there are far more tooth dimensions than independent axes of variation. 


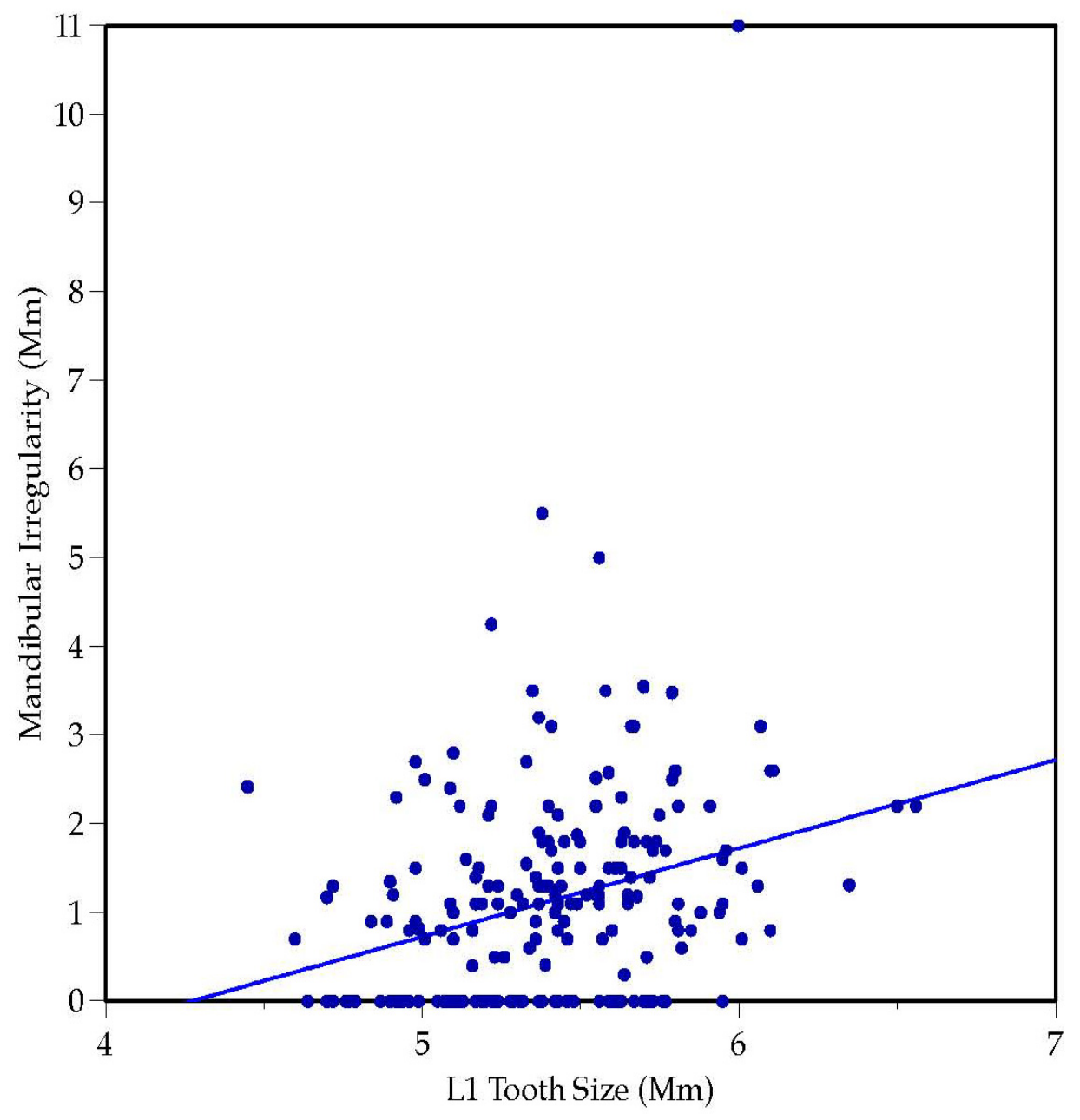

Figure 71. Plot between mesiodistal tooth size of L1 and mandibular irregularity.

As tooth size increases, so does the typical irregularity. 


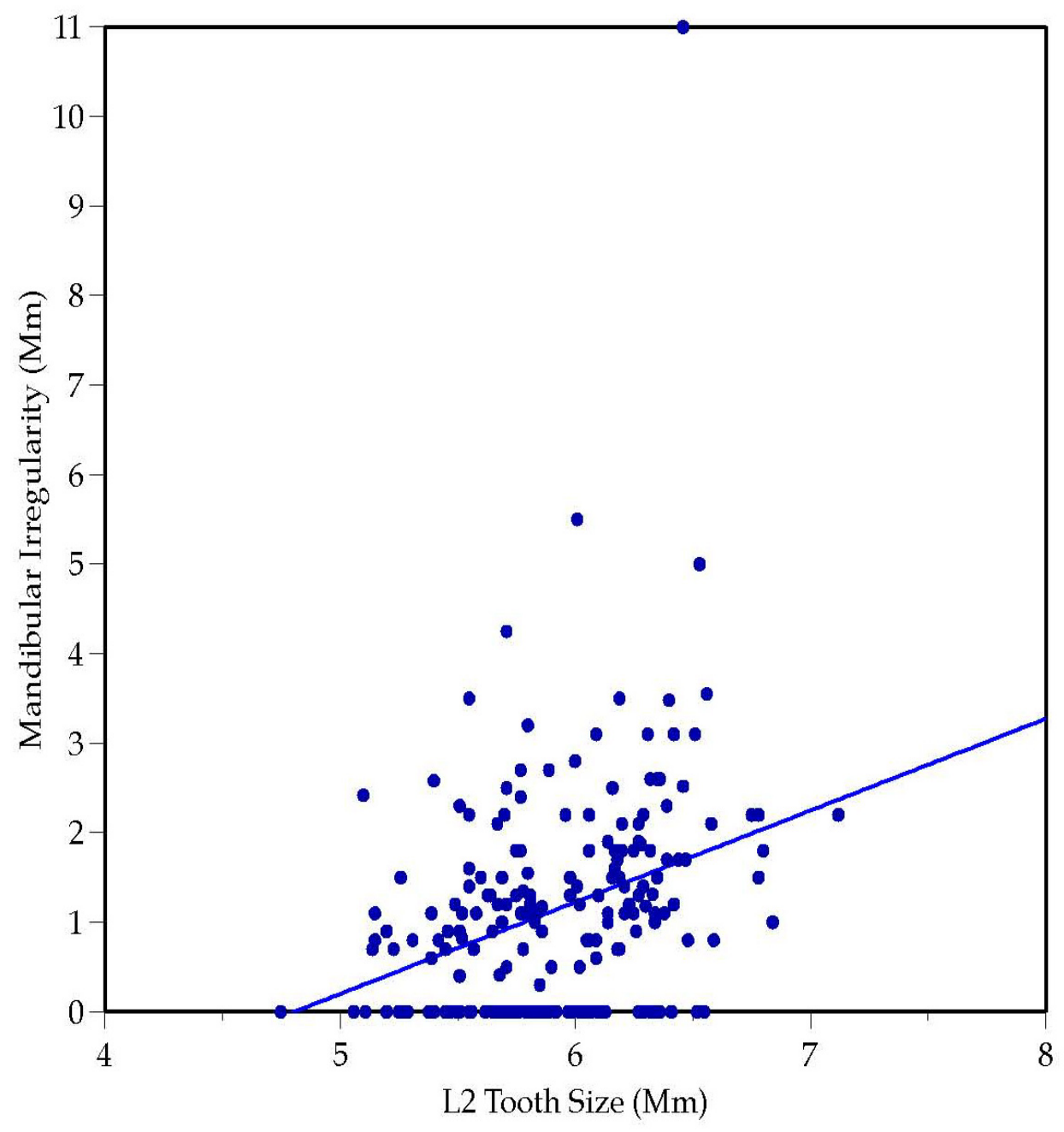

Figure 72. Plot between mesiodistal tooth size of L2 and mandibular irregularity.

As tooth size increases, so does the typical irregularity. 


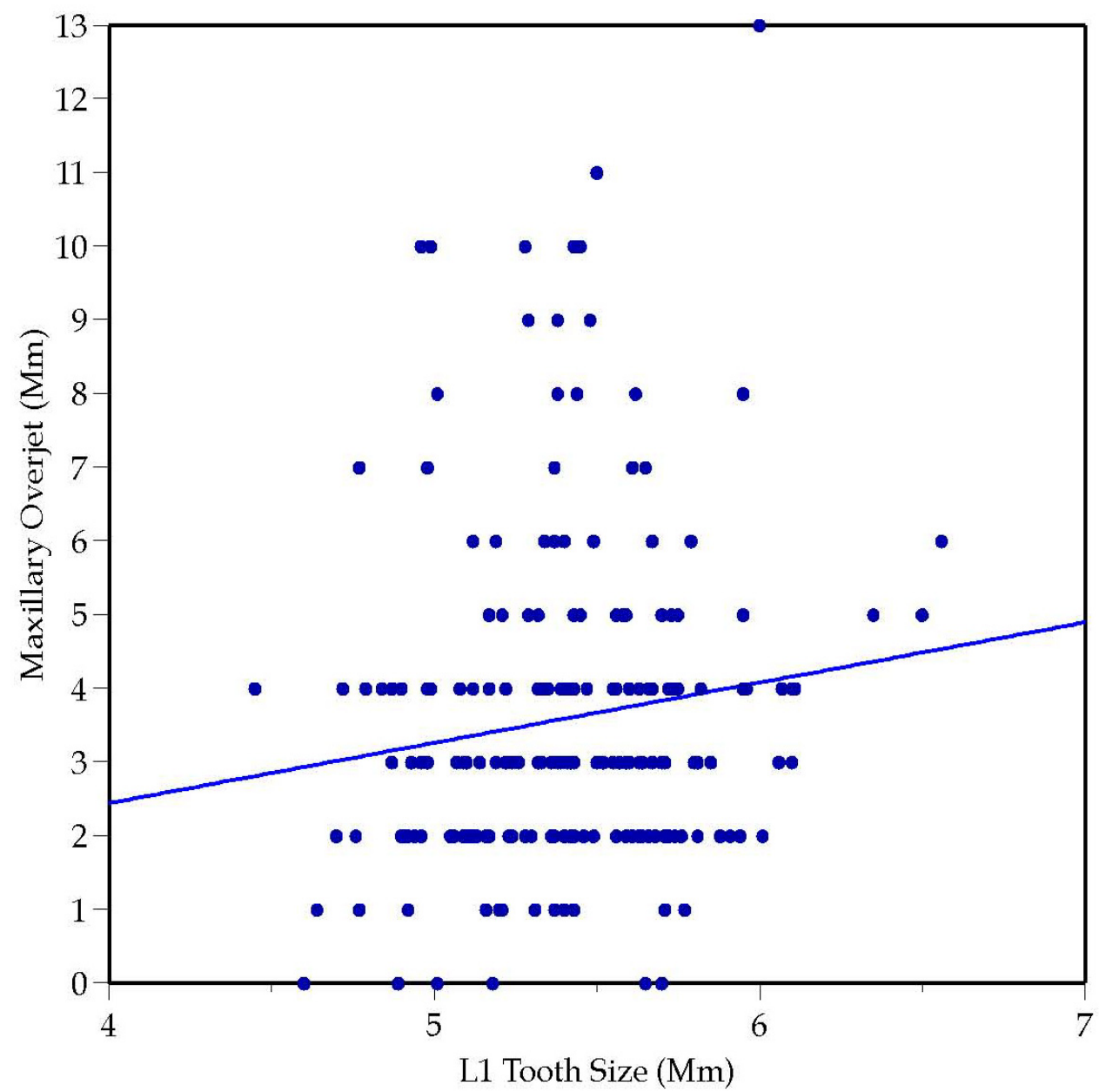

Figure 73. Plot between mesiodistal tooth size of L1 and maxillary overjet.

As tooth size increases, so does the typical size of the overjet. 


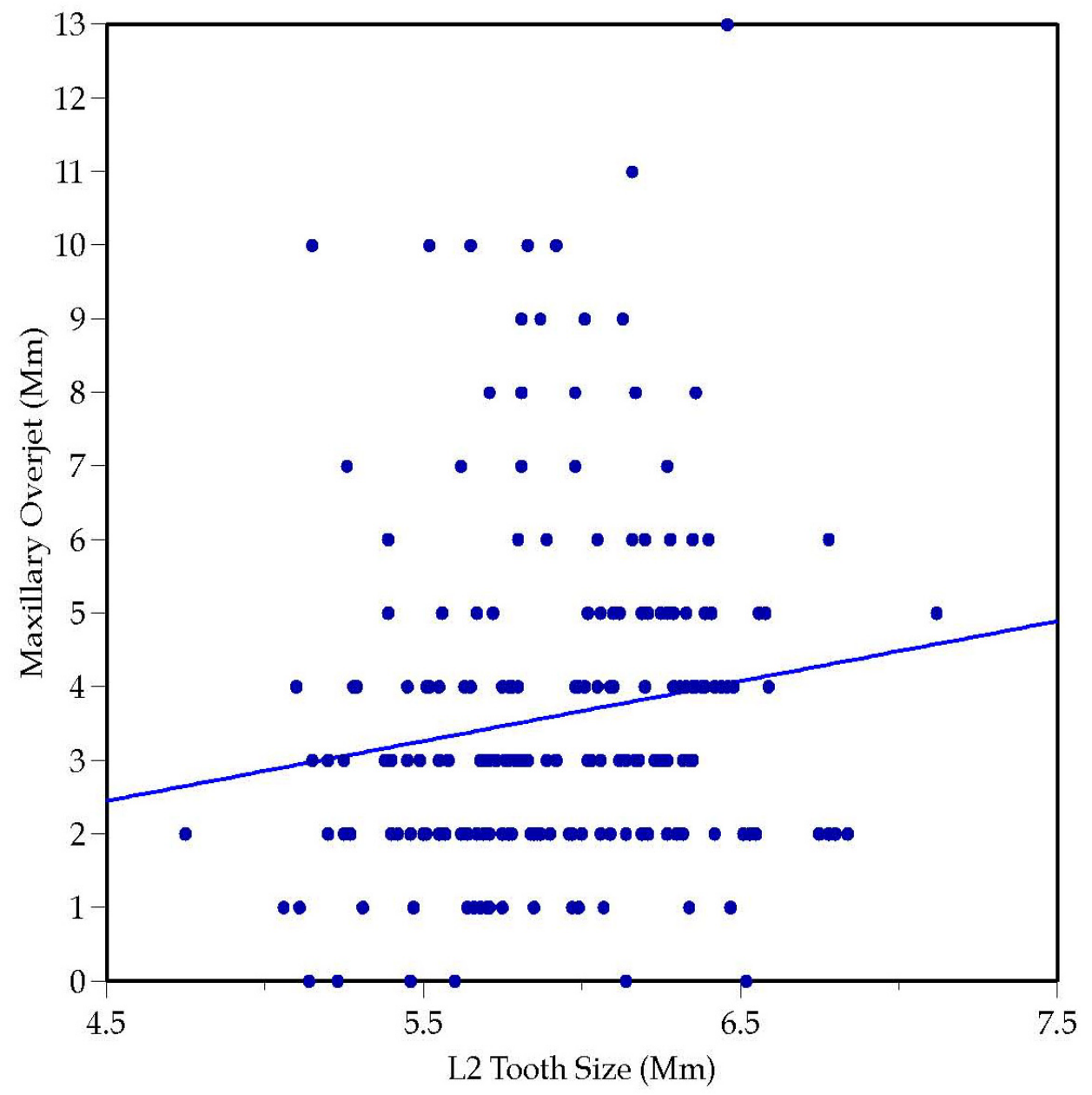

Figure 74. Plot between mesiodistal tooth size of L2 and maxillary overjet.

As tooth size increases, so does the typical size of the overjet. 


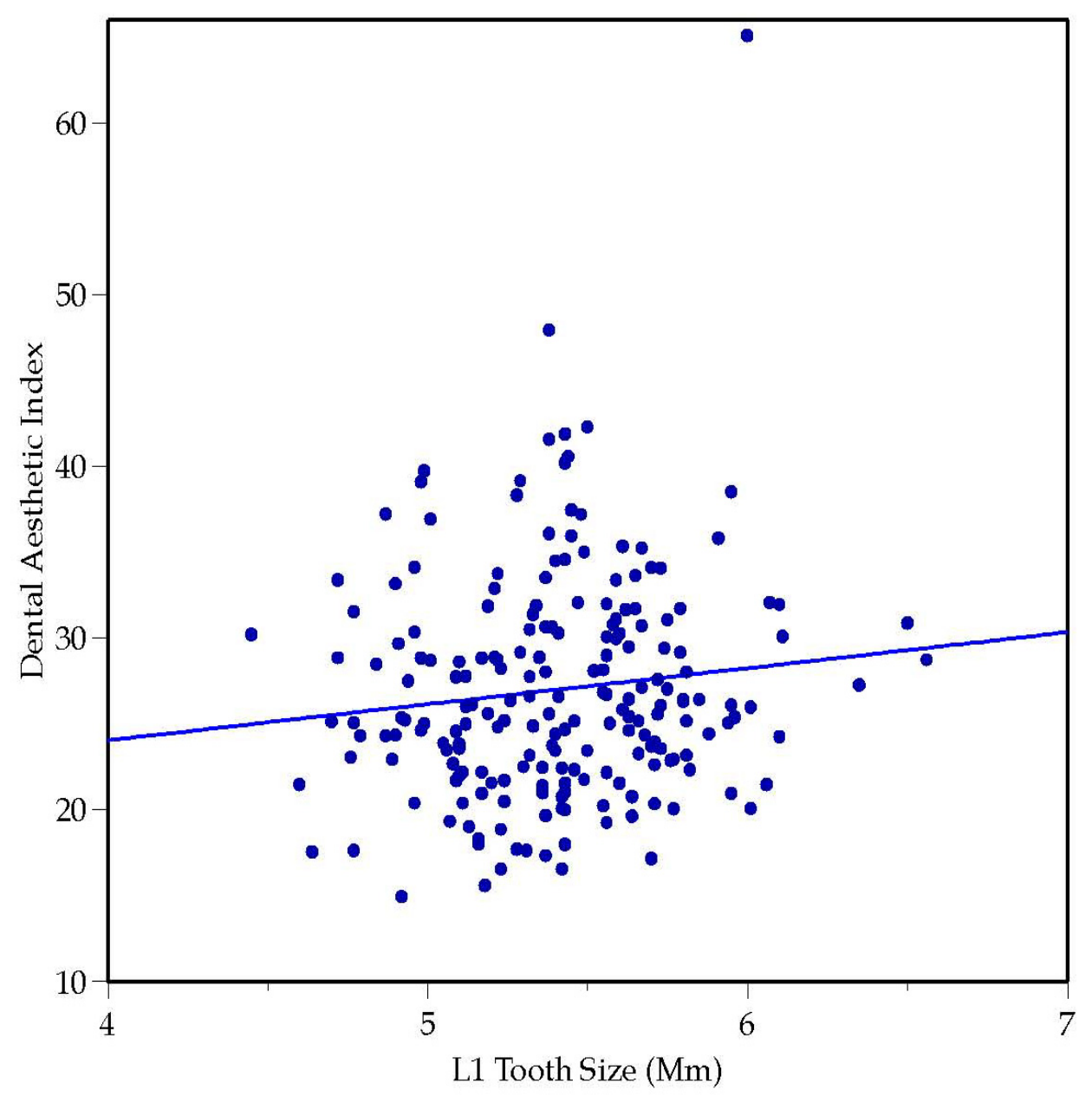

Figure 75. Plot between mesiodistal tooth size of L1 and DAI score.

As tooth size increases, so does the typical DAI score. 


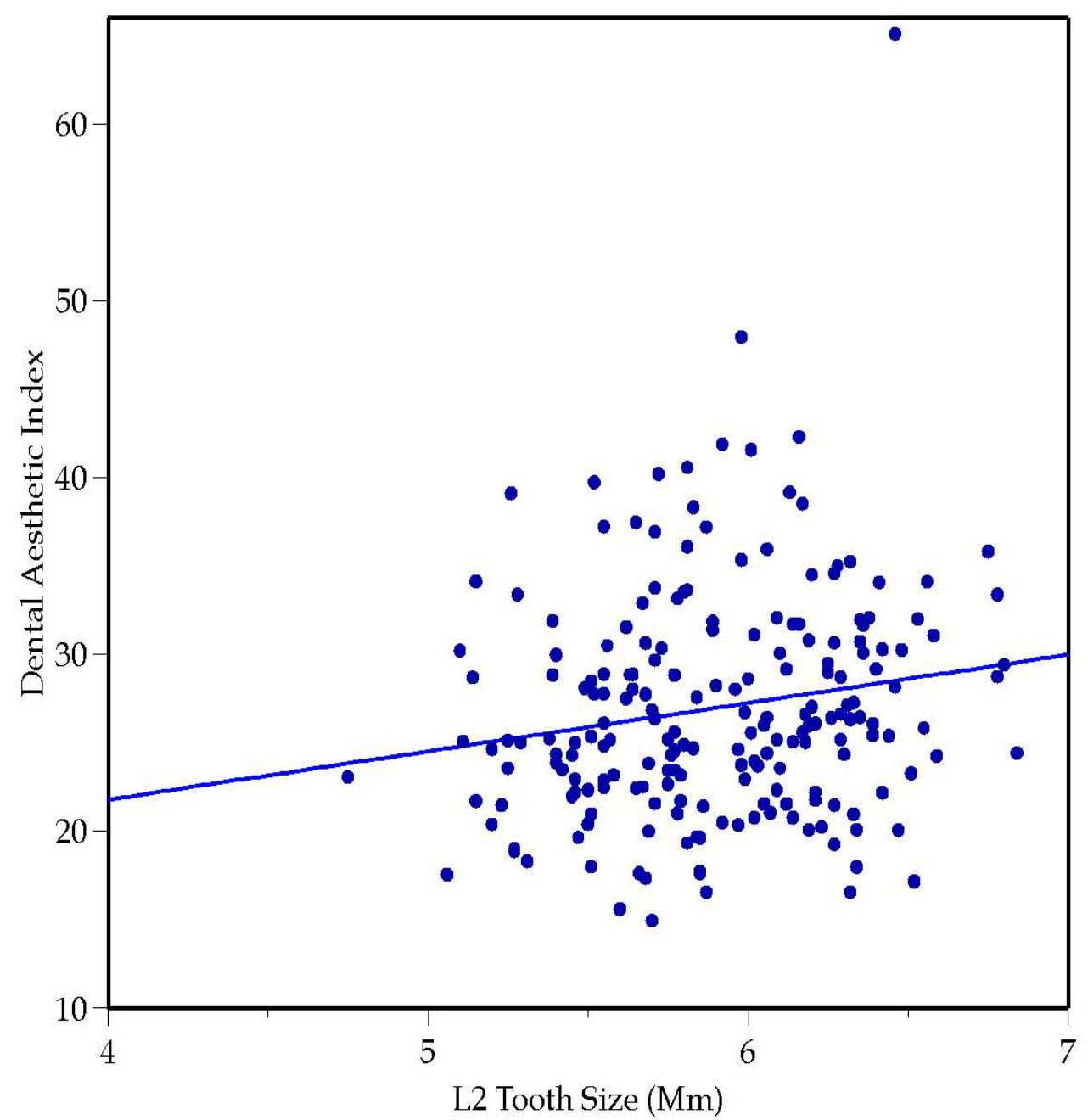

Figure 76. Plot between mesiodistal tooth size of L2 and DAI score.

As tooth size increases, so does the typical DAI score. 
Table 6. Matrix of Pearson correlation coefficients for all 14 mesiodistal crown dimensions taken pairwise.

\begin{tabular}{|c|c|c|c|c|c|c|c|c|c|c|c|c|c|c|}
\hline \multicolumn{8}{|c|}{ Maxilla } & \multicolumn{7}{|c|}{ Mandible } \\
\hline & I1 & $\mathrm{I} 2$ & $\mathrm{C}$ & P1 & P2 & M1 & M2 & I1 & $\mathrm{I} 2$ & $\mathrm{C}$ & $\mathrm{P} 1$ & P2 & M1 & M2 \\
\hline \multicolumn{15}{|c|}{ Maxilla } \\
\hline I1 & 1.00 & 0.46 & 0.60 & 0.47 & 0.43 & 0.48 & 0.43 & 0.66 & 0.67 & 0.59 & 0.48 & 0.36 & 0.46 & 0.39 \\
\hline I1 & 0.46 & 1.00 & 0.51 & 0.49 & 0.40 & 0.35 & 0.32 & 0.50 & 0.54 & 0.40 & 0.52 & 0.38 & 0.39 & 0.32 \\
\hline $\mathrm{C}$ & 0.60 & 0.51 & 1.00 & 0.54 & 0.46 & 0.44 & 0.44 & 0.53 & 0.62 & 0.72 & 0.58 & 0.46 & 0.46 & 0.41 \\
\hline P1 & 0.46 & 0.49 & 0.54 & 1.00 & 0.68 & 0.47 & 0.47 & 0.52 & 0.53 & 0.48 & 0.76 & 0.62 & 0.43 & 0.43 \\
\hline P2 & 0.43 & 0.40 & 0.46 & 0.68 & 1.00 & 0.48 & 0.53 & 0.49 & 0.43 & 0.44 & 0.61 & 0.66 & 0.43 & 0.43 \\
\hline M1 & 0.48 & 0.35 & 0.44 & 0.47 & 0.48 & 1.00 & 0.70 & 0.49 & 0.48 & 0.46 & 0.44 & 0.49 & 0.71 & 0.57 \\
\hline M2 & 0.43 & 0.32 & 0.44 & 0.47 & 0.53 & 0.70 & 1.00 & 0.43 & 0.39 & 0.39 & 0.50 & 0.55 & 0.62 & 0.68 \\
\hline \multicolumn{15}{|c|}{ Mandible } \\
\hline I1 & 0.66 & 0.50 & 0.53 & 0.52 & 0.49 & 0.49 & 0.43 & 1.00 & 0.75 & 0.58 & 0.53 & 0.51 & 0.52 & 0.42 \\
\hline $\mathrm{I} 2$ & 0.67 & 0.54 & 0.62 & 0.53 & 0.43 & 0.48 & 0.39 & 0.75 & 1.00 & 0.65 & 0.53 & 0.44 & 0.53 & 0.37 \\
\hline $\mathrm{C}$ & 0.59 & 0.40 & 0.72 & 0.48 & 0.44 & 0.46 & 0.39 & 0.58 & 0.65 & 1.00 & 0.56 & 0.48 & 0.52 & 0.39 \\
\hline P1 & 0.48 & 0.52 & 0.58 & 0.76 & 0.61 & 0.44 & 0.50 & 0.53 & 0.53 & 0.56 & 1.00 & 0.64 & 0.46 & 0.47 \\
\hline P2 & 0.36 & 0.38 & 0.46 & 0.62 & 0.66 & 0.49 & 0.55 & 0.51 & 0.44 & 0.48 & 0.64 & 1.00 & 0.51 & 0.49 \\
\hline M1 & 0.46 & 0.39 & 0.46 & 0.43 & 0.43 & 0.71 & 0.62 & 0.52 & 0.53 & 0.52 & 0.46 & 0.51 & 1.00 & 0.63 \\
\hline M2 & 0.39 & 0.32 & 0.41 & 0.43 & 0.43 & 0.57 & 0.68 & 0.42 & 0.37 & 0.39 & 0.47 & 0.49 & 0.63 & 1.00 \\
\hline
\end{tabular}


There are several multivariate statistical methods that reduce this statistical redundancy. We pursued a stepwire linear regression approach to predict crown size from the compilation of 14 measures of malocclusion. (Cohen and Cohen 1975; Freund and Littell 1991).

The method can be illustrated using the composite variable of the sum of the 14 mesiodistal crown dimensions. This addresses the interesting question of whether a person's "tooth size" taken globally (as the sum of 14 tooth types) is statistically dependent on one or more measures of dental malocclusion.

Results for summed crown sizes are listed in Table 7. These values show the "final solution" of the stepwise procedure. At step 0 the univariate F-ratio for all 14 measures of malocclusion with tooth size was determined. Step 1 selected the variable with the largest F-ratio, which in this case was interdental spacing and the 13 other F-ratios were recalculated on the basis of spacing, being in the predictive model. At step 2, there was one statistically significantly F-ratio contingent on having accounted for spacing; this was mandibular irregularity. Again, the program recalculates all of the other F-ratios, now contingent on having spacing and mandibular irregularity in the model. The new "contingent" F-ratios take into account the covariance of the variables already entered into the model, so redundant statistical interdependencies among the predictor variables are accounted for. Each variable, then, has to contribute significant independent covariance to the model in order to be entered in the stepwise procedure. The stepwise procedure progresses until none of the remaining variables contributes significant additional information. Typically this stepwise procedure consists of only a few "steps" because most variables have very little unique information to contribute to the variation in the dependent (tooth size) variable. In this example, only two measures of malocclusion, (1) spacing and (2) mandibular irregularity, are predictive of summed tooth size. The other 12 measures of malocclusion contain trivial statistical information about summed tooth size that is not already accounted for by the two variables in the model, and the stepwise procedure ceases. In other words, two variables (spacing and mandibular irregularity) are conjointly the "best" predictors of summed tooth size from among those tested.

In fact, the forward and backward stepping procedure used here does not ensure that the optimal combination of predictors is discovered - though it generally is. There is a "brute force" procedure incorporated in the JMP software that will (as a selectable option) test every possible combination of predictor variables (Freund and Littell 1991). This "brute force" procedure was tested in many instances, and as commonly found - the stepping procedure arrives at the same solution. 
Table 7. Results of stepwise multiple regression analysis predicting summed mesiodistal widths of all 14 tooth types from measures of malocclusion.

\begin{tabular}{lcrll}
\hline Variable & Estimate & \multicolumn{1}{c}{ SSQ } & F-Ratio & P-Value \\
\hline Intercept & 110.313 & 0.0000 & 0.00 & 1.0000 \\
Total displacements & 0 & 0.5513 & 0.02 & 0.8773. \\
Total rotations & 0 & 0.8599 & 0.04 & 0.8471 \\
Total overlapping & 0 & 18.0653 & 0.79 & 0.3762 \\
Total D+R+O & 0 & 3.2099 & 0.14 & 0.7094 \\
Crowding & 0 & 22.8180 & 0.99 & 0.3198 \\
Spacing & -2.259 & 309.1153 & 13.47 & 0.0003 \\
Diastema & 0 & 2.8633 & 0.12 & 0.7248 \\
Maxillary irregularity & 0 & 3.1213 & 0.14 & 0.7132 \\
Mandibular irregularity & 0.632 & 121.4144 & 5.29 & 0.0224 \\
Maxillaryt overjet & 0 & 28.0018 & 1.22 & 0.2703 \\
Mandibular overjet & 0 & 2.2794 & 0.10 & 0.7535 \\
Openbite & 0 & 45.9624 & 2.01 & 0.1575 \\
AP relationship & 0 & 35.2387 & 1.54 & 0.2161 \\
DAI score & 0 & 0.0417 & 0.00 & 0.9661 \\
\hline
\end{tabular}


With reference to the results in Table 7, the two predictor variables collectively account for $11 \%$ of the variation in tooth size. While far from the majority of the variance in tooth size, the statistical association is highly significant statistically. Moreover, one would not expect "much" of the variation in tooth size to be explained by dental malocclusion.

The "Estimate" column is Table 7 lists additional information: One, the signs of the estimates show that (1) there is a negative association between spacing and tooth size and (2) the association between tooth size and mandibular irregularity is positive. This simply reinforces the bivariate findings described previously. Two, regression coefficient for spacing $(-2.3)$ is much larger than for mandibular irregularity (0.6), meaning that, of these two variables, spacing plays the larger role in accounting for the variance in tooth size. Other information from the procedure, namely, the individual $\mathrm{R}^{2}$, further interpret this. Spacing by itself accounts for $9 \%$ of the variance summed tooth size; the addition of mandibular irregularity to the model only added another $2 \%$, for a total of $11 \%$.

\section{Maxillary Crown Dimensions}

Table 8 lists the final stepwise results for the central incisor. Of the 14 potential predictors, three were entered into the prediction model. Of these, spacing has the largest coefficient, which is, of course, negative. The recurrent statistical impact of interdental spacing as seen, for example, among the bivariate tests described in an earlier section, suggest that the small mesiodistal crown diameters may define a distinctive subset of the patients studied here. That is, our focus was to evaluate the effect of large crown diameters on raising the risk of malocclusions. Yet, the recurrent, statistically imposing associations between small crown sizes and spacing suggest that these patients constitute an influential subset of the tooth size distributions.

The predictive model developed in Table 8 shows that (1) interdental spacing has an important negative association with crown size but, secondarily, (2) mandibular irregularity and, to a lesser extent, the severity of maxillary overjet also is predictive of U1 size. Collectively, the three measures of malocclusion explain $12.5 \%$ of the variance in U1 width in the statistical sense, though, again, spacing accounts for most of this variance $\left(R^{2}=8 \%\right)$.

Just one measure of malocclusion has a significant association with U2 size (Table 9), namely crowding. That is, once crowding has been entered in the stepwise procedure, no other measure of malocclusion has significant covariation with $\mathrm{U} 2$ size that is not already explained by the variation due to 
Table 8. Results of stepwise multiple regression analysis predicting mesiodistal width of the maxillary central incisor from measures of malocclusion.

\begin{tabular}{lllll}
\hline Variable & Estimate & SSQ & F-Ratio & P-Value \\
\hline Intercept & 8.486 & 0.0000 & 0.00 & 1.0000 \\
Total displacements & 0 & 0.2534 & 0.92 & 0.3387 \\
Total rotations & 0 & 0.1025 & 0.37 & 0.5432 \\
Total overlapping & 0 & 0.2139 & 0.78 & 0.3796 \\
Total D+R+O & 0 & 0.2895 & 1.05 & 0.3064 \\
Crowding & 0 & 0.3978 & 1.45 & 0.2304 \\
Spacing & -0.173 & 1.7953 & 6.52 & 0.0114 \\
Diastema & 0 & 0.1497 & 0.54 & 0.4624 \\
Maxillary irregularity & 0 & 0.2948 & 1.07 & 0.3021 \\
Mandibular irregularity & 0.082 & 1.8622 & 6.76 & 0.0100 \\
Maxillary overjet & 0.040 & 1.5292 & 5.55 & 0.0194 \\
Mandibular overjet & 0 & 0.1354 & 0.49 & 0.4846 \\
Openbite & 0 & 0.4377 & 1.59 & 0.2083 \\
AP relationship & 0 & 1.3889 & 1.42 & 0.2357 \\
DAI score & 0 & 0.0848 & 0.31 & 0.5803 \\
\hline
\end{tabular}


Table 9. Results of stepwise multiple regression analysis predicting mesiodistal width of the maxillary lateral incisor from measures of malocclusion.

\begin{tabular}{lllll}
\hline Variable & Estimate & SSQ & F-Ratio & P-Value \\
\hline Intercept & 6.300 & 0.0000 & 0.00 & 1.0000 \\
Total displacements & 0 & 0.0382 & 0.12 & 0.7332 \\
Total rotations & 0 & 0.1305 & 0.40 & 0.5388 \\
Total overlapping & 0 & 0.4424 & 1.36 & 0.2456 \\
Total D+R+O & 0 & 0.0443 & 0.14 & 0.7137 \\
Crowding & 0.242 & 6.8426 & 20.94 & 0.0000 \\
Spacing & 0 & 0.7205 & 2.22 & 0.1380 \\
Diastema & 0 & 0.2067 & 0.63 & 0.4278 \\
Maxillary irregularity & 0 & 0.6858 & 2.11 & 0.1479 \\
Mandibular irregularity & 0 & 0.2805 & 0.86 & 0.3555 \\
Maxillary overjet & 0 & 0.1594 & 0.49 & 0.4863 \\
Mandibular overjet & 0 & 0.0040 & 0.01 & 0.9119 \\
Openbite & 0 & 0.5906 & 1.81 & 0.1795 \\
AP relationship & 0 & 1.3692 & 4.26 & 0.0404 \\
DAI score & 0 & 0.0240 & 0.07 & 0.7873 \\
\hline
\end{tabular}


crowding. By itself, crowding accounts for a quarter (24\%) of the variance in U2 size, which seems striking. On the other hand, this strong association probably is tied to the unusually high variability in U2 size among whites (Polder et al. 2004, Albashaireh and Khader 2006). We would predict much lower associations among other social groups where U2 is not so prone to size reduction, pegging, microdonty, congenital absence, and the like. It should not go unnoticed that the high variability in $\mathrm{U} 2$ width is a common source of Bolton discrepancies, leading to failure of proper intercuspation but also, evidently, to enhanced risk of anterior crowding.

Mesiodistal width of the upper canine (Table 10) is significantly predicted by just one variable of those tested, namely crowding. This association accounts for $16 \%$ in MD variation in canine width, which suggests that canine size-its placement in the arch-merits more critical attention. There seem to be several impinging issues here. One, the permanent canine is much larger mesiodistally than the primary canine it replaces. Two, it erupts late - during the second transition (van der Linden and Duterloo 1976) and has to erupt into a confined space. Data (Moorrees 1959) suggest that canine eruption pushes the earliererupting incisors medially, thereby instigating their irregularity and crowding (if not already underway).

Predictors of first premolar size (Table 11) are few, just interdental spacing. On the other hand, this association accounts for a fifth of the variation in premolar crown size $\left(R^{2}=19 \%\right)$. At face value, these results suggest that mesiodistal crown length of $U 4$ has an appreciable influence on crowding spacing in the anterior region. Its negative association here with anterior spacing suggests that, when $\mathrm{U} 4$ is short mediodistally, this raises the likelihood of interdental spacing in the anterior segment. Conversely, a mediodistally elongated first premolar would reduce spacing. Since U4 emerges late (10 to 12 years of age; van der Linden and Duterloo 1976), the effect on the anterior segment likewise should be late in childhood, which coincides with the pre-teen changes documented by Moorrees (1959).

Stepwise results for the second premolar, likewise implicate spacing as the prime associate (Table 12). Again the relationship is negative, which sensibly means that mesiodistally longer U5 diminish the likelihood of spacing. A couple of the interesting developmental features here are (1) that later-erupting teeth in the midarch effect occlusion in the anterior segment and (2) that the supposed mesial forces act around the "corner" of the arch (Southard et al. 1989). This latter feature implies more than just the linear vectors of pressure, and may well implicate the complexes of supragingivel interdental fibers. 
Table 10. Results of stepwise multiple regression analysis predicting mesiodistal width of the maxillary canine from measures of malocclusion.

\begin{tabular}{lllll}
\hline Variable & Estimate & SSQ & F-Ratio & P-Value \\
\hline Intercept & 7.60 & 0.0000 & 0.00 & 1.0000 \\
Total displacements & 0 & 0.0907 & 0.45 & 0.5046 \\
Total rotations & 0 & 0.0078 & 0.04 & 0.8452 \\
Total overlapping & 0 & 0.3293 & 1.63 & 0.2029 \\
Total D+R+O & 0 & 0.1177 & 0.58 & 0.4471 \\
Crowding & 0.157 & 2.8606 & 14.13 & 0.0002 \\
Spacing & 0 & 0.2326 & 1.15 & 0.2848 \\
Diastema & 0 & 0.1657 & 0.82 & 0.3669 \\
Maxillary irregularity & 0 & 0.0717 & 0.35 & 0.5530 \\
Mandibular irregularity & 0 & 0.1302 & 0.64 & 0.4239 \\
Maxillary overjet & 0 & 0.5896 & 2.94 & 0.0879 \\
Mandibular overjet & 0 & 0.0010 & 0.01 & 0.9434 \\
Openbite & 0 & 0.2215 & 1.10 & 0.2967 \\
AP relationship & 0 & 0.2280 & 1.13 & 0.2897 \\
DAI score & 0 & 0.1838 & 0.91 & 0.3420 \\
\hline
\end{tabular}


Table 11. Results of stepwise multiple regression analysis predicting mesiodistal width of the maxillary first premolar from measures of malocclusion.

\begin{tabular}{lclll}
\hline Variable & Estimate & SSQ & F-Ratio & P-Value \\
\hline Intercept & 6.968 & 0.0000 & 0.00 & 1.0000 \\
Total displacements & 0 & 0.0001 & 0.00 & 0.9836 \\
Total rotations & 0 & 0.0821 & 0.50 & 0.4811 \\
Total overlapping & 0 & 0.1726 & 1.05 & 0.3066 \\
Total D+R+O & 0 & 0.0707 & 0.43 & 0.5133 \\
Crowding & 0 & 0.5492 & 3.38 & 0.0674 \\
Spacing & -0.193 & 2.4334 & 14.81 & 0.0002 \\
Diastema & 0 & 0.0251 & 0.15 & 0.6971 \\
Maxillary irregularity & 0 & 0.0011 & 0.01 & 0.9337 \\
Mandibular irregularity & 0 & 0.2711 & 1.65 & 0.1998 \\
Maxillary overjet & 0 & 0.6111 & 3.77 & 0.0536 \\
Mandibular overjet & 0 & 0.0199 & 0.12 & 0.7289 \\
Openbite & 0 & 0.1557 & 0.95 & 0.3317 \\
AP relationship & 0 & 0.4446 & 2.73 & 0.1001 \\
DAI score & 0 & 0.1303 & 0.79 & 0.3745 \\
\hline
\end{tabular}


Table 12. Results of stepwise multiple regression analysis predicting mesiodistal width of the maxillary second premolar from measures of malocclusion.

\begin{tabular}{lllll}
\hline Variable & Estimate & SSQ & F-Ratio & P-Value \\
\hline Intercept & 6.927 & 0.0000 & 0.00 & 1.0000 \\
Total displacements & 0 & 0.4105 & 2.29 & 0.1322 \\
Total rotations & 0 & 0.1345 & 0.74 & 0.3898 \\
Total overlapping & 0 & 0.0503 & 0.28 & 0.5991 \\
Total D+R+O & 0 & 0.0058 & 0.03 & 0.8584 \\
Crowding & 0 & 0.2397 & 1.33 & 0.2506 \\
Spacing & -0.164 & 1.7511 & 9.69 & 0.0021 \\
Diastema & 0 & 0.0966 & 0.53 & 0.4662 \\
Maxillary irregularity & 0 & 0.0310 & 0.17 & 0.6798 \\
Mandibular irregularity & 0 & 0.1893 & 1.05 & 0.3074 \\
Maxillary overjet & 0.027 & 0.7653 & 4.23 & 0.0409 \\
Mandibular overjet & 0 & 0.0112 & 0.06 & 0.8041 \\
Openbite & 0 & 0.3408 & 1.89 & 0.1704 \\
AP relationship & 0 & 0.3587 & 1.99 & 0.1595 \\
DAI score & 0 & 0.4441 & 2.47 & 0.1173 \\
\hline
\end{tabular}


Moving distally to the molar region (Table 13), effects on malocclusions in this anterior segment are, not surprisingly, weak. For U6 (Table 13) mandibular irregularity is the only statistically significant predictor, and the explained variance $\left(\mathrm{R}^{2}=2 \%\right)$ is trivial.

This theme - that distally positioned teeth have little influence on anterior malocclusion - is reflected as well in the U7 model (Table 14). Here, there is the negative association with spacing that is seen several times previously. The association is weak $\left(\mathrm{R}^{2}=3 \%\right)$. The noteworthy feature is, perhaps, that there is any statistically discernable effect at all this far back in the arch. Alternatively, these stepwise models do not preclude the associations being indirect. For example, this association with U7 (Table 14) may be due to U7's positive size covariation with anterior teeth.

\section{Mandibular Crown Dimensions}

Two measures of malocclusion were significantly associated with L1 size, namely crowding and mandibular irregularity (Table 15), but of these crowding clearly is the more strongly associated. The relatively weak association between L1 size and mandibular irregularity may be due to the method of quantifying irregularity (Jenny and Cons 1996), where it is merely scored as present or absent in each arcade.

Mesiodistal crown size of L2 has significant associations with three measures of malocclusion (Table 16), namely overlapping, spacing and mandibular irregularity. The associations with overlapping and malocclusion are positive, so broader L2 incisors increase the risk of anterior overlapping and of mandibular irregularity. Conversely, the negative regression coefficient with interdental spacing (Table 17) denotes a negative relationship, where narrower L2 widths are associated with greater spacing. Mesiodistal width of L3 is significantly associated with just one of the 14 measures of malocclusion. This is a negative association.

Because "spacing" commonly occurs as a significant variable in these analyses, it merits evaluating the relationship in a bit more detail. "Spacing" is scored in a course fashion (Jenny and Cons 1996), with only three ordinal grades namely none (score of 0 ), one jaw with spacing (score of 1 ), or both jaws with spacing (score of 2). Still, cases with the largest canine diameters tend to have no spacing, canine size is intermediate when one arch exhibits spacing, and crown size is smallest where there is spacing in both arches. Mean canine widths associated with these three grades of spacing are $6.8 \mathrm{~mm}, 6.7 \mathrm{~mm}$, and $6.3 \mathrm{~mm}$, 
Table 13. Results of stepwise multiple regression analysis predicting mesiodistal width of the maxillary first molar from measures of malocclusion.

\begin{tabular}{lclll}
\hline Variable & Estimate & SSQ & F-Ratio & P-Value \\
\hline Intercept & 10.270 & 0.0000 & 0.00 & 1.0000 \\
Total displacements & 0 & 0.0792 & 0.26 & 0.6108 \\
Total rotations & 0 & 0.0001 & 0.00 & 0.9861 \\
Total overlapping & 0 & 0.3706 & 1.22 & 0.2705 \\
Total D+R+O & 0 & 0.1393 & 0.46 & 0.4997 \\
Crowding & 0 & 0.3919 & 1.29 & 0.2571 \\
Spacing & 0 & 0.7319 & 2.43 & 0.1209 \\
Diastema & 0 & 0.0390 & 0.13 & 0.7210 \\
Maxillary irregularity & 0 & 0.4357 & 1.44 & 0.2320 \\
Mandibular irregularity & 0.066 & 1.4309 & 4.71 & 0.0312 \\
Maxillary overjet & 0 & 0.0031 & 0.01 & 0.9194 \\
Mandibular overjet & 0 & 0.1913 & 0.63 & 0.4288 \\
Openbite & 0 & 0.5227 & 1.73 & 0.1904 \\
AP relationship & 0 & 0.0315 & 0.10 & 0.7483 \\
DAI score & 0 & 0.0680 & 0.22 & 0.6374 \\
\hline
\end{tabular}


Table 14. Results of stepwise multiple regression analysis predicting mesiodistal width of the maxillary second molar from measures of malocclusion.

\begin{tabular}{lclll}
\hline Variable & Estimate & SSQ & F-Ratio & P-Value \\
\hline Intercept & 9.794 & 0.0000 & 0.00 & 1.0000 \\
Total displacements & 0 & 0.1373 & 0.41 & 0.5208 \\
Total rotations & 0 & 0.1105 & 0.33 & 0.5645 \\
Total overlapping & 0 & 0.0609 & 1.18 & 0.6690 \\
Total D+R+O & 0 & 0.0239 & 0.07 & 0.7890 \\
Crowding & 0 & 0.0006 & 0.00 & 0.9651 \\
Spacing & -0.174 & 1.9729 & 5.96 & 0.0154 \\
Diastema & 0 & 0.0766 & 0.23 & 0.6315 \\
Maxillary irregularity & 0 & 0.0071 & 0.02 & 0.8836 \\
Mandibular irregularity & 0 & 0.3481 & 1.05 & 0.3062 \\
Maxillary overjet & 0 & 0.0191 & 0.06 & 0.8106 \\
Mandibular overjet & 0 & 0.0452 & 0.14 & 0.7127 \\
Openbite & 0 & 0.3543 & 1.07 & 0.3018 \\
AP relationship & 0 & 0.0163 & 0.05 & 0.8252 \\
DAI score & 0 & 0.0013 & 0.00 & 0.9500 \\
\hline
\end{tabular}


Table 15. Results of stepwise multiple regression analysis predicting mesiodistal width of the mandibular central incisor from measures of malocclusion.

\begin{tabular}{lllll}
\hline Variable & Estimate & SSQ & F-Ratio & P-Value \\
\hline Intercept & 5.159 & 0.0000 & 0.00 & 1.0000 \\
Total displacements & 0 & 0.1427 & 1.27 & 0.2610 \\
Total rotations & 0 & 0.1813 & 1.62 & 0.2048 \\
Total overlapping & 0 & 0.1105 & 0.98 & 0.3228 \\
Total D+R+O & 0 & 0.0159 & 0.14 & 0.7075 \\
Crowding & 0.141 & 1.8179 & 16.17 & 0.0001 \\
Spacing & 0 & 0.1864 & 1.66 & 0.1986 \\
Diastema & 0 & 0.0174 & 0.15 & 0.6952 \\
Maxillary irregularity & 0 & 0.0872 & 0.77 & 0.3799 \\
Mandibular irregularity & 0.042 & 0.4627 & 4.12 & 0.0438 \\
Maxillary overjet & 0 & 0.1369 & 1.22 & 0.2709 \\
Mandibular overjet & 0 & 0.0319 & 0.28 & 0.5956 \\
Openbite & 0 & 0.0215 & 0.19 & 0.6632 \\
AP relationship & 0 & 0.1853 & 1.65 & 0.2000 \\
DAI score & 0 & 0.0078 & 0.07 & 0.7925 \\
\hline
\end{tabular}


Table 16. Results of stepwise multiple regression analysis predicting mesiodistal width of the mandibular lateral incisor from measures of malocclusion.

\begin{tabular}{lllll}
\hline Variable & Estimate & SSQ & F-Ratio & P-Value \\
\hline Intercept & 5.776 & 0.0000 & 0.00 & 1.0000 \\
Total displacements & 0 & 0.0332 & 0.24 & 0.6282 \\
Total rotations & 0 & 0.0769 & 0.55 & 0.4612 \\
Total overlapping & 0.020 & 0.5056 & 3.59 & 0.0595 \\
Total D+R+O & 0 & 0.0038 & 0.03 & 0.8703 \\
Crowding & 0 & 0.0172 & 0.12 & 0.7279 \\
Spacing & -0.127 & 0.8029 & 5.71 & 0.0178 \\
Diastema & 0 & 0.0124 & 0.09 & 0.7678 \\
Maxillary irregularity & 0 & 0.0150 & 0.11 & 0.7452 \\
Mandibular irregularity & 0.068 & 1.1637 & 8.27 & 0.0045 \\
Maxillary overjet & 0 & 0.2745 & 1.96 & 0.1631 \\
Mandibular overjet & 0 & 0.3432 & 2.46 & 0.1186 \\
Openbite & 0 & 0.0796 & 0.56 & 0.4534 \\
AP relationship & 0 & 0.2067 & 1.47 & 0.2264 \\
DAI score & 0 & 0.0738 & 0.52 & 0.4703 \\
\hline
\end{tabular}


Table 17. Results of stepwise multiple regression analysis predicting mesiodistal width of the mandibular canine from measures of malocclusion.

\begin{tabular}{lclll}
\hline Variable & Estimate & SSQ & F-Ratio & P-Value \\
\hline Intercept & 6.782 & 0.0000 & 0.00 & 1.0000 \\
Total displacements & 0 & 0.0775 & 0.38 & 0.5382 \\
Total rotations & 0 & 0.0359 & 0.18 & 0.6751 \\
Total overlapping & 0 & 0.1533 & 0.75 & 0.3864 \\
Total D+R+O & 0 & 0.1208 & 0.59 & 0.4419 \\
Crowding & 0 & 0.1589 & 0.78 & 0.3777 \\
Spacing & -0.165 & 1.7793 & 8.76 & 0.0034 \\
Diastema & 0 & 0.0556 & 0.27 & 0.6022 \\
Maxillary irregularity & 0 & 0.0015 & 0.01 & 0.9326 \\
Mandibular irregularity & 0 & 0.3144 & 1.55 & 0.2143 \\
Maxillary overjet & 0 & 0.5769 & 2.87 & 0.0920 \\
Mandibular overjet & 0 & 0.0376 & 0.18 & 0.6682 \\
Openbite & 0 & 0.1739 & 0.86 & 0.3562 \\
AP relationship & 0 & 0.0002 & 0.00 & 0.9769 \\
DAI score & 0 & 0.1314 & 0.65 & 0.4226 \\
\hline
\end{tabular}


respectively, which supports the visual impression from Figure 74 that the principal difference is the small crown sizes of those with interdental spacing in both arches.

The stepwise analysis for L4 (Table 18) identifies just one significant association with malocclusion, namely a positive association between the degree of crowding and L4 diameter.

Interdental spacing surfaces again is the only predictor here for L5 size (Table 19). It is perhaps noteworthy that tooth size in the midarch plays any detectable role in dental malocclusion. Mean mesiodistal diameters of the second premolars (L5) are $7.2 \mathrm{~mm}$ in those without spacing and, likewise, 7.2 $\mathrm{mm}$ in those with spacing in one arch. When, however, there is spacing in both arches, mean L5 size drops to $6.7 \mathrm{~mm}$, which is the main source of the significant association.

Notably, this same measure of interdental spacing is the one and only significant predictor of L6 size (Table 20) and of L7 size (Table 21). So too, it is the smaller crown dimensions in subjects with spacing in both jaws that produce the statistically significant associations. The three mesiodistal crown means for L6 are $11.1 \mathrm{~mm}$ (spacing 0), $11.1 \mathrm{~mm}$ (spacing 1), and $10.6 \mathrm{~mm}$ (spacing 2). The means for L7 are $10.3 \mathrm{~mm}$ (spacing 0); $10.3 \mathrm{~mm}$ (spacing 1), and $9.7 \mathrm{~mm}$ (spacing 2).

These several comparisons show that the effect of spacing is widespread. Indeed, "spacing" entered 10 of the 14 stepwise models, making it the most pervasive predictor of tooth size in these analyses. But, what we are looking at is almost certainly the recurrence of the same effect: Because tooth crown diameters all are significantly and positively intercorrelated (Harris and Bailit 1988); people with one small tooth type tend to be the same people with other small tooth types. "Tooth size" should be viewed as an over-arching generic feature of an individual, not a collection of independent crown dimensions. One measure of this is to assess the relationship between (1) the three grades of "spacing" against (2) summed mesiodistal sizes of all 14 tooth types (Figure 77). Most subjects in the sample have no spacing $($ mean $=111.1 \mathrm{~mm})$, and most of the rest have crowding in just one arch (means $=110.0 \mathrm{~mm}$ ). Indeed, just 13 of the 207 people in the study have spacing in both arches, and they also have significantly smaller crown sizes (mean $=104.3 \mathrm{~mm}$ ).

Concern might be raised here that we have not controlled for sexual dimorphism. Conceivably, subjects with very small teeth might just be females. Table 22 lists the results of a two-way Ancova, where the sex of the subject is 
Table 18. Results of stepwise multiple regression analysis predicting mesiodistal width of the mandibular first premolar from measures of malocclusion.

\begin{tabular}{lclll}
\hline Variable & Estimate & SSQ & F-Ratio & P-Value \\
\hline Intercept & 6.90 & 0.0000 & 0.00 & 1.0000 \\
Total displacements & 0 & 0.0819 & 0.51 & 0.4775 \\
Total rotations & 0 & 0.0337 & 0.21 & 0.6488 \\
Total overlapping & 0 & 0.0038 & 0.02 & 0.8777 \\
Total D+R+O & 0 & 0.0028 & 0.02 & 0.8948 \\
Crowding & 0.129 & 1.9307 & 11.98 & 0.0007 \\
Spacing & 0 & 0.3305 & 2.06 & 0.1527 \\
Diastema & 0 & 0.1685 & 1.05 & 0.3077 \\
Maxillary irregularity & 0 & 0.0933 & 0.58 & 0.4482 \\
Mandibular irregularity & 0 & 0.0781 & 0.48 & 0.4877 \\
Maxillary overjet & 0 & 0.0648 & 0.40 & 0.5275 \\
Mandibular overjet & 0 & 0.0546 & 0.34 & 0.5619 \\
Openbite & 0 & 0.0811 & 0.50 & 0.4794 \\
AP relationship & 0 & 0.2100 & 1.30 & 0.2547 \\
DAI score & 0 & 0.0911 & 0.56 & 0.4537 \\
\hline
\end{tabular}


Table 19. Results of stepwise multiple regression analysis predicting mesiodistal width of the mandibular second premolar from measures of malocclusion.

\begin{tabular}{lllll}
\hline Variable & Estimate & SSQ & F-Ratio & P-Value \\
\hline Intercept & 7.236 & 0.0000 & 0.00 & 1.0000 \\
Total displacements & 0 & 0.0362 & 0.19 & 0.6628 \\
Total rotations & 0 & 0.0250 & 0.13 & 0.7169 \\
Total overlapping & 0 & 0.0016 & 0.01 & 0.9265 \\
Total D+R+O & 0 & 0.0003 & 0.00 & 0.9677 \\
Crowding & 0 & 0.0002 & 0.00 & 0.9724 \\
Spacing & -0.190 & 2.3572 & 12.46 & 0.0005 \\
Diastema & 0 & 0.0041 & 0.02 & 0.8831 \\
Maxillary irregularity & 0 & 0.0139 & 0.07 & 0.7869 \\
Mandibular irregularity & 0 & 0.0145 & 0.08 & 0.7824 \\
Maxillary overjet & 0 & 0.4172 & 2.22 & 0.1379 \\
Mandibular overjet & 0 & 0.0177 & 0.09 & 0.7605 \\
Openbite & 0 & 0.0065 & 0.03 & 0.8534 \\
AP relationship & 0 & 0.0917 & 0.48 & 0.4876 \\
DAI score & 0 & 0.1162 & 0.61 & 0.4346 \\
\hline
\end{tabular}


Table 20. Results of stepwise multiple regression analysis predicting mesiodistal width of the mandibular first molar from measures of malocclusion.

\begin{tabular}{lllll}
\hline Variable & Estimate & SSQ & F-Ratio & P-Value \\
\hline Intercept & 11.113 & 0.0000 & 0.00 & 1.0000 \\
Total displacements & 0 & 0.0010 & 0.00 & 0.9564 \\
Total rotations & 0 & 0.0070 & 0.02 & 0.8842 \\
Total overlapping & 0 & 0.0041 & 0.01 & 0.9114 \\
Total D+R+O & 0 & 0.0016 & 0.01 & 0.9453 \\
Crowding & 0 & 0.1451 & 0.44 & 0.5074 \\
Spacing & -0.189 & 2.3454 & 7.156 & 0.0081 \\
Diastema & 0 & 0.0001 & 0.00 & 0.9845 \\
Maxillary irregularity & 0 & 0.0137 & 0.04 & 0.8384 \\
Mandibular irregularity & 0 & 0.3718 & 1.13 & 0.2882 \\
Maxillary overjet & 0 & 0.0414 & 0.13 & 0.7233 \\
Mandibular overjet & 0 & 0.0285 & 0.09 & 0.7688 \\
Openbite & 0 & 0.0049 & 0.02 & 0.9028 \\
AP relationship & 0 & 0.0321 & 0.10 & 0.7554 \\
DAI score & 0 & 0.0210 & 0.06 & 0.8010 \\
\hline
\end{tabular}


Table 21. Results of stepwise multiple regression analysis predicting mesiodistal width of the mandibular second molar from measures of malocclusion.

\begin{tabular}{lclll}
\hline Variable & Estimate & SSQ & F-Ratio & P-Value \\
\hline Intercept & 10.291 & 0.0000 & 0.00 & 1.0000 \\
Total displacements & 0 & 0.0583 & 0.16 & 0.6931 \\
Total rotations & 0 & 1.2175 & 3.31 & 0.0703 \\
Total overlapping & 0 & 0.0618 & 0.17 & 0.6846 \\
Total D+R+O & 0 & 0.3441 & 0.93 & 0.3373 \\
Crowding & 0 & 0.0063 & 0.02 & 0.8966 \\
Spacing & -0.186 & 2.2559 & 6.07 & 0.0146 \\
Diastema & 0 & 0.3684 & 0.99 & 0.3207 \\
Maxillary irregularity & 0 & 0.5828 & 1.57 & 0.2114 \\
Mandibular irregularity & 0 & 0.8663 & 2.35 & 0.1272 \\
Maxillary overjet & 0 & 0.4774 & 1.29 & 0.2582 \\
Mandibular overjet & 0 & 0.6160 & 1.66 & 0.1988 \\
Openbite & 0 & 0.9992 & 2.71 & 0.1013 \\
AP relationship & 0 & 1.2499 & 3.40 & 0.0666 \\
DAI score & 0 & 1.1537 & 3.14 & 0.0781 \\
\hline
\end{tabular}




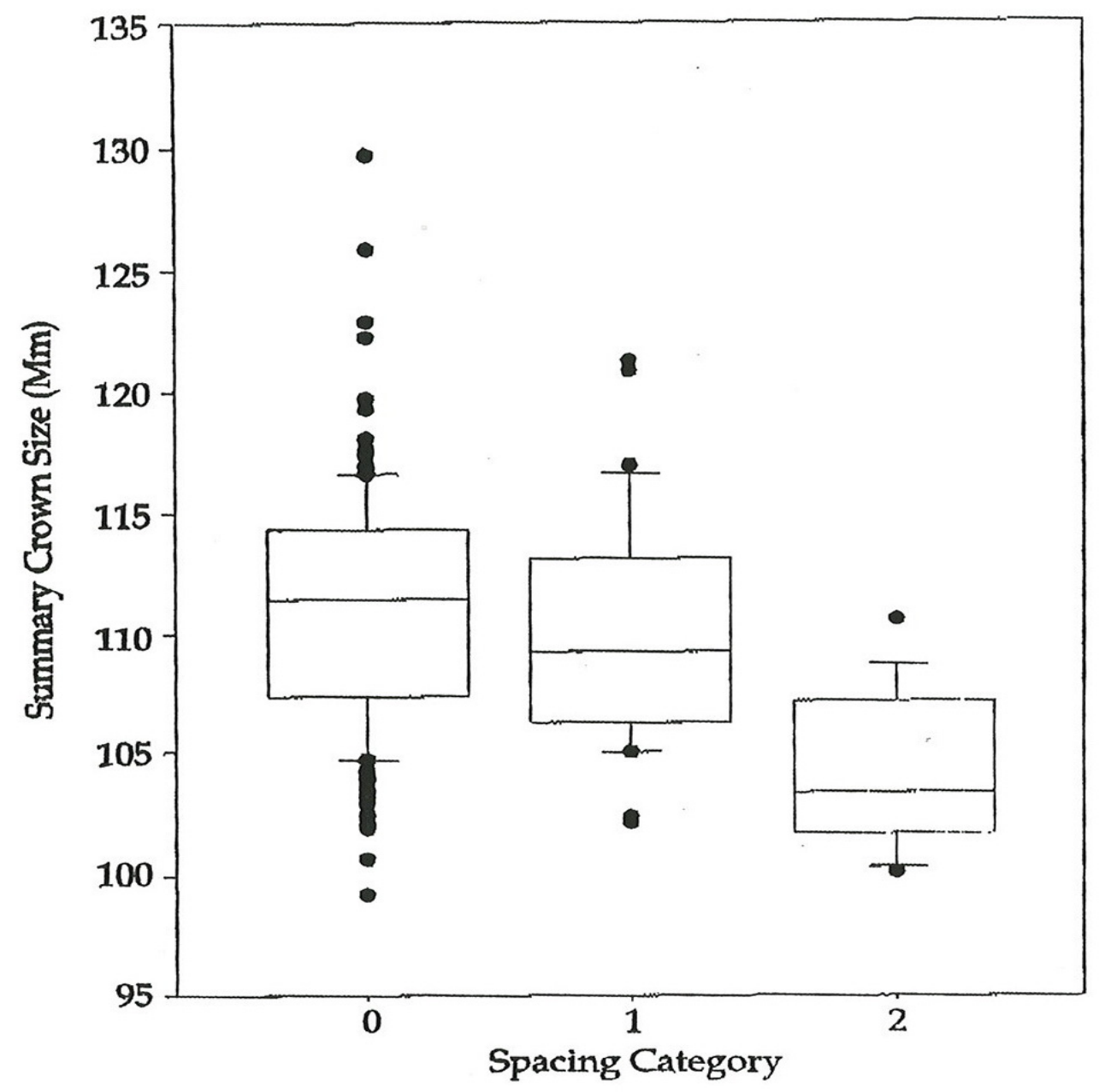

Figure 77. Plot showing the relationship between the three grades of "spacing" against the summed mesiodistal sizes of all 14 tooth types. 
Table 22. Analysis of variance testing for a difference in summed tooth size among grades of spacing while controlling for sexual dimprohism.

\begin{tabular}{lcccr}
\hline Source & df & SSQ & F-Ratio & P-Value \\
\hline Sex & 1 & 288.703 & 13.7771 & 0.0003 \\
Spacing & 2 & 582.455 & 13.892 & $<0.0001$ \\
Sex-x-Spacing & 2 & 41.096. & 0.980 & 0.3770 \\
\hline
\end{tabular}


taken out of the comparison among grades of spacing. Sexual dimorphism is highly significant for the sum of 14 mesiodistal crown diameters; however, this effect is independent of the fact disclosed above that those with interdental spacing tend to have smaller tooth crowns $(\mathrm{P}<0.0001)$. 


\section{CHAPTER V. DISCUSSION}

Malocclusions are quite common in the United States and other industrialized countries (e.g., Kelly and Harvey 1977; Brunelle et al. 1996). Indeed, only about one teenager in ten has a naturally-occurring good occlusion. Such high frequencies naturally raise the question of what factors are responsible for malocclusion - and for their high prevalence in modern populations. Corruccini and Potter (1980) and Harris and Smith (1980) showed that the common tooth-based sorts of malocclusion (e.g., crowding, rotations, overlapping) have no discernible genetic basis and, instead, are the result of environmental issues (Corruccini 1999). Harris and Johnson (1991), among others, suggest that malocclusions should be viewed as developing from some combination of two broad sources; one is skeletal, which has a familial (genetic) basis (e.g., Nakata et al. 1974 a,b; Harris 1975), and the other is dental, which seems to be controlled predominately by the environment (Beecher and Corruccini 1983).

Added to this mix is the issue of tooth size. Mesiodistal crown diameters can affect the risk of dental malocclusions, as mesiodistally broader teeth obviously require greater arch size for proper arrangements. Due to several converging issues the effects of crown size are probably of increasing relevance. Townsend and others (Dempsey et al. 1995; Hughes et al. 2007) document that tooth sizes are under appreciable genetic control. Begg's classic argument (1954) is that, in the past, interproximal attrition due to substantial grit in the diet was accommodative in that it reduced mesiodistal crown dimensions such that TSASDs were uncommon. The grit introduced by the stone-milling of grains led to substantive attrition into the 20th century in many industrialized societies (Brothwell 1981; Brook et al. 2006).

Nowadays, several trends seem to conspire towards high frequencies of dental malrelationships. Improved childhood health (decreased morbidity) and enhanced nutrition have led to secular trends of increasing tooth size (Garn et al. 1968; Ebeling et al. 1973; Harris et al. 2001), while, conversely, diminished chewing stress during tooth eruption phases of childhood fails to stimulate tooth alignment and, thereby, fails to stimulate alveolar bone growth (e.g., Watt and Williams 1952; Beecher and Corruccini 1981, 1983; Ciochon et al. 1997; Mavropoulos et al. 2005). Thirdly, the absence of interproximal attrition exacerbates the risk of dental malocclusions.

Discussions of dental malocclusions in the orthodontic literature often are couched as developmental competitions between tooth crown dimensions and 
alveolar bone support. The issue commonly is described as an "either-or" question - whether crowding is a tooth-size or an arch-size problem. This "competition" seems to us to misconstrue the developmental events. The erupting tooth, specifically the dental follicle, produces the molecular signals that stimulate alveolar bone growth as the tooth erupts (Wise et al. 2002). At later ages, alveolar remodeling occurs along with tooth movement, but its capacity to proliferate is greatly restricted (e.g., Reitan and Rygh 1994; Krishnan and Davidovitch 2006; Masella and Meister 2006). Alveolar bone proliferates to surround and support the teeth. However, when teeth erupt into crowded, overlapping positions, the alveolus does not need to grow more than supports their crowded, constrained locations. Alveolar growth is sufficient to this step. The problem occurs when the clinician wants to move the teeth into alignment, which characteristically requires additional arch perimeter and, thereby, more bony support. At present, we do no know how to engineer bone growth (guided tissue generation) to harmonize the occlusion. The alternative is that extractions and/or IPR (interproximal reduction) are the viable alternatives. The point, however, is that the "fault" of malocclusion seldom is inadequate bone growth; bone growth is almost invariably sufficient to support the teeth in their maloccluded positions. Research studies that conclude that there is less alveolar support when teeth erupt in crowded overlapping positions compared to naturally occurring good occlusions seem to overlook this developmental consideration. In our opinion, inadequate alveolar bone growth is rarely the cause of a TSASD but, rather the consequence.

It is not readily apparent how the mesiodistal sizes of late-emerging teeth in the buccal segment can influence the crowding already existing among the early-emerging anterior teeth. One explanation is that the various tooth types show the same sorts of positive associations because tooth sizes are highly intercorrelated (Moorrees and Reed 1964; Potter et al. 1968) and are congruent within an individual (Bolton 1962).

Numerous studies have looked at the relationship between tooth size and dental crowding. Some studies conclude that mesiodistal tooth size is larger in subjects with dental crowding (Lundström 1969; Fastlicht 1970; Norderval et al. 1975; Doris et al. 1981; Smith et al. 1982; Gilmore and Little 1984). Almost all of the studies that have tested for an effect of crown size have compared just two samples, one with crowding and the other without (Lindström 1949; Doris et al. 1981; Melo et al. 2001). Those with crowding tended to have larger crowns requiring more arch space for alignment. The present study extended this design to test whether there are graded responses between increasing tooth size and increasing severity of malocclusion. That is, (1) can an association be documented between a subject's tooth size and his extent of tooth-based 
malocclusion (rotations, displacements, crowding) and (2) what is the nature of the association? The test design takes the sexual dimorphism of tooth dimensions into account, so that tooth sizes between the sexes do not confound interpretations.

Fastlicht (1970) tested whether orthodontic treatment influenced the crowding of the mandibular incisors. He found a strong statistical correlation between mesiodistal widths of the mandibular and the maxillary incisors with crowding. He showed that where there were larger mesiodistal widths, there was more crowding. The present study found similar results, showing that the displacement of the maxillary canines was significantly related to the size of the maxillary lateral incisor and canine. This is anticipated as the maxillary canine is the last tooth anterior to the maxillary first molar to erupt and may have insufficient space to erupt into its proper position in the dental arch.

Norderval et al. (1975) compared a group with well aligned mandibular front teeth with a group exhibiting mandibular anterior crowding. The group with crowding had significantly broader mesiodistal diameters $(P<0.05)$ of the incisors.

Bernabé and Flores-Mir (2006) compared the mediodistal and buccolingual crown dimensions among samples grouped as mild crowding, moderate crowding, and no crowding. Statistically significant average difference was found between each of these three groups. This agrees with the present study where crowding is significantly related to mesiodistal size of both maxillary and mandibular central incisors.

The fundamental issue in this study was to search for statistical associations between tooth size and the extent of the malocclusion; a positive "dose-response" relationship between increasing crown size and each measure of crowding. Each of the 10 measures of occlusal variation was tested against each of the mesiodistal crown dimensions of the 14 tooth types. All the regressive coefficients between tooth size and the extent of malocclusion are positive, showing that larger crown dimensions are significantly predictive of crowding; this includes displacements, rotations, and dental overlapping. There were two exceptions where the coefficients were negative, spacing and diastema. In these cases the smaller the teeth the greater the risk of interdental spacing. The common theme is that broader teeth require more space, which increases the risk and degree of dental malocclusion. Our speculation is that tooth-size arch-size discrepancies are on the increase because of secular trends toward larger crown sizes, with little enhancement of the supporting jaw structure. 


\section{CHAPTER VI. SUMMARY AND CONCLUSIONS}

Malocclusion has been demonstrated to be multifactorial in origin. Most of the studies that have tested for an effect of crown size have compared just two samples, one with crowding and the other without. Those groups with crowding tend to have larger crowns requiring more space for alignment. Our study examined mesiodistal tooth size as a contributing factor to malocclusion and the nature of that association.

We mesasured the mesiodistal width of both maxillary and mandibular teeth from central incisor to second molar on pretreatment orthodontic casts. The casts used were from the University of Tennessee department of orthodontics. The subjects were from 11 to 25 years of age. Data was collected by one observer. Major findings include:

1. Generally, the association between crowding and malocclusion does have a dose response relationship. Subjects with malocclusion have a tendency toward greater tooth size.

2. The relationship is based on a continuum of relative tooth size and degree of malocclusion, rather than the distinct groupings, "crowded" and "not crowded."

3. Specifically, the association between mesiodistal tooth size and malocclusion becomes stronger as the teeth exhibiting larger mesiodistal dimensions are located nearer the midline of the dental arch. 


\section{LIST OF REFERENCES}

Adams CP. A comparison of 15 year old children with excellent occlusion and with crowding of the teeth, Angle Class I malocclusion, in respect of face size and shape and tooth size. Swed Dent J Suppl 1982;15:11-26.

Albashaireh ZS, Khader YS. The prevalence and pattern of hypodontia of the permanent teeth and crown size and shape deformity affecting upper lateral incisors in a sample of Jordanian dental patients. Community Dent Health 2006;23:239-43.

Al-Khateeb S, Abu Alhaija E. Tooth size discrepancies and arch parameters among different malocclusions in a Jordanian sample. Angle Orthod 2006;76:459-65.

Alkofide E, Hashim H. Intermaxillary tooth size discrepancies among different malocclusion classes: a comparative study. J Clin Ped Dent 2002;26:383-7.

Angle EH. Treatment of malocclusion of teeth. Philadelphia: S. S. White Dental Manufacturing Company, 1970:26.

Arya B, Savara B, Thomas D, Clarkson Q. Relations of sex and occlusion to mesiodistal tooth size. Am J Orthod 1974;66:479-86.

Basaran G, Selek M, Hamamci O, Akkus Z. Intermaxillary Bolton tooth size discrepancies among different malocclusion groups. Angle Orthod 2006;76:26-30.

Beecher RM, Corruccini RS. Effects of dietary consistency on craniofacial and occlusal development in the rat. Angle Orthod 1981;51:61-9.

Beecher RM, Corruccini RS, Freeman M. Craniofacial correlates of dietary consistency in a nonhuman primate. J Craniofac Genet Dev Biol 1983;3:193-202.

Begg PR. Stone age man's dentition. Am J Orthod 1954;40:298-312,373-83, 462-75,517-31. 
Bernabé E, Flores-Mir C. Dental morphology and crowding: a multivariate approach. Angle Orthod 2006;76:20-5.

Birkeland K, Boe OE, Wisth PJ. The relationship between occlusion and satisfaction with dental appearance in orthodontically treated and untreated groups: a longitudinal study. Eur J Orthod 2000;22:509-18.

Birleland K, Katle A, Lovgreen S, Boe OE, Wisth PJ. Factors influencing the decision about orthodontic treatment. J Orofac Orthop 1999;60:292-307.

Bolton WA. Disharmony in tooth size and its relation to the analysis and treatment of malocclusion. Angle Orthod 1958;28:113-30.

Brothwell DR. Digging up bones. 3rd ed. Ithaca: Cornell University Press, 1981.

Brook AH, Underhill C, Foo LK, Hector M. Approximal attrition and permanent tooth crown size in a Romano-British population. Dental Anthropology 2006;19:23-8.

Brook PH, Shaw WC. The development of an index of orthodontic treatment priority. Eur J Orthod 1989:11;309-20.

Brunelle JA, Bhat M, Lipton JA. Prevalence and distribution of selected occlusal characteristics in the US population, 1988-1991.

J Dent Res 1996;75:706-13.

Chi J, Johnson M, Harkness M. Age changes in orthodontic treatment need: a longitudinal study of 10- and 13- year-old children using the Dental Aesthetic Index. Aus Orthod J 2000;16:150-6.

Ciochon RL, Nisbett RA, Corruccini RS. Dietary consistency and craniofacial development related to masticatory function in minipigs. J Craniofac Genet Dev Biol 1997;17:96-102.

Cohen J, Cohen P. Applied multiple regression/correlation analysis for the behavioral sciences. New York: John Wiley \& Sons, 1975.

Corruccini RS. How anthropology informs the orthodontic diagnosis of malocclusions' causes. Lewiston, NY: Edwin Mellen Press, 1999. 
Corruccini RS. Australian aboriginal tooth succession, interproximal attrition, and Begg's theory. Am J Orthod Dentofac Orthop 1990;97:349-57.

Corruccini RS, Potter RH. Genetic analysis of occlusal variation in twins. Am J Orthod 1980;78:140-54.

Crosby DR, Alexander CG. The occurrence of tooth size discrepancies among different malocclusion groups. Am J Orthod Dentofacial Orthop 1989;95:457-61.

Dahlberg AA. The changing dentition of man. J Am Dent Assoc 1945;32:676-90.

Dahlberg AA. The dentition of American Indians. In: Laughlin WS, Editor. The physical anthropology of the American Indian. New York: Viking Fund Inc., 1951, p 138-76.

Dahlberg G. Statistical methods for medical and biological students. London: George Allen and Unwin, Ltd, 1940.

Dempsey PJ, Townsend GC, Martin NG, Neale MC. Genetic covariance structure of incisor crown size in twins. J Dent Res 1995;74:1389-98.

Doris JM, Dernard BW, Kuftinec MM. A biometric study of tooth size and dental crowding. Am J Orthod 1981;79:326-36.

Ebeling CF, Ingervall B, Hedegard B, Lewin T. Secular changes in tooth size in Swedish men. Acta Odont Scand 1973;31:141-7.

Esa R, Razak IA, Allister JH. Epidemiology of malocclusion and orthodontic treatment need of 12-13 year old Malaysian school children. Community Dent Health 2001;18:31-6.

Fastlicht J. Crowding of mandibular incisors. Am J Orthod 1970;58:156-63.

Freeman JE, Maskeroni AJ, Lorton L. Frequency of Bolton tooth-size discrepancies among orthodontic patients. Am J Orthod Dentofacial Orthop 1996;110:24-7.

Garn SM, Lewis AB, Kerewsky R. Sex difference in tooth size. J Dent Res 1964;43:306. 
Garn SM, Lewis AB, Swindler DR. Genetic control of sexual dimorphism in tooth size. J Dent Res 1967;46:963-72.

Garn SM, Lewis AB, Walenga A. Evidence for a secular trend in tooth size over two generations. J Dent Res 1968;47:503.

Gavan JA. The consistency of anthropometric measurements. Am J Phys Anthropol 1950;8:417-26.

Gilmore CA, Little RM. Mandibular incisor dimensions and crowding. Am J Orthod 1984;86:493-502.

Grainger RM. Orthodontic treatment priority index. National Center for Health Statistics Series 2, no. 25. Washington, DC: Public Health Service, 1967.

Harris EF. Interpreting heritability estimates in the orthodontic literature. Semin Orthod 2008;14:125-34.

Harris EF, Bodford K. Bilateral asymmetry in the tooth relationships of orthodontic patients. Angle Orthod 2007;77:779-86.

Harris EF, Potter RH, Lin J. A secular trend in tooth size in urban Chinese assessed from two-generation family data. Am J Phys Anthropol 2001;115:312-8.

Harris EF, Johnson MG. Hertability of craniometric and occlusal variables: a longitudinal sib analysis. Am J Dentofacial Orthop 1991;99:258-68.

Harris EF, Bailit HL. A principal components analysis of human odontometrics. Am J Phys Anthropol 1988;75:87-99.

Harris EF, Vaden JL, Williams RA. Lower incisor space analysis: A contrast of methodologies. Am J Orthod Dentofacial Orthop 1987;92:375-80.

Harris EF, Smith RJ. A study of occlusion and arch widths in families. Am J Orthod 1980;78:155-63. 
Harris JE. Genetic factors in the growth of the head: inheritance of the craniofacial complex and malocclusion. Dent Clin N Am 1975;19:151-60.

Helm S. Epideminology and public health aspects of malocclusion. J Dent Res Spec 1977:56;C27-C31.

Horowitz SL, Osborne RH, DeGeorge FV. Hereditary factors in tooth dimensions, a study of the anterior teeth of twins. Angle Orthod 1958;28:87-93.

Houston WJB. The Analysis of errors in orthodontic measurements. Am J Orthod 1983;83:382-90.

Howe RP, McNamara JA Jr, O'Connor KA. An examination of dental crowding and its relationship to tooth size and arch dimensions. Am J Orthod 1983;83:363-73.

Hughes TE, Bockmann MR, Seow K, Gotjamaos T, Gully N, Richards LC, Townsend GC. Strong genetic control of emergence of human primary incisors. J Dent Res 2007;86:1160-5.

Jenny J, Cons NC, Kohout FJ, Jakobson J. Predicting handicapping malocclusion using the Dental Aesthetic Index (DAI). Int Dent J 1993:43;128-32.

Jenny J, Cons NC. Establishing malocclusion severity levels on the Dental Aesthetic Index (DAI) scale. Aus Dent J 1996;41:43-6.

Johnson M, Harkness M. Prevalance of malocclusion and orthodontic treatment need in 10-year-old New Zealand children. Aus Orthod J 2000;16:1-8.

Keene A, Engel G. The mandibular dental arch, part IV: prediction and prevention of lower anterior relapse. Angle Orthod 1979;49:173-80.

Kelly JE, Harvey CR. An assessment of the occlusion of the teeth of youths 12-17 years. United States. Rockville MD: Health Resources Administration, National Center for Health Statistics; 1977. 
Kerosuo H, Enezi SA, Kerosuo E, Abdulkarim E. Association between normative and self-perceived orthodontic treatment need among Arab high school students. Am J Orthod Dent Orthop 2004;125:375-8.

Kieser JA, Groeneveld HT. The reliability of human orthotometric data. J Dent Assoc S Afr 1991;46:267-70.

Kieser JA, Groeneveld HT. Measurement error in human dental mensuration. Ann Hum Biol 1990;17:523-8.

Kisely S, Howell K, Green J. Pathways to orthodontic care. J Public Health Med 1997;19:148-55.

Krishnan V, Davidovich Z. Cellular, molecular, and tissue-level reactions to orthodontic force. Am J Orthod Dentofacial Orthop 2006;129:469 el-32.

Laino A, Quaremba G, Paduano S, Stanzione S. Prevalence of tooth size discrepancy among different malocclusion groups. Prog Orthod 2003;4:37-44.

Little RM. The irregularity index: a quantitative score of mandibular anterior alignment. Am J Orthod 1975;68:554-63.

Lundström A. Aetiology of crowding of the teeth (based on studies of twins and on morphological investigation) and its bearing on orthodontic treatment (expansion or extraction). Eur Orthod Soc 1951;176-90.

Lundström A. Tooth size and occlusion in twins. New York: S Karger, 1948.

Mandall NA, McCord JF, Blinkhorn AS, Worthington HV, O'Brien KD. Perceived aesthetic impact of malocclusion and oral selfperceptions in 14-15-year-old Asian and Caucasian children in Greater Manchester. Eur J Orthod 1999;21:175-83.

Masella RS, Meister M. Current concepts in the biology of orthodontic tooth movement. Am J Orthod Dentofacial Orthop 2006;129:458-68. 
Mavropoulos A, Ammann P, Bresin A, Kiliaridis S. Masticatory demands induce region-specific changes in mandibular bone density in growing rats. Angle Orthod 2005;75:625-30.

McCann J, Burden DJ. An investigation of tooth size in Northern Irish people with bimaxillary dental protrusion. Eur J Orthod 1996;18:617-21.

McKeown M. The diagnosis of incipient arch crowding in children. NZ Dent J 1981;77:93-6.

Melo L, Ono Y, Takagi Y. Indicators of mandibular dental crowding in mixed dentition. Pediatr Dent 2001;23:118-22.

Mills LF. Arch width, arch length, and tooth size in young adult males. Angle Orthod 1964;34:124-9.

Moorrees CFA, Reed RB. Correlations of tooth size. Arch Oral Biol 1964;9:685-97.

Moorrees CFA. The dentition of the growing child. Cambridge: Harvard University Press; 1959.

Moorrees CFA, Thomsen SO, Jensen E, Yen PK. Mesiodistal crown diameters of the deciduous and permanent teeth in individuals. J Dent Res 1957;36:39-47.

Mossey PA. The heritability of malocclusion: part 1. Genetics, principles and terminology. Br J Orthod 1999;26:103-13.

Mossey PA. The heritability of malocclusion: part 2. The influence of genetics in malocclusion. Br J Orthod 1999;26:195-203.

Nakata M, Yu P-L, Davis B, Nance WE. Genmetic determinants of craniofacial morphology: a twin study. Ann Hum Genet 1974;37:431-42.

Nakata M, Yu P-L, Nance WE. Multivariate analysis of cranio-facial measurements in twin and family data. Am J Phys Anthropol 1974;41:423-30.

Nance HN. The limitations of orthodontic treatment. Am J Orthod Oral Surg 1947;33:177-223. 
Neff CW. Tailored occlusion with the anterior coefficient. Am J Orthod 1949;35:309-14.

Nie Q, Lin J. Comparison of intermaxillary tooth size discrepancies among different malocclusion groups. Am J Orthod Dentofacial Orthop 1999;116:539-44.

Norderval K, Wisth PJ, Doe OE. Mandibular anterior crowding in relation to tooth size and craniofacial morphology. Scand J Dent Res 1975;83:267-73.

$\mathrm{O}^{\prime}$ Brien KD, McComb JL, Fox N, Wright J. Factors influencing the uptake of orthodontic treatment. Br J Orthod 1996;23:331-4.

Otuyemi OD, Ogunyinka A, Dosumu O, Cons NC, Jenny J. Malocclusion and orthodontic treatment need of secondary school students in Nigeria according to the dental aesthetic index (DAI). Int Dent J 1999;49:203-10.

Paredes V, Gandia JL, Cibrian R. Do Bolton's ratios apply to a Spanish population. Am J Orthod Dentofacial Orthop 2006;129:428-30.

Peck S, Peck H. Crown dimensions and mandibular incisor alignment. Angle Orthod 1972;42:148-53.

Polder BJ, Van'tHof MA, Van der Linden FP, Kuijpers-Jagtman AM. A meta-analysis of the prevalence of dental agenesis of permanent teeth. Community Dent Oral Epidemiol 2004;32:217-26.

Potter RHY, Yu P-L, Dahlberg AA, Merritt AD, and Conneally PM. Genetic studies of tooth size factors in Pima Indian families. Am J Hum Genet 1968;20:89-99.

Proffit WR. On the aetiology of malocclusion. The Northcroft lecture, 1985 presented to the British Society for the Study of Orthodontics, Oxford, April 18, 1985. Br J Orthod 1986;13:1-11.

Proffit WR. Contemporary orthodontics, 3rd ed. St. Louis: Mosby Year Book, 1999. 
Radnzic D. Dental crowding and its relationship to mesiodistal crown diameters and arch dimensions. Am J Orthod Dentofacial Orthop 1988;94:50-6.

Reitan K, Rygh P. Biomechanical principles and reactions. In: Graber TM, Vanarsdall RL, eds. Orthodontics: current principles and techniques, 2nd ed. St Louis: CV Mosby, 1994, p 96-192.

Seipel CM. Variation of tooth position. Svensk Tandlakara 1946;39:5-174.

Selmler-Oslen R. An odontometrical study on the Norwegian Lapps. Thesis published by Det. Norske Videnskaps-Akadem, Jacop, Dybuad. (cited Lundtröm 1954).

Shaw A, Elcock C, Brook AH. Incisor crown shape and crowding. Am J Orthod Dentofacial Orthop 2003;123:562-7.

Sheats RD, McGorray SP, Keeling SD, Wheeler TT, King GJ. Occlusal traits and perceptions of orthodontic need in eighth grade students. Angle Orthod 1998;68:107-14.

Smith S, Buschang P, Watanabe E. Interarch tooth size relationships of 3 populations: "Does Bolton's analysis apply?" Am J Orthod Dentofacial Orthop 2000;117:169-74.

Solal RR, Rohlf FJ. Biometry: the principles and practice of statistics in biological research, 3rd ed. San Francisco: WH Freeman and Company, 1995.

Southard TE, Behrents RG, Tolley EA. The anterior component of force. Part 2. relationship with dental malalignment. Am J Orthod Dentofacial Orthop 1989;97:41-4.

Sperry TP, Worms FW, Isaacson RJ, Speidel TM. Tooth-size discrepancy in mandibular prognathism. Am J Orthod 1977;72:183-90.

Stenvik A, Espeland L, Derset GP, Eriksen HM. Attitudes to malocclusion among 18- and 35-year-old Norwegians. Community Dent Oral Epidemiol 1996;24:390-3. 
Stricker G, Clifford E, Cohen LK, Gidden DB, Meskin LH, Evans CA. Psychological aspects of craniofacial disfigurement. Am J Orthod 1979:76;410-22.

Tsai HH. Dental crowding in primary dentition and its relationship to arch and crown dimensions. J Dent Child 2003;70:164-9.

Tukey JW. Exploratory data analysis. Reading, Mass: Addison-Wesley, 1977.

Utermohle CJ, Zegura SL, Heathcole GM. Multiple observers, humidity and choice of precision statistics: factors influencing craniometric data quality. Am J Phys Anthropol 1983;61:85-95.

van der Linden FPGM, Duterloo HS. Development of the human dentition: an atlas. Hagerstown, MD: Harper and Row, Publishers, 1976.

Watt DG, Williams HM. The effects of the physical consistency of food on the growth and development of the mandible and maxilla of the rat. Am J Orthod 1951;37:895-928.

Willens G, DeBruyne I, Verdonck A, Fieuws S. Prevalence of dentofacial characteristics in a Belgian orthodontic population. Clin Oral Invest 2001;5:220-6.

Winer BJ, Brown DR, Michels KM. Statistical princiles in experimental design. New York: McGraw-Hill; 1991.

Wide GE, Frazier-Bowers S, D'Souza RN. Cellular, molecular, and genetic determinants of tooth eruption. Crit Rev Oral Biol Med 2002;13:323-34

Zeisz RC, Nuckools J. Dental anatomy: The form and function of the permanent teeth and the form and function of the deciduous teeth. St. Louis: Mosby; 1949. 


\section{APPENDIX. \\ ANCOVA RESULTS \\ Statistical results, by measure \\ of malocclusion and tooth type}


Table A-1. ANCOVA results testing for associations between mesiodistal width of the maxillary central incisor and U3 displacements.

\begin{tabular}{llc}
\hline Source & F-Ratio & P-Value \\
\hline \multicolumn{2}{c}{ Among Subjects } \\
U1-Size & 3.5376 & 0.0614 \\
Sex & 2.5180 & 0.1141 \\
U1-Size-x-Sex & 0.9087 & 0.3416 \\
\multicolumn{2}{c}{ Within Subjects } \\
Side & 2.4263 & 0.1209 \\
Side-x-U1-Size & 2.2353 & 0.1364 \\
Side-x-Sex & 0.0382 & 0.8453 \\
Side-x-U1-Size-x-Sex & 1.9612 & 0.1629 \\
\hline
\end{tabular}

* df for each test is 1 and 203.

Means, Sexes Pooled

U_L3 dis $\quad 0.38$

U_R3 dis $\quad 0.41$

Means, by Sex

Boys $\quad 0.330 .30$

Girls $\quad 0.480 .42$ 
Table A-2. ANCOVA results testing for associations between mesiodistal width of the maxillary central incisor and $\mathrm{U} 2$ displacements.

\begin{tabular}{lcc}
\hline Source & F-Ratio $^{*}$ & P-Value \\
\hline U1-Size & \multicolumn{2}{c}{ Among Subjects } \\
Sex & 2.3262 & 0.1288 \\
U1-Size-x-Sex & 6.1700 & 0.0138 \\
& 0.0194 & 0.8894 \\
Side & \multicolumn{2}{c}{ Within Subjects } \\
Side-x-U1-Size & 0.4254 & 0.5150 \\
Side-x-Sex & 0.3861 & 0.5350 \\
Side-x-U1-Size-x-Sex & 0.0622 & 0.8033 \\
S & 2.6798 & 0.1032 \\
\hline
\end{tabular}

* df for each test is 1 and 203.

Means, Sexes Pooled

\begin{tabular}{lll} 
U_L2 dis & 0.41 & \\
U_R2 dis & 0.37 & \\
& Means, by Sex & \\
\multicolumn{4}{r}{ U_R2 dis } & U_L2 dis \\
Boys & 0.280 .32 & \\
Girls & 0.480 .49 &
\end{tabular}


Table A-3. ANCOVA results testing for associations between mesiodistal width of the maxillary central incisor and U1 displacements.

\begin{tabular}{llc}
\hline Source & F-Ratio & P-Value \\
\hline & \multicolumn{2}{c}{ Among Subjects } \\
U1-Size & 5.8228 & 0.0167 \\
Sex & 0.2423 & 0.6231 \\
U1-Size-x-Sex & 0.0102 & 0.9197 \\
\multicolumn{2}{c}{ Within Subjects } \\
Side & 2.1556 & 0.1436 \\
Side-x-U1-Size & 2.2360 & 0.1364 \\
Side-x-Sex & 1.4661 & 0.2274 \\
Side-x-U1-Size-x-Sex & 0.2071 & 0.6495 \\
\hline
\end{tabular}

* df for each test is 1 and 203.

Means, Sexes Pooled

$\begin{array}{lll}\text { U_L1 dis } & 0.36 & \\ \text { U_R1 dis } & 0.34 & \\ & \text { Means, by Sex } & \\ & \text { U_R1 dis } & \text { U_L1 dis } \\ \text { Boys } & 0.350 .32 & \\ \text { Girls } & 0.330 .40 & \end{array}$


Table A-4. ANCOVA results testing for associations between mesiodistal width of the maxillary central incisor and L3 displacements.

\begin{tabular}{llc}
\hline Source & F-Ratio $^{*}$ & P-Value \\
\hline & \multicolumn{2}{c}{ Among Subjects } \\
U1-Size & 4.8653 & 0.0285 \\
Sex & 3.8462 & 0.0512 \\
U1-Size-x-Sex & 0.0878 & 0.7673 \\
\multicolumn{2}{c}{ Within Subjects } \\
Side & 0.1763 & 0.6750 \\
Side-x-U1-Size & 0.1842 & 0.6682 \\
Side-x-Sex & 2.5029 & 0.1152 \\
Side-x-U1-Size-x-Sex & 0.1918 & 0.6619 \\
\hline
\end{tabular}

* df for each test is 1 and 203.

Means, Sexes Pooled

\begin{tabular}{lll} 
L_L3 dis & 0.23 & \\
L_R3 dis & 0.23 & \\
\multicolumn{4}{c}{ Means, by Sex } \\
\multicolumn{4}{c}{ L_R3 dis } & L_L3 dis \\
Boys & 0.140 .20 & \\
Girls & 0.330 .26
\end{tabular}


Table A-5. ANCOVA results testing for associations between mesiodistal width of the maxillary central incisor and L2 displacements.

\begin{tabular}{lcc}
\hline Source & F-Ratio* & P-Value \\
\hline \multicolumn{3}{c}{ Among Subjects } \\
U1-Size & 18.9529 & $<0.0001$ \\
Sex & 4.0880 & 0.0445 \\
U1-Size-x-Sex & 1.2794 & 0.2593 \\
\multicolumn{2}{c}{ Within Subjects } \\
Side & 1.1504 & 0.2847 \\
Side-x-U1-Size & 1.3060 & 0.2545 \\
Side-x-Sex & 0.6421 & 0.4239 \\
Side-x-U1-Size-x-Sex & 0.7807 & 0.3780 \\
\hline
\end{tabular}

* df for each test is 1 and 203.

Means, Sexes Pooled

\begin{tabular}{lrr} 
L_L2 dis & 0.47 & \\
L_R2 dis & 0.51 & \\
& Means, by Sex & \\
\multicolumn{4}{c}{ L_R2 dis } & L_L2 dis \\
Boys & 0.430 .42 & \\
Girls & 0.630 .55 &
\end{tabular}


Table A-6. ANCOVA results testing for associations between mesiodistal width of the maxillary central incisor and L1 displacements.

\begin{tabular}{llc}
\hline Source & F-Ratio* & P-Value \\
\hline & \multicolumn{2}{c}{ Among Subjects } \\
U1-Size & 2.3891 & 0.1237 \\
Sex & 0.1088 & 0.7419 \\
U1-Size-x-Sex & 3.4616 & 0.0643 \\
\multicolumn{2}{c}{ Within Subjects } \\
Side & 4.0198 & 0.0463 \\
Side-x-U1-Size & 4.2766 & 0.0399 \\
Side-x-Sex & 0.0934 & 0.7602 \\
Side-x-U1-Size-x-Sex & 1.1379 & 0.2874 \\
\hline
\end{tabular}

* df for each test is 1 and 203.

Means, Sexes Pooled

\begin{tabular}{|c|c|c|}
\hline L_L1 dis & 0.17 & \\
\hline L_R1 dis & 0.22 & \\
\hline \multicolumn{3}{|c|}{ Means, by Sex } \\
\hline & L_R1 dis & L_L1 dis \\
\hline Boys & 0.220 .17 & \\
\hline Girls & 0.190 .16 & \\
\hline
\end{tabular}


Table A-7. ANCOVA results testing for associations between mesiodistal width of the maxillary central incisor and U3 rotations.

\begin{tabular}{llc}
\hline Source & F-Ratio* & P-Value \\
\hline \multicolumn{2}{c}{ Among Subjects } \\
U1-Size & 0.0212 & 0.8844 \\
Sex & 3.8477 & 0.0512 \\
U1-Size-x-Sex & 0.6381 & 0.4253 \\
\multicolumn{2}{c}{} \\
Side & \multicolumn{2}{c}{ Within Subjects } \\
Side-x-U1-Size & 0.0015 & 0.9693 \\
Side-x-Sex & 0.0025 & 0.9605 \\
Side-x-U1-Size-x-Sex & 0.3108 & 0.5778 \\
S & 1.4019 & 0.2378 \\
\hline
\end{tabular}

* df for each test is 1 and 203.

Means, Sexes Pooled

$\begin{array}{lll}\text { U_L3 rot } & 0.22 & \\ \text { U_R3 rot } & 0.22 & \\ & \text { Means, by Sex } & \\ & \text { U_R3 rot } & \text { U_L3 rot } \\ \text { Boys } & 0.180 .15 & \\ \text { Girls } & 0.260 .28 & \end{array}$


Table A-8. ANCOVA results testing for associations between mesiodistal width of the maxillary central incisor and $\mathrm{U} 2$ rotations.

\begin{tabular}{llc}
\hline Source & F-Ratio* & P-Value \\
\hline \multicolumn{2}{c}{ Among Subjects } \\
U1-Size & 0.5083 & 0.4767 \\
Sex & 0.4839 & 0.4875 \\
U1-Size-x-Sex & 0.2001 & 0.6551 \\
\multicolumn{2}{c}{ Within Subjects } \\
Side & 0.0137 & 0.9069 \\
Side-x-U1-Size & 0.0051 & 0.9432 \\
Side-x-Sex & 0.0000 & 0.9953 \\
Side-x-U1-Size-x-Sex & 0.2304 & 0.6317 \\
\hline
\end{tabular}

* df for each test is 1 and 203.

Means, Sexes Pooled

$\begin{array}{lll}\text { U_L2 rot } & 0.42 & \\ \text { U_R2 rot } & 0.39 & \\ & \text { Means, by Sex } & \\ & \text { U_R2 rot } & \text { U_L2 rot } \\ \text { Boys } & 0.370 .40 & \\ \text { Girls } & 0.420 .45 & \end{array}$


Table A-9. ANCOVA results testing for associations between mesiodistal width of the maxillary central incisor and $\mathrm{U} 1$ rotations.

\begin{tabular}{llc}
\hline Source & F-Ratio* & P-Value \\
\hline & \multicolumn{2}{c}{ Among Subjects } \\
U1-Size & 1.6355 & 0.2024 \\
Sex & 0.2025 & 0.6532 \\
U1-Size-x-Sex & 0.5015 & 0.4796 \\
\multicolumn{2}{c}{ Within Subjects } \\
Side & 2.2066 & 0.1390 \\
Side-x-U1-Size & 2.2706 & 0.1334 \\
Side-x-Sex & 0.4808 & 0.4889 \\
Side-x-U1-Size-x-Sex & 0.1641 & 0.6858 \\
\hline
\end{tabular}

* df for each test is 1 and 203.

Means, Sexes Pooled

$\begin{array}{lll}\text { U_L1 rot } & 0.49 & \\ \text { U_R1 rot } & 0.47 & \\ & \text { Means, by Sex } & \\ & \text { U_R1 rot } & \text { U_L1 rot } \\ \text { Boys } & 0.480 .46 & \\ \text { Girls } & 0.480 .54 & \end{array}$


Table A-10. ANCOVA results testing for associations between mesiodistal width of the maxillary central incisor and L3 rotations.

\begin{tabular}{llc}
\hline Source & F-Ratio $^{*}$ & P-Value \\
\hline \multicolumn{2}{c}{ Among Subjects } \\
U1-Size & 8.8001 & 0.0034 \\
Sex & 6.9989 & 0.0088 \\
U1-Size-x-Sex & 0.7419 & 0.3901 \\
\multicolumn{2}{c}{ Within Subjects } \\
Side & 4.4052 & 0.0371 \\
Side-x-U1-Size & 4.6327 & 0.0325 \\
Side-x-Sex & 4.0172 & 0.0464 \\
Side-x-U1-Size-x-Sex & 0.2542 & 0.6147 \\
\hline
\end{tabular}

* df for each test is 1 and 203.

Means, Sexes Pooled

$\begin{array}{lll}\text { L_L3 rot } & 0.35 & \\ \text { L_R3 rot } & 0.39 & \\ & \text { Means, by Sex } & \\ & \text { L_R3 rot } & \text { L_L3 rot } \\ \text { Boys } & 0.260 .31 & \\ \text { Girls } & 0.540 .41 & \end{array}$


Table A-11. ANCOVA results testing for associations between mesiodistal width of the maxillary central incisor and L2 rotations.

\begin{tabular}{lcc}
\hline Source & F-Ratio* & \multicolumn{2}{c}{ P-Value } \\
\hline \multicolumn{2}{c}{ Among Subjects } \\
U1-Size & 5.3262 & 0.0220 \\
Sex & 1.4471 & 0.2304 \\
U1-Size-x-Sex & 3.8289 & 0.0517 \\
\multicolumn{2}{c}{} \\
Side & \multicolumn{2}{c}{ Within Subjects } \\
Side-x-U1-Size & 0.7305 & 0.3937 \\
Side-x-Sex & 0.4932 & 0.4833 \\
Side-x-U1-Size-x-Sex & 0.3355 & 0.5631 \\
\hline
\end{tabular}

* df for each test is 1 and 203.

Means, Sexes Pooled

$\begin{array}{ll}\text { L_L2 rot } & 0.23 \\ \text { L_R2 rot } & 0.35\end{array}$

L_R2 rot 0.35

Means, by Sex

Boys $\quad 0.320 .23$

L_R2 rot L_L2 rot

Girls $\quad 0.420 .27$ 
Table A-12. ANCOVA results testing for associations between mesiodistal width of the maxillary central incisor and L1 rotations.

\begin{tabular}{llc}
\hline Source & F-Ratio* & P-Value \\
\hline & \multicolumn{2}{c}{ Among Subjects } \\
U1-Size & 1.6622 & 0.1988 \\
Sex & 2.1361 & 0.1454 \\
U1-Size-x-Sex & 0.2453 & 0.6209 \\
\multicolumn{2}{c}{ Within Subjects } \\
Side & 0.3982 & 0.5287 \\
Side-x-U1-Size & 0.3542 & 0.5524 \\
Side-x-Sex & 0.6124 & 0.4348 \\
Side-x-U1-Size-x-Sex & 1.1534 & 0.2841 \\
\hline
\end{tabular}

* df for each test is 1 and 203.

Means, Sexes Pooled

$\begin{array}{lll}\text { L_L1 rot } & 0.34 & \\ \text { L_R1 rot } & 0.38 & \\ & \text { Means, by Sex } & \\ & \text { L_R1 rot } & \text { L_L1 rot } \\ \text { Boys } & 0.430 .37 & \\ \text { Girls } & 0.310 .32 & \end{array}$


Table A-13. ANCOVA results testing for associations between mesiodistal width of the maxillary central incisor and U2-U3 overlap.

\begin{tabular}{llc}
\hline Source & F-Ratio* & P-Value \\
\hline \multicolumn{2}{c}{ Among Subjects } \\
U1-Size & 2.8172 & 0.0948 \\
Sex & 6.7772 & 0.0099 \\
U1-Size-x-Sex & 0.0506 & 0.8222 \\
\multicolumn{2}{c}{} \\
Side & \multicolumn{2}{c}{ Within Subjects } \\
Side-x-U1-Size & 1.7119 & 0.1922 \\
Side-x-Sex & 1.6323 & 0.2028 \\
Side-x-U1-Size-x-Sex & 0.0993 & 0.7529 \\
\hline
\end{tabular}

* $\mathrm{df}$ for each test is 1 and 203.

Means, Sexes Pooled

$$
\begin{array}{ll}
\text { U_L2-3 lapping } & 0.37 \\
\text { U_R3-2 lapping } & 0.38 \\
\text { Means, by Sex } &
\end{array}
$$

$\begin{array}{lll}\text { Boys } & 0.27 & 0.27 \\ \text { Girls } & 0.50 & 0.46\end{array}$

U_R3-2 lapping U_L2-3 lapping 
Table A-14. ANCOVA results testing for associations between mesiodistal width of the maxillary central incisor and U1-U2 overlap.

\begin{tabular}{lcc}
\hline Source & F-Ratio* & \multicolumn{2}{c}{ P-Value } \\
\hline \multicolumn{2}{c}{ Among Subjects } \\
U1-Size & 0.3522 & 0.5535 \\
Sex & 8.0703 & 0.0050 \\
U1-Size-x-Sex & 0.6330 & 0.4272 \\
\multicolumn{2}{c}{} \\
Side & \multicolumn{2}{c}{ Within Subjects } \\
Side-x-U1-Size & 4.8037 & 0.0295 \\
Side-x-Sex & 4.6935 & 0.0314 \\
Side-x-U1-Size-x-Sex & 0.0534 & 0.8175 \\
\hline
\end{tabular}

*df for each test is 1 and 203.

Means, Sexes Pooled

$\begin{array}{ll}\text { U_L1-2 lapping } & 0.31 \\ \text { U_R2-1 lapping } & 0.35 \\ \text { Means, by Sex } & \end{array}$

Boys

U_R2-1 lapping U_L1-2 lapping

Girls

$0.24 \quad 0.22$

$0.42 \quad 0.42$ 
Table A-15. ANCOVA results testing for associations between mesiodistal width of the maxillary central incisor and L2-L3 overlap.

\begin{tabular}{lcc}
\hline Source & F-Ratio $^{*}$ & P-Value \\
\hline \multicolumn{3}{c}{ Among Subjects } \\
U1-Size & 23.2040 & $<0.0001$ \\
Sex & 4.1925 & 0.0419 \\
U1-Size-x-Sex & 0.1444 & 0.7044 \\
\multicolumn{2}{c}{ Within Subjects } \\
Side & 0.5212 & 0.4712 \\
Side-x-U1-Size & 0.6281 & 0.4290 \\
Side-x-Sex & 0.0227 & 0.8804 \\
Side-x-U1-Size-x-Sex & 0.1959 & 0.6585 \\
\hline
\end{tabular}

* df for each test is 1 and 203.

Means, Sexes Pooled

$\begin{array}{ll}\text { L_L2-3 lapping } & 0.65 \\ \text { L_R3-2 lapping } & 0.71 \\ \text { Means, by Sex } & \end{array}$

Boys

L_R3-2 lapping L_L2-3 lapping

Girls

0.610 .56

$0.81 \quad 0.74$ 
Table A-16. ANCOVA results testing for associations between mesiodistal width of the maxillary central incisor and L1-L2 overlap.

\begin{tabular}{llc}
\hline Source & F-Ratio $^{*}$ & P-Value \\
\hline & \multicolumn{2}{c}{ Among Subjects } \\
U1-Size & 4.0805 & 0.0447 \\
Sex & 1.1278 & 0.2895 \\
U1-Size-x-Sex & 0.1453 & 0.7034 \\
\multicolumn{2}{c}{ Within Subjects } \\
Side & 1.7706 & 0.1848 \\
Side-x-U1-Size & 1.7178 & 0.1915 \\
Side-x-Sex & 0.2503 & 0.6174 \\
Side-x-U1-Size-x-Sex & 0.0174 & 0.8952 \\
\hline
\end{tabular}

* df for each test is 1 and 203.

Means, Sexes Pooled

L_L1-2 lapping $\quad 0.46$

L_R2-1 lapping $\quad 0.47$

Means, by Sex

Boys

L_R2-1 lapping L_L1-2 lapping

Girls

0.440 .45

0.520 .49 
Table A-17. ANCOVA results testing for associations between mesiodistal width of the maxillary central incisor and total displacements.

\begin{tabular}{lrcrr}
\hline Term & Estimate & SE & t-Test & P-Value \\
\hline Intercept & -12.079 & 3.841 & -3.14 & 0.0019 \\
U1-Size & 1.865 & 0.444 & 4.20 & $<0.0001$ \\
Sex & -0.632 & 0.239 & -2.64 & 0.0089 \\
Interaction & 0.055 & 0.444 & 0.12 & 0.9017 \\
\hline
\end{tabular}

Table A-18. ANCOVA results testing for associations between mesiodistal width of the maxillary central incisor and total rotations.

\begin{tabular}{lrccc}
\hline Term & Estimate & SE & t-Test & P-Value \\
\hline Intercept & -4.927 & 2.886 & -1.71 & 0.0893 \\
U1-Size & 1.067 & 0.334 & 3.20 & 0.0016 \\
Sex & -0.376 & 0.180 & -2.09 & 0.0378 \\
Interaction & -0.473 & 0.334 & -1.42 & 0.1577 \\
\hline
\end{tabular}

Table A-19. ANCOVA results testing for associations between mesiodistal width of the maxillary central incisor and maxillary R1-L1 overlap.

\begin{tabular}{lcccc}
\hline Term & Estimate & SE & t-Test & P-Value \\
\hline Intercept & -1.148 & 0.540 & -2.13 & 0.0346 \\
U1-Size & 0.162 & 0.062 & 2.60 & 0.0100 \\
Sex & -0.032 & 0.034 & -0.95 & 0.3441 \\
Interaction & -0.117 & 0.062 & -1.87 & 0.0632 \\
\hline
\end{tabular}


Table A-20. ANCOVA results testing for associations between mesiodistal width of the maxillary central incisor and mandibular R1-L1 overlap.

\begin{tabular}{lcccc}
\hline Term & Estimate & SE & t-Test & P-Value \\
\hline Intercept & -0.154 & 0.567 & -0.27 & 0.7861 \\
U1-Size & 0.062 & 0.066 & 0.94 & 0.3470 \\
Sex & 0.094 & 0.035 & 2.67 & 0.0083 \\
Interaction & 0.011 & 0.066 & 0.16 & 0.8722 \\
\hline
\end{tabular}

Table A-21. ANCOVA results testing for associations between mesiodistal width of the maxillary central incisor and total overlapping.

\begin{tabular}{lcccc}
\hline Term & Estimate & SE & t-Test & P-Value \\
\hline Intercept & -9.439 & 3.550 & -2.66 & 0.0085 \\
U1-Size & 1.590 & 0.410 & 3.87 & 0.0001 \\
Sex & -0.591 & 0.221 & -2.67 & 0.0081 \\
Interaction & 0.049 & 0.410 & 0.12 & 0.9053 \\
\hline
\end{tabular}

Table A-22. ANCOVA results testing for associations between mesiodistal width of the maxillary central incisor and total displacements, rotations, and overlapping.

\begin{tabular}{lrcrc}
\hline Term & Estimate & $\mathrm{SE}$ & $\mathrm{t}$-Test & P-Value \\
\hline Intercept & -26.445 & 8.731 & -3.03 & 0.0028 \\
U1-Size & 4.522 & 1.009 & 4.48 & $<0.0001$ \\
Sex & -1.599 & 0.544 & -2.94 & 0.0037 \\
Interaction & -0.369 & 1.009 & -0.37 & 0.7148 \\
\hline
\end{tabular}


Table A-23. ANCOVA results testing for associations between mesiodistal width of the maxillary central incisor and crowding.

\begin{tabular}{lcccc}
\hline Term & Estimate & SE & t-Test & P-Value \\
\hline Intercept & -2.549 & 0.825 & -3.09 & 0.0023 \\
U1-Size & 0.455 & 0.095 & 4.77 & $<0.0001$ \\
Sex & -0.165 & 0.051 & -3.21 & 0.0015 \\
Interaction & -0.094 & 0.095 & -0.99 & 0.3253 \\
\hline
\end{tabular}

Table A-24. ANCOVA results testing for associations between mesiodistal width of the maxillary central incisor and spacing.

\begin{tabular}{lrcrc}
\hline Term & Estimate & SE & t-Test & P-Value \\
\hline Intercept & 2.628 & 0.635 & 4.14 & $<0.0001$ \\
U1-Size & -0.275 & 0.073 & -3.75 & 0.0002 \\
Sex & 0.074 & 0.040 & 1.88 & 0.0612 \\
Interaction & 0.074 & 0.073 & 1.01 & 0.3142 \\
\hline
\end{tabular}

Table A-25. ANCOVA results testing for associations between mesiodistal width of the maxillary central incisor and diastema.

\begin{tabular}{lrcrc}
\hline Term & Estimate & $\mathrm{SE}$ & $\mathrm{t}$-Test & P-Value \\
\hline Intercept & 2.143 & 0.656 & 3.27 & 0.0013 \\
U1-Size & -0.222 & 0.076 & -2.93 & 0.0037 \\
Sex & 0.056 & 0.041 & 1.37 & 0.1724 \\
Interaction & 0.111 & 0.076 & 1.47 & 0.1428 \\
\hline
\end{tabular}


Table A-26. ANCOVA results testing for associations between mesiodistal width of the maxillary central incisor and maxillary incisor irregularity.

\begin{tabular}{lrcrc}
\hline Term & Estimate & SE & t-Test & P-Value \\
\hline Intercept & -3.190 & 1.551 & -2.06 & 0.0410 \\
U1-Size & 0.533 & 0.179 & 2.97 & 0.0033 \\
Sex & -0.202 & 0.097 & -2.09 & 0.0377 \\
Interaction & 0.022 & 0.179 & 0.12 & 0.9037 \\
\hline
\end{tabular}

Table A-27. ANCOVA results testing for associations between mesiodistal width of the maxillary central incisor and mandibular incisor irregularity.

\begin{tabular}{lrcrc}
\hline Term & Estimate & SE & t-Test & P-Value \\
\hline Intercept & -5.346 & 1.399 & -3.82 & 0.0002 \\
U1-Size & 0.749 & 0.162 & 4.64 & $<0.0001$ \\
Sex & -0.152 & 0.087 & -1.75 & 0.0821 \\
Interaction & -0.181 & 0.162 & -1.12 & 0.2637 \\
\hline
\end{tabular}

Table A-28. ANCOVA results testing for associations between mesiodistal width of the maxillary central incisor and maxillary overjet.

\begin{tabular}{lcccc}
\hline Term & Estimate & $\mathrm{SE}$ & $\mathrm{t}$-Test & P-Value \\
\hline Intercept & -5.447 & 2.566 & -2.12 & 0.0350 \\
U1-Size & 1.049 & 0.297 & 3.54 & 0.0005 \\
Sex & -0.215 & 0.160 & -1.35 & 0.1794 \\
Interaction & -0.403 & 0.297 & -1.36 & 0.1756 \\
\hline
\end{tabular}


Table A-29. ANCOVA results testing for associations between mesiodistal width of the maxillary central incisor and mandibular overjet.

\begin{tabular}{lrccc}
\hline Term & Estimate & SE & t-Test & P-Value \\
\hline Intercept & -0.452 & 0.315 & -1.43 & 0.1533 \\
U1-Size & 0.058 & 0.036 & 1.58 & 0.1151 \\
Sex & -0.049 & 0.020 & -2.47 & 0.0144 \\
Interaction & -0.058 & 0.036 & -1.58 & 0.1151 \\
\hline
\end{tabular}

Table A-30. ANCOVA results testing for associations between mesiodistal width of the maxillary central incisor and openbite.

\begin{tabular}{lcccc}
\hline Term & Estimate & SE & t-Test & P-Value \\
\hline Intercept & -0.214 & 0.533 & -0.40 & 0.6888 \\
U1-Size & 0.035 & 0.062 & 0.56 & 0.5755 \\
Sex & 0.005 & 0.033 & 0.16 & 0.8756 \\
Interaction & -0.107 & 0.062 & -1.73 & 0.0852 \\
\hline
\end{tabular}

Table A-31. ANCOVA results testing for associations between mesiodistal width of the maxillary central incisor and anteroposterior relationship.

\begin{tabular}{lcccc}
\hline Term & Estimate & SE & t-Test & P-Value \\
\hline Intercept & 0.514 & 0.821 & 0.63 & 0.5321 \\
U1-Size & 0.029 & 0.095 & 0.30 & 0.7609 \\
Sex & 0.033 & 0.051 & 0.64 & 0.5214 \\
Interaction & -0.116 & 0.095 & -1.22 & 0.2228 \\
\hline
\end{tabular}


Table A-32. ANCOVA results testing for associations between mesiodistal width of the maxillary central incisor and DAI score.

\begin{tabular}{lrcrc}
\hline Term & Estimate & SE & t-Test & P-Value \\
\hline Intercept & 2.491 & 7.395 & 0.34 & 0.7366 \\
U1-Size & 2.852 & 0.855 & 3.34 & 0.0010 \\
Sex & -0.743 & 0.462 & -1.61 & 0.1091 \\
Interaction & -1.331 & 0.855 & -1.56 & 0.1209 \\
\hline
\end{tabular}


Table A-33. ANCOVA results testing for associations between mesiodistal width of the maxillary lateral incisor and U3 displacements.

\begin{tabular}{lcc}
\hline Source & F-Ratio* & P-Value \\
\hline \multicolumn{3}{c}{ Among Subjects } \\
U2-Size & 4.2803 & 0.0398 \\
Sex & 1.7976 & 0.1815 \\
U2-Size-x-Sex & 0.4548 & 0.5008 \\
\multicolumn{3}{c}{ Within Subjects } \\
Side & 0.2040 & 0.6520 \\
Side-x-U2-Size & 0.2848 & 0.5941 \\
Side-x-Sex & 0.4447 & 0.5056 \\
Side-x-U2-Size-x-Sex & 8.8045 & 0.0034 \\
* & \\
*df for each test is 1 and 203. \\
Means, Sexes Pooled \\
U_R3 dis 0.41 \\
U_L3 dis 0.38 \\
Means, by Sex \\
\multicolumn{3}{c}{ U_R3 dis } \\
Girl U_L3 dis
\end{tabular}


Table A-34. ANCOVA results testing for associations between mesiodistal width of the maxillary lateral incisor and U2 displacements.

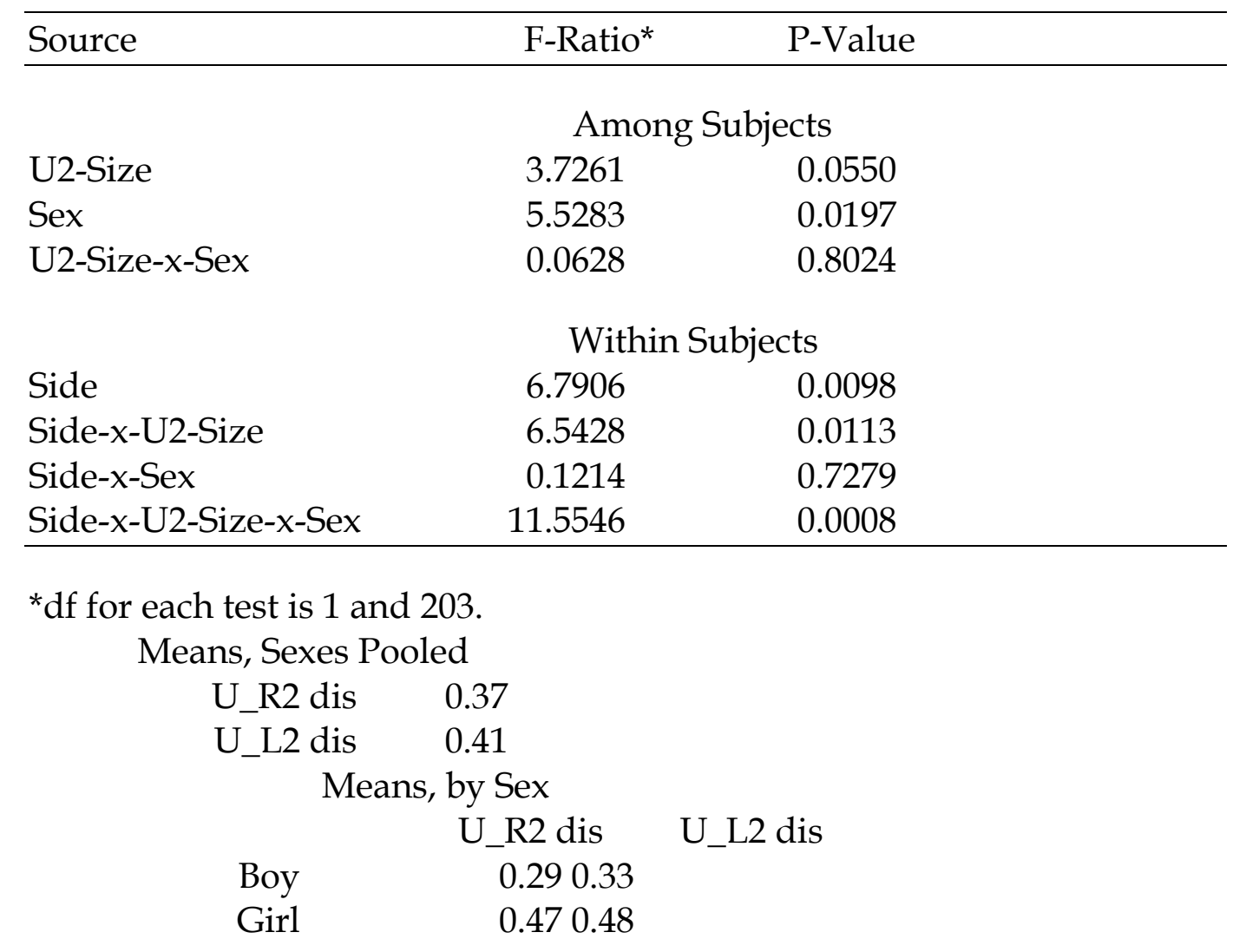


Table A-35. ANCOVA results testing for associations between mesiodistal width of the maxillary lateral incisor and U1 displacements.

\begin{tabular}{|c|c|c|}
\hline Source & F-Ratio* & P-Value \\
\hline & \multicolumn{2}{|c|}{ Among Subjects } \\
\hline U2-Size & 1.6922 & 0.1948 \\
\hline Sex & 0.0003 & 0.9855 \\
\hline \multirow[t]{2}{*}{ U2-Size-x-Sex } & 0.0287 & 0.8657 \\
\hline & \multicolumn{2}{|c|}{ Within Subjects } \\
\hline Side & 0.3784 & 0.5391 \\
\hline Side-x-U2-Size & 0.3498 & 0.5549 \\
\hline Side-x-Sex & 0.5941 & 0.4417 \\
\hline Side-x-U2-Size-x-Sex & 0.8796 & 0.3494 \\
\hline \multicolumn{3}{|c|}{ * df for each test is 1 and 203.} \\
\hline U_R1 dis & \multicolumn{2}{|l|}{0.34} \\
\hline U_L1 dis & \multicolumn{2}{|l|}{0.36} \\
\hline \multicolumn{3}{|c|}{ Means, by Sex } \\
\hline & \multicolumn{2}{|l|}{ U_R1 dis } \\
\hline Boy & 0.360 .34 & \\
\hline Girl & 0.330 .37 & \\
\hline
\end{tabular}


Table A-36. ANCOVA results testing for associations between mesiodistal width of the maxillary lateral incisor and L3 displacements.

\begin{tabular}{|c|c|c|}
\hline Source & F-Ratio* & P-Value \\
\hline & \multicolumn{2}{|c|}{ Among Subjects } \\
\hline U2-Size & 0.2222 & 0.6379 \\
\hline Sex & 2.1213 & 0.1468 \\
\hline \multirow[t]{2}{*}{ U2-Size-x-Sex } & 3.1223 & 0.0787 \\
\hline & \multicolumn{2}{|c|}{ Within Subjects } \\
\hline Side & 0.1621 & 0.6877 \\
\hline Side-x-U2-Size & 0.1767 & 0.6746 \\
\hline Side-x-Sex & 2.4492 & 0.1191 \\
\hline Side-x-U2-Size-x-Sex & 1.7712 & 0.1847 \\
\hline \multicolumn{3}{|c|}{ *df for each test is 1 and 203} \\
\hline L_R3 dis & \multicolumn{2}{|l|}{0.23} \\
\hline L_L3 dis & \multicolumn{2}{|l|}{0.23} \\
\hline \multicolumn{3}{|c|}{ Means, by Sex } \\
\hline & \multicolumn{2}{|l|}{ L_R3 dis } \\
\hline Boy & 0.150 .21 & \\
\hline Girl & 0.300 .23 & \\
\hline
\end{tabular}


Table A-37. ANCOVA results testing for associations between mesiodistal width of the maxillary lateral incisor and L2 displacements.

\begin{tabular}{llc}
\hline Source & F-Ratio* $^{*}$ & P-Value \\
\hline & \multicolumn{2}{c}{ Among Subjects } \\
U2-Size & 5.1285 & 0.0246 \\
Sex & 1.1895 & 0.2767 \\
U2-Size-x-Sex & 0.0006 & 0.9809 \\
& \multicolumn{2}{c}{} \\
Side & Within Subjects \\
Side-x-U2-Size & 0.1934 & 0.6606 \\
Side-x-Sex & 0.2710 & 0.6032 \\
Side-x-U2-Size-x-Sex & 0.3097 & 0.5785 \\
\hline
\end{tabular}

*df for each test is 1 and 203.

Means, Sexes Pooled

\begin{tabular}{lrr} 
L_R2 dis & 0.51 & \\
L_L2 dis & 0.47 & \\
& Means, by Sex \\
\multicolumn{4}{c}{ L_R2 dis } & L_L2 dis \\
Boy & 0.460 .44 & \\
Girl & 0.570 .51 &
\end{tabular}


Table A-38. ANCOVA results testing for associations between mesiodistal width of the maxillary lateral incisor and L1 displacements.

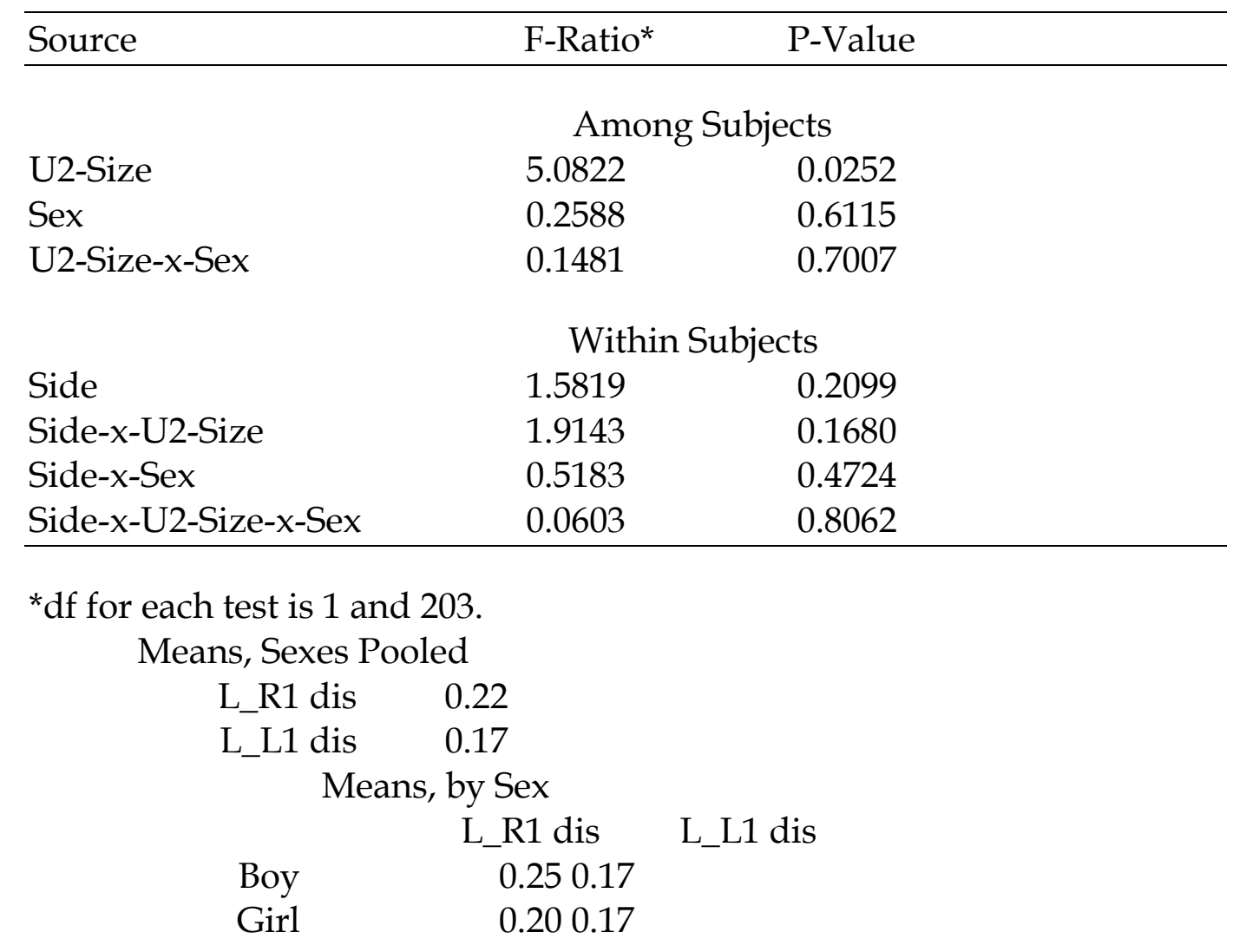


Table A-39. ANCOVA results testing for associations between mesiodistal width of the maxillary lateral incisor and U3 rotations.

\begin{tabular}{|c|c|c|}
\hline Source & F-Ratio* & P-Value \\
\hline & \multicolumn{2}{|c|}{ Among Subjects } \\
\hline U2-Size & 0.2508 & 0.6171 \\
\hline Sex & 4.0719 & 0.0449 \\
\hline \multirow[t]{2}{*}{ U2-Size-x-Sex } & 0.1278 & 0.7211 \\
\hline & \multicolumn{2}{|c|}{ Within Subjects } \\
\hline Side & 0.0001 & 0.9915 \\
\hline Side-x-U2-Size & 0.0000 & 0.9979 \\
\hline Side-x-Sex & 0.3582 & 0.5502 \\
\hline Side-x-U2-Size-x-Sex & 0.0104 & 0.9189 \\
\hline \multicolumn{3}{|c|}{ * $\mathrm{df}$ for each test is 1 and 203} \\
\hline \multicolumn{3}{|c|}{ Means, Sexes Pooled } \\
\hline U_R3 rot & \multicolumn{2}{|l|}{0.22} \\
\hline U_L3 rot & \multicolumn{2}{|l|}{0.22} \\
\hline \multicolumn{3}{|c|}{ Means, by Sex } \\
\hline \multicolumn{3}{|c|}{ U_R3 rot } \\
\hline Boy & \\
\hline Girl & \multicolumn{2}{|l|}{0.260 .29} \\
\hline
\end{tabular}


Table A-40. ANCOVA results testing for associations between mesiodistal width of the maxillary lateral incisor and $\mathrm{U} 2$ rotations.

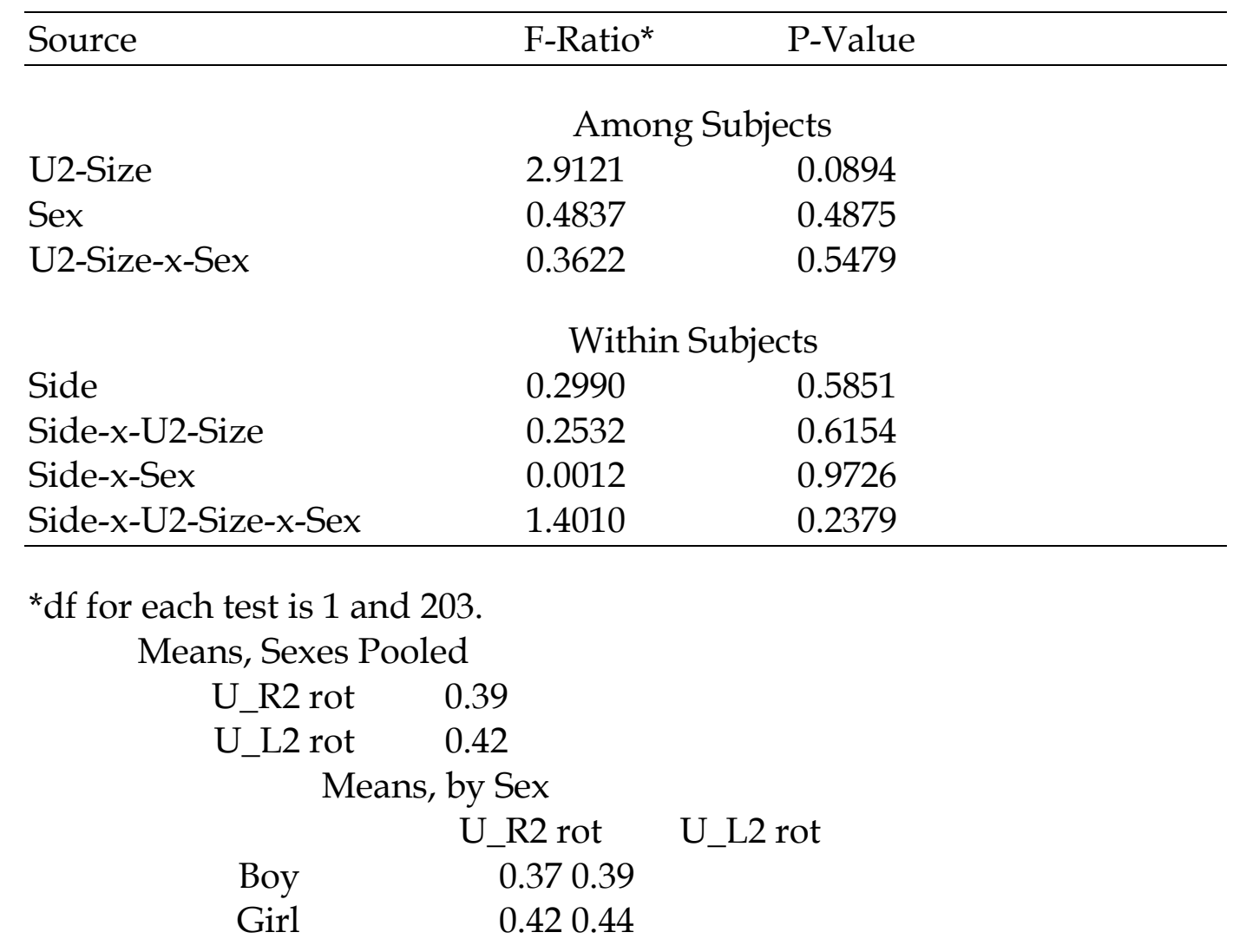


Table A-41. ANCOVA results testing for associations between mesiodistal width of the maxillary lateral incisor and U1 rotations.

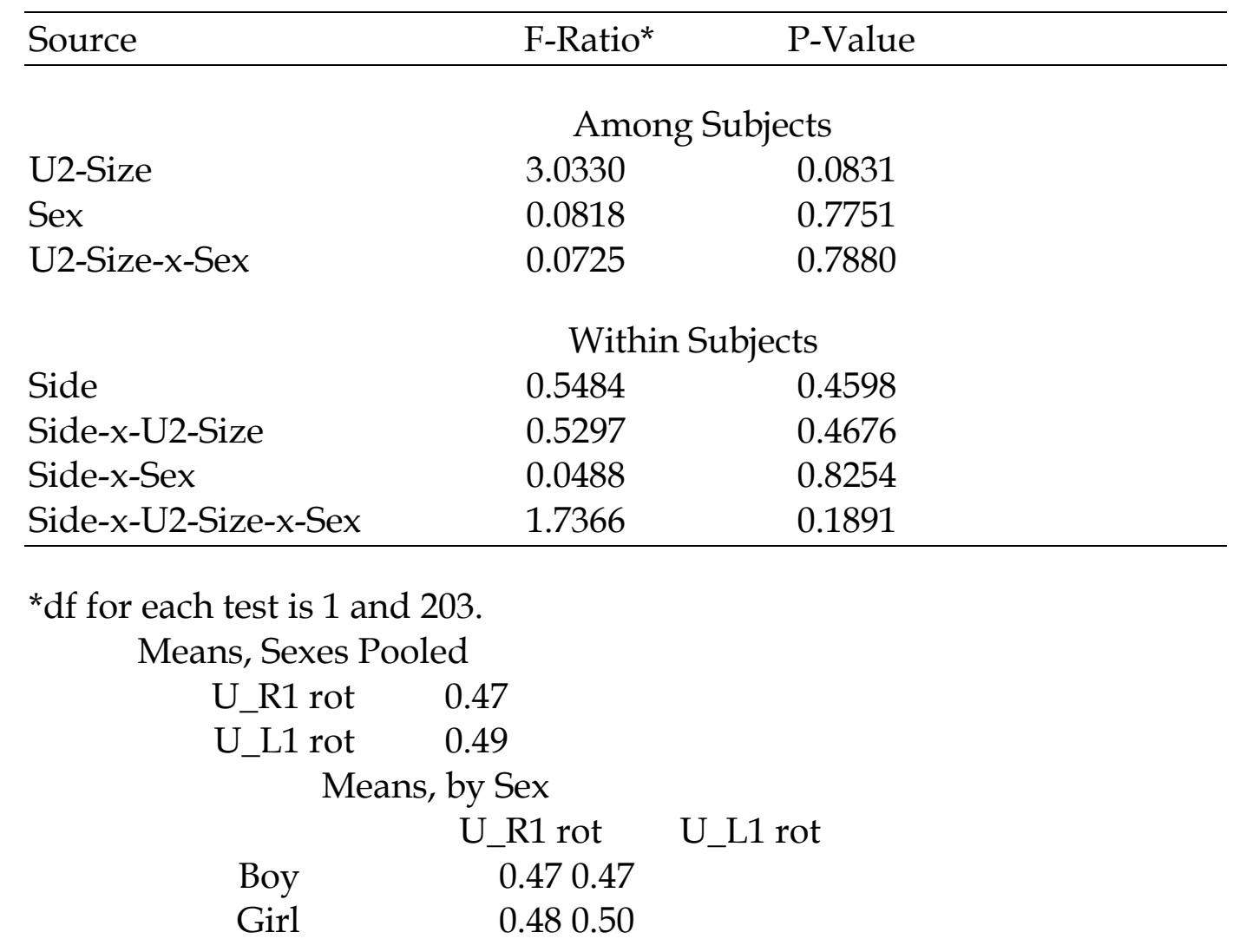


Table A-42. ANCOVA results testing for associations between mesiodistal width of the maxillary lateral incisor and L3 rotations.

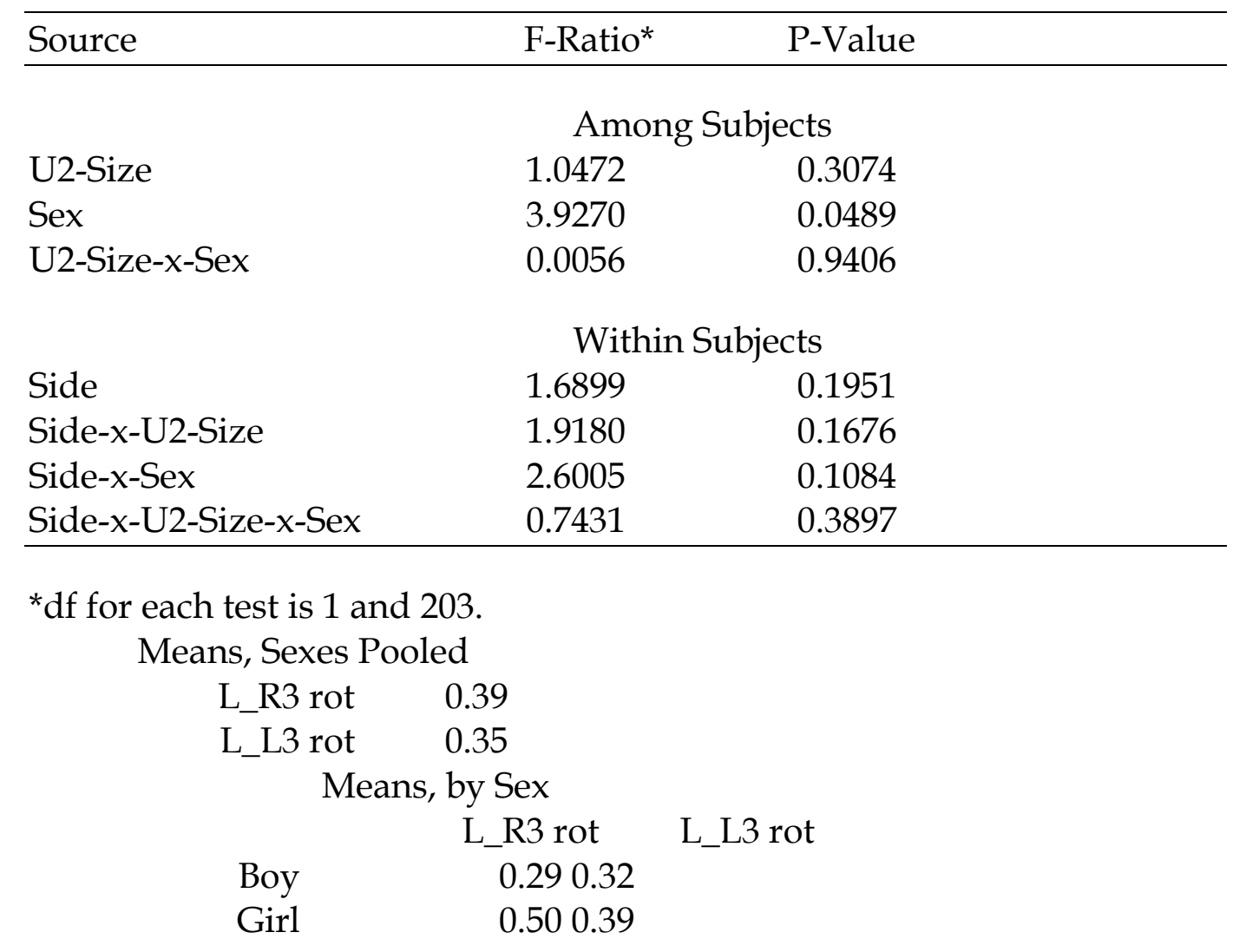


Table A-43. ANCOVA results testing for associations between mesiodistal width of the maxillary lateral incisor and $\mathrm{L} 2$ rotations.

\begin{tabular}{|c|c|c|}
\hline Source & F-Ratio* & P-Value \\
\hline & \multicolumn{2}{|c|}{ Among Subjects } \\
\hline U2-Size & 0.7958 & 0.3734 \\
\hline Sex & 0.4635 & 0.4968 \\
\hline \multirow[t]{2}{*}{ U2-Size-x-Sex } & 1.4732 & 0.2263 \\
\hline & \multicolumn{2}{|c|}{ Within Subjects } \\
\hline Side & 0.0209 & 0.8852 \\
\hline Side-x-U2-Size & 0.1397 & 0.7090 \\
\hline Side-x-Sex & 0.6885 & 0.4076 \\
\hline Side-x-U2-Size-x-Sex & 0.0413 & 0.8393 \\
\hline \multicolumn{3}{|c|}{ * $\mathrm{df}$ for each test is 1 and 203} \\
\hline L_R2 rot & \multicolumn{2}{|l|}{0.35} \\
\hline L_L2 rot & \multicolumn{2}{|l|}{0.23} \\
\hline \multicolumn{3}{|c|}{ Means, by Sex } \\
\hline & \multicolumn{2}{|l|}{ L_R2 rot } \\
\hline Boy & 0.320 .23 & \\
\hline Girl & 0.400 .24 & \\
\hline
\end{tabular}


Table A-44. ANCOVA results testing for associations between mesiodistal width of the maxillary lateral incisor and L1 rotations.

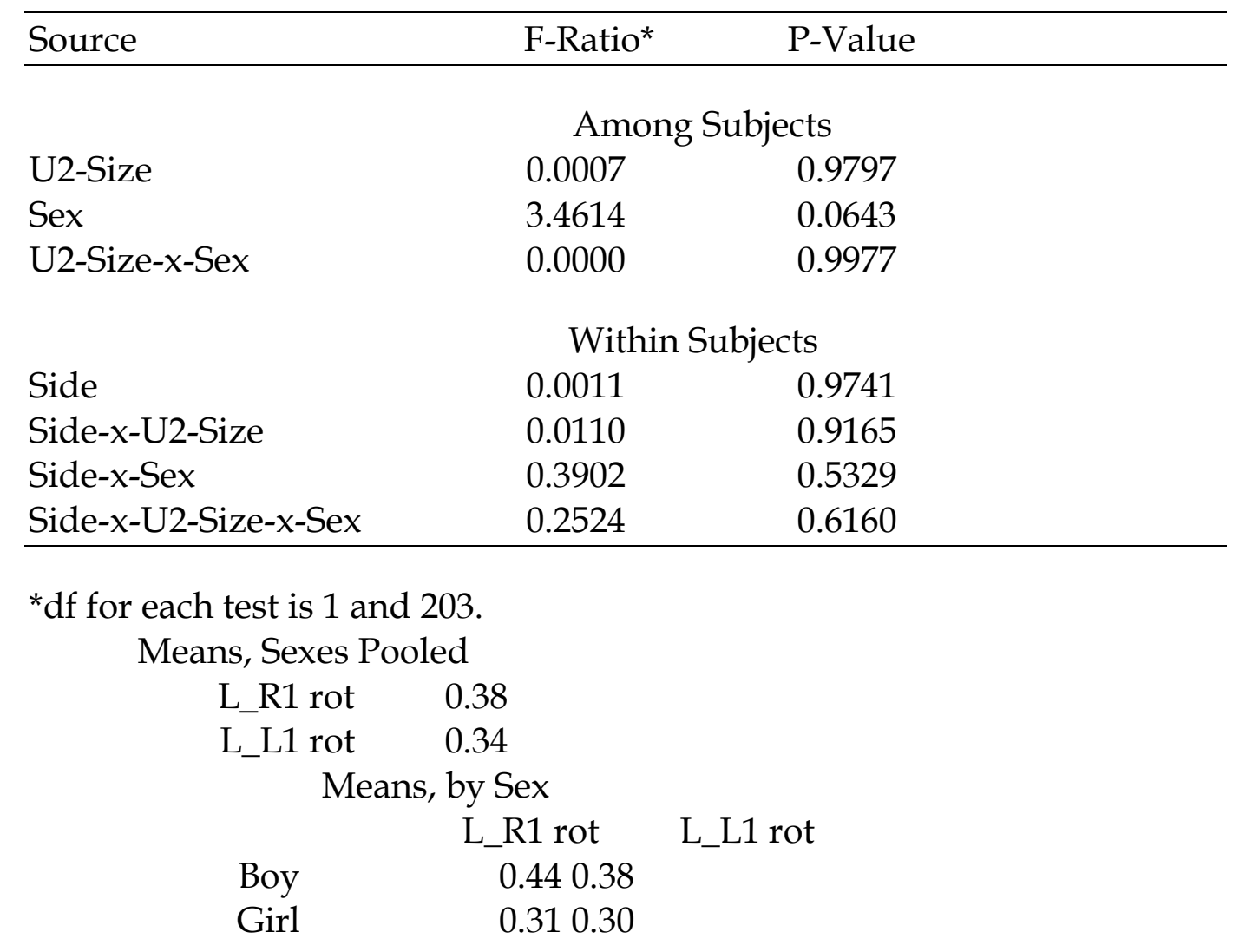


Table A-45. ANCOVA results testing for associations between mesiodistal width of the maxillary lateral incisor and U2-U3 overlap.

\begin{tabular}{llc}
\hline Source & F-Ratio* $^{*}$ & P-Value \\
\hline & \multicolumn{2}{c}{ Among Subjects } \\
U2-Size & 6.4025 & 0.0122 \\
Sex & 6.2854 & 0.0130 \\
U2-Size-x-Sex & 0.0088 & 0.9253 \\
& \multicolumn{2}{c}{} \\
Side & Within Subjects \\
Side-x-U2-Size & 0.1837 & 0.6687 \\
Side-x-Sex & 0.2224 & 0.6377 \\
Side-x-U2-Size-x-Sex & 0.5232 & 0.4703 \\
\hline
\end{tabular}

* $\mathrm{df}$ for each test is 1 and 203.

Means, Sexes Pooled

$\begin{array}{ll}\text { U_R3-2 lapping } & 0.38 \\ \text { U_L2-3 lapping } & 0.37 \\ \text { Means, by Sex } & \end{array}$

Boy

U_R3-2 lapping U_L2-3 lapping

Girl

0.270 .29

0.510 .45 
Table A-46. ANCOVA results testing for associations between mesiodistal width of the maxillary lateral incisor and U1-U2 overlap.

\begin{tabular}{|c|c|c|}
\hline Source & F-Ratio* & P-Value \\
\hline \multicolumn{3}{|c|}{ Among Subjects } \\
\hline U2-Size & 4.8170 & 0.0293 \\
\hline Sex & 9.1870 & 0.0028 \\
\hline U2-Size-x-Sex & 0.7472 & 0.3884 \\
\hline \multicolumn{3}{|c|}{ Within Subjects } \\
\hline Side & 0.1775 & 0.6739 \\
\hline Side-x-U2-Size & 0.2555 & 0.6138 \\
\hline Side-x-Sex & 0.1779 & 0.6736 \\
\hline Side-x-U2-Size-x-Sex & 1.5613 & 0.2129 \\
\hline \multicolumn{3}{|l|}{$\begin{array}{c}\text { *df for each test is } 1 \text { and } 203 . \\
\text { Means, Sexes Pooled }\end{array}$} \\
\hline U_R2-1 lapping & 0.35 & \\
\hline U_L1-2 lapping & 0.31 & \\
\hline \multicolumn{3}{|c|}{ Means, by Sex } \\
\hline & U_R2-1 lapping & U_L1-2 lapping \\
\hline Boy & 0.250 .23 & \\
\hline Girl & 0.460 .41 & \\
\hline
\end{tabular}


Table A-47. ANCOVA results testing for associations between mesiodistal width of the maxillary lateral incisor and L2-L3 overlap.

\begin{tabular}{|c|c|c|}
\hline Source & F-Ratio* & P-Value \\
\hline \multicolumn{3}{|c|}{ Among Subjects } \\
\hline U2-Size & 7.6456 & 0.0062 \\
\hline Sex & 1.1035 & 0.2947 \\
\hline U2-Size-x-Sex & 0.3883 & 0.5339 \\
\hline \multicolumn{3}{|c|}{ Within Subjects } \\
\hline Side & 0.0137 & 0.9070 \\
\hline Side-x-U2-Size & 0.0528 & 0.8185 \\
\hline Side-x-Sex & 0.0013 & 0.9718 \\
\hline Side-x-U2-Size-x-Sex & 0.3115 & 0.5774 \\
\hline \multicolumn{3}{|l|}{$\begin{array}{c}\text { *df for each test is } 1 \text { and } 203 . \\
\text { Means, Sexes Pooled }\end{array}$} \\
\hline L_R3-2 lapping & 0.71 & \\
\hline L_L2-3 lapping & 0.65 & \\
\hline \multicolumn{3}{|c|}{ Means, by Sex } \\
\hline & L_R3-2 lapping & L_L2-3 lapping \\
\hline Boy & 0.670 .60 & \\
\hline Girl & 0.760 .70 & \\
\hline
\end{tabular}


Table A-48. ANCOVA results testing for associations between mesiodistal width of the maxillary lateral incisor and L1-L2 overlap.

\begin{tabular}{lcc}
\hline Source & F-Ratio* & P-Value \\
\hline \multicolumn{3}{c}{ Among Subjects } \\
U2-Size & 10.2793 & 0.0016 \\
Sex & 0.7761 & 0.3794 \\
U2-Size-x-Sex & 1.1748 & 0.2797 \\
\multicolumn{3}{c}{ Within Subjects } \\
Side & 1.3443 & 0.2476 \\
Side-x-U2-Size & 1.2992 & 0.2557 \\
Side-x-Sex & 0.5713 & 0.4506 \\
Side-x-U2-Size-x-Sex & 0.0083 & 0.9273 \\
\hline \multicolumn{3}{c}{} \\
*df for each test is 1 and 203. \\
Means, Sexes Pooled \\
L_R2-1 lapping \\
L_L1-2 lapping \\
Means, by Sex \\
\multicolumn{2}{c}{ L_R2-1 lapping } \\
Boy L_L1-2 lapping \\
Girl 0.47 \\
0
\end{tabular}


Table A-49. ANCOVA results testing for associations between mesiodistal width of the maxillary lateral incisor and total displacements.

\begin{tabular}{lcccc}
\hline Term & Estimate & SE & t-Test & P-Value \\
\hline Intercept & -3.8710 & 2.6614 & -1.45 & 0.1474 \\
U2 & 1.2004 & 0.4002 & 3.00 & 0.0030 \\
Sex & -0.4381 & 0.2368 & -1.85 & 0.0658 \\
Interaction & 0.2287 & 0.4002 & 0.57 & 0.5683 \\
\hline
\end{tabular}

Table A-50. ANCOVA results testing for associations between mesiodistal width of the maxillary lateral incisor and total rotations.

\begin{tabular}{lrcrc}
\hline Term & Estimate & SE & t-Test & P-Value \\
\hline Intercept & 0.2842 & 1.9888 & 0.14 & 0.8865 \\
U2 & 0.5992 & 0.2991 & 2.00 & 0.0464 \\
Sex & -0.2576 & 0.1770 & -1.46 & 0.1471 \\
Interaction & 0.0088 & 0.2991 & 0.03 & 0.9766 \\
\hline
\end{tabular}

Table A-51. ANCOVA results testing for associations between mesiodistal width of the maxillary lateral incisor and maxillary R1-L1 overlap.

\begin{tabular}{lcccc}
\hline Term & Estimate & SE & t-Test & P-Value \\
\hline Intercept & -0.7290 & 0.3672 & -1.99 & 0.0485 \\
U2 & 0.1467 & 0.0552 & 2.66 & 0.0085 \\
Sex & -0.0171 & 0.0327 & -0.52 & 0.6003 \\
Interaction & -0.0399 & 0.0552 & -0.72 & 0.4709 \\
\hline
\end{tabular}


Table A-52. ANCOVA results testing for associations between mesiodistal width of the maxillary lateral incisor and mandibular R1-L1 overlap.

\begin{tabular}{lcccc}
\hline Term & Estimate & SE & t-Test & P-Value \\
\hline Intercept & -0.6707 & 0.3784 & -1.77 & 0.0778 \\
U2 & 0.1590 & 0.0569 & 2.79 & 0.0057 \\
Sex & 0.0933 & 0.0337 & 2.77 & 0.0061 \\
Interaction & -0.0125 & 0.0569 & -0.22 & 0.8259 \\
\hline
\end{tabular}

Table A-53. ANCOVA results testing for associations between mesiodistal width of the maxillary lateral incisor and total overlapping.

\begin{tabular}{lccrr}
\hline Term & Estimate & SE & t-Test & P-Value \\
\hline Intercept & -6.3852 & 2.3866 & -2.68 & 0.0081 \\
U2 & 1.6211 & 0.3589 & 4.52 & $<0.0001$ \\
Sex & -0.4632 & 0.2124 & -2.18 & 0.0303 \\
Interaction & -0.1691 & 0.3589 & -0.47 & 0.6381 \\
\hline
\end{tabular}

Table A-54. ANCOVA results testing for associations between mesiodistal width of the maxillary lateral incisor and total displacements, rotations, and overlapping.

\begin{tabular}{lrcrc}
\hline Term & Estimate & $\mathrm{SE}$ & $\mathrm{t}$-Test & P-Value \\
\hline Intercept & -9.9720 & 6.0047 & -1.66 & 0.0983 \\
U2 & 3.4207 & 0.9030 & 3.79 & 0.0002 \\
Sex & -1.1588 & 0.5343 & -2.17 & 0.0312 \\
Interaction & 0.0685 & 0.9030 & 0.08 & 0.9396 \\
\hline
\end{tabular}


Table A-55. ANCOVA results testing for associations between mesiodistal width of the maxillary lateral incisor and crowding.

\begin{tabular}{lcccc}
\hline Term & Estimate & SE & t-Test & P-value \\
\hline Intercept & -1.3905 & 0.5565 & -2.50 & 0.0133 \\
U2 & 0.4188 & 0.0837 & 5.00 & $<0.0001$ \\
Sex & -0.1254 & 0.0495 & -2.53 & 0.0121 \\
Interaction & -0.1070 & 0.0837 & -1.28 & 0.2026 \\
\hline
\end{tabular}

Table A-56. ANCOVA results testing for associations between mesiodistal width of the maxillary lateral incisor and spacing.

\begin{tabular}{lrcrr}
\hline Term & Estimate & SE & t-Test & P-Value \\
\hline Intercept & 2.0586 & 0.4257 & 4.84 & $<0.0001$ \\
U2 & -0.2729 & 0.0640 & -4.26 & $<0.0001$ \\
Sex & 0.0516 & 0.0379 & 1.36 & 0.1746 \\
Interaction & 0.1001 & 0.0640 & 1.56 & 0.1195 \\
\hline
\end{tabular}

Table A-57. ANCOVA results testing for associations between mesiodistal width of the maxillary lateral incisor and diastema.

\begin{tabular}{lrcrc}
\hline Term & Estimate & $\mathrm{SE}$ & $\mathrm{t}$-Test & P-Value \\
\hline Intercept & 1.4089 & 0.4464 & 3.16 & 0.0018 \\
U2 & -0.1782 & 0.0671 & -2.66 & 0.0086 \\
Sex & 0.0346 & 0.0397 & 0.87 & 0.3844 \\
Interaction & 0.0773 & 0.0671 & 1.15 & 0.2508 \\
\hline
\end{tabular}


Table A-58. ANCOVA results testing for associations between mesiodistal width of the maxillary lateral incisor and maxillary incisor irregularity.

\begin{tabular}{lcccc}
\hline Term & Estimate & SE & t-Test & P-Value \\
\hline Intercept & -2.1855 & 1.0397 & -2.10 & 0.0368 \\
U2 & 0.5438 & 0.1564 & 3.48 & 0.0006 \\
Sex & -0.1588 & 0.0925 & -1.72 & 0.0876 \\
Interaction & 0.1794 & 0.1564 & 1.15 & 0.2525 \\
\hline
\end{tabular}

Table A-59. ANCOVA results testing for associations between mesiodistal width of the maxillary lateral incisor and mandibular incisor irregularity.

\begin{tabular}{lrcrc}
\hline Term & Estimate & SE & t-Test & P-Value \\
\hline Intercept & -1.6873 & 0.9774 & -1.73 & 0.0858 \\
U2 & 0.4243 & 0.1470 & 2.89 & 0.0043 \\
Sex & -0.0701 & 0.0870 & -0.81 & 0.4213 \\
Interaction & 0.0197 & 0.1470 & 0.13 & 0.8936 \\
\hline
\end{tabular}

Table A-60. ANCOVA results testing for associations between mesiodistal width of the maxillary lateral incisor and maxillary overjet.

\begin{tabular}{lcccc}
\hline Term & Estimate & $\mathrm{SE}$ & $\mathrm{t}$-Test & P-Value \\
\hline Intercept & 1.8715 & 1.7902 & 1.05 & 0.2971 \\
U2 & 0.2596 & 0.2692 & 0.96 & 0.3360 \\
Sex & -0.0793 & 0.1593 & -0.50 & 0.6193 \\
Interaction & 0.0503 & 0.2692 & 0.19 & 0.8520 \\
\hline
\end{tabular}


Table A-61. ANCOVA results testing for associations between mesiodistal width of the maxillary lateral incisor and mandibular overjet.

\begin{tabular}{lcccc}
\hline Term & Estimate & SE & t-Test & P-Value \\
\hline Intercept & -0.1128 & 0.2157 & -0.52 & 0.6016 \\
U2 & 0.0233 & 0.0324 & 0.72 & 0.4743 \\
Sex & -0.0415 & 0.0192 & -2.16 & 0.0319 \\
Interaction & -0.0233 & 0.0324 & -0.72 & 0.4743 \\
\hline
\end{tabular}

Table A-62. ANCOVA results testing for associations between mesiodistal width of the maxillary lateral incisor and openbite.

\begin{tabular}{lcccc}
\hline Term & Estimate & SE & t-Test & P-Value \\
\hline Intercept & -0.3544 & 0.3625 & -0.98 & 0.3295 \\
U2 & 0.0638 & 0.0545 & 1.17 & 0.2432 \\
Sex & 0.0068 & 0.0322 & 0.21 & 0.8320 \\
Interaction & 0.0309 & 0.0545 & 0.57 & 0.5712 \\
\hline
\end{tabular}

Table A-63. ANCOVA results testing for associations between mesiodistal width of the maxillary lateral incisor and anteroposterior relationship.

\begin{tabular}{lcccc}
\hline Term & Estimate & SE & t-Test & P-Value \\
\hline Intercept & 1.7536 & 0.5473 & 3.20 & 0.0016 \\
U2 & -0.1502 & 0.0823 & -1.82 & 0.0695 \\
Sex & 0.0463 & 0.0487 & 0.95 & 0.3431 \\
Interaction & -0.1649 & 0.0823 & -2.00 & 0.0465 \\
\hline
\end{tabular}


Table A-64. ANCOVA results testing for associations between mesiodistal width of the maxillary lateral incisor and DAI score.

\begin{tabular}{lcccc}
\hline Term & Estimate & $\mathrm{SE}$ & $\mathrm{t}$-Test & P-Value \\
\hline Intercept & 20.7000 & 5.1314 & 4.03 & $<0.0001$ \\
U2 & 0.9506 & 0.7717 & 1.23 & 0.2194 \\
Sex & -0.3648 & 0.4566 & -0.80 & 0.4253 \\
Interaction & 0.1717 & 0.7717 & 0.22 & 0.8241 \\
\hline
\end{tabular}


Table A-65. ANCOVA results testing for associations between mesiodistal width of the maxillary canine and U3 displacements.

\begin{tabular}{llc}
\hline Source & F-Ratio* & P-Value \\
\hline & \multicolumn{2}{c}{ Among Subjects } \\
U3-Size & 7.9819 & 0.0052 \\
Sex & 4.3359 & 0.0386 \\
U3-Size-x-Sex & 0.1031 & 0.7485 \\
& \multicolumn{2}{c}{ Within Subjects } \\
Side & 0.1688 & 0.6816 \\
Side-x-U3-Size & 0.1287 & 0.7201 \\
Side-x-Sex & 0.1895 & 0.6638 \\
Side-x-U3-Size-x-Sex & 0.8033 & 0.3712 \\
\hline
\end{tabular}

* $\mathrm{df}$ for each test is 1 and 203.

Means, Sexes Pooled
U_R3 dis $\quad 0.41$
U_L3 dis 0.38

Means, by Sex

U_R3 dis U_L3 dis

Boy $\quad 0.310 .29$

Girl $\quad 0.520 .45$ 
Table A-66. ANCOVA results testing for associations between mesiodistal width of the maxillary canine and U2 displacements.

\begin{tabular}{llc}
\hline Source & F-Ratio* & P-Value \\
\hline \multicolumn{3}{c}{ Among Subjects } \\
U3-Size & 2.0287 & 0.1559 \\
Sex & 6.3508 & 0.0125 \\
U3-Size-x-Sex & 0.0702 & 0.7914 \\
& \multicolumn{2}{c}{ Within Subjects } \\
Side & 3.2939 & 0.0710 \\
Side-x-U3-Size & 3.2631 & 0.0723 \\
Side-x-Sex & 0.5557 & 0.4569 \\
Side-x-U3-Size-x-Sex & 4.5645 & 0.0338 \\
\hline
\end{tabular}

* $\mathrm{df}$ for each test is 1 and 203.

Means, Sexes Pooled
U_R2 dis $\quad 0.37$
U_L2 dis $\quad 0.41$

Means, by Sex

U_R3 dis U_L3 dis

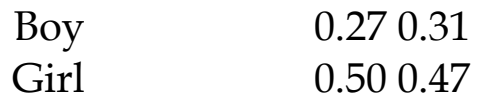


Table A-67. ANCOVA results testing for associations between mesiodistal width of the maxillary canine and U1 displacements.

\begin{tabular}{llc}
\hline Source & F-Ratio* & P-Value \\
\hline \multicolumn{2}{c}{ Among Subjects } \\
U3-Size & 0.6983 & 0.4043 \\
Sex & 0.0268 & 0.8702 \\
U3-Size-x-Sex & 0.0819 & 0.7751 \\
& \multicolumn{2}{c}{ Within Subjects } \\
Side & 0.0219 & 0.8824 \\
Side-x-U3-Size & 0.0279 & 0.8675 \\
Side-x-Sex & 0.7044 & 0.4023 \\
Side-x-U3-Size-x-Sex & 0.0071 & 0.9330 \\
\hline
\end{tabular}

* $\mathrm{df}$ for each test is 1 and 203.

Means, Sexes Pooled

$\begin{array}{ll}\text { U_R1 dis } & 0.34 \\ \text { U_L1 dis } & 0.36\end{array}$

Means, by Sex

U_R3 dis U_L3 dis

Boy $\quad 0.360 .34$

Girl $\quad 0.330 .39$ 
Table A-68. ANCOVA results testing for associations between mesiodistal width of the maxillary canine and L3 displacements.

\begin{tabular}{llc}
\hline Source & F-Ratio* & P-Value \\
\hline \multicolumn{2}{c}{ Among Subjects } \\
U3-Size & 1.7836 & 0.1832 \\
Sex & 3.2118 & 0.0746 \\
U3-Size-x-Sex & 4.9983 & 0.0265 \\
& \multicolumn{2}{c}{ Within Subjects } \\
Side & 0.1706 & 0.6800 \\
Side-x-U3-Size & 0.1604 & 0.6892 \\
Side-x-Sex & 1.6335 & 0.2027 \\
Side-x-U3-Size-x-Sex & 0.1933 & 0.6607 \\
\hline
\end{tabular}

* df for each test is 1 and 203.

Means, Sexes Pooled

$\begin{array}{cc}\text { L_R3 dis } & 0.23 \\ \text { L_L3 dis } & 0.23 \\ \text { ns, by Sex } & \end{array}$

Means, by Sex

$\begin{array}{ll}\text { Boy } & 0.130 .17 \\ \text { Girl } & 0.290 .23\end{array}$


Table A-69. ANCOVA results testing for associations between mesiodistal width of the maxillary canine and L2 displacements.

\begin{tabular}{lcc}
\hline Source & F-Ratio* & P-Value \\
\hline \multicolumn{3}{c}{ Among Subjects } \\
U3-Size & 11.1600 & 0.0010 \\
Sex & 4.0868 & 0.0445 \\
U3-Size-x-Sex & 0.0192 & 0.8899 \\
& \multicolumn{2}{c}{ Within Subjects } \\
Side & 0.0002 & 0.9885 \\
Side-x-U3-Size & 0.0021 & 0.9635 \\
Side-x-Sex & 0.2285 & 0.6331 \\
Side-x-U3-Size-x-Sex & 1.0581 & 0.3049 \\
\hline
\end{tabular}

*df for each test is 1 and 203.

Means, Sexes Pooled

$\begin{array}{cc}\text { L_R2 dis } & 0.51 \\ \text { L_L2 dis } & 0.47 \\ \text { ns, by Sex } & \end{array}$

Means, by Sex

$\begin{array}{ll}\text { Boy } & 0.410 .41 \\ \text { Girl } & 0.610 .57\end{array}$


Table A-70. ANCOVA results testing for associations between mesiodistal width of the maxillary canine and L1 displacements.

\begin{tabular}{llc}
\hline Source & F-Ratio* & P-Value \\
\hline \multicolumn{2}{c}{ Among Subjects } \\
U3-Size & 2.5939 & 0.1088 \\
Sex & 0.0129 & 0.9096 \\
U3-Size-x-Sex & 0.0778 & 0.7806 \\
& \multicolumn{2}{c}{ Within Subjects } \\
Side & 1.0523 & 0.3062 \\
Side-x-U3-Size & 1.2591 & 0.2631 \\
Side-x-Sex & 0.1524 & 0.6966 \\
Side-x-U3-Size-x-Sex & 0.8978 & 0.3445 \\
\hline
\end{tabular}

* $\mathrm{df}$ for each test is 1 and 203.

Means, Sexes Pooled

$\begin{array}{cc}\text { L_R1 dis } & 0.22 \\ \text { L_L1 dis } & 0.17 \\ \text { ns, by Sex } & \end{array}$

Means, by Sex

Boy $\quad 0.240 .16$

Girl $\quad 0.220 .17$ 
Table A-71. ANCOVA results testing for associations between mesiodistal width of the maxillary canine and $\mathrm{U} 3$ rotations.

\begin{tabular}{lcc}
\hline Source & F-Ratio* & P-Value \\
\hline \multicolumn{3}{c}{ Among Subjects } \\
U3-Size & 0.0241 & 0.8768 \\
Sex & 3.5415 & 0.0613 \\
U3-Size-x-Sex & 0.0064 & 0.9361 \\
& \multicolumn{2}{c}{ Within Subjects } \\
Side & 0.0001 & 0.9925 \\
Side-x-U3-Size & 0.0000 & 0.9959 \\
Side-x-Sex & 0.3088 & 0.5790 \\
Side-x-U3-Size-x-Sex & 0.3146 & 0.5755 \\
\hline
\end{tabular}

* $\mathrm{df}$ for each test is 1 and 203.

Means, Sexes Pooled

$\begin{array}{ll}\text { U_R3 rot } & 0.22 \\ \text { U_L3 rot } & 0.22\end{array}$

Means, by Sex

Boy $\quad 0.180 .16$

Girl $\quad 0.260 .28$ 
Table A-72. ANCOVA results testing for associations between mesiodistal width of the maxillary canine and $\mathrm{U} 2$ rotations.

\begin{tabular}{llc}
\hline Source & F-Ratio* & P-Value \\
\hline & \multicolumn{2}{c}{ Among Subjects } \\
U3-Size & 8.3544 & 0.0043 \\
Sex & 2.3672 & 0.1255 \\
U3-Size-x-Sex & 0.0170 & 0.8964 \\
& \multicolumn{2}{c}{ Within Subjects } \\
Side & 0.0639 & 0.8007 \\
Side-x-U3-Size & 0.0489 & 0.8252 \\
Side-x-Sex & 0.0038 & 0.9509 \\
Side-x-U3-Size-x-Sex & 0.0208 & 0.8854 \\
\hline
\end{tabular}

* $\mathrm{df}$ for each test is 1 and 203.

Means, Sexes Pooled
U_R2 rot
0.39
U_L2 rot 0.42

Means, by Sex

$\begin{array}{lrl} & \text { U_R3 dis } & \text { U_L3 dis } \\ \text { Boy } & 0.340 .37 & \\ \text { Girl } & 0.460 .48\end{array}$


Table A-73. ANCOVA results testing for associations between mesiodistal width of the maxillary canine and U1 rotations.

\begin{tabular}{llc}
\hline Source & F-Ratio* & P-Value \\
\hline & \multicolumn{2}{c}{ Among Subjects } \\
U3-Size & 0.2027 & 0.6530 \\
Sex & 0.0692 & 0.7928 \\
U3-Size-x-Sex & 0.0005 & 0.9814 \\
& \multicolumn{2}{c}{ Within Subjects } \\
Side & 0.0442 & 0.8337 \\
Side-x-U3-Size & 0.0490 & 0.8250 \\
Side-x-Sex & 0.1272 & 0.7218 \\
Side-x-U3-Size-x-Sex & 0.0574 & 0.8110 \\
\hline
\end{tabular}

* $\mathrm{df}$ for each test is 1 and 203.

Means, Sexes Pooled
U_R1 rot $\quad 0.47$
U_L1 rot 0.49

Means, by Sex

U_R3 dis U_L3 dis

$\begin{array}{ll}\text { Boy } & 0.470 .47 \\ \text { Girl } & 0.480 .51\end{array}$


Table A-74. ANCOVA results testing for associations between mesiodistal width of the maxillary canine and L3 rotations.

\begin{tabular}{llc}
\hline Source & F-Ratio* & P-Value \\
\hline & \multicolumn{2}{c}{ Among Subjects } \\
U3-Size & 7.3094 & 0.0074 \\
Sex & 7.6915 & 0.0061 \\
U3-Size-x-Sex & 0.9795 & 0.3235 \\
\multicolumn{2}{c}{ Within Subjects } \\
Side & 0.0304 & 0.8617 \\
Side-x-U3-Size & 0.0503 & 0.8227 \\
Side-x-Sex & 2.1070 & 0.1482 \\
Side-x-U3-Size-x-Sex & 0.0523 & 0.8194 \\
\hline
\end{tabular}

*df for each test is 1 and 203.

Means, Sexes Pooled

$\begin{array}{cc}\text { L_R3 rot } & 0.39 \\ \text { L_L3 rot } & 0.35 \\ \text { Le, by Sex } & \end{array}$

Means, by Sex

U_R3 dis U_L3 dis

Boy $\quad 0.270 .29$

Girl $\quad 0.550 .44$ 
Table A-75. ANCOVA results testing for associations between mesiodistal width of the maxillary canine and L2 rotations.

\begin{tabular}{llc}
\hline Source & F-Ratio* & P-Value \\
\hline & \multicolumn{2}{c}{ Among Subjects } \\
U3-Size & 1.9423 & 0.1649 \\
Sex & 1.1354 & 0.2879 \\
U3-Size-x-Sex & 1.2857 & 0.2582 \\
& \multicolumn{2}{c}{ Within Subjects } \\
Side & 0.1218 & 0.7274 \\
Side-x-U3-Size & 0.2416 & 0.6236 \\
Side-x-Sex & 0.8458 & 0.3588 \\
Side-x-U3-Size-x-Sex & 0.0117 & 0.9138 \\
\hline
\end{tabular}

* $\mathrm{df}$ for each test is 1 and 203.

Means, Sexes Pooled

$\begin{array}{cc}\text { L_R2 rot } & 0.35 \\ \text { L_L2 rot } & 0.23 \\ \text { Lans, by Sex } & \end{array}$

Means, by Sex

$\begin{array}{lll}\text { Boy } & 0.31 & 0.23 \\ \text { Girl } & 0.43 & 0.25\end{array}$


Table A-76. ANCOVA results testing for associations between mesiodistal width of the maxillary canine and L1 rotations.

\begin{tabular}{llc}
\hline Source & F-Ratio $^{*}$ & P-Value \\
\hline \multicolumn{2}{c}{ Among Subjects } \\
U3-Size & 1.3966 & 0.2387 \\
Sex & 4.8038 & 0.0295 \\
U3-Size-x-Sex & 1.3850 & 0.2406 \\
& \multicolumn{2}{c}{ Within Subjects } \\
Side & 0.0842 & 0.7719 \\
Side-x-U3-Size & 0.0607 & 0.8056 \\
Side-x-Sex & 0.4687 & 0.4944 \\
Side-x-U3-Size-x-Sex & 0.0356 & 0.8506 \\
\hline
\end{tabular}

*df for each test is 1 and 203.

Means, Sexes Pooled

$\begin{array}{cc}\text { L_R1 rot } & 0.38 \\ \text { L_L1 rot } & 0.34 \\ \text { ns, by Sex } & \end{array}$

Means, by Sex

$\begin{array}{lll}\text { Boy } & 0.44 & 0.37 \\ \text { Girl } & 0.28 & 0.28\end{array}$


Table A-77. ANCOVA results testing for associations between mesiodistal width of the maxillary canine and U2-U3 overlap.

\begin{tabular}{llc}
\hline Source & F-Ratio* & P-Value \\
\hline \multicolumn{3}{c}{ Among Subjects } \\
U3-Size & 6.7177 & 0.0102 \\
Sex & 9.2401 & 0.0027 \\
U3-Size-x-Sex & 0.0981 & 0.7544 \\
& \multicolumn{2}{c}{ Within Subjects } \\
Side & 0.0572 & 0.8112 \\
Side-x-U3-Size & 0.0359 & 0.8500 \\
Side-x-Sex & 0.3339 & 0.5640 \\
Side-x-U3-Size-x-Sex & 2.3874 & 0.1239 \\
\hline
\end{tabular}

* $\mathrm{df}$ for each test is 1 and 203.

Means, Sexes Pooled

U_R3-2 lapping $\quad 0.38$

U_L2-3 lapping $\quad 0.37$

Means, by Sex

U_R3 dis U_L3 dis

Boy $\quad 0.250 .24$

Girl $\quad 0.540 .46$ 
Table A-78. ANCOVA results testing for associations between mesiodistal width of the maxillary canine and U1-U2 overlap.

\begin{tabular}{|c|c|c|}
\hline Source & F-Ratio* & P-Value \\
\hline & \multicolumn{2}{|c|}{ Among Subjects } \\
\hline U3-Size & 6.7976 & 0.0098 \\
\hline Sex & 12.7908 & 0.0004 \\
\hline \multirow[t]{2}{*}{ U3-Size-x-Sex } & 0.0330 & 0.8561 \\
\hline & \multicolumn{2}{|c|}{ Within Subjects } \\
\hline Side & 0.2953 & 0.5874 \\
\hline Side-x-U3-Size & 0.2447 & 0.6214 \\
\hline Side-x-Sex & 0.0275 & 0.8685 \\
\hline Side-x-U3-Size-x-Sex & 0.0375 & 0.8466 \\
\hline
\end{tabular}

* $\mathrm{df}$ for each test is 1 and 203.

Means, Sexes Pooled

U_R2-1 lapping $\quad 0.35$

U_L1-2 lapping $\quad 0.31$

Means, by Sex

U_R3 dis U_L3 dis

Boy $\quad 0.220 .19$

Girl $\quad 0.480 .43$ 
Table A-79. ANCOVA results testing for associations between mesiodistal width of the maxillary canine and L2-L3 overlap.

\begin{tabular}{lcc}
\hline Source & F-Ratio* & P-Value \\
\hline \multicolumn{3}{c}{ Among Subjects } \\
U3-Size & 20.7044 & $<0.0001$ \\
Sex & 5.6409 & 0.0185 \\
U3-Size-x-Sex & 0.4657 & 0.4958 \\
\multicolumn{3}{c}{} \\
Side & \multicolumn{2}{c}{ Within Subjects } \\
Side-x-U3-Size & 0.1816 & 0.6705 \\
Side-x-Sex & 0.2640 & 0.6079 \\
Side-x-U3-Size-x-Sex & 0.0197 & 0.8884 \\
\hline
\end{tabular}

*df for each test is 1 and 203.

Means, Sexes Pooled

$\begin{array}{ll}\text { L_R3-2 lapping } & 0.71 \\ \text { L_L2-3 lapping } & 0.65\end{array}$

Means, by Sex

U_R3 dis U_L3 dis

Boy $\quad 0.600 .53$

Girl $\quad 0.830 .75$ 
Table A-80. ANCOVA results testing for associations between mesiodistal width of the maxillary canine and L1-L2 overlap.

\begin{tabular}{llc}
\hline Source & F-Ratio* & P-Value \\
\hline \multicolumn{2}{c}{ Among Subjects } \\
U3-Size & 4.0556 & 0.0453 \\
Sex & 1.5371 & 0.2165 \\
U3-Size-x-Sex & 0.3862 & 0.5350 \\
\multicolumn{2}{c}{ Within Subjects } \\
Side 1.0086 & 0.3164 & \\
Side-x-U3-Size & 1.0131 & 0.3153 \\
Side-x-Sex & 0.1983 & 0.6565 \\
Side-x-U3-Size-x-Sex & 0.6239 & 0.4305 \\
\hline
\end{tabular}

*df for each test is 1 and 203.

Means, Sexes Pooled

L_R2-1 lapping $\quad 0.47$

L_L1-2 lapping $\quad 0.46$

Means, by Sex

U_R3 dis U_L3 dis

Boy $\quad 0.430 .45$

Girl $\quad 0.520 .51$ 
Table A-81. ANCOVA results testing for associations between mesiodistal width of the maxillary canine and total displacements.

\begin{tabular}{lcccc}
\hline Term & Estimate & SE & t-Test & P-Value \\
\hline Intercept & -10.7012 & 4.2522 & -2.52 & 0.0126 \\
U3-Size & 1.8877 & 0.5449 & 3.46 & 0.0006 \\
Sex & -0.6768 & 0.2508 & -2.70 & 0.0075 \\
Interaction & 0.3633 & 0.5449 & 0.67 & 0.5057 \\
\hline
\end{tabular}

Table A-82. ANCOVA results testing for associations between mesiodistal width of the maxillary canine and total rotations.

\begin{tabular}{lcccc}
\hline Term & Estimate & SE & t-Test & P-Value \\
\hline Intercept & -3.6868 & 3.1851 & -1.16 & 0.2484 \\
U3-Size & 1.0210 & 0.4082 & 2.50 & 0.0132 \\
Sex & -0.3916 & 0.1879 & -2.08 & 0.0384 \\
Interaction & -0.1769 & 0.4082 & -0.43 & 0.6652 \\
\hline
\end{tabular}

Table A-83. ANCOVA results testing for associations between mesiodistal width of the maxillary canine and maxillary R1-L1 overlap.

\begin{tabular}{lcccc}
\hline Term & Estimate & SE & t-Test & P-Value \\
\hline Intercept & -0.5514 & 0.5988 & -0.92 & 0.3582 \\
U3-Size & 0.1013 & 0.0767 & 1.32 & 0.1881 \\
Sex & -0.0249 & 0.0353 & -0.71 & 0.4812 \\
Interaction & 0.0102 & 0.0767 & 0.13 & 0.8949 \\
\hline
\end{tabular}


Table A-84. ANCOVA results testing for associations between mesiodistal width of the maxillary canine and mandibular R1-L1 overlap.

\begin{tabular}{lcccc}
\hline Term & Estimate & SE & t-Test & P-Value \\
\hline Intercept & -0.4704 & 0.6166 & -0.76 & 0.4464 \\
U3-Size & 0.1080 & 0.0790 & 1.37 & 0.1733 \\
Sex & 0.0854 & 0.0364 & 2.35 & 0.0198 \\
Interaction & 0.0598 & 0.0790 & 0.76 & 0.4503 \\
\hline
\end{tabular}

Table A-85. ANCOVA results testing for associations between mesiodistal width of the maxillary canine and total overlapping.

\begin{tabular}{lrcrr}
\hline Term & Estimate & SE & t-Test & P-Value \\
\hline Intercept & -13.4738 & 3.8228 & -3.52 & 0.0005 \\
U3-Size & 2.2781 & 0.4899 & 4.65 & $<0.0001$ \\
Sex & -0.7412 & 0.2255 & -3.29 & 0.0012 \\
Interaction & 0.2095 & 0.4899 & 0.43 & 0.6693 \\
\hline
\end{tabular}

Table A-86. ANCOVA results testing for associations between mesiodistal width of the maxillary canine and total displacements, rotations, and overlapping.

\begin{tabular}{lcccc}
\hline Term & Estimate & $\mathrm{SE}$ & $\mathrm{t}$-Test & P-Value \\
\hline Intercept & -27.8618 & 9.5820 & -2.91 & 0.0040 \\
U3-Size & 5.1868 & 1.2279 & 4.22 & $<0.0001$ \\
Sex & -1.8096 & 0.5651 & -3.20 & 0.0016 \\
Interaction & 0.3960 & 1.2279 & 0.32 & 0.7474 \\
\hline
\end{tabular}


Table A-87. ANCOVA results testing for associations between mesiodistal width of the maxillary canine and crowding.

\begin{tabular}{lcccc}
\hline Term & Estimate & SE & t-Test & P-Value \\
\hline Intercept & -3.1551 & 0.8924 & -3.54 & 0.0005 \\
U3-Size & 0.5846 & 0.1144 & 5.11 & $<0.0001$ \\
Sex & -0.1978 & 0.0526 & -3.76 & 0.0002 \\
Interaction & -0.1893 & 0.1144 & -1.66 & 0.0994 \\
\hline
\end{tabular}

Table A-88. ANCOVA results testing for associations between mesiodistal width of the maxillary canine and spacing.

\begin{tabular}{lrcrc}
\hline Term & Estimate & SE & t-Test & P-Value \\
\hline Intercept & 2.9298 & 0.6897 & 4.25 & $<0.0001$ \\
U3-Size & -0.3452 & 0.0884 & -3.91 & 0.0001 \\
Sex & 0.0928 & 0.0407 & 2.28 & 0.0235 \\
Interaction & 0.1299 & 0.0884 & 1.47 & 0.1431 \\
\hline
\end{tabular}

Table A-89. ANCOVA results testing for associations between mesiodistal width of the maxillary canine and diastema.

\begin{tabular}{lrcrc}
\hline Term & Estimate & $\mathrm{SE}$ & $\mathrm{t}$-Test & P-Value \\
\hline Intercept & 2.1951 & 0.7183 & 3.06 & 0.0025 \\
U3-Size & -0.2532 & 0.0921 & -2.75 & 0.0065 \\
Sex & 0.0661 & 0.0424 & 1.56 & 0.1202 \\
Interaction & 0.0832 & 0.0921 & 0.90 & 0.3669 \\
\hline
\end{tabular}


Table A-90. ANCOVA results testing for associations between mesiodistal width of the maxillary canine and maxillary incisor irregularity.

\begin{tabular}{lcccc}
\hline Term & Estimate & SE & t-Test & P-Value \\
\hline Intercept & -3.7915 & 1.6945 & -2.24 & 0.0263 \\
U3-Size & 0.6697 & 0.2171 & 3.08 & 0.0023 \\
Sex & -0.2375 & 0.0999 & -2.38 & 0.0184 \\
Interaction & -0.0463 & 0.2171 & -0.21 & 0.8313 \\
\hline
\end{tabular}

Table A-91. ANCOVA results testing for associations between mesiodistal width of the maxillary canine and mandibular incisor irregularity.

\begin{tabular}{lcccc}
\hline Term & Estimate & SE & t-Test & P-Value \\
\hline Intercept & -3.1678 & 1.5759 & -2.01 & 0.0457 \\
U3-Size & 0.5480 & 0.2019 & 2.71 & 0.0072 \\
Sex & -0.1348 & 0.0929 & -1.45 & 0.1485 \\
Interaction & 0.0898 & 0.2019 & 0.44 & 0.6569 \\
\hline
\end{tabular}

Table A-92. ANCOVA results testing for associations between mesiodistal width of the maxillary canine and maxillary overjet.

\begin{tabular}{lcccc}
\hline Term & Estimate & $\mathrm{SE}$ & $\mathrm{t}$-Test & P-Value \\
\hline Intercept & -2.9772 & 2.8462 & -1.05 & 0.2968 \\
U3-Size & 0.8505 & 0.3647 & 2.33 & 0.0207 \\
Sex & -0.2070 & 0.1679 & -1.23 & 0.2189 \\
Interaction & -0.4223 & 0.3647 & -1.16 & 0.2483 \\
\hline
\end{tabular}


Table A-93. ANCOVA results testing for associations between mesiodistal width of the maxillary canine and mandibular overjet.

\begin{tabular}{lcccc}
\hline Term & Estimate & SE & t-Test & P-Value \\
\hline Intercept & -0.4757 & 0.3445 & -1.38 & 0.1688 \\
U3-Size & 0.0675 & 0.0441 & 1.53 & 0.1280 \\
Sex & -0.0516 & 0.0203 & -2.54 & 0.0119 \\
Interaction & -0.0675 & 0.0441 & -1.53 & 0.1280 \\
\hline
\end{tabular}

Table A-94. ANCOVA results testing for associations between mesiodistal width of the maxillary canine and openbite.

\begin{tabular}{lcccc}
\hline Term & Estimate & SE & t-Test & P-Value \\
\hline Intercept & 0.7424 & 0.5841 & 1.27 & 0.2052 \\
U3-Size & -0.0854 & 0.0748 & -1.14 & 0.2555 \\
Sex & 0.0246 & 0.0344 & 0.72 & 0.4753 \\
Interaction & -0.0302 & 0.0748 & -0.40 & 0.6868 \\
\hline
\end{tabular}

Table A-95. ANCOVA results testing for associations between mesiodistal width of the maxillary canine and anteroposterior relationship.

\begin{tabular}{lcccc}
\hline Term & Estimate & SE & t-Test & P-Value \\
\hline Intercept & -0.1402 & 0.8869 & -0.16 & 0.8746 \\
U3-Size & 0.1191 & 0.1137 & 1.05 & 0.2957 \\
Sex & 0.0163 & 0.0523 & 0.31 & 0.7555 \\
Interaction & -0.2593 & 0.1137 & -2.28 & 0.0236 \\
\hline
\end{tabular}


Table A-96. ANCOVA results testing for associations between mesiodistal width of the maxillary canine and DAI score.

\begin{tabular}{lcccc}
\hline Term & Estimate & SE & t-Test & P-Value \\
\hline Intercept & 8.7728 & 8.1649 & 1.07 & 0.2839 \\
U3-Size & 2.3678 & 1.0463 & 2.26 & 0.0247 \\
Sex & -0.7087 & 0.4816 & -1.47 & 0.1427 \\
Interaction & -1.5229 & 1.0463 & -1.46 & 0.1471 \\
\hline
\end{tabular}


Table A-97. ANCOVA results testing for associations between mesiodistal width of the maxillary first premolar and U3 displacements.

\begin{tabular}{lll}
\hline Source & F-Ratio* & P-Value \\
\hline \multicolumn{2}{c}{ Among Subjects } \\
U4-Size & 0.0323 & 0.8576 \\
Sex & 1.2886 & 0.2576 \\
U4-Size-x-Sex & 0.0015 & 0.969 \\
& \multicolumn{2}{c}{ Within Subjects } \\
Side 0.0288 & 0.8654 & 0.8355 \\
Side-x-U4-Size & 0.0433 & 0.5415 \\
Side-x-Sex & 0.374 & 0.6138 \\
Side-x-U4-Size-x-Sex & 0.2555 &
\end{tabular}

* $\mathrm{df}$ for each test is 1 and 203.

Means, Sexes Pooled

\begin{tabular}{lrr} 
U_R2 dis & 0.37 \\
U_L2 dis & 0.41 & \\
& Means, by Sex & \\
\multicolumn{4}{r}{ U_R2 dis } & U_L2 dis \\
Boy & 0.290 .34 & \\
Girl & 0.460 .49 &
\end{tabular}


Table A-98. ANCOVA results testing for associations between mesiodistal width of the maxillary first premolar and U2 displacements.

\begin{tabular}{llc}
\hline Source & F-Ratio* & P-Value \\
\hline & \multicolumn{2}{c}{ Among Subjects } \\
U4-Size & 0.0323 & 0.8576 \\
Sex & 4.6426 & 0.0324 \\
U4-Size-x-Sex & 0.2225 & 0.6376 \\
& \multicolumn{2}{c}{ Within Subjects } \\
Side & 1.6437 & 0.2013 \\
Side-x-U4-Size & 1.5222 & 0.2187 \\
Side-x-Sex & 0.0376 & 0.8464 \\
Side-x-U4-Size-x-Sex & 1.2473 & 0.2654 \\
\hline
\end{tabular}

*df for each test is 1 and 203.

Means, Sexes Pooled

$\begin{array}{ll}\text { U_R3 dis } & 0.41 \\ \text { U_L3 dis } & 0.38\end{array}$

Means, by Sex

U_R3 dis U_L3 dis

Boy $\quad 0.35 \quad 0.34$

Girl $\quad 0.47 \quad 0.41$ 
Table A-99. ANCOVA results testing for associations between mesiodistal width of the maxillary first premolar and U1 displacements.

\begin{tabular}{llc}
\hline Source & F-Ratio* & P-Value \\
\hline & \multicolumn{2}{c}{ Among Subjects } \\
U4-Size & 0.8903 & 0.3465 \\
Sex & 0.0036 & 0.9525 \\
U4-Size-x-Sex & 0.0616 & 0.8042 \\
& \multicolumn{2}{c}{ Within Subjects } \\
Side & 0.0001 & 0.9933 \\
Side-x-U4-Size & 0.0003 & 0.9873 \\
Side-x-Sex & 0.6982 & 0.4044 \\
Side-x-U4-Size-x-Sex & 0.0925 & 0.7614 \\
\hline
\end{tabular}

* $\mathrm{df}$ for each test is 1 and 203.

Means, Sexes Pooled

U_R1 dis $\quad 0.34$

U_L1 dis $\quad 0.36$

Means, by Sex

U_R1 dis U_L1 dis

Boy $\quad 0.360 .34$

Girl $\quad 0.320 .37$ 
Table A-100. ANCOVA results testing for associations between mesiodistal width of the maxillary first premolar and L3 displacements.

\begin{tabular}{llc}
\hline Source & F-Ratio* & P-Value \\
\hline \multicolumn{2}{c}{ Among Subjects } \\
U4-Size & 0.7676 & 0.3820 \\
Sex & 2.2233 & 0.1375 \\
U4-Size-x-Sex & 1.2563 & 0.2637 \\
& \multicolumn{2}{c}{ Within Subjects } \\
Side & 0.3693 & 0.5441 \\
Side-x-U4-Size & 0.3668 & 0.5454 \\
Side-x-Sex & 2.1165 & 0.1473 \\
Side-x-U4-Size-x-Sex & 0.0084 & 0.9271 \\
\hline
\end{tabular}

* $\mathrm{df}$ for each test is 1 and 203.

Means, Sexes Pooled

\begin{tabular}{lrr} 
L_R3 dis & 0.23 \\
L_L3 dis & 0.23 & \\
\multicolumn{4}{c}{ Means, by Sex } \\
\multicolumn{4}{c}{ L_R3 dis } & L_L3 dis \\
Boy & 0.150 .21 & \\
Girl & 0.300 .24 &
\end{tabular}


Table A-101. ANCOVA results testing for associations between mesiodistal width of the maxillary first premolar and L2 displacements.

\begin{tabular}{lcc}
\hline Source & F-Ratio* & P-Value \\
\hline \multicolumn{2}{c}{ Among Subjects } \\
U4-Size & 5.0154 & 0.0262 \\
Sex & 1.1508 & 0.2847 \\
U4-Size-x-Sex & 0.2410 & 0.6240 \\
& \multicolumn{2}{c}{ Within Subjects } \\
Side & 0.0384 & 0.8448 \\
Side-x-U4-Size & 0.0209 & 0.8853 \\
Side-x-Sex & 0.2392 & 0.6253 \\
Side-x-U4-Size-x-Sex & 1.0024 & 0.3179 \\
\hline
\end{tabular}

* $\mathrm{df}$ for each test is 1 and 203.

Means, Sexes Pooled

\begin{tabular}{lrr} 
L_R2 dis & 0.51 \\
L_L2 dis & 0.47 \\
& & \\
\multicolumn{4}{c}{ Means, by Sex } \\
Boy & L_R2 dis & L_L2 dis \\
Girl & 0.460 .44 & \\
& 0.560 .51 &
\end{tabular}


Table A-102. ANCOVA results testing for associations between mesiodistal width of the maxillary first premolar and L1 displacements.

\begin{tabular}{llc}
\hline Source & F-Ratio* & P-Ralue \\
\hline \multicolumn{3}{c}{ Among Subjects } \\
U4-Size & 0.1468 & 0.7020 \\
Sex & 0.4854 & 0.4868 \\
U4-Size-x-Sex & 0.0409 & 0.8399 \\
& \multicolumn{2}{c}{ Within Subjects } \\
Side & 0.8265 & 0.3644 \\
Side-x-U4-Size & 0.9926 & 0.3203 \\
Side-x-Sex & 0.5812 & 0.4467 \\
Side-x-U4-Size-x-Sex & 0.2940 & 0.5883 \\
\hline
\end{tabular}

* $\mathrm{df}$ for each test is 1 and 203.

Means, Sexes Pooled

\begin{tabular}{lrr} 
L_R1 dis & 0.22 & \\
L_L1 dis & 0.17 & \\
& Means, by Sex \\
\multicolumn{4}{c}{} & L_R1 dis & L_L1 dis \\
Boy & 0.250 .17 & \\
Girl & 0.190 .17 &
\end{tabular}


Table A-103. ANCOVA results testing for associations between mesiodistal width of the maxillary first premolar and U3 rotations.

\begin{tabular}{llc}
\hline Source & F-Ratio* & P-Value \\
\hline \multicolumn{2}{c}{ Among Subjects } \\
U4-Size & 0.3104 & 0.5781 \\
Sex & 4.5206 & 0.0347 \\
U4-Size-x-Sex & 0.5716 & 0.4505 \\
& \multicolumn{2}{c}{ Within Subjects } \\
Side & 0.2872 & 0.5926 \\
Side-x-U4-Size & 0.2984 & 0.5855 \\
Side-x-Sex & 0.4249 & 0.5153 \\
Side-x-U4-Size-x-Sex & 0.0787 & 0.7794 \\
\hline
\end{tabular}

* $\mathrm{df}$ for each test is 1 and 203.

Means, Sexes Pooled

$\begin{array}{ll}\text { U_R3 rot } & 0.22 \\ \text { U_L3 rot } & 0.22\end{array}$

Means, by Sex

U_R3 rot U_L3 rot

Boy $\quad 0.170 .16$

Girl $\quad 0.260 .29$ 
Table A-104. ANCOVA results testing for associations between mesiodistal width of the maxillary first premolar and $\mathrm{U} 2$ rotations.

\begin{tabular}{lcc}
\hline Source & F-Ratio* & P-Value \\
\hline \multicolumn{3}{c}{ Among Subjects } \\
U4-Size & 0.2566 & 0.6130 \\
Sex & 0.2205 & 0.6392 \\
U4-Size-x-Sex & 0.1245 & 0.7246 \\
& \multicolumn{2}{c}{ Within Subjects } \\
Side & 0.0850 & 0.7709 \\
Side-x-U4-Size & 0.1138 & 0.7362 \\
Side-x-Sex & 0.0034 & 0.9538 \\
Side-x-U4-Size-x-Sex & 1.9843 & 0.1605 \\
\hline
\end{tabular}

* $\mathrm{df}$ for each test is 1 and 203.

Means, Sexes Pooled

$\begin{array}{ll}\text { U_R2 rot } & 0.39 \\ \text { U_L2 rot } & 0.42\end{array}$

Means, by Sex

U_R2 rot U_L2 rot

Boy $\quad 0.370 .40$

Girl $\quad 0.400 .44$ 
Table A-105. ANCOVA results testing for associations between mesiodistal width of the maxillary first premolar and U1 rotations.

\begin{tabular}{llc}
\hline Source & F-Ratio* & P-Value \\
\hline \multicolumn{2}{c}{ Among Subjects } \\
U4-Size & 1.7269 & 0.1903 \\
Sex & 0.0545 & 0.8157 \\
U4-Size-x-Sex & 0.0336 & 0.8548 \\
\multicolumn{2}{c}{ Within Subjects } \\
Side & \multicolumn{2}{c}{0.5177} \\
Side-x-U4-Size & 0.4199 & 0.5274 \\
Side-x-Sex & 0.4008 & 0.8127 \\
Side-x-U4-Size-x-Sex & 0.0563 & 0.6729 \\
\hline
\end{tabular}

* $\mathrm{df}$ for each test is 1 and 203.

Means, Sexes Pooled

$\begin{array}{lll}\text { U_R1 rot } & 0.47 & \\ \text { U_L1 rot } & 0.49 & \\ & \text { Means, by Sex } & \\ & \text { U_R1 rot } & \text { U_L1 rot } \\ \text { Boy } & 0.470 .47 & \\ \text { Girl } & 0.480 .50 & \end{array}$


Table A-107. ANCOVA results testing for associations between mesiodistal width of the maxillary first premolar and L2 rotations.

\begin{tabular}{llc}
\hline Source & F-Ratio* & P-Value \\
\hline \multicolumn{2}{c}{ Among Subjects } \\
U4-Size & 2.7094 & 0.1013 \\
Sex 0.5625 & 0.4541 & \\
U4-Size-x-Sex & 1.8345 & 0.1771 \\
\multicolumn{3}{c}{ Within Subjects } \\
Side 0.7860 & \multicolumn{2}{c}{} \\
Side-x-U4-Size & 1.3764 & 0.2956 \\
Side-x-Sex & 0.8124 & 0.3685 \\
Side-x-U4-Size-x-Sex & 2.1242 & 0.1465 \\
\hline
\end{tabular}

*df for each test is 1 and 203.

Means, Sexes Pooled

\begin{tabular}{lll} 
L_R2 rot & 0.35 & \\
L_L2 rot & 0.23 & \\
& Means, by Sex & \\
\multicolumn{4}{c}{ L_R2 rot } & L_L2 rot \\
Boy & 0.320 .23 & \\
Girl & 0.400 .23 &
\end{tabular}


Table A-108. ANCOVA results testing for associations between mesiodistal width of the maxillary first premolar and L1 rotations.

\begin{tabular}{llc}
\hline Source & F-Ratio* & P-Value \\
\hline \multicolumn{2}{c}{ Among Subjects } \\
U4-Size & 0.8362 & 0.3616 \\
Sex 3.1939 & 0.0754 & \\
U4-Size-x-Sex & 1.3145 & 0.2529 \\
\multicolumn{2}{c}{ Within Subjects } \\
Side 0.1836 & 0.6687 & 0.6279 \\
Side-x-U4-Size & 0.2357 & 0.5586 \\
Side-x-Sex & 0.3432 & 0.3772 \\
Side-x-U4-Size-x-Sex & 0.7831 & \\
\hline
\end{tabular}

*df for each test is 1 and 203.

Means, Sexes Pooled

\begin{tabular}{lcc} 
L_R1 rot & 0.38 & \\
L_L1 rot & 0.34 & \\
& Means, by Sex & \\
\multicolumn{4}{c}{ L_R1 rot } & L_L1 rot \\
Boy & 0.430 .37 & \\
Girl & 0.310 .30 &
\end{tabular}


Table A-109. ANCOVA results testing for associations between mesiodistal width of the maxillary first premolar and U2-U3 overlap.

\begin{tabular}{llc}
\hline Source & F-Ratio* $^{*}$ & P-Value \\
\hline \multicolumn{2}{c}{ Among Subjects } \\
U4-Size & 0.2016 & 0.6539 \\
Sex & 5.1005 & 0.0250 \\
U4-Size-x-Sex & 0.0034 & 0.9538 \\
\multicolumn{2}{c}{ Within Subjects } \\
Side & 0.0896 & 0.7650 \\
Side-x-U4-Size & 0.1040 & 0.7474 \\
Side-x-Sex & 0.4904 & 0.4845 \\
Side-x-U4-Size-x-Sex & 0.6847 & 0.4089 \\
\hline
\end{tabular}

*df for each test is 1 and 203.

Means, Sexes Pooled

$\begin{array}{ll}\text { U_R3-2 lapping } & 0.38 \\ \text { U_L2-3 lapping } & 0.37 \\ \text { Means, by Sex } & \end{array}$

Boy

U_R3-2 lapping U_L2-3 lapping

Girl

0.280 .30

0.490 .44 
Table A-110. ANCOVA results testing for associations between mesiodistal width of the maxillary first premolar and U1-U2 overlap.

\begin{tabular}{lcc}
\hline Source & F-Ratio* $^{*}$ & P-Value \\
\hline \multicolumn{2}{c}{ Among Subjects } \\
U4-Size & 0.0143 & 0.9050 \\
Sex & 7.7425 & 0.0059 \\
U4-Size-x-Sex & 0.3132 & 0.5763 \\
\multicolumn{3}{c}{ Within Subjects } \\
Side & 0.4202 & 0.5176 \\
Side-x-U4-Size & 0.3641 & 0.5469 \\
Side-x-Sex & 0.0936 & 0.7600 \\
Side-x-U4-Size-x-Sex & 0.1852 & 0.6674 \\
\hline
\end{tabular}

*df for each test is 1 and 203.

Means, Sexes Pooled

$\begin{array}{ll}\text { U_R2-1 lapping } & 0.35 \\ \text { U_L1-2 lapping } & 0.31 \\ \text { Means, by Sex } & \end{array}$

$\begin{array}{ll}\text { Boy } & 0.250 .23 \\ \text { Girl } & 0.450 .40\end{array}$ 
Table A-111. ANCOVA results testing for associations between mesiodistal width of the maxillary first premolar and L2-L3 overlap.

\begin{tabular}{llc}
\hline Source & F-Ratio* & P-Value \\
\hline \multicolumn{2}{c}{ Among Subjects } \\
U4-Size & 9.4220 & 0.0024 \\
Sex & 1.1374 & 0.2875 \\
U4-Size-x-Sex & 1.8102 & 0.1800 \\
& \multicolumn{2}{c}{ Within Subjects } \\
Side & 1.2552 & 0.2639 \\
Side-x-U4-Size & 1.4426 & 0.2311 \\
Side-x-Sex & 0.0034 & 0.9538 \\
Side-x-U4-Size-x-Sex & 0.0201 & 0.8875 \\
\hline
\end{tabular}

* $\mathrm{df}$ for each test is 1 and 203.

Means, Sexes Pooled

\begin{tabular}{lrr} 
L_R3-2 lapping & 0.71 & \\
L_L2-3 lapping & 0.65 & \\
Means, by Sex & \\
\multicolumn{4}{c}{ L_R3-2 lapping } & L_L2-3 lapping \\
Boy & 0.66 & 0.60 \\
Girl & 0.760 .69 &
\end{tabular}


Table A-112. ANCOVA results testing for associations between mesiodistal width of the maxillary first premolar and L1-L2 overlap.

\begin{tabular}{lcc}
\hline Source & F-Ratio* & P-Value \\
\hline \multicolumn{3}{c}{ Among Subjects } \\
U4-Size & 10.3587 & 0.0015 \\
Sex & 0.7370 & 0.3916 \\
U4-Size-x-Sex & 0.4192 & 0.5181 \\
& \multicolumn{2}{c}{ Within Subjects } \\
Side & 0.6691 & 0.4143 \\
Side-x-U4-Size & 0.6331 & 0.4271 \\
Side-x-Sex & 0.6486 & 0.4215 \\
Side-x-U4-Size-x-Sex & 2.1003 & 0.1488 \\
\hline
\end{tabular}

*df for each test is 1 and 203.

Means, Sexes Pooled

\begin{tabular}{lrr} 
L_R2-1 lapping & 0.47 & \\
L_L1-2 lapping & 0.46 & \\
Means, by Sex & \\
\multicolumn{4}{r}{ L_R2-1 lapping } & L_L1-2 lapping \\
Boy & 0.440 .46 & \\
Girl & 0.520 .47
\end{tabular}


Table A-113. ANCOVA results testing for associations between mesiodistal width of the maxillary first premolar and total displacements.

\begin{tabular}{lcccc}
\hline Term & Estimate & SE & t-Test & P-Value \\
\hline Intercept & -3.4039 & 4.1240 & -0.83 & 0.4101 \\
U4-Size & 0.7697 & 0.4228 & 1.82 & 0.1517 \\
Sex & -1.0569 & 4.1240 & -0.26 & 0.7980 \\
Interaction & 0.0610 & 0.4228 & 0.14 & 0.8854 \\
\hline
\end{tabular}

Table A-114. ANCOVA results testing for associations between mesiodistal width of the maxillary first premolar and total rotations.

\begin{tabular}{lcccc}
\hline Term & Estimate & SE & t-Test & P-Value \\
\hline Intercept & -1.692 & 2.966 & -0.57 & 0.5690 \\
U4-Size & 0.860 & 0.428 & 2.01 & 0.0459 \\
Sex & -0.255 & 0.177 & -1.44 & 0.1504 \\
Interaction & -0.029 & 0.428 & -0.07 & 0.9460 \\
\hline
\end{tabular}

Table A-115. ANCOVA results testing for associations between mesiodistal width of the maxillary first premolar and maxillary R1-L1 overlap.

\begin{tabular}{lcccc}
\hline Term & Estimate & $\mathrm{SE}$ & $\mathrm{t}$-Test & P-Value \\
\hline Intercept & -0.451 & 0.554 & -0.81 & 0.4172 \\
U4-Size & 0.101 & 0.080 & 1.26 & 0.2108 \\
Sex & -0.012 & 0.033 & -0.37 & 0.7116 \\
Interaction & -0.073 & 0.080 & -0.91 & 0.3616 \\
\hline
\end{tabular}


Table A-116. ANCOVA results testing for associations between mesiodistal width of the maxillary first premolar and mandibular R1-L1 overlap.

\begin{tabular}{lrcrc}
\hline Term & Estimate & $\mathrm{SE}$ & $\mathrm{t}$-Test & P-Value \\
\hline Intercept & 0.8642 & 0.5870 & 1.47 & 0.1425 \\
U4-Size & -0.0491 & 0.0602 & -0.82 & 0.4155 \\
Sex & 0.3251 & 0.5870 & 0.55 & 0.5803 \\
Interaction & -0.0222 & 0.0602 & -0.37 & 0.7131 \\
\hline
\end{tabular}

Table A-117. ANCOVA results testing for associations between mesiodistal width of the maxillary first premolar and total overlapping.

\begin{tabular}{lcccc}
\hline Term & Estimate & SE & t-Test & P-Value \\
\hline Intercept & -6.3852 & 2.3866 & -2.68 & 0.0081 \\
U4-Size & 1.6211 & 0.3589 & 4.52 & 0.0054 \\
Sex & -0.4632 & 0.2124 & -2.18 & 0.0303 \\
Interaction & -0.1691 & 0.3589 & -0.47 & 0.6381 \\
\hline
\end{tabular}

Table A-118. ANCOVA results testing for associations between mesiodistal width of the maxillary first premolar and total displacements, rotations, and overlapping.

\begin{tabular}{lcccc}
\hline Term & Estimate & $\mathrm{SE}$ & $\mathrm{t}$-Test & P-Value \\
\hline Intercept & -1.714 & 4.043 & -0.42 & 0.6721 \\
U4-Size & 0.840 & 0.584 & 1.44 & 0.0167 \\
Sex & -0.399 & 0.241 & -1.65 & 0.0996 \\
Interaction & 0.147 & 0.584 & 0.25 & 0.8010 \\
\hline
\end{tabular}


Table A-119. ANCOVA results testing for associations between mesiodistal width of the maxillary first premolar and crowding.

\begin{tabular}{lcccc}
\hline Term & Estimate & SE & t-Test & P-Value \\
\hline Intercept & -2.203 & 0.842 & -2.62 & 0.0095 \\
U4-Size & 0.519 & 0.122 & 4.27 & $<0.0001$ \\
Sex & -0.121 & 0.050 & -2.40 & 0.0172 \\
Interaction & -0.189 & 0.122 & -1.56 & 0.1213 \\
\hline
\end{tabular}

Table A-120. ANCOVA results testing for associations between mesiodistal width of the maxillary first premolar and spacing.

\begin{tabular}{lcccc}
\hline Term & Estimate & $\mathrm{SE}$ & $\mathrm{t}$-Test & P-Value \\
\hline Intercept & 2.934 & 0.634 & 4.63 & $<0.0001$ \\
U4-Size & -0.388 & 0.092 & -4.24 & $<0.0001$ \\
Sex & 0.050 & 0.038 & 1.33 & 0.1840 \\
Interaction & 0.177 & 0.092 & 1.93 & 0.0553 \\
& & & & \\
\hline
\end{tabular}

Table A-121. ANCOVA results testing for associations between mesiodistal width of the maxillary first premolar and diastema.

\begin{tabular}{lcccc}
\hline Term & Estimate & $\mathrm{SE}$ & $\mathrm{t}$-Test & P-Value \\
\hline Intercept & 1.731 & 0.662 & 2.61 & 0.0096 \\
U4-Size & -0.218 & 0.096 & -2.28 & 0.0235 \\
Sex & 0.033 & 0.039 & 0.83 & 0.4099 \\
Interaction & 0.243 & 0.096 & 2.54 & 0.0118 \\
\hline
\end{tabular}


Table A-122. ANCOVA results testing for associations between mesiodistal width of the maxillary first premolar and maxillary incisor irregularity.

\begin{tabular}{lcccc}
\hline Term & Estimate & $\mathrm{SE}$ & $\mathrm{t}$-Test & P-Value \\
\hline Intercept & -0.687 & 1.599 & -0.43 & 0.6678 \\
U4-Size & 0.306 & 0.231 & 1.33 & 0.1863 \\
Sex & -0.138 & 0.095 & -1.45 & 0.1484 \\
Interaction & 0.008 & 0.231 & 0.03 & 0.9740 \\
\hline
\end{tabular}

Table A-123. ANCOVA results testing for associations between mesiodistal width of the maxillary first premolar and mandibular incisor irregularity.

\begin{tabular}{lcccc}
\hline Term & Estimate & SE & t-Test & P-Value \\
\hline Intercept & -2.022 & 1.468 & -1.38 & 0.1699 \\
U4-Size & 0.454 & 0.212 & 2.14 & 0.0333 \\
Sex & -0.062 & 0.088 & -0.71 & 0.4785 \\
Interaction & 0.150 & 0.212 & 0.71 & 0.4797 \\
\hline
\end{tabular}

Table A-124. ANCOVA results testing for associations between mesiodistal width of the maxillary first premolar and maxillary overjet.

\begin{tabular}{lcccc}
\hline Term & Estimate & $\mathrm{SE}$ & $\mathrm{t}$-Test & P-Value \\
\hline Intercept & -1.507 & 2.648 & -0.57 & 0.5700 \\
U4-Size & 0.740 & 0.383 & 1.94 & 0.0544 \\
Sex & -0.093 & 0.158 & -0.59 & 0.5549 \\
Interaction & -0.420 & 0.383 & -1.10 & 0.2739 \\
\hline
\end{tabular}


Table A-125. ANCOVA results testing for associations between mesiodistal width of the maxillary first premolar and mandibular overjet.

\begin{tabular}{lcccc}
\hline Term & Estimate & SE & t-Test & P-Value \\
\hline Intercept & -0.252 & 0.321 & -0.78 & 0.4339 \\
U4-Size & 0.042 & 0.046 & 0.91 & 0.3615 \\
Sex & -0.042 & 0.019 & -2.18 & 0.0305 \\
Interaction & -0.042 & 0.046 & -0.91 & 0.3615 \\
\hline
\end{tabular}

Table A-126. ANCOVA results testing for associations between mesiodistal width of the maxillary first premolar and openbite.

\begin{tabular}{lccrc}
\hline Term & Estimate & SE & t-Test & P-Value \\
\hline Intercept & 0.406 & 0.542 & 0.75 & 0.4549 \\
U4-Size & -0.048 & 0.078 & -0.62 & 0.5386 \\
Sex & 0.013 & 0.032 & 0.39 & 0.6994 \\
Interaction & -0.041 & 0.078 & -0.53 & 0.5995 \\
\hline
\end{tabular}

Table A-127. ANCOVA results testing for associations between mesiodistal width of the maxillary first premolar and anteroposterior relationship.

\begin{tabular}{lccrc}
\hline Term & Estimate & $\mathrm{SE}$ & $\mathrm{t}$-Test & P-Value \\
\hline Intercept & 1.864 & 0.828 & 2.25 & 0.0255 \\
U4-Size & -0.161 & 0.120 & -1.35 & 0.1799 \\
Sex & 0.044 & 0.049 & 0.89 & 0.3764 \\
Interaction & -0.069 & 0.120 & -0.58 & 0.5650 \\
\hline
\end{tabular}


Table A-128. ANCOVA results testing for associations between mesiodistal width of the maxillary first premolar and DAI score.

\begin{tabular}{lcccc}
\hline Term & Estimate & SE & t-Test & P-Value \\
\hline Intercept & 20.795 & 7.671 & 2.71 & 0.0073 \\
U4-Size & 0.901 & 1.108 & 0.81 & 0.4172 \\
Sex & -0.343 & 0.457 & -0.75 & 0.4536 \\
Interaction & -0.276 & 1.108 & -0.25 & 0.8033 \\
\hline
\end{tabular}


Table A-129. ANCOVA results testing for associations between mesiodistal width of the maxillary second premolar and U3 displacements.

\begin{tabular}{lcc}
\hline Source & F-Ratio* & P-Value \\
\hline \multicolumn{2}{c}{ Among Subjects } \\
U5-Size & 0.1629 & 0.6869 \\
Sex & 1.3915 & 0.2395 \\
U5-Size-x-Sex & 0.2709 & 0.6033 \\
& \multicolumn{2}{c}{ Within Subjects } \\
Side & 0.9803 & 0.3233 \\
Side-x-U5-Size & 0.8776 & 0.3500 \\
Side-x-Sex & 0.1905 & 0.6630 \\
Side-x-U5-Size-x-Sex & 2.2355 & 0.1364 \\
\hline
\end{tabular}

*df for each test is 1 and 203.

Means, Sexes Pooled

U_R3 dis $\quad 0.41$

U_L3 dis 0.38

Means, by Sex

$\begin{array}{ll}\text { Boy } & 0.360 .34 \\ \text { Girl } & 0.480 .41\end{array}$


Table A-130. ANCOVA results testing for associations between mesiodistal width of the maxillary second premolar and $\mathrm{U} 2$ displacements.

\begin{tabular}{llc}
\hline Source & F-Ratio* & P-Value \\
\hline \multicolumn{2}{c}{ Among Subjects } \\
U5-Size & 0.1422 & 0.7065 \\
Sex & 4.7473 & 0.0305 \\
U5-Size-x-Sex & 0.0097 & 0.9216 \\
& \multicolumn{2}{c}{ Within Subjects } \\
Side & 1.1513 & 0.2846 \\
Side-x-U5-Size & 1.0528 & 0.3061 \\
Side-x-Sex & 0.0642 & 0.8003 \\
Side-x-U5-Size-x-Sex & 1.0513 & 0.3064 \\
\hline
\end{tabular}

*df for each test is 1 and 203.

Means, Sexes Pooled

\begin{tabular}{lrr} 
U_R2 dis & 0.37 & \\
U_L2 dis & 0.41 & \\
& Means, by Sex \\
\multicolumn{4}{c}{ U_R2 dis } & U_L2 dis \\
Boy & 0.290 .33 & \\
Girl & 0.460 .48 &
\end{tabular}


Table A-131. ANCOVA results testing for associations between mesiodistal width of the maxillary second premolar and U1 displacements.

\begin{tabular}{llc}
\hline Source & F-Ratio* & P-Value \\
\hline \multicolumn{2}{c}{ Among Subjects } \\
U5-Size & 0.1557 & 0.6936 \\
Sex & 0.0065 & 0.9358 \\
U5-Size-x-Sex & 0.0088 & 0.9252 \\
& \multicolumn{2}{c}{ Within Subjects } \\
Side & 0.7907 & 0.3749 \\
Side-x-U5-Size & 0.8649 & 0.3535 \\
Side-x-Sex & 0.9923 & 0.3204 \\
Side-x-U5-Size-x-Sex & 1.6773 & 0.1968 \\
\hline
\end{tabular}

*df for each test is 1 and 203.

Means, Sexes Pooled

\begin{tabular}{lrr} 
U_R1 dis & 0.34 & \\
U_L1 dis & 0.36 & \\
& Means, by Sex \\
\multicolumn{4}{c}{ U_R1 dis } & U_L1 dis \\
Boy & 0.360 .35 & \\
Girl & 0.310 .38 &
\end{tabular}


Table A-132. ANCOVA results testing for associations between mesiodistal width of the maxillary second premolar and L3 displacements.

\begin{tabular}{llc}
\hline Source & F-Ratio* & P-Value \\
\hline \multicolumn{2}{c}{ Among Subjects } \\
U5-Size & 0.5778 & 0.4480 \\
Sex & 1.5821 & 0.2099 \\
U5-Size-x-Sex & 1.2727 & 0.2606 \\
& \multicolumn{2}{c}{ Within Subjects } \\
Side & 1.1339 & 0.2882 \\
Side-x-U5-Size & 1.1075 & 0.2939 \\
Side-x-Sex & 1.7677 & 0.1852 \\
Side-x-U5-Size-x-Sex & 0.9828 & 0.3227 \\
\hline
\end{tabular}

*df for each test is 1 and 203.

Means, Sexes Pooled

\begin{tabular}{lrr} 
L_R3 dis & 0.23 & \\
L_L3 dis & 0.23 & \\
& Means, by Sex \\
\multicolumn{4}{c}{ L_R3 dis } & L_L3 dis \\
Boy & 0.160 .21 & \\
Girl & 0.290 .23 &
\end{tabular}


Table A-133. ANCOVA results testing for associations between mesiodistal width of the maxillary second premolar and L2 displacements.

\begin{tabular}{llc}
\hline Source & F-Ratio* & P-Value \\
\hline & \multicolumn{2}{c}{ Among Subjects } \\
U5-Size & 1.0815 & 0.2996 \\
Sex & 1.0351 & 0.3102 \\
U5-Size-x-Sex & 4.5387 & 0.0343 \\
& \multicolumn{2}{c}{ Within Subjects } \\
Side & 0.3656 & 0.5461 \\
Side-x-U5-Size & 0.2930 & 0.5889 \\
Side-x-Sex & 0.1669 & 0.6833 \\
Side-x-U5-Size-x-Sex & 0.0397 & 0.8422 \\
\hline
\end{tabular}

*df for each test is 1 and 203.

Means, Sexes Pooled

\begin{tabular}{lll} 
L_R2 dis & 0.51 & \\
L_L2 dis & 0.47 & \\
& Means, by Sex \\
\multicolumn{4}{c}{ L_R2 dis } & L_L2 dis \\
Boy & 0.450 .42 & \\
Girl & 0.550 .49 &
\end{tabular}


Table A-134. ANCOVA results testing for associations between mesiodistal width of the maxillary second premolar and L1 displacements.

\begin{tabular}{llc}
\hline Source & F-Ratio* & P-Value \\
\hline \multicolumn{2}{c}{ Among Subjects } \\
U5-Size & 0.8520 & 0.3571 \\
Sex & 0.7836 & 0.3771 \\
U5-Size-x-Sex & 0.0224 & 0.8813 \\
& \multicolumn{2}{c}{ Within Subjects } \\
Side & 0.3236 & 0.5701 \\
Side-x-U5-Size & 0.4473 & 0.5044 \\
Side-x-Sex & 0.5346 & 0.4655 \\
Side-x-U5-Size-x-Sex & 0.6174 & 0.4329 \\
\hline
\end{tabular}

*df for each test is 1 and 203.

Means, Sexes Pooled

\begin{tabular}{lrr} 
L_R1 dis & 0.22 & \\
L_L1 dis & 0.17 & \\
& Means, by Sex \\
\multicolumn{4}{c}{ Boy } & L_R1 dis & L_L1 dis \\
Girl & 0.260 .17 & \\
& 0.190 .16 &
\end{tabular}


Table A-135. ANCOVA results testing for associations between mesiodistal width of the maxillary second premolar and U3 rotations.

\begin{tabular}{lcc}
\hline Source & F-Ratio* & P-Value \\
\hline & \multicolumn{2}{c}{ Among Subjects } \\
U5-Size & 1.8728 & 0.1727 \\
Sex & 5.2575 & 0.0229 \\
U5-Size-x-Sex & 0.0016 & 0.9679 \\
& \multicolumn{2}{c}{ Within Subjects } \\
Side & 0.6017 & 0.4388 \\
Side-x-U5-Size & 0.6357 & 0.4262 \\
Side-x-Sex & 0.5428 & 0.4621 \\
Side-x-U5-Size-x-Sex & 0.9970 & 0.3192 \\
\hline
\end{tabular}

*df for each test is 1 and 203.

Means, Sexes Pooled

$\begin{array}{lll}\text { U_R3 rot } & 0.22 & \\ \text { U_L3 rot } & 0.22 & \\ & \text { Means, by Sex } \\ & \text { U_R3 rot } & \text { U_L3 rot } \\ \text { Boy } & 0.170 .15 & \\ \text { Girl } & 0.260 .30 & \end{array}$


Table A-136. ANCOVA results testing for associations between mesiodistal width of the maxillary second premolar and $\mathrm{U} 2$ rotations.

\begin{tabular}{lcc}
\hline Source & F-Ratio* & P-Value \\
\hline \multicolumn{3}{c}{ Among Subjects } \\
U5-Size & 0.0204 & 0.8865 \\
Sex & 0.2344 & 0.6288 \\
U5-Size-x-Sex & 1.1634 & 0.2820 \\
& \multicolumn{2}{c}{ Within Subjects } \\
Side & 0.1077 & 0.7432 \\
Side-x-U5-Size & 0.0757 & 0.7834 \\
Side-x-Sex & 0.0004 & 0.9845 \\
Side-x-U5-Size-x-Sex & 1.3527 & 0.2462 \\
\hline
\end{tabular}

*df for each test is 1 and 203.

Means, Sexes Pooled

$\begin{array}{lrr}\text { U_R2 rot } & 0.39 \\ \text { U_L2 rot } & 0.42 & \\ & \text { Means, by Sex } \\ & \text { U_R2 rot } & \text { U_L2 rot } \\ \text { Boy } & 0.360 .40 & \\ \text { Girl } & 0.400 .44 & \end{array}$


Table A-137. ANCOVA results testing for associations between mesiodistal width of the maxillary second premolar and U1 rotations.

\begin{tabular}{lcc}
\hline Source & F-Ratio* & P-Value \\
\hline & \multicolumn{2}{c}{ Among Subjects } \\
U5-Size & 0.8313 & 0.3630 \\
Sex & 0.0660 & 0.7975 \\
U5-Size-x-Sex & 1.3203 & 0.2519 \\
& \multicolumn{2}{c}{ Within Subjects } \\
Side & 0.0025 & 0.9603 \\
Side-x-U5-Size & 0.0050 & 0.9435 \\
Side-x-Sex & 0.0953 & 0.7579 \\
Side-x-U5-Size-x-Sex & 0.0237 & 0.8777 \\
\hline
\end{tabular}

*df for each test is 1 and 203.

Means, Sexes Pooled

$\begin{array}{lrr}\text { U_R1 rot } & 0.47 & \\ \text { U_L1 rot } & 0.49 & \\ & \text { Means, by Sex } \\ & \text { U_R1 rot } & \text { U_L1 rot } \\ \text { Boy } & 0.460 .46 & \\ \text { Girl } & 0.470 .50 & \end{array}$


Table A-138. ANCOVA results testing for associations between mesiodistal width of the maxillary second premolar and L3 rotations.

\begin{tabular}{llc}
\hline Source & F-Ratio* & \multicolumn{2}{c}{ P-Value } \\
\hline & \multicolumn{2}{c}{ Among Subjects } \\
U5-Size & 7.6866 & 0.0061 \\
Sex & 5.5999 & 0.0189 \\
U5-Size-x-Sex & 0.1755 & 0.6758 \\
& \multicolumn{2}{c}{ Within Subjects } \\
Side & 0.0265 & 0.8709 \\
Side-x-U5-Size & 0.0135 & 0.9077 \\
Side-x-Sex & 2.0263 & 0.1561 \\
Side-x-U5-Size-x-Sex & 0.3011 & 0.5838 \\
\hline
\end{tabular}

*df for each test is 1 and 203.

Means, Sexes Pooled

\begin{tabular}{lll} 
L_R3 rot & 0.39 & \\
L_L3 rot & 0.35 & \\
& Means, by Sex & \\
\multicolumn{4}{c}{ L_R3 rot } & L_L3 rot \\
Boy & 0.270 .31 & \\
Girl & 0.510 .41 &
\end{tabular}


Table A-139. ANCOVA results testing for associations between mesiodistal width of the maxillary second premolar and L2 rotations.

\begin{tabular}{llc}
\hline Source & F-Ratio* & P-Value \\
\hline & \multicolumn{2}{c}{ Among Subjects } \\
U5-Size & 0.8376 & 0.3612 \\
Sex & 0.5439 & 0.4617 \\
U5-Size-x-Sex & 0.1883 & 0.6648 \\
& \multicolumn{2}{c}{ Within Subjects } \\
Side & 0.0002 & 0.9891 \\
Side-x-U5-Size & 0.0231 & 0.8794 \\
Side-x-Sex & 0.6555 & 0.4191 \\
Side-x-U5-Size-x-Sex & 0.0111 & 0.9161 \\
\hline
\end{tabular}

*df for each test is 1 and 203.

Means, Sexes Pooled

\begin{tabular}{lll} 
L_R2 rot & 0.35 & \\
L_L2 rot & 0.23 & \\
& Means, by Sex & \\
\multicolumn{4}{c}{ L_R2 rot } & L_L2 rot \\
Boy & 0.320 .23 & \\
Girl & 0.400 .24 &
\end{tabular}


Table A-140. ANCOVA results testing for associations between mesiodistal width of the maxillary second premolar and L1 rotations.

\begin{tabular}{lcc}
\hline Source & F-Ratio* & P-Value \\
\hline & \multicolumn{2}{c}{ Among Subjects } \\
U5-Size & 0.0689 & 0.7932 \\
Sex & 3.2808 & 0.0716 \\
U5-Size-x-Sex & 1.7729 & 0.1845 \\
& \multicolumn{2}{c}{ Within Subjects } \\
Side & 0.8188 & 0.3666 \\
Side-x-U5-Size & 0.9481 & 0.3314 \\
Side-x-Sex & 0.2093 & 0.6478 \\
Side-x-U5-Size-x-Sex & 1.0183 & 0.3141 \\
\hline
\end{tabular}

*df for each test is 1 and 203.

Means, Sexes Pooled

$\begin{array}{lcc}\text { L_R1 rot } & 0.38 & \\ \text { L_L1 rot } & 0.34 & \\ & \text { Means, by Sex } \\ & \text { L_R1 rot } & \text { L_L1 rot } \\ \text { Boy } & 0.430 .37 & \\ \text { Girl } & 0.310 .29 & \end{array}$


Table A-141. ANCOVA results testing for associations between mesiodistal width of the maxillary second premolar and U2-U3 overlap.

\begin{tabular}{lcc}
\hline Source & F-Ratio* & P-Value \\
\hline & \multicolumn{2}{c}{ Among Subjects } \\
U5-Size & 0.8459 & 0.3588 \\
Sex & 5.5489 & 0.0194 \\
U5-Size-x-Sex & 0.0780 & 0.7804 \\
& \multicolumn{2}{c}{ Within Subjects } \\
Side & 0.0166 & 0.8978 \\
Side-x-U5-Size & 0.0242 & 0.8764 \\
Side-x-Sex & 0.4760 & 0.4910 \\
Side-x-U5-Size-x-Sex & 0.4122 & 0.5216 \\
\hline
\end{tabular}

*df for each test is 1 and 203.

Means, Sexes Pooled

$\begin{array}{ll}\text { U_R3-2 lapping } & 0.38 \\ \text { U_L2-3 lapping } & 0.37 \\ \text { Means, by Sex } & \end{array}$

Boy

U_R3-2 lapping U_L2-3 lapping

Girl

0.280 .29

0.500 .44 
Table A-142. ANCOVA results testing for associations between mesiodistal width of the maxillary second premolar and U1-U2 overlap.

\begin{tabular}{lcc}
\hline Source & F-Ratio & P-Value \\
\hline & \multicolumn{2}{c}{ Among Subjects } \\
U5-Size & 1.0051 & 0.3173 \\
Sex & 8.5520 & 0.0038 \\
U5-Size-x-Sex & 1.5640 & 0.2125 \\
& \multicolumn{2}{c}{ Within Subjects } \\
Side & 1.0112 & 0.3158 \\
Side-x-U5-Size & 0.9297 & 0.3361 \\
Side-x-Sex & 0.0370 & 0.8476 \\
Side-x-U5-Size-x-Sex & 0.6007 & 0.4392 \\
\hline
\end{tabular}

*df for each test is 1 and 203.

Means, Sexes Pooled

\begin{tabular}{lrr} 
U_R2-1 lapping & 0.35 & \\
U_L1-2 lapping & 0.31 & \\
\multicolumn{4}{c}{ Means, by Sex } \\
Boy & U_R2-1 lapping & U_L1-2 lapping \\
Girl & 0.240 .22 & \\
& 0.440 .41 &
\end{tabular}


Table A-143. ANCOVA results testing for associations between mesiodistal width of the maxillary second premolar and L2-L3 overlap.

\begin{tabular}{lcc}
\hline Source & F-Ratio* & \multicolumn{2}{c}{ P-Value } \\
\hline \multicolumn{2}{c}{ Among Subjects } \\
U5-Size & 11.1290 & 0.0010 \\
Sex & 1.7797 & 0.1837 \\
\hline U5-Size-x-Sex & 1.3146 & 0.2529 \\
& \multicolumn{2}{c}{ Within Subjects } \\
Side & 0.0000 & 0.9974 \\
Side-x-U5-Size & 0.0104 & 0.9188 \\
Side-x-Sex & 0.0010 & 0.9744 \\
Side-x-U5-Size-x-Sex & 1.4393 & 0.2317 \\
\hline
\end{tabular}

*df for each test is 1 and 203.

Means, Sexes Pooled

\begin{tabular}{ll} 
L_R3-2 lapping & 0.71 \\
L_L2-3 lapping & 0.65 \\
\multicolumn{2}{c}{ Means, by Sex }
\end{tabular}

Boy

L_R3-2 lapping L_L2-3 lapping

Girl

0.650 .58

0.770 .70 
Table A-144. ANCOVA results testing for associations between mesiodistal width of the maxillary second premolar and L1-L2 overlap.

\begin{tabular}{llc}
\hline Source & F-Ratio* $^{*}$ & P-Value \\
\hline & \multicolumn{2}{c}{ Among Subjects } \\
U5-Size & 0.1644 & 0.6855 \\
Sex & 0.3578 & 0.5504 \\
U5-Size-x-Sex & 0.0815 & 0.7755 \\
& \multicolumn{2}{c}{ Within Subjects } \\
Side & 1.2682 & 0.2614 \\
Side-x-U5-Size & 1.1852 & 0.2776 \\
Side-x-Sex & 0.4884 & 0.4855 \\
Side-x-U5-Size-x-Sex & 4.4202 & 0.0367 \\
\hline
\end{tabular}

*df for each test is 1 and 203.

Means, Sexes Pooled

\begin{tabular}{lrr} 
L_R2-1 lapping & 0.47 & \\
L_L1-2 lapping & 0.46 & \\
Means, by Sex & \\
\multicolumn{4}{c}{ L_R2-1 lapping } & L_L1-2 lapping \\
Boy & 0.450 .45 & \\
Girl & 0.510 .46 &
\end{tabular}


Table A-145. ANCOVA results testing for associations between mesiodistal width of the maxillary second premolar and total displacements.

\begin{tabular}{lccrc}
\hline Term & Estimate & $\mathrm{SE}$ & $\mathrm{t}$-Test & P-Value \\
\hline Intercept & 2.639 & 3.752 & 0.70 & 0.4827 \\
U5-Size & 0.214 & 0.561 & 0.38 & 0.7027 \\
Sex & -0.381 & 0.244 & -1.56 & 0.1209 \\
Interaction & 0.449 & 0.561 & 0.80 & 0.4243 \\
\hline
\end{tabular}

Table A-146. ANCOVA results testing for associations between mesiodistal width of the maxillary second premolar and total rotations.

\begin{tabular}{lcccc}
\hline Term & Estimate & SE & t-Test & P-Value \\
\hline Intercept & -1.907 & 2.729 & -0.70 & 0.4854 \\
U5-Size & 0.918 & 0.408 & 2.25 & 0.0254 \\
Sex & -0.289 & 0.178 & -1.62 & 0.1057 \\
Interaction & 0.418 & 0.408 & 1.03 & 0.3060 \\
\hline
\end{tabular}

Table A-147. ANCOVA results testing for associations between mesiodistal width of the maxillary second premolar and maxillary R1-L1 overlap.

\begin{tabular}{lcccc}
\hline Term & Estimate & $\mathrm{SE}$ & $\mathrm{t}$-Test & P-Value \\
\hline Intercept & -0.355 & 0.513 & -0.69 & 0.4904 \\
U5-Size & 0.089 & 0.077 & 1.16 & 0.2474 \\
Sex & -0.015 & 0.033 & -0.44 & 0.6596 \\
Interaction & 0.027 & 0.077 & 0.35 & 0.7235 \\
\hline
\end{tabular}


Table A-148. ANCOVA results testing for associations between mesiodistal width of the maxillary second premolar and mandibular R1L1 overlap.

\begin{tabular}{lcccc}
\hline Term & Estimate & $\mathrm{SE}$ & $\mathrm{t}$-Test & P-Value \\
\hline Intercept & 0.123 & 0.531 & 0.23 & 0.8175 \\
U5-Size & 0.039 & 0.079 & 0.50 & 0.6196 \\
Sex & 0.100 & 0.035 & 2.89 & 0.0043 \\
Interaction & -0.044 & 0.079 & -0.56 & 0.5780 \\
\hline
\end{tabular}

Table A-149. ANCOVA results testing for associations between mesiodistal width of the maxillary second premolar and total overlapping.

\begin{tabular}{lccrc}
\hline Term & Estimate & SE & t-Test & P-Value \\
\hline Intercept & -3.724 & 3.400 & -1.10 & 0.2747 \\
U5-Size & 1.205 & 0.508 & 2.37 & 0.0186 \\
Sex & -0.454 & 0.221 & -2.05 & 0.0418 \\
Interaction & 0.401 & 0.508 & 0.79 & 0.4308 \\
\hline
\end{tabular}

Table A-150. ANCOVA results testing for associations between mesiodistal width of the maxillary second premolar and total displacements, rotations, and overlapping.

\begin{tabular}{lcccc}
\hline Term & Estimate & $\mathrm{SE}$ & $\mathrm{t}$-Test & P-Value \\
\hline Intercept & -2.992 & 8.482 & -0.35 & 0.7246 \\
U5-Size & 2.338 & 1.268 & 1.84 & 0.0666 \\
Sex & -1.123 & 0.553 & -2.03 & 0.0434 \\
Interaction & 1.268 & 1.268 & 1.00 & 0.3182 \\
\hline
\end{tabular}


Table A-151. ANCOVA results testing for associations between mesiodistal width of the maxillary second premolar and crowding.

\begin{tabular}{lcccc}
\hline Term & Estimate & SE & t-Test & P-Value \\
\hline Intercept & -1.276 & 0.792 & -1.61 & 0.1087 \\
U5-Size & 0.398 & 0.118 & 3.36 & 0.0009 \\
Sex & -0.130 & 0.052 & -2.51 & 0.0127 \\
Interaction & -0.052 & 0.118 & -0.44 & 0.6638 \\
\hline
\end{tabular}

Table A-152. ANCOVA results testing for associations between mesiodistal width of the maxillary second premolar and spacing.

\begin{tabular}{lcccc}
\hline Term & Estimate & SE & t-Test & P-Value \\
\hline Intercept & 2.191 & 0.597 & 3.67 & 0.0003 \\
U5-Size & -0.291 & 0.089 & -3.26 & 0.0013 \\
Sex & 0.057 & 0.039 & 1.46 & 0.1454 \\
Interaction & 0.101 & 0.089 & 1.14 & 0.2566 \\
\hline
\end{tabular}

Table A-153. ANCOVA results testing for associations between mesiodistal width of the maxillary second premolar and diastema.

\begin{tabular}{lcccc}
\hline Term & Estimate & $\mathrm{SE}$ & $\mathrm{t}$-Test & P-Value \\
\hline Intercept & 1.708 & 0.618 & 2.76 & 0.0063 \\
U5-Size & -0.222 & 0.092 & -2.40 & 0.0174 \\
Sex & 0.040 & 0.040 & 1.00 & 0.3180 \\
Interaction & 0.044 & 0.092 & 0.48 & 0.6346 \\
\hline
\end{tabular}


Table A-154. ANCOVA results testing for associations between mesiodistal width of the maxillary second premolar and maxillary incisor irregularity.

\begin{tabular}{lcccc}
\hline Term & Estimate & SE & t-Test & P-Value \\
\hline Intercept & 0.141 & 1.483 & 0.09 & 0.9245 \\
U5-Size & 0.194 & 0.222 & 0.87 & 0.3834 \\
Sex & -0.141 & 0.097 & -1.46 & 0.1466 \\
Interaction & -0.022 & 0.222 & -0.10 & 0.9222 \\
\hline
\end{tabular}

Table A-155. ANCOVA results testing for associations between mesiodistal width of the maxillary second premolar and mandibular incisor irregularity.

\begin{tabular}{lcccc}
\hline Term & Estimate & SE & t-Test & P-Value \\
\hline Intercept & 0.370 & 1.367 & 0.27 & 0.7870 \\
U5-Size & 0.110 & 0.204 & 0.54 & 0.5911 \\
Sex & -0.052 & 0.089 & -0.58 & 0.5613 \\
Interaction & 0.319 & 0.204 & 1.56 & 0.1194 \\
\hline
\end{tabular}

Table A-156. ANCOVA results testing for associations between mesiodistal width of the maxillary second premolar and maxillary overjet.

\begin{tabular}{lcccc}
\hline Term & Estimate & $\mathrm{SE}$ & $\mathrm{t}$-Test & P-Value \\
\hline Intercept & -1.329 & 2.447 & -0.54 & 0.5876 \\
U5-Size & 0.741 & 0.366 & 2.03 & 0.0441 \\
Sex & -0.120 & 0.159 & -0.75 & 0.4534 \\
Interaction & -0.313 & 0.366 & -0.85 & 0.3937 \\
\hline
\end{tabular}


Table A-157. ANCOVA results testing for associations between mesiodistal width of the maxillary second premolar and mandibular overjet.

\begin{tabular}{lcccc}
\hline Term & Estimate & $\mathrm{SE}$ & $\mathrm{t}$-Test & P-Value \\
\hline Intercept & -0.119 & 0.298 & -0.40 & 0.6905 \\
U5-Size & 0.024 & 0.044 & 0.54 & 0.5897 \\
Sex & -0.042 & 0.019 & -2.16 & 0.0321 \\
Interaction & -0.024 & 0.044 & -0.54 & 0.5897 \\
\hline
\end{tabular}

Table A-158. ANCOVA results testing for associations between mesiodistal width of the maxillary second premolar and openbite.

\begin{tabular}{lccrc}
\hline Term & Estimate & SE & t-Test & P-Value \\
\hline Intercept & 0.459 & 0.502 & 0.91 & 0.3614 \\
U5-Size & -0.058 & 0.075 & -0.77 & 0.4407 \\
Sex & 0.015 & 0.033 & 0.46 & 0.6482 \\
Interaction & -0.018 & 0.075 & -0.25 & 0.8055 \\
\hline
\end{tabular}

Table A-159. ANCOVA results testing for associations between mesiodistal width of the maxillary second premolar and anteroposterior relationship.

\begin{tabular}{lccrc}
\hline Term & Estimate & $\mathrm{SE}$ & $\mathrm{t}$-Test & P-Value \\
\hline Intercept & 1.112 & 0.768 & 1.45 & 0.1492 \\
U5-Size & -0.053 & 0.115 & -0.46 & 0.6431 \\
Sex & 0.041 & 0.050 & 0.82 & 0.4112 \\
Interaction & -0.118 & 0.115 & -1.03 & 0.3038 \\
\hline
\end{tabular}


Table A-160. ANCOVA results testing for associations between mesiodistal width of the maxillary second premolar and DAI score.

\begin{tabular}{lcccc}
\hline Term & Estimate & SE & t-Test & P-Value \\
\hline Intercept & 22.670 & 7.095 & 3.20 & 0.0016 \\
U5-Size & 0.657 & 1.060 & 0.62 & 0.5363 \\
Sex & -0.358 & 0.462 & -0.77 & 0.4395 \\
Interaction & -0.559 & 1.060 & -0.53 & 0.5984 \\
\hline
\end{tabular}


Table A-161. ANCOVA results testing for associations between mesiodistal width of the maxillary first molar and U3 displacements.

\begin{tabular}{llc}
\hline Source & F-Ratio* & P-Value \\
\hline & \multicolumn{2}{c}{ Among Subjects } \\
U6_Size & 2.0189 & 0.1569 \\
Sex & 2.1074 & 0.1481 \\
U6-Size-x-Sex & 0.5362 & 0.4648 \\
& \multicolumn{2}{c}{ Within Subjects } \\
Side & 0.1420 & 0.7067 \\
Side-x-U6-Size & 0.1153 & 0.7346 \\
Side-x-Sex & 0.2398 & 0.6249 \\
Side-x-U6-Size-x-Sex & 0.2391 & 0.6254 \\
\hline
\end{tabular}

* $\mathrm{df}$ for each test is 1 and 203.

Means, Sexes Pooled

\begin{tabular}{lrr} 
U_R3 dis & 0.41 & \\
U_L3 dis & 0.38 & \\
& Means, by Sex \\
\multicolumn{4}{c}{ U_R3 dis } & U_L3 dis \\
Boy & 0.330 .32 & \\
Girl & 0.480 .42 &
\end{tabular}


Table A-162. ANCOVA results testing for associations between mesiodistal width of the maxillary first molar and $\mathrm{U} 2$ displacements.

\begin{tabular}{llc}
\hline Source & F-Ratio* & P-Value \\
\hline & \multicolumn{2}{c}{ Among Subjects } \\
U6_Size & 3.2430 & 0.0732 \\
Sex & 6.5656 & 0.0111 \\
U6-Size-x-Sex & 0.7544 & 0.3861 \\
& \multicolumn{2}{c}{ Within Subjects } \\
Side & 0.6467 & 0.4222 \\
Side-x-U6-Size & 0.5862 & 0.4448 \\
Side-x-Sex & 0.0747 & 0.7848 \\
Side-x-U6-Size-x-Sex & 0.4856 & 0.4867 \\
\hline
\end{tabular}

*df for each test is 1 and 203.

Means, Sexes Pooled

\begin{tabular}{lrr} 
U_R2 dis & 0.37 \\
U_L2 dis & 0.41 & \\
& Means, by Sex \\
\multicolumn{4}{c}{ U_R2 dis } & U_L2 dis \\
Boy & 0.280 .33 & \\
Girl & 0.490 .51 &
\end{tabular}


Table A-163. ANCOVA results testing for associations between mesiodistal width of the maxillary first molar and U1 displacements.

\begin{tabular}{llc}
\hline Source & F-Ratio* & P-Value \\
\hline & \multicolumn{2}{c}{ Among Subjects } \\
U6_Size & 0.0833 & 0.7732 \\
Sex & 0.0073 & 0.9319 \\
U6-Size-x-Sex & 2.2739 & 0.1331 \\
& \multicolumn{2}{c}{ Within Subjects } \\
Side & 0.7665 & 0.3823 \\
Side-x-U6-Size & 0.7610 & 0.3841 \\
Side-x-Sex & 0.3334 & 0.5643 \\
Side-x-U6-Size-x-Sex & 1.4109 & 0.2363 \\
\hline
\end{tabular}

*df for each test is 1 and 203.

Means, Sexes Pooled

\begin{tabular}{lrr} 
U_R1 dis & 0.34 \\
U_L1 dis & 0.36 & \\
& & \\
\multicolumn{4}{c}{ Means, by Sex } \\
Boy & U_R1 dis & U_L1 dis \\
Girl & 0.350 .33 & \\
Girl & 0.320 .35 &
\end{tabular}


Table A-164. ANCOVA results testing for associations between mesiodistal width of the maxillary first molar and L3 displacements.

\begin{tabular}{llc}
\hline Source & F-Ratio* & P-Value \\
\hline & \multicolumn{2}{c}{ Among Subjects } \\
U6_Size & 0.0164 & 0.8983 \\
Sex & 1.9309 & 0.1662 \\
U6-Size-x-Sex & 0.6970 & 0.4048 \\
& \multicolumn{2}{c}{ Within Subjects } \\
Side & 0.0115 & 0.9148 \\
Side-x-U6-Size & 0.0063 & 0.9367 \\
Side-x-Sex & 2.1736 & 0.1419 \\
Side-x-U6-Size-x-Sex & 3.2756 & 0.0718 \\
\hline
\end{tabular}

*df for each test is 1 and 203.

Means, Sexes Pooled

\begin{tabular}{lll} 
L_R3 dis & 0.23 & \\
L_L3 dis & 0.23 & \\
& Means, by Sex \\
\multicolumn{4}{c}{ L_R3 dis } & L_L3 dis \\
Boy & 0.160 .20 & \\
Girl & 0.300 .23 &
\end{tabular}


Table A-165. ANCOVA results testing for associations between mesiodistal width of the maxillary first molar and L2 displacements.

\begin{tabular}{lcc}
\hline Source & F-Ratio* & P-Value \\
\hline & \multicolumn{2}{c}{ Among Subjects } \\
U6_Size & 5.8871 & 0.0161 \\
Sex & 2.1144 & 0.1475 \\
U6-Size-x-Sex & 1.1724 & 0.2802 \\
& \multicolumn{2}{c}{ Within Subjects } \\
Side & 0.8497 & 0.3577 \\
Side-x-U6-Size & 0.9301 & 0.3360 \\
Side-x-Sex & 0.5403 & 0.4632 \\
Side-x-U6-Size-x-Sex & 0.3207 & 0.5718 \\
\hline
\end{tabular}

*df for each test is 1 and 203.

Means, Sexes Pooled

\begin{tabular}{lrr} 
L_R2 dis & 0.51 & \\
L_L2 dis & 0.47 & \\
& Means, by Sex \\
\multicolumn{4}{c}{ L_R2 dis } & L_L2 dis \\
Boy & 0.420 .42 & \\
Girl & 0.580 .51 &
\end{tabular}


Table A-166. ANCOVA results testing for associations between mesiodistal width of the maxillary first molar and L1 displacements.

\begin{tabular}{lcc}
\hline Source & F-Ratio* & P-Value \\
\hline & \multicolumn{2}{c}{ Among Subjects } \\
U6_Size & 0.1654 & 0.6846 \\
Sex & 0.3874 & 0.5344 \\
U6-Size-x-Sex & 2.0393 & 0.1548 \\
& \multicolumn{2}{c}{ Within Subjects } \\
Side & 2.1840 & 0.1410 \\
Side-x-U6-Size & 2.4252 & 0.1210 \\
Side-x-Sex & 0.1864 & 0.6663 \\
Side-x-U6-Size-x-Sex & 0.2044 & 0.6517 \\
\hline
\end{tabular}

*df for each test is 1 and 203.

Means, Sexes Pooled

\begin{tabular}{lrr} 
L_R1 dis & 0.22 & \\
L_L1 dis & 0.17 & \\
& Means, by Sex \\
\multicolumn{4}{c}{ L_R1 dis } & L_L1 dis \\
Boy & 0.240 .16 & \\
Girl & 0.190 .15 &
\end{tabular}


Table A-167. ANCOVA results testing for associations between mesiodistal width of the maxillary first molar and U3 rotations.

\begin{tabular}{lcc}
\hline Source & F-Ratio* & P-Value \\
\hline & \multicolumn{2}{c}{ Among Subjects } \\
U6_Size & 0.0667 & 0.7965 \\
Sex & 4.3015 & 0.0393 \\
U6-Size-x-Sex & 0.0740 & 0.7859 \\
& \multicolumn{2}{c}{ Within Subjects } \\
Side & 0.0285 & 0.8660 \\
Side-x-U6-Size & 0.0342 & 0.8534 \\
Side-x-Sex & 0.4024 & 0.5265 \\
Side-x-U6-Size-x-Sex & 0.4035 & 0.5260 \\
\hline
\end{tabular}

*df for each test is 1 and 203.

Means, Sexes Pooled

$\begin{array}{lll}\text { U_R3 rot } & 0.22 & \\ \text { U_L3 rot } & 0.22 & \\ & \text { Means, by Sex } \\ & \text { U_R3 rot } & \text { U_L3 rot } \\ \text { Boy } & 0.170 .16 & \\ \text { Girl } & 0.260 .29 & \end{array}$


Table A-168. ANCOVA results testing for associations between mesiodistal width of the maxillary first molar and $\mathrm{U} 2$ rotations.

\begin{tabular}{llc}
\hline Source & F-Ratio* & P-Value \\
\hline & \multicolumn{2}{c}{ Among Subjects } \\
U6_Size & 0.0106 & 0.9181 \\
Sex & 0.2832 & 0.5952 \\
U6-Size-x-Sex & 0.0653 & 0.7985 \\
& \multicolumn{2}{c}{ Within Subjects } \\
Side & 2.1700 & 0.1423 \\
Side-x-U6-Size & 2.0459 & 0.1542 \\
Side-x-Sex & 0.1163 & 0.7334 \\
Side-x-U6-Size-x-Sex & 0.5870 & 0.4445 \\
\hline
\end{tabular}

*df for each test is 1 and 203.

Means, Sexes Pooled

\begin{tabular}{lrr} 
U_R2 rot & 0.39 & \\
U_L2 rot & 0.42 & \\
& Means, by Sex & \\
\multicolumn{4}{c}{ U_R2 rot } & U_L2 rot \\
Boy & 0.360 .42 & \\
Girl & 0.420 .44 &
\end{tabular}


Table A-169. ANCOVA results testing for associations between mesiodistal width of the maxillary first molar and U1 rotations.

\begin{tabular}{lcc}
\hline Source & F-Ratio* & P-Value \\
\hline & \multicolumn{2}{c}{ Among Subjects } \\
U6_Size & 0.1359 & 0.7128 \\
Sex & 0.0379 & 0.8459 \\
U6-Size-x-Sex & 0.3589 & 0.5498 \\
& \multicolumn{2}{c}{ Within Subjects } \\
Side & 0.0029 & 0.9570 \\
Side-x-U6-Size & 0.0023 & 0.9616 \\
Side-x-Sex & 0.0738 & 0.7862 \\
Side-x-U6-Size-x-Sex & 0.5009 & 0.4799 \\
\hline
\end{tabular}

*df for each test is 1 and 203.

Means, Sexes Pooled

Overall Means

$\begin{array}{lrr}\text { U_R1 rot } & 0.47 & \\ \text { U_L1 rot } & 0.49 & \\ & \text { Means, by Sex } & \\ & \text { U_R1 rot } & \text { U_L1 rot } \\ \text { Boy } & 0.470 .46 & \\ \text { Girl } & 0.470 .49 & \end{array}$


Table A-170. ANCOVA results testing for associations between mesiodistal width of the maxillary first molar and L3 rotations.

\begin{tabular}{llc}
\hline Source & F-Ratio* & P-Value \\
\hline & \multicolumn{2}{c}{ Among Subjects } \\
U6_Size & 1.8032 & 0.1808 \\
Sex & 4.7036 & 0.0313 \\
U6-Size-x-Sex & 1.2560 & 0.2637 \\
& \multicolumn{2}{c}{ Within Subjects } \\
Side & 0.7170 & 0.3981 \\
Side-x-U6-Size & 0.8081 & 0.3698 \\
Side-x-Sex & 2.7511 & 0.0987 \\
Side-x-U6-Size-x-Sex & 0.4396 & 0.5080 \\
\hline
\end{tabular}

*df for each test is 1 and 203.

Means, Sexes Pooled

\begin{tabular}{lll} 
L_R3 rot & 0.39 & \\
L_L3 rot & 0.35 & \\
& Means, by Sex & \\
\multicolumn{4}{c}{ L_R3 rot } & L_L3 rot \\
Boy & 0.270 .30 & \\
Girl & 0.500 .38 &
\end{tabular}


Table A-171. ANCOVA results testing for associations between mesiodistal width of the maxillary first molar and L2 rotations.

\begin{tabular}{llc}
\hline Source & F-Ratio* & P-Value \\
\hline & \multicolumn{2}{c}{ Among Subjects } \\
U6_Size & 1.7601 & 0.1861 \\
Sex & 0.8482 & 0.3582 \\
U6-Size-x-Sex & 1.2613 & 0.2627 \\
& \multicolumn{2}{c}{ Within Subjects } \\
Side & 0.0870 & 0.7683 \\
Side-x-U6-Size & 0.1907 & 0.6628 \\
Side-x-Sex & 0.7869 & 0.3761 \\
Side-x-U6-Size-x-Sex & 0.4406 & 0.5076 \\
\hline
\end{tabular}

*df for each test is 1 and 203.

Means, Sexes Pooled

\begin{tabular}{lll} 
L_R2 rot & 0.35 & \\
L_L2 rot & 0.23 & \\
& Means, by Sex \\
\multicolumn{4}{c}{ L_R2 rot } & L_L2 rot \\
Boy & 0.320 .23 & \\
Girl & 0.420 .24 &
\end{tabular}


Table A-172. ANCOVA results testing for associations between mesiodistal width of the maxillary first molar and L1 rotations.

\begin{tabular}{llc}
\hline Source & F-Ratio* & P-Value \\
\hline & \multicolumn{2}{c}{ Among Subjects } \\
U6_Size & 0.9051 & 0.3425 \\
Sex & 4.2482 & 0.0406 \\
U6-Size-x-Sex & 0.4796 & 0.4894 \\
& \multicolumn{2}{c}{ Within Subjects } \\
Side & 0.6085 & 0.4363 \\
Side-x-U6-Size & 0.6604 & 0.4174 \\
Side-x-Sex & 0.1731 & 0.6778 \\
Side-x-U6-Size-x-Sex & 0.8374 & 0.3612 \\
\hline
\end{tabular}

*df for each test is 1 and 203.

Means, Sexes Pooled

\begin{tabular}{lll} 
L_R1 rot & 0.38 & \\
L_L1 rot & 0.34 & \\
& Means, by Sex & \\
\multicolumn{4}{c}{ L_R1 rot } & L_L1 rot \\
Boy & 0.430 .39 & \\
Girl & 0.300 .29 &
\end{tabular}


Table A-173. ANCOVA results testing for associations between mesiodistal width of the maxillary first molar and U2-U3 overlap.

\begin{tabular}{llc}
\hline Source & F-Ratio* & P-Value \\
\hline & \multicolumn{2}{c}{ Among Subjects } \\
U6_Size & 3.0070 & 0.0844 \\
Sex & 6.8074 & 0.0098 \\
U6-Size-x-Sex & 0.5517 & 0.4585 \\
& \multicolumn{2}{c}{ Within Subjects } \\
Side & 0.0851 & 0.7708 \\
Side-x-U6-Size & 0.0957 & 0.7573 \\
Side-x-Sex & 0.5286 & 0.4680 \\
Side-x-U6-Size-x-Sex & 0.0168 & 0.8969 \\
\hline
\end{tabular}

*df for each test is 1 and 203.

Means, Sexes Pooled

$\begin{array}{ll}\text { U_R3-2 lapping } & 0.38 \\ \text { U_L2-3 lapping } & 0.37 \\ \text { Means, by Sex } & \end{array}$

$\begin{array}{lll}\text { Boy } & 0.25 & 0.28 \\ \text { Girl } & 0.50 & 0.45\end{array}$

U_R3-2 lapping U_L2-3 lapping 
Table A-174. ANCOVA results testing for associations between mesiodistal width of the maxillary first molar and U1-U2 overlap.

\begin{tabular}{llc}
\hline Source & F-Ratio* & P-Value \\
\hline & \multicolumn{2}{c}{ Among Subjects } \\
U6_Size & 0.0252 & 0.8740 \\
Sex & 7.4486 & 0.0069 \\
U6-Size-x-Sex & 0.0077 & 0.9303 \\
& \multicolumn{2}{c}{ Within Subjects } \\
Side & 0.2013 & 0.6541 \\
Side-x-U6-Size & 0.2299 & 0.6321 \\
Side-x-Sex & 0.2220 & 0.6381 \\
Side-x-U6-Size-x-Sex & 0.6301 & 0.4282 \\
\hline
\end{tabular}

*df for each test is 1 and 203.

Means, Sexes Pooled

$\begin{array}{lrr}\text { U_R2-1 lapping } & 0.35 & \\ \text { U_L1-2 lapping } & 0.31 & \\ \text { Means, by Sex } \\ \text { Boy } & \text { U_R2-1 lapping } & \text { U_L1-2 lapping } \\ \text { Girl } & 0.24 & 0.24 \\ & 0.45 & 0.40\end{array}$


Table A-175. ANCOVA results testing for associations between mesiodistal width of the maxillary first molar and L2-L3 overlap.

\begin{tabular}{lcc}
\hline Source & F-Ratio $^{*}$ & P-Value \\
\hline \multicolumn{2}{c}{ Among Subjects } \\
U6_Size & 10.3054 & 0.0015 \\
Sex & 2.4681 & 0.1177 \\
U6-Size-x-Sex & 1.0436 & 0.3082 \\
& \multicolumn{2}{c}{ Within Subjects } \\
Side & 3.7039 & 0.0557 \\
Side-x-U6-Size & 4.0455 & 0.0456 \\
Side-x-Sex & 0.2234 & 0.6370 \\
Side-x-U6-Size-x-Sex & 1.4900 & 0.2236 \\
\hline
\end{tabular}

*df for each test is 1 and 203.

Means, Sexes Pooled

\begin{tabular}{lrr} 
L_R3-2 lapping & 0.71 & \\
L_L2-3 lapping & 0.65 & \\
Means, by Sex & \\
\multicolumn{4}{c}{ L_R3-2 lapping } & L_L2-3 lapping \\
Boy & 0.630 .57 & \\
Girl & 0.800 .70
\end{tabular}


Table A-176. ANCOVA results testing for associations between mesiodistal width of the maxillary first molar and L1-L2 overlap.

\begin{tabular}{llc}
\hline Source & F-Ratio* & P-Value \\
\hline & \multicolumn{2}{c}{ Among Subjects } \\
U6_Size & 0.6176 & 0.4328 \\
Sex & 0.5113 & 0.4754 \\
U6-Size-x-Sex & 1.2284 & 0.2690 \\
& \multicolumn{2}{c}{ Within Subjects } \\
Side & 1.2206 & 0.2705 \\
Side-x-U6-Size & 1.1881 & 0.2770 \\
Side-x-Sex & 0.3223 & 0.5709 \\
Side-x-U6-Size-x-Sex & 0.0303 & 0.8619 \\
\hline
\end{tabular}

*df for each test is 1 and 203.

Means, Sexes Pooled

\begin{tabular}{lrr} 
L_R2-1 lapping & 0.47 & \\
L_L1-2 lapping & 0.46 & \\
Means, by Sex & \\
\multicolumn{4}{c}{ L_R2-1 lapping } & L_L1-2 lapping \\
Boy & 0.430 .45 & \\
Girl & 0.500 .46 &
\end{tabular}


Table A-177. ANCOVA results testing for associations between mesiodistal width of the maxillary first molar and total displacements.

\begin{tabular}{lcccc}
\hline Term & Estimate & SE & t-Test & P-Value \\
\hline Intercept & -5.522 & 4.595 & -1.20 & 0.2309 \\
U6-Size & 0.923 & 0.444 & 2.08 & 0.0390 \\
Sex & -0.495 & 0.246 & -2.01 & 0.0456 \\
Interaction & 0.554 & 0.444 & 1.25 & 0.2140 \\
\hline
\end{tabular}

Table A-178. ANCOVA results testing for associations between mesiodistal width of the maxillary first molar and total rotations.

\begin{tabular}{lcccc}
\hline Term & Estimate & SE & t-Test & P-Value \\
\hline Intercept & 0.926 & 3.428 & 0.27 & 0.7873 \\
U6-Size & 0.320 & 0.331 & 0.97 & 0.3356 \\
Sex & -0.266 & 0.183 & -1.45 & 0.1485 \\
Interaction & 0.177 & 0.331 & 0.53 & 0.5942 \\
\hline
\end{tabular}

Table A-179. ANCOVA results testing for associations between mesiodistal width of the maxillary first molar and maxillary R1-L1 overlap.

\begin{tabular}{lcccc}
\hline Term & Estimate & $\mathrm{SE}$ & $\mathrm{t}$-Test & P-Value \\
\hline Intercept & -0.972 & 0.633 & -1.54 & 0.1261 \\
U6-Size & 0.118 & 0.061 & 1.93 & 0.0546 \\
Sex & -0.025 & 0.034 & -0.75 & 0.4553 \\
Interaction & -0.062 & 0.061 & -1.01 & 0.3128 \\
\hline
\end{tabular}


Table A-180. ANCOVA results testing for associations between mesiodistal width of the maxillary first molar and mandibular R1-L1 overlap.

\begin{tabular}{lccrc}
\hline Term & Estimate & SE & t-Test & P-Value \\
\hline Intercept & 0.480 & 0.660 & 0.73 & 0.4675 \\
U6-Size & -0.010 & 0.064 & -0.15 & 0.8811 \\
Sex & 0.104 & 0.035 & 2.96 & 0.0035 \\
Interaction & 0.010 & 0.064 & 0.15 & 0.8817 \\
\hline
\end{tabular}

Table A-181. ANCOVA results testing for associations between mesiodistal width of the maxillary first molar and total overlapping.

\begin{tabular}{lccrc}
\hline Term & Estimate & SE & t-Test & P-Value \\
\hline Intercept & -6.139 & 4.210 & -1.46 & 0.1463 \\
U6-Size & 1.010 & 0.407 & 2.48 & 0.0139 \\
Sex & -0.507 & 0.225 & -2.25 & 0.0256 \\
Interaction & 0.361 & 0.407 & 0.89 & 0.3762 \\
\hline
\end{tabular}

Table A-182. ANCOVA results testing for associations between mesiodistal width of the maxillary first molar and total displacements, rotations, and overlapping.

\begin{tabular}{lrrrc}
\hline Term & Estimate & \multicolumn{1}{c}{ SE } & t-Test & P-Value \\
\hline Intercept & -10.735 & 10.477 & -1.02 & 0.3068 \\
U6-Size & 2.253 & 1.013 & 2.22 & 0.0272 \\
Sex & -1.268 & 0.561 & -2.26 & 0.0248 \\
Interaction & 1.092 & 1.013 & 1.08 & 0.2824 \\
\hline
\end{tabular}


Table A-183. ANCOVA results testing for associations between mesiodistal width of the maxillary first molar and crowding.

\begin{tabular}{lcccc}
\hline Term & Estimate & SE & t-Test & P-Value \\
\hline Intercept & -1.244 & 0.993 & -1.25 & 0.2117 \\
U6-Size & 0.254 & 0.096 & 2.65 & 0.0087 \\
Sex & -0.136 & 0.053 & -2.56 & 0.0111 \\
Interaction & -0.036 & 0.096 & -0.38 & 0.7057 \\
\hline
\end{tabular}

Table A-184. ANCOVA results testing for associations between mesiodistal width of the maxillary first molar and spacing.

\begin{tabular}{lcccc}
\hline Term & Estimate & SE & t-Test & P-Value \\
\hline Intercept & 2.061 & 0.751 & 2.74 & 0.0066 \\
U6-Size & -0.175 & 0.073 & -2.41 & 0.0169 \\
Sex & 0.060 & 0.040 & 1.49 & 0.1380 \\
Interaction & 0.019 & 0.073 & 0.26 & 0.7936 \\
\hline
\end{tabular}

Table A-185. ANCOVA results testing for associations between mesiodistal width of the maxillary first molar and diastema.

\begin{tabular}{lccrc}
\hline Term & Estimate & SE & t-Test & P-Value \\
\hline Intercept & 0.946 & 0.777 & 1.22 & 0.2246 \\
U6-Size & -0.070 & 0.075 & -0.93 & 0.3552 \\
Sex & 0.034 & 0.042 & 0.81 & 0.4176 \\
Interaction & 0.035 & 0.075 & 0.46 & 0.6445 \\
\hline
\end{tabular}


Table A-186. ANCOVA results testing for associations between mesiodistal width of the maxillary first molar and maxillary incisor irregularity.

\begin{tabular}{lccrc}
\hline Term & Estimate & $\mathrm{SE}$ & $\mathrm{t}$-Test & P-Value \\
\hline Intercept & -2.571 & 1.821 & -1.41 & 0.1596 \\
U6-Size & 0.386 & 0.176 & 2.19 & 0.0294 \\
Sex & -0.181 & 0.097 & -1.86 & 0.0647 \\
Interaction & 0.061 & 0.176 & 0.35 & 0.7296 \\
\hline
\end{tabular}

Table A-187. ANCOVA results testing for associations between mesiodistal width of the maxillary first molar and mandibular incisor irregularity.

\begin{tabular}{lcccc}
\hline Term & Estimate & SE & t-Test & P-Value \\
\hline Intercept & -2.769 & 1.682 & -1.65 & 0.1012 \\
U6-Size & 0.375 & 0.163 & 2.31 & 0.0221 \\
Sex & -0.097 & 0.090 & -1.08 & 0.2814 \\
Interaction & 0.135 & 0.163 & 0.83 & 0.4081 \\
\hline
\end{tabular}

Table A-188. ANCOVA results testing for associations between mesiodistal width of the maxillary first molar and maxillary overjet.

\begin{tabular}{lcccc}
\hline Term & Estimate & SE & t-Test & P-Value \\
\hline Intercept & 1.591 & 3.069 & 0.52 & 0.6046 \\
U6-Size & 0.195 & 0.297 & 0.66 & 0.5124 \\
Sex & 0.508 & 3.069 & 0.17 & 0.8687 \\
Interaction & -0.058 & 0.297 & -0.20 & 0.8453 \\
\hline
\end{tabular}


Table A-189. ANCOVA results testing for associations between mesiodistal width of the maxillary first molar and mandibular overjet.

\begin{tabular}{lcccc}
\hline Term & Estimate & SE & t-Test & P-Value \\
\hline Intercept & 0.097 & 0.370 & 0.26 & 0.7928 \\
U6-Size & -0.006 & 0.036 & -0.16 & 0.8753 \\
Sex & -0.097 & 0.370 & -0.26 & 0.7928 \\
Interaction & 0.006 & 0.036 & 0.16 & 0.8753 \\
\hline
\end{tabular}

Table A-190. ANCOVA results testing for associations between mesiodistal width of the maxillary first molar and openbite.

\begin{tabular}{lccrc}
\hline Term & Estimate & $\mathrm{SE}$ & $\mathrm{t}$-Test & P-Value \\
\hline Intercept & 0.515 & 0.621 & 0.83 & 0.4081 \\
U6-Size & -0.042 & 0.060 & -0.70 & 0.4833 \\
Sex & 0.606 & 0.621 & 0.98 & 0.3301 \\
Interaction & -0.057 & 0.060 & -0.95 & 0.3433 \\
\hline
\end{tabular}

Table A-191. ANCOVA results testing for associations between mesiodistal width of the maxillary first molar and anteroposterior relationship.

\begin{tabular}{lcccc}
\hline Term & Estimate & $\mathrm{SE}$ & $\mathrm{t}$-Test & P-Value \\
\hline Intercept & 1.057 & 0.956 & 1.11 & 0.2701 \\
U6-Size & -0.030 & 0.092 & -0.32 & 0.7499 \\
Sex & 0.369 & 0.956 & 0.39 & 0.6999 \\
Interaction & -0.032 & 0.092 & -0.34 & 0.7323 \\
\hline
\end{tabular}


Table A-192. ANCOVA results testing for associations between mesiodistal width of the maxillary first molar and DAI score.

\begin{tabular}{lcccc}
\hline Term & Estimate & $\mathrm{SE}$ & $\mathrm{t}$-Test & P-Value \\
\hline Intercept & 19.747 & 8.805 & 2.24 & 0.0260 \\
U6-Size & 0.704 & 0.851 & 0.83 & 0.4091 \\
Sex & 0.567 & 8.805 & 0.06 & 0.9487 \\
Interaction & -0.094 & 0.851 & -0.11 & 0.9119 \\
\hline
\end{tabular}


Table A-193. ANCOVA results testing for associations between mesiodistal width of the maxillary second molar and U3 displacements.

\begin{tabular}{lcc}
\hline Source & F-Ratio* & \multicolumn{2}{c}{ P-Value } \\
\hline \multicolumn{2}{c}{ Among Subjects } \\
U7-Size & 0.1514 & 0.6976 \\
Sex & 0.0182 & 0.8929 \\
U7-Size-x-Sex & 0.0421 & 0.8376 \\
& \multicolumn{2}{c}{ Within Subjects } \\
Side & 0.0444 & 0.8333 \\
Side-x-U7-Size & 0.0227 & 0.8804 \\
Side-x-Sex & 3.8286 & 0.0518 \\
Side-x-U7-Size-x-Sex & 3.9650 & 0.0478 \\
\hline
\end{tabular}

* df for each test is 1 and 203.

Means, Sexes Pooled

U_R3 dis $\quad 0.41$

U_L3 dis 0.38

Means, by Sex

$\begin{array}{lrr} & \text { U_R3 dis } & \text { U_L3 dis } \\ \text { Boy } & 0.360 .33 & \\ \text { Girl } & 0.490 .41 & \end{array}$


Table A-194. ANCOVA results testing for associations between mesiodistal width of the maxillary second molar and U2 displacements.

\begin{tabular}{lcc}
\hline Source & F-Ratio* & P-Value \\
\hline & \multicolumn{2}{c}{ Among Subjects } \\
U7-Size & 4.0255 & 0.0461 \\
Sex & 2.5542 & 0.1116 \\
U7-Size-x-Sex & 3.0693 & 0.0813 \\
& \multicolumn{2}{c}{ Within Subjects } \\
Side & 0.5267 & 0.4688 \\
Side-x-U7-Size & 0.4653 & 0.4959 \\
Side-x-Sex & 0.5997 & 0.4396 \\
Side-x-U7-Size-x-Sex & 0.6211 & 0.4316 \\
\hline
\end{tabular}

*df for each test is 1 and 203.

Means, Sexes Pooled

$\begin{array}{ll}\text { U_R2 dis } & 0.37 \\ \text { U_L2 dis } & 0.41\end{array}$

Means, by Sex

$\begin{array}{lr}\text { Boy } & 0.290 .33 \\ \text { Girl } & 0.490 .51\end{array}$


Table A-195. ANCOVA results testing for associations between mesiodistal width of the maxillary second molar and U1 displacements.

\begin{tabular}{lcc}
\hline Source & F-Ratio* & P-Value \\
\hline & \multicolumn{2}{c}{ Among Subjects } \\
U7-Size & 0.0000 & 0.9946 \\
Sex & 0.5763 & 0.4486 \\
U7-Size-x-Sex & 0.5906 & 0.4431 \\
& \multicolumn{2}{c}{ Within Subjects } \\
Side & 0.2563 & 0.6132 \\
Side-x-U7-Size & 0.2338 & 0.6292 \\
Side-x-Sex & 0.0013 & 0.9712 \\
Side-x-U7-Size-x-Sex & 0.0062 & 0.9374 \\
\hline
\end{tabular}

*df for each test is 1 and 203.

Means, Sexes Pooled

U_R1 dis $\quad 0.34$

U_L1 dis $\quad 0.36$

Means, by Sex

$\begin{array}{lr}\text { Boy } & 0.360 .34 \\ \text { Girl } & 0.320 .36\end{array}$


Table A-196. ANCOVA results testing for associations between mesiodistal width of the maxillary second molar and L3 displacements.

\begin{tabular}{llc}
\hline Source & F-Ratio* & P-Value \\
\hline & \multicolumn{2}{c}{ Among Subjects } \\
U7-Size & 0.8968 & 0.3448 \\
Sex & 0.1064 & 0.7447 \\
U7-Size-x-Sex & 0.0539 & 0.8166 \\
& \multicolumn{2}{c}{ Within Subjects } \\
Side & 0.8343 & 0.3621 \\
Side-x-U7-Size & 0.7998 & 0.3722 \\
Side-x-Sex & 1.5257 & 0.2182 \\
Side-x-U7-Size-x-Sex & 1.7255 & 0.1905 \\
\hline
\end{tabular}

* $\mathrm{df}$ for each test is 1 and 203.

Means, Sexes Pooled

$\begin{array}{ll}\text { L_R3 dis } & 0.23 \\ \text { L_L3 dis } & 0.23\end{array}$

Means, by Sex

$\begin{array}{ll}\text { Boy } & 0.160 .20 \\ \text { Girl } & 0.310 .24\end{array}$


Table A-197. ANCOVA results testing for associations between mesiodistal width of the maxillary second molar and L2 displacements.

\begin{tabular}{lcc}
\hline Source & F-Ratio* & \multicolumn{2}{c}{ P-Value } \\
\hline & \multicolumn{2}{c}{ Among Subjects } \\
U7-Size & 4.3200 & 0.0389 \\
Sex & 1.0126 & 0.3155 \\
U7-Size-x-Sex & 0.8654 & 0.3533 \\
& \multicolumn{2}{c}{ Within Subjects } \\
Side & 0.0287 & 0.8656 \\
Side-x-U7-Size & 0.0163 & 0.8985 \\
Side-x-Sex & 1.3223 & 0.2515 \\
Side-x-U7-Size-x-Sex & 1.2611 & 0.2628 \\
\hline
\end{tabular}

* $\mathrm{df}$ for each test is 1 and 203.

Means, Sexes Pooled

L_R2 dis $\quad 0.51$

L_L2 dis $\quad 0.47$

Means, by Sex

$\begin{array}{ll}\text { Boy } & 0.440 .43 \\ \text { Girl } & 0.570 .52\end{array}$


Table A-198. ANCOVA results testing for associations between mesiodistal width of the maxillary second molar and L1 displacements.

\begin{tabular}{llc}
\hline Source & F-Ratio* & P-Value \\
\hline \multicolumn{2}{c}{ Among Subjects } \\
U7-Size & 0.4996 & 0.4805 \\
Sex & 0.6732 & 0.4129 \\
U7-Size-x-Sex & 0.7310 & 0.3936 \\
& \multicolumn{2}{c}{ Within Subjects } \\
Side & 1.3025 & 0.2551 \\
Side-x-U7-Size & 1.4951 & 0.2228 \\
Side-x-Sex & 0.0021 & 0.9638 \\
Side-x-U7-Size-x-Sex & 0.0064 & 0.9364 \\
\hline
\end{tabular}

*df for each test is 1 and 203.

Means, Sexes Pooled
L_R1 dis
0.22
L_L1 dis $\quad 0.17$

Means, by Sex

$\begin{array}{ll}\text { Boy } & 0.240 .17 \\ \text { Girl } & 0.190 .16\end{array}$


Table A-199. ANCOVA results testing for associations between mesiodistal width of the maxillary second molar and $\mathrm{U} 3$ rotations.

\begin{tabular}{lcc}
\hline Source & F-Ratio* & P-Value \\
\hline & \multicolumn{2}{c}{ Among Subjects } \\
U7-Size & 0.5376 & 0.4643 \\
Sex & 0.0523 & 0.8194 \\
U7-Size-x-Sex & 0.0097 & 0.9217 \\
& \multicolumn{2}{c}{ Within Subjects } \\
Side & 0.0047 & 0.9452 \\
Side-x-U7-Size & 0.0039 & 0.9505 \\
Side-x-Sex & 0.0217 & 0.8829 \\
Side-x-U7-Size-x-Sex & 0.0129 & 0.9098 \\
\hline
\end{tabular}

*df for each test is 1 and 203.

Means, Sexes Pooled

$\begin{array}{ll}\text { U_R3 rot } & 0.22 \\ \text { U_L3 rot } & 0.22\end{array}$

Means, by Sex

$\begin{array}{ll}\text { Boy } & 0.170 .15 \\ \text { Girl } & 0.260 .29\end{array}$


Table A-200. ANCOVA results testing for associations between mesiodistal width of the maxillary second molar and $\mathrm{U} 2$ rotations.

\begin{tabular}{lcc}
\hline Source & F-Ratio* & P-Value \\
\hline & \multicolumn{2}{c}{ Among Subjects } \\
U7-Size & 0.4441 & 0.5059 \\
Sex & 0.6154 & 0.4337 \\
U7-Size-x-Sex & 0.6512 & 0.4206 \\
& \multicolumn{2}{c}{ Within Subjects } \\
Side & 0.0124 & 0.9113 \\
Side-x-U7-Size & 0.0326 & 0.8570 \\
Side-x-Sex & 6.1110 & 0.0143 \\
Side-x-U7-Size-x-Sex & 6.1394 & 0.0140 \\
\hline
\end{tabular}

*df for each test is 1 and 203.

Means, Sexes Pooled

$\begin{array}{ll}\text { U_R2 rot } & 0.39 \\ \text { U_L2 rot } & 0.42\end{array}$

Means, by Sex

$\begin{array}{ll}\text { Boy } & 0.370 .42 \\ \text { Girl } & 0.400 .46\end{array}$


Table A-201. ANCOVA results testing for associations between mesiodistal width of the maxillary second molar and U1 rotations.

\begin{tabular}{llc}
\hline Source & F-Ratio* & P-Value \\
\hline & \multicolumn{2}{c}{ Among Subjects } \\
U7-Size & 0.0272 & 0.8691 \\
Sex & 0.2623 & 0.6091 \\
U7-Size-x-Sex & 0.2542 & 0.6147 \\
& \multicolumn{2}{c}{ Within Subjects } \\
Side & 1.6016 & 0.2071 \\
Side-x-U7-Size & 1.5659 & 0.2122 \\
Side-x-Sex & 0.0288 & 0.8655 \\
Side-x-U7-Size-x-Sex & 0.0283 & 0.8665 \\
\hline
\end{tabular}

*df for each test is 1 and 203.

Means, Sexes Pooled

$$
\begin{array}{ll}
\text { U_R1 rot } & 0.47 \\
\text { U_L1 rot } & 0.49
\end{array}
$$

Means, by Sex

$\begin{array}{lr}\text { Boy } & 0.470 .48 \\ \text { Girl } & 0.480 .49\end{array}$


Table A-202. ANCOVA results testing for associations between mesiodistal width of the maxillary second molar and L3 rotations.

\begin{tabular}{llc}
\hline Source & F-Ratio* & \multicolumn{2}{c}{ P-Value } \\
\hline & \multicolumn{2}{c}{ Among Subjects } \\
U7-Size & 1.0257 & 0.3124 \\
Sex & 0.4619 & 0.4975 \\
U7-Size-x-Sex & 0.3109 & 0.5777 \\
& \multicolumn{2}{c}{ Within Subjects } \\
Side & 1.3456 & 0.2474 \\
Side-x-U7-Size & 1.5114 & 0.2203 \\
Side-x-Sex & 2.1234 & 0.1466 \\
Side-x-U7-Size-x-Sex & 2.4344 & 0.1203 \\
\hline
\end{tabular}

*df for each test is 1 and 203.

Means, Sexes Pooled

L_R3 rot $\quad 0.39$

L_L3 rot 0.35

Means, by Sex

Boy

L_R3 rot L_L3 rot

Girl

0.280 .30

0.510 .38 
Table A-203. ANCOVA results testing for associations between mesiodistal width of the maxillary second molar and L2 rotations.

\begin{tabular}{llc}
\hline Source & F-Ratio* & P-Value \\
\hline & \multicolumn{2}{c}{ Among Subjects } \\
U7-Size & 0.2863 & 0.5932 \\
Sex & 1.3208 & 0.2518 \\
U7-Size-x-Sex & 1.4192 & 0.2349 \\
& \multicolumn{2}{c}{ Within Subjects } \\
Side & 2.1248 & 0.1465 \\
Side-x-U7-Size & 2.5923 & 0.1089 \\
Side-x-Sex & 0.0042 & 0.9484 \\
Side-x-U7-Size-x-Sex & 0.0174 & 0.8951 \\
\hline
\end{tabular}

*df for each test is 1 and 203.

Means, Sexes Pooled

$\begin{array}{ll}\text { L_R2 rot } & 0.35 \\ \text { L_L2 rot } & 0.23\end{array}$

Means, by Sex

Boy

L_R2 rot L_L2 rot

Girl

0.310 .25

0.410 .23 
Table A-204. ANCOVA results testing for associations between mesiodistal width of the maxillary second molar and L1 rotations.

\begin{tabular}{llc}
\hline Source & F-Ratio* & P-Value \\
\hline & \multicolumn{2}{c}{ Among Subjects } \\
U7-Size & 0.0622 & 0.8033 \\
Sex & 1.9524 & 0.1639 \\
U7-Size-x-Sex & 2.2825 & 0.1324 \\
& \multicolumn{2}{c}{ Within Subjects } \\
Side & 1.8821 & 0.1716 \\
Side-x-U7-Size & 2.0296 & 0.1558 \\
Side-x-Sex & 0.0092 & 0.9236 \\
Side-x-U7-Size-x-Sex & 0.0060 & 0.9385 \\
\hline
\end{tabular}

*df for each test is 1 and 203.

Means, Sexes Pooled

L_R1 rot $\quad 0.38$

L_L1 rot $\quad 0.34$

Means, by Sex

Boy

L_R1 rot L_L1 rot

Girl

0.430 .37

0.310 .28 
Table A-205. ANCOVA results testing for associations between mesiodistal width of the maxillary second molar and U2-U3 overlap.

\begin{tabular}{lcc}
\hline Source & F-Ratio* & P-Value \\
\hline & \multicolumn{2}{c}{ Among Subjects } \\
U7-Size & 0.9633 & 0.3275 \\
Sex & 0.0462 & 0.8299 \\
U7-Size-x-Sex & 0.1277 & 0.7212 \\
& \multicolumn{2}{c}{ Within Subjects } \\
Side & 0.1199 & 0.7295 \\
Side-x-U7-Size & 0.1455 & 0.7033 \\
Side-x-Sex & 1.6441 & 0.2012 \\
Side-x-U7-Size-x-Sex & 1.7616 & 0.1859 \\
\hline
\end{tabular}

*df for each test is 1 and 203. Means, Sexes Pooled U_R3-2 lapping $\quad 0.38$ U_L2-3 lapping $\quad 0.37$

Means, by Sex

$\begin{array}{rr}\text { U_R3-2 lapping } & \text { U_L2-3 lapping } \\ 0.280 .29 & \\ 0.510 .44 & \end{array}$


Table A-206. ANCOVA results testing for associations between mesiodistal width of the maxillary second molar and U1-U2 overlap.

\begin{tabular}{llc}
\hline Source & F-Ratio* & P-Value \\
\hline & \multicolumn{2}{c}{ Among Subjects } \\
U7-Size & 0.0276 & 0.8681 \\
Sex & 0.1637 & 0.6862 \\
U7-Size-x-Sex & 0.0602 & 0.8064 \\
& \multicolumn{2}{c}{ Within Subjects } \\
Side & 0.3457 & 0.5572 \\
Side-x-U7-Size & 0.3209 & 0.5717 \\
Side-x-Sex & 3.8482 & 0.0512 \\
Side-x-U7-Size-x-Sex & 3.7994 & 0.0526 \\
\hline
\end{tabular}

* $\mathrm{df}$ for each test is 1 and 203. Means, Sexes Pooled U_R2-1 lapping $\quad 0.35$ U_L1-2 lapping $\quad 0.31$

Means, by Sex

U_R2-1 lapping U_L1-2 lapping

Boy

0.250 .24

Girl

0.430 .41 
Table A-207. ANCOVA results testing for associations between mesiodistal width of the maxillary second molar and L2-L3 overlap.

\begin{tabular}{|c|c|c|}
\hline Source & F-Ratio* & P-Value \\
\hline & \multicolumn{2}{|c|}{ Among Subjects } \\
\hline U7-Size & 10.4266 & 0.0014 \\
\hline Sex & 1.0558 & 0.3054 \\
\hline \multirow[t]{2}{*}{ U7-Size-x-Sex } & 0.8867 & 0.3475 \\
\hline & \multicolumn{2}{|c|}{ Within Subjects } \\
\hline Side & 2.7926 & 0.0962 \\
\hline Side-x-U7-Size & 3.1184 & 0.0789 \\
\hline Side-x-Sex & 1.4311 & 0.2330 \\
\hline Side-x-U7-Size-x-Sex & 1.4803 & 0.2251 \\
\hline
\end{tabular}

*df for each test is 1 and 203. Means, Sexes Pooled L_R3-2 lapping $\quad 0.71$ L_L2-3 lapping $\quad 0.65$

Means, by Sex

$\begin{array}{ll}\text { Boy } & 0.640 .58 \\ \text { Girl } & 0.790 .70\end{array}$


Table A-208. ANCOVA results testing for associations between mesiodistal width of the maxillary second molar and L1-L2 overlap.

\begin{tabular}{llc}
\hline Source & F-Ratio & P-Value \\
\hline & \multicolumn{2}{c}{ Among Subjects } \\
U7-Size & 0.8461 & 0.3587 \\
Sex & 0.0619 & 0.8038 \\
U7-Size-x-Sex & 0.0424 & 0.8372 \\
& \multicolumn{2}{c}{ Within Subjects } \\
Side & 0.8045 & 0.3708 \\
Side-x-U7-Size & 0.7678 & 0.3819 \\
Side-x-Sex & 0.2667 & 0.6061 \\
Side-x-U7-Size-x-Sex & 0.3097 & 0.5785 \\
\hline
\end{tabular}

*df for each test is 1 and 203. Means, Sexes Pooled

L_R2-1 lapping $\quad 0.47$

L_L1-2 lapping $\quad 0.46$

Means, by Sex

$\begin{array}{ll}\text { Boy } & 0.440 .45 \\ \text { Girl } & 0.510 .47\end{array}$


Table A-209. ANCOVA results testing for associations between mesiodistal width of the maxillary second molar and total displacements.

\begin{tabular}{lcccc}
\hline Term & Estimate & SE & t-Test & P-Value \\
\hline Intercept & -3.4039 & 4.1240 & -0.83 & 0.4101 \\
U7-Size & 0.7697 & 0.4228 & 1.82 & 0.0702 \\
Sex & -1.0569 & 4.1240 & -0.26 & 0.7980 \\
Interaction & 0.0610 & 0.4228 & 0.14 & 0.8854 \\
\hline
\end{tabular}

Table A-210. ANCOVA results testing for associations between mesiodistal width of the maxillary second molar and total rotations.

\begin{tabular}{lrcrc}
\hline Term & Estimate & $\mathrm{SE}$ & $\mathrm{t}$-Test & P-Value \\
\hline Intercept & 2.6359 & 3.0615 & 0.86 & 0.3903 \\
U7-Size & 0.1656 & 0.3139 & 0.53 & 0.5983 \\
Sex & -0.9125 & 3.0615 & -0.30 & 0.7660 \\
Interaction & 0.0688 & 0.3139 & 0.22 & 0.8267 \\
\hline
\end{tabular}

Table A-211. ANCOVA results testing for associations between mesiodistal width of the maxillary second molar and maxillary R1-L1 overlap.

\begin{tabular}{lcccc}
\hline Term & Estimate & $\mathrm{SE}$ & $\mathrm{t}$-Test & P-Value \\
\hline Intercept & -0.5211 & 0.5654 & -0.92 & 0.3578 \\
U7-Size & 0.0793 & 0.0580 & 1.37 & 0.1730 \\
Sex & 0.7150 & 0.5654 & 1.26 & 0.2074 \\
Interaction & -0.0752 & 0.0580 & -1.30 & 0.1958 \\
\hline
\end{tabular}


Table A-212. ANCOVA results testing for associations between mesiodistal width of the maxillary second molar and mandibular R1-L1 overlap.

\begin{tabular}{lrcrc}
\hline Term & Estimate & $\mathrm{SE}$ & $\mathrm{t}$-Test & P-Value \\
\hline Intercept & 0.8642 & 0.5870 & 1.47 & 0.1425 \\
U7-Size & -0.0491 & 0.0602 & -0.82 & 0.4155 \\
Sex & 0.3251 & 0.5870 & 0.55 & 0.5803 \\
Interaction & -0.0222 & 0.0602 & -0.37 & 0.7131 \\
\hline
\end{tabular}

Table A-213. ANCOVA results testing for associations between mesiodistal width of the maxillary second molar and total overlapping.

\begin{tabular}{lcccc}
\hline Term & Estimate & SE & t-Test & P-Value \\
\hline Intercept & -3.0454 & 3.7829 & -0.81 & 0.4217 \\
U7-Size & 0.7587 & 0.3878 & 1.96 & 0.0518 \\
Sex & -0.9779 & 3.7829 & -0.26 & 0.7963 \\
Interaction & 0.0532 & 0.3878 & 0.14 & 0.8911 \\
\hline
\end{tabular}

Table A-214. ANCOVA results testing for associations between mesiodistal width of the maxillary second molar and total displacements, rotations, and overlapping.

\begin{tabular}{lcccc}
\hline Term & Estimate & SE & t-Test & P-Value \\
\hline Intercept & -3.8134 & 9.4122 & -0.41 & 0.6858 \\
U7-Size & 1.6941 & 0.9650 & 1.76 & 0.0807 \\
Sex & -2.9472 & 9.4122 & -0.31 & 0.7545 \\
Interaction & 0.1830 & 0.9650 & 0.19 & 0.8498 \\
\hline
\end{tabular}


Table A-215. ANCOVA results testing for associations between mesiodistal width of the maxillary second molar and crowding.

\begin{tabular}{lcccc}
\hline Term & Estimate & SE & t-Test & P-Value \\
\hline Intercept & -0.4792 & 0.8863 & -0.54 & 0.5893 \\
U7-Size & 0.1926 & 0.0909 & 2.12 & 0.0353 \\
Sex & 1.2458 & 0.8863 & 1.41 & 0.1614 \\
Interaction & -0.1406 & 0.0909 & -1.55 & 0.1235 \\
\hline
\end{tabular}

Table A-216. ANCOVA results testing for associations between mesiodistal width of the maxillary second molar and spacing.

\begin{tabular}{lcccc}
\hline Term & Estimate & SE & t-Test & P-Value \\
\hline Intercept & 2.0927 & 0.6652 & 3.15 & 0.0019 \\
U7-Size & -0.1896 & 0.0682 & -2.78 & 0.0060 \\
Sex & -0.6851 & 0.6652 & -1.03 & 0.3043 \\
Interaction & 0.0763 & 0.0682 & 1.12 & 0.2645 \\
\hline
\end{tabular}

Table A-217. ANCOVA results testing for associations between mesiodistal width of the maxillary second molar and diastema.

\begin{tabular}{lcccc}
\hline Term & Estimate & $\mathrm{SE}$ & $\mathrm{t}$-Test & P-Value \\
\hline Intercept & 1.5752 & 0.6871 & 2.29 & 0.0229 \\
U7-Size & -0.1382 & 0.0704 & -1.96 & 0.0511 \\
Sex & -0.2116 & 0.6871 & -0.31 & 0.7584 \\
Interaction & 0.0259 & 0.0704 & 0.37 & 0.7133 \\
\hline
\end{tabular}


Table A-218. ANCOVA results testing for associations between mesiodistal width of the maxillary second molar and maxillary incisor irregularity.

\begin{tabular}{lcccc}
\hline Term & Estimate & $\mathrm{SE}$ & $\mathrm{t}$-Test & P-Value \\
\hline Intercept & -0.5902 & 1.6341 & -0.36 & 0.7183 \\
U7-Size & 0.2094 & 0.1675 & 1.25 & 0.2129 \\
Sex & 1.2608 & 1.6341 & 0.77 & 0.4413 \\
Interaction] & -0.1450 & 0.1675 & -0.87 & 0.3877 \\
\hline
\end{tabular}

Table A-219. ANCOVA results testing for associations between mesiodistal width of the maxillary second molar and mandibular incisor irregularity.

\begin{tabular}{lcccc}
\hline Term & Estimate & SE & t-Test & P-Value \\
\hline Intercept & -1.4923 & 1.5081 & -0.99 & 0.3236 \\
U7-Size & 0.2673 & 0.1546 & 1.73 & 0.0854 \\
Sex & -1.2815 & 1.5081 & -0.85 & 0.3965 \\
Interaction & 0.1235 & 0.1546 & 0.80 & 0.4253 \\
\hline
\end{tabular}

Table A-220. ANCOVA results testing for associations between mesiodistal width of the maxillary second molar and maxillary overjet.

\begin{tabular}{lcccc}
\hline Term & Estimate & SE & t-Test & P-Value \\
\hline Intercept & 2.8039 & 2.7321 & 1.03 & 0.3060 \\
U7-Size & 0.0845 & 0.2801 & 0.30 & 0.7633 \\
Sex & 2.3066 & 2.7321 & 0.84 & 0.3995 \\
Interaction & -0.2443 & 0.2801 & -0.87 & 0.3841 \\
\hline
\end{tabular}


Table A-221. ANCOVA results testing for associations between mesiodistal width of the maxillary second molar and mandibular overjet.

\begin{tabular}{lcccc}
\hline Term & Estimate & SE & t-Test & P-Value \\
\hline Intercept & -0.3065 & 0.3280 & -0.93 & 0.3510 \\
U7-Size & 0.0360 & 0.0336 & 1.07 & 0.2853 \\
Sex & 0.3065 & 0.3280 & 0.93 & 0.3510 \\
Interaction & -0.0360 & 0.0336 & -1.07 & 0.2853 \\
\hline
\end{tabular}

Table A-222. ANCOVA results testing for associations between mesiodistal width of the maxillary second molar and openbite.

\begin{tabular}{lrcrc}
\hline Term & Estimate & $\mathrm{SE}$ & $\mathrm{t}$-Test & P-Value \\
\hline Intercept & 0.5753 & 0.5541 & 1.04 & 0.3004 \\
U7-Size & -0.0514 & 0.0568 & -0.91 & 0.3663 \\
Sex & 0.2765 & 0.5541 & 0.50 & 0.6183 \\
Interaction] & -0.0266 & 0.0568 & -0.47 & 0.6399 \\
\hline
\end{tabular}

Table A-223. ANCOVA results testing for associations between mesiodistal width of the maxillary second molar and anteroposterior relationship.

\begin{tabular}{lrcrc}
\hline Term & Estimate & $\mathrm{SE}$ & $\mathrm{t}$-Test & P-Value \\
\hline Intercept & 0.9409 & 0.8518 & 1.10 & 0.2706 \\
U7-Size & -0.0194 & 0.0873 & -0.22 & 0.8244 \\
Sex & 0.3751 & 0.8518 & 0.44 & 0.6601 \\
Interaction & -0.0344 & 0.0873 & -0.39 & 0.6941 \\
\hline
\end{tabular}


Table A-224. ANCOVA results testing for associations between mesiodistal width of the maxillary second molar and DAI score.

\begin{tabular}{lcccc}
\hline Term & Estimate & $\mathrm{SE}$ & $\mathrm{t}$-Test & P-Value \\
\hline Intercept & 26.6387 & 7.8401 & 3.40 & 0.0008 \\
U7-Size & 0.0489 & 0.8038 & 0.06 & 0.9515 \\
Sex & 7.4953 & 7.8401 & 0.96 & 0.3402 \\
Interaction & -0.8013 & 0.8038 & -1.00 & 0.3200 \\
\hline
\end{tabular}


Table A-225. ANCOVA results testing for associations between mesiodistal width of the mandibular central incisor and U3 displacements.

\begin{tabular}{llc}
\hline Source & F-Ratio* & P-Value \\
\hline \multicolumn{2}{c}{ Among Subjects } \\
L1-Size & 1.7098 & 0.1925 \\
Sex & 0.5932 & 0.4421 \\
L1-Size-x-Sex & 0.7422 & 0.3900 \\
& \multicolumn{2}{c}{ Within Subjects } \\
Side & 3.1878 & 0.0757 \\
Side-x-L1-Size & 2.9904 & 0.0853 \\
Side-x-Sex & 1.7609 & 0.1860 \\
Side-x-L1-Size-x-Sex & 1.8048 & 0.1806 \\
\hline
\end{tabular}

*df for each test is 1 and 203.

Means, Sexes Pooled

\begin{tabular}{lll} 
U_R3 dis & 0.41 & \\
U_L3 dis & 0.38 & \\
& Means, by Sex \\
\multicolumn{4}{c}{ U_R3 dis } & U_L3 dis \\
Boy & 0.360 .33 & \\
Girl & 0.490 .43 &
\end{tabular}


Table A-226. ANCOVA results testing for associations between mesiodistal width of the mandibular central incisor and $\mathrm{U} 2$ displacements.

\begin{tabular}{llc}
\hline Source & F-Ratio* & P-Value \\
\hline \multicolumn{2}{c}{ Among Subjects } \\
L1-Size & 3.3149 & 0.0701 \\
Sex & 0.9079 & 0.3418 \\
L1-Size-x-Sex & 1.2513 & 0.2646 \\
& \multicolumn{2}{c}{ Within Subjects } \\
Side & 0.9273 & 0.3367 \\
Side-x-L1-Size & 0.8707 & 0.3519 \\
Side-x-Sex & 5.2797 & 0.0226 \\
Side-x-L1-Size-x-Sex & 5.3651 & 0.0215 \\
\hline
\end{tabular}

* df for each test is 1 and 203.

Means, Sexes Pooled

\begin{tabular}{lrr} 
U_R2 dis & 0.37 & \\
U_L2 dis & 0.41 & \\
& Means, by Sex \\
\multicolumn{4}{c}{ U_R2 dis } & U_L2 dis \\
Boy & 0.290 .33 & \\
Girl & 0.490 .50 &
\end{tabular}


Table A-227. ANCOVA results testing for associations between mesiodistal width of the mandibular central incisor and U1 displacements.

\begin{tabular}{llc}
\hline Source & F-Ratio* & P-Value \\
\hline \multicolumn{2}{c}{ Among Subjects } \\
L1-Size & 2.2326 & 0.1367 \\
Sex & 0.2344 & 0.6288 \\
L1-Size-x-Sex & 0.2248 & 0.6359 \\
& \multicolumn{2}{c}{ Within Subjects } \\
Side & 0.7399 & 0.3907 \\
Side-x-L1-Size & 0.7935 & 0.3741 \\
Side-x-Sex & 0.1070 & 0.7439 \\
Side-x-L1-Size-x-Sex & 0.1550 & 0.6942 \\
\hline
\end{tabular}

*df for each test is 1 and 203.

Means, Sexes Pooled

U_R1 dis $\quad 0.34$

U_L1 dis $\quad 0.36$

Means, by Sex

U_R1 dis U_L1 dis

$\begin{array}{ll}\text { Boy } & 0.350 .33 \\ \text { Girl } & 0.320 .38\end{array}$ 
Table A-228. ANCOVA results testing for associations between mesiodistal width of the mandibular central incisor and L3 displacements.

\begin{tabular}{llc}
\hline Source & F-Ratio* & P-Value \\
\hline \multicolumn{2}{c}{ Among Subjects } \\
L1-Size & 3.5484 & 0.0610 \\
Sex & 0.1841 & 0.6684 \\
L1-Size-x-Sex & 0.2992 & 0.5850 \\
& \multicolumn{2}{c}{ Within Subjects } \\
Side & 2.0682 & 0.1519 \\
Side-x-L1-Size & 2.1437 & 0.1447 \\
Side-x-Sex & 2.2724 & 0.1333 \\
Side-x-L1-Size-x-Sex & 2.6492 & 0.1052 \\
\hline
\end{tabular}

*df for each test is 1 and 203.

Means, Sexes Pooled

\begin{tabular}{lll} 
L_R3 dis & 0.23 & \\
L_L3 dis & 0.23 & \\
\multicolumn{4}{c}{ Means, by Sex } \\
Boy & L_R3 dis & L_L3 dis \\
Girl & 0.150 .21 & \\
& 0.330 .24 &
\end{tabular}


Table A-229. ANCOVA results testing for associations between mesiodistal width of the mandibular central incisor and L2 displacements.

\begin{tabular}{lcc}
\hline Source & F-Ratio $^{*}$ & P-Value \\
\hline \multicolumn{3}{c}{ Among Subjects } \\
L1-Size & 17.8539 & $<0.0001$ \\
Sex & 0.2766 & 0.5995 \\
L1-Size-x-Sex & 0.4107 & 0.5223 \\
& \multicolumn{2}{c}{ Within Subjects } \\
Side & 0.1777 & 0.6738 \\
Side-x-L1-Size & 0.1398 & 0.7089 \\
Side-x-Sex & 1.3292 & 0.2503 \\
Side-x-L1-Size-x-Sex & 1.2667 & 0.2617 \\
\hline
\end{tabular}

*df for each test is 1 and 203.

Means, Sexes Pooled

\begin{tabular}{lrr} 
L_R2 dis & 0.51 & \\
L_L2 dis & 0.47 & \\
& Means, by Sex \\
\multicolumn{4}{c}{ L_R2 dis } & L_L2 dis \\
Boy & 0.440 .42 & \\
Girl & 0.600 .55 &
\end{tabular}


Table A-230. ANCOVA results testing for associations between mesiodistal width of the mandibular central incisor and L1 displacements.

\begin{tabular}{llc}
\hline Source & F-Ratio & P-Value \\
\hline \multicolumn{2}{c}{ Among Subjects } \\
L1-Size & 1.2995 & 0.2557 \\
Sex & 0.6198 & 0.4321 \\
L1-Size-x-Sex & 0.6730 & 0.4130 \\
& \multicolumn{2}{c}{ Within Subjects } \\
Side & 4.9063 & 0.0279 \\
Side-x-L1-Size & 5.3167 & 0.0221 \\
Side-x-Sex & 0.0240 & 0.8770 \\
Side-x-L1-Size-x-Sex & 0.0327 & 0.8568 \\
\hline
\end{tabular}

* $\mathrm{df}$ for each test is 1 and 203.

Means, Sexes Pooled

\begin{tabular}{lll} 
L_R1 dis & 0.22 & \\
L_L1 dis & 0.17 & \\
& Means, by Sex \\
\multicolumn{4}{c}{ L_R1 dis } & L_L1 dis \\
Boy & 0.240 .17 & \\
Girl & 0.200 .16 &
\end{tabular}


Table A-231. ANCOVA results testing for associations between mesiodistal width of the mandibular central incisor and $\mathrm{U} 3$ rotations.

\begin{tabular}{llc}
\hline Source & F-Ratio* & P-Value \\
\hline & \multicolumn{2}{c}{ Among Subjects } \\
L1-Size & 0.0251 & 0.8743 \\
Sex & 0.0029 & 0.9573 \\
L1-Size-x-Sex & 0.0070 & 0.9334 \\
& \multicolumn{2}{c}{ Within Subjects } \\
Side & 0.0026 & 0.9593 \\
Side-x-L1-Size & 0.0019 & 0.9650 \\
Side-x-Sex & 1.5681 & 0.2119 \\
Side-x-L1-Size-x-Sex & 1.4716 & 0.2265 \\
\hline
\end{tabular}

* $\mathrm{df}$ for each test is 1 and 203.

Means, Sexes Pooled

$\begin{array}{lll}\text { U_R3 rot } & 0.22 & \\ \text { U_L3 rot } & 0.22 & \\ & \text { Means, by Sex } \\ & \text { U_R3 rot } & \text { U_L3 rot } \\ \text { Boy } & 0.180 .15 & \\ \text { Girl } & 0.270 .29 & \end{array}$


Table A-232. ANCOVA results testing for associations between mesiodistal width of the mandibular central incisor and $\mathrm{U} 2$ rotations.

\begin{tabular}{llc}
\hline Source & F-Ratio* & P-Value \\
\hline & \multicolumn{2}{c}{ Among Subjects } \\
L1-Size & 2.2336 & 0.1366 \\
Sex & 0.1782 & 0.6733 \\
L1-Size-x-Sex & 0.2271 & 0.6342 \\
& \multicolumn{2}{c}{ Within Subjects } \\
Side & 4.4288 & 0.0366 \\
Side-x-L1-Size & 4.2864 & 0.0397 \\
Side-x-Sex & 0.1749 & 0.6762 \\
Side-x-L1-Size-x-Sex & 0.1980 & 0.6569 \\
\hline
\end{tabular}

*df for each test is 1 and 203.

Means, Sexes Pooled

\begin{tabular}{lll} 
U_R2 rot & 0.39 & \\
U_L2 rot & 0.42 & \\
& Means, by Sex \\
\multicolumn{4}{c}{ U_R2 rot } & U_L2 rot \\
Boy & 0.360 .40 & \\
Girl & 0.440 .44 &
\end{tabular}


Table A-233. ANCOVA results testing for associations between mesiodistal width of the mandibular central incisor and $\mathrm{U} 1$ rotations.

\begin{tabular}{llc}
\hline Source & F-Ratio* & P-Value \\
\hline \multicolumn{2}{c}{ Among Subjects } \\
L1-Size & 4.7790 & 0.0300 \\
Sex & 0.4198 & 0.5178 \\
L1-Size-x-Sex & 0.3742 & 0.5414 \\
& \multicolumn{2}{c}{ Within Subjects } \\
Side & 0.0226 & 0.8808 \\
Side-x-L1-Size & 0.0179 & 0.8937 \\
Side-x-Sex & 0.0567 & 0.8120 \\
Side-x-L1-Size-x-Sex & 0.0486 & 0.8257 \\
\hline
\end{tabular}

* $\mathrm{df}$ for each test is 1 and 203.

Means, Sexes Pooled

\begin{tabular}{lll} 
U_R1 rot & 0.47 & \\
U_L1 rot & 0.49 & \\
& Means, by Sex & \\
\multicolumn{4}{c}{ U_R1 rot } & U_L1 rot \\
Boy & 0.460 .45 & \\
Girl & 0.480 .51 &
\end{tabular}


Table A-234. ANCOVA results testing for associations between mesiodistal width of the mandibular central incisor and L3 rotations.

\begin{tabular}{lcc}
\hline Source & F-Ratio* & P-Value \\
\hline \multicolumn{3}{c}{ Among Subjects } \\
L1-Size & 5.7607 & 0.0173 \\
Sex & 0.0530 & 0.8182 \\
L1-Size-x-Sex & 0.1495 & 0.6994 \\
& \multicolumn{2}{c}{ Within Subjects } \\
Side & 16.8348 & $<0.0001$ \\
Side-x-L1-Size & 17.5465 & $<0.0001$ \\
Side-x-Sex & 3.5783 & 0.0600 \\
Side-x-L1-Size-x-Sex & 4.1979 & 0.0418 \\
\hline
\end{tabular}

*df for each test is 1 and 203.

Means, Sexes Pooled

\begin{tabular}{lll} 
L_R3 rot & 0.39 & \\
L_L3 rot & 0.35 & \\
& Means, by Sex & \\
\multicolumn{4}{c}{ L_R3 rot } & L_L3 rot \\
Boy & 0.270 .31 & \\
Girl & 0.540 .38 &
\end{tabular}


Table A-235. ANCOVA results testing for associations between mesiodistal width of the mandibular central incisor and $\mathrm{L} 2$ rotations.

\begin{tabular}{lcc}
\hline Source & F-Ratio* & P-Value \\
\hline \multicolumn{2}{c}{ Among Subjects } \\
L1-Size & 11.7520 & 0.0007 \\
Sex & 0.5784 & 0.4478 \\
L1-Size-x-Sex & 0.7161 & 0.3984 \\
& \multicolumn{2}{c}{ Within Subjects } \\
Side & 0.0362 & 0.8492 \\
Side-x-L1-Size & 0.0011 & 0.9733 \\
Side-x-Sex & 0.5571 & 0.4563 \\
Side-x-L1-Size-x-Sex & 0.4838 & 0.4875 \\
\hline
\end{tabular}

*df for each test is 1 and 203.

Means, Sexes Pooled

\begin{tabular}{lll} 
L_R2 rot & 0.35 & \\
L_L2 rot & 0.23 & \\
& Means, by Sex & \\
\multicolumn{4}{c}{ L_R2 rot } & L_L2 rot \\
Boy & 0.300 .22 & \\
Girl & 0.410 .26 &
\end{tabular}


Table A-236. ANCOVA results testing for associations between mesiodistal width of the mandibular central incisor and L1 rotations.

\begin{tabular}{lcc}
\hline Source & F-Ratio* & P-Value \\
\hline \multicolumn{3}{c}{ Among Subjects } \\
L1-Size & 3.8540 & 0.0510 \\
Sex & 2.2842 & 0.1322 \\
L1-Size-x-Sex & 2.5898 & 0.1091 \\
& \multicolumn{2}{c}{ Within Subjects } \\
Side & 0.6027 & 0.4385 \\
Side-x-L1-Size & 0.7023 & 0.4030 \\
Side-x-Sex & 0.1552 & 0.6940 \\
Side-x-L1-Size-x-Sex & 0.1325 & 0.7162 \\
\hline
\end{tabular}

*df for each test is 1 and 203.

Means, Sexes Pooled

$\begin{array}{lcc}\text { L_R1 rot } & 0.38 & \\ \text { L_L1 rot } & 0.34 & \\ & \text { Means, by Sex } \\ & \text { L_R1 rot } & \text { L_L1 rot } \\ \text { Boy } & 0.420 .36 & \\ \text { Girl } & 0.320 .30 & \end{array}$


Table A-237. ANCOVA results testing for associations between mesiodistal width of the mandibular central incisor and U2-U3 overlap.

\begin{tabular}{lcc}
\hline Source & F-Ratio* & P-Value \\
\hline \multicolumn{2}{c}{ Among Subjects } \\
L1-Size & 5.8401 & 0.0165 \\
Sex & 2.2224 & 0.1376 \\
L1-Size-x-Sex & 2.7934 & 0.0962 \\
& \multicolumn{2}{c}{ Within Subjects } \\
Side & 1.7994 & 0.1813 \\
Side-x-L1-Size & 1.7322 & 0.1896 \\
Side-x-Sex & 0.1805 & 0.6714 \\
Side-x-L1-Size-x-Sex & 0.2034 & 0.6525 \\
\hline
\end{tabular}

*df for each test is 1 and 203.

Means, Sexes Pooled

$\begin{array}{ll}\text { U_R3-2 lapping } & 0.38 \\ \text { U_L2-3 lapping } & 0.37 \\ \text { Means, by Sex } & \end{array}$

Boy

U_R3-2 lapping U_L2-3 lapping

Girl

0.280 .28

0.520 .48 
Table A-238. ANCOVA results testing for associations between mesiodistal width of the mandibular central incisor and U1-U2 overlap.

\begin{tabular}{llc}
\hline Source & F-Ratio $^{*}$ & P-Value \\
\hline \multicolumn{2}{c}{ Among Subjects } \\
L1-Size & 3.0197 & 0.0838 \\
Sex & 1.8515 & 0.1751 \\
L1-Size-x-Sex & 1.3339 & 0.2495 \\
& \multicolumn{2}{c}{ Within Subjects } \\
Side & 0.0000 & 0.9998 \\
Side-x-L1-Size & 0.0031 & 0.9560 \\
Side-x-Sex & 0.0344 & 0.8531 \\
Side-x-L1-Size-x-Sex & 0.0441 & 0.8338 \\
\hline
\end{tabular}

*df for each test is 1 and 203.

Means, Sexes Pooled

$\begin{array}{ll}\text { U_R2-1 lapping } & 0.35 \\ \text { U_L1-2 lapping } & 0.31 \\ \text { Means, by Sex } & \end{array}$

Boy

U_R2-1 lapping U_L1-2 lapping

Girl

0.230 .21

0.460 .40 
Table A-239. ANCOVA results testing for associations between mesiodistal width of the mandibular central incisor and L2-L3 overlap.

\begin{tabular}{lcc}
\hline Source & F-Ratio* & P-Value \\
\hline \multicolumn{3}{c}{ Among Subjects } \\
L1-Size & 33.4639 & $<0.0001$ \\
Sex & 0.6692 & 0.4143 \\
L1-Size-x-Sex & 0.4723 & 0.4927 \\
& \multicolumn{2}{c}{ Within Subjects } \\
Side & 11.1283 & 0.0010 \\
Side-x-L1-Size & 11.7642 & 0.0007 \\
Side-x-Sex & 0.0573 & 0.8111 \\
Side-x-L1-Size-x-Sex & 0.0794 & 0.7784 \\
\hline
\end{tabular}

*df for each test is 1 and 203.

Means, Sexes Pooled

\begin{tabular}{lrr} 
L_R3-2 lapping & 0.71 & \\
L_L2-3 lapping & 0.65 & \\
Means, by Sex & \\
\multicolumn{4}{c}{ L_R3-2 lapping } & L_L2-3 lapping \\
Boy & 0.610 .58 & \\
Girl & 0.820 .72 &
\end{tabular}


Table A-240. ANCOVA results testing for associations between mesiodistal width of the mandibular central incisor and L1-L2 overlap.

\begin{tabular}{lcc}
\hline Source & F-Ratio $^{*}$ & P-Value \\
\hline \multicolumn{2}{c}{ Among Subjects } \\
L1-Size & 15.1042 & 0.0001 \\
Sex & 0.2059 & 0.6505 \\
L1-Size-x-Sex & 0.2943 & 0.5881 \\
& \multicolumn{2}{c}{ Within Subjects } \\
Side & 0.0010 & 0.9754 \\
Side-x-L1-Size & 0.0037 & 0.9515 \\
Side-x-Sex & 0.9580 & 0.3289 \\
Side-x-L1-Size-x-Sex & 1.0754 & 0.3010 \\
\hline
\end{tabular}

*df for each test is 1 and 203.

Means, Sexes Pooled

\begin{tabular}{|c|c|c|}
\hline L_R2-1 lapping & 0.47 & \\
\hline L_L1-2 lapping & 0.46 & \\
\hline \multicolumn{3}{|c|}{ Means, by Sex } \\
\hline & L_R2-1 lapping & L_L1-2 lapping \\
\hline Boy & 0.420 .44 & \\
\hline Girl & 0.540 .48 & \\
\hline
\end{tabular}


Table A-241. ANCOVA results testing for associations between mesiodistal width of the mandibular central incisor and total displacements.

\begin{tabular}{lrcrc}
\hline Term & Estimate & $\mathrm{SE}$ & $\mathrm{t}$-Test & P-Value \\
\hline Intercept & -8.7843 & 3.6016 & -2.44 & 0.0156 \\
L1 & 2.3935 & 0.6667 & 3.59 & 0.0004 \\
Sex & 2.1005 & 3.6016 & 0.58 & 0.5604 \\
Interaction & -0.4890 & 0.6667 & -0.73 & 0.4641 \\
\hline
\end{tabular}

Table A-242. ANCOVA results testing for associations between mesiodistal width of the mandibular central incisor and total rotations.

\begin{tabular}{lccrc}
\hline Term & Estimate & SE & t-Test & P-Value \\
\hline Intercept & -7.3144 & 2.6112 & -2.80 & 0.0056 \\
L1 & 2.1426 & 0.4833 & 4.43 & $<0.0001$ \\
Sex & -0.6636 & 2.6112 & -0.25 & 0.7996 \\
Interaction & 0.0531 & 0.4833 & 0.11 & 0.9127 \\
\hline
\end{tabular}

Table A-243. ANCOVA results testing for associations between mesiodistal width of the mandibular central incisor and maxillary R1-L1 overlap.

\begin{tabular}{lcccc}
\hline Term & Estimate & $\mathrm{SE}$ & $\mathrm{t}$-Test & P-Value \\
\hline Intercept & -0.6916 & 0.5049 & -1.37 & 0.1722 \\
L1 & 0.1735 & 0.0935 & 1.86 & 0.0649 \\
Sex & 0.2208 & 0.5049 & 0.44 & 0.6624 \\
Interaction & -0.0447 & 0.0935 & -0.48 & 0.6327 \\
\hline
\end{tabular}


Table A-244. ANCOVA results testing for associations between mesiodistal width of the mandibular central incisor and mandibular R1-L1 overlap.

\begin{tabular}{lcccc}
\hline Term & Estimate & $\mathrm{SE}$ & $\mathrm{t}$-Test & P-Value \\
\hline Intercept & -1.0618 & 0.5117 & -2.08 & 0.0392 \\
L1 & 0.2658 & 0.0947 & 2.81 & 0.0055 \\
Sex & -0.6414 & 0.5117 & -1.25 & 0.2115 \\
Interaction & 0.1343 & 0.0947 & 1.42 & 0.1578 \\
\hline
\end{tabular}

Table A-245. ANCOVA results testing for associations between mesiodistal width of the mandibular central incisor and total overlapping.

\begin{tabular}{lrcrc}
\hline Term & Estimate & SE & t-Test & P-Value \\
\hline Intercept & -13.6621 & 3.1643 & -4.32 & $<0.0001$ \\
L1 & 3.3366 & 0.5857 & 5.70 & $<0.0001$ \\
Sex & -0.6826 & 3.1643 & -0.22 & 0.8294 \\
Interaction & 0.0140 & 0.5857 & 0.02 & 0.9810 \\
\hline
\end{tabular}

Table A-246. ANCOVA results testing for associations between mesiodistal width of the mandibular central incisor and total displacements, rotations, and overlapping.

\begin{tabular}{lcccc}
\hline Term & Estimate & $\mathrm{SE}$ & $\mathrm{t}$-Test & P-Value \\
\hline Intercept & -29.7607 & 7.9280 & -3.75 & 0.0002 \\
L1 & 7.8728 & 1.4675 & 5.36 & $<0.0001$ \\
Sex & 0.7544 & 7.9280 & 0.10 & 0.9243 \\
Interaction & -0.4219 & 1.4675 & -0.29 & 0.7740 \\
\hline
\end{tabular}


Table A-247. ANCOVA results testing for associations between mesiodistal width of the mandibular central incisor and crowding.

\begin{tabular}{lcccc}
\hline Term & Estimate & SE & t-Test & P-Value \\
\hline Intercept & -3.2503 & 0.7354 & -4.42 & $<0.0001$ \\
L1 & 0.8590 & 0.1361 & 6.31 & $<0.0001$ \\
Sex & 0.4132 & 0.7354 & 0.56 & 0.5749 \\
Interaction & -0.1066 & 0.1361 & -0.78 & 0.4346 \\
\hline
\end{tabular}

Table A-248. ANCOVA results testing for associations between mesiodistal width of the mandibular central incisor and spacing.

\begin{tabular}{lcccc}
\hline Term & Estimate & SE & t-Test & P-Value \\
\hline Intercept & 2.9875 & 0.5738 & 5.21 & $<0.0001$ \\
L1 & -0.5084 & 0.1062 & -4.79 & $<0.0001$ \\
Sex & -0.8559 & 0.5738 & -1.49 & 0.1374 \\
Interaction & 0.1718 & 0.1062 & 1.62 & 0.1073 \\
\hline
\end{tabular}

Table A-249. ANCOVA results testing for associations between mesiodistal width of the mandibular central incisor and diastema.

\begin{tabular}{lcccc}
\hline Term & Estimate & $\mathrm{SE}$ & $\mathrm{t}$-Test & P-Value \\
\hline Intercept & 1.9073 & 0.6041 & 3.16 & 0.0018 \\
L1 & -0.3134 & 0.1118 & -2.80 & 0.0056 \\
Sex & -1.1627 & 0.6041 & -1.92 & 0.0557 \\
Interaction & 0.2239 & 0.1118 & 2.00 & 0.0466 \\
\hline
\end{tabular}


Table A-250. ANCOVA results testing for associations between mesiodistal width of the mandibular central incisor and maxillary incisor irregularity.

\begin{tabular}{lcccc}
\hline Term & Estimate & SE & t-Test & P-Value \\
\hline Intercept & -2.2031 & 1.4441 & -1.53 & 0.1287 \\
L1 & 0.6762 & 0.2673 & 2.53 & 0.0122 \\
Sex & 1.0209 & 1.4441 & 0.71 & 0.4804 \\
Interaction & -0.2216 & 0.2673 & -0.83 & 0.4082 \\
\hline
\end{tabular}

Table A-251. ANCOVA results testing for associations between mesiodistal width of the mandibular central incisor and mandibular incisor irregularity.

\begin{tabular}{lcccc}
\hline Term & Estimate & SE & t-Test & P-Value \\
\hline Intercept & -4.7615 & 1.2947 & -3.68 & 0.0003 \\
L1 & 1.0938 & 0.2397 & 4.56 & $<0.0001$ \\
Sex & 1.1092 & 1.2947 & 0.86 & 0.3926 \\
Interaction & -0.2284 & 0.2397 & -0.95 & 0.3418 \\
\hline
\end{tabular}

Table A-252. ANCOVA results testing for associations between mesiodistal width of the mandibular central incisor and maxillary overjet.

\begin{tabular}{lcccc}
\hline Term & Estimate & $\mathrm{SE}$ & $\mathrm{t}$-Test & P-Value \\
\hline Intercept & -1.4257 & 2.4180 & -0.59 & 0.5561 \\
L1 & 0.9350 & 0.4476 & 2.09 & 0.0380 \\
Sex & 1.8411 & 2.4180 & 0.76 & 0.4473 \\
Interaction & -0.3653 & 0.4476 & -0.82 & 0.4153 \\
\hline
\end{tabular}


Table A-253. ANCOVA results testing for associations between mesiodistal width of the mandibular central incisor and mandibular overjet.

\begin{tabular}{lcccc}
\hline Term & Estimate & $\mathrm{SE}$ & $\mathrm{t}$-Test & P-Value \\
\hline Intercept & -0.4699 & 0.2905 & -1.62 & 0.1074 \\
L1 & 0.0957 & 0.0538 & 1.78 & 0.0766 \\
Sex & 0.4699 & 0.2905 & 1.62 & 0.1074 \\
Interaction & -0.0957 & 0.0538 & -1.78 & 0.0766 \\
\hline
\end{tabular}

Table A-254. ANCOVA results testing for associations between mesiodistal width of the mandibular central incisor and openbite.

\begin{tabular}{lcccc}
\hline Term & Estimate & SE & t-Test & P-Value \\
\hline Intercept & -0.0772 & 0.4936 & -0.16 & 0.8759 \\
L1 & 0.0292 & 0.0914 & 0.32 & 0.7494 \\
Sex & 0.7478 & 0.4936 & 1.51 & 0.1314 \\
Interaction & -0.1369 & 0.0914 & -1.50 & 0.1356 \\
\hline
\end{tabular}

Table A-255. ANCOVA results testing for associations between mesiodistal width of the mandibular central incisor and anteroposterior relationship.

\begin{tabular}{lrcrc}
\hline Term & Estimate & $\mathrm{SE}$ & $\mathrm{t}$-Test & P-Value \\
\hline Intercept & 1.5684 & 0.7553 & 2.08 & 0.0391 \\
L1 & -0.1495 & 0.1398 & -1.07 & 0.2861 \\
Sex & 1.0469 & 0.7553 & 1.39 & 0.1672 \\
Interaction & -0.1849 & 0.1398 & -1.32 & 0.1874 \\
\hline
\end{tabular}


Table A-256. ANCOVA results testing for associations between mesiodistal width of the mandibular central incisor and DAI score.

\begin{tabular}{lcccc}
\hline Term & Estimate & $\mathrm{SE}$ & $\mathrm{t}$-Test & P-Value \\
\hline Intercept & 12.8777 & 6.9323 & 1.86 & 0.0647 \\
L1 & 2.6399 & 1.2832 & 2.06 & 0.0409 \\
Sex & 8.2019 & 6.9323 & 1.18 & 0.2381 \\
Interaction & -1.6115 & 1.2832 & -1.26 & 0.2106 \\
\hline
\end{tabular}


Table A-257. ANCOVA results testing for associations between mesiodistal width of the mandibular lateral incisor and U3 displacements.

\begin{tabular}{llc}
\hline Source & F-Ratio* & P-Value \\
\hline & \multicolumn{2}{c}{ Among Subjects } \\
L2-Size & 2.3615 & 0.1259 \\
Sex & 1.2715 & 0.2608 \\
L2-Size-x-Sex & 1.0715 & 0.3018 \\
& \multicolumn{2}{c}{ Within Subjects } \\
Side & 2.0535 & 0.1534 \\
Side-x-L2-Size & 1.8918 & 0.1705 \\
Side-x-Sex & 2.6725 & 0.1036 \\
Side-x-L2-Size-x-Sex & 2.7606 & 0.0982 \\
\hline
\end{tabular}

* $\mathrm{df}$ for each test is 1 and 203.

Means, Sexes Pooled

U_R3 dis $\quad 0.41$

U_L3 dis $\quad 0.38$

Means, by Sex

$\begin{array}{lrl} & \text { Boy } & 0.340 .32 \\ \text { Girl } & 0.470 .41\end{array}$


Table A-258. ANCOVA results testing for associations between mesiodistal width of the mandibular lateral incisor and U2 displacements.

\begin{tabular}{llc}
\hline Source & F-Ratio* & P-Value \\
\hline \multicolumn{2}{c}{ Among Subjects } \\
L2-Size & 6.1262 & 0.0141 \\
Sex & 0.2168 & 0.6420 \\
L2-Size-x-Sex & 0.4089 & 0.5233 \\
& \multicolumn{2}{c}{ Within Subjects } \\
Side & 3.8872 & 0.0500 \\
Side-x-L2-Size & 3.7535 & 0.0541 \\
Side-x-Sex & 7.3560 & 0.0073 \\
Side-x-L2-Size-x-Sex & 7.5043 & 0.0067 \\
\hline
\end{tabular}

* $\mathrm{df}$ for each test is 1 and 203.

Means, Sexes Pooled

$\begin{array}{ll}\text { U_R2 dis } & 0.37 \\ \text { U_L2 dis } & 0.41\end{array}$

Means, by Sex

$\begin{array}{lr}\text { Boy } & 0.280 .32 \\ \text { Girl } & 0.490 .49\end{array}$


Table A-259. ANCOVA results testing for associations between mesiodistal width of the mandibular lateral incisor and U1 displacements.

\begin{tabular}{llc}
\hline Source & F-Ratio* & P-Value \\
\hline \multicolumn{2}{c}{ Among Subjects } \\
L2-Size & 1.0635 & 0.3036 \\
Sex & 0.2798 & 0.5974 \\
L2-Size-x-Sex & 0.2793 & 0.5978 \\
& \multicolumn{2}{c}{ Within Subjects } \\
Side & 0.5008 & 0.4799 \\
Side-x-L2-Size & 0.5263 & 0.4690 \\
Side-x-Sex & 0.7309 & 0.3936 \\
Side-x-L2-Size-x-Sex & 0.6255 & 0.4299 \\
\hline
\end{tabular}

* $\mathrm{df}$ for each test is 1 and 203.

Means, Sexes Pooled

U_R1 dis $\quad 0.34$

U_L1 dis $\quad 0.36$

Means, by Sex

$\begin{array}{lr}\text { Boy } & 0.360 .33 \\ \text { Girl } & 0.320 .37\end{array}$


Table A-260. ANCOVA results testing for associations between mesiodistal width of the mandibular lateral incisor and L3 displacements.

\begin{tabular}{llc}
\hline Source & F-Ratio* & P-Value \\
\hline & \multicolumn{2}{c}{ Among Subjects } \\
L2-Size & 8.1833 & 0.0047 \\
Sex & 1.0472 & 0.3074 \\
L2-Size-x-Sex & 0.8023 & 0.3715 \\
& \multicolumn{2}{c}{ Within Subjects } \\
Side & 0.2017 & 0.6538 \\
Side-x-L2-Size & 0.1944 & 0.6598 \\
Side-x-Sex & 0.1357 & 0.7130 \\
Side-x-L2-Size-x-Sex & 0.2177 & 0.6413 \\
\hline
\end{tabular}

* $\mathrm{df}$ for each test is 1 and 203.

Means, Sexes Pooled

$\begin{array}{ll}\text { L_R3 dis } & 0.23 \\ \text { L_L3 dis } & 0.23\end{array}$

Means, by Sex

$\begin{array}{lrl} & \text { L_R3 dis } & \text { L_L3 dis } \\ \text { Boy } & 0.140 .20 & \\ \text { Girl } & 0.310 .25 & \end{array}$


Table A-261. ANCOVA results testing for associations between mesiodistal width of the mandibular lateral incisor and L2 displacements.

\begin{tabular}{lcc}
\hline Source & F-Ratio* & P-Value \\
\hline \multicolumn{3}{c}{ Among Subjects } \\
L2-Size & 25.1451 & $<0.0001$ \\
Sex & 0.0376 & 0.8465 \\
L2-Size-x-Sex & 0.0953 & 0.7579 \\
& \multicolumn{2}{c}{ Within Subjects } \\
Side & 0.3564 & 0.5512 \\
Side-x-L2-Size & 0.4239 & 0.5158 \\
Side-x-Sex & 1.3222 & 0.2515 \\
Side-x-L2-Size-x-Sex & 1.2301 & 0.2687 \\
\hline
\end{tabular}

*df for each test is 1 and 203.

Means, Sexes Pooled

L_R2 dis $\quad 0.51$

L_L2 dis $\quad 0.47$

Means, by Sex

$\begin{array}{ll}\text { Boy } & 0.430 .43 \\ \text { Girl } & 0.590 .54\end{array}$


Table A-262. ANCOVA results testing for associations between mesiodistal width of the mandibular lateral incisor and L1 displacements.

\begin{tabular}{llc}
\hline Source & F-Ratio* & P-Value \\
\hline & \multicolumn{2}{c}{ Among Subjects } \\
L2-Size & 2.8685 & 0.0919 \\
Sex & 0.8057 & 0.3704 \\
L2-Size-x-Sex & 0.8653 & 0.3534 \\
& \multicolumn{2}{c}{ Within Subjects } \\
Side & 4.1360 & 0.0433 \\
Side-x-L2-Size & 4.5452 & 0.0342 \\
Side-x-Sex & 0.0350 & 0.8518 \\
Side-x-L2-Size-x-Sex & 0.0228 & 0.8800 \\
\hline
\end{tabular}

* $\mathrm{df}$ for each test is 1 and 203.

Means, Sexes Pooled

L_R1 dis $\quad 0.22$

L_L1 dis $\quad 0.17$

Means, by Sex

$\begin{array}{ll}\text { Boy } & 0.240 .17 \\ \text { Girl } & 0.200 .17\end{array}$


Table A-263. ANCOVA results testing for associations between mesiodistal width of the mandibular lateral incisor and U3 rotations.

\begin{tabular}{llc}
\hline Source & F-Ratio* & P-Value \\
\hline & \multicolumn{2}{c}{ Among Subjects } \\
L2-Size & 0.0027 & 0.9587 \\
Sex & 0.0820 & 0.7749 \\
L2-Size-x-Sex & 0.0215 & 0.8837 \\
& \multicolumn{2}{c}{ Within Subjects } \\
Side & 0.0312 & 0.8600 \\
Side-x-L2-Size & 0.0309 & 0.8606 \\
Side-x-Sex & 1.0241 & 0.3128 \\
Side-x-L2-Size-x-Sex & 0.9408 & 0.3332 \\
\hline
\end{tabular}

* df for each test is 1 and 203.

Means, Sexes Pooled

$\begin{array}{ll}\text { U_R3 rot } & 0.22 \\ \text { U_L3 rot } & 0.22\end{array}$

Means, by Sex

$\begin{array}{ll}\text { Boy } & 0.180 .15 \\ \text { Girl } & 0.260 .29\end{array}$


Table A-264. ANCOVA results testing for associations between mesiodistal width of the mandibular lateral incisor and $\mathrm{U} 2$ rotations.

\begin{tabular}{llc}
\hline Source & F-Ratio* & P-Value \\
\hline & \multicolumn{2}{c}{ Among Subjects } \\
L2-Size & 3.2601 & 0.0725 \\
Sex & 0.2225 & 0.6377 \\
L2-Size-x-Sex & 0.2775 & 0.5989 \\
& \multicolumn{2}{c}{ Within Subjects } \\
Side & 1.1080 & 0.2938 \\
Side-x-L2-Size & 1.0219 & 0.3133 \\
Side-x-Sex & 0.0002 & 0.9899 \\
Side-x-L2-Size-x-Sex & 0.0005 & 0.9825 \\
\hline
\end{tabular}

* df for each test is 1 and 203.

Means, Sexes Pooled

$\begin{array}{ll}\text { U_R2 rot } & 0.39 \\ \text { U_L2 rot } & 0.42\end{array}$

Means, by Sex

$\begin{array}{ll}\text { Boy } & 0.360 .40 \\ \text { Girl } & 0.430 .45\end{array}$


Table A-265. ANCOVA results testing for associations between mesiodistal width of the mandibular lateral incisor and U1 rotations.

\begin{tabular}{lcc}
\hline Source & F-Ratio* & P-Value \\
\hline & \multicolumn{2}{c}{ Among Subjects } \\
L2-Size & 4.0381 & 0.0458 \\
Sex & 0.3688 & 0.5443 \\
L2-Size-x-Sex & 0.4051 & 0.5252 \\
& \multicolumn{2}{c}{ Within Subjects } \\
Side & 0.3167 & 0.5742 \\
Side-x-L2-Size & 0.3321 & 0.5650 \\
Side-x-Sex & 0.4671 & 0.4951 \\
Side-x-L2-Size-x-Sex & 0.4321 & 0.5117 \\
\hline
\end{tabular}

* $\mathrm{df}$ for each test is 1 and 203.

Means, Sexes Pooled

$$
\begin{array}{ll}
\text { U_R1 rot } & 0.47 \\
\text { U_L1 rot } & 0.49
\end{array}
$$

Means, by Sex

$\begin{array}{lrl} & \text { U_R1 rot } & \text { U_L1 rot } \\ \text { Boy } & 0.470 .46 & \\ \text { Girl } & 0.490 .52 & \end{array}$


Table A-266. ANCOVA results testing for associations between mesiodistal width of the mandibular lateral incisor and L3 rotations.

\begin{tabular}{lcc}
\hline Source & F-Ratio* & P-Value \\
\hline \multicolumn{2}{c}{ Among Subjects } \\
L2-Size & 11.7829 & 0.0007 \\
Sex & 1.6299 & 0.2032 \\
L2-Size-x-Sex & 2.0883 & 0.1500 \\
& \multicolumn{2}{c}{ Within Subjects } \\
Side & 1.8783 & 0.1720 \\
Side-x-L2-Size & 2.0642 & 0.1523 \\
Side-x-Sex & 0.5881 & 0.4440 \\
Side-x-L2-Size-x-Sex & 0.7786 & 0.3786 \\
\hline
\end{tabular}

* df for each test is 1 and 203.

Means, Sexes Pooled

L_R3 rot $\quad 0.39$

L_L3 rot 0.35

Means, by Sex

Boy

L_R3 rot L_L3 rot

Girl

0.280 .31

0.530 .41 
Table A-267. ANCOVA results testing for associations between mesiodistal width of the mandibular lateral incisor and L2 rotations.

\begin{tabular}{llc}
\hline Source & F-Ratio* & P-Value \\
\hline \multicolumn{2}{c}{ Among Subjects } \\
L2-Size & 9.3500 & 0.0025 \\
Sex & 2.4267 & 0.1208 \\
L2-Size-x-Sex & 2.6631 & 0.1042 \\
& \multicolumn{2}{c}{ Within Subjects } \\
Side & 0.7128 & 0.3995 \\
Side-x-L2-Size & 1.0601 & 0.3044 \\
Side-x-Sex & 1.0002 & 0.3184 \\
Side-x-L2-Size-x-Sex & 1.1355 & 0.2879 \\
\hline
\end{tabular}

* df for each test is 1 and 203.

Means, Sexes Pooled

$\begin{array}{ll}\text { L_R2 rot } & 0.35 \\ \text { L_L2 rot } & 0.23\end{array}$

Means, by Sex

$\begin{array}{ll}\text { Boy } & 0.310 .23 \\ \text { Girl } & 0.420 .24\end{array}$


Table A-268. ANCOVA results testing for associations between mesiodistal width of the mandibular lateral incisor and L1 rotations.

\begin{tabular}{llc}
\hline Source & F-Ratio* & P-Value \\
\hline \multicolumn{2}{c}{ Among Subjects } \\
L2-Size & 0.0711 & 0.7901 \\
Sex & 0.1445 & 0.7042 \\
L2-Size-x-Sex & 0.2538 & 0.6149 \\
& \multicolumn{2}{c}{ Within Subjects } \\
Side & 0.5405 & 0.4631 \\
Side-x-L2-Size & 0.6396 & 0.4248 \\
Side-x-Sex & 0.3035 & 0.5823 \\
Side-x-L2-Size-x-Sex & 0.2669 & 0.6060 \\
\hline
\end{tabular}

* $\mathrm{df}$ for each test is 1 and 203.

Means, Sexes Pooled

L_R1 rot $\quad 0.38$

L_L1 rot $\quad 0.34$

Means, by Sex

Boy

L_R1 rot L_L1 rot

Girl

0.440 .37

0.310 .29 
Table A-269. ANCOVA results testing for associations between mesiodistal width of the mandibular lateral incisor and U2-U3 overlap.

\begin{tabular}{llc}
\hline Source & F-Ratio* $^{*}$ & P-Value \\
\hline \multicolumn{2}{c}{ Among Subjects } \\
L2-Size & 3.1740 & 0.0763 \\
Sex & 0.7905 & 0.3750 \\
L2-Size-x-Sex & 0.5165 & 0.4732 \\
& \multicolumn{2}{c}{ Within Subjects } \\
Side & 1.1038 & 0.2947 \\
Side-x-L2-Size & 1.0302 & 0.3113 \\
Side-x-Sex & 1.9755 & 0.1614 \\
Side-x-L2-Size-x-Sex & 2.0765 & 0.1511 \\
\hline
\end{tabular}

* $\mathrm{df}$ for each test is 1 and 203. Means, Sexes Pooled U_R3-2 lapping $\quad 0.38$ U_L2-3 lapping $\quad 0.37$

Means, by Sex

U_R3-2 lapping U_L2-3 lapping

Boy

0.280 .27

Girl

0.500 .45 
Table A-270. ANCOVA results testing for associations between mesiodistal width of the mandibular lateral incisor and U1-U2 overlap.

\begin{tabular}{llc}
\hline Source & F-Ratio* & P-Value \\
\hline & \multicolumn{2}{c}{ Among Subjects } \\
L2-Size & 3.2929 & 0.0711 \\
Sex & 0.1679 & 0.6825 \\
L2-Size-x-Sex & 0.0403 & 0.8410 \\
& \multicolumn{2}{c}{ Within Subjects } \\
Side & 0.0296 & 0.8637 \\
Side-x-L2-Size & 0.0121 & 0.9126 \\
Side-x-Sex & 0.4900 & 0.4847 \\
Side-x-L2-Size-x-Sex & 0.5241 & 0.4699 \\
\hline
\end{tabular}

*df for each test is 1 and 203. Means, Sexes Pooled U_R2-1 lapping $\quad 0.35$ U_L1-2 lapping $\quad 0.31$

Means, by Sex

U_R2-1 lapping U_L1-2 lapping

Boy

0.250 .22

Girl

0.460 .41 
Table A-271. ANCOVA results testing for associations between mesiodistal width of the mandibular lateral incisor and L2-L3 overlap.

\begin{tabular}{lcc}
\hline Source & F-Ratio* $^{*}$ & P-Value \\
\hline \multicolumn{2}{c}{ Among Subjects } \\
L2-Size & 35.9868 & $<0.0001$ \\
Sex & 0.8300 & 0.3634 \\
L2-Size-x-Sex & 0.6264 & 0.4296 \\
& \multicolumn{2}{c}{ Within Subjects } \\
Side & 8.0521 & 0.0050 \\
Side-x-L2-Size & 8.6347 & 0.0037 \\
Side-x-Sex & 0.5163 & 0.4732 \\
Side-x-L2-Size-x-Sex & 0.5572 & 0.4563 \\
\hline
\end{tabular}

*df for each test is 1 and 203. Means, Sexes Pooled L_R3-2 lapping $\quad 0.71$ L_L2-3 lapping $\quad 0.65$

Means, by Sex

$\begin{array}{ll}\text { Boy } & 0.630 .58 \\ \text { Girl } & 0.80\end{array}$


Table A-272. ANCOVA results testing for associations between mesiodistal width of the mandibular lateral incisor and L1-L2 overlap.

\begin{tabular}{lcc}
\hline Source & F-Ratio & \multicolumn{2}{c}{ P-Value } \\
\hline \multicolumn{3}{c}{ Among Subjects } \\
L2-Size & 17.3411 & $<0.0001$ \\
Sex & 0.0197 & 0.8885 \\
L2-Size-x-Sex & 0.0497 & 0.8239 \\
& \multicolumn{2}{c}{ Within Subjects } \\
Side & 0.0156 & 0.9008 \\
Side-x-L2-Size & 0.0241 & 0.8768 \\
Side-x-Sex & 1.4727 & 0.2263 \\
Side-x-L2-Size-x-Sex & 1.6259 & 0.2037 \\
\hline
\end{tabular}

*df for each test is 1 and 203. Means, Sexes Pooled

L_R2-1 lapping $\quad 0.47$

L_L1-2 lapping $\quad 0.46$

Means, by Sex

$\begin{array}{ll}\text { Boy } & 0.430 .45 \\ \text { Girl } & 0.540 .48\end{array}$


Table A-273. ANCOVA results testing for associations between mesiodistal width of the mandibular lateral incisor and total displacements.

\begin{tabular}{lcccc}
\hline Term & Estimate & SE & t-Test & P-Value \\
\hline Intercept & -10.8407 & 3.4006 & -3.19 & 0.0017 \\
L2-Size & 2.5275 & 0.5757 & 4.39 & $<0.0001$ \\
Sex & -2.5989 & 3.4006 & -0.76 & 0.4456 \\
Interaction & 0.3512 & 0.5757 & 0.61 & 0.5425 \\
\hline
\end{tabular}

Table A-274. ANCOVA results testing for associations between mesiodistal width of the mandibular lateral incisor and total rotations.

\begin{tabular}{lcccc}
\hline Term & Estimate & SE & t-Test & P-Value \\
\hline Intercept & -6.1933 & 2.5244 & -2.45 & 0.0150 \\
L2-Size & 1.7769 & 0.4273 & 4.16 & $<0.0001$ \\
Sex & 3.3032 & 2.5244 & 1.31 & 0.1922 \\
Interaction & -0.6160 & 0.4273 & -1.44 & 0.1510 \\
\hline
\end{tabular}

Table A-275. ANCOVA results testing for associations between mesiodistal width of the mandibular lateral incisor and maxillary R1-L1 overlap.

\begin{tabular}{lcccc}
\hline Term & Estimate & $\mathrm{SE}$ & $\mathrm{t}$-Test & P-Value \\
\hline Intercept & -0.5323 & 0.4864 & -1.09 & 0.2751 \\
L2-Size & 0.1318 & 0.0823 & 1.60 & 0.1110 \\
Sex & 0.3614 & 0.4864 & 0.74 & 0.4583 \\
Interaction & -0.0640 & 0.0823 & -0.78 & 0.4378 \\
\hline
\end{tabular}


Table A-276. ANCOVA results testing for associations between mesiodistal width of the mandibular lateral incisor and mandibular R1-L1 overlap.

\begin{tabular}{lrcrc}
\hline Term & Estimate & SE & t-Test & P-Value \\
\hline Intercept & -0.5842 & 0.4994 & -1.17 & 0.2434 \\
L2-Size & 0.1631 & 0.0845 & 1.93 & 0.0551 \\
Sex & -0.2898 & 0.4994 & -0.58 & 0.5623 \\
Interaction & 0.0648 & 0.0845 & 0.77 & 0.4443 \\
\hline
\end{tabular}

Table A-277. ANCOVA results testing for associations between mesiodistal width of the mandibular lateral incisor and total overlapping.

\begin{tabular}{lcccc}
\hline Term & Estimate & SE & t-Test & P-Value \\
\hline Intercept & -12.2492 & 3.0505 & -4.02 & $<0.0001$ \\
L2-Size & 2.8091 & 0.5164 & 5.44 & $<0.0001$ \\
Sex & -2.3795 & 3.0505 & -0.78 & 0.4363 \\
Interaction & 0.3112 & 0.5164 & 0.60 & 0.5474 \\
\hline
\end{tabular}

Table A-278. ANCOVA results testing for associations between mesiodistal width of the mandibular lateral incisor and total displacements, rotations, and overlapping.

\begin{tabular}{lcccc}
\hline Term & Estimate & SE & t-Test & P-Value \\
\hline Intercept & -29.2831 & 7.5810 & -3.86 & 0.0002 \\
L2-Size & 7.1135 & 1.2833 & 5.54 & $<0.0001$ \\
Sex & -1.6752 & 7.5810 & -0.22 & 0.8253 \\
Interaction & 0.0464 & 1.2833 & 0.04 & 0.9712 \\
\hline
\end{tabular}


Table A-279. ANCOVA results testing for associations between mesiodistal width of the mandibular lateral incisor and crowding.

\begin{tabular}{lcccc}
\hline Term & Estimate & $\mathrm{SE}$ & $\mathrm{t}$-Test & P-Value \\
\hline Intercept & -2.6704 & 0.7192 & -3.71 & 0.0003 \\
L2-Size & 0.6869 & 0.1217 & 5.64 & $<0.0001$ \\
Sex & 0.3154 & 0.7192 & 0.44 & 0.6614 \\
Interaction & -0.0777 & 0.1217 & -0.64 & 0.5242 \\
\hline
\end{tabular}

Table A-280. ANCOVA results testing for associations between mesiodistal width of the mandibular lateral incisor and spacing.

\begin{tabular}{lccrc}
\hline Term & Estimate & SE & t-Test & P-Value \\
\hline Intercept & 3.0331 & 0.5488 & 5.53 & $<0.0001$ \\
L2-Size & -0.4723 & 0.0929 & -5.08 & $<0.0001$ \\
Sex & -0.8741 & 0.5488 & -1.59 & 0.1127 \\
Interaction & 0.1590 & 0.0929 & 1.71 & 0.0885 \\
\hline
\end{tabular}

Table A-281. ANCOVA results testing for associations between mesiodistal width of the mandibular lateral incisor and diastema.

\begin{tabular}{lcccc}
\hline Term & Estimate & $\mathrm{SE}$ & $\mathrm{t}$-Test & P-Value \\
\hline Intercept & 1.8348 & 0.5835 & 3.14 & 0.0019 \\
L2-Size & -0.2732 & 0.0988 & -2.77 & 0.0062 \\
Sex & -0.8592 & 0.5835 & -1.47 & 0.1425 \\
Interaction & 0.1525 & 0.0988 & 1.54 & 0.1243 \\
\hline
\end{tabular}


Table A-282. ANCOVA results testing for associations between mesiodistal width of the mandibular lateral incisor and maxillary incisor irregularity.

\begin{tabular}{lcccc}
\hline Term & Estimate & $\mathrm{SE}$ & $\mathrm{t}$-Test & P-Value \\
\hline Intercept & -3.3096 & 1.3664 & -2.42 & 0.0163 \\
L2-Size & 0.8013 & 0.2313 & 3.46 & 0.0006 \\
Sex & -1.1294 & 1.3664 & -0.83 & 0.4095 \\
Interaction & 0.1613 & 0.2313 & 0.70 & 0.4863 \\
\hline
\end{tabular}

Table A-283. ANCOVA results testing for associations between mesiodistal width of the mandibular lateral incisor and mandibular incisor irregularity.

\begin{tabular}{lcccc}
\hline Term & Estimate & SE & t-Test & P-Value \\
\hline Intercept & -5.1494 & 1.2287 & -4.19 & $<0.0001$ \\
L2-Size & 1.0630 & 0.2080 & 5.11 & $<0.0001$ \\
Sex & -0.2570 & 1.2287 & -0.21 & 0.8345 \\
Interaction & 0.0246 & 0.2080 & 0.12 & 0.9058 \\
\hline
\end{tabular}

Table A-284. ANCOVA results testing for associations between mesiodistal width of the mandibular lateral incisor and maxillary overjet.

\begin{tabular}{lcccc}
\hline Term & Estimate & $\mathrm{SE}$ & $\mathrm{t}$-Test & P-Value \\
\hline Intercept & -1.5587 & 2.3243 & -0.67 & 0.5032 \\
L2-Size & 0.8742 & 0.3935 & 2.22 & 0.0274 \\
Sex & 0.4432 & 2.3243 & 0.19 & 0.8490 \\
Interaction & -0.0952 & 0.3935 & -0.24 & 0.8091 \\
\hline
\end{tabular}


Table A-285. ANCOVA results testing for associations between mesiodistal width of the mandibular lateral incisor and mandibular overjet.

\begin{tabular}{lcccc}
\hline Term & Estimate & $\mathrm{SE}$ & $\mathrm{t}$-Test & P-Value \\
\hline Intercept & -0.7901 & 0.2726 & -2.90 & 0.0042 \\
L2-Size & 0.1421 & 0.0461 & 3.08 & 0.0024 \\
Sex & 0.7901 & 0.2726 & 2.90 & 0.0042 \\
Interaction & -0.1421 & 0.0461 & -3.08 & 0.0024 \\
\hline
\end{tabular}

Table A-286. ANCOVA results testing for associations between mesiodistal width of the mandibular lateral incisor and openbite.

\begin{tabular}{lcccc}
\hline Term & Estimate & SE & t-Test & P-Value \\
\hline Intercept & -0.0998 & 0.4755 & -0.21 & 0.8340 \\
L2-Size & 0.0300 & 0.0805 & 0.37 & 0.7100 \\
Sex & 0.6219 & 0.4755 & 1.31 & 0.1923 \\
Interaction & -0.1039 & 0.0805 & -1.29 & 0.1984 \\
\hline
\end{tabular}

Table A-287. ANCOVA results testing for associations between mesiodistal width of the mandibular lateral incisor and anteroposterior relationship.

\begin{tabular}{lcccc}
\hline Term & Estimate & $\mathrm{SE}$ & $\mathrm{t}$-Test & P-Value \\
\hline Intercept & 1.3493 & 0.7311 & 1.85 & 0.0664 \\
L2-Size & -0.1019 & 0.1238 & -0.82 & 0.4115 \\
Sex & 0.0860 & 0.7311 & 0.12 & 0.9064 \\
Interaction & -0.0071 & 0.1238 & -0.06 & 0.9541 \\
\hline
\end{tabular}


Table A-288. ANCOVA results testing for associations between mesiodistal width of the mandibular lateral incisor and DAI score.

\begin{tabular}{lrrrc}
\hline Term & Estimate & $\mathrm{SE}$ & $\mathrm{t}$-Test & P-Value \\
\hline Intercept & 9.5372 & 6.6403 & 1.44 & 0.1525 \\
L2-Size & 2.9629 & 1.1241 & 2.64 & 0.0090 \\
Sex & 0.9743 & 6.6403 & 0.15 & 0.8835 \\
Interaction & -0.2488 & 1.1241 & -0.22 & 0.8250 \\
\hline
\end{tabular}


Table A-289. ANCOVA results testing for associations between mesiodistal width of the mandibular canine and U3 displacements.

\begin{tabular}{llc}
\hline Source & F-Ratio* & P-Value \\
\hline \multicolumn{3}{c}{ Among Subjects } \\
L3-Size & 4.0087 & 0.0466 \\
Sex & 0.0968 & 0.7560 \\
L3-Size-x-Sex & 0.1825 & 0.6697 \\
& \multicolumn{2}{c}{ Within Subjects } \\
Side & 1.4804 & 0.2251 \\
Side-L3-Size & 1.3448 & 0.2476 \\
Side-Sex & 0.1978 & 0.6569 \\
Side-L3-Size-x-Sex & 0.1965 & 0.6581 \\
\hline
\end{tabular}

*df for each test is 1 and 203.

Means, Sexes Pooled
U_R3 dis $\quad 0.41$

U_L3 dis $\quad 0.38$

Means, by Sex

$\begin{array}{lr}\text { Boy } & 0.330 .30 \\ \text { Girl } & 0.510 .47\end{array}$


Table A-290. ANCOVA results testing for associations between mesiodistal width of the mandibular canine and U2 displacements.

\begin{tabular}{llc}
\hline Source & F-Ratio* & P-Value \\
\hline \multicolumn{3}{c}{ Among Subjects } \\
L3-Size & 0.9436 & 0.3325 \\
Sex & 0.0013 & 0.9714 \\
L3-Size-x-Sex & 0.0340 & 0.8539 \\
& \multicolumn{2}{c}{ Within Subjects } \\
Side & 1.8516 & 0.1751 \\
Side-L3-Size & 1.7741 & 0.1844 \\
Side-Sex & 1.8017 & 0.1810 \\
Side-L3-Size-x-Sex & 1.8778 & 0.1721 \\
\hline
\end{tabular}

*df for each test is 1 and 203.

Means, Sexes Pooled

$\begin{array}{ll}\text { U_R2 dis } & 0.37 \\ \text { U_L2 dis } & 0.41\end{array}$

Means, by Sex

$\begin{array}{ll}\text { Boy } & 0.280 .32 \\ \text { Girl } & 0.500 .48\end{array}$


Table A-291. ANCOVA results testing for associations between mesiodistal width of the mandibular canine and U1 displacements.

\begin{tabular}{llc}
\hline Source & F-Ratio* & P-Value \\
\hline \multicolumn{2}{c}{ Among Subjects } \\
L3-Size & 0.0821 & 0.7747 \\
Sex & 0.1577 & 0.6917 \\
L3-Size-x-Sex & 0.1422 & 0.7065 \\
& \multicolumn{2}{c}{ Within Subjects } \\
Side & 0.5593 & 0.4554 \\
Side-L3-Size & 0.6483 & 0.4217 \\
Side-Sex & 2.2540 & 0.1348 \\
Side-L3-Size-x-Sex & 2.4256 & 0.1209 \\
\hline
\end{tabular}

*df for each test is 1 and 203.

Means, Sexes Pooled

U_R1 dis $\quad 0.34$

U_L1 dis $\quad 0.36$

Means, by Sex

$\begin{array}{lrl} & \text { U_R1 dis } & \text { U_L1 dis } \\ \text { Boy } & 0.370 .36 & \\ \text { Girl } & 0.300 .40\end{array}$


Table A-292. ANCOVA results testing for associations between mesiodistal width of the mandibular canine and L3 displacements.

\begin{tabular}{lcc}
\hline Source & F-Ratio* & P-Value \\
\hline & \multicolumn{2}{c}{ Among Subjects } \\
L3-Size & 2.7615 & 0.0981 \\
Sex & 1.1265 & 0.2898 \\
L3-Size-x-Sex & 0.8559 & 0.3560 \\
& \multicolumn{2}{c}{ Within Subjects } \\
Side & 0.0522 & 0.8195 \\
Side-L3-Size & 0.0601 & 0.8066 \\
Side-Sex & 0.2473 & 0.6195 \\
Side-L3-Size-x-Sex & 0.3434 & 0.5585 \\
\hline
\end{tabular}

*df for each test is 1 and 203.

Means, Sexes Pooled

$\begin{array}{ll}\text { L_R3 dis } & 0.23 \\ \text { L_L3 dis } & 0.23\end{array}$

Means, by Sex

$\begin{array}{lr}\text { Boy } & 0.130 .18 \\ \text { Girl } & 0.320 .24\end{array}$


Table A-293. ANCOVA results testing for associations between mesiodistal width of the mandibular canine and L2 displacements.

\begin{tabular}{llc}
\hline Source & F-Ratio* & P-Value \\
\hline & \multicolumn{2}{c}{ Among Subjects } \\
L3-Size & 4.1846 & 0.0421 \\
Sex & 0.0297 & 0.8633 \\
L3-Size-x-Sex & 0.0044 & 0.9473 \\
& \multicolumn{2}{c}{ Within Subjects } \\
Side & 0.2733 & 0.6017 \\
Side-L3-Size & 0.2749 & 0.6006 \\
Side-Sex & 5.4134 & 0.0210 \\
Side-L3-Size-x-Sex & 5.2459 & 0.0230 \\
\hline
\end{tabular}

*df for each test is 1 and 203.

Means, Sexes Pooled

L_R2 dis $\quad 0.51$

L_L2 dis $\quad 0.47$

Means, by Sex

$\begin{array}{ll}\text { Boy } & 0.410 .42 \\ \text { Girl } & 0.570 .57\end{array}$


Table A-294. ANCOVA results testing for associations between mesiodistal width of the mandibular canine and L1 displacements.

\begin{tabular}{llc}
\hline Source & F-Ratio* & P-Value \\
\hline & \multicolumn{2}{c}{ Among Subjects } \\
L3-Size & 1.1207 & 0.2910 \\
Sex & 0.1177 & 0.7319 \\
L3-Size-x-Sex & 0.1248 & 0.7242 \\
& \multicolumn{2}{c}{ Within Subjects } \\
Side & 0.1479 & 0.7009 \\
Side-L3-Size & 0.2102 & 0.6471 \\
Side-Sex & 0.0027 & 0.9585 \\
Side-L3-Size-x-Sex & 0.0078 & 0.9298 \\
\hline
\end{tabular}

*df for each test is 1 and 203.

Means, Sexes Pooled

$\begin{array}{ll}\text { L_R1 dis } & 0.22 \\ \text { L_L1 dis } & 0.17 \\ & \end{array}$

Means, by Sex

$\begin{array}{ll}\text { Boy } & 0.230 .16 \\ \text { Girl } & 0.200 .17\end{array}$


Table A-295. ANCOVA results testing for associations between mesiodistal width of the mandibular canine and U3 rotations.

\begin{tabular}{llc}
\hline Source & F-Ratio* & P-Value \\
\hline \multicolumn{2}{c}{ Among Subjects } \\
L3-Size & 0.1479 & 0.7010 \\
Sex & 0.0513 & 0.8211 \\
L3-Size-x-Sex & 0.1106 & 0.7398 \\
& \multicolumn{2}{c}{ Within Subjects } \\
Side & 0.0660 & 0.7975 \\
Side-L3-Size & 0.0808 & 0.7764 \\
Side-Sex & 2.0496 & 0.1538 \\
Side-L3-Size-x-Sex & 1.9374 & 0.1655 \\
\hline
\end{tabular}

*df for each test is 1 and 203.

Means, Sexes Pooled

$\begin{array}{ll}\text { U_R3 rot } & 0.22 \\ \text { U_L3 rot } & 0.22\end{array}$

Means, by Sex

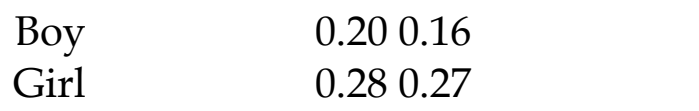


Table A-296. ANCOVA results testing for associations between mesiodistal width of the mandibular canine and $\mathrm{U} 2$ rotations.

\begin{tabular}{llc}
\hline Source & F-Ratio* & P-Value \\
\hline \multicolumn{2}{c}{ Among Subjects } \\
L3-Size & 2.9597 & 0.0869 \\
Sex & 0.0194 & 0.8894 \\
L3-Size-x-Sex & 0.0460 & 0.8304 \\
& \multicolumn{2}{c}{ Within Subjects } \\
Side & 2.3521 & 0.1267 \\
Side-L3-Size & 2.1930 & 0.1402 \\
Side-Sex & 0.0206 & 0.8861 \\
Side-L3-Size-x-Sex & 0.0106 & 0.9180 \\
\hline
\end{tabular}

*df for each test is 1 and 203.

Means, Sexes Pooled

$\begin{array}{ll}\text { U_R2 rot } & 0.39 \\ \text { U_L2 rot } & 0.42\end{array}$

Means, by Sex

$\begin{array}{ll}\text { Boy } & 0.330 .39 \\ \text { Girl } & 0.460 .46\end{array}$


Table A-297. ANCOVA results testing for associations between mesiodistal width of the mandibular canine and U1 rotations.

\begin{tabular}{lcc}
\hline Source & F-Ratio* & P-Value \\
\hline & \multicolumn{2}{c}{ Among Subjects } \\
L3-Size & 0.8113 & 0.3688 \\
Sex & 0.9180 & 0.3391 \\
L3-Size-x-Sex & 0.9604 & 0.3283 \\
& \multicolumn{2}{c}{ Within Subjects } \\
Side & 0.0078 & 0.9297 \\
Side-L3-Size & 0.0007 & 0.9787 \\
Side-Sex & 2.5733 & 0.1102 \\
Side-L3-Size-x-Sex & 2.5793 & 0.1098 \\
\hline
\end{tabular}

*df for each test is 1 and 203.

Means, Sexes Pooled

$\begin{array}{ll}\text { U_R1 rot } & 0.47 \\ \text { U_L1 rot } & 0.49\end{array}$

Means, by Sex

$\begin{array}{ll}\text { Boy } & 0.460 .50 \\ \text { Girl } & 0.49\end{array}$


Table A-298. ANCOVA results testing for associations between mesiodistal width of the mandibular canine and L3 rotations.

\begin{tabular}{lcc}
\hline Source & F-Ratio* & P-Value \\
\hline \multicolumn{2}{c}{ Among Subjects } \\
L3-Size & 3.4185 & 0.0659 \\
Sex & 1.9156 & 0.1679 \\
L3-Size-x-Sex & 2.3406 & 0.1276 \\
& \multicolumn{2}{c}{ Within Subjects } \\
Side & 0.0411 & 0.8395 \\
Side-L3-Size & 0.0665 & 0.7968 \\
Side-Sex & 0.0743 & 0.7854 \\
Side-L3-Size-x-Sex & 0.1302 & 0.7186 \\
\hline
\end{tabular}

* $\mathrm{df}$ for each test is 1 and 203.

Means, Sexes Pooled

L_R3 rot 0.39

L_L3 rot $\quad 0.35$

Means, by Sex

Boy

L_R3 rot L_L3 rot

Girl $\quad 0.560 .44$ 
Table A-299. ANCOVA results testing for associations between mesiodistal width of the mandibular canine and L2 rotations.

\begin{tabular}{llc}
\hline Source & F-Ratio* & P-Value \\
\hline & \multicolumn{2}{c}{ Among Subjects } \\
L3-Size & 4.7080 & 0.0312 \\
Sex & 0.5310 & 0.4670 \\
L3-Size-x-Sex & 0.6637 & 0.4162 \\
& \multicolumn{2}{c}{ Within Subjects } \\
Side & 0.1037 & 0.7478 \\
Side-L3-Size & 0.0357 & 0.8504 \\
Side-Sex & 0.3850 & 0.5356 \\
Side-L3-Size-x-Sex & 0.3302 & 0.5662 \\
\hline
\end{tabular}

* $\mathrm{df}$ for each test is 1 and 203.

Means, Sexes Pooled

$\begin{array}{cc}\text { L_R2 rot } & 0.35 \\ \text { L_L2 rot } & 0.23\end{array}$

Means, by Sex

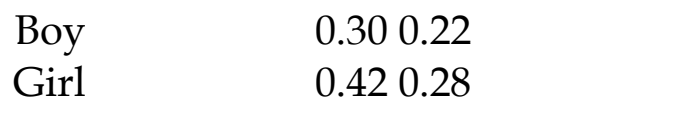


Table A-300. ANCOVA results testing for associations between mesiodistal width of the mandibular canine and L1 rotations.

\begin{tabular}{lcc}
\hline Source & F-Ratio* & P-Value \\
\hline & \multicolumn{2}{c}{ Among Subjects } \\
L3-Size & 0.0711 & 0.7900 \\
Sex & 0.6170 & 0.4331 \\
L3-Size-x-Sex & 0.7960 & 0.3733 \\
& \multicolumn{2}{c}{ Within Subjects } \\
Side & 0.3484 & 0.5557 \\
Side-L3-Size & 0.4054 & 0.5250 \\
Side-Sex & 0.0119 & 0.9132 \\
Side-L3-Size-x-Sex & 0.0079 & 0.9291 \\
\hline
\end{tabular}

* $\mathrm{df}$ for each test is 1 and 203.

Means, Sexes Pooled

L_R1 rot $\quad 0.38$

L_L1 rot $\quad 0.34$

Means, by Sex

$\begin{array}{lll}\text { Boy } & 0.430 .37 \\ \text { Girl } & 0.30 & 0.28\end{array}$


Table A-301. ANCOVA results testing for associations between mesiodistal width of the mandibular canine and U2-U3 overlap.

\begin{tabular}{llc}
\hline Source & F-Ratio* & P-Value \\
\hline & \multicolumn{2}{c}{ Among Subjects } \\
L3-Size & 1.0382 & 0.3094 \\
Sex & 0.0540 & 0.8165 \\
L3-Size-x-Sex & 0.0057 & 0.9399 \\
& \multicolumn{2}{c}{ Within Subjects } \\
Side & 0.1466 & 0.7022 \\
Side-L3-Size & 0.1893 & 0.6639 \\
Side-Sex & 1.8816 & 0.1717 \\
Side-L3-Size-x-Sex & 1.9861 & 0.1603 \\
\hline
\end{tabular}

*df for each test is 1 and 203. Means, Sexes Pooled U_R3-2 lapping $\quad 0.38$ U_L2-3 lapping $\quad 0.37$

Means, by Sex

U_R3-2 lapping U_L2-3 lapping

Boy

0.270 .27

Girl

0.530 .44 
Table A-302. ANCOVA results testing for associations between mesiodistal width of the mandibular canine and U1-U2 overlap.

\begin{tabular}{llc}
\hline Source & F-Ratio* & P-Value \\
\hline & \multicolumn{2}{c}{ Among Subjects } \\
L3-Size & 0.0132 & 0.9086 \\
Sex & 0.2855 & 0.5937 \\
L3-Size-x-Sex & 0.1351 & 0.7135 \\
& \multicolumn{2}{c}{ Within Subjects } \\
Side & 1.1450 & 0.2859 \\
Side-L3-Size & 1.0735 & 0.3014 \\
Side-Sex & 0.6413 & 0.4242 \\
Side-L3-Size-x-Sex & 0.6411 & 0.4242 \\
\hline
\end{tabular}

*df for each test is 1 and 203. Means, Sexes Pooled U_R2-1 lapping $\quad 0.35$ U_L1-2 lapping $\quad 0.31$

Means, by Sex

U_R2-1 lapping U_L1-2 lapping

Boy

0.250 .22

Girl

0.430 .42 
Table A-303. ANCOVA results testing for associations between mesiodistal width of the mandibular canine and L2-L3 overlap.

\begin{tabular}{lcc}
\hline Source & F-Ratio* & P-Value \\
\hline \multicolumn{3}{c}{ Among Subjects } \\
L3-Size & 12.0329 & 0.0006 \\
Sex & 0.1492 & 0.6997 \\
L3-Size-x-Sex & 0.0595 & 0.8075 \\
& \multicolumn{2}{c}{ Within Subjects } \\
Side & 0.6786 & 0.4111 \\
Side-L3-Size & 0.8465 & 0.3586 \\
Side-Sex & 1.0524 & 0.3062 \\
Side-L3-Size-x-Sex & 1.0779 & 0.3004 \\
\hline
\end{tabular}

*df for each test is 1 and 203. Means, Sexes Pooled L_R3-2 lapping $\quad 0.71$ L_L2-3 lapping $\quad 0.65$

Means, by Sex

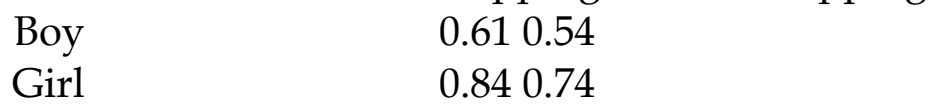


Table A-304. ANCOVA results testing for associations between mesiodistal width of the mandibular canine and L1-L2 overlap.

\begin{tabular}{llc}
\hline Source & F-Ratio* & P-Value \\
\hline & \multicolumn{2}{c}{ Among Subjects } \\
L3-Size & 4.6412 & 0.0324 \\
Sex & 0.6360 & 0.4261 \\
L3-Size-x-Sex & 0.7738 & 0.3801 \\
& \multicolumn{2}{c}{ Within Subjects } \\
Side & 0.5164 & 0.4732 \\
Side-L3-Size & 0.4798 & 0.4893 \\
Side-Sex & 0.0273 & 0.8690 \\
Side-L3-Size-x-Sex & 0.0379 & 0.8458 \\
\hline
\end{tabular}

*df for each test is 1 and 203. Means, Sexes Pooled

L_R2-1 lapping $\quad 0.47$

L_L1-2 lapping $\quad 0.46$

Means, by Sex

$\begin{array}{ll}\text { Boy } & 0.430 .44 \\ \text { Girl } & 0.550 .51\end{array}$


Table A-305. ANCOVA results testing for associations between mesiodistal width of the mandibular canine and total displacements.

\begin{tabular}{lcccc}
\hline Term & Estimate & SE & t-Test & P-Value \\
\hline Intercept & -5.1731 & 4.1359 & -1.25 & 0.2125 \\
L3-Size & 1.3778 & 0.6199 & 2.22 & 0.0273 \\
Sex & -0.6092 & 4.1359 & -0.15 & 0.8830 \\
Interaction & -0.0012 & 0.6199 & 0.00 & 0.9984 \\
\hline
\end{tabular}

Table A-306. ANCOVA results testing for associations between mesiodistal width of the mandibular canine and total rotations.

\begin{tabular}{lcccc}
\hline Term & Estimate & $\mathrm{SE}$ & $\mathrm{t}$-Test & P-Value \\
\hline Intercept & -2.9207 & 3.0494 & -0.96 & 0.3393 \\
L3-Size & 1.0815 & 0.4570 & 2.37 & 0.0189 \\
Sex & 3.3723 & 3.0494 & 1.11 & 0.2701 \\
Interaction & -0.5630 & 0.4570 & -1.23 & 0.2194 \\
\hline
\end{tabular}

Table A-307. ANCOVA results testing for associations between mesiodistal width of the mandibular canine and maxillary R1-L1 overlap.

\begin{tabular}{lcccc}
\hline Term & Estimate & SE & t-Test & P-Value \\
\hline Intercept & -0.1571 & 0.5742 & -0.27 & 0.7847 \\
L3-Size & 0.0590 & 0.0861 & 0.69 & 0.4940 \\
Sex & -0.0888 & 0.5742 & -0.15 & 0.8772 \\
Interaction & 0.0104 & 0.0861 & 0.12 & 0.9041 \\
\hline
\end{tabular}


Table A-308. ANCOVA results testing for associations between mesiodistal width of the mandibular canine and mandibular R1-L1 overlap.

\begin{tabular}{lcccc}
\hline Term & Estimate & SE & t-Test & P-Value \\
Intercept & -0.8494 & 0.5852 & -1.45 & 0.1482 \\
L3-Size & 0.1824 & 0.0877 & 2.08 & 0.0388 \\
Sex & -0.0856 & 0.5852 & -0.15 & 0.8839 \\
Interaction & 0.0231 & 0.0877 & 0.26 & 0.7929 \\
\hline
\end{tabular}

Table A-309. ANCOVA results testing for associations between mesiodistal width of the mandibular canine and total overlapping.

\begin{tabular}{lcccc}
\hline Term & Estimate & SE & t-Test & P-Value \\
\hline Intercept & -6.4766 & 3.7626 & -1.72 & 0.0867 \\
L3-Size & 1.6077 & 0.5639 & 2.85 & 0.0048 \\
Sex & -0.8929 & 3.7626 & -0.24 & 0.8127 \\
Interaction & 0.0348 & 0.5639 & 0.06 & 0.9508 \\
\hline
\end{tabular}

Table A-310. ANCOVA results testing for associations between mesiodistal width of the mandibular canine and total displacements, rotations, and overlapping.

\begin{tabular}{lrccc}
\hline Term & Estimate & $\mathrm{SE}$ & $\mathrm{t}$-Test & P-Value \\
\hline Intercept & -14.5704 & 9.3619 & -1.56 & 0.1212 \\
L3-Size & 4.0669 & 1.4031 & 2.90 & 0.0042 \\
Sex & 1.8702 & 9.3619 & 0.20 & 0.8419 \\
Interaction & -0.5294 & 1.4031 & -0.38 & 0.7063 \\
\hline
\end{tabular}


Table A-311. ANCOVA results testing for associations between mesiodistal width of the mandibular canine and crowding.

\begin{tabular}{lcccc}
\hline Term & Estimate & SE & t-Test & P-Value \\
\hline Intercept & -2.1935 & 0.8729 & -2.51 & 0.0127 \\
L3-Size & 0.5372 & 0.1308 & 4.11 & $<0.0001$ \\
Sex & 1.4135 & 0.8729 & 1.62 & 0.1069 \\
Interaction & -0.2393 & 0.1308 & -1.83 & 0.0688 \\
\hline
\end{tabular}

Table A-312. ANCOVA results testing for associations between mesiodistal width of the mandibular canine and spacing.

\begin{tabular}{lcccc}
\hline Term & Estimate & $\mathrm{SE}$ & $\mathrm{t}$-Test & P-Value \\
\hline Intercept & 3.1443 & 0.6529 & 4.82 & $<0.0001$ \\
L3-Size & -0.4361 & 0.0979 & -4.46 & $<0.0001$ \\
Sex & -1.6111 & 0.6529 & -2.47 & 0.0144 \\
Interaction & 0.2563 & 0.0979 & 2.62 & 0.0095 \\
\hline
\end{tabular}

Table A-313. ANCOVA results testing for associations between mesiodistal width of the mandibular canine and diastema.

\begin{tabular}{lcccc}
\hline Term & Estimate & $\mathrm{SE}$ & $\mathrm{t}$-Test & P-Value \\
\hline Intercept & 2.3868 & 0.6819 & 3.50 & 0.0006 \\
L3-Size & -0.3258 & 0.1022 & -3.19 & 0.0017 \\
Sex & -1.3399 & 0.6819 & -1.96 & 0.0508 \\
Interaction & 0.2113 & 0.1022 & 2.07 & 0.0399 \\
\hline
\end{tabular}


Table A-314. ANCOVA results testing for associations between mesiodistal width of the mandibular canine and maxillary incisor irregularity.

\begin{tabular}{lcccc}
\hline Term & Estimate & $\mathrm{SE}$ & $\mathrm{t}$-Test & P-Value \\
\hline Intercept & -0.8756 & 1.6465 & -0.53 & 0.5955 \\
L3-Size & 0.3408 & 0.2468 & 1.38 & 0.1688 \\
Sex & -0.6526 & 1.6465 & -0.40 & 0.6922 \\
Interaction & 0.0689 & 0.2468 & 0.28 & 0.7803 \\
\hline
\end{tabular}

Table A-315. ANCOVA results testing for associations between mesiodistal width of the mandibular canine and mandibular incisor irregularity.

\begin{tabular}{lccrc}
\hline Term & Estimate & SE & t-Test & P-Value \\
\hline Intercept & -2.0228 & 1.5147 & -1.34 & 0.1832 \\
L3-Size & 0.4661 & 0.2270 & 2.05 & 0.0413 \\
Sex & -0.5800 & 1.5147 & -0.38 & 0.7022 \\
Interaction & 0.0669 & 0.2270 & 0.29 & 0.7685 \\
\hline
\end{tabular}

Table A-316. ANCOVA results testing for associations between mesiodistal width of the mandibular canine and maxillary overjet.

\begin{tabular}{lcccc}
\hline Term & Estimate & $\mathrm{SE}$ & $\mathrm{t}$-Test & P-Value \\
\hline Intercept & -1.4952 & 2.7380 & -0.55 & 0.5856 \\
L3-Size & 0.7602 & 0.4104 & 1.85 & 0.0654 \\
Sex & 0.7999 & 2.7380 & 0.29 & 0.7705 \\
Interaction & -0.1489 & 0.4104 & -0.36 & 0.7172 \\
\hline
\end{tabular}


Table A-317. ANCOVA results testing for associations between mesiodistal width of the mandibular canine and mandibular overjet.

\begin{tabular}{lcccc}
\hline Term & Estimate & $\mathrm{SE}$ & $\mathrm{t}$-Test & P-Value \\
\hline Intercept & -0.2879 & 0.3315 & -0.87 & 0.3861 \\
L3-Size & 0.0501 & 0.0497 & 1.01 & 0.3148 \\
Sex & 0.2879 & 0.3315 & 0.87 & 0.3861 \\
Interaction & -0.0501 & 0.0497 & -1.01 & 0.3148 \\
\hline
\end{tabular}

Table A-318. ANCOVA results testing for associations between mesiodistal width of the mandibular canine and openbite.

\begin{tabular}{lcccc}
\hline Term & Estimate & $\mathrm{SE}$ & $\mathrm{t}$-Test & P-Value \\
\hline Intercept & 0.3730 & 0.5587 & 0.67 & 0.5052 \\
L3-Size & -0.0432 & 0.0837 & -0.52 & 0.6068 \\
Sex & 0.4488 & 0.5587 & 0.80 & 0.4228 \\
Interaction & -0.0639 & 0.0837 & -0.76 & 0.4463 \\
\hline
\end{tabular}

Table A-319. ANCOVA results testing for associations between mesiodistal width of the mandibular canine and anteroposterior relationship.

\begin{tabular}{lcccc}
\hline Term & Estimate & $\mathrm{SE}$ & $\mathrm{t}$-Test & P-Value \\
\hline Intercept & 0.3682 & 0.8546 & 0.43 & 0.6671 \\
L3-Size & 0.0619 & 0.1281 & 0.48 & 0.6296 \\
Sex & 1.3973 & 0.8546 & 1.64 & 0.1036 \\
Interaction & -0.2036 & 0.1281 & -1.59 & 0.1135 \\
\hline
\end{tabular}


Table A-320. ANCOVA results testing for associations between mesiodistal width of the mandibular canine and DAI score.

\begin{tabular}{lcccc}
\hline Term & Estimate & $\mathrm{SE}$ & $\mathrm{t}$-Test & P-Value \\
\hline Intercept & 18.6217 & 7.9080 & 2.35 & 0.0195 \\
L3-Size & 1.2558 & 1.1852 & 1.06 & 0.2906 \\
Sex & 1.7817 & 7.9080 & 0.23 & 0.8220 \\
Interaction & -0.3443 & 1.1852 & -0.29 & 0.7717 \\
\hline
\end{tabular}


Table A-321. ANCOVA results testing for associations between mesiodistal width of the mandibular first premolar and U3 displacements.

\begin{tabular}{lcc}
\hline Source & F-Ratio* & P-Value \\
\hline \multicolumn{2}{c}{ Among Subjects } \\
L4-Size & 0.9420 & 0.3329 \\
Sex & 0.0662 & 0.7972 \\
L4-Size-x-Sex & 0.1067 & 0.7443 \\
& \multicolumn{2}{c}{ Within Subjects } \\
Side & 0.0216 & 0.8832 \\
Side-x-L4-Size & 0.0343 & 0.8532 \\
Side-x-Sex & 0.4704 & 0.4936 \\
Side-x-L4-Size-x-Sex & 0.5170 & 0.4729 \\
\hline
\end{tabular}

* $\mathrm{df}$ for each test is 1 and 203.

Means, Sexes Pooled

\begin{tabular}{lrr} 
U_R3 dis & 0.41 \\
U_L3 dis & 0.38 & \\
& Means, by Sex \\
\multicolumn{4}{c}{ U_R3 dis } & U_L3 dis \\
Boy & 0.340 .34 & \\
Girl & 0.480 .41 &
\end{tabular}


Table A-322. ANCOVA results testing for associations between mesiodistal width of the mandibular first premolar and U2 displacements.

\begin{tabular}{lcc}
\hline Source & F-Ratio* & P-Value \\
\hline \multicolumn{2}{c}{ Among Subjects } \\
L4-Size & 0.1605 & 0.6891 \\
Sex & 0.0296 & 0.8635 \\
L4-Size-x-Sex & 0.0026 & 0.9591 \\
& \multicolumn{2}{c}{ Within Subjects } \\
Side & 2.9386 & 0.0880 \\
Side-x-L4-Size & 2.8055 & 0.0955 \\
Side-x-Sex & 2.7743 & 0.0973 \\
Side-x-L4-Size-x-Sex & 2.8286 & 0.0941 \\
\hline
\end{tabular}

* $\mathrm{df}$ for each test is 1 and 203.

Means, Sexes Pooled

U_R2 dis $\quad 0.37$

U_L2 dis 0.41

Means, by Sex

U_R2 dis U_L2 dis

$\begin{array}{ll}\text { Boy } & 0.290 .33 \\ \text { Girl } & 0.460 .48\end{array}$ 
Table A-323. ANCOVA results testing for associations between mesiodistal width of the mandibular first premolar and U1 displacements.

\begin{tabular}{llc}
\hline Source & F-Ratio* & P-Value \\
\hline \multicolumn{2}{c}{ Among Subjects } \\
L4-Size & 0.0130 & 0.9092 \\
Sex & 0.1348 & 0.7139 \\
L4-Size-x-Sex & 0.1279 & 0.7210 \\
& \multicolumn{2}{c}{ Within Subjects } \\
Side & 0.5513 & 0.4587 \\
Side-x-L4-Size & 0.5059 & 0.4777 \\
Side-x-Sex & 1.1767 & 0.2793 \\
Side-x-L4-Size-x-Sex & 1.2624 & 0.2625 \\
\hline
\end{tabular}

* $\mathrm{df}$ for each test is 1 and 203.

Means, Sexes Pooled

$\begin{array}{lrr}\text { U_R1 dis } & 0.34 & \\ \text { U_L1 dis } & 0.36 & \\ & \text { Means, by Sex } \\ & \text { U_R1 dis } & \text { U_L1 dis } \\ \text { Boy } & 0.360 .35 & \\ \text { Girl } & 0.320 .37 & \end{array}$


Table A-324. ANCOVA results testing for associations between mesiodistal width of the mandibular first premolar and L3 displacements.

\begin{tabular}{lcc}
\hline Source & F-Ratio* & P-Value \\
\hline \multicolumn{2}{c}{ Among Subjects } \\
L4-Size & 2.7370 & 0.0996 \\
Sex & 1.4101 & 0.2364 \\
L4-Size-x-Sex & 1.1975 & 0.2751 \\
& \multicolumn{2}{c}{ Within Subjects } \\
Side & 1.5562 & 0.2137 \\
Side-x-L4-Size & 1.5238 & 0.2185 \\
Side-x-Sex & 0.9644 & 0.3272 \\
Side-x-L4-Size-x-Sex & 1.1116 & 0.2930 \\
\hline
\end{tabular}

*df for each test is 1 and 203.

Means, Sexes Pooled

\begin{tabular}{lll} 
L_R3 dis & 0.23 & \\
L_L3 dis & 0.23 & \\
& Means, by Sex \\
\multicolumn{4}{c}{ L_R3 dis } & L_L3 dis \\
Boy & 0.150 .20 & \\
Girl & 0.300 .24 &
\end{tabular}


Table A-325. ANCOVA results testing for associations between mesiodistal width of the mandibular first premolar and L2 displacements.

\begin{tabular}{llc}
\hline Source & F-Ratio* & P-Value \\
\hline \multicolumn{3}{c}{ Among Subjects } \\
L4-Size & 3.1644 & 0.0768 \\
Sex & 0.4480 & 0.5040 \\
L4-Size-x-Sex & 0.3680 & 0.5448 \\
& \multicolumn{2}{c}{ Within Subjects } \\
Side & 0.6805 & 0.4104 \\
Side-x-L4-Size & 0.6080 & 0.4365 \\
Side-x-Sex & 1.1098 & 0.2934 \\
Side-x-L4-Size-x-Sex & 1.0631 & 0.3037 \\
\hline
\end{tabular}

*df for each test is 1 and 203.

Means, Sexes Pooled

\begin{tabular}{lrr} 
L_R2 dis & 0.51 & \\
L_L2 dis & 0.47 & \\
& Means, by Sex \\
\multicolumn{4}{c}{ L_R2 dis } & L_L2 dis \\
Boy & 0.450 .43 & \\
Girl & 0.560 .51 &
\end{tabular}


Table A-326. ANCOVA results testing for associations between mesiodistal width of the mandibular first premolar and L1 displacements.

\begin{tabular}{lcc}
\hline Source & F-Ratio & P-Value \\
\hline \multicolumn{2}{c}{ Among Subjects } \\
L4-Size & 0.4870 & 0.4861 \\
Sex & 0.5840 & 0.4457 \\
L4-Size-x-Sex & 0.5313 & 0.4669 \\
& \multicolumn{2}{c}{ Within Subjects } \\
Side & 0.0004 & 0.9834 \\
Side-x-L4-Size & 0.0109 & 0.9170 \\
Side-x-Sex & 0.7907 & 0.3749 \\
Side-x-L4-Size-x-Sex & 0.7090 & 0.4008 \\
\hline
\end{tabular}

* $\mathrm{df}$ for each test is 1 and 203.

Means, Sexes Pooled

\begin{tabular}{lll} 
L_R1 dis & 0.22 & \\
L_L1 dis & 0.17 & \\
& Means, by Sex \\
\multicolumn{4}{c}{ L_R1 dis } & L_L1 dis \\
Boy & 0.250 .17 & \\
Girl & 0.200 .17 &
\end{tabular}


Table A-327. ANCOVA results testing for associations between mesiodistal width of the mandibular first premolar and U3 rotations.

\begin{tabular}{lcc}
\hline Source & F-Ratio* & P-Value \\
\hline \multicolumn{2}{c}{ Among Subjects } \\
L4-Size & 0.5304 & 0.4673 \\
Sex & 1.3100 & 0.2537 \\
L4-Size-x-Sex & 1.0465 & 0.3075 \\
& \multicolumn{2}{c}{ Within Subjects } \\
Side & 0.6127 & 0.4347 \\
Side-x-L4-Size & 0.6058 & 0.4373 \\
Side-x-Sex & 0.2650 & 0.6073 \\
Side-x-L4-Size-x-Sex & 0.2380 & 0.6262 \\
\hline
\end{tabular}

*df for each test is 1 and 203.

Means, Sexes Pooled

$\begin{array}{lll}\text { U_R3 rot } & 0.22 & \\ \text { U_L3 rot } & 0.22 & \\ & \text { Means, by Sex } \\ & \text { U_R3 rot } & \text { U_L3 rot } \\ \text { Boy } & 0.170 .15 & \\ \text { Girl } & 0.260 .28 & \end{array}$


Table A-328. ANCOVA results testing for associations between mesiodistal width of the mandibular first premolar and $\mathrm{U} 2$ rotations.

\begin{tabular}{llc}
\hline Source & F-Ratio* & P-Value \\
\hline \multicolumn{2}{c}{ Among Subjects } \\
L4-Size & 1.1426 & 0.2864 \\
Sex & 1.6858 & 0.1956 \\
L4-Size-x-Sex & 1.6316 & 0.2029 \\
& \multicolumn{2}{c}{ Within Subjects } \\
Side & 0.2608 & 0.6101 \\
Side-x-L4-Size & 0.2226 & 0.6376 \\
Side-x-Sex & 0.2403 & 0.6245 \\
Side-x-L4-Size-x-Sex & 0.2370 & 0.6269 \\
\hline
\end{tabular}

*df for each test is 1 and 203.

Means, Sexes Pooled

$\begin{array}{lll}\text { U_R2 rot } & 0.39 & \\ \text { U_L2 rot } & 0.42 & \\ & \text { Means, by Sex } \\ & \text { U_R2 rot } & \text { U_L2 rot } \\ \text { Boy } & 0.370 .40 & \\ \text { Girl } & 0.400 .43 & \end{array}$


Table A-329. ANCOVA results testing for associations between mesiodistal width of the mandibular first premolar and U1 rotations.

\begin{tabular}{lcc}
\hline Source & F-Ratio* & P-Value \\
\hline \multicolumn{2}{c}{ Among Subjects } \\
L4-Size & 5.1382 & 0.0245 \\
Sex & 0.9833 & 0.3226 \\
L4-Size-x-Sex & 1.0313 & 0.3111 \\
& \multicolumn{2}{c}{ Within Subjects } \\
Side & 2.3478 & 0.1270 \\
Side-x-L4-Size & 2.3045 & 0.1306 \\
Side-x-Sex & 0.2250 & 0.6357 \\
Side-x-L4-Size-x-Sex & 0.2210 & 0.6388 \\
\hline
\end{tabular}

*df for each test is 1 and 203.

Means, Sexes Pooled

$\begin{array}{lll}\text { U_R1 rot } & 0.47 & \\ \text { U_L1 rot } & 0.49 & \\ & \text { Means, by Sex } \\ & \text { U_R1 rot } & \text { U_L1 rot } \\ \text { Boy } & 0.470 .47 & \\ \text { Girl } & 0.500 .51 & \end{array}$


Table A-330. ANCOVA results testing for associations between mesiodistal width of the mandibular first premolar and L3 rotations.

\begin{tabular}{llc}
\hline Source & F-Ratio* & P-Value \\
\hline & \multicolumn{2}{c}{ Among Subjects } \\
L4-Size & 2.7757 & 0.0972 \\
Sex & 0.3377 & 0.5618 \\
L4-Size-x-Sex & 0.4871 & 0.4860 \\
& \multicolumn{2}{c}{ Within Subjects } \\
Side & 0.5535 & 0.4577 \\
Side-x-L4-Size & 0.6275 & 0.4292 \\
Side-x-Sex & 0.1415 & 0.7072 \\
Side-x-L4-Size-x-Sex & 0.2140 & 0.6441 \\
\hline
\end{tabular}

* $\mathrm{df}$ for each test is 1 and 203.

Means, Sexes Pooled

\begin{tabular}{lll} 
L_R3 rot & 0.39 & \\
L_L3 rot & 0.35 & \\
& Means, by Sex & \\
\multicolumn{4}{c}{ L_R3 rot } & L_L3 rot \\
Boy & 0.280 .31 & \\
Girl & 0.510 .40 &
\end{tabular}


Table A-331. ANCOVA results testing for associations between mesiodistal width of the mandibular first premolar and L2 rotations.

\begin{tabular}{llc}
\hline Source & F-Ratio* & P-Value \\
\hline & \multicolumn{2}{c}{ Among Subjects } \\
L4-Size & 7.1857 & 0.0080 \\
Sex & 3.8393 & 0.0514 \\
L4-Size-x-Sex & 4.0498 & 0.0455 \\
& \multicolumn{2}{c}{ Within Subjects } \\
Side & 0.4555 & 0.5005 \\
Side-x-L4-Size & 0.6721 & 0.4133 \\
Side-x-Sex & 0.3983 & 0.5287 \\
Side-x-L4-Size-x-Sex & 0.4635 & 0.4968 \\
\hline
\end{tabular}

*df for each test is 1 and 203.

Means, Sexes Pooled

\begin{tabular}{lll} 
L_R2 rot & 0.35 & \\
L_L2 rot & 0.23 & \\
& Means, by Sex & \\
\multicolumn{4}{c}{ L_R2 rot } & L_L2 rot \\
Boy & 0.310 .23 & \\
Girl & 0.420 .25 &
\end{tabular}


Table A-332. ANCOVA results testing for associations between mesiodistal width of the mandibular first premolar and L1 rotations.

\begin{tabular}{llc}
\hline Source & F-Ratio* & P-Value \\
\hline \multicolumn{3}{c}{ Among Subjects } \\
L4-Size & 0.2131 & 0.6448 \\
Sex & 0.0547 & 0.8153 \\
L4-Size-x-Sex & 0.1102 & 0.7403 \\
& \multicolumn{2}{c}{ Within Subjects } \\
Side & 0.2072 & 0.6495 \\
Side-x-L4-Size & 0.2527 & 0.6157 \\
Side-x-Sex & 0.0120 & 0.9127 \\
Side-x-L4-Size-x-Sex & 0.0063 & 0.9371 \\
\hline
\end{tabular}

*df for each test is 1 and 203.

Means, Sexes Pooled

$\begin{array}{lcc}\text { L_R1 rot } & 0.38 & \\ \text { L_L1 rot } & 0.34 & \\ & \text { Means, by Sex } \\ & \text { L_R1 rot } & \text { L_L1 rot } \\ \text { Boy } & 0.430 .37 & \\ \text { Girl } & 0.310 .30 & \end{array}$


Table A-333. ANCOVA results testing for associations between mesiodistal width of the mandibular first premolar and U2-U3 overlap.

\begin{tabular}{lcc}
\hline Source & F-Ratio* & P-Value \\
\hline \multicolumn{2}{c}{ Among Subjects } \\
L4-Size & 1.3824 & 0.2411 \\
Sex & 0.0534 & 0.8176 \\
L4-Size-x-Sex & 0.1314 & 0.7174 \\
& \multicolumn{2}{c}{ Within Subjects } \\
Side & 1.3412 & 0.2482 \\
Side-x-L4-Size & 1.4042 & 0.2374 \\
Side-x-Sex & 1.7409 & 0.1885 \\
Side-x-L4-Size-x-Sex & 1.8623 & 0.1739 \\
\hline
\end{tabular}

*df for each test is 1 and 203.

Means, Sexes Pooled

$\begin{array}{ll}\text { U_R3-2 lapping } & 0.38 \\ \text { U_L2-3 lapping } & 0.37 \\ \text { Means, by Sex } & \end{array}$

Boy

U_R3-2 lapping U_L2-3 lapping

Girl

0.280 .29

0.510 .44 
Table A-334. ANCOVA results testing for associations between mesiodistal width of the mandibular first premolar and U1-U2 overlap.

\begin{tabular}{llc}
\hline Source & F-Ratio* & P-Value \\
\hline \multicolumn{2}{c}{ Among Subjects } \\
L4-Size & 0.1405 & 0.7082 \\
Sex & 2.6676 & 0.1040 \\
L4-Size-x-Sex & 2.1958 & 0.1399 \\
& \multicolumn{2}{c}{ Within Subjects } \\
Side & 0.0093 & 0.9231 \\
Side-x-L4-Size & 0.0033 & 0.9545 \\
Side-x-Sex & 0.3064 & 0.5805 \\
Side-x-L4-Size-x-Sex & 0.2845 & 0.5943 \\
\hline
\end{tabular}

*df for each test is 1 and 203.

Means, Sexes Pooled

$\begin{array}{ll}\text { U_R2-1 lapping } & 0.35 \\ \text { U_L1-2 lapping } & 0.31 \\ \text { Means, by Sex } & \end{array}$

Boy

U_R2-1 lapping U_L1-2 lapping

Girl

0.250 .23

0.440 .39 
Table A-335. ANCOVA results testing for associations between mesiodistal width of the mandibular first premolar and L2-L3 overlap.

\begin{tabular}{lcc}
\hline Source & F-Ratio $^{*}$ & P-Value \\
\hline \multicolumn{3}{c}{ Among Subjects } \\
L4-Size & 11.1701 & 0.0010 \\
Sex & 0.4036 & 0.5259 \\
L4-Size-x-Sex & 0.3179 & 0.5735 \\
& \multicolumn{2}{c}{ Within Subjects } \\
Side & 1.7702 & 0.1848 \\
Side-x-L4-Size & 1.9888 & 0.1600 \\
Side-x-Sex & 1.0082 & 0.3165 \\
Side-x-L4-Size-x-Sex & 1.0235 & 0.3129 \\
\hline
\end{tabular}

* $\mathrm{df}$ for each test is 1 and 203.

Means, Sexes Pooled

\begin{tabular}{lrr} 
L_R3-2 lapping & 0.71 & \\
L_L2-3 lapping & 0.65 & \\
Means, by Sex & \\
\multicolumn{4}{r}{ L_R3-2 lapping } & L_L2-3 lapping \\
Boy & 0.650 .59 & \\
Girl & 0.780 .70
\end{tabular}


Table A-336. ANCOVA results testing for associations between mesiodistal width of the mandibular first premolar and L1-L2 overlap.

\begin{tabular}{llc}
\hline Source & F-Ratio* & P-Value \\
\hline & \multicolumn{2}{c}{ Among Subjects } \\
L4-Size & 7.6361 & 0.0062 \\
Sex & 2.0863 & 0.1502 \\
L4-Size-x-Sex & 2.2378 & 0.1362 \\
& \multicolumn{2}{c}{ Within Subjects } \\
Side & 0.3486 & 0.5556 \\
Side-x-L4-Size & 0.3182 & 0.5733 \\
Side-x-Sex & 1.9567 & 0.1634 \\
Side-x-L4-Size-x-Sex & 2.0755 & 0.1512 \\
\hline
\end{tabular}

*df for each test is 1 and 203.

Means, Sexes Pooled

\begin{tabular}{|c|c|c|}
\hline L_R2-1 lapping & 0.47 & \\
\hline L_L1-2 lapping & 0.46 & \\
\hline \multicolumn{3}{|c|}{ Means, by Sex } \\
\hline & L_R2-1 lapping & L_L1-2 lapping \\
\hline Boy & 0.440 .46 & \\
\hline Girl & 0.530 .48 & \\
\hline
\end{tabular}


Table A-337. ANCOVA results testing for associations between mesiodistal width of the mandibular first premolar and total displacements.

\begin{tabular}{lcccc}
\hline Term & Estimate & $\mathrm{SE}$ & $\mathrm{t}$-Test & P-Value \\
\hline Intercept & -2.7943 & 4.3819 & -0.64 & 0.5244 \\
L4-Size & 0.9737 & 0.6197 & 1.57 & 0.1177 \\
Sex & -1.0974 & 4.3819 & -0.25 & 0.8025 \\
Interaction & 0.0952 & 0.6197 & 0.15 & 0.8781 \\
\hline
\end{tabular}

Table A-338. ANCOVA results testing for associations between mesiodistal width of the mandibular first premolar and total rotations.

\begin{tabular}{lcccc}
\hline Term & Estimate & SE & t-Test & P-Value \\
\hline Intercept & -3.6507 & 3.2036 & -1.14 & 0.2558 \\
L4-Size & 1.1191 & 0.4530 & 2.47 & 0.0143 \\
Sex & 1.2157 & 3.2036 & 0.38 & 0.7047 \\
Interaction & -0.2124 & 0.4530 & -0.47 & 0.6397 \\
\hline
\end{tabular}

Table A-339. ANCOVA results testing for associations between mesiodistal width of the mandibular first premolar and maxillary R1-L1 overlap.

\begin{tabular}{lcccc}
\hline Term & Estimate & $\mathrm{SE}$ & $\mathrm{t}$-Test & P-Value \\
\hline Intercept & -0.8912 & 0.5995 & -1.49 & 0.1387 \\
L4-Size & 0.1607 & 0.0848 & 1.90 & 0.0595 \\
Sex & 0.5051 & 0.5995 & 0.84 & 0.4005 \\
Interaction & -0.0739 & 0.0848 & -0.87 & 0.3847 \\
\hline
\end{tabular}


Table A-340. ANCOVA results testing for associations between mesiodistal width of the mandibular first premolar and mandibular R1-L1 overlap.

\begin{tabular}{lcccc}
\hline Term & Estimate & SE & t-Test & P-Value \\
\hline Intercept & -0.4529 & 0.6212 & -0.73 & 0.4668 \\
L4-Size & 0.1187 & 0.0878 & 1.35 & 0.1781 \\
Sex & 0.6159 & 0.6212 & 0.99 & 0.3227 \\
Interaction & -0.0734 & 0.0878 & -0.84 & 0.4041 \\
\hline
\end{tabular}

Table A-341. ANCOVA results testing for associations between mesiodistal width of the mandibular first premolar and total overlapping.

\begin{tabular}{lcccc}
\hline Term & Estimate & $\mathrm{SE}$ & $\mathrm{t}$-Test & P-Value \\
\hline Intercept & -7.2940 & 3.9632 & -1.84 & 0.0672 \\
L4-Size & 1.6462 & 0.5605 & 2.94 & 0.0037 \\
Sex & -0.0076 & 3.9632 & 0.00 & 0.9985 \\
Interaction & -0.0642 & 0.5605 & -0.11 & 0.9089 \\
\hline
\end{tabular}

Table A-342. ANCOVA results testing for associations between mesiodistal width of the mandibular first premolar and total displacements, rotations, and overlapping.

\begin{tabular}{lcccc}
\hline Term & Estimate & $\mathrm{SE}$ & $\mathrm{t}$-Test & P-Value \\
\hline Intercept & -13.7391 & 9.8816 & -1.39 & 0.1659 \\
L4-Size & 3.7390 & 1.3975 & 2.68 & 0.0081 \\
Sex & 0.1106 & 9.8816 & 0.01 & 0.9911 \\
Interaction & -0.1814 & 1.3975 & -0.13 & 0.8968 \\
\hline
\end{tabular}


Table A-343. ANCOVA results testing for associations between mesiodistal width of the mandibular first premolar and crowding.

\begin{tabular}{lcccc}
\hline Term & Estimate & SE & t-Test & P-Value \\
\hline Intercept & -2.5143 & 0.9145 & -2.75 & 0.0065 \\
L4-Size & 0.5522 & 0.1293 & 4.27 & $<0.0001$ \\
Sex & 1.6420 & 0.9145 & 1.80 & 0.0741 \\
Interaction & -0.2507 & 0.1293 & -1.94 & 0.0540 \\
\hline
\end{tabular}

Table A-344. ANCOVA results testing for associations between mesiodistal width of the mandibular first premolar and spacing.

\begin{tabular}{lcccc}
\hline Term & Estimate & $\mathrm{SE}$ & $\mathrm{t}$-Test & P-Value \\
\hline Intercept & 3.1134 & 0.6881 & 4.52 & $<0.0001$ \\
L4-Size & -0.4061 & 0.0973 & -4.17 & $<0.0001$ \\
Sex & -1.8434 & 0.6881 & -2.68 & 0.0080 \\
Interaction & 0.2687 & 0.0973 & 2.76 & 0.0063 \\
\hline
\end{tabular}

Table A-345. ANCOVA results testing for associations between mesiodistal width of the mandibular first premolar and diastema.

\begin{tabular}{lcccc}
\hline Term & Estimate & SE & t-Test & P-Value \\
\hline Intercept & 2.5006 & 0.7115 & 3.51 & 0.0005 \\
L4-Size & -0.3230 & 0.1006 & -3.21 & 0.0015 \\
Sex & -2.0401 & 0.7115 & -2.87 & 0.0046 \\
Interaction & 0.2942 & 0.1006 & 2.92 & 0.0038 \\
\hline
\end{tabular}


Table A-346. ANCOVA results testing for associations between mesiodistal width of the mandibular first premolar and maxillary incisor irregularity.

\begin{tabular}{lcccc}
\hline Term & Estimate & SE & t-Test & P-Value \\
\hline Intercept & -0.3898 & 1.7354 & -0.22 & 0.8225 \\
L4-Size & 0.2562 & 0.2454 & 1.04 & 0.2977 \\
Sex & -1.0861 & 1.7354 & -0.63 & 0.5321 \\
Interaction & 0.1334 & 0.2454 & 0.54 & 0.5872 \\
\hline
\end{tabular}

Table A-347. ANCOVA results testing for associations between mesiodistal width of the mandibular first premolar and mandibular incisor irregularity.

\begin{tabular}{lcccc}
\hline Term & Estimate & SE & t-Test & P-Value \\
\hline Intercept & -2.0604 & 1.5939 & -1.29 & 0.1976 \\
L4-Size & 0.4493 & 0.2254 & 1.99 & 0.0476 \\
Sex & -1.0034 & 1.5939 & -0.63 & 0.5297 \\
Interaction & 0.1317 & 0.2254 & 0.58 & 0.5597 \\
\hline
\end{tabular}

Table A-348. ANCOVA results testing for associations between mesiodistal width of the mandibular first premolar and maxillary overjet.

\begin{tabular}{lcccc}
\hline Term & Estimate & $\mathrm{SE}$ & $\mathrm{t}$-Test & P-Value \\
\hline Intercept & 0.7036 & 2.8983 & 0.24 & 0.8084 \\
L4-Size & 0.4102 & 0.4099 & 1.00 & 0.3181 \\
Sex & 1.2049 & 2.8983 & 0.42 & 0.6780 \\
Interaction & -0.1826 & 0.4099 & -0.45 & 0.6564 \\
\hline
\end{tabular}


Table A-349. ANCOVA results testing for associations between mesiodistal width of the mandibular first premolar and mandibular overjet.

\begin{tabular}{lcccc}
\hline Term & Estimate & SE & t-Test & P-Value \\
\hline Intercept & -0.0236 & 0.3499 & -0.07 & 0.9463 \\
L4-Size & 0.0091 & 0.0495 & 0.18 & 0.8549 \\
Sex & 0.0236 & 0.3499 & 0.07 & 0.9463 \\
Interaction & -0.0091 & 0.0495 & -0.18 & 0.8549 \\
\hline
\end{tabular}

Table A-350. ANCOVA results testing for associations between mesiodistal width of the mandibular first premolar and openbite.

\begin{tabular}{lcccc}
\hline Term & Estimate & SE & t-Test & P-Value \\
\hline Intercept & 0.2317 & 0.5861 & 0.40 & 0.6930 \\
L4-Size & -0.0218 & 0.0829 & -0.26 & 0.7931 \\
Sex & 0.8352 & 0.5861 & 1.43 & 0.1557 \\
Interaction & -0.1163 & 0.0829 & -1.40 & 0.1621 \\
\hline
\end{tabular}

Table A-351. ANCOVA results testing for associations between mesiodistal width of the mandibular first premolar and anteroposterior relationship.

\begin{tabular}{lrcrc}
\hline Term & Estimate & $\mathrm{SE}$ & $\mathrm{t}$-Test & P-Value \\
\hline Intercept & 1.7201 & 0.9005 & 1.91 & 0.0575 \\
L4-Size & -0.1369 & 0.1274 & -1.08 & 0.2836 \\
Sex & 0.4424 & 0.9005 & 0.49 & 0.6237 \\
Interaction & -0.0561 & 0.1274 & -0.44 & 0.6603 \\
\hline
\end{tabular}


Table A-352. ANCOVA results testing for associations between mesiodistal width of the mandibular first premolar and DAI score.

\begin{tabular}{lcccc}
\hline Term & Estimate & $\mathrm{SE}$ & $\mathrm{t}$-Test & P-Value \\
\hline Intercept & 26.8413 & 8.3394 & 3.22 & 0.0015 \\
L4-Size & 0.0216 & 1.1794 & 0.02 & 0.9854 \\
Sex & -2.8090 & 8.3394 & -0.34 & 0.7366 \\
Interaction & 0.3532 & 1.1794 & 0.30 & 0.7649 \\
\hline
\end{tabular}


Table A-353. ANCOVA results testing for associations between mesiodistal width of the mandibular second premolar and U3 displacements.

\begin{tabular}{lcc}
\hline Source & F-Ratio* & P-Value \\
\hline \multicolumn{2}{c}{ Among Subjects } \\
L5-Size & 0.2594 & 0.6111 \\
Sex & 0.1822 & 0.6699 \\
L5-Size-x-Size & 0.2508 & 0.6171 \\
& \multicolumn{2}{c}{ Within Subjects } \\
Side & 0.0199 & 0.8878 \\
Side-x-L5-Size & 0.0101 & 0.9201 \\
Side-x-Sex & 0.3449 & 0.5577 \\
Side-x-L5-Size-Sex & 0.3899 & 0.5331 \\
\hline
\end{tabular}

*df for each test is 1 and 203.

Means, Sexes Pooled

\begin{tabular}{lll} 
U_R3 dis & 0.41 & \\
U_L3 dis & 0.38 & \\
& Means, by Sex \\
\multicolumn{4}{c}{ U_R3 dis } & U_L3 dis \\
Boy & 0.350 .34 & \\
Girl & 0.480 .41 &
\end{tabular}


Table A-354. ANCOVA results testing for associations between mesiodistal width of the mandibular second premolar and U2 displacements.

\begin{tabular}{llc}
\hline Source & F-Ratio* & P-Value \\
\hline \multicolumn{2}{c}{ Among Subjects } \\
L5-Size & 0.1898 & 0.6636 \\
Sex & 0.1490 & 0.6999 \\
L5-Size-x-Size & 0.0637 & 0.8011 \\
& \multicolumn{2}{c}{ Within Subjects } \\
Side & 1.5357 & 0.2167 \\
Side-x-L5-Size & 1.4082 & 0.2367 \\
Side-x-Sex & 0.0325 & 0.8571 \\
Side-x-L5-Size-Sex & 0.0377 & 0.8462 \\
\hline
\end{tabular}

*df for each test is 1 and 203.

Means, Sexes Pooled

\begin{tabular}{lrr} 
U_R2 dis & 0.36 \\
U_L2 dis & 0.41 & \\
& Means, by Sex \\
\multicolumn{4}{c}{ U_R2 dis } & U_L2 dis \\
Boy & 0.280 .33 & \\
Girl & 0.450 .48 &
\end{tabular}


Table A-355. ANCOVA results testing for associations between mesiodistal width of the mandibular second premolar and U1 displacements.

\begin{tabular}{lcc}
\hline Source & F-Ratio* & P-Value \\
\hline \multicolumn{2}{c}{ Among Subjects } \\
L5-Size & 0.1445 & 0.7043 \\
Sex & 0.4902 & 0.4847 \\
L5-Size-x-Size & 0.4839 & 0.4874 \\
& \multicolumn{2}{c}{ Within Subjects } \\
Side & 0.0440 & 0.8340 \\
Side-x-L5-Size & 0.0315 & 0.8593 \\
Side-x-Sex & 1.3841 & 0.2408 \\
Side-x-L5-Size-Sex & 1.5123 & 0.2202 \\
\hline
\end{tabular}

*df for each test is 1 and 203.

Means, Sexes Pooled

\begin{tabular}{lrr} 
U_R1 dis & 0.34 & \\
U_L1 dis & 0.35 & \\
& Means, by Sex & \\
\multicolumn{4}{r}{ B_R1 dis } & U_L1 dis \\
Boy & 0.360 .35 & \\
Girl & 0.320 .37 &
\end{tabular}


Table A-356. ANCOVA results testing for associations between mesiodistal width of the mandibular second premolar and L3 displacements.

\begin{tabular}{lcc}
\hline Source & F-Ratio* & P-Value \\
\hline \multicolumn{2}{c}{ Among Subjects } \\
L5-Size & 0.9123 & 0.3406 \\
Sex & 1.8031 & 0.1808 \\
L5-Size-x-Size & 1.5683 & 0.2119 \\
& \multicolumn{2}{c}{ Within Subjects } \\
Side & 0.6172 & 0.4330 \\
Side-x-L5-Size & 0.5925 & 0.4424 \\
Side-x-Sex & 3.4034 & 0.0665 \\
Side-x-L5-Size-Sex & 3.7555 & 0.0540 \\
\hline
\end{tabular}

*df for each test is 1 and 203.

Means, Sexes Pooled

$\begin{array}{lcl}\text { L_R3 dis } & 0.22 \\ \text { L_L3 dis } & 0.22 \\ \text { Means, by Sex } \\ \text { L_R3 dis } \quad \text { L_L3 dis } \\ \text { Boy } & 0.150 .20 \\ \text { Girl } & 0.300 .23\end{array}$


Table A-357. ANCOVA results testing for associations between mesiodistal width of the mandibular second premolar and L2 displacements.

\begin{tabular}{llc}
\hline Source & F-Ratio* & P-Value \\
\hline \multicolumn{2}{c}{ Among Subjects } \\
L5-Size & 0.7652 & 0.3827 \\
Sex & 3.4914 & 0.0631 \\
L5-Size-x-Size & 3.2913 & 0.0711 \\
& \multicolumn{2}{c}{ Within Subjects } \\
Side & 0.2835 & 0.5950 \\
Side-x-L5-Size & 0.2259 & 0.6351 \\
Side-x-Sex & 0.0117 & 0.9139 \\
Side-x-L5-Size-Sex & 0.0066 & 0.9354 \\
\hline
\end{tabular}

*df for each test is 1 and 203.

Means, Sexes Pooled

\begin{tabular}{lll} 
L_R2 dis & 0.51 & \\
L_L2 dis & 0.47 & \\
& Means, by Sex \\
\multicolumn{4}{r}{ L_R2 dis } & L_L2 dis \\
Boy & 0.450 .43 & \\
Girl & 0.550 .49 &
\end{tabular}


Table A-358. ANCOVA results testing for associations between mesiodistal width of the mandibular second premolar and L1 displacements.

\begin{tabular}{lcc}
\hline Source & F-Ratio* & P-Value \\
\hline \multicolumn{2}{c}{ Among Subjects } \\
L5-Size & 0.5587 & 0.4556 \\
Sex & 0.0038 & 0.9510 \\
L5-Size-x-Size & 0.0005 & 0.9823 \\
& \multicolumn{2}{c}{ Within Subjects } \\
Side & 0.0563 & 0.8127 \\
Side-x-L5-Size & 0.1163 & 0.7335 \\
Side-x-Sex & 4.7646 & 0.0302 \\
Side-x-L5-Size-Sex & 4.5708 & 0.0337 \\
\hline
\end{tabular}

*df for each test is 1 and 203.

Means, Sexes Pooled

\begin{tabular}{lll} 
L_R1 dis & 0.22 & \\
L_L1 dis & 0.16 & \\
& Means, by Sex \\
\multicolumn{4}{c}{ L_R1 dis } & L_L1 dis \\
Boy & 0.250 .16 & \\
Girl & 0.190 .16 &
\end{tabular}


Table A-359. ANCOVA results testing for associations between mesiodistal width of the mandibular second premolar and U3 rotations.

\begin{tabular}{lcc}
\hline Source & F-Ratio* & P-Value \\
\hline \multicolumn{2}{c}{ Among Subjects } \\
L5-Size & 0.8025 & 0.3714 \\
Sex & 0.8659 & 0.3532 \\
L5-Size-x-Size & 0.6375 & 0.4256 \\
& \multicolumn{2}{c}{ Within Subjects } \\
Side & 0.1237 & 0.7255 \\
Side-x-L5-Size & 0.1353 & 0.7134 \\
Side-x-Sex & 0.6896 & 0.4073 \\
Side-x-L5-Size-Sex & 0.7610 & 0.3840 \\
\hline
\end{tabular}

* $\mathrm{df}$ for each test is 1 and 203.

Means, Sexes Pooled

$\begin{array}{ccc}\text { U_R3 rot } & 0.21 \\ \text { U_L3 rot } & 0.22 \\ \text { Means, by Sex } \\ \text { U_R3 rot } \quad \text { U_L3 rot } \\ \text { Boy } & 0.170 .15 \\ \text { Girl } & 0.250 .29\end{array}$


Table A-360. ANCOVA results testing for associations between mesiodistal width of the mandibular second premolar and $\mathrm{U} 2$ rotations.

\begin{tabular}{llc}
\hline Source & F-Ratio* & P-Value \\
\hline & \multicolumn{2}{c}{ Among Subjects } \\
L5-Size & 0.1203 & 0.7290 \\
Sex & 0.5807 & 0.4469 \\
L5-Size-x-Size & 0.5399 & 0.4633 \\
& \multicolumn{2}{c}{ Within Subjects } \\
Side & 2.6645 & 0.1042 \\
Side-x-L5-Size & 2.5262 & 0.1135 \\
Side-x-Sex & 1.2761 & 0.2600 \\
Side-x-L5-Size-Sex & 1.2585 & 0.2633 \\
\hline
\end{tabular}

* $\mathrm{df}$ for each test is 1 and 203.

Means, Sexes Pooled

$\begin{array}{lrr}\text { U_R2 rot } & 0.39 & \\ \text { U_L2 rot } & 0.42 & \\ & \text { Means, by Sex } \\ & \text { U_R2 rot } & \text { U_L2 rot } \\ \text { Boy } & 0.360 .40 & \\ \text { Girl } & 0.400 .43 & \end{array}$


Table A-361. ANCOVA results testing for associations between mesiodistal width of the mandibular second premolar and U1 rotations.

\begin{tabular}{lcc}
\hline Source & F-Ratio* & P-Value \\
\hline \multicolumn{2}{c}{ Among Subjects } \\
L5-Size & 0.7841 & 0.3769 \\
Sex & 0.4413 & 0.5072 \\
L5-Size-x-Size & 0.4260 & 0.5147 \\
& \multicolumn{2}{c}{ Within Subjects } \\
Side & 0.6158 & 0.4335 \\
Side-x-L5-Size & 0.5912 & 0.4428 \\
Side-x-Sex & 0.0050 & 0.9436 \\
Side-x-L5-Size-Sex & 0.0034 & 0.9534 \\
\hline
\end{tabular}

*df for each test is 1 and 203.

Means, Sexes Pooled

$\begin{array}{lll}\text { U_R1 rot } & 0.47 & \\ \text { U_L1 rot } & 0.48 & \\ & \text { Means, by Sex } \\ & \text { U_R1 rot } & \text { U_L1 rot } \\ \text { Boy } & 0.460 .47 & \\ \text { Girl } & 0.470 .49 & \end{array}$


Table A-362. ANCOVA results testing for associations between mesiodistal width of the mandibular second premolar and L3 rotations.

\begin{tabular}{lcc}
\hline Source & F-Ratio & P-Value \\
\hline \multicolumn{2}{c}{ Among Subjects } \\
L5-Size & 2.7262 & 0.1003 \\
Sex & 0.0546 & 0.8155 \\
L5-Size-x-Size & 0.1318 & 0.7169 \\
& \multicolumn{2}{c}{ Within Subjects } \\
Side & 1.8662 & 0.1734 \\
Side-x-L5-Size & 2.0151 & 0.1573 \\
Side-x-Sex & 0.0533 & 0.8176 \\
Side-x-L5-Size-Sex & 0.0170 & 0.8964 \\
\hline
\end{tabular}

*df for each test is 1 and 203.

Means, Sexes Pooled

\begin{tabular}{lll} 
L_R3 rot & 0.38 & \\
L_L3 rot & 0.35 & \\
& Means, by Sex & \\
\multicolumn{4}{c}{ L_R3 rot } & L_L3 rot \\
Boy & 0.280 .31 & \\
Girl & 0.500 .39 &
\end{tabular}


Table A-363. ANCOVA results testing for associations between mesiodistal width of the mandibular second premolar and L2 rotations.

\begin{tabular}{lcc}
\hline Source & F-Ratio & P-Value \\
\hline \multicolumn{2}{c}{ Among Subjects } \\
L5-Size & 1.6627 & 0.1987 \\
Sex & 0.2000 & 0.6552 \\
L5-Size-x-Size & 0.1620 & 0.6877 \\
& \multicolumn{2}{c}{ Within Subjects } \\
Side & 0.6211 & 0.4316 \\
Side-x-L5-Size & 0.4140 & 0.5207 \\
Side-x-Sex & 2.1031 & 0.1485 \\
Side-x-L5-Size-Sex & 1.9883 & 0.1600 \\
\hline
\end{tabular}

*df for each test is 1 and 203.

Means, Sexes Pooled

\begin{tabular}{lll} 
L_R2 rot & 0.35 & \\
L_L2 rot & 0.23 & \\
& Means, by Sex & \\
\multicolumn{4}{c}{ L_R2 rot } & L_L2 rot \\
Boy & 0.300 .22 & \\
Girl & 0.380 .23 &
\end{tabular}


Table A-364. ANCOVA results testing for associations between mesiodistal width of the mandibular second premolar and L1 rotations.

\begin{tabular}{llc}
\hline Source & F-Ratio & P-Value \\
\hline \multicolumn{2}{c}{ Among Subjects } \\
L5-Size & 0.4260 & 0.5147 \\
Sex & 5.5147 & 0.0198 \\
L5-Size-x-Size & 6.0805 & 0.0145 \\
& \multicolumn{2}{c}{ Within Subjects } \\
Side & 0.6398 & 0.4247 \\
Side-x-L5-Size & 0.7410 & 0.3904 \\
Side-x-Sex & 0.5578 & 0.4560 \\
Side-x-L5-Size-Sex & 0.5131 & 0.4746 \\
\hline
\end{tabular}

* $\mathrm{df}$ for each test is 1 and 203.

Means, Sexes Pooled

\begin{tabular}{lll} 
L_R1 rot & 0.37 & \\
L_L1 rot & 0.33 & \\
& Means, by Sex & \\
\multicolumn{4}{c}{ L_R1 rot } & L_L1 rot \\
Boy & 0.420 .36 & \\
Girl & 0.300 .28 &
\end{tabular}


Table A-365. ANCOVA results testing for associations between mesiodistal width of the mandibular second premolar and U2-U3 overlap.

\begin{tabular}{llc}
\hline Source & F-Ratio* $^{*}$ & P-Value \\
\hline \multicolumn{2}{c}{ Among Subjects } \\
L5-Size & 0.3513 & 0.5540 \\
Sex & 0.1506 & 0.6984 \\
L5-Size-x-Size & 0.2811 & 0.5966 \\
& \multicolumn{2}{c}{ Within Subjects } \\
Side & 0.8300 & 0.3634 \\
Side-x-L5-Size & 0.8751 & 0.3507 \\
Side-x-Sex & 0.1397 & 0.7090 \\
Side-x-L5-Size-Sex & 0.1789 & 0.6727 \\
\hline
\end{tabular}

*df for each test is 1 and 203.

Means, Sexes Pooled

$\begin{array}{ll}\text { U_R3-2 lapping } & 0.38 \\ \text { U_L2-3 lapping } & 0.36 \\ \text { Means, by Sex } & \end{array}$

Boy

U_R3-2 lapping U_L2-3 lapping

Girl

0.270 .30

0.500 .44 
Table A-366. ANCOVA results testing for associations between mesiodistal width of the mandibular second premolar and U1-U2 overlap.

\begin{tabular}{lcc}
\hline Source & F-Ratio & P-Value \\
\hline \multicolumn{2}{c}{ Among Subjects } \\
L5-Size & 0.0329 & 0.8563 \\
Sex & 0.5949 & 0.4414 \\
L5-Size-x-Size & 0.3616 & 0.5483 \\
& \multicolumn{2}{c}{ Within Subjects } \\
Side & 0.6167 & 0.4332 \\
Side-x-L5-Size & 0.6894 & 0.4073 \\
Side-x-Sex & 0.7592 & 0.3846 \\
Side-x-L5-Size-Sex & 0.7138 & 0.3992 \\
\hline
\end{tabular}

* $\mathrm{df}$ for each test is 1 and 203.

Means, Sexes Pooled

$\begin{array}{ll}\text { U_R2-1 lapping } & 0.34 \\ \text { U_L1-2 lapping } & 0.31 \\ \text { Means, by Sex } & \end{array}$

Boy

U_R2-1 lapping U_L1-2 lapping

Girl

$\begin{array}{ll}0.244 & 0.23 \\ 0.448 & 0.39\end{array}$


Table A-367. ANCOVA results testing for associations between mesiodistal width of the mandibular second premolar and L2-L3 overlap.

\begin{tabular}{lcc}
\hline Source & F-Ratio & P-Value \\
\hline \multicolumn{2}{c}{ Among Subjects } \\
L5-Size & 10.8117 & 0.0012 \\
Sex & 2.6739 & 0.1036 \\
L5-Size-x-Size & 2.4544 & 0.1188 \\
& \multicolumn{2}{c}{ Within Subjects } \\
Side & 3.3416 & 0.0690 \\
Side-x-L5-Size & 3.7076 & 0.0556 \\
Side-x-Sex & 1.7523 & 0.1871 \\
Side-x-L5-Size-Sex & 1.7916 & 0.1822 \\
\hline
\end{tabular}

* $\mathrm{df}$ for each test is 1 and 203.

Means, Sexes Pooled

\begin{tabular}{|c|c|c|}
\hline L_R3-2 lapping & 0.71 & \\
\hline L_L2-3 lapping & 0.65 & \\
\hline \multicolumn{3}{|c|}{ Means, by Sex } \\
\hline & L_R3-2 lapping & L_L2-3 lapping \\
\hline Boy & 0.650 .59 & \\
\hline Girl & 0.760 .69 & \\
\hline
\end{tabular}


Table A-368. ANCOVA results testing for associations between mesiodistal width of the mandibular second premolar and L1-L2 overlap.

\begin{tabular}{lcc}
\hline Source & F-Ratio & \multicolumn{2}{c}{ P-Value } \\
\hline \multicolumn{2}{c}{ Among Subjects } \\
L5-Size & 1.5438 & 0.2155 \\
Sex & 0.4098 & 0.5228 \\
L5-Size-x-Size & 0.3592 & 0.5496 \\
& \multicolumn{2}{c}{ Within Subjects } \\
Side & 0.5473 & 0.4603 \\
Side-x-L5-Size & 0.4998 & 0.4804 \\
Side-x-Sex & 6.2455 & 0.0132 \\
Side-x-L5-Size-Sex & 6.5274 & 0.0114 \\
\hline
\end{tabular}

* $\mathrm{df}$ for each test is 1 and 203.

Means, Sexes Pooled

\begin{tabular}{lrr} 
L_R2-1 lapping & 0.47 & \\
L_L1-2 lapping & 0.46 & \\
Means, by Sex & \\
\multicolumn{4}{c}{ L_R2-1 lapping } & L_L1-2 lapping \\
Boy & 0.440 .45 & \\
Girl & 0.510 .45 &
\end{tabular}


Table A-369. ANCOVA results testing for associations between mesiodistal width of the mandibular second premolar and total displacements.

\begin{tabular}{lcccc}
\hline Term & Estimate & $\mathrm{SE}$ & $\mathrm{t}$-Test & P-Value \\
\hline Intercept & -0.15911 & 3.935166 & -0.04 & 0.9678 \\
L5 & 0.59065 & 0.546191 & 1.08 & 0.2808 \\
Sex & -2.91699 & 3.935166 & -0.74 & 0.4594 \\
Interaction & 0.350531 & 0.546191 & 0.64 & 0.5217 \\
\hline
\end{tabular}

Table A-370. ANCOVA results testing for associations between mesiodistal width of the mandibular second premolar and total rotations.

\begin{tabular}{lcccc}
\hline Term & Estimate & SE & t-Test & P-Value \\
\hline Intercept & -0.968 & 2.873752 & -0.34 & 0.7366 \\
L5 & 0.722673 & 0.398869 & 1.81 & 0.0715 \\
Sex & -4.71371 & 2.873752 & -1.64 & 0.1025 \\
Interaction & 0.619692 & 0.398869 & 1.55 & 0.1218 \\
\hline
\end{tabular}

Table A-371. ANCOVA results testing for associations between mesiodistal width of the mandibular second premolar and maxillary R1L1 overlap.

\begin{tabular}{lclcc}
\hline Term & Estimate & $\mathrm{SE}$ & $\mathrm{t}$-Test & P-Value \\
\hline Intercept & -0.0651 & 0.540644 & -0.12 & 0.9043 \\
L5 & 0.04244 & 0.07504 & 0.57 & 0.5723 \\
Sex & -0.26972 & 0.540644 & -0.5 & 0.6184 \\
Interaction & 0.036075 & 0.07504 & 0.48 & 0.6312 \\
\hline
\end{tabular}


Table A-372. ANCOVA results testing for associations between mesiodistal width of the mandibular second premolar and mandibular R1-L1 overlap.

\begin{tabular}{lccrc}
\hline Term & Estimate & SE & t-Test & P-Value \\
Intercept & 0.182247 & 0.557551 & 0.33 & 0.7441 \\
L5 & 0.027445 & 0.077387 & 0.35 & 0.7232 \\
Sex & -0.36158 & 0.557551 & -0.65 & 0.5174 \\
Interaction & 0.064446 & 0.077387 & 0.83 & 0.4059 \\
\hline
\end{tabular}

Table A-373. ANCOVA results testing for associations between mesiodistal width of the mandibular second premolar and total overlapping.

\begin{tabular}{lcccc}
\hline Term & Estimate & SE & t-Test & P-Value \\
\hline Intercept & -3.16644 & 3.583089 & -0.88 & 0.3779 \\
L5 & 1.043029 & 0.497323 & 2.1 & 0.0372 \\
Sex & -3.98874 & 3.583089 & -1.11 & 0.2669 \\
Interaction & 0.496391 & 0.497323 & 1 & 0.3194 \\
\hline
\end{tabular}

Table A-374. ANCOVA results testing for associations between mesiodistal width of the mandibular second premolar and total displacements, rotations, and overlapping.

\begin{tabular}{lcccc}
\hline Term & Estimate & SE & t-Test & P-Value \\
\hline Intercept & -4.29354 & 8.907965 & -0.48 & 0.6303 \\
L5 & 2.356352 & 1.236402 & 1.91 & 0.0581 \\
Sex & -11.6194 & 8.907965 & -1.3 & 0.1936 \\
Interaction & 1.466614 & 1.236402 & 1.19 & 0.2369 \\
\hline
\end{tabular}


Table A-375. ANCOVA results testing for associations between mesiodistal width of the mandibular second premolar and crowding.

\begin{tabular}{lcccc}
\hline Term & Estimate & SE & t-Test & P-Value \\
\hline Intercept & -0.66655 & 0.842755 & -0.79 & 0.4299 \\
L5 & 0.284796 & 0.116972 & 2.43 & 0.0158 \\
Sex & -0.167 & 0.842755 & -0.2 & 0.8431 \\
Interaction & 0.007171 & 0.116972 & 0.06 & 0.9512 \\
\hline
\end{tabular}

Table A-376. ANCOVA results testing for associations between mesiodistal width of the mandibular second premolar and spacing.

\begin{tabular}{lcccc}
\hline Term & Estimate & SE & t-Test & P-Value \\
\hline Intercept & 2.521934 & 0.624594 & 4.04 & $<0.0001$ \\
L5 & -0.31557 & 0.086692 & -3.64 & 0.0003 \\
Sex & 0.000402 & 0.624594 & 0 & 0.9995 \\
Interaction & 0.007211 & 0.086692 & 0.08 & 0.9338 \\
\hline
\end{tabular}

Table A-377. ANCOVA results testing for associations between mesiodistal width of the mandibular second premolar and diastema.

\begin{tabular}{lcccc}
\hline Term & Estimate & SE & t-Test & P-Value \\
\hline Intercept & 1.469757 & 0.652851 & 2.25 & 0.0254 \\
L5 & -0.17242 & 0.090614 & -1.9 & 0.0585 \\
Sex & -0.04669 & 0.652851 & -0.07 & 0.9431 \\
Interaction & 0.011112 & 0.090614 & 0.12 & 0.9025 \\
\hline
\end{tabular}


Table A-378. ANCOVA results testing for associations between mesiodistal width of the mandibular second premolar and maxillary incisor irregularity.

\begin{tabular}{lclcc}
\hline Term & Estimate & SE & t-Test & P-Value \\
\hline Intercept & -0.80751 & 1.551687 & -0.52 & 0.6033 \\
L5 & 0.312004 & 0.21537 & 1.45 & 0.149 \\
Sex & 0.193186 & 1.551687 & 0.12 & 0.901 \\
Interaction & -0.04684 & 0.21537 & -0.22 & 0.828 \\
\hline
\end{tabular}

Table A-379. ANCOVA results testing for associations between mesiodistal width of the mandibular second premolar and mandibular incisor irregularity.

\begin{tabular}{lcccc}
\hline Term & Estimate & SE & t-Test & P-Value \\
\hline Intercept & -0.87026 & 1.435008 & -0.61 & 0.5449 \\
L5 & 0.276261 & 0.199175 & 1.39 & 0.167 \\
Sex & -1.77407 & 1.435008 & -1.24 & 0.2178 \\
Interaction & 0.238606 & 0.199175 & 1.2 & 0.2323 \\
\hline
\end{tabular}

Table A-380. ANCOVA results testing for associations between mesiodistal width of the mandibular second premolar and maxillary overjet.

\begin{tabular}{lclrc}
\hline Term & Estimate & SE & t-Test & P-Value \\
\hline Intercept & 0.277668 & 2.58432 & 0.11 & 0.9145 \\
L5 & 0.463918 & 0.358697 & 1.29 & 0.1974 \\
Sex & 1.831259 & 2.58432 & 0.71 & 0.4794 \\
Interaction & -0.26727 & 0.358697 & -0.75 & 0.4571 \\
\hline
\end{tabular}


Table A-381. ANCOVA results testing for associations between mesiodistal width of the mandibular second premolar and mandibular overjet.

\begin{tabular}{lcccc}
\hline Term & Estimate & SE & t-Test & P-Value \\
\hline Intercept & -0.01058 & 0.313061 & -0.03 & 0.9731 \\
L5 & 0.007093 & 0.043452 & 0.16 & 0.8705 \\
Sex & 0.010575 & 0.313061 & 0.03 & 0.9731 \\
Interaction & -0.00709 & 0.043452 & -0.16 & 0.8705 \\
\hline
\end{tabular}

Table A-382. ANCOVA results testing for associations between mesiodistal width of the mandibular second premolar and openbite.

\begin{tabular}{lcccc}
\hline Term & Estimate & SE & t-Test & P-Value \\
\hline Intercept & -0.13667 & 0.527446 & -0.26 & 0.7958 \\
L5 & 0.02878 & 0.073208 & 0.39 & 0.6946 \\
Sex & -0.06029 & 0.527446 & -0.11 & 0.9091 \\
Interaction & 0.009654 & 0.073208 & 0.13 & 0.8952 \\
\hline
\end{tabular}

Table A-383. ANCOVA results testing for associations between mesiodistal width of the mandibular second premolar and anteroposterior relationship.

\begin{tabular}{lclcc}
\hline Term & Estimate & $\mathrm{SE}$ & $\mathrm{t}$-Test & P-Value \\
\hline Intercept & 1.350807 & 0.80394 & 1.68 & 0.0944 \\
L5 & -0.08263 & 0.111585 & -0.74 & 0.4599 \\
Sex & 1.293278 & 0.80394 & 1.61 & 0.1092 \\
Interaction & -0.17414 & 0.111585 & -1.56 & 0.1202 \\
\hline
\end{tabular}


Table A-384. ANCOVA results testing for associations between mesiodistal width of the mandibular second premolar and DAI score.

\begin{tabular}{lcccc}
\hline Term & Estimate & $\mathrm{SE}$ & $\mathrm{t}$-Test & P-Value \\
\hline Intercept & 22.30307 & 7.445733 & 3 & 0.0031 \\
L5 & 0.661295 & 1.033448 & 0.64 & 0.523 \\
Sex & 4.852641 & 7.445733 & 0.65 & 0.5153 \\
Interaction & -0.72322 & 1.033448 & -0.7 & 0.4848 \\
\hline
\end{tabular}


Table A-385. ANCOVA results testing for associations between mesiodistal width of the mandibular first molar and U3 displacements.

\begin{tabular}{llc}
\hline Source & F-Ratio $^{*}$ & P-Value \\
\hline \multicolumn{2}{c}{ Among Subjects } \\
L6-Size & 0.1732 & 0.6777 \\
Sex & 0.9253 & 0.3372 \\
L6-Size-x-Sex & 0.8102 & 0.3691 \\
& \multicolumn{2}{c}{ Within Subjects } \\
Side & 0.0659 & 0.7977 \\
Side-x-L6-Size & 0.0430 & 0.8360 \\
Side-x-Sex & 1.6221 & 0.2043 \\
Side-x-L6-Size-Sex & 1.6944 & 0.1945 \\
\hline
\end{tabular}

*df for each test is 1 and 203.

Means, Sexes Pooled

\begin{tabular}{lrr} 
U_R3 dis & 0.41 & \\
U_L3 dis & 0.38 & \\
& Means, by Sex \\
\multicolumn{4}{c}{ U_R3 dis } & U_L3 dis \\
Boy & 0.340 .32 & \\
Girl & 0.470 .40 &
\end{tabular}


Table A-386. ANCOVA results testing for associations between mesiodistal width of the mandibular first molar and U2 displacements.

\begin{tabular}{lcc}
\hline Source & F-Ratio* & P-Value \\
\hline \multicolumn{2}{c}{ Among Subjects } \\
L6-Size & 3.3982 & 0.0667 \\
Sex & 2.9444 & 0.0877 \\
L6-Size-x-Sex & 3.4416 & 0.0650 \\
& \multicolumn{2}{c}{ Within Subjects } \\
Side & 1.3277 & 0.2506 \\
Side-x-L6-Size & 1.2625 & 0.2625 \\
Side-x-Sex & 1.2455 & 0.2657 \\
Side-x-L6-Size-Sex & 1.2948 & 0.2565 \\
\hline
\end{tabular}

*df for each test is 1 and 203.

Means, Sexes Pooled

\begin{tabular}{lrr} 
U_R2 dis & 0.37 & \\
U_L2 dis & 0.41 & \\
& Means, by Sex \\
\multicolumn{4}{c}{ U_R2 dis } & U_L2 dis \\
Boy & 0.290 .34 & \\
Girl & 0.510 .52 &
\end{tabular}


Table A-387. ANCOVA results testing for associations between mesiodistal width of the mandibular first molar and U1 displacements.

\begin{tabular}{llc}
\hline Source & F-Ratio* & P-Value \\
\hline \multicolumn{2}{c}{ Among Subjects } \\
L6-Size & 0.0187 & 0.8912 \\
Sex & 0.6673 & 0.4150 \\
L6-Size-x-Sex & 0.6778 & 0.4113 \\
& \multicolumn{2}{c}{ Within Subjects } \\
Side & 0.0040 & 0.9495 \\
Side-x-L6-Size & 0.0023 & 0.9616 \\
Side-x-Sex & 0.0889 & 0.7659 \\
Side-x-L6-Size-Sex & 0.0661 & 0.7973 \\
\hline
\end{tabular}

*df for each test is 1 and 203.

Means, Sexes Pooled

$\begin{array}{lrr}\text { U_R1 dis } & 0.34 & \\ \text { U_L1 dis } & 0.36 & \\ & \text { Means, by Sex } \\ & \text { U_R1 dis } & \text { U_L1 dis } \\ \text { Boy } & 0.360 .34 & \\ \text { Girl } & 0.310 .36 & \end{array}$


Table A-388. ANCOVA results testing for associations between mesiodistal width of the mandibular first molar and L3 displacements.

\begin{tabular}{lcc}
\hline Source & F-Ratio* & P-Value \\
\hline \multicolumn{2}{c}{ Among Subjects } \\
L6-Size & 0.7528 & 0.3866 \\
Sex & 0.0973 & 0.7554 \\
L6-Size-x-Sex & 0.0521 & 0.8197 \\
& \multicolumn{2}{c}{ Within Subjects } \\
Side & 0.4510 & 0.5026 \\
Side-x-L6-Size & 0.3955 & 0.5301 \\
Side-x-Sex & 6.8470 & 0.0095 \\
Side-x-L6-Size-Sex & 7.2233 & 0.0078 \\
\hline
\end{tabular}

*df for each test is 1 and 203.

Means, Sexes Pooled

\begin{tabular}{lll} 
L_R3 dis & 0.23 & \\
L_L3 dis & 0.23 & \\
& Means, by Sex \\
\multicolumn{4}{c}{ L_R3 dis } & L_L3 dis \\
Boy & 0.170 .19 & \\
Girl & 0.320 .23 &
\end{tabular}


Table A-389. ANCOVA results testing for associations between mesiodistal width of the mandibular first molar and L2 displacements.

\begin{tabular}{llc}
\hline Source & F-Ratio* & P-Value \\
\hline \multicolumn{2}{c}{ Among Subjects } \\
L6-Size & 0.8586 & 0.3552 \\
Sex & 0.0150 & 0.9025 \\
L6-Size-x-Sex & 0.0043 & 0.9476 \\
& \multicolumn{2}{c}{ Within Subjects } \\
Side & 0.0046 & 0.9461 \\
Side-x-L6-Size & 0.0074 & 0.9314 \\
Side-x-Sex & 4.1641 & 0.0426 \\
Side-x-L6-Size-Sex & 4.0633 & 0.0451 \\
\hline
\end{tabular}

*df for each test is 1 and 203.

Means, Sexes Pooled

\begin{tabular}{lrr} 
L_R2 dis & 0.51 & \\
L_L2 dis & 0.47 & \\
& Means, by Sex \\
\multicolumn{4}{c}{ L_R2 dis } & L_L2 dis \\
Boy & 0.440 .45 & \\
Girl & 0.560 .52 &
\end{tabular}


Table A-390. ANCOVA results testing for associations between mesiodistal width of the mandibular first molar and L1 displacements.

\begin{tabular}{lcc}
\hline Source & F-Ratio* & P-Value \\
\hline \multicolumn{2}{c}{ Among Subjects } \\
L6-Size & 1.5608 & 0.2130 \\
Sex & 3.3512 & 0.0686 \\
L6-Size-x-Sex & 3.4263 & 0.0656 \\
& \multicolumn{2}{c}{ Within Subjects } \\
Side & 1.8425 & 0.1762 \\
Side-x-L6-Size & 2.0608 & 0.1527 \\
Side-x-Sex & 0.1831 & 0.6692 \\
Side-x-L6-Size-Sex & 0.1654 & 0.6847 \\
\hline
\end{tabular}

*df for each test is 1 and 203.

Means, Sexes Pooled

\begin{tabular}{lrr} 
L_R1 dis & 0.22 & \\
L_L1 dis & 0.17 & \\
& Means, by Sex \\
\multicolumn{4}{c}{ L_R1 dis } & L_L1 dis \\
Boy & 0.230 .16 & \\
Girl & 0.200 .16 &
\end{tabular}


Table A-391. ANCOVA results testing for associations between mesiodistal width of the mandibular first molar and U3 rotations.

\begin{tabular}{lcc}
\hline Source & F-Ratio* & P-Value \\
\hline \multicolumn{2}{c}{ Among Subjects } \\
L6-Size & 0.0011 & 0.9737 \\
Sex & 1.6585 & 0.1993 \\
L6-Size-x-Sex & 1.4040 & 0.2374 \\
& \multicolumn{2}{c}{ Within Subjects } \\
Side & 0.0328 & 0.8565 \\
Side-x-L6-Size & 0.0346 & 0.8526 \\
Side-x-Sex & 0.0326 & 0.8568 \\
Side-x-L6-Size-Sex & 0.0219 & 0.8826 \\
\hline
\end{tabular}

*df for each test is 1 and 203.

Means, Sexes Pooled

\begin{tabular}{lll} 
U_R3 rot & 0.22 & \\
U_L3 rot & 0.22 & \\
& Means, by Sex \\
\multicolumn{4}{c}{ U_R3 rot } & U_L3 rot \\
Boy & 0.170 .15 & \\
Girl & 0.250 .28 &
\end{tabular}


Table A-392. ANCOVA results testing for associations between mesiodistal width of the mandibular first molar and $\mathrm{U} 2$ rotations.

\begin{tabular}{llc}
\hline Source & F-Ratio* & P-Value \\
\hline \multicolumn{2}{c}{ Among Subjects } \\
L6-Size & 0.3926 & 0.5317 \\
Sex & 0.5707 & 0.4508 \\
L6-Size-x-Sex & 0.6273 & 0.4293 \\
& \multicolumn{2}{c}{ Within Subjects } \\
Side & 1.6035 & 0.2069 \\
Side-x-L6-Size & 1.5135 & 0.2200 \\
Side-x-Sex & 0.1578 & 0.6916 \\
Side-x-L6-Size-Sex & 0.1445 & 0.7042 \\
\hline
\end{tabular}

*df for each test is 1 and 203.

Means, Sexes Pooled

$\begin{array}{lll}\text { U_R2 rot } & 0.39 & \\ \text { U_L2 rot } & 0.42 & \\ & \text { Means, by Sex } \\ & \text { U_R2 rot } & \text { U_L2 rot } \\ \text { Boy } & 0.360 .41 & \\ \text { Girl } & 0.430 .45 & \end{array}$


Table A-393. ANCOVA results testing for associations between mesiodistal width of the mandibular first molar and U1 rotations.

\begin{tabular}{llc}
\hline Source & F-Ratio* & P-Value \\
\hline \multicolumn{2}{c}{ Among Subjects } \\
L6-Size & 0.3309 & 0.5658 \\
Sex & 0.1210 & 0.7283 \\
L6-Size-x-Sex & 0.1313 & 0.7175 \\
& \multicolumn{2}{c}{ Within Subjects } \\
Side & 1.3621 & 0.2445 \\
Side-x-L6-Size & 1.3460 & 0.2473 \\
Side-x-Sex & 0.2102 & 0.6471 \\
Side-x-L6-Size-Sex & 0.2129 & 0.6450 \\
\hline
\end{tabular}

*df for each test is 1 and 203.

Means, Sexes Pooled

\begin{tabular}{lll} 
U_R1 rot & 0.47 & \\
U_L1 rot & 0.49 & \\
& Means, by Sex & \\
\multicolumn{4}{c}{ U_R1 rot } & U_L1 rot \\
Boy & 0.470 .48 & \\
Girl & 0.490 .50 &
\end{tabular}


Table A-394. ANCOVA results testing for associations between mesiodistal width of the mandibular first molar and L3 rotations.

\begin{tabular}{llc}
\hline Source & F-Ratio* & P-Value \\
\hline \multicolumn{2}{c}{ Among Subjects } \\
L6-Size & 0.0960 & 0.7570 \\
Sex & 0.0072 & 0.9324 \\
L6-Size-x-Sex & 0.0340 & 0.8540 \\
& \multicolumn{2}{c}{ Within Subjects } \\
Side & 1.0116 & 0.3157 \\
Side-x-L6-Size & 1.1261 & 0.2899 \\
Side-x-Sex & 0.5638 & 0.4536 \\
Side-x-L6-Size-Sex & 0.7085 & 0.4009 \\
\hline
\end{tabular}

*df for each test is 1 and 203.

Means, Sexes Pooled

\begin{tabular}{lll} 
L_R3 rot & 0.39 & \\
L_L3 rot & 0.35 & \\
& Means, by Sex & \\
\multicolumn{4}{c}{ L_R3 rot } & L_L3 rot \\
Boy & 0.290 .32 & \\
Girl & 0.510 .38 &
\end{tabular}


Table A-395. ANCOVA results testing for associations between mesiodistal width of the mandibular first molar and L2 rotations.

\begin{tabular}{lcc}
\hline Source & F-Ratio* & P-Value \\
\hline \multicolumn{2}{c}{ Among Subjects } \\
L6-Size & 2.7644 & 0.0979 \\
Sex & 3.2528 & 0.0728 \\
L6-Size-x-Sex & 3.4615 & 0.0643 \\
& \multicolumn{2}{c}{ Within Subjects } \\
Side & 0.0180 & 0.8933 \\
Side-x-L6-Size & 0.0674 & 0.7954 \\
Side-x-Sex & 0.0661 & 0.7974 \\
Side-x-L6-Size-Sex & 0.0456 & 0.8312 \\
\hline
\end{tabular}

*df for each test is 1 and 203.

Means, Sexes Pooled

\begin{tabular}{lrr} 
L_R2 rot & 0.35 & \\
L_L2 rot & 0.23 & \\
& Means, by Sex \\
\multicolumn{4}{c}{ L_R2 rot } & L_L2 rot \\
Boy & 0.320 .24 & \\
Girl & 0.420 .26 &
\end{tabular}


Table A-396. ANCOVA results testing for associations between mesiodistal width of the mandibular first molar and L1 rotations.

\begin{tabular}{llc}
\hline Source & F-Ratio* & P-Value \\
\hline \multicolumn{2}{c}{ Among Subjects } \\
L6-Size & 0.0002 & 0.9902 \\
Sex & 2.1642 & 0.1428 \\
L6-Size-x-Sex & 2.4562 & 0.1186 \\
& \multicolumn{2}{c}{ Within Subjects } \\
Side & 2.9284 & 0.0886 \\
Side-x-L6-Size & 3.0370 & 0.0829 \\
Side-x-Sex & 0.8764 & 0.3503 \\
Side-x-L6-Size-Sex & 0.8893 & 0.3468 \\
\hline
\end{tabular}

*df for each test is 1 and 203.

Means, Sexes Pooled

\begin{tabular}{lcc} 
L_R1 rot & 0.38 & \\
L_L1 rot & 0.34 & \\
& Means, by Sex & \\
\multicolumn{4}{c}{ L_R1 rot } & L_L1 rot \\
Boy & 0.410 .38 & \\
Girl & 0.300 .28 &
\end{tabular}


Table A-397. ANCOVA results testing for associations between mesiodistal width of the mandibular first molar and U2-U3 overlap.

\begin{tabular}{llc}
\hline Source & F-Ratio $^{*}$ & P-Value \\
\hline \multicolumn{2}{c}{ Among Subjects } \\
L6-Size & 0.3108 & 0.5778 \\
Sex & 0.2384 & 0.6259 \\
L6-Size-x-Sex & 0.1358 & 0.7129 \\
& \multicolumn{2}{c}{ Within Subjects } \\
Side & 0.8695 & 0.3522 \\
Side-x-L6-Size & 0.9303 & 0.3359 \\
Side-x-Sex & 0.9689 & 0.3261 \\
Side-x-L6-Size-Sex & 1.0686 & 0.3025 \\
\hline
\end{tabular}

*df for each test is 1 and 203.

Means, Sexes Pooled

$\begin{array}{ll}\text { U_R3-2 lapping } & 0.38 \\ \text { U_L2-3 lapping } & 0.37 \\ \text { Means, by Sex } & \end{array}$

Boy

U_R3-2 lapping U_L2-3 lapping

Girl

0.270 .29

0.510 .43 
Table A-398. ANCOVA results testing for associations between mesiodistal width of the mandibular first molar and U1-U2 overlap.

\begin{tabular}{llc}
\hline Source & F-Ratio* & P-Value \\
\hline \multicolumn{2}{c}{ Among Subjects } \\
L6-Size & 0.0000 & 0.9963 \\
Sex & 0.2310 & 0.6313 \\
L6-Size-x-Sex & 0.3856 & 0.5353 \\
& \multicolumn{2}{c}{ Within Subjects } \\
Side & 0.0358 & 0.8502 \\
Side-x-L6-Size & 0.0488 & 0.8253 \\
Side-x-Sex & 0.3576 & 0.5505 \\
Side-x-L6-Size-Sex & 0.3328 & 0.5647 \\
\hline
\end{tabular}

*df for each test is 1 and 203.

Means, Sexes Pooled

\begin{tabular}{lrr} 
U_R2-1 lapping & 0.35 & \\
U_L1-2 lapping & 0.31 & \\
\multicolumn{4}{c}{ Means, by Sex } \\
Boy & U_R2-1 lapping & U_L1-2 lapping \\
Girl & 0.250 .24 & \\
& 0.450 .41 &
\end{tabular}


Table A-399. ANCOVA results testing for associations between mesiodistal width of the mandibular first molar and L2-L3 overlap.

\begin{tabular}{llc}
\hline Source & F-Ratio* & P-Value \\
\hline \multicolumn{3}{c}{ Among Subjects } \\
L6-Size & 6.3981 & 0.0122 \\
Sex & 0.0304 & 0.8618 \\
L6-Size-x-Sex & 0.0095 & 0.9224 \\
& \multicolumn{2}{c}{ Within Subjects } \\
Side & 2.1288 & 0.1461 \\
Side-x-L6-Size & 2.3966 & 0.1232 \\
Side-x-Sex & 1.7428 & 0.1883 \\
Side-x-L6-Size-Sex & 1.8013 & 0.1811 \\
\hline
\end{tabular}

*df for each test is 1 and 203.

Means, Sexes Pooled

\begin{tabular}{lrr} 
L_R3-2 lapping & 0.71 & \\
L_L2-3 lapping & 0.65 & \\
Means, by Sex & \\
\multicolumn{4}{c}{ L_R3-2 lapping } & L_L2-3 lapping \\
Boy & 0.650 .58 & \\
Girl & 0.810 .70 &
\end{tabular}


Table A-400. ANCOVA results testing for associations between mesiodistal width of the mandibular first molar and L1-L2 overlap.

\begin{tabular}{llc}
\hline Source & F-Ratio* & P-Value \\
\hline \multicolumn{2}{c}{ Among Subjects } \\
L6-Size & 1.5699 & 0.2117 \\
Sex & 0.0003 & 0.9854 \\
L6-Size-x-Sex & 0.0041 & 0.9487 \\
& \multicolumn{2}{c}{ Within Subjects } \\
Side & 0.2263 & 0.6348 \\
Side-x-L6-Size & 0.2158 & 0.6428 \\
Side-x-Sex & 0.0064 & 0.9361 \\
Side-x-L6-Size-Sex & 0.0019 & 0.9654 \\
\hline
\end{tabular}

*df for each test is 1 and 203.

Means, Sexes Pooled

\begin{tabular}{|c|c|c|}
\hline L_R2-1 lapping & 0.47 & \\
\hline L_L1-2 lapping & 0.46 & \\
\hline \multicolumn{3}{|c|}{ Means, by Sex } \\
\hline & L_R2-1 lapping & L_L1-2 lapping \\
\hline Boy & 0.430 .45 & \\
\hline Girl & 0.520 .48 & \\
\hline
\end{tabular}


Table A-401. ANCOVA results testing for associations between mesiodistal width of the mandibular first molar and total displacements.

\begin{tabular}{lcccc}
\hline Term & Estimate & SE & t-Test & P-Value \\
\hline Intercept & -3.0271 & 4.7823 & -0.63 & 0.5275 \\
L6 & 0.6424 & 0.4320 & 1.49 & 0.1386 \\
Sex & -2.2902 & 4.7823 & -0.48 & 0.6325 \\
Interaction & 0.1643 & 0.4320 & 0.38 & 0.7041 \\
\hline
\end{tabular}

Table A-402. ANCOVA results testing for associations between mesiodistal width of the mandibular first molar and total rotations.

\begin{tabular}{lcccc}
\hline Term & Estimate & SE & t-Test & P-Value \\
\hline Intercept & 0.1957 & 3.5321 & 0.06 & 0.9559 \\
L6 & 0.3690 & 0.3191 & 1.16 & 0.2488 \\
Sex & 0.9048 & 3.5321 & 0.26 & 0.7981 \\
Interaction & -0.1074 & 0.3191 & -0.34 & 0.7367 \\
\hline
\end{tabular}

Table A-403. ANCOVA results testing for associations between mesiodistal width of the mandibular first molar and maxillary R1-L1 overlap.

\begin{tabular}{lcccc}
\hline Term & Estimate & $\mathrm{SE}$ & $\mathrm{t}$-Test & P-Value \\
\hline Intercept & -0.2435 & 0.6569 & -0.37 & 0.7113 \\
L6 & 0.0448 & 0.0593 & 0.75 & 0.4513 \\
Sex & 0.6380 & 0.6569 & 0.97 & 0.3325 \\
Interaction & -0.0591 & 0.0593 & -1.00 & 0.3203 \\
\hline
\end{tabular}


Table A-404. ANCOVA results testing for associations between mesiodistal width of the mandibular first molar and mandibular R1-L1 overlap.

\begin{tabular}{lcccc}
\hline Term & Estimate & $\mathrm{SE}$ & $\mathrm{t}$-Test & P-Value \\
\hline Intercept & 0.1128 & 0.6799 & 0.17 & 0.8684 \\
L6 & 0.0241 & 0.0614 & 0.39 & 0.6952 \\
Sex & -0.1491 & 0.6799 & -0.22 & 0.8266 \\
Interaction & 0.0224 & 0.0614 & 0.37 & 0.7153 \\
\hline
\end{tabular}

Table A-405. ANCOVA results testing for associations between mesiodistal width of the mandibular first molar and total overlapping.

\begin{tabular}{lcccc}
\hline Term & Estimate & SE & t-Test & P-Value \\
\hline Intercept & -3.3446 & 4.3844 & -0.76 & 0.4464 \\
L6 & 0.6970 & 0.3961 & 1.76 & 0.0799 \\
Sex & 0.0521 & 4.3844 & 0.01 & 0.9905 \\
Interaction & -0.0483 & 0.3961 & -0.12 & 0.9032 \\
\hline
\end{tabular}

Table A-406. ANCOVA results testing for associations between mesiodistal width of the mandibular first molar and total displacements, rotations, and overlapping.

\begin{tabular}{lrrrc}
\hline Term & Estimate & \multicolumn{1}{c}{$\mathrm{SE}$} & $\mathrm{t}$-Test & P-Value \\
\hline Intercept & -6.1761 & 10.8922 & -0.57 & 0.5713 \\
L6 & 1.7084 & 0.9839 & 1.74 & 0.0840 \\
Sex & -1.3333 & 10.8922 & -0.12 & 0.9027 \\
Interaction & 0.0086 & 0.9839 & 0.01 & 0.9930 \\
\hline
\end{tabular}


Table A-407. ANCOVA results testing for associations between mesiodistal width of the mandibular first molar and crowding.

\begin{tabular}{lcccc}
\hline Term & Estimate & SE & t-Test & P-Value \\
\hline Intercept & -0.4386 & 1.0285 & -0.43 & 0.6702 \\
L6 & 0.1665 & 0.0929 & 1.79 & 0.0746 \\
Sex & 1.3492 & 1.0285 & 1.31 & 0.1910 \\
Interaction & -0.1336 & 0.0929 & -1.44 & 0.1521 \\
\hline
\end{tabular}

Table A-408. ANCOVA results testing for associations between mesiodistal width of the mandibular first molar and spacing.

\begin{tabular}{lcccc}
\hline Term & Estimate & SE & t-Test & P-Value \\
\hline Intercept & 2.6117 & 0.7679 & 3.40 & 0.0008 \\
L6 & -0.2130 & 0.0694 & -3.07 & 0.0024 \\
Sex & 0.1594 & 0.7679 & 0.21 & 0.8358 \\
Interaction & -0.0080 & 0.0694 & -0.12 & 0.9079 \\
\hline
\end{tabular}

Table A-409. ANCOVA results testing for associations between mesiodistal width of the mandibular first molar and diastema.

\begin{tabular}{lcccc}
\hline Term & Estimate & $\mathrm{SE}$ & $\mathrm{t}$-Test & P-Value \\
\hline Intercept & 1.6356 & 0.7950 & 2.06 & 0.0409 \\
L6 & -0.1279 & 0.0718 & -1.78 & 0.0764 \\
Sex & -0.6380 & 0.7950 & -0.80 & 0.4231 \\
Interaction & 0.0618 & 0.0718 & 0.86 & 0.3907 \\
\hline
\end{tabular}


Table A-410. ANCOVA results testing for associations between mesiodistal width of the mandibular first molar and maxillary incisor irregularity.

\begin{tabular}{lcccc}
\hline Term & Estimate & $\mathrm{SE}$ & $\mathrm{t}$-Test & P-Value \\
\hline Intercept & -1.2999 & 1.8905 & -0.69 & 0.4925 \\
L6 & 0.2476 & 0.1708 & 1.45 & 0.1487 \\
Sex & 0.2311 & 1.8905 & 0.12 & 0.9028 \\
Interaction & -0.0361 & 0.1708 & -0.21 & 0.8330 \\
\hline
\end{tabular}

Table A-411. ANCOVA results testing for associations between mesiodistal width of the mandibular first molar and mandibular incisor irregularity.

\begin{tabular}{lcccc}
\hline Term & Estimate & SE & t-Test & P-Value \\
\hline Intercept & -2.2898 & 1.7445 & -1.31 & 0.1908 \\
L6 & 0.3090 & 0.1576 & 1.96 & 0.0513 \\
Sex & -0.1449 & 1.7445 & -0.08 & 0.9339 \\
Interaction & 0.0044 & 0.1576 & 0.03 & 0.9777 \\
\hline
\end{tabular}

Table A-412. ANCOVA results testing for associations between mesiodistal width of the mandibular first molar and maxillary overjet.

\begin{tabular}{lrrrc}
\hline Term & Estimate & SE & t-Test & P-Value \\
\hline Intercept & 2.3768 & 3.1656 & 0.75 & 0.4536 \\
L6 & 0.1106 & 0.2860 & 0.39 & 0.6994 \\
Sex & 0.1339 & 3.1656 & 0.04 & 0.9663 \\
Interaction & -0.0195 & 0.2860 & -0.07 & 0.9456 \\
\hline
\end{tabular}


Table A-413. ANCOVA results testing for associations between mesiodistal width of the mandibular first molar and mandibular overjet.

\begin{tabular}{lcccc}
\hline Term & Estimate & SE & t-Test & P-Value \\
\hline Intercept & -0.3857 & 0.3790 & -1.02 & 0.3100 \\
L6 & 0.0391 & 0.0342 & 1.14 & 0.2549 \\
Sex & 0.3857 & 0.3790 & 1.02 & 0.3100 \\
Interaction & -0.0391 & 0.0342 & -1.14 & 0.2549 \\
\hline
\end{tabular}

Table A-414. ANCOVA results testing for associations between mesiodistal width of the mandibular first molar and openbite.

\begin{tabular}{lrcrc}
\hline Term & Estimate & SE & t-Test & P-Value \\
\hline Intercept & 0.0866 & 0.6397 & 0.14 & 0.8925 \\
L6 & -0.0002 & 0.0578 & 0.00 & 0.9967 \\
Sex & 0.8892 & 0.6397 & 1.39 & 0.1660 \\
Interaction & -0.0794 & 0.0578 & -1.37 & 0.1707 \\
\hline
\end{tabular}

Table A-415. ANCOVA results testing for associations between mesiodistal width of the mandibular first molar and anteroposterior relationship.

\begin{tabular}{lrcrc}
\hline Term & Estimate & $\mathrm{SE}$ & $\mathrm{t}$-Test & P-Value \\
\hline Intercept & 1.1299 & 0.9843 & 1.15 & 0.2524 \\
L6 & -0.0336 & 0.0889 & -0.38 & 0.7056 \\
Sex & 0.7299 & 0.9843 & 0.74 & 0.4592 \\
Interaction & -0.0621 & 0.0889 & -0.70 & 0.4857 \\
\hline
\end{tabular}


Table A-416. ANCOVA results testing for associations between mesiodistal width of the mandibular first molar and DAI score.

\begin{tabular}{lcccc}
\hline Term & Estimate & $\mathrm{SE}$ & $\mathrm{t}$-Test & P-Value \\
\hline Intercept & 23.7271 & 9.0746 & 2.61 & 0.0096 \\
L6 & 0.3072 & 0.8197 & 0.37 & 0.7082 \\
Sex & 6.8454 & 9.0746 & 0.75 & 0.4515 \\
Interaction & -0.6515 & 0.8197 & -0.79 & 0.4277 \\
\hline
\end{tabular}


Table A-417. ANCOVA results testing for associations between mesiodistal width of the mandibular second molar and U3 displacements.

\begin{tabular}{lcc}
\hline Source & F-Ratio* & P-Value \\
\hline \multicolumn{2}{c}{ Among Subjects } \\
L7-Size & 0.8974 & 0.3446 \\
Sex & 0.0901 & 0.7643 \\
L7-Size-x-Sex & 0.0597 & 0.8072 \\
& \multicolumn{2}{c}{ Within Subjects } \\
Side & 0.0611 & 0.8051 \\
Side-x-L7-Size & 0.0397 & 0.8423 \\
Side-x-Sex & 1.3997 & 0.2382 \\
Side-x-L7-Size-x-Sex & 1.4812 & 0.2250 \\
\hline
\end{tabular}

* $\mathrm{df}$ for each test is 1 and 203.

Means, Sexes Pooled

$\begin{array}{lrr}\text { U_R3 dis } & 0.41 \\ \text { U_L3 dis } & 0.38 & \\ & \\ \text { Means, } & \text { by Sex } \\ & \text { U_R3 dis } & \text { U_L3 dis } \\ \text { Boy } & 0.360 .34 & \\ \text { Girl } & 0.470 .40 & \end{array}$


Table A-418. ANCOVA results testing for associations between mesiodistal width of the mandibular second molar and $\mathrm{U} 2$ displacements.

\begin{tabular}{lcc}
\hline Source & F-Ratio* & P-Value \\
\hline \multicolumn{2}{c}{ Among Subjects } \\
L7-Size & 0.1543 & 0.6948 \\
Sex & 1.5815 & 0.2100 \\
L7-Size-x-Sex & 1.9089 & 0.1686 \\
& \multicolumn{2}{c}{ Within Subjects } \\
Side & 0.8133 & 0.3682 \\
Side-x-L7-Size & 0.7307 & 0.3936 \\
Side-x-Sex & 0.4582 & 0.4992 \\
Side-x-L7-Size-x-Sex & 0.4775 & 0.4904 \\
\hline
\end{tabular}

*df for each test is 1 and 203.

Means, Sexes Pooled

\begin{tabular}{lrr} 
U_R2 dis & 0.37 \\
U_L2 dis & 0.41 & \\
& Means, by Sex & \\
\multicolumn{4}{c}{ U_R2 dis } & U_L2 dis \\
Boy & 0.300 .35 & \\
Girl & 0.460 .49 &
\end{tabular}


Table A-419. ANCOVA results testing for associations between mesiodistal width of the mandibular second molar and U1 displacements.

\begin{tabular}{lcc}
\hline Source & F-Ratio* & P-Value \\
\hline \multicolumn{2}{c}{ Among Subjects } \\
L7-Size & 0.1425 & 0.7062 \\
Sex & 0.3069 & 0.5802 \\
L7-Size-x-Sex & 0.3021 & 0.5832 \\
& \multicolumn{2}{c}{ Within Subjects } \\
Side & 1.1331 & 0.2884 \\
Side-x-L7-Size & 1.1981 & 0.2750 \\
Side-x-Sex & 0.3022 & 0.5831 \\
Side-x-L7-Size-x-Sex & 0.3739 & 0.5415 \\
\hline
\end{tabular}

*df for each test is 1 and 203.

Means, Sexes Pooled

U_R1 dis $\quad 0.34$

U_L1 dis $\quad 0.36$

Means, by Sex

U_R1 dis U_L1 dis

$\begin{array}{ll}\text { Boy } & 0.370 .35 \\ \text { Girl } & 0.320 .38\end{array}$ 
Table A-420. ANCOVA results testing for associations between mesiodistal width of the mandibular second molar and L3 displacements.

\begin{tabular}{llc}
\hline Source & F-Ratio* & P-Value \\
\hline \multicolumn{2}{c}{ Among Subjects } \\
L7-Size & 0.0013 & 0.9712 \\
Sex & 0.0173 & 0.8955 \\
L7-Size-x-Sex & 0.0024 & 0.9610 \\
& \multicolumn{2}{c}{ Within Subjects } \\
Side & 0.0703 & 0.7911 \\
Side-x-L7-Size & 0.0641 & 0.8004 \\
Side-x-Sex & 0.5976 & 0.4404 \\
Side-x-L7-Size-x-Sex & 0.7409 & 0.3904 \\
\hline
\end{tabular}

*df for each test is 1 and 203.

Means, Sexes Pooled

\begin{tabular}{lll} 
L_R3 dis & 0.23 & \\
L_L3 dis & 0.23 & \\
& Means, by Sex \\
\multicolumn{4}{c}{ L_R3 dis } & L_L3 dis \\
Boy & 0.160 .21 & \\
Girl & 0.300 .24 &
\end{tabular}


Table A-421. ANCOVA results testing for associations between mesiodistal width of the mandibular second molar and L2 displacements.

\begin{tabular}{lcc}
\hline Source & F-Ratio* & P-Value \\
\hline \multicolumn{2}{c}{ Among Subjects } \\
L7-Size & 5.3667 & 0.0215 \\
Sex & 0.0393 & 0.8430 \\
L7-Size-x-Sex & 0.0150 & 0.9025 \\
& \multicolumn{2}{c}{ Within Subjects } \\
Side & 0.1519 & 0.6971 \\
Side-x-L7-Size & 0.1870 & 0.6659 \\
Side-x-Sex & 2.0358 & 0.1552 \\
Side-x-L7-Size-x-Sex & 1.9414 & 0.1650 \\
\hline
\end{tabular}

* $\mathrm{df}$ for each test is 1 and 203.

Means, Sexes Pooled

\begin{tabular}{lrr} 
L_R2 dis & 0.51 & \\
L_L2 dis & 0.47 & \\
& Means, by Sex \\
\multicolumn{4}{c}{ L_R2 dis } & L_L2 dis \\
Boy & 0.440 .44 & \\
Girl & 0.570 .52 &
\end{tabular}


Table A-422. ANCOVA results testing for associations between mesiodistal width of the mandibular second molar and L1 displacements.

\begin{tabular}{llc}
\hline Source & F-Ratio* & P-Value \\
\hline \multicolumn{2}{c}{ Among Subjects } \\
L7-Size & 4.4361 & 0.0364 \\
Sex & 0.5327 & 0.4663 \\
L7-Size-x-Sex & 0.5653 & 0.4530 \\
& \multicolumn{2}{c}{ Within Subjects } \\
Side & 1.0540 & 0.3058 \\
Side-x-L7-Size & 1.2618 & 0.2626 \\
Side-x-Sex & 1.5165 & 0.2196 \\
Side-x-L7-Size-x-Sex & 1.4245 & 0.2341 \\
\hline
\end{tabular}

*df for each test is 1 and 203.

Means, Sexes Pooled

\begin{tabular}{lll} 
L_R1 dis & 0.22 & \\
L_L1 dis & 0.17 & \\
& Means, by Sex \\
\multicolumn{4}{c}{ L_R1 dis } & L_L1 dis \\
Boy & 0.240 .16 & \\
Girl & 0.200 .17 &
\end{tabular}


Table A-423. ANCOVA results testing for associations between mesiodistal width of the mandibular second molar and U3 rotations.

\begin{tabular}{llc}
\hline Source & F-Ratio* & P-Value \\
\hline & \multicolumn{2}{c}{ Among Subjects } \\
L7-Size & 0.1016 & 0.7502 \\
Sex & 0.0579 & 0.8100 \\
L7-Size-x-Sex & 0.0132 & 0.9087 \\
& \multicolumn{2}{c}{ Within Subjects } \\
Side & 1.2823 & 0.2588 \\
Side-x-L7-Size & 1.2880 & 0.2577 \\
Side-x-Sex & 0.4526 & 0.5019 \\
Side-x-L7-Size-x-Sex & 0.3913 & 0.5323 \\
\hline
\end{tabular}

* $\mathrm{df}$ for each test is 1 and 203.

Means, Sexes Pooled

$\begin{array}{lll}\text { U_R3 rot } & 0.22 & \\ \text { U_L3 rot } & 0.22 & \\ & \text { Means, by Sex } \\ & \text { U_R3 rot } & \text { U_L3 rot } \\ \text { Boy } & 0.180 .15 & \\ \text { Girl } & 0.260 .29 & \end{array}$


Table A-424. ANCOVA results testing for associations between mesiodistal width of the mandibular second molar and $\mathrm{U} 2$ rotations.

\begin{tabular}{lcc}
\hline Source & F-Ratio* & P-Value \\
\hline \multicolumn{2}{c}{ Among Subjects } \\
L7-Size & 0.9184 & 0.3390 \\
Sex & 0.8889 & 0.3469 \\
L7-Size-x-Sex & 0.9297 & 0.3361 \\
& \multicolumn{2}{c}{ Within Subjects } \\
Side & 0.0062 & 0.9373 \\
Side-x-L7-Size & 0.0199 & 0.8879 \\
Side-x-Sex & 5.3052 & 0.0223 \\
Side-x-L7-Size-x-Sex & 5.3273 & 0.0220 \\
\hline
\end{tabular}

* $\mathrm{df}$ for each test is 1 and 203.

Means, Sexes Pooled

$\begin{array}{lrr}\text { U_R2 rot } & 0.39 & \\ \text { U_L2 rot } & 0.42 & \\ & \text { Means, by Sex } \\ & \text { U_R2 rot } & \text { U_L2 rot } \\ \text { Boy } & 0.380 .42 & \\ \text { Girl } & 0.400 .45 & \end{array}$


Table A-425. ANCOVA results testing for associations between mesiodistal width of the mandibular second molar and U1 rotations.

\begin{tabular}{lcc}
\hline Source & F-Ratio* & P-Value \\
\hline \multicolumn{2}{c}{ Among Subjects } \\
L7-Size & 0.0663 & 0.7971 \\
Sex & 0.4933 & 0.4833 \\
L7-Size-x-Sex & 0.4993 & 0.4806 \\
& \multicolumn{2}{c}{ Within Subjects } \\
Side & 0.5919 & 0.4426 \\
Side-x-L7-Size & 0.5686 & 0.4517 \\
Side-x-Sex & 0.0293 & 0.8643 \\
Side-x-L7-Size-x-Sex & 0.0261 & 0.8717 \\
\hline
\end{tabular}

* $\mathrm{df}$ for each test is 1 and 203.

Means, Sexes Pooled

$\begin{array}{lll}\text { U_R1 rot } & 0.47 & \\ \text { U_L1 rot } & 0.49 & \\ & \text { Means, by Sex } & \\ & \text { U_R1 rot } & \text { U_L1 rot } \\ \text { Boy } & 0.480 .49 & \\ \text { Girl } & 0.480 .50 & \end{array}$


Table A-426. ANCOVA results testing for associations between mesiodistal width of the mandibular second molar and L3 rotations.

\begin{tabular}{lcc}
\hline Source & F-Ratio* & P-Value \\
\hline & \multicolumn{2}{c}{ Among Subjects } \\
L7-Size & 1.4928 & 0.2232 \\
Sex & 0.0926 & 0.7612 \\
L7-Size-x-Sex & 0.1624 & 0.6874 \\
& \multicolumn{2}{c}{ Within Subjects } \\
Side & 0.8713 & 0.3517 \\
Side-x-L7-Size & 0.9871 & 0.3216 \\
Side-x-Sex & 0.7661 & 0.3825 \\
Side-x-L7-Size-x-Sex & 0.9479 & 0.3314 \\
\hline
\end{tabular}

*df for each test is 1 and 203.

Means, Sexes Pooled

\begin{tabular}{lll} 
L_R3 rot & 0.39 & \\
L_L3 rot & 0.35 & \\
& Means, by Sex \\
\multicolumn{4}{c}{ L_R3 rot } & L_L3 rot \\
Boy & 0.300 .33 & \\
Girl & 0.490 .38 &
\end{tabular}


Table A-427. ANCOVA results testing for associations between mesiodistal width of the mandibular second molar and L2 rotations.

\begin{tabular}{lcc}
\hline Source & F-Ratio* & P-Value \\
\hline \multicolumn{3}{c}{ Among Subjects } \\
L7-Size & 0.9037 & 0.3429 \\
Sex & 0.2211 & 0.6387 \\
L7-Size-x-Sex & 0.2657 & 0.6068 \\
& \multicolumn{2}{c}{ Within Subjects } \\
Side & 0.8431 & 0.3596 \\
Side-x-L7-Size & 1.1171 & 0.2918 \\
Side-x-Sex & 1.8538 & 0.1749 \\
Side-x-L7-Size-x-Sex & 1.7008 & 0.1937 \\
\hline
\end{tabular}

* $\mathrm{df}$ for each test is 1 and 203.

Means, Sexes Pooled

\begin{tabular}{lll} 
L_R2 rot & 0.35 & \\
L_L2 rot & 0.23 & \\
& Means, by Sex & \\
\multicolumn{4}{c}{ L_R2 rot } & L_L2 rot \\
Boy & 0.310 .24 & \\
Girl & 0.400 .24 &
\end{tabular}


Table A-428. ANCOVA results testing for associations between mesiodistal width of the mandibular second molar and L1 rotations.

\begin{tabular}{lcc}
\hline Source & F-Ratio* & \multicolumn{2}{c}{ P-Value } \\
\hline \multicolumn{2}{c}{ Among Subjects } \\
L7-Size & 0.0323 & 0.8575 \\
Sex & 5.6281 & 0.0186 \\
L7-Size-x-Sex & 6.1911 & 0.0136 \\
& \multicolumn{2}{c}{ Within Subjects } \\
Side & 3.5347 & 0.0615 \\
Side-x-L7-Size & 3.7259 & 0.0550 \\
Side-x-Sex & 0.0713 & 0.7898 \\
Side-x-L7-Size-x-Sex & 0.0808 & 0.7765 \\
\hline
\end{tabular}

* $\mathrm{df}$ for each test is 1 and 203.

Means, Sexes Pooled

\begin{tabular}{lll} 
L_R1 rot & 0.38 & \\
L_L1 rot & 0.34 & \\
& Means, by Sex & \\
\multicolumn{4}{c}{ L_R1 rot } & L_L1 rot \\
Boy & 0.420 .37 & \\
Girl & 0.300 .28 &
\end{tabular}


Table A-429. ANCOVA results testing for associations between mesiodistal width of the mandibular second molar and U2-U3 overlap.

\begin{tabular}{lcc}
\hline Source & F-Ratio* & P-Value \\
\hline \multicolumn{2}{c}{ Among Subjects } \\
L7-Size & 0.6373 & 0.4256 \\
Sex & 0.9993 & 0.3187 \\
L7-Size-x-Sex & 1.2629 & 0.2624 \\
& \multicolumn{2}{c}{ Within Subjects } \\
Side & 0.4556 & 0.5004 \\
Side-x-L7-Size & 0.4986 & 0.4809 \\
Side-x-Sex & 1.2394 & 0.2669 \\
Side-x-L7-Size-x-Sex & 1.3490 & 0.2468 \\
\hline
\end{tabular}

* $\mathrm{df}$ for each test is 1 and 203.

Means, Sexes Pooled

$\begin{array}{ll}\text { U_R3-2 lapping } & 0.38 \\ \text { U_L2-3 lapping } & 0.37 \\ \text { Means, by Sex } & \end{array}$

Boy

U_R3-2 lapping U_L2-3 lapping

Girl

0.300 .31

0.500 .43 
Table A-430. ANCOVA results testing for associations between mesiodistal width of the mandibular second molar and U1-U2 overlap.

\begin{tabular}{lcc}
\hline Source & F-Ratio & P-Value \\
\hline \multicolumn{2}{c}{ Among Subjects } \\
L7-Size & 0.1531 & 0.6960 \\
Sex & 0.0009 & 0.9761 \\
L7-Size-x-Sex & 0.0361 & 0.8494 \\
& \multicolumn{2}{c}{ Within Subjects } \\
Side & 1.0244 & 0.3127 \\
Side-x-L7-Size & 0.9750 & 0.3246 \\
Side-x-Sex & 4.3634 & 0.0380 \\
Side-x-L7-Size-x-Sex & 4.3241 & 0.0388 \\
\hline
\end{tabular}

*df for each test is 1 and 203.

Means, Sexes Pooled

$\begin{array}{ll}\text { U_R2-1 lapping } & 0.35 \\ \text { U_L1-2 lapping } & 0.31 \\ \text { Means, by Sex } & \end{array}$

Boy

U_R2-1 lapping U_L1-2 lapping

Girl

0.250 .24

0.440 .41 
Table A-431. ANCOVA results testing for associations between mesiodistal width of the mandibular second molar and L2-L3 overlap.

\begin{tabular}{lcc}
\hline Source & F-Ratio* & P-Value \\
\hline \multicolumn{2}{c}{ Among Subjects } \\
L7-Size & 5.5270 & 0.0197 \\
Sex & 0.1410 & 0.7077 \\
L7-Size-x-Sex & 0.0932 & 0.7605 \\
& \multicolumn{2}{c}{ Within Subjects } \\
Side & 6.4824 & 0.0116 \\
Side-x-L7-Size & 6.9739 & 0.0089 \\
Side-x-Sex & 1.3869 & 0.2403 \\
Side-x-L7-Size-x-Sex & 1.4487 & 0.2301 \\
\hline
\end{tabular}

* df for each test is 1 and 203.

Means, Sexes Pooled

\begin{tabular}{|c|c|c|}
\hline L_R3-2 lapping & 0.71 & \\
\hline L_L2-3 lapping & 0.65 & \\
\hline \multicolumn{3}{|c|}{ Means, by Sex } \\
\hline & L_R3-2 lapping & L_L2-3 lapping \\
\hline Boy & 0.650 .60 & \\
\hline Girl & 0.780 .69 & \\
\hline
\end{tabular}


Table A-432. ANCOVA results testing for associations between mesiodistal width of the mandibular second molar and L1-L2 overlap.

\begin{tabular}{lcc}
\hline Source & F-Ratio* & P-Value \\
\hline \multicolumn{2}{c}{ Among Subjects } \\
L7-Size & 1.7323 & 0.1896 \\
Sex & 0.1915 & 0.6622 \\
L7-Size-x-Sex & 0.1536 & 0.6955 \\
& \multicolumn{2}{c}{ Within Subjects } \\
Side & 0.0906 & 0.7637 \\
Side-x-L7-Size & 0.1068 & 0.7441 \\
Side-x-Sex & 0.9245 & 0.3374 \\
Side-x-L7-Size-x-Sex & 1.0354 & 0.3101 \\
\hline
\end{tabular}

* df for each test is 1 and 203.

Means, Sexes Pooled

\begin{tabular}{|c|c|c|}
\hline L_R2-1 lapping & 0.47 & \\
\hline L_L1-2 lapping & 0.46 & \\
\hline \multicolumn{3}{|c|}{ Means, by Sex } \\
\hline & L_R2-1 lapping & L_L1-2 lapping \\
\hline Boy & 0.430 .46 & \\
\hline Girl & 0.520 .46 & \\
\hline
\end{tabular}


Table A-433. ANCOVA results testing for associations between mesiodistal width of the mandibular second molar and total displacements.

\begin{tabular}{lcccc}
\hline Term & Estimate & SE & t-Test & P-Value \\
\hline Intercept & 0.8261 & 4.0872 & 0.20 & 0.8400 \\
L7-Size & 0.3214 & 0.3988 & 0.81 & 0.4212 \\
Sex & 0.8419 & 4.0872 & 0.21 & 0.8370 \\
Interaction & -0.1214 & 0.3988 & -0.30 & 0.7611 \\
\hline
\end{tabular}

Table A-434. ANCOVA results testing for associations between mesiodistal width of the mandibular second molar and total rotations.

\begin{tabular}{lrcrc}
\hline Term & Estimate & SE & t-Test & P-Value \\
\hline Intercept & 6.1932 & 3.0133 & 2.06 & 0.0411 \\
L7-Size & -0.1882 & 0.2940 & -0.64 & 0.5229 \\
Sex & 0.5258 & 3.0133 & 0.17 & 0.8617 \\
Interaction & -0.0709 & 0.2940 & -0.24 & 0.8096 \\
\hline
\end{tabular}

Table A-435. ANCOVA results testing for associations between mesiodistal width of the mandibular second molar and maxillary R1-L1 overlap.

\begin{tabular}{lcccc}
\hline Term & Estimate & $\mathrm{SE}$ & $\mathrm{t}$-Test & P-Value \\
\hline Intercept & -0.4326 & 0.5572 & -0.78 & 0.4385 \\
L7-Size & 0.0666 & 0.0544 & 1.23 & 0.2220 \\
Sex & 0.6971 & 0.5572 & 1.25 & 0.2124 \\
Interaction & -0.0696 & 0.0544 & -1.28 & 0.2020 \\
\hline
\end{tabular}


Table A-436. ANCOVA results testing for associations between mesiodistal width of the mandibular second molar and mandibular R1-L1 overlap.

\begin{tabular}{lcccc}
\hline Term & Estimate & SE & t-Test & P-Value \\
\hline Intercept & 0.1005 & 0.5785 & 0.17 & 0.8622 \\
L7-Size & 0.0274 & 0.0565 & 0.48 & 0.6285 \\
Sex & -0.1149 & 0.5785 & -0.20 & 0.8427 \\
Interaction & 0.0210 & 0.0565 & 0.37 & 0.7104 \\
\hline
\end{tabular}

Table A-437. ANCOVA results testing for associations between mesiodistal width of the mandibular second molar and total overlapping.

\begin{tabular}{lcccc}
\hline Term & Estimate & SE & t-Test & P-Value \\
\hline Intercept & -0.0628 & 3.7468 & -0.02 & 0.9866 \\
L7-Size & 0.4329 & 0.3656 & 1.18 & 0.2378 \\
Sex & 0.9251 & 3.7468 & 0.25 & 0.8052 \\
Interaction & -0.1306 & 0.3656 & -0.36 & 0.7213 \\
\hline
\end{tabular}

Table A-438. ANCOVA results testing for associations between mesiodistal width of the mandibular second molar and total displacements, rotations, and overlapping.

\begin{tabular}{lcccc}
\hline Term & Estimate & SE & t-Test & P-Value \\
\hline Intercept & 6.9565 & 9.3287 & 0.75 & 0.4567 \\
L7-Size & 0.5662 & 0.9102 & 0.62 & 0.5347 \\
Sex & 2.2928 & 9.3287 & 0.25 & 0.8061 \\
Interaction & -0.3230 & 0.9102 & -0.35 & 0.7231 \\
\hline
\end{tabular}


Table A-439. ANCOVA results testing for associations between mesiodistal width of the mandibular second molar and crowding.

\begin{tabular}{lcccc}
\hline Term & Estimate & SE & t-Test & P-Value \\
\hline Intercept & -0.2738 & 0.8757 & -0.31 & 0.7549 \\
L7-Size & 0.1628 & 0.0854 & 1.90 & 0.0582 \\
Sex & 1.0578 & 0.8757 & 1.21 & 0.2285 \\
Interaction & -0.1148 & 0.0854 & -1.34 & 0.1806 \\
\hline
\end{tabular}

Table A-440. ANCOVA results testing for associations between mesiodistal width of the mandibular second molar and spacing.

\begin{tabular}{lcccc}
\hline Term & Estimate & SE & t-Test & P-Value \\
\hline Intercept & 2.0175 & 0.6571 & 3.07 & 0.0024 \\
L7-Size & -0.1724 & 0.0641 & -2.69 & 0.0078 \\
Sex & -0.1921 & 0.6571 & -0.29 & 0.7703 \\
Interaction & 0.0240 & 0.0641 & 0.37 & 0.7083 \\
\hline
\end{tabular}

Table A-441. ANCOVA results testing for associations between mesiodistal width of the mandibular second molar and diastema.

\begin{tabular}{lcccc}
\hline Term & Estimate & $\mathrm{SE}$ & $\mathrm{t}$-Test & P-Value \\
\hline Intercept & 1.7946 & 0.6738 & 2.66 & 0.0084 \\
L7-Size & -0.1525 & 0.0657 & -2.32 & 0.0214 \\
Sex & 0.2292 & 0.6738 & 0.34 & 0.7341 \\
Interaction & -0.0184 & 0.0657 & -0.28 & 0.7794 \\
\hline
\end{tabular}


Table A-442. ANCOVA results testing for associations between mesiodistal width of the mandibular second molar and maxillary incisor irregularity.

\begin{tabular}{lcccc}
\hline Term & Estimate & $\mathrm{SE}$ & $\mathrm{t}$-Test & P-Value \\
\hline Intercept & 1.6609 & 1.6151 & 1.03 & 0.3050 \\
L7-Size & -0.0211 & 0.1576 & -0.13 & 0.8936 \\
Sex & 1.0990 & 1.6151 & 0.68 & 0.4970 \\
Interaction & -0.1194 & 0.1576 & -0.76 & 0.4494 \\
\hline
\end{tabular}

Table A-443. ANCOVA results testing for associations between mesiodistal width of the mandibular second molar and mandibular incisor irregularity.

\begin{tabular}{lcccc}
\hline Term & Estimate & SE & t-Test & P-Value \\
\hline Intercept & -2.1873 & 1.4805 & -1.48 & 0.1411 \\
L7-Size & 0.3237 & 0.1445 & 2.24 & 0.0261 \\
Sex & -0.1374 & 1.4805 & -0.09 & 0.9262 \\
Interaction & 0.0056 & 0.1445 & 0.04 & 0.9691 \\
\hline
\end{tabular}

Table A-444. ANCOVA results testing for associations between mesiodistal width of the mandibular second molar and maxillary overjet.

\begin{tabular}{lrcrc}
\hline Term & Estimate & $\mathrm{SE}$ & $\mathrm{t}$-Test & P-Value \\
\hline Intercept & 6.3345 & 2.6685 & 2.37 & 0.0185 \\
L7-Size & -0.2627 & 0.2604 & -1.01 & 0.3142 \\
Sex & 4.4119 & 2.6685 & 1.65 & 0.0998 \\
Interaction & -0.4343 & 0.2604 & -1.67 & 0.0969 \\
\hline
\end{tabular}


Table A-445. ANCOVA results testing for associations between mesiodistal width of the mandibular second molar and mandibular overjet.

\begin{tabular}{lcccc}
\hline Term & Estimate & SE & t-Test & P-Value \\
\hline Intercept & -0.6007 & 0.3187 & -1.88 & 0.0609 \\
L7-Size & 0.0633 & 0.0311 & 2.03 & 0.0432 \\
Sex & 0.6007 & 0.3187 & 1.88 & 0.0609 \\
Interaction & -0.0633 & 0.0311 & -2.03 & 0.0432 \\
\hline
\end{tabular}

Table A-446. ANCOVA results testing for associations between mesiodistal width of the mandibular second molar and openbite.

\begin{tabular}{lrcrc}
\hline Term & Estimate & SE & t-Test & P-Value \\
\hline Intercept & 0.8423 & 0.5415 & 1.56 & 0.1214 \\
L7-Size & -0.0746 & 0.0528 & -1.41 & 0.1595 \\
Sex & 0.7451 & 0.5415 & 1.38 & 0.1704 \\
Interaction & -0.0709 & 0.0528 & -1.34 & 0.1810 \\
\hline
\end{tabular}

Table A-447. ANCOVA results testing for associations between mesiodistal width of the mandibular second molar and anteroposterior relationship.

\begin{tabular}{lcccc}
\hline Term & Estimate & $\mathrm{SE}$ & $\mathrm{t}$-Test & P-Value \\
\hline Intercept & 2.1991 & 0.8295 & 2.65 & 0.0087 \\
L7-Size & -0.1407 & 0.0809 & -1.74 & 0.0836 \\
Sex & 1.0161 & 0.8295 & 1.22 & 0.2220 \\
Interaction & -0.0941 & 0.0809 & -1.16 & 0.2466 \\
\hline
\end{tabular}


Table A-448. ANCOVA results testing for associations between mesiodistal width of the mandibular second molar and DAI score.

\begin{tabular}{lccrc}
\hline Term & Estimate & $\mathrm{SE}$ & $\mathrm{t}$-Test & P-Value \\
\hline Intercept & 38.9652 & 7.5756 & 5.14 & $<0.0001$ \\
L7-Size & -1.1481 & 0.7392 & -1.55 & 0.1219 \\
Sex & 17.8924 & 7.5756 & 2.36 & 0.0191 \\
Interaction & -1.7651 & 0.7392 & -2.39 & 0.0179 \\
\hline
\end{tabular}




\section{VITA}

John Robert Zang-Bodis was born in Bad Axe, Michigan on December 29, 1979. He grew up in Harbor Beach, Michigan with his parents and sister, and graduated from Harbor Beach Community High School in June of 1998. That September he entered The University of Michigan, and in 2001 was admitted to the University of Michigan, School of Dentistry. He received his Doctor of Dental Surgery degree in May of 2005. Upon graduation he entered a General Practice Residency at Loyola University Medical Center in Chicago, Illinois; and received a certificate of completion in June of 2006. In August of 2006 he began his orthodontic training at the University of Tennessee Health Science Center and is expected to receive a Master of Dental Science degree in May 2009. 